Al130? 198040

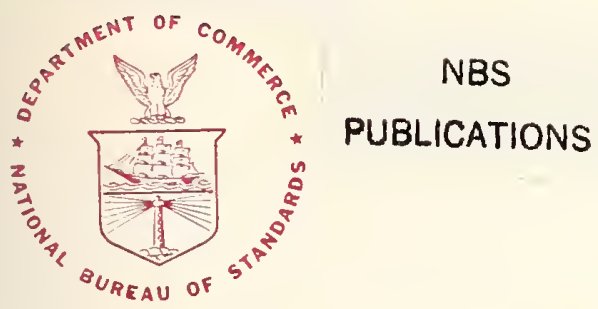

\title{
Measurement \\ of Electrical Quantities in Pulse Power Systems
}




\section{NATIONAL BUREAU OF STANDARDS}

The National Bureau of Standards' was established by an act of Congress on March 3, 1901. The Bureau's overall goal is to strengthen and advance the Nation's science and technology and facilitate their effective application for public benefit. To this end, the Bureau conducts research and provides: (1) a basis for the Nation's physical measurement system, (2) scientific and technological services for industry and government, (3) a technical basis for equity in trade, and (4) technical services to promote public safety. The Bureau's technical work is performed by the National Measurement Laboratory, the National Engineering Laboratory, and the Institute for Computer Sciences and Technology.

THE NATIONAL MEASUREMENT LABORATORY provides the national system of physical and chemical and materials measurement; coordinates the system with measurement systems of other nations and furnishes essential services leading to accurate and uniform physical and chemical measurement throughout the Nation's scientific community, industry, and commerce; conducts materials research leading to improved methods of measurement, standards, and data on the properties of materials needed by industry, commerce, educational institutions, and Government; provides advisory and research services to other Government agencies; develops, produces, and distributes Standard Reference Materials; and provides calibration services. The Laboratory consists of the following centers:

$$
\begin{aligned}
& \text { Absolute Physical Quantities }{ }^{2} \text { - Radiation Research - Chemical Physics - } \\
& \text { Analytical Chemistry - Materials Science }
\end{aligned}
$$

THE NATIONAL ENGINEERING LABORATORY provides technology and technical services to the public and private sectors to address national needs and to solve national problems; conducts research in engineering and applied science in support of these efforts; builds and maintains competence in the necessary disciplines required to carry out this research and technical service; develops engineering data and measurement capabilities; provides engineering measurement traceability services; develops test methods and proposes engineering standards and code changes; develops and proposes new engineering practices; and develops and improves mechanisms to transfer results of its research to the ultimate user. The Laboratory consists of the following centers:

Applied Mathematics - Electronics and Electrical Engineering ${ }^{2}$ - Manufacturing Engineering - Building Technology - Fire Research - Chemical Engineering ${ }^{2}$

THE INSTITUTE FOR COMPUTER SCIENCES AND TECHNOLOGY conducts research and provides scientific and technical services to aid Federal agencies in the selection, acquisition, application, and use of computer technology to improve effectiveness and economy in Government operations in accordance with Public Law 89-306 (40 U.S.C. 759), relevant Executive Orders, and other directives; carries out this mission by managing the Federal Information Processing Standards Program, developing Federal ADP standards guidelines, and managing Federal participation in ADP voluntary standardization activities; provides scientific and technological advisory services and assistance to Federal agencies; and provides the technical foundation for computer-related policies of the Federal Government. The Institute consists of the following centers:

Programming Science and Technology-Computer Systems Engineering.

'Headquarlers and Laboralories al Gaithersburg, MD, unless olherwise noted; mailing address Washington, DC 20234.

${ }^{2}$ Some divisions within the center are located at Boulder, CO 80303. 


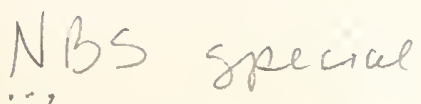

parcecation

\section{Measurement of Electrical Quantities in Pulse Power Systems}

Proceedings of the Workshop on Measurement of Electrical Quantities in Pulse Power Systems held at the National Bureau of Standards, Boulder, Colorado, March 2-4, 1981

R. H. McKnight and

R. E. Hebner, Jr., Editors

Center for Electronics and Electrical Engineering

National Engineering Laboratory

National Bureau of Standards

Washington, DC 20234

Sponsored by:

The Air Force Office of Scientific Research; the Defense Advanced Research Project Agency, Directed Energy Office; the Department of Energy, Office of Inertial Fusion; the Naval Surface Weapons Center; the U.S. Army, Harry Diamond Laboratories; and the National Bureau of Standards, Center for Electronics and Electrical Engineering.

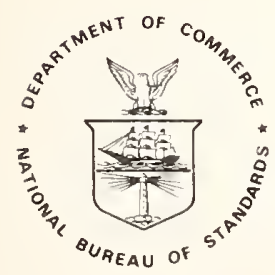

U.S. DEPARTMENT OF COMMERCE, Malcolm Baldrige, Secretary NATIONAL BUREAU OF STANDARDS, Ernest Ambler, Director 
Library of Congress Catalog Card Number: 82-600535

National Bureau of Standards Special Publication 628

Nat. Bur. Stand. (U.S.), Spec. Pubi. 628, 420 pages, (June 1982)

CODEN: XNBSAV

U.S. GOVERNMENT PRINTING OFFICE

WASHINGTON: 1982

For sale by the Superintendent of Documents, U.S. Government Printing Office, Washington, D.C. 20402 Price $\$ 9.50$

(Add 25 percent for other than U.S. mailing) 


\section{Foreword}

Pulse power systems find application in a wide range of industrial, educational, and government organizations. As these systems are extended to higher power levels and to more critical applications, there is an increasing requirement for accurate electrical measurements. These measurements are necessary in the design, the operation and the procurement testing of pulse power systems.

To bring together active researchers in the pulse power field, a workshop on Measurement of Electrical Quantities in Pulse Power Systems was organized and held at NBS-Boulder on March 2-4, 1981. These proceedings are a record of that workshop.

The purposes of the workshop were (1) to disseminate information on modern measurement techniques, (2) define the state-of-the-art of measurement of electrical quantities in pulse power systems, and (3) identify specific areas in which improvements in measurements are required. The attendees were representatives from industry, university, and government.

The workshop was sponsored by the Air Force Office of Scientific Research; the Defense Advanced Research Project Agency; the Department of Energy; the Naval Surface Weapons Center; Harry Diamond Laboratories; and the National Bureau of Standards, Center for Electronics and Electrical Engineering. Without this sponsorship, the workshop and publication of these proceedings would not have been possible. An acknowledgment must also go to the organizing committee whose efforts contributed significantly to the overall success of the workshop. 


\section{Preface}

The purpose of these proceedings is to provide a record of the workshop on Measurement of Electrical Quantities in Pulse Power Systems. Papers to be presented at the meeting were selected on the basis of a review by the organizing committee of contributed abstracts. Some of the papers presented at the workshop are included here by abstract only because the final manuscripts were not submitted by the authors.

The papers were organized and presented in four topical areas: (1) Voltage Measurements, (2) Power and Energy Measurements, (3) Current Measurements, and (4) Data Acquisition.

Open discussion periods were held at the end of each technical session. These discussions were recorded, transcribed, and returned to the discussers for editing. What appears in the proceedings are those edited comments. A number of figures and references have been added for clarity.

The papers have been reproduced as received from the author(s). During the discussions and technical presentations, trade names were used. In general, they were included to specify adequately the experimental procedures used. Such identification does not imply that the materials or equipment identified are necessarily the best available for the purpose.

Ronald H. Mcknight Robert E. Hebner, Jr. Editors 


\begin{abstract}
The Workshop on Measurement of Electrical Quantities in Pulse Power Systems addressed measurements in pulse systems having the characteristics of pulse duration less than a millisecond, system voltages greater than 10 kilovolts, and system currents greater than 10 ki Toamperes. The presented papers were divided into four categories: voltage measurements, current measurements, power and energy measurements, and data acquisition. Included are discussions of applications of conventional measurement techniques and state-of-the-art systems.
\end{abstract}

Key words: current measurements; electrical measurements; electromagnetic pulse; fusion; nuclear effects simulation; particle beam technology; pulse power; transients; voltage measurements. 
Organizing Committee

The following individuals were members of the organizing committee and acted as reviewers for abstracts submitted in response to the announcement of the workshop.

J.R. Bayless - Defense Advanced Research Projects Agency

M. Di Capua - Physics International

A. H. Guenther - Air Force Weapons Laboratory

R. L. Gullickson - Defense Nuclear Agency

D. G. Gritton - Lawrence Livermore Laboratory

R. E. Hebner, Jr. - National Bureau of Standards

M. Kristiansen - Texas Tech University

R. Lamb - Harry Diamond Laboratories

R. A. Lawton - National Bureau of Standards

R. H. McKnight - National Bureau of Standards

E. Nolting - Naval Surface Weapons Center

D. Pellinen - GCA Corporation

K. R. Prestwich - Sandia Laboratories

C. E. Rossi - Department of Energy

W. J. Sarjeant - LoS Alamos Scientific Laboratory

J. Shannon - Maxwell Laboratories

J. D. Shipman, Jr. - Sachs/Freeman Associates

\section{Special Acknowledgments}

Several individuals by virtue of their extra efforts contributed significantly to the success of the workshop. Ken Prestwich was instrumental in securing keynote speakers for the various technical sessions. Bob Lawton's assistance in coordinating arrangements for facilities at the NBS-Boulder complex was most helpful. The conference secretary, Sandra Kelley, provided invaluable support throughout the process of organizing, arranging, and completing the workshop. 


\section{Session A - Voltage Measurements}

Electro-optical Pulsed Voltage Measurements,

J. E. Thompson

High-Voltage Probe for Liquid Immersion,

N. W. Harris

Experimental Comparison of Step Response and Ramp Response Measurements in Free-Standing Dividers,

R. E. Hebner, Jr.

A Comparison of Three Different Designs of Resistor Divider, T. R. McComb, M.M.C. Collins, and W. J. Sarjeant

A 100-kV, 2-ns-Risetime, dc-Coupled Probe,

J. Power, W. Nunnally, and D. Young

Discussion 1

Calibration of Capacitive Voltage Probes in Water-Dielectric, High-Power Pulse Generators,

M. Wilkinson and E. Chu

Measurement of Transient Potentials in Coaxial Transmission Lines Using Coaxial Dividers,

N. Fujimoto, S. A. Boggs, and R. C. Madge

A Voltage Monitor for Magnetically-Insulated Transmission Lines,

R. W. Stinnett

Measurement of Magnetically Insulated Low Voltage Using a Thomson Parabola Charged Particle Analyzer,

T. D. Stanley and R. W. Stinnett

Discussion 2

$$
\text { Session B - Power and Energy Measurements }
$$

Ion Current and Voltage Determinations by Nuclear Techniques, F. C. Young

Electrical Measurement Techniques Used at the Casino Facility, E. Nolting, R. Martin, and M. Ruppalt 
Indirect Measurements by Computer Simulation,

R. A. Hill

133

Calibration of the Blackjack 5 Pulse Generator Output Power, R. Richardson, E. Chu, W. Clark, J. Shannon, and M. Wilkinson

Discussion 3

Session C - Current Measurements

Rogowski Coils, Fluxmeters, and Resistors for Pulsed Current Measurements,

M. S. Di Capua

Precise Measurement of Current in Pulsed Power Systems,

M. E. Wilmer and P. A. Pearson

194

Low-Inductance Shunts for Measuring Large Pulsed Currents, W. F. Praeg

Measuring Fast Pulse Currents Using Low Inductance Current Viewing Resistors and DI/DT Probes,

C. A. Muehlenweg and G. McDuff

Wide-Frequency Range Current Transformers and Application to Pulsed Power Systems,

J. M. Anderson

Discussion 4

Data Acquisition and Processing Techniques,

R. E. Kolibas, P. A. Corbiere, and J. J. Moriarty

A Technique for Measuring Beam Current Density in the Antares Electron Gun (abstract),

W. R. Scarlett

Thomson Spectrometer Measurement of Heavy Ion Beams Produced by a Pulse Powered Plasma Focus Device,

M. J. Rhee

Current Measuring Diagnostic Techniques for High DI/DT

Particle Beam Accelerators (abstract),

D. H. McDaniel

Proton Current Measurements Using the Prompt Gamma Ray Diagnostic Technique,

R. J. Leeper, E.J.T. Burns, D. L. Johnson, and W. M. McMurtry 
The Measurement of Pulsed 디ectric Currents by the

Faraday Effect,

J. Katzenstein, W. Caton, and G. M. Wilkinson

Cavity Current Monitors,

J. Shannon, E. Chu, R. Richardson, M. Wilkinson,

and C. Trivelpiece

Discussion 5

300

$$
\text { Session D - Data Acquisition }
$$

High Voltage Monitoring and Control Through Fiber Optics,

J. G. Stewart, Jr. and W. A. Petty

Remote Command Data Link Provides Enhanced Simulator

Performance in High EMP Environment,

S. Lyons

A Fiber Optic Monitoring System for Antares Pulse Power System (abstract),

M. E. Thuot and W. R. Scarlett

Discussion 6

Data Recording Techniques for the Sandia Particle Beam

Fusion Accelerator,

W. B. Boyer and E. L. Neau

An Evaluation of Digital Recording Equipment and Numerical Correction Techniques in Impulse Measurement, R. Malewski, T. R. McComb, and M.M.C. Collins

Analysis of a Power System Transient Recording Laboratory, R. E. Poliner, and T. J. Reed

Application Problems Using Instrumentation Amplifiers in the Pulse Power Environment,

E. E. Cunningham

Discussion 7

Digital Correction of Cable Attenuation Losses,

C. Trivelpiece, R. Richardson, J. Shannon, and J. B. Smith

Precision Picosecond-Microsecond Electromagnetic Waveform Measurements at NBS,

R. A. Lawton

Discussion 8 

Session A - Voltage Measurements

\title{
ELECTRO-OPTICAL PULSED VOLTAGE MEASUREMENTS
}

\author{
J. E. Thompson
}

College of Engineering

University of South Carolina

Columbia, SC 29208

Pulsed voltages are typically measured using resistive or capacitive voltage dividers. Problems associated with these types of measurements include the effects of stray capacitance, oscillations, noise pickup, and ground loops. Many of these problems can be reduced and measurement frequency response improved by using electro-optical measurement techniques. The most useful optical techniques for voltage measurement are the Kerr and Pockels effects. The relevant characteristics of these two effects are discussed together with optical techniques useful for utilizing the Kerr effect to measure pulsed voltages. Frequency response, accuracy and calibration of the measurement techniques are discussed in the context of high voltage measurements. Specific electro-optical measurement techniques and devices including cross polarizer analyzers, interferometric measurements, matched impedance Kerr cells, a sampling transient analyzer, and a traveling wave measurement technique are discussed.

Key Words: accuracy, calibration, electro-optical measurements, frequency response, interferometric measurements, Kerr effect, Pocke1s effect, polarization.

\section{Introduction}

Pulsed, high voltage measurements are typically made using resistive or capacitive voltage dividers. The frequency response, and hence the fastest measureable risetime, are limited by stray capacitance and inductance associated with the divider circuitry. Typical, minimum measureable risetime, obtainable with a carefully designed divider, is greater than 1 nanosecond. The signal fidelity and signal to noise ratio, obtainable with lumped parameter divider techniques, is also adversely effected by ground loops and electromagnetic interference.

Improved pulsed voltage measurements, with respect to time response, noise immunity, and sensitivity can be obtained using electro-optical measurement techniques. The general arrangement necessary for electro- 
optical measurements is shown in Figure 1 and consists of a polarized light source, an electro-optical device, a polarization analyzer, and a light detector.

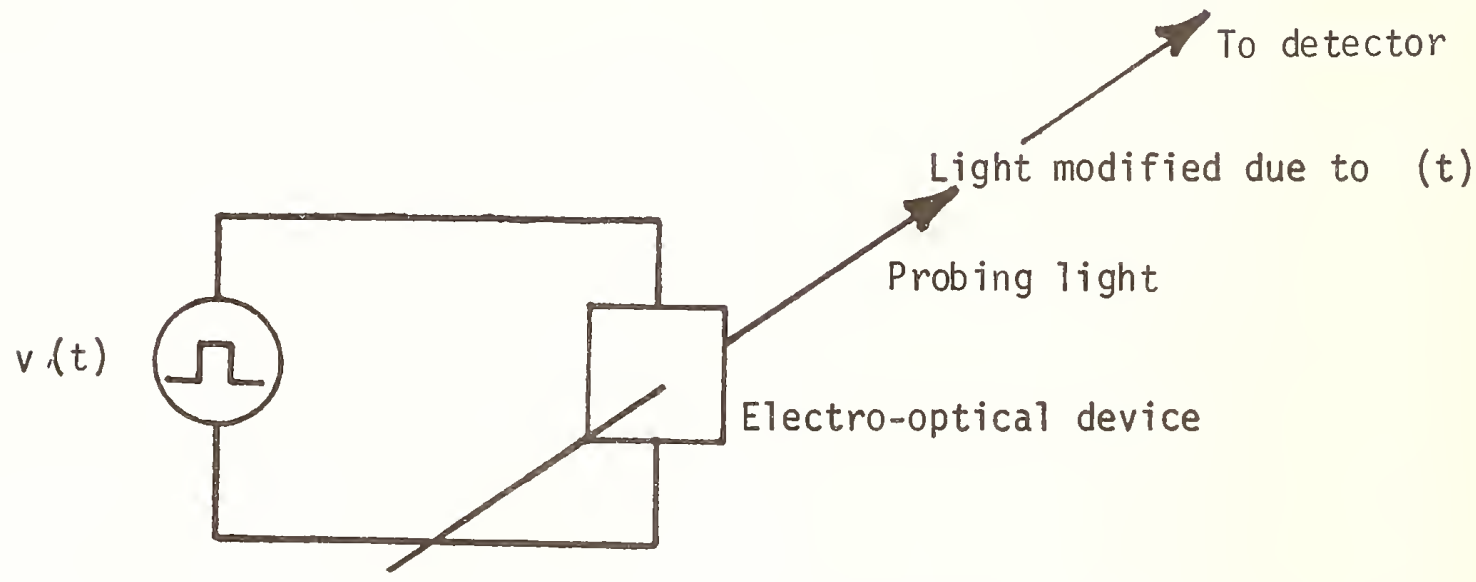

Figure 1. Electro-optical Voltage Measurement.

Voltage $v(t)$ to be measured, is applied across the electro-optical device. Polarized light is passed through the device while the voltage is applied. The light polarization state is modified, due to the relevant electro-optical effect (usually the Kerr or Pockels effect), in a manner dependent upon $v(t)$. The modified polarization state of the light is passed through an analyzer, which produces a relationship between polarization state and light intensity. The output light intensity, which is now related to $v(t)$, is detected and recorded.

The general electro-optical technique described produces a light intensity which is dependent upon $v(t)$. The measurement technique has several advantages compared to more conventional, resistive or capacitive divider techniques. The electro-optical measuring device, shown in Figure 1, is connected directly, in parallel, with $v(t)$. No divider circuitry is required. This reduces oscillations and risetime degradation associated with stray inductance and capacitance. The frequency response, for a carefully designed system, is limited by the capacitance of the electro-optical device and the characteristic relaxation time of the measurement medium used. Risetimes of less than one nanosecond have been directly observed using electro-optical techniques. System risetime responses as fast as tens of picoseconds have been projected as a realistic measurement capability.

The light source and detector are not physically connected to $v(t)$. This permits rather complete ground loop and electro-magnetic interference isolation. This isolation capability implies that improved signal to noise ratios and signal fidelity can be produced. Additionally, as will be shown in this report, the sensitivity of electrooptical measurement techniques improves at higher voltages. This makes it possible to measure voltage amplitudes with precision on the order of $1 \%$. 
This paper will describe the electro-optical effects most useful for pulsed voltage measurements and optical techniques for producing light intensity variations which can be related to $v(t)$. Practical measurement systems and typical results will also be presented.

\section{Electro-optical Effects}

Several electro-optical effects exist which can be used to measure voltage. The most useful of these are the Kerr and Pockels effects. The polarization state of light, which passes through electrically stressed a Kerr or Pockels effect medium, is modified in a manner dependent upon the value of the voltage applied. The Pockels effect has been used to measure voltages in the range of tens of kilovolts [1]. This effect utilizes relatively expensive crystals such as KDP, KD*P, and $\mathrm{LiNbO}_{3}$ and is 1 imited to voltage measurements in this range, unless voltage division techniques are utilized. The Kerr effect utilizes electro-optical liquids, such as nitrobenzene, and can be used, directly, at voltage levels of several hundred kilovolts. The Kerr effect is therefore most appropriate for pulsed, high field measurements.

The general arrangement for electro-optical measurement has been shown in Figure 1. The electro-optical device shown consists, for high voltage Kerr effect measurements, of an electrode structure filled with Kerr effect liquid. A simple arrangement for the "Kerr ce11", electrooptical device is shown in Figure 2 and consists of 2 parallel plate electrodes separated by a volume filled with Kerr effect fluid.

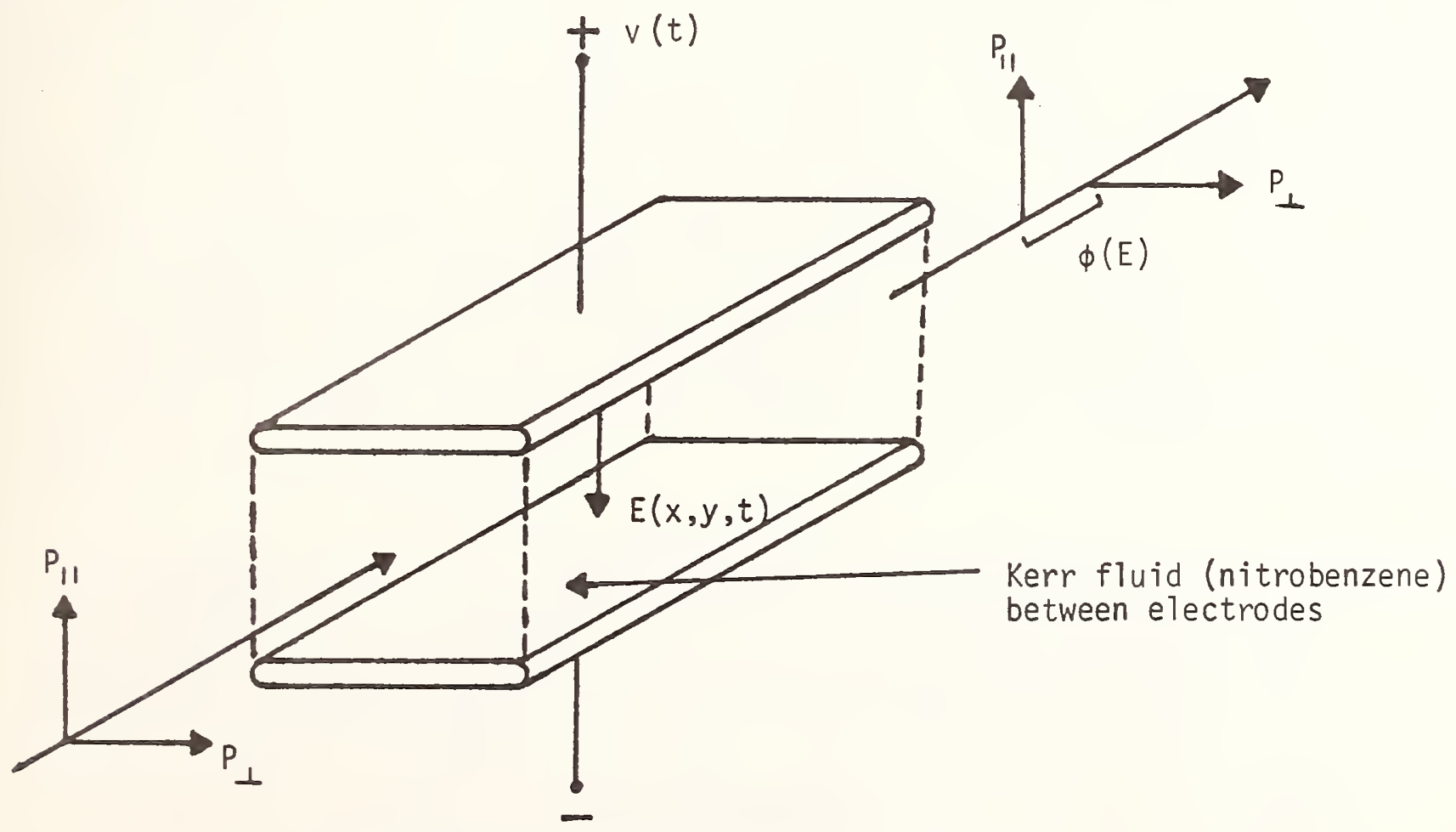

Figure 2. Kerr Electro-optical Effect. 
Linearly pclarized light, having polarization components parallel, $P_{\mathbf{l}}$, and perpendicular, $P_{I}$, to the applied field, $\bar{E}$, is passed through the Kerr cel1. A phase shift $\phi$ is introduced, because of the Kerr effect, between the orthogonal light components. This introduced phase is related to the local value of the electric field at a point $x, y$ and time $t$ according to,

$$
\phi(x, y, t)=2 \pi K L \bar{E}(x, y, t) \cdot \bar{E}(x, y, t),
$$

where $K$ is constant(the Kerr coefficient) and $L$ is the length of the Kerr cell in the direction of light propagation. The electric field, $\bar{E}$, can be assumed, for short pulses having a duration of less than 100 $\mu S$, to be uniform in the central electrode region and equal to $v(t) / d$, where $d$ is the electrode separation. The applied voltage is therefore given, in terms of $\phi$, by

$$
v(t)=\left[\frac{\phi(t)}{2 K L d^{2}}\right]^{1 / 2} .
$$

This implies that measurement of $\phi(t)$, in the uniform field region, will determine $v(t)$. It should be noted that his relationship neglects the effects of fringing fields at the ends of the Kerr cell. These effects can be neglected for most Kerr cells of practical lengths. A quantitative analysis concerning the effects of fringing is given by Thatcher [2].

3. Measurement of $\phi$, Polarizing Light Intensity Variations Indicative of $v(t)$

The applied voltage $v(t)$ is related to $\phi(t)$. The quantity $\phi(t)$ can be measured in several ways. The most common technique involves the use of crossed polarizers. An alternate technique, which has advantages for certain applications, uses a polarization interferometer. This section will present the general measurement principles and will discuss waveform shape, interpretation, calibration, errors, and temporal response considerations.

\subsection{Cross Polarizer Measurements}

The cross polarizer analyzer arrangement necessary for measurina $\phi(t)$ is shown in Fiqure 3 and consists of 1 inear polarizers $P_{45^{\circ}}$ and $P-45^{\circ}$ and quarter wave plates $Q_{0}$ and $Q_{90}$, placed before and after the Kerr cel1. The polarizer $P_{45}$ linearly polarizes the incoming light into components $P_{\|}$and $P_{\perp}$, oriented parallel and perpendicularly to the applied field E. Polarizer $P_{-45}$ is oriented orthogonally to $P_{45}$

such that no light will pass through $P_{-45}$, if the Kerr cell is isotropic. The Kerr liquid, however, becomes anisotropic as voltage is applied. It can be shown that the intensity of the transmitted light is given by, 


$$
I(x, y, t)=I_{m} \sin ^{2}[\phi(x, y, t) / 2],
$$

where $I_{m}$ is a constant and corresponds to the maximum transmitted light intensity.

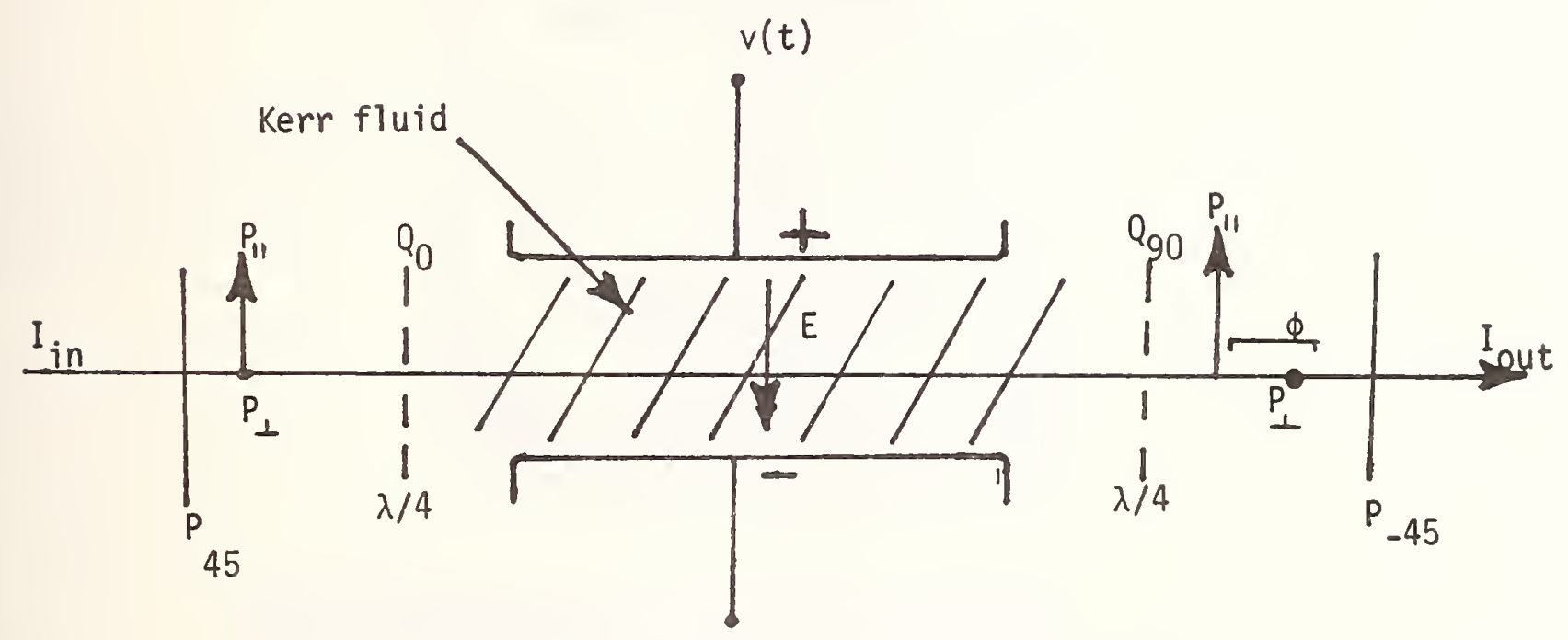

Figure 3. Cross Polarizer, Kerr Electro-Optical Measurements.

The quarter wave plates $Q_{0}$ and $Q_{90}$ convert the linearly polarized light to circularly polarized light and back again to linearly polarized light. Their purpose is to make the transmitted ligith intensity independent of the direction of $\bar{E}$. This is described in detail in reference [3].

The spatial variation implied by Eq. 1 is shown in Figure 4 .

The photograph shows the shadow of the electrodes and dark curves, or fringes, corresponding to the minima of Eq. 3. The electric field at any location $x, y$ can be determined from the data of Figure 4 by using Eq. 3 along each of the fringes.

The electric field at one point, located in a region of uniform field, can be determined by measuring the intensity transmitted at that point, as a function of time. A typical applied voltage, to be electro-optically measured, and the corresponding, transmitted intensity is depicted in Figure 5. The voltage rises to a flat top and then falls to zero. The output intensity, in terms of $v(t)$, is given, using Eqs. 2 and 3 as,

$$
I(t)=I_{m} \sin ^{2}\left[\frac{\pi K l v^{2}(t)}{d^{2}}\right] \text {. }
$$




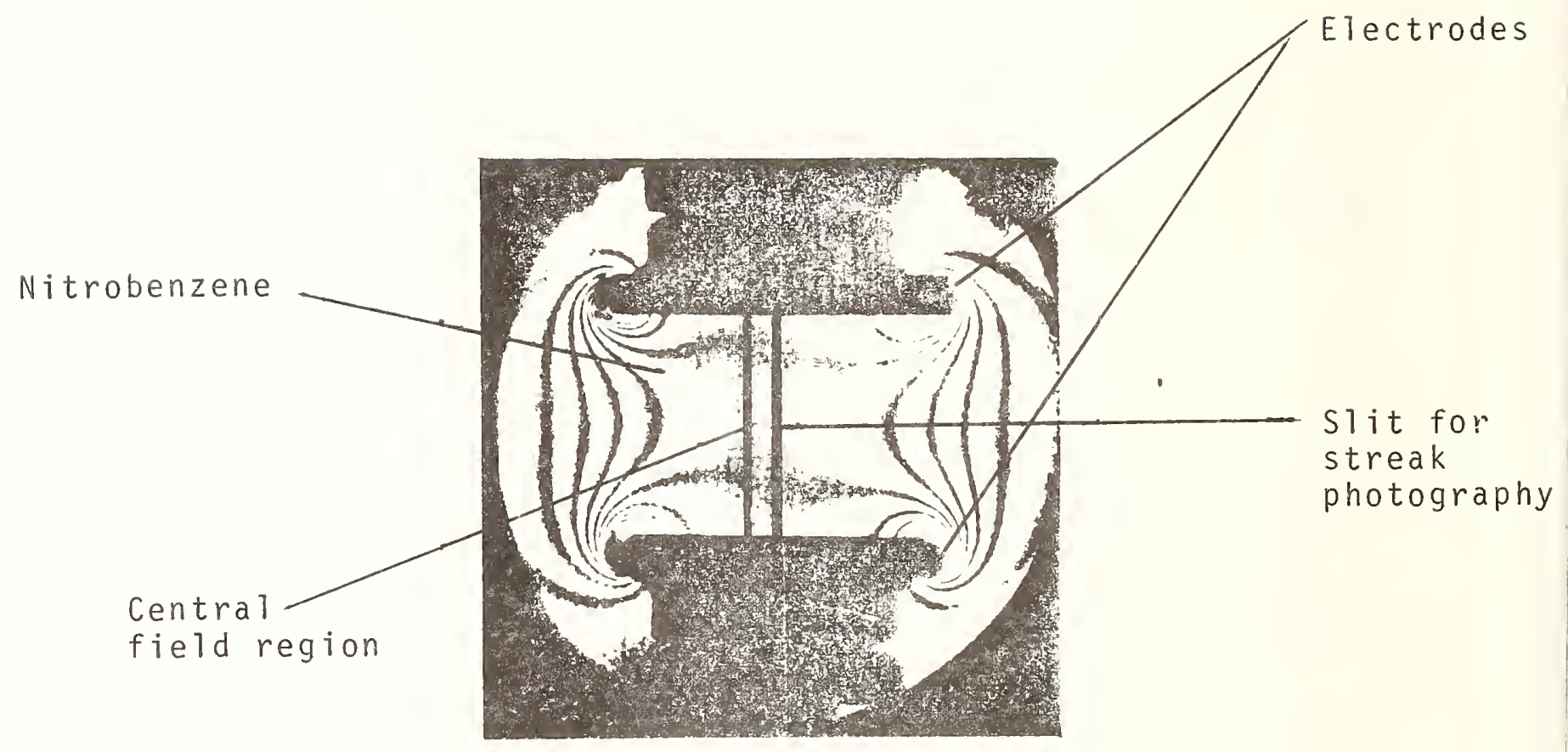

Figure 4. Crossed Polarizer Output Intensity [10].

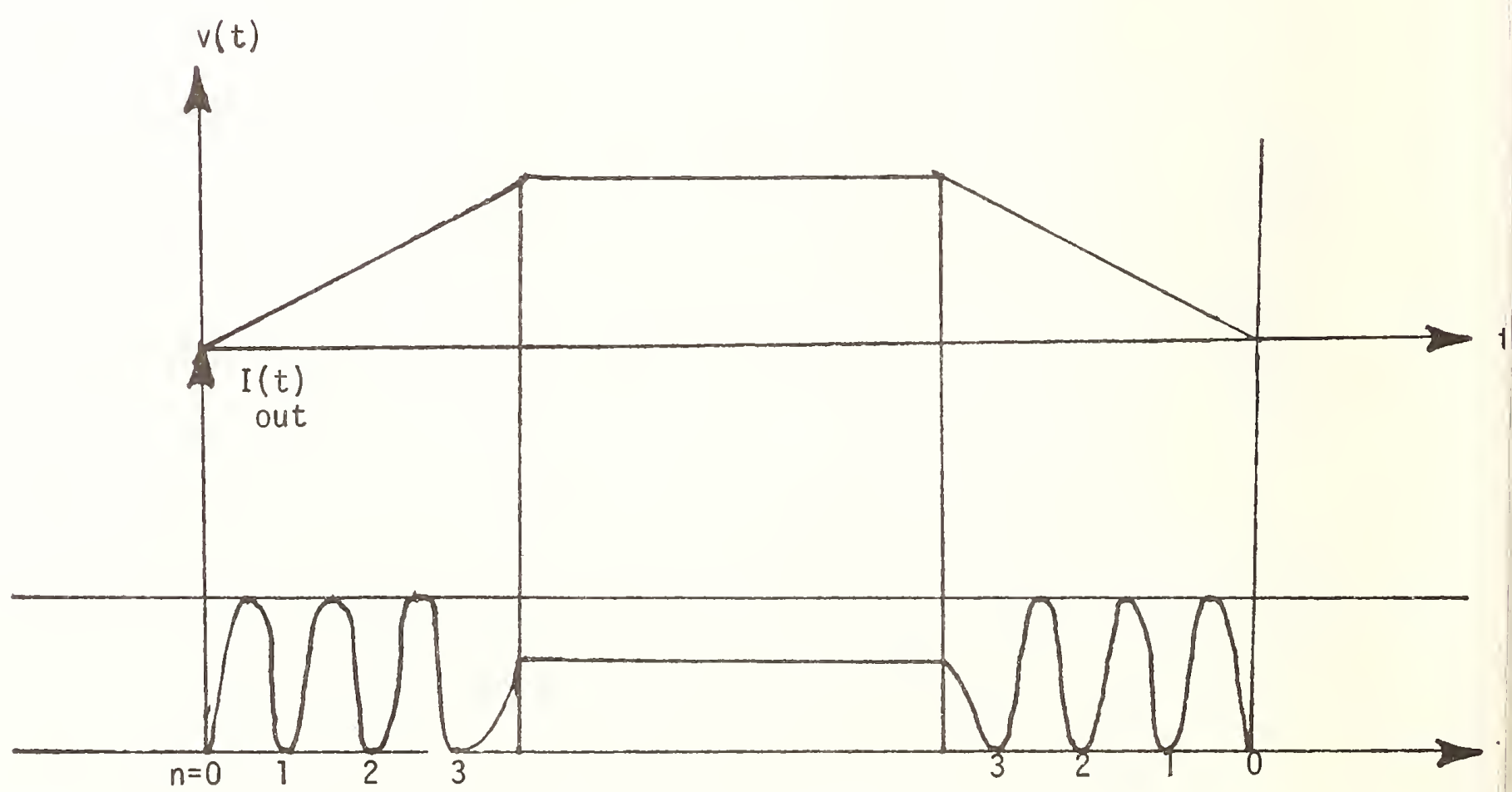

Figure 5. Cross Polarizer Output 
An actual $I(t)$ is shown in Fiqure 6 together with $v(t)$ as measured using a resistive divider. It can be seen that $I(t)$ experiences maxima and minima as the voltage increases and decreases in time. The $I(t)$ of Figures 5 and 6 can be related to $v(t)$, by solving Eq. 4 for $v(t)$,

$$
v(t)=\frac{d^{2}}{\pi k L} \sin ^{-1}\left[\frac{I(t)}{I_{m}}\right] \text {. }
$$

This inversion can be performed, for a $11 t$, for we 11 behaved waveforms and be can be automated for predictable waveshapes such as pulses. The inversion, however, becomes somewhat ambiguous for complicated temporal waveshapes, such as damped sinusoids.

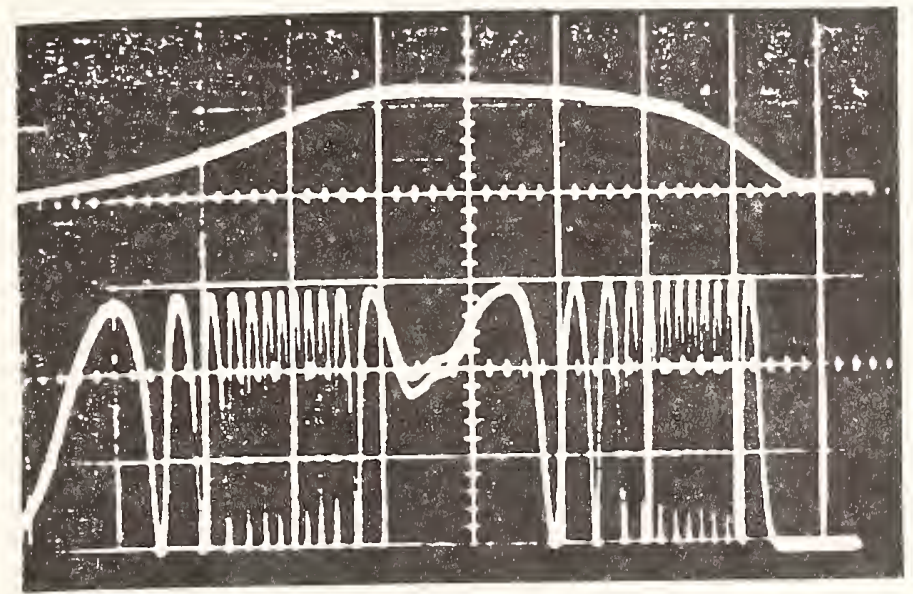

Resistive divider

Kerr fringes

Figure 6. Cross Polarizer Kerr Electro-optical Effect Voltage Measurements (Hebner,et al [1]). 
The $v(t)$ can also be determined from the $I(t)$ shown in Figure 6 by noting that $I(t)$ experiences minima when,

$$
\frac{\pi K L v^{2}(t)}{d^{2}}=n \pi ; n=0,1,2, \ldots
$$

The number of minima or "fringes" can be counted, as a function of time, to yield,

$$
v(t)=d\left[\frac{n(t)}{K L}\right]^{\frac{3}{2}} .
$$

It should be noted that even though Eq. 7 was derived assuming integer values of $n$, partial fringe shifts can be measured such that the value of $v(t)$ at times not corresponding to minima can also be determined. Partial fringe shifts is small as $1 / 4$ are easily measureable. The results shown in Eq. 7, therefore, imply that $v(t)$ can be determined by counting the number of fringes produced, as a function of time, $n(t)$. This counting can also be automated.

\subsubsection{Measurement Accuracy}

Equation 7 shows that the voltage is proportional to the square root of the number of fringes observed. This implies that the change in $n$, $\Delta n$, for a change in $v, \Delta v$, is given by,

$$
\Delta n=\frac{2 K L}{d^{2}} v \Delta v .
$$

This, in turn, implies that $\Delta n$, for a given $\Delta v$, increases as the voltage increases. The sensitivity of the measured $n$ to a change in $v$ therefore increases at higher voltage levels. As an example, assuming $v=100 \mathrm{kV}$, $L=0.7 \mathrm{~m}, d=0.002 \mathrm{~m}$ and a minimum detectable $\Delta n$ of 0.25 , implies a minimum, measureable $\Delta v$ of $154 \mathrm{~V}$. The resolution implied is therefore 154/ $100 \mathrm{kV}=0.15 \%$. The resolution and sensitivity to peak voltage values therefore improves substantially at higher voltages. This of course is a positive attribute for hiah voltage pulse measurements and is an advantage of the electro-optical technique.

\subsubsection{Calibration}

The Kerr effect measurement can be calibrated by applying a voltage value to produce the second minimum, corresponding to $n=1$ of Eq. 6 . This voltage value, $V_{1}$, is termed the cell constant and, from Eq. 7, is given by, 


$$
v_{1}=\left[\frac{d^{2}}{K L}\right]^{1 / 2} .
$$

The voltage $V_{1}$ is typically applied using a calibrated source or measured using a calibrated, resistive divider. Voltage values can therefore be determined, after calibration, in terms of $V_{1}$ according to,

$$
v(t)=V_{1}[n(t)]^{\frac{1}{2}}
$$

\subsubsection{Measurement Errors}

Errors, associated with the electro-optical measurement of $v(t)$, arise due to errors in measurement of $V_{1}$ and errors involved in the measurement of $\phi$. Using Eqs. 2 and 10, it can be shown that the percentage error in measuring $v, \Delta v / v$, in terms of measurement errors in $V_{7}, \Delta V_{7} / V_{7}$, and $\phi, \Delta \phi / \phi$, is given by

$$
\frac{\Delta v}{v}= \pm \frac{\Delta V_{1}}{V_{1}} \pm \frac{1}{2} \frac{\Delta \phi}{\phi} .
$$

The values of $\Delta V / V_{1}$ and $1 / 2 \Delta \phi / \phi$ are typical1y $1 \%$ each [4]. This implies that the error associated with measurement of $v$ is on the order of a few percent.

\subsubsection{Temporal Response}

The temporal response of the electro-optical measurement technique is determined by the responses of the Kerr ce11, the Kerr cel1 1eads, the light detector and recorder. The frequency response of the Kerr cel1 is determined by the capacitance of the Kerr cel1, for lumped Kerr cel1 measurements, and the intrinsic relaxation time of the Kerr liquid for distributed Kerr cell measurements. The Kerr cell liquid is typically nitrobenzene, which has a high relative permittivity of approximately 36 . The resulting capacitance, $C$, of a nitrobenzene Kerr cell is, therefore, on the order of tens of picofarads. The risetime capability of lumped Kerr cells of this type is determined primarily by the Kerr cell capacitance and the circuitry used to connect the Kerr cell to the voltage being measured. The risetime of Kerr cells driven with a transmission line is given by $Z_{0} C$, where $Z_{0}$ is the characteristic impedance of the line, and is on the order of a few nanoseconds for $50 \Omega$ cable. Lower impedance, coaxial or parallel plate, transmission line connections result in faster risetimes. It should therefore be clear, that for lumped Kerr cells, connected to the voltage to be measured with a transmission line, low capacitance and low driving impedance are necessary to accomplish less than nanosecond responses. It should be noted that for proper 
operation, the Kerr cell should terminate the line in its characteristic impedance. Bergman has satisfactorily used a Kerr cell and transmission line connections to measure voltage risetimes on the order of $1 \mathrm{~ns}$ [5].

The connection between the lumped Kerr cell and $v(t)$ can be made with inductive leads. The measurement frequency response is, in this case, limited by the resonant frequency of the leads and the Kerr cell capacitance. This resonant frequency is on the order of $1 \times 10^{8} \mathrm{~Hz}$. which implies a risetime capability of $3.5 \mathrm{~ns}$ [6].

The frequency response of Kerr effect is intrinsically limited by the natural relaxation time of the liquid used. This time is on the order of 50ps for nitrobenzene, as 1ittle as $2 \mathrm{ps}$ for $\mathrm{CS}_{2}$, and is the ultimate limit to risetimes, measureable using the Kerr effect [7]. This 1 imit is, however, applicable only if capacitance and inductive effects are negligible; as in the case of a travelling wave measurement system to be discussed in a later section of this paper.

Equations 7 and 8 imply that the sensitivity of the measurement technique increases at higher field values due to the fact that more fringes are created, per field increment, at high voltages. This implies that a large number, $N$, of fringes can be created during the measured pulse risetime.

This is illustrated in the detected output shown in Figures 5 and 6 . This further implies that the frequency response of the 1 ight detector used must be on the order of $\mathrm{N}$ times the frequency response implied by the measured pulse risetime. There, therefore, must be a compromise between sensitivity/accuracy, or equivalentiy number of fringes, and measureable risetime capability of the electro-optical system.

The frequency response of available photo detectors, capable of driving a low impedance oscilloscope or coaxial cable is limited. Improved frequency response capability can be attained by using an image converter camera as the detector. This is accomplished by streaking the fringe pattern observed as $I_{\text {out }}$. A typical fringe pattern, obtained using the arrangement of Figure 3 and a pulsed laser, is shown in Figure 7. The observed intensity distribution is again given by Eq. 3. Fringes, corresponding to the minima of Eq. 3, are seen to occur in space and are indicative of the fact that the electric field distribution, in the region shown, is spatilaly nonuniform. Few fringes are observed in the indicated, central region, where the field is most uniform. The intensity transmitted through the slit will be alternately bright and dark as the electric field increases from a zero to a peak value. This temporal change in intensity can be detected using an image converter camera to streak the transmitted fringe pattern. Results of this type are shown in Figure 7. A uniform field in the slit region would produce vertical alternating bright and dark bands of the field increases from 0 , at $0 \mu \mathrm{s}$, to a peak value, at $4.8 \mu \mathrm{s}$. The observed bending of the fringes is due to the fact that the field, in the sit region, is spatially nonuniform. The fringes are observed to remain relatively straight in the indicated central region. These unbent 
fringes can be used to determine the temporal performance of the electric field, $E(t)$, in the central region and hence the applied voltage, $v(t)$. This is done by counting the number of fringes, $n(t)$, as a function of time, and using Eq. 10.

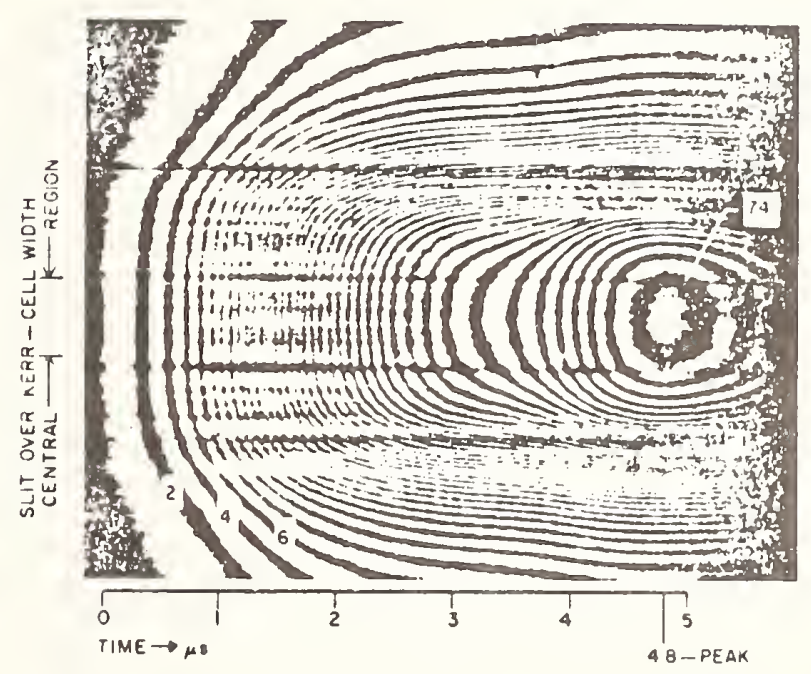

Figure 7. Streaked Cross Polarizer Kerr Electro-optical Voltage Measurement (Hebner, et a] [ ]).

The image converter camera, used as a detector, produces measurement data shown in Figure 7, using the crossed polarizer arrangement of Figure 3. This compares to analogous measurement data, obtained using a photodetector, shown in Figure 6. The advantages of the image converter measurement is the fast writing speed available. Using writing speeds of $0.1 \mathrm{~mm} / \mathrm{ps}$ it is possible to discern a relatively large number of fringes, during the pulse risetime, in a short time period. Good sensitivity, accuracy, and superior risetime capability are therefore achievable.

The temporal response discussions have addressed the shortestrisetime or time variation which can be measured. It should, however, be noted that there is a limit to the longest pulse which can be effectively measured. Long pulses cause volume space charge accumulation in the Kerr measurement fluid. This charqe distorts the inter-electro electric field and complicates measurement results [8]. Volume space charge effects are not important for nanosecond duration pulses. The pulse duration at which the effects do become important depends on the resistivity of the Kerr fluid. Higher resistivity values are required for longer pulses. Reasonable care can produce nitrobenzene bulk resistivity in the rance of $10^{9} \Omega-\mathrm{cm}$. Resistivities, on this order, permit the measurement of pulse lengths up to several hundred microseconds.

\subsection{Interferometric Phase Measurements}

The phase difference $\phi$, introduced due to the Kerr effect, can be measured using the crossed polarizer arranaement of Fiqure 3 . The rela- 
tionship between $\phi, E, v$, and $n$ have been presented in this paper. This phase measurement technique produces a fringe pattern in time, as shown in Figure 6 or a fringe pattern in space, as shown in Fiqure 4, which can be streaked in time to hield measurement data, as shown in Figure 7. The temporal data of Figures 6 and 7 are interpretable for simple waveshapes such as flat pulses with little oscillatory overshoot or undershoot. However, electro-optical results produced by complicated waveforms, such as damped sinusoids, or pulses with oscillatory components, are difficult to interpret. This arises due to the fact that the relationship between $v(t)$ and $I(t)$ as given by Eq. 4, is nonlinear and multivalued. Certain of the ambiguities, associated with the relationship between $v$ and $I$, can be eliminated using two probing wavelengths, simultaneousiy, to produce two separate sets of measurement data similar to that shown in Figure 6 or by using a Kerr cell having two probed lengths [9].

An alternate technique for measuring $\phi$, which can produce more interpretable temporal voltage measurements and sensitive spatial field measurement, utilizes the polarization analyzer shown in Fiqure $\mathcal{E}$. Linearly polarized 1ight, having components, parallel $P_{11}$, and perpendicular, $P_{1}$, to the applied field are passed through the Kerr fluid, as shown in Figure 2. The light, having a phase shift $\phi$, between the orthogonal components, then enters the analyzer. The analyzer produces output beams $P$ and $P$ in the two interferometer paths, using the polarizers and halfwave plate shown. These two beams are then recombined, using the beam splitter shown, to produce a fringe pattern indicative of $\phi$. The detailed operation of the analyzer, as used for field measurements is given in reference [10].Typical results obtained using a pulsed laser are shown in Figure 9. The interferogram shows the shadow of the two excitation electrodes and fringes formed between the electrodes in the nitrobenzene filled region. The position of the fringes is related to the local field value. Interference patterns of the type shown in Figure 9 are useful for measuring field spatial distributions, at a specific time. These fringes can also be streaked in time, in a manner analogous to that used to obtain the results of Figure 7, by positioning a vertical slit, as described previously. Results obtained in this manner are shown in Figure 10. Figure $10(a)$ shows, as the upper trace, the $v(t)$ to be measured and, as the lower trace, the measurement of $I(t)$ using the crossed polarizer technique of Figure 3. It should be clear that some interpretation difficulties exist for relating $I(t)$ to $v(t)$ in this case. Similar measurement results, obtained using the analyzer of Figure 8 and fringes, as shown in Figure 9, streaked in time, are shown in Figure 1?(b). It can be observed that each streaked fringe has a temporal shape which is clearly related to the excitation waveshape. It can be shown that the measured field values are given by,

$$
E(t)=\left[\begin{array}{ll}
\frac{1}{\delta x K L} & \Delta x(t)
\end{array}\right]^{1 / 2},
$$

where $\delta x$ is the spacing between undiviated fringes (corresponding to zero field) and $\Delta x(t)$ is the measured deviation of the fringes [0]. The auantity 


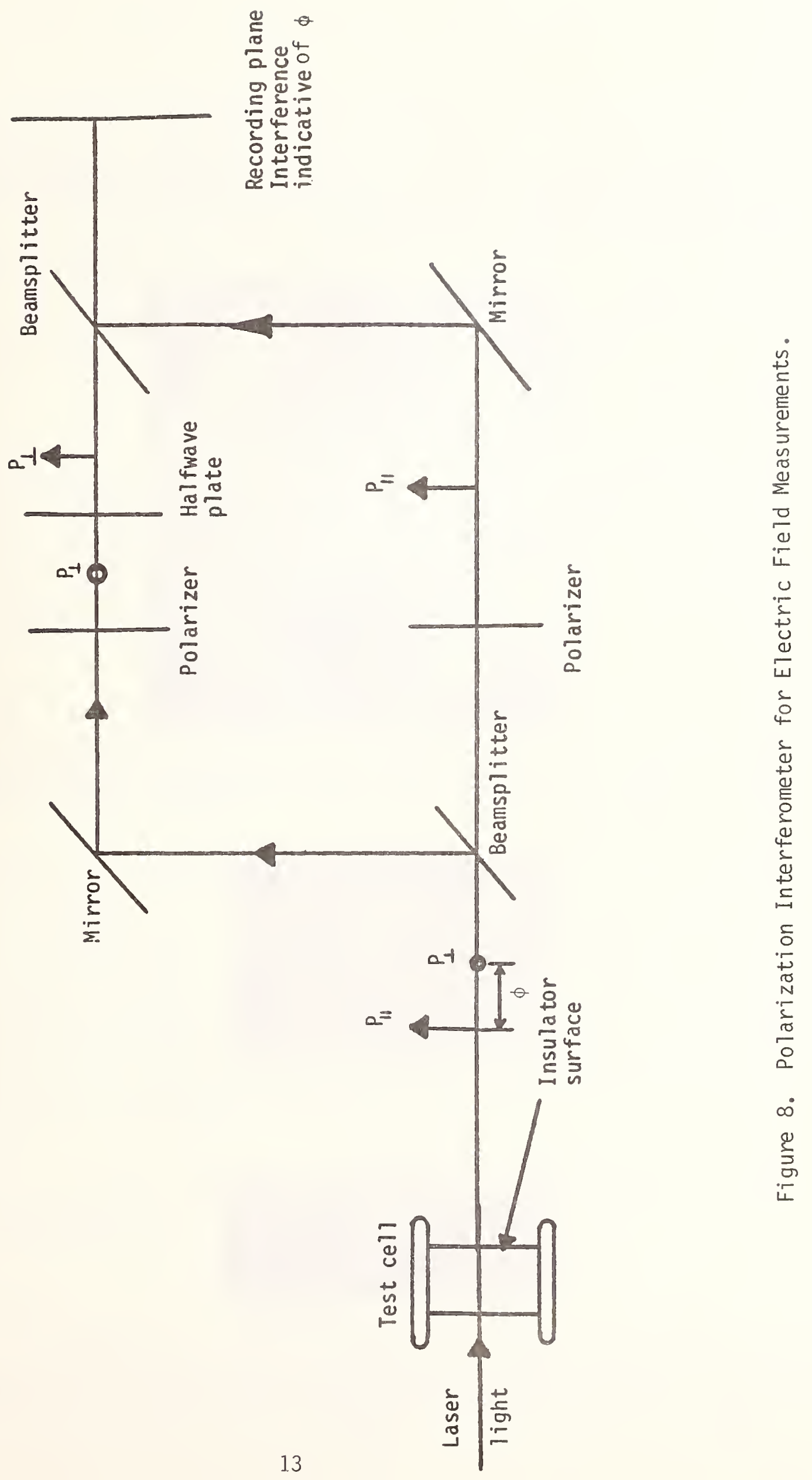


$\Delta x(t)$ can be directly measured from the interferogram to yield the temporal performance of $E(t)$. The voltage $v(t)$ is clearly related to $E(t)$ for uniform field electrode geometries.

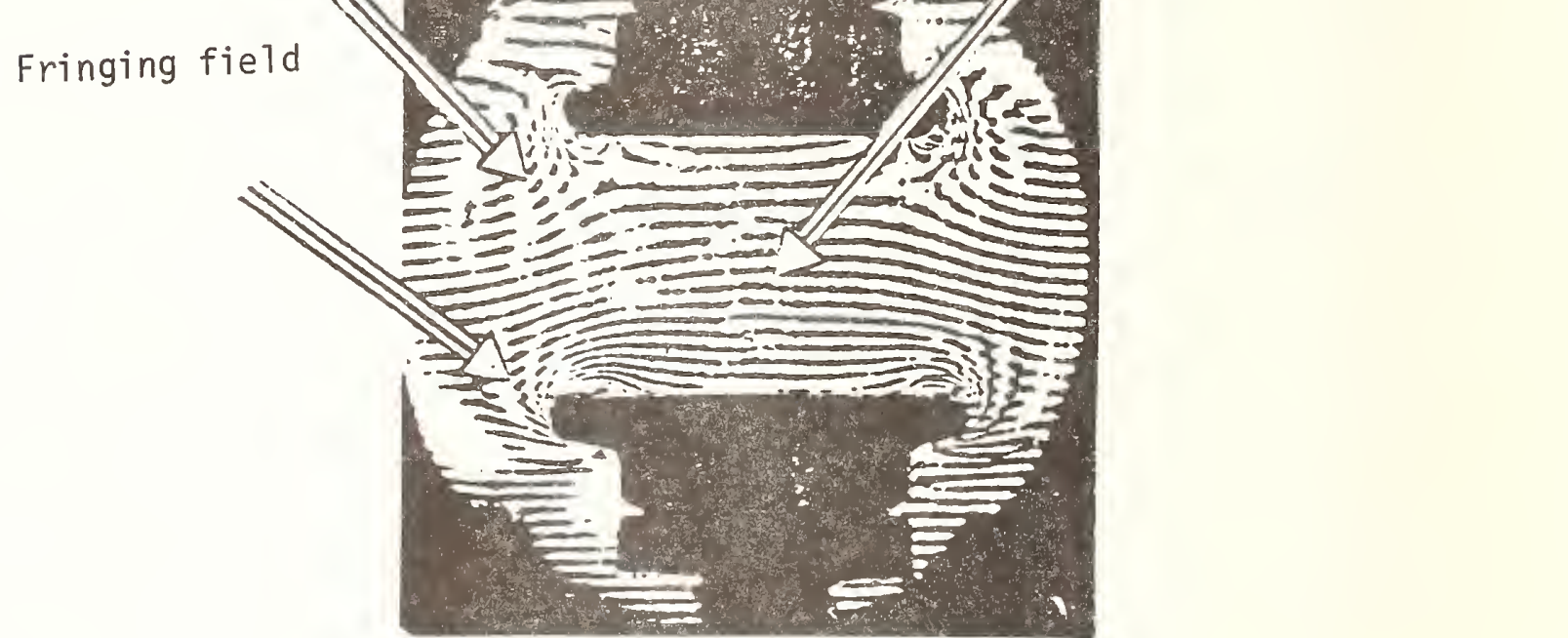

Figure 9. Typical Interferogram of Field Distribution Between Parallel Electrodes 1101.

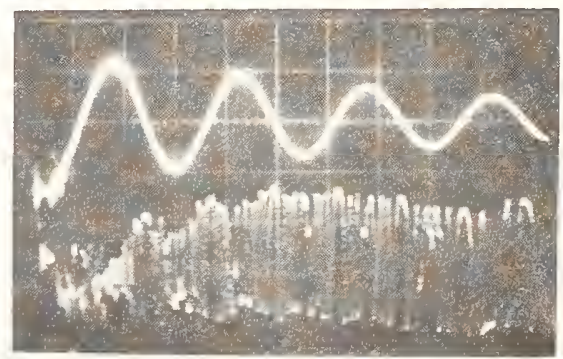

$5 \mathrm{kV} / \mathrm{cm}$

$20 \mu \mathrm{s} / \mathrm{cm}$

(a) Voltage Divider and Cross Polarizer

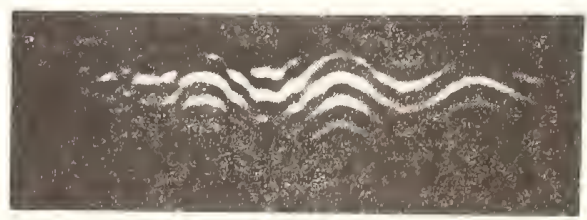

$40 \mu \mathrm{s} / \mathrm{cm}$

(日) Interferometric

Figure 10. Cross Polarizer and Interferometric Measurements [10]. 
The streaked interferometric measurments, illustrated in Figure $10(b)$, also have sensitivity that increases with $v$. The results can be viewed somewhat directly, similar to an oscilloscope trace. There is therefore less ambiguity in relating $I(t)$ to $v(t)$. It should, however, be clear that additional expense and complication is associated with this technique primarily due to the need for an image converter camera. The technique if however attractive for certain applications where complicated, fast pulses are to be measured. Results to date have been produced, utilizing this technique, with subnanosecond resolution[11]. It should however be possible to extend this to lops resolution, using the fast streak cameras (10ps/mm) currently available.

\section{Typical Pulse Measurement Systems}

The general principles assocaited with electro-optical voltage measurements have been discussed in the previous sections. Several practical systems, used by various researchers, will be described in this section. The first utilizes on impedance matched Kerr cell to improve the risetime and a sampling pulsed laser. The second uses a pulsed laser and optical delay lines to determine fast risetimes. The last technique utilizes a coaxial geometry appropriate to very fast traveling wave measurements.

\subsection{Impedance Matched Kerr Cel1}

Voltage measuring Kerr cells are primarily capacitive. The frequency response of these cells is, therefore, limited by the time required to charge this capacitance or by resonant oscillations between the capacitance and lead inductance.

Bergman and Kolleogy have used impedance matched Kerr cell to measure fast voltage behavior associated with a discharge in $\mathrm{N}_{2}$ gas [5]. The measurement Kerr cell is shown in Figure 11 and consists of a parallel plate transmission line which is fed by a coaxial cable connected to the voltage being measured. The voltage pulse propagates from the source, through the cable and the Kerr cell, and out another load coaxial line. The dimensions of the nitrobenzene filled Kerr cell are chosen to produce a parallel plate transmission line having the same characteristic impedance as the driving and load cables. No reflections, therefore, occur. The risetime of the response is therefore not limited by the capacitive charging, line impedance, or by oscillations due to the Kerr cell capacitance and lead inductance.

The cell shown in Figure 11 has been used in conjunction with a pulsed dye laser to permit voltage measuréments at discrete lns times. The temporal resolution capability is, therefore, on the order of $1 \mathrm{~ns}$.

\subsection{Optical Sampling Transient Analyzer}

A nitrobenzene filled transmission. line has been used by Davies and Nelson to measure the risetimes of fast pulses [12]. This system is shown in Figure 12. The voltage to be measured propagates down the 1 ine. Light 
from a pulsed laser travels through fiber optic cable and enters the line, through linear polarizers, passes through the nitrobenzene, and exits on the other side of the line, through crossed polarizers. The light entry and exit parts are displaced along the line as shown. The Kerr effect at these entry ports, therefore, measures the pulse voltage at various times, which can be related to the risetime and propagation velocity of the wave. A larger or smaller time interval of the excitation can be measured, with a given port separation, by using fiber optical cables of different

lengths. The frequency response of this system is on the order of $1 \mathrm{GHz}$.

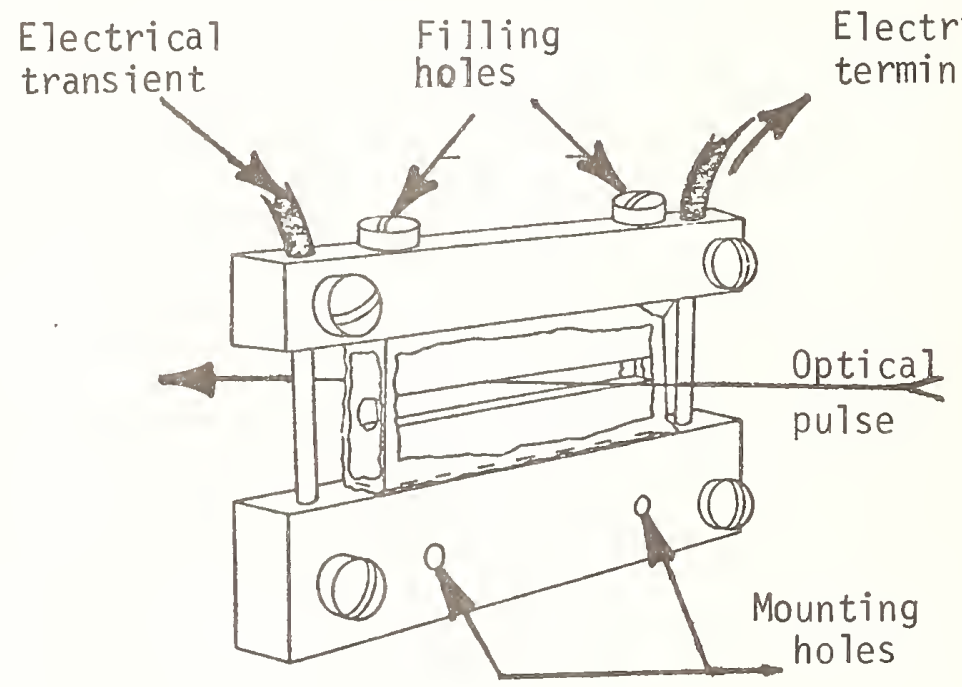

Figure 11. Schematic Drawing of the Kerr Cell with Electrical Connections Indicated [5].

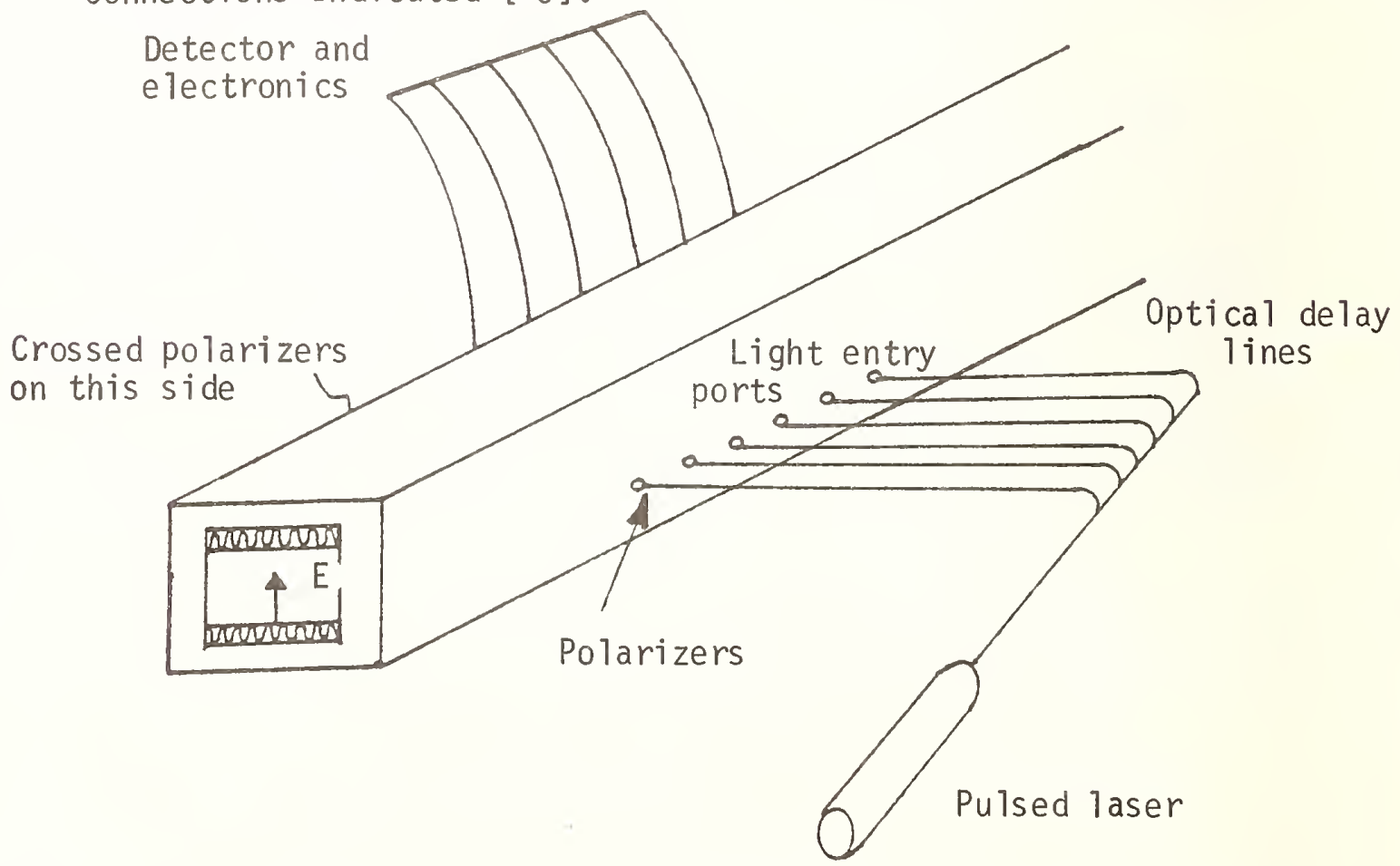

Figure 12. Optical Sampling Transient Analyzer (Davies and Nelson [12]). 


\subsection{Coaxial Field Measurements}

Wunsch, Sanchez, and Green have used the Kerr effect to measure the electric fields within pulse excited coaxial cylinders[7]. The experimental arrangement is shown in Figure 13 and consists of a water filled coaxial transmission line with optical input and output ports. The electromagnetic pulse, to be measured, propagates down the line. Light is directed through a polarizer into the area between the coaxial cylinders and propagates in the same direction as the pulse. The light beam is positioned nearer the center conductor to be in a higher field region to improve measurement sensitivity. The polarization state of the light changes (due to the Kerr effect of water) as the pulse fills the volume between the two mirrors. The risetime of the optical response is therefore dependent upon the risetime of the pulse, the propagation time between the mirrors, and the response time of the Kerr fluid. Megavolt signals have been measured with this system. The system risetime is on the order of $10 \mathrm{~ns}$ and is 1 imited due to the optical detector being used.

The frequency response of the system shown in Figure 13 can be improved substantially if $\mathrm{CS}_{2}$ liquid is used. The pulse and light propagation velocity for $\mathrm{CS}_{2}$ are approximately the same. A travelling wave measurement system therefore results. The frequency response is therefore limited only by the response time of the $\mathrm{CS}_{2}$ which is on the order of $2 \mathrm{ps}$. No measurements using $\mathrm{CS}_{2}$ were taken; however, results were obtained using other liquids including nitrobenzene, water, and transformer oil. [7]

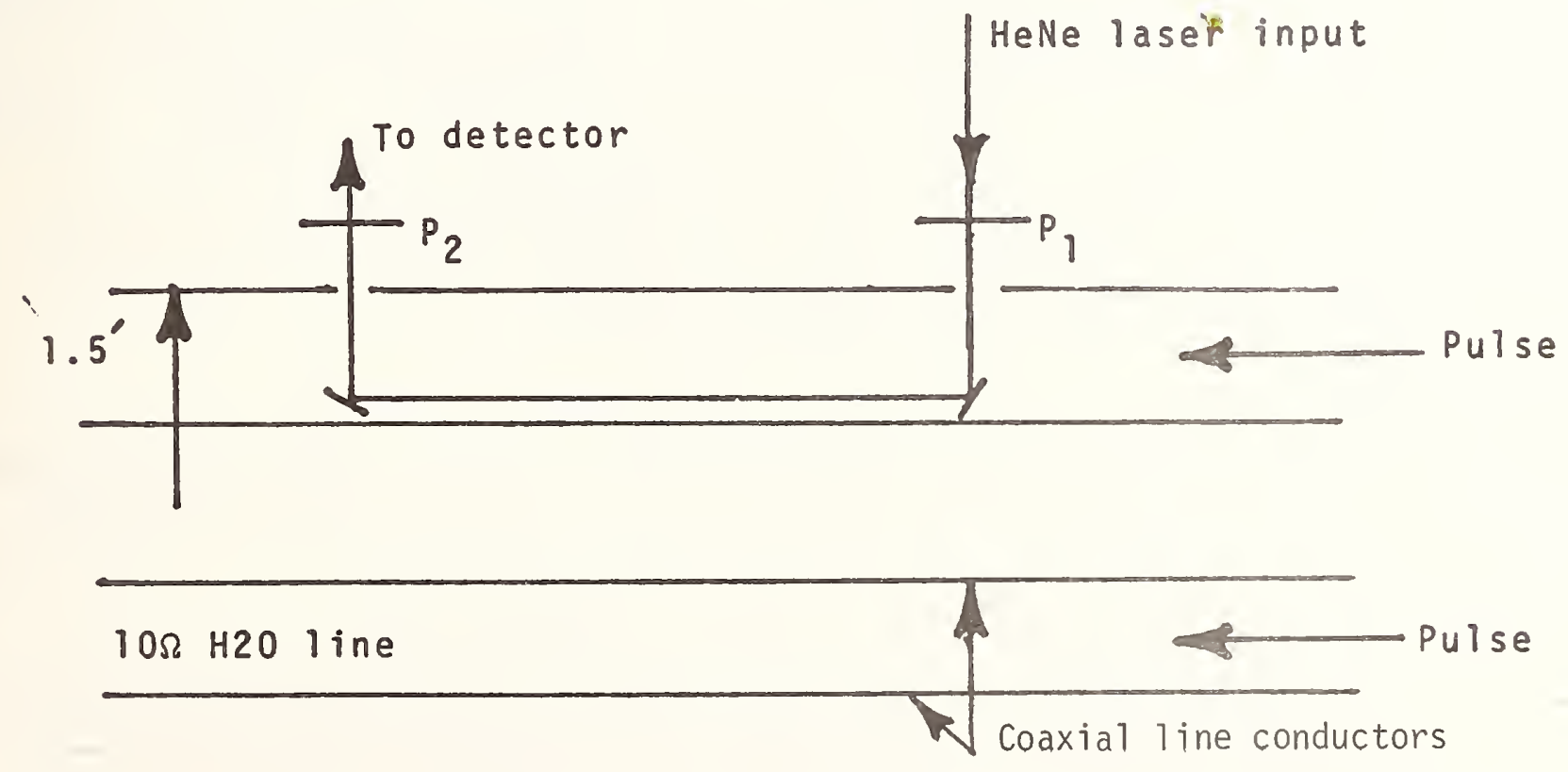

Figure 13. Megavolt $\mathrm{H}_{2} \mathrm{O}$ Measurements (Wunsch [7 ]). 


\section{Summary and Conclusions}

Fast pulse voltage measurements are typically done using capacitive and resistive voltage dividers. However, the time response of these systems is limited due to stray capacitance and inductance effects which either slow the observed risetime or produce oscillations for fast pulses. Additionally, these techniques are susceptible to ground loops and electromagnetic interference.

Electro-optical measurement techniques utilizing either the Pockels or Kerr effects can be used to measure pulsed voltages. The Kerr effect is more appropriate for pulsed high voltages. Kerr effect measurement techniques have no ground loops and can be effectively snielded from EMI. Kerr effect measurements do not typically incorporate divider circuits. The frequency response is therefore determined by the geometry of the Kerr ce11 used. Lumped measurement systems, where the Kerr measurement ce11 is capacitive, can be used to measure voltage risetimes in the nanosecond regime. Faster responses are possible using the Kerr cell is a transmission line geometry. The ultimate frequency response for this arrangement can be much less than 1ns.

Kerr effect techniques, in addition to being rather noise immune and very fast, are particularly accurate at high voltages. Because of the quadratic relationship between the voltage being measured and the detected light intensity, the measurement accuracy improves substantially at high voltages.

Electro-optical measurement techniques have definite advantages for pulsed, high voltage measurements. It should, however, be noted that they are typically more difficult to implement than more conventional techniques. The techniques require a laser, a Kerr cell, and detector system. U1trafast pulses can require an image converter camera. However, for certain applications and situations, the added complications are warranted. It is also probable that for some subnanosecond voltage measurements, electro-optical techniques, used in conjunction with an image converter camera, may be the only techniques with sufficient frequency response and noise immunity which can be used successfully.

\section{References}

[1] R. L. Hebner, Jr.,R. A. Malewski, E. C. Cassidy, "Optical Measurements at High Voltage Levels," Proc. IEEE, Vol. 65, pp. 1524-1548 (1977).

[2] P.D. Thatcher, "Optical Effects of Fringing Fields in Kerr Cells," IEEE Trans. E1ec. Insul., Vol. EI-11, pp. 40-50 (1976).

[3] E.C. Cassidy, N. N. Cones, S. R. Booker, "Development and Evaluation of Electro-optical High Voltage Pulse Measurement Techniques," IEEE Trans. on Instr. and Measurement, Vol. IM-19, pp. 395-402 (1970).

[4] R.E. Hebner, Jr. and M. Misakian, "Calibration of Hiqh Voltage Pulse Measurement Systems Based on the Kerr Effect", NBS Report NBSIR 77-1317, Sept. 16, 1977. 
[5] E.E. Bergmann and G. P. Koleogy, "Measurement of Nanosecond HV Transients with the Kerr Effect," Rev. Sci. Instr., Vol. 48, pp. 1641-1644 (1977).

[6] A.J. Schwab, "Compact High Power $\mathrm{N}_{2}$ Laser: Circuit Theory and Design", IEEE Journal of Quantum ETectronics, Vol. CE-12, pp. 183-188 (1976).

[7] D.C.Wunsch, A.S. Sanchez, and E.W. Green," Nanosecond High Voltage Measurements", CPEM 74 Digest: Conf. on Precision Electromagnetic Measurements, London, England, 1-5 July, 1974 (London, England: IEE 1974) pp. 82-83.

[8] E.C. Cassidy and R.E. Hebner, "Experimental Study of the Behavior of Nitrobenzene under Varied High Voltage Conditions", Annual Report, Conf. on Elec. Insul. and Diel. Phen:, National Academy of Sciences, Washington, D. C., pp. 37-44 (1973).

[9] W. Botticher, Ch. Homann, and H. Hukner, "Electro-optical Measurement of Current and Voltage in Fast, Hiah Pressure Glow Discharaes", J. Phys. I: Sci. Instr., Vol. 11, pp. 248-250 (1978).

[10] J.E. Thompson, M. Kristiansen, and M. D. Hagler, "Optical Measurement of High Electric and Magnetic Fields", IEEE Trans. on Instr. and Meas., Vol. 25, pp. 1-7 (1976).

[11] J.E. Thompson J. Lin, K. Mikkelson, and M. Kristiansen, "Investigation of Fast Insulator Surface Flashover in Vacuum," IEEE Trans= actions on Plasma Science, Vol. PS-8, pp. 191-i97 (1980).

[12] T.J.Davies and M.A. Nelson, "Ontical Sampling Transient Analyzer System," presented at Conf. on Laser Engr. and Applications, Washington, D.C.; 1975. 


\author{
HIGH VOLTAGE PROBE FOR LIQUID IMMERSION \\ Neville W. Harris \\ Ion Physics Company \\ Burlington, MA $0 \div 803$
}

\begin{abstract}
A capacitive voltage divider for the measurement of very high voltage transients in liquid-filled transmission lines has been developed. The probe is suitable for pulse lengths in the range $10 \mathrm{nsec}$ to 1 usec, and peak voltages between $50 \mathrm{kV}$ and $10 \mathrm{MV}$. The divider automatically compensates for changes in dielectric constant and loss.
\end{abstract}

Key Words: Voltage monitor; capacity divider; pulse voltage monitor; high voltage divider; waterline voltage monitor.

\title{
1. Introduction
}

The development of a very high power, short duration pulse generator operating at voltages in excess of $400 \mathrm{kV}$ required a fast high voltage probe. The probe described is suitable for use in liquid dielectrics at electrical field stress levels near breakdown and compensates automatically for changes in dielectric constant and resistivity.

The $400 \mathrm{kV}$ pulse generator employed the usual technique of charging a transmission line to twice the required voltage and discharging into a matched load. This resulted in a flat topped pulse with very short rise and fall times. Powers up to $10^{12} \mathrm{~W}$ with pulse lengths of the order of 50 nsec were generated in this fashion. The transmission line, in this case, was a coaxial line filled with water stressed close to its breakdown limit. A suitable voltage monitor had to be immune to the very severe interference fields radiated by the generator and had not to reduce the breakdown voltage of the coaxial line. 
2. Principle of Operation

Figure 1 illustrates the principle of operation. A cylindrical

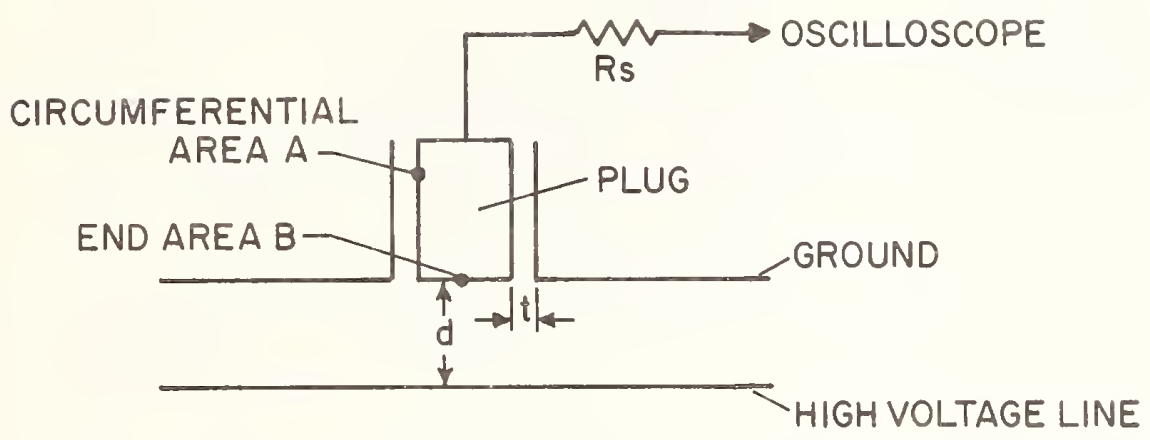

Figure 1. PRINCIPLE OF OPERATION

plug is mounted concentrically into a recess in the grounded conductor of a transmission line. The plug end is flush with the grounded conductor surface. The dielectric fluid filling the transmission line also fills the capacitive monitor. The plug to grounded conductor distance is t and opposing area A. The plug to high voltage conductor distance is d and end area B. The voltage division ratio is then

$$
\frac{\mathrm{Ad}}{\mathrm{Bt}}
$$

and the field division ratio is simply $B / A$.

If the field stress in the medium is near breakdown level then the field in the monitor can be reduced to safe levels by making $B>A$. B should be significantly larger than A to take care of mounting alignment errors and local field enhancement.

Note trat the conductivity and the dielectric constant of the fluid do not need to be. known. This means that temperature, impurity and frequency effects are automatically compensated. Nonlinear effects in the fluid are compensated properly only when area $B$ is similar to area A.

The resistor $\mathrm{R}_{\mathrm{S}}$ between the plug and the monitor cable reduces the sensitivity of the monitor and increases it bandwidth. Care must be taken that self capacity of the resistor, or its capacity to its holder do not degrade the bandwidth.

\section{Construction}

Figure 2 shows the construction of the voltage divider A cylindrical plug was mounted on an insulating sheet which was in turn fixed to a backing plate. A resistor was 


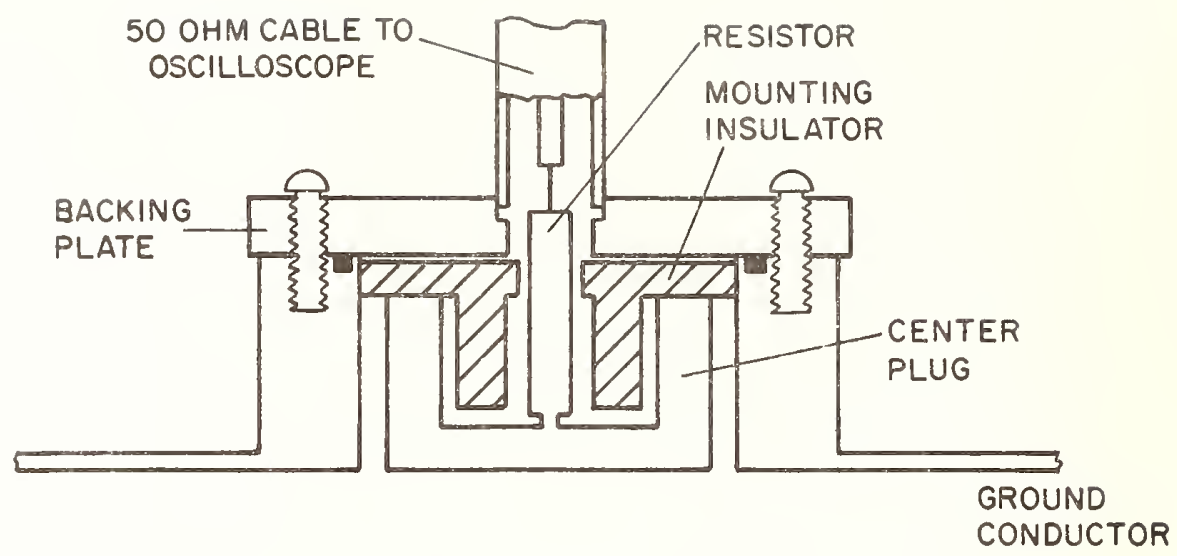

TRANSMISSION LINE

Figure 2. CONSTRUCTION OF MONITOR

connected between the plug and a $50 \Omega$ monitor cable. This assembly was then inserted into a recess in the ground conductor of the transmission line and sealed with an O-ring as shown. The diameter of the plug was close to that of the recess, and the face flush with inner surface of the ground conductor. Centering the plug in the recess can be accomplished using grooves in the insulator as the gap $t$ is usually in the range 0.2-1 mm. Accurate centering may also be accomplished by the use of insulating centering screws; see Figure 3.

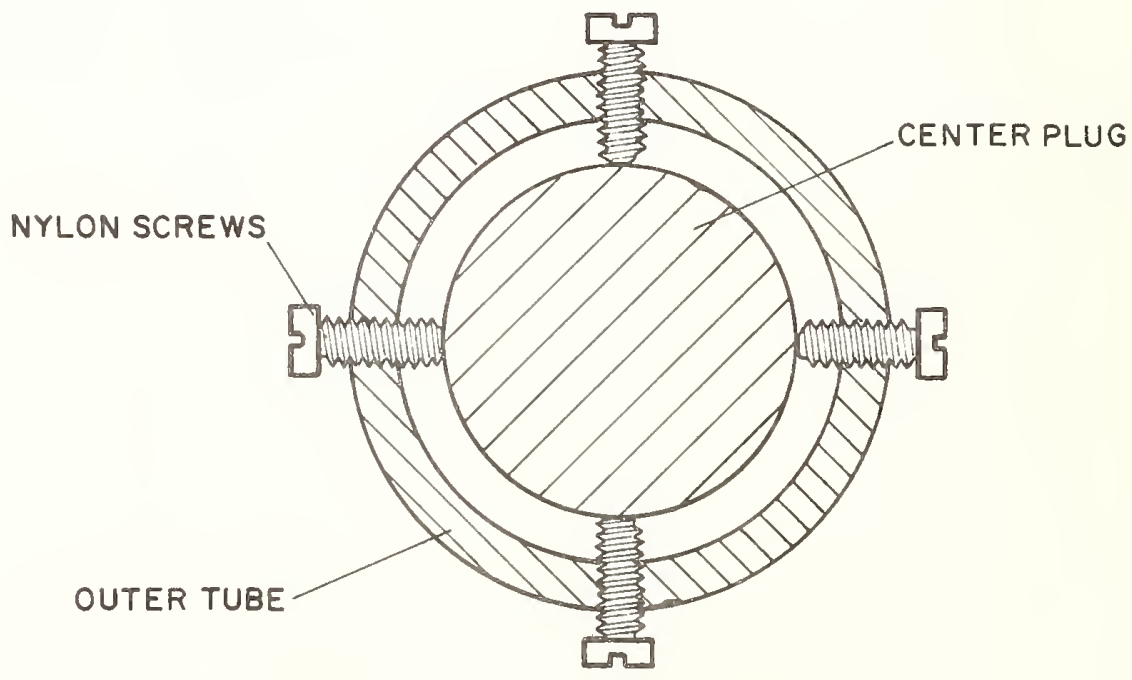

Figure 3. USE OF CENTERING SCREWS 


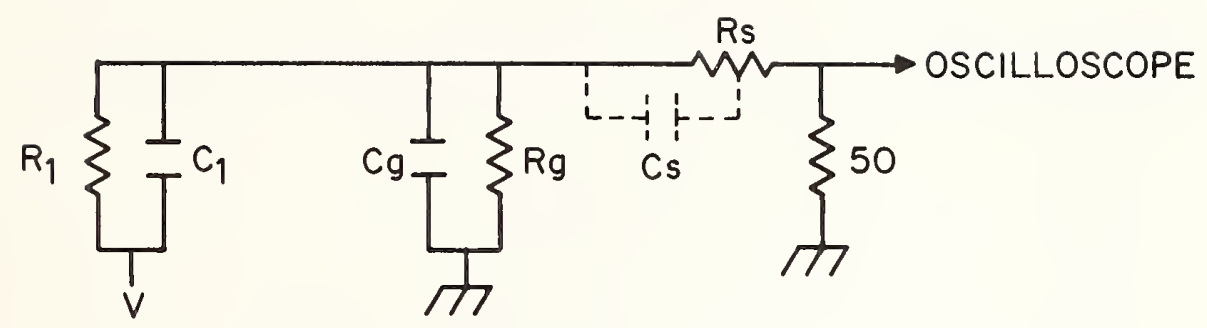

Figure 4. EQUIVAIENT CIRCUIT OF DIVIDER

The equivalent circuit for the divider is shown in Figure 4. $\mathrm{C}_{I}, \mathrm{C}_{\mathrm{g}}, \mathrm{C}_{\mathrm{S}}$, and $\mathrm{R}_{\mathrm{S}}$ are the capacity between plug and line, capacity between plug and ground, stray capacity between resistor $R_{S}$ and plug and value of the series resistance, respectively. $\mathrm{C}_{\mathrm{g}}$ is readily calculable from the formula for concentric cylinders, as the end effects are very small. Similarly with $\mathrm{C}_{l}$, the plug and the inner conductor of the transmission line form a parallel plate capacitor with the ground conductor acting as a guard ring. We can assume $\mathrm{C}_{\mathrm{g}}$ to be much greater than $\mathrm{C}_{l}$. The effect of finite fluid ressistivity is modeled in the equivalent circuit by $R_{1}$ and $\mathrm{R}_{\mathrm{g}}$, note that $\mathrm{R}_{1} \mathrm{C}_{1}=\mathrm{R}_{\mathrm{g}} \mathrm{C}_{\mathrm{g}}$.

Neglecting losses, fluid resistivity and assuming $\mathrm{C}_{\mathrm{g}} \gg \mathrm{C}_{1}$ the output of the divider, $\mathrm{U}$, into the cable for an input step function voltage $\mathrm{V}$ is

$$
U=V \frac{C_{1}}{C_{g}} \frac{50}{\left(R_{S}+50\right)} \exp \left[\frac{-t}{\left(R_{S}+50\right) C_{g}}\right],
$$

The low frequency limit of the divider is determined by the decay time constant, $\left(R_{S}+50\right) C_{g}$. The high frequency response of the probe is limited by transit time effects along the plug and the reactance of the resistor $R_{S}$. However, if the value of $R_{S}$ is suitable chosen, (e.g., about $1 \mathrm{k} \Omega$ ) the effects of resistor shunt capacity and series inductance are negligible.

The pulse generator, for which the probe was designed, uses water as the dielectric due to the high energy density storage capabilities of water. However, water suffers from the disadvantages that the dielectric constant varies with frequency and temperature, and in particular, the resistivity depends markedly on impurity concentration.

The divider output $U$ for an input step function $V$ is more complicated than that given by $\mathrm{Eq}$. (2), if we take fluid conductivity into account. 


$$
\mathrm{U}=\frac{\mathrm{V} 50}{\left(\mathrm{R}_{\mathrm{S}}+50\right)}\left[\frac{1-\exp \left(\mathrm{Yt} / \mathrm{C}_{\mathrm{E}}+\mathrm{C}_{I}\right)}{\mathrm{Y} \mathrm{R}_{1}}+\frac{\mathrm{C}_{7}}{\mathrm{C}_{\mathrm{g}}+\mathrm{C}_{I}} \exp \frac{-\mathrm{Yt}}{\left(\mathrm{C}_{\mathrm{g}}+\mathrm{C}_{1}\right)}\right],
$$

where

$$
Y=R_{1}^{-1}+R_{g}^{-1}+\left(R_{S}+50\right)^{-1}
$$

For times much shorter than the decay time constant, the divider output is equal to that given in Eq. (2). For times comparable with this time constant, the output decays more slowly and is not asymptotic to zero. Hence an increase in the conductivity of the dielectric increases the bandwidth of the divider and does not affect its calibration or division ratio.

The contribution to $C_{\mathrm{g}}$ made by the mounting insulator is negligible if the insulator thickness is very much greater than the gap between the plug and the recess in which it is mounted. Consequently, a change in the dielectric medium affects $\mathrm{C}_{\mathrm{g}}$ and $\mathrm{C}_{1}$ in the same ratio and does not affect the calibration. The probe blends in smoothly with the ground conductor of a transmission line and does not degrade the standoff voltage capabilities of the line. The body of the probe is an almost complete shield which keeps out unwanted electromagnetic fields. Pickup of interference signals is further minimized by the symmetrical, coaxial construction of the inner conductor and series resistor. The probe is suitable for pulse lengths between 10 nsec and 1 usec with outputs in excess of $100 \mathrm{~V}$ and a signal to noise ratio better than $35 \mathrm{~dB}$.

\section{Results}

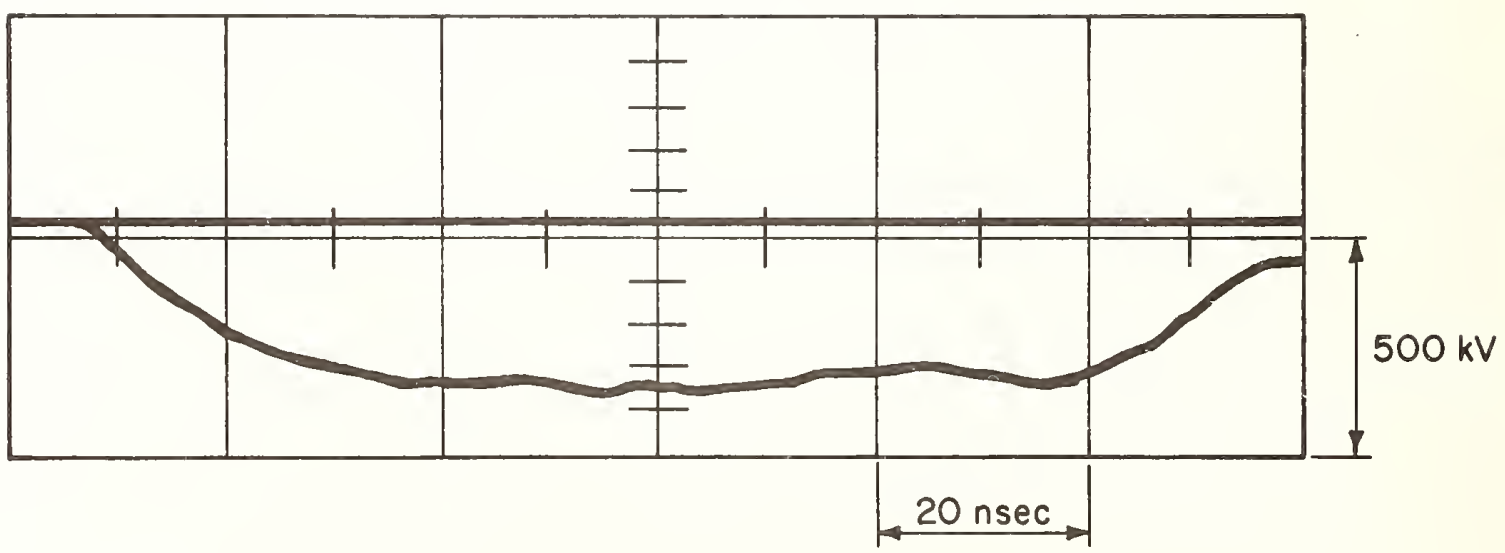

Figure 5. MONITOR OUTPUT 
Figure 5 shows a typical waveform from the monitor. When the generator was connected to a resistive load, this capacitive monitor agreed with the current monitor fitted and had substantially less 'hash'.

The best calibration procedure involves launching a known pulsed voltage onto the transmission line. Sensitivity can be increased, for the purposes of calibration, by reducing the transmission line spacing $d$. The alternative method of calibration, measuring the capacities $\mathrm{C}_{\mathrm{g}}$ and $\mathrm{C}_{1}$, is less satisfactory due to films on the electrode surfaces. Errors and malfunction of the monitor can be caused by the presence of foreign matter in the body of the monitor. In water systems, these can be either air bubbles or solid particles. Little trouble was experienced with either bubbles or particles as the monitor was mounted near (but not at) the bottom of the horizontally mounted coaxial transmission line. A crude but effective method of flushing the monitor was simple to loosen momentarily the mounting screws, allowing water to flow past the O-ring.

\section{Conclusions}

A simple capacitive voltage monitor that depends for its division ratio on geometry, not material properties has been described. It was used in a high power, high voltage (400 kV) pulse generator and gave a clean reliable signal. 


\title{
EXPERIMENTAL COMPARISON OF STEP-RESPONSE AND RAMP-RESPONSE
} MEASUREMENTS IN FREESTANDING DIVIDERS

\author{
Robert E. Hebner \\ Electrosystems Division \\ National Bureau of Standards \\ Washington, D.C. 20234
}

It is conventionally assumed that the magnitude and the waveshape of a high voltage pulse can be determined from measurements of the divider ratio, the step response, and the output voltage. This assumption has been verified experimentally by measuring the input with a second, reference divider which introduces negligible distortion in the applied waveform. The validity of the approach had been demonstrated for pulses ranging from less than a kilovolt to more than a megavolt on microsecond time scales. Specifically, in a system designed to measure pulses with peak values up to $4 \mathrm{MV}$, the differences between the calculated and measured voltages were typically less than three percent. The testing of the reference divider, the step response measurements, the data analysis and the high voltage testing of the system are described.

Key words: divider; high voltage measurements; impulse; step response.

\section{Introduction}

Standardized techniques to calibrate impulse voltage measurement systems are important in such applications as insuring that measurements made in a number of different laboratories are consistent or providing the buyer and the seller of pulsed voltage apparatus a technical basis for insuring that procurement specifications are met. In recognition of these and/or related advantages, a standard (IEEE 4) [1] has been developed to provide a consistent approach to the high voltage measurements required by the electric power industry. For pulse measurements, the fundamental approach used in this standard is to determine the divider ratio--a single real number by which the output voltage is multiplied to determine the input voltage. In addition, it is verified that the transfer characteristics are frequency independent over a frequency range suitable for the voltage pulse which is to be measured. In general, the ratio is determined using a low voltage technique, e.g., impedance measurement. The frequency independence is inferred from a measurement of the step response of the device under test. This report describes an attempt to extend the approach to a very large system by correcting the waveform using the measured step response of the item under test [2]. 


\section{Experimental Background}

The desired measurements required a variety of test and measurement circuits to be developed. The most critical component was a well-characterized divider which could be used as a reference. The divider used was a counter-wound, resistive divider rated at 1 MV in air. The divider ratio was determined under low voltage by measuring the dc voltage ratio, the resistance ratio, and the step response. The ratio was determined at high voltage by comparison with an electro-optic Kerr system.

The equivalent circuit of the divider is shown in figure 1. The high impedance element is $R_{H}$, the low impedance element is $R_{L}$, the terminator is $R_{T}, R_{1}$ and $R_{2}$ are the resistances of the cable which connects the divider to a measuring instrument, and $R_{D}$ is a damping resistor in the high voltage lead. To determine the voltage ratio, a direct voltage $V_{j}$ was applied to the input and both the input voltage and the output voltage $V_{0}$ were measured. The direct voltage ratio was verified by measuring the individual resistances $R_{D}, R_{H}, R_{L}, R_{T}$, $R_{2}$, and $R_{T}$. The step response was determined by applying a voltage step to the input and measuring the response at the output terminals. The step was generated by applying a dc voltage to the input and shorting it to ground using a mercury-wetted reed relay.

One obvious concern with the measurement of the step response is that the circuit in which the response is measured is not the same as the circuit in which high voltage pulse is measured. A fundamental reason for this difference is that while the relay is typically only a few centimeters high, the item under test can be several meters high. The different configuration leads to different capacitance and inductance associated with the high voltage lead and thus a difference in the response.

The ratio was determined at high voltage by measuring the same pulse with the divider under test and with a measurement system based on the electro-optic Kerr effect $[3,4,5]$. The Kerr system offers the advantages of different errors from dividers, optical coupling between the high and the low voltage portion of the circuit, and increased precision with increased voltage.

A summary of the ratio determinations is given in table 1 .

Table 1. Summary of Standard Divider Ratio Determinations

\begin{tabular}{lccc} 
Measurement Method & Ratio & $\%$ Deviation from Average \\
\cline { 1 - 2 } DC Voltage & $10620: 1$ & +0.2 \\
Resistance & $10620: 1$ & +0.2 \\
Step Response & $10590: 1$ & -0.1 \\
Kerr System & $10560: 1$ & -0.4
\end{tabular}




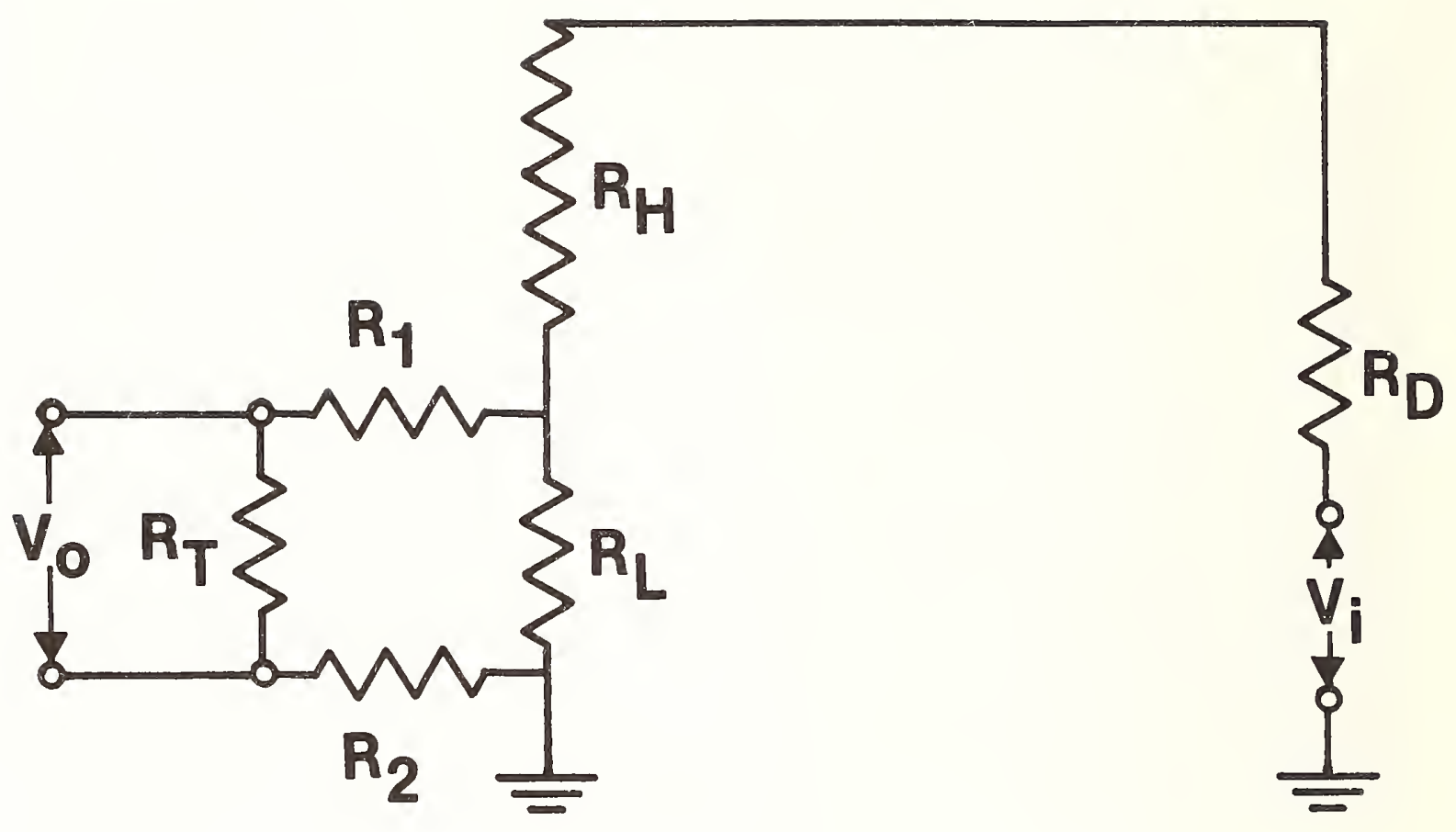

Fig. 1 Equivalent circuit of the reference divider including the damping resistor $R_{D}$, the terminator $R_{T}$, and cable resistances $R_{1}$ and $R_{2}$. 
The deviations in these data are about a factor of two larger than could be attained by very careful measurement, but are small enough for the present application.

\section{Measurements on Divider Under Test}

The experimental approach is first to measure the step response of the divider under test. Then a voltage pulse is measured using both the divider under test and the standard divider. Using the measured output from the reference divider and the step response of the divider under test, the anticipated output from the divider under test is calculated. To verify that the measured response of the divider under test is appropriate for the voltage pulse to be measured, the calculated and measured outputs are compared.

A typical test setup used to calibrate a $4 \mathrm{MV}$ divider is shown in figure 2. Typical results are summarized in table 2.

Table 2. Typical Comparison of Calculated and Measured Waveforms

\begin{tabular}{cccc}
$\begin{array}{c}\text { Time } \\
(\mu \mathrm{s})\end{array}$ & $\begin{array}{c}\text { Calculated } \\
\text { Waveform }(V)\end{array}$ & $\begin{array}{c}\text { Measured } \\
\text { Waveform }(V)\end{array}$ & $\begin{array}{c}\text { Difference } \\
\% \text { of Peak }\end{array}$ \\
\cline { 2 - 3 } 0.2 & 20.7 & 23.7 & -1.0 \\
0.4 & 61.8 & 63.0 & -0.4 \\
0.6 & 118.0 & 115.0 & +1.0 \\
0.8 & 190.0 & 184.0 & +2.0 \\
1.0 & 271.0 & 260.0 & +3.0
\end{tabular}

The calculated waveform is determined by solving Duhamel's integral,

$$
v_{0}(t)=\frac{1}{N} \int_{0}^{t} \theta^{\prime}(\tau) v_{i}(t-\tau) d \tau,
$$

where $N$ is the divider ratio, $V_{0}$ is the calculated output voltage, $\tau$ is an integration variable, $O^{\prime}(\tau)$ is the derivative of the measured step response, and $V_{i}$ is the $h i g h$ voltage applied to the divider.

The high voltage $V_{j}$ is determined by multiplying the output voltage from the reference divider by the reference divider ratio. For the measured output to agree with the calculated output, it is necessary to measure accurately the output of the reference divider, the ratio of the reference divider, the output from the divider under test and the step response of the divider under test. In a large number of measurements, the disagreement between the measured and calculated output from the divider under test ranged between $0.5 \%$ and $7 \%$ of the peak voltage. These measurements, therefore, suggest that the divider under test is suitable for the measurement of the waveshapes used if uncertainties of several percent can be tolerated. 


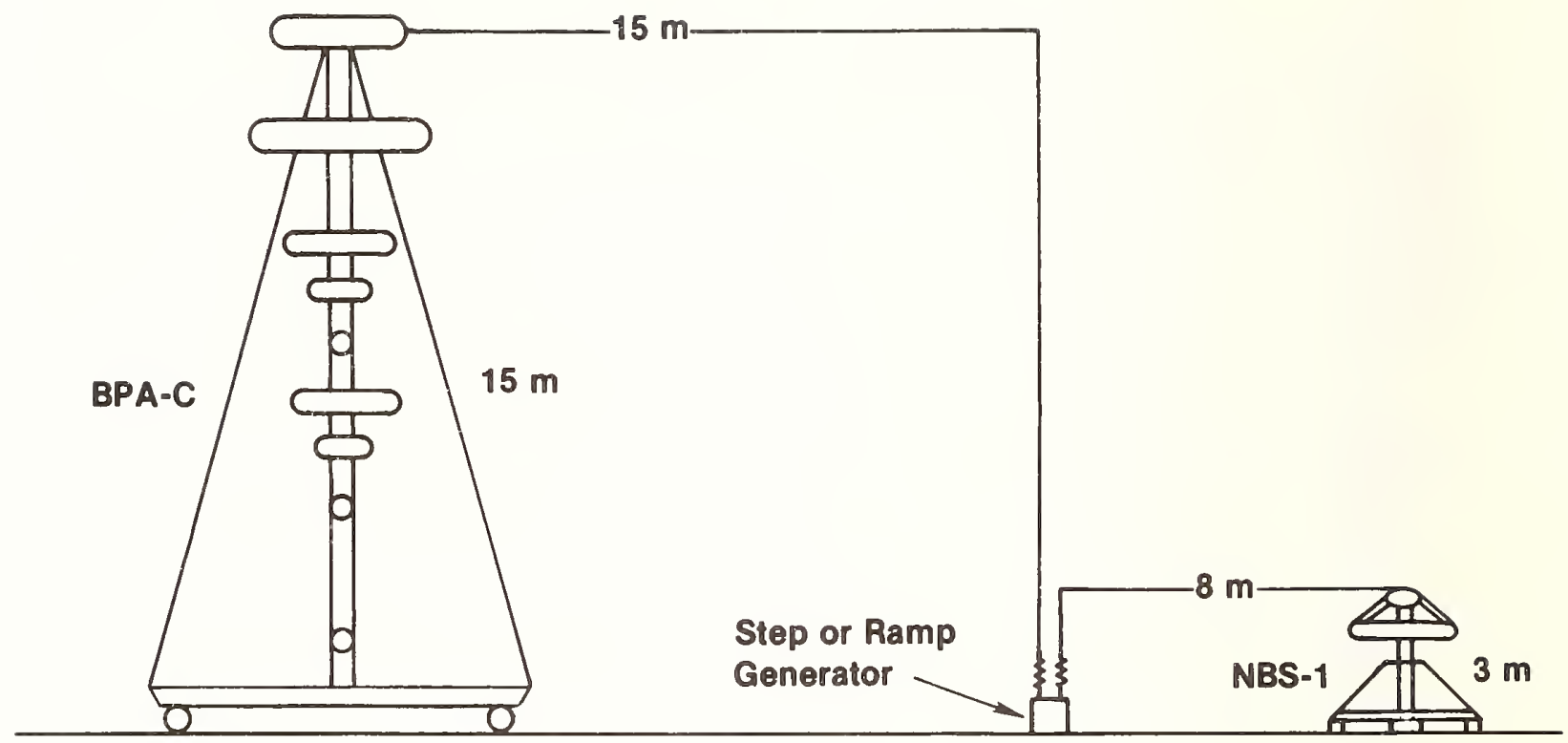

Fig. 2 Typical test set-up for step response and low-voltage ramp response measurements. 
These tests were performed using a voltage pulse which had a linear rise of about 1 us. The voltage was "chopped" at $1 \mu \mathrm{s}$, so that the input voltage rapidly (i.e., in a time short compared to $1 \mu \mathrm{s})$ fell to zero. A low voltage pulse generator, with an output impedance of $1 \Omega$ and a peak output voltage of $1 \mathrm{kV}$ was used for many of the tests. Typical measurements of a single ramp voltage are shown in figure 3 . Tests were also made at high voltage, up to about $1 \mathrm{MV}$, by using a sphere-gap to chop a standard lightning impulse near its peak. The inaccuracies in the high voltage measurements also ranged up to $7 \%$ of the peak voltage.

\section{Summary}

A technique to verify the accuracy of pulse measurement systems for front-chopped voltage waveforms, the most difficult to measure, according to the general guidelines of IEEE 4 has been applied to a 4-MV system. The approach has been verified for particular sets of conditions in interlaboratory comparisons. The technique has been used to measure pulses longer than a few microseconds and with peak voltages up to a few megavolts.

This work reviewed the extension of the approach to systems designed to measure pulses with peak values up to 4 MV. Techniques to verify accurate measurement for pulses of higher voltage and/or shorter times have yet to be developed and evaluated. The evaluation is important to prove that the verification technique is valid for a range of pulse shapes, pulse amplitudes, and circuit configurations.

\section{References}

[1] The Institute of Electrical and Electronics Engineers, Inc., IEEE Standard Techniques for High-Voltage Testing, (New York: Wiley-Interscience, 1978).

[2] R. E. Hebner, D. L. Hillhouse, and R. A. Bullock, "Evaluation of a Multimegavolt Impulse Measurement System," Nat. Bur. Stand. (U.S.), NBSIR 79-1933, 1979.

[3] E. C. Cassidy, W. E. Anderson, and S. R. Booker, "Recent Refinements and Developments in Kerr System Electrical Measurement Techniques," IEEE Trans. Instrum. Meas., Vo1. IM-21, pp 505-510, 1972.

[4] R. E. Hebner, E. C. Cassidy, and J. E. Jones, "Improved Techniques for the Measurement of High-Voltage Impulses Using the Electrooptic Kerr Effect," IEEE Trans. Instrum. Meas., Vol. IM-24, pp 361-366, 1975.

[5] R. E. Hebner, R. A. Malewski, and E. C. Cassidy, "Optical Methods of Electricai Measurements," Proc. IEEE, Vol. 65, pp 1524-1548, 1977. 


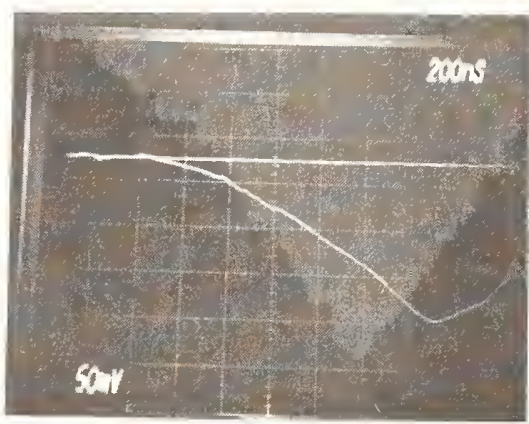

\section{Low Voltage Ramp Measurements}

$B P A=C$

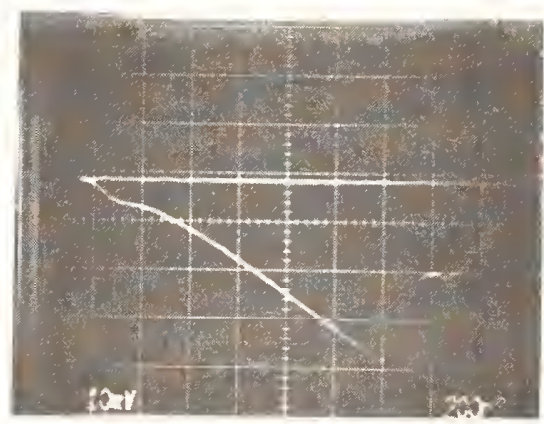

NBS-1

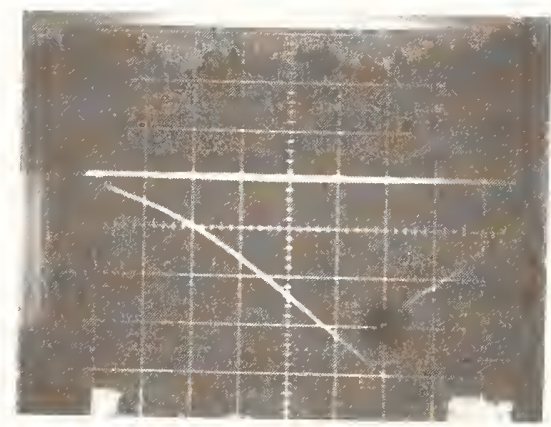

\section{Direct}

Fig. 3 The low voltage measured using the divider under test $(B P A-C)$, the reference divider $(N B S-1)$ and direct measurement without an external divider. 
6. Sponsor Acknowledgement

Partial support for this work was obtained from the Bonneville Power Administration, U.S. Department of Energy. 


\section{A COMPARISON OF THREE DIFFERENT DESIGNS OF RESISTOR DIVIDER}

T.R. McComb and M.M.C. Collins,

National Research Council of Canada, Division of Electrical Engineering, Ottawa, Ontario, Canada, K1A OR8
W.J. Sarjeant*

University of California, Electronics Division, Los Alamos National Lab., Los Alamos, N.M., 87545

This paper describes a comparison of dividers developed in the Laser Physics Section at NRC, with dividers developed in the Power Engineering Section, NRCC. Requirements on the design of high voltage impulse measuring systems, which are to be used to measure short-duration impulse voltages, are discussed briefly using transmission line concepts. Developments in the field of Power Engineering are summarized and the classification of impulse measuring systems into two-component and three-component systems is outlined. Four different dividers, whose response times lie in the range $1.4-15 \mathrm{~ns}$, are described. Comparative measurements of the crest value of linearly-rising impulses chopped on the front have been made at a single voltage; these measurements have been corrected for the effects of system response time. Measurements of the crest value of a trapezoidal pulse have been made using three of these dividers and the results are presented in the form of a calibration curve covering the range $100-400 \mathrm{kV}$. Estimates of the correction needed to allow for the dividers' response times are calculated on the basis of rectilinear approximations to the trapezoidal pulse.

Key Words: Impulse measuring systems; resistor dividers; comparative measurements; design; dividers; voltage measurement; response time errors.

\section{Introduction}

Research over the past 30 years has led to the development of new International and American Standards [1,2] on high voltage test techniques. The recommendations of these standards for impulse measuring

* Formerly with National Research Council of Canada, Division of Physics, Ottawa, Ontario, Canada, K1A OR6 
systems are based on the measurement of the Standard Lightning Impulse whose waveform is given by

$$
v=k\left(e^{-\alpha t}-e^{-\beta t}\right)
$$

where $\alpha=1 / 68.5 \mu \mathrm{s}$ and $\beta=1 / 0.405 \mu \mathrm{s}$ and $k$ is the efficiency of the generator.

This gives a virtual front time ( 1.67 times the time interval between the instants when the impulse is $30 \%$ and $90 \%$ of crest value) of $1.2 \mu \mathrm{s}$ and a time to half-value of $50 \mu \mathrm{s}$. The accuracies of measurement required by these standards are crest voltage $\pm 3 \%$, front time $\pm 10 \%$ and time to half-value $\pm 10 \%$. Accepted procedures for, and advice on, meeting these requirements are given in the standards.

The purpose of an impulse measuring system is to provide a reduced copy of the input voltage waveform and this requires that the ratio of the system is constant throughout the frequency range of interest. The design of a satisfactory measuring system entails determining this frequency range and ensuring that the ratio of the system is constant for this range. In practical circuits high voltage impulses are generated using switching gaps and these gaps generate fast pulses which propagate through the circuit. Whether oscillations are generated and how long they persist is mainly determined by the reflection coefficients in the system. A full analysis of the circuit involves treating interconnections as transmission lines and treating apparatus as transmission lines for twice the travel time of the apparatus $[3,4,5]$.

An impulse measuring system can be thought of as a two component system - a transmission line (the high voltage lead) and a termination (the divider, recorder, and the cable interconnecting them). The divider impedance will not usually be equal to the line impedance and hence there will be a reflection generated at the divider which will lead to oscillations in the circuit. These can be reduced by the insertion of a terminating resistor in the high voltage lead at the test object and this gives a three component system. When the terminating resistor matches the surge impedance of the lead no oscillations will be generated in the measuring system.

\section{Errors Caused by the Response Time of the System}

Because of the response time of the measuring system the output will not immediately follow the input. There is no general method of applying a simple correction for this. In the special case of a linearly rising voltage it can be shown that the error in amplitude $\delta(t)$ at time $(t)$ is given by

$$
\delta(t)=S \int_{0}^{t}[1-g(t)] d t
$$


[Appendix $4 B$ of reference 2] where $S$ is the rate of change of voitage and $g(t)$ is the normalized step response. After a sufficient time, known as the settling time, $\delta(t)$ is approximately equal to $\delta(\infty)$. For any linearly rising voltage which lasts longer than the settling time the value of $S$ can be read from the output and the necessary correction calculated.

Standards impose further restrictions on the response characteristics when the system is used to measure impulses chopped on the front. The initial distortion time ( $T_{0}$ - $i l l u s t r a t e d$ in Figure 1 ) must be less than one twentieth of the time to chopping.

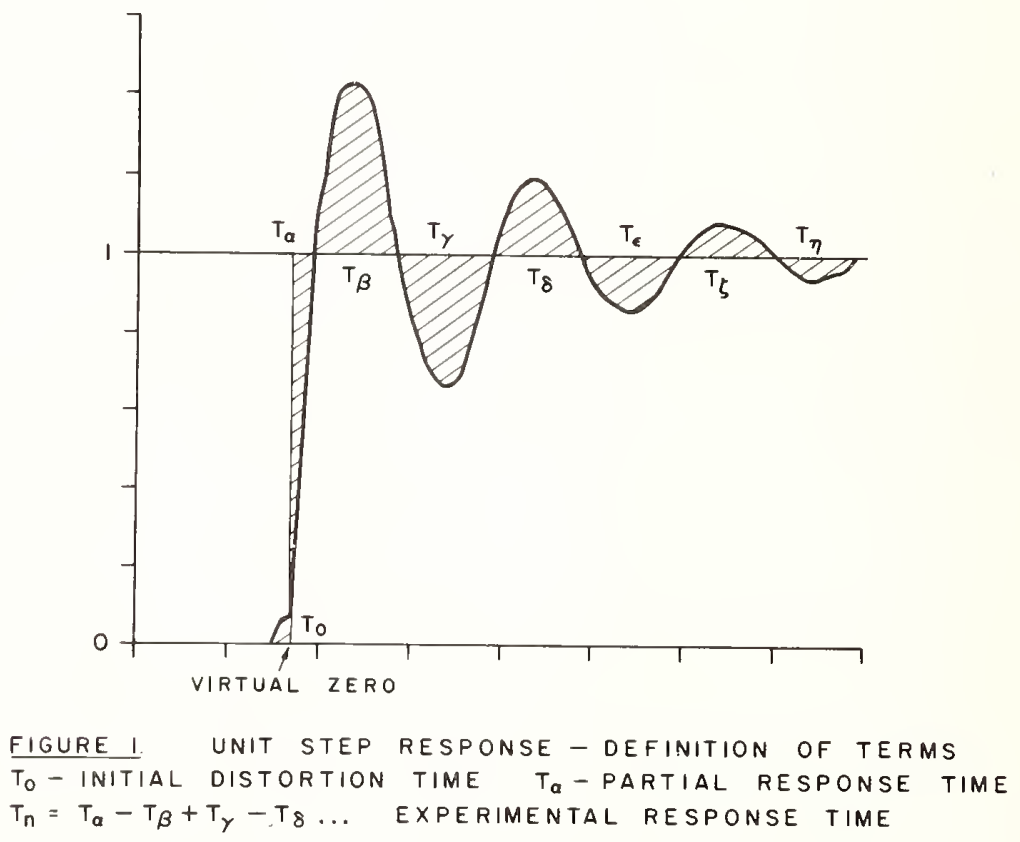

3. Design of Resistor Dividers

\subsection{Principles of Design}

An ideal resistor divider would have a high voltage arm and a low voltage arm whose impedances are purely resistive in the frequency range of interest.

In practice this condition cannot be achieved for the high voltage arm because the resistor will have distributed stray capacitance to ground. For a resistor mounted vertically on the ground plane the distribution of capacitance is non-linear and the stray capacitance of the lower parts of the high voltage arm will have to charge through the upper parts of the resistor giving a long settling time. The addition of a shielding ring or plate at the top of the high voltage arm will provide a capacitative path for this charging current. The shape and 
position of the shield (or shields) can be adjusted to give a more uniform field distribution which not only improves the response characteristics but also increases the voltage rating [6].

A purely resistive low voltage arm is readily achieved by using a good quality, high frequency, coaxial cable terminated in a resistor whose resistance is equal to the surge impedance of the cable. When direct input to the deflection plates of the oscilloscope is used, as in this work, a damping circuit must be inserted between the oscilloscope plates and the cable terminating resistor.

The total resistance of the divider must be sufficiently high to avoid excessive loading and to provide a suitable division ratio. However, the distribution of stray capacitance of a well designed screened divider will still have some non-linearity and this residual effect will cause the divider response time to increase with divider resistance. A nominal value of $25 \Omega / \mathrm{kV}$ [6] offers a practical compromise on the conflicting requirements of acceptable loading and fast response time.

\section{$3.2^{\circ}$ Resistor Dividers}

These principles have been applied to the design of four dividers which are shown in Figure 2. The low voltage arm for each of these dividers was $30 \mathrm{~m}$ of RGT7/U terminated in an $8 \mathrm{~W} 52 \Omega$ carbon composition resistor.

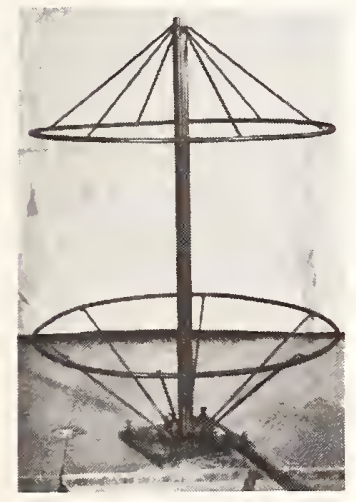

(a)

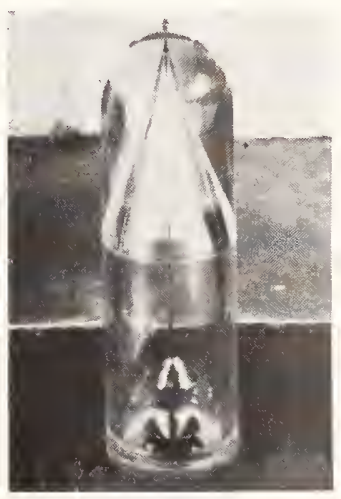

(b)

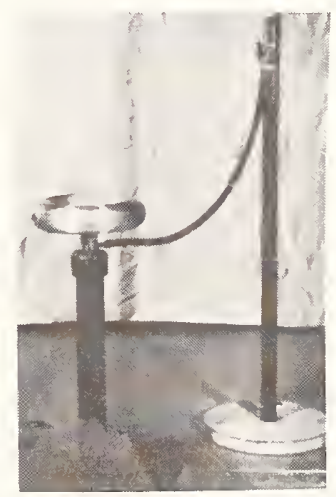

(c)

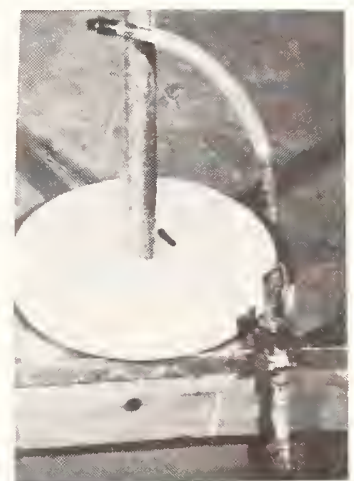

(d)

\section{FIGURE 2. RESISTOR DIVIDERS}

(a) WIRE - WOUND, AIR - INSULATED DIVIDER

(b) WIRE - WOUND, PRESSURIZED DIVIDER

(c) SILICON CARBIDE DIVIDER

(d) COPPER SULPHATE DIVIDER WITH MINI-PROBE 


\subsubsection{Power Engineering Section Dividers}

The wire-wound, air-insulated divider shown in Figure 2(a) was designed to measure full lightning impulses and lightning impulses chopped near crest [7]. The high voltage arm was a wire-wound resistor with a non-inductive winding. Conical shields were mounted at the top and at the bottom of the high voltage arm. The divider is rated at $350 \mathrm{kV}$ for standard lightning impulses.

The pressurized divider shown in Figure 2(b) was designed to measure chopped impulses with risetimes of from $50 \mathrm{~ns}$ to $1 \mu \mathrm{s}$ [7]. This divider is a faster version of the air-insulated divider and was made by reducing the divider size by a factor of eight and insulating it in is an atmosphere of nitrogen at a pressure of $300 \mathrm{kPa}$ [7]. The pressurized divider is rated at $350 \mathrm{kV}$ for impulses chopped at 1 us .

\subsubsection{Laser Physics Section Dividers}

Two different dividers were developed for.use in pulsed laser systems to measure impulses which are typically trapezoidal in shape and have durations from 100 ns to $5 \mu \mathrm{s}$. One of these used a silicon carbide resistor as the high voltage arm, shown in Figure 2(c), while the other used a copper sulphate resistor, shown in Figure 2(d).

The silicon carbide divider used a Union Carbide Type AS $6.8 \mathrm{k} \Omega$ resistor which was impregnated with, and contained in, a silicon dielectric fluid (Dow Corning DC-200, viscosity $20 \mathrm{~mm}^{2} / \mathrm{s}$ ) to reduce local damage at high voltage stress. The resistor length was $0.45 \mathrm{~m}$ and the value after impregnation was $10 \mathrm{k} \Omega$. Bulk silicon carbide resistors offer high power dissipation and low inductance but they can be nonlinear at high stress levels. This divider was used with a flat shield and a damping lead which consisted of six Allan Bradley type HB $2 \mathrm{~W}$ carbide composition resistors each $10 \Omega$ and each separated by 6 in. of \#22 AWG wire and encased in Tygon tubing which had been filled with silicon oil. This divider was rated at $300 \mathrm{kV}$ for pulses shorter than 5 us.

The copper sulphate divider was a two-stage divider with the second stage using the terminated cable as a low voltage arm. The high voltage arm of this divider consisted of a solution of copper sulphate in distilled water contained in a length $(0.3 \mathrm{~m})$ of Tygon tubing $\left(\frac{1}{4}\right.$ in. I.D.). Connections were made by immersing cylindrical rolls of high purity copper wire gauze in the solution and the solution concentration was adjusted to give a resistance of $3.0 \mathrm{k} \Omega \pm 5 \%$. The low voltage arm was a mini-probe [8] in parallel with one or more $330 \Omega$ carbon $2 W$ resistors as required to limit the voltage on the mini-probe to less than $20 \mathrm{kV}$. The high voltage arm of the mini-probe consisted of a lossy transmission line terminated in a carbon $2 \mathrm{~W}$ resistor. This resistor was partly covered by a grounded shield and the shielding was adjusted to give the best response time (estimated as $30 \mathrm{ps}$ [8]). When immersed in oil, this two-stage divider was rated at $400 \mathrm{kV}$ for pulses shorter than 1 us. 


\section{Impulse Measuring Systems}

\subsection{Common Components}

The cables, matching circuits, recording units, and ground returns were the same for all systems used. Each cable was $30 \mathrm{~m}$ of RG17/U terminated in a low-inductance 'cartwheel', made from four $220 \Omega(10 \%)$ carbon $2 W$ resistors selected to give a $52 \Omega$ total resistance. Experience indicates that these resistors must be pulse conditioned before use. Oscillations in the connections between the terminating resistance and the oscilloscope deflection plates are damped by the circuit. The surge oscilloscopes were Southern Instrument (circa 1960) osci110scopes which had been extensively modified at NRCC to improve their shielding and Tinearity [9].

\subsection{Matching Resistor}

The ordinary type of non-inductive resistor, obtained by winding two resistors on the same former in opposite directions, was unsatisfactory when used as a matching resistor in a three component system because of its residual inductance. A satisfactory design [3] was achieved by the use of four such resistors in parallel, arranged in the form of the spokes in a wheel, the hub forming one terminal and the rim the other. To reduce the stress on each resistor, two of these cartwheels were used in series.

\subsection{Grounding}

The entire circuit was mounted on a large ground plane $(7.8 \mathrm{~m}$ by $13.5 \mathrm{~m}$ ) which consisted of interconnected rectangular sheets ( $1.3 \mathrm{~m}$ by $2.7 \mathrm{~m}$ ) of brass or aluminum. These sheets could be easily moved and clipped together to give a ground plane appropriate to the circuit in use. Each oscilloscope was grounded directly by the sheath of the RG $17 / \mathrm{U}$ to the ground plane at the base of the divider being used with it and indirectly by the stray capacitance to ground. Each oscilloscope was isolated from the line by inserting a choke in each power lead and by an isolation transformer. No information is available on the nature of the danger posed by fast pulses travelling through ground systems although this is a common problem in high voltage laboratories and gasinsulated sub-stations. In this work the operator was either isolated from other grounds or from the oscilloscope during the impulse discharge time.

\subsection{Description of Systems}

The main features of each system are given in Table 1. The wirewound, air-insulated divider and the pressurized divider were used in three component systems with a high voltage lead of bare wire (\#14 AWG). Both the laser physics dividers were designed to be used as voltage probes without any additional leads and they were used in this manner for some of the measurements. For the calibration with trapezoidal 
impulses they were used with an additional wire lead to ensure that there were no proximity effects.

\section{TABLE 1 IMPULSE MEASURING SYSTEMS}

\begin{tabular}{|c|c|c|c|c|}
\hline $\begin{array}{c}\text { System } \\
\text { Identification }\end{array}$ & $\begin{array}{l}\text { Divider } \\
\text { Used }\end{array}$ & $\begin{array}{l}\text { No. of } \\
\text { Components }\end{array}$ & $\begin{array}{c}\text { Terminating } \\
\text { Resistor } \\
(\Omega)\end{array}$ & $\begin{array}{l}\text { Length of } \\
\text { H.V. Lead } \\
\quad(\mathrm{m})\end{array}$ \\
\hline I & $\begin{array}{l}\text { Wire-wound, } \\
\text { Insulated in high } \\
\text { pressure Nitrogen }\end{array}$ & 3 & 215 & 2.1 \\
\hline $\begin{array}{l}\text { I I }(\mathrm{a}) \\
(\mathrm{b})\end{array}$ & $\begin{array}{l}\text { Wire-Wound } \\
\text { Air-Insulated }\end{array}$ & 3 & $\begin{array}{l}215 \\
235\end{array}$ & 2.1 \\
\hline III & $\begin{array}{l}\text { Wire-Wound } \\
\text { Air-Insulated }\end{array}$ & 3 & 432 & 1.8 \\
\hline IV & Silicon carbide & 2 & - & $\begin{array}{l}\text { No addition- } \\
\text { al Lead }\end{array}$ \\
\hline V & Silicon Carbide & 2 & - & 2.4 \\
\hline VI & $\begin{array}{l}\text { Copper Sulphate } \\
\text { and secondary } \\
\text { divider }\end{array}$ & 2 & - & $\begin{array}{l}\text { No addition- } \\
\text { a] Lead }\end{array}$ \\
\hline VI I & $\begin{array}{l}\text { Copper Sulphate } \\
\text { and secondary } \\
\text { divider }\end{array}$ & 2 & - & 2.3 \\
\hline
\end{tabular}

5. Measurements

\subsection{Response Time Measurements}

A voltage step with a risetime of less than 1 ns was applied to each impulse measuring system using a mercury-wetted reed relay as prescribed in the standards [1,2]. The response was recorded using a Tektronix WP2200 Transient Digitizer System which converted the analog signal into 9 bit words with 512 words per sweep at sweep speeds down to 5 ns. This system was used to normalize the responses and integrate the unit step response to give the response parameters listed in Table 2 . Corrections for the travel times of the additional vertical lead were made using standard procedures [1,2]. Measurements were also made using a high voltage step generated with a high pressure chopping gap [7].

\subsection{Ramp Measurements}

A linearly rising pulse was generated using the circuit shown in Figure 3. This circuit was laid out in an approximate straight line and the reference measuring system was connected in the same line. The other measuring systems were connected. one at a time, at right angles to the reference system to minimize coupling betweer the measuring systems. 


\section{TABLE II RESPONSE CHARACTERISTICS}

$\begin{array}{lccc}\text { System } & \begin{array}{c}\text { Response Time } \\ \left(\int_{0}^{T} S[1-g(t)] d t\right)\end{array} & \begin{array}{c}\text { Settling Time } \\ \left(T_{S}\right)\end{array} & \begin{array}{c}\text { Rise Time } \\ \left(T_{10 \%}-T_{90 \%}\right)\end{array} \\ & n s & n s & n s \\ \text { I } & -2.0 & 65 & 1.7 \\ \text { II (a) } & 7.6 & 85 & 12 \\ \text { (b) } & 7.8 & 40 & 28 \\ \text { II I } & 10 & 40 & 19 * \\ \text { IV } & 4.5 & 200 & 3 \\ \text { V } & -3.5 & 15 & 1.7 \\ \text { VI } & 1.5 & 280 & 2.5 \\ \text { VII } & -6.3 & & \end{array}$

* equivalent partial response time [2] is $0.3 \mathrm{~ns}$

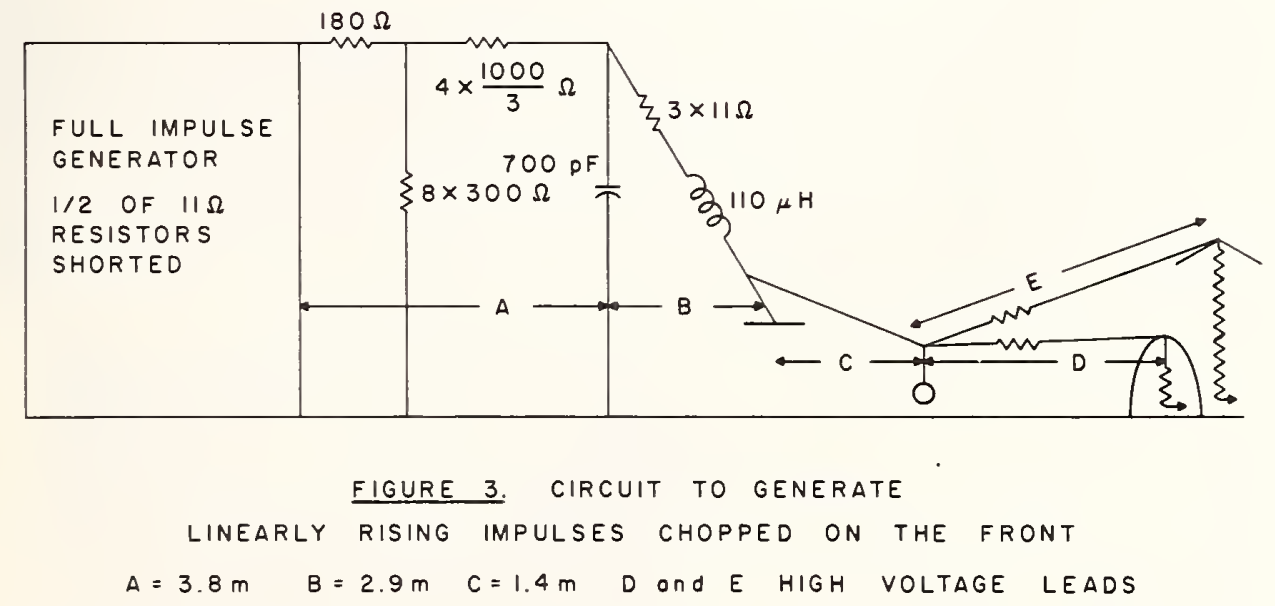

Ramp measurements were performed for linearly rising pulses with crest values of $\pm 100 \mathrm{kV}$ and response time corrections were made. Illustrative records are shown in Figure 4 and results are given in Table III.

\subsection{Calibration With Trapezoidal Impulses}

For a comparative calibration to be of value, it must be carried out with the impulse shapes with which the divider being calibrated is to be used. The usual working pulse for the laser physics dividers is trapezoidal in shape, with extremely fast risetimes and falltimes, achieved by using oil-immersed gaps for chopping. Similar pulses are generated by the circuit shown in Figure 5 but the risetimes and falltimes of the pulse are slower than those of the working pulse because of 
the increased breakdown time of the air gaps. As the crest voltage was increased the trapezoidal pulse became more triangular in form and this is illustrated in Figure 6. Comparative values are plotted in Figure 7. By using rectilinear approximations to the impulse the approximate size of response time errors can be calculated and these corrections are indicated in Figure 7.

\section{TABLE II I RAMP MEASUREMENTS - CORRECTED MEASUREMENTS}

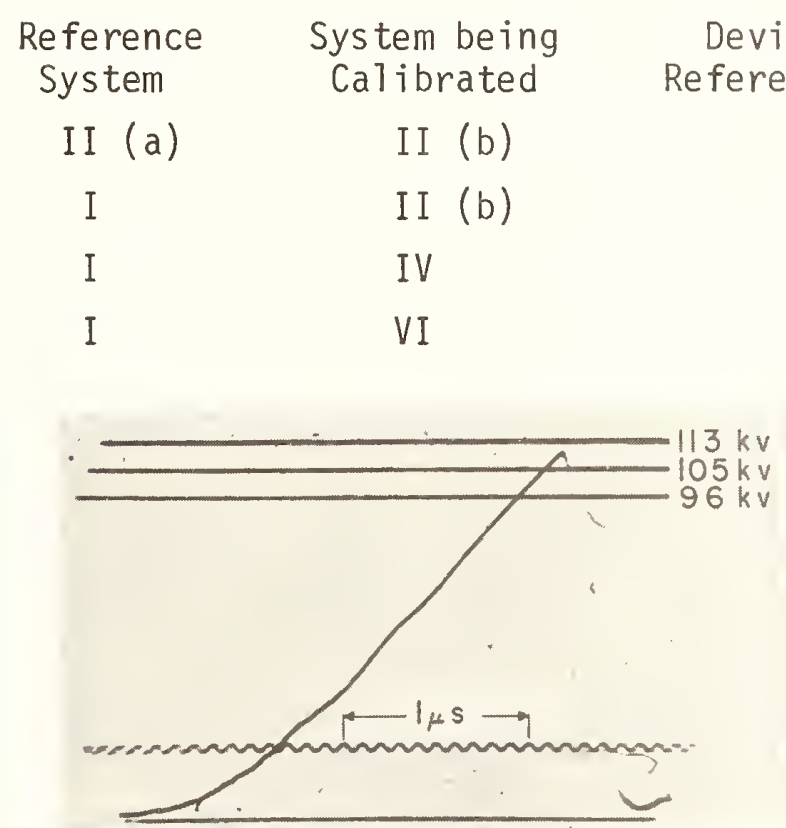

(a)

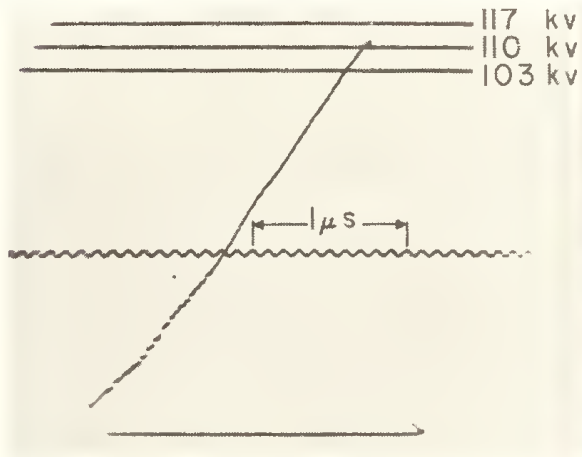

(c)

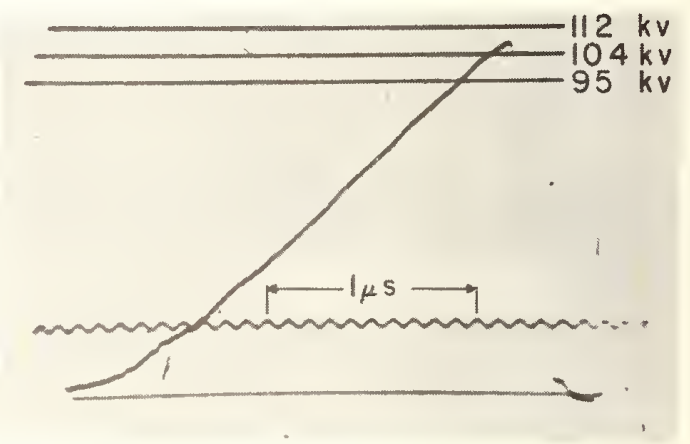

(b)

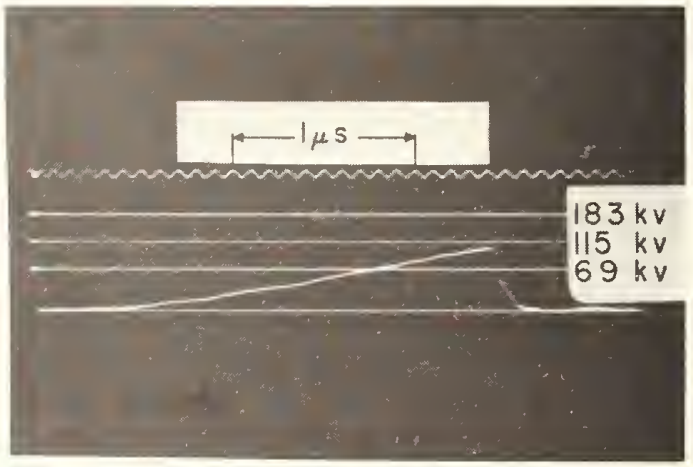

(d)

FIGURE 4. RAMP MEASUREMENTS

(a) WIRE-WOUND, AIR INSULATED DIVIDER

(b) WIRE - WOUND, PRESSURIZED DIVIDER

(c) SILICON CARBIDE DIVIDER

(d) COPPER SULPHATE DIVIDER WITH MINI-PROBE 


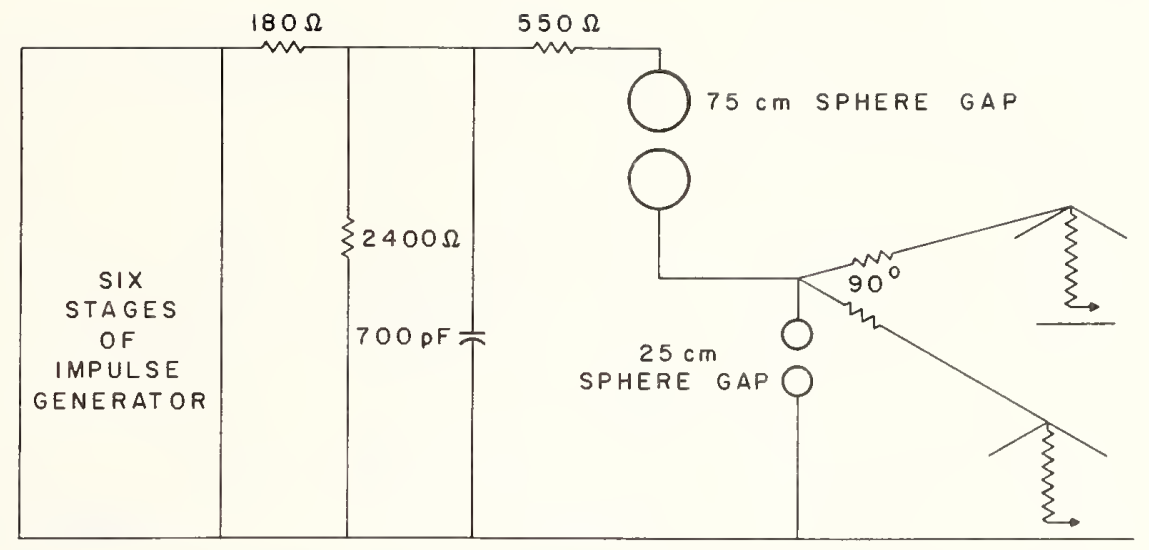

FIGURE 5. CIRCUIT TO GENERATE TRAPEZOIDAL IMPULSES

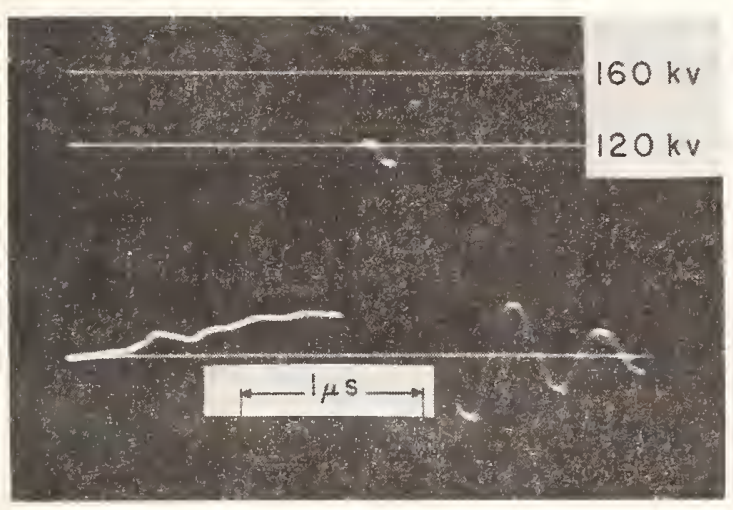

(a)

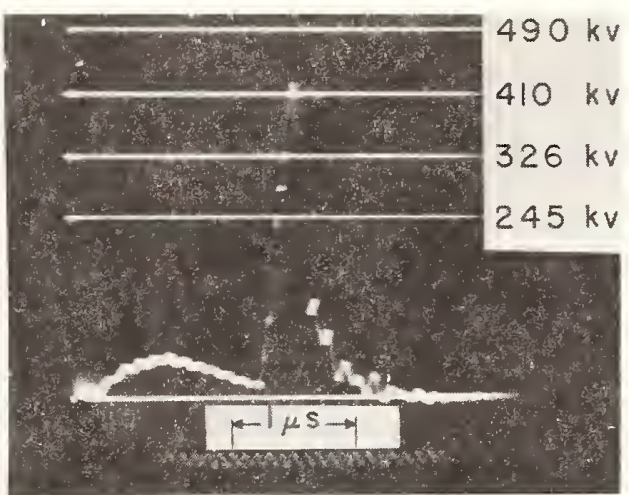

(b)

\section{FIGURE 6. CALIBRATION IMPULSES}

$\begin{array}{ll}\text { (a) TRAPEZOIDAL (b) TRIANGULAR } & \text { (b) TRUA }\end{array}$

\section{Calibration and Accuracy}

Each oscillogram is calibrated by the addition of a baseline and one or more voltage lines supplied from a dc calibration source whose total error is $<0.03 \%$. The non- linearity of the screen is $<1 \%$ for deflections of $< \pm 300 \mathrm{~V}$. The effect of this non-linearity can be further reduced by using two calibration lines which straddle the crest value.

The oscillograms were read on an optical comparator at a magnification of 10 . The resolution of the optical comparator was $10^{-4}$ inches and the accuracy was approximately $2 \cdot 10^{-4} \mathrm{in}$. Reproducibility of a single setting was $4 \times 10^{-4}$ in. This led to approximately $0.1 \%$ maximum reading error for peaks which caused a deflection of approximately half 


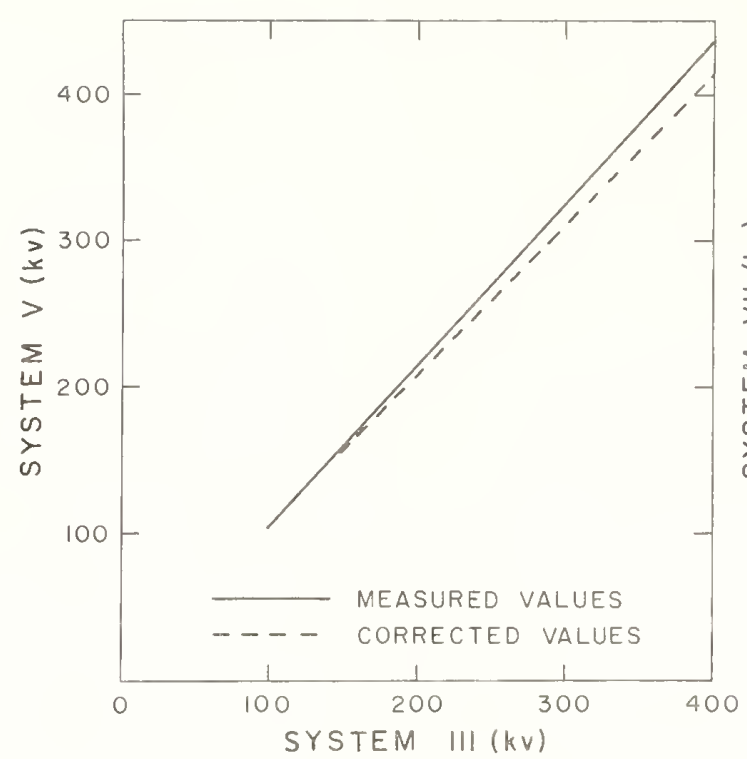

(a)

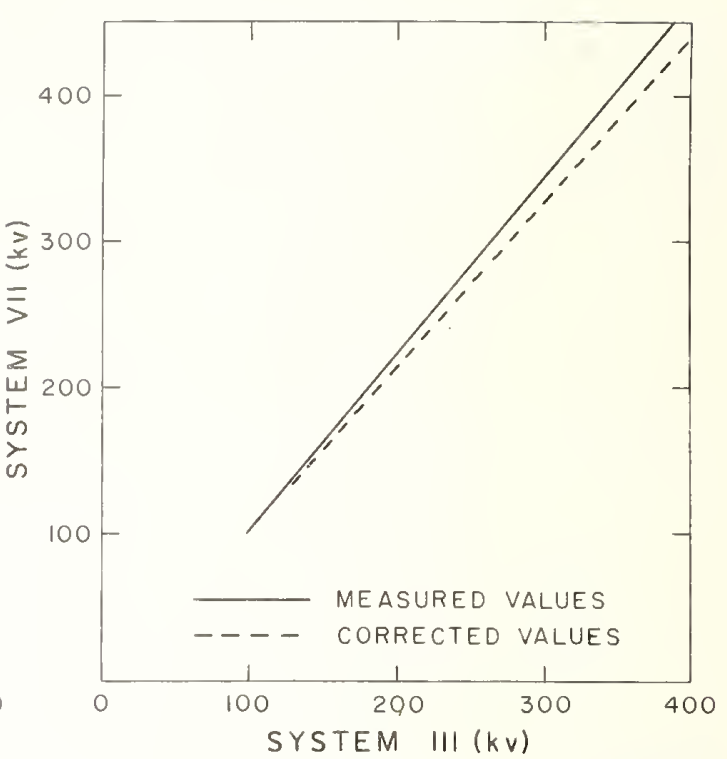

(b)

(a) SILICON CARBIDE DIVIDER

(b) COPPER SULPHATE DIVIDER WITH MINI-PROBE

full scale. System error has been evaluated by comparing measurements made by two versions of System II. The measurements by the quasiidentical systems disagreed by an average value of $0.8 \%$ with a standard deviation of $0.5 \%$. This error has been shown to be caused by the oscilloscopes. The standard deviation is caused mainly by the ripple on the high voltage supply to the CRO tube but no explanation has been found for the average discrepancy being non-zero. This error is smal1 compared with the errors caused by the response time of the calibration with the trapezoidal pulse. For the corrected values obtained from the ramp measurement this difference in the oscilloscope is significant.

Each oscillogram was calibrated with approximately 30 cycles of a stable oscillation at a suitable frequency. This reduced the error in the time scale to approximately $1 \frac{1}{2} \%$ of the full scale deflection. The total error in the response times is estimated at approximately $5 \%$.

\section{Conclusions}

These measurements show that, for the case of linearly rising impulses, whose duration is longer than the settling time of the measuring system, corrections can be made for errors caused by the response time of the measuring system. These corrections give results which agree to within 2 to $4 \%$. However for trapezoidal and triangular impulses with oscillations no simple correction can be made and differences as high as $15 \%$ at $400 \mathrm{kV}$ have been measured. Approximate calculation of errors caused by the response time give values as high as $7 \%$ but these can only be taken as indicative of the order of magnitude of the corrections needed. 


\section{References}

[1] International Electrotechnical Commission - Standard 60 - High Voltage Test Techniques.

[2] IEEE Standard Techniques for High Voltage Testing. IEEE Standard 4 - 1978.

[3] F.C. Creed, T. Kawamura and G. Newi, Step Response of Measuring Systems for High Impulse Voltages. IEEE Transactions on Power Apparatus and Systems, PAS-86, No. 11, pp. 1408-1420, Nov. 1967.

[4] F.C. Creed and M.M.C. Collins, The Systems Concept in Generating High-Voltage Impulses. IEEE Transactions on Power Apparatus and Systems, PAS-89, No. 7, pp. 1383-1387, Sept/0ct. 1970.

[5] F.C. Creed and M.M.C. Collins, Transient Impedance of High-Voltage Impulse Generating Systems. IEEE Transactions on Power Apparatus and Systems, PAS-89, No. 7, pp. 1387-1393, Sept/0ct. 1970.

[6] P.F. Goosens and P.G. Provoost, Sources of Error in the Recording of High Surge Voltages by Means of the Cathode-Ray Oscillograph. Bull. Schweiz: Elektrotechn. Verein, 37(7), pp. 175-184, 1946. Translated in NRC Report TT-552.

[7] F.C. Creed and M.M.C.Collins, The Measurement of Short-Duration Impulse Voltages. IEEE Transactions on Communications and Electronics, CE69, pp. 621-630, 1963.

[8] W.J. Sarjeant and A.J. Alcock, High-Voltage Probe System with Subnanosecond Rise Time. Rev. Sci. Instrum. Vol. 47, No. 10, pp. 12831287, October 1976.

[9] F.C. Creed, An Improved Cathode-Ray Oscillograph for Impulse Recording. Bulletin of the Radio and Electrical Engineering Division, National Research Council of Canada, Vol. 11, pp. 7-11, Oct/Dec. 1961 . 


\author{
A 100-kV, 2-ns RISETIME, dc-COUPLED PROBE \\ J. Power, W. Nunnally, and D. Young \\ Los Alamos National Laboratory \\ Los Alamos, New Mexico 87545
}

\begin{abstract}
A high-voltage probe has been designed and constructed; it is unique because it may be used for both high-voltage dc and for transient measurements. The probe is rated at $100-\mathrm{kV}$ dc with a short transient rating of 150-kV peak. System risetime is less than $2 \mathrm{~ns}$. The input impedance is 1000 Mis shunted by $13 \mathrm{pF}$. A distributed RC network providing the desired attenuation is housed in a $25.4-\mathrm{cm}-d i a m$ cylindrical housing. A 15.2-m length of coaxial cable connects the probe to a termination network at the oscilloscope input. The oscilloscope input impedance must be at least $100 \mathrm{k} \Omega$ to maintain the correct dc attenuation ratio.

Key words: dc-coupled probe; 100-kV rating; 2-ns risetime

\section{Introduction}

A high-voltage probe was needed to study the electrical breakdown characteristics of various gases. The measurements to be made required a 100-kV probe with a risetime of about 2 ns and with dc response. Therefore, an effort to develop such a probe was initiated.

Most high-voltage probes are either resistive or capacitive dividers, as illustrated in Figure 1. Both of these type of probes can be built to have good risetimes, but neither is appropriate for both dc and fast transient measurements. For good transient response, the resistive divider must be made to have a resistance value that is far too low for high-voltage dc measurements. Purely resistive and capacitive probes are both simple types of a more general divider in which the voltage division is accomplished by any arbitrary network such that the division ratio is constant as a function of frequency. A new probe was developed based on the simple schematic in Figure 2. There are essentially two probes in parallel, a high-resistance dc probe and a much lower impedance, RCcoupled ac probe. The probe is most easily understood by considering it as made of three similar sections. First is the high-voltage section
\end{abstract}




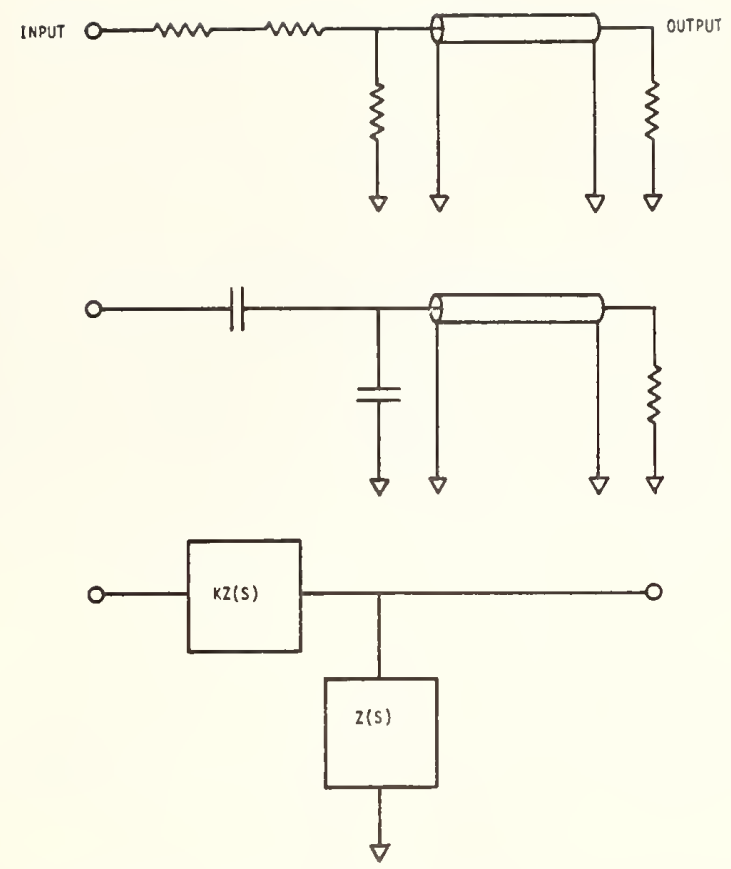

Figure 1. Resistive, capacitive, and general dividers.

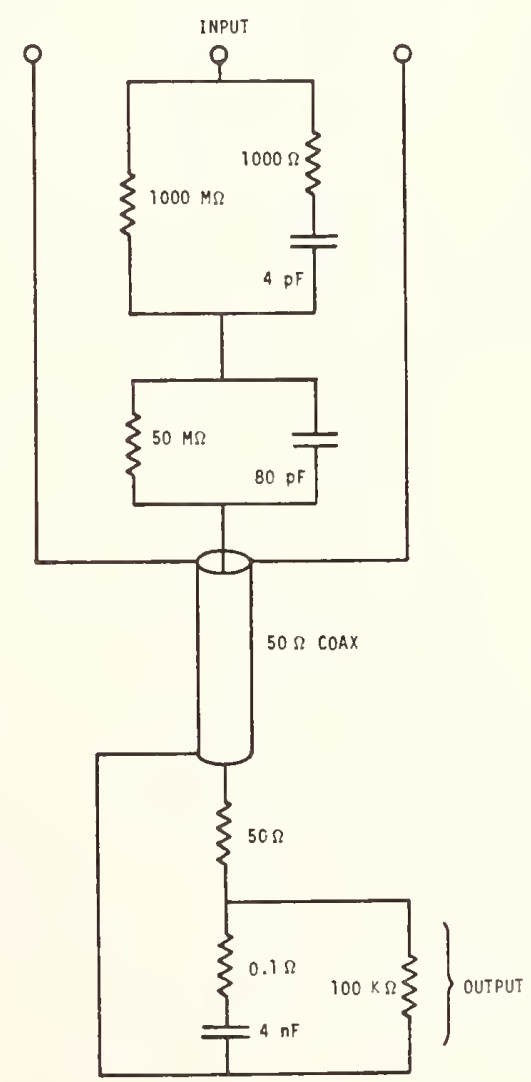

Figure 2. Simplified probe schematic. 
consisting of the 1000-M $\Omega$ resistor in parallel with the $1000-\Omega$ resistor and the 4-pF capacitor. Section two is a coupling network including the coaxial output cable. Section three is the termination and the output is viewed across this section with a high input impedance oscilloscope. The dc portion, consisting of the high-resistance components, functions as a standard resistive divider. The ac probe is made up of three RC networks in series, each having the same time constant, and hence, the division ratio is constant as a function of frequency. There are no small resistors and capacitors rated to $100 \mathrm{kV}$, so the high-voltage section is really 10 identical sections in series, as shown in Figure 3 . The highvoltage section is housed in a 48.3-cm-1ong, 25.4-cm-diam aluminum cylinder and must be viewed as a distribution network for proper high-frequency analysis.

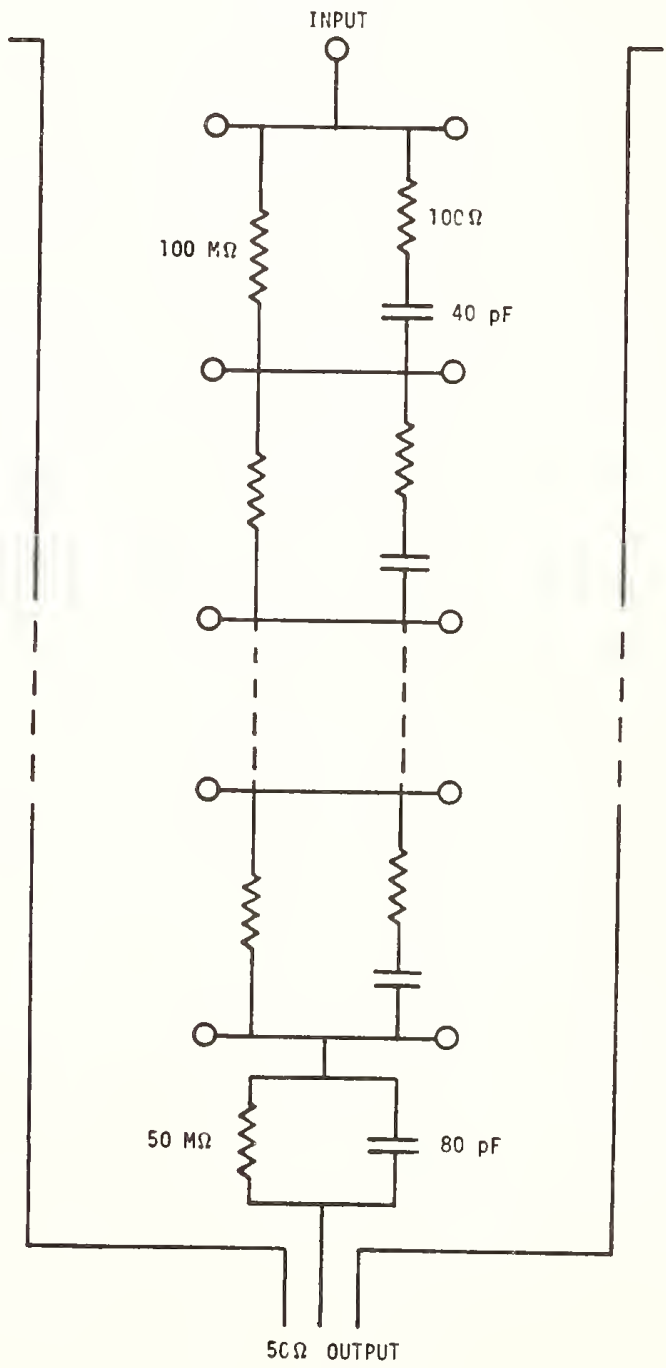

Figure 3. High-voltage probe divider network. 


\section{Computer Analysis}

The probe was simulated using a NET-2 circuit analysis program. Figure 4 shows the model used for each of the 10 high-voltage sections, as well as the input and output sections. The model also included a 15.2-m-long, 50- $\Omega$ transmission line, which is not shown in Figure 4. Pertinent stray capacities and inductances are included in the analysis. The component values of the ac portion of the probe were optimized based on the computer prediction response. Figure 5 shows the predicted output using a 300-ps risetime, Gaussian input pulse. The probe is designed to drive a high input impedance oscilloscope plug-in with a limited bandwidth; hence, the initial ringing at about $1200 \mathrm{MHz}$ is not observed. Based on the computer output, one would expect a minimum risetime of about 1 ns.

\section{Prototype Testing}

A prototype probe was built to test the computer predictions.

\subsection{Probe Components}

Standard components are used in the high-voltage divider. The capacitors are 40-pF, 5-kV, NPO ceramic rf capacitors, which were tested

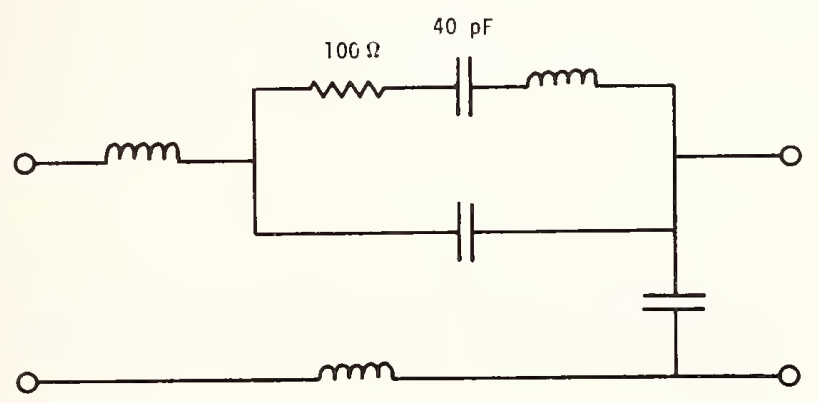

HIGH FREQUENCY

MODEL FCR INDIVIDUAL

PROBE DIVIDER SECTION

PULSE GENERATOR AND

INPUT COUPLING

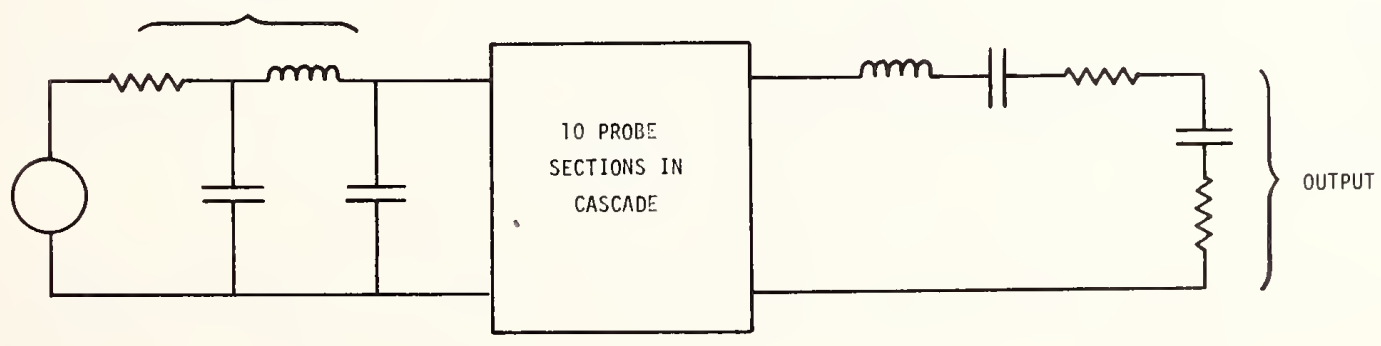

Figure 4. Model used for probe high-frequency analysis. 


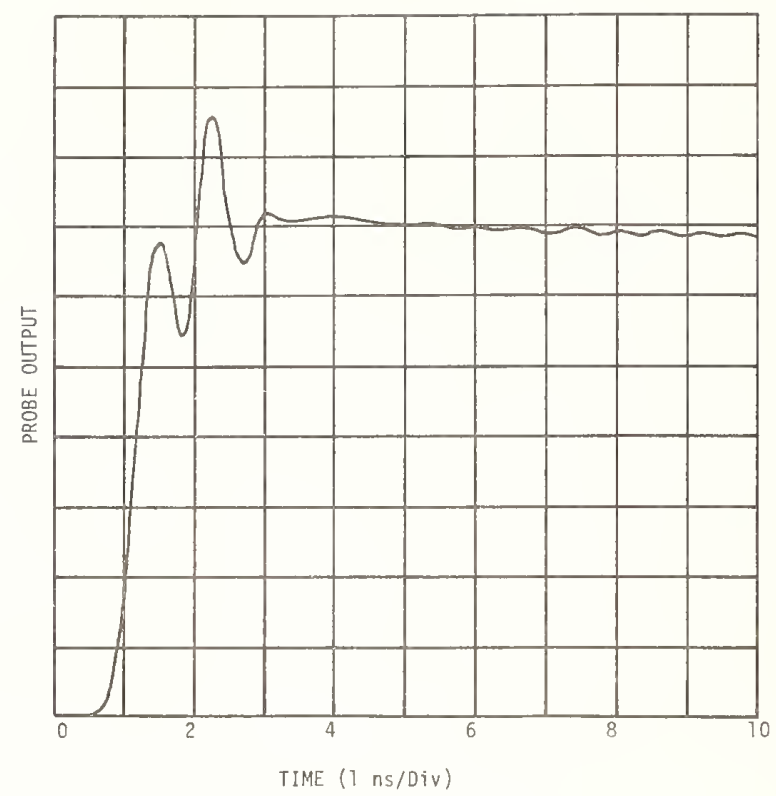

Figure 5. Computer model output.

to $20-\mathrm{kV}$ dc. The resistors are $100-\Omega, 1-W$ Allen-Bradley carbon composition units. These were tested to $30 \mathrm{kV}$ in a narrow pulse RC discharging circuit. All high-voltage divider components are housed in an oil-filled acrylic tube.

\subsection{Pulse Generation and Input Coupling}

Fast risetime pulses were generated using a mercury pulser with two Tektronix 113 delay cables used as the charge lines. This apparatus gives subnanosecond risetime, $1500 \mathrm{~V}$ pulses into $50 \Omega$.

Coupling to the probe was made through a $25-\Omega$ transmission line tapered to the 25.4-cm-diam of the probe body. The transmission line was terminated with $25 \Omega$ instead of $50 \Omega$ to 7 ower the source impedance driving the probe. A resistive divider was built into one of the terminating resistors of the transition section. This was done to monitor the actual input to the high-voltage probe. The risetime of the monitor probe is about $300 \mathrm{ps}$, as shown in Figure 6.

\subsection{Output Cable Termination}

The probe output cable is terminated with a 50- $\Omega$ series resistor, a $0.1-\Omega$ resistor, and a 40-nF capacitor in series to ground. The output is viewed across the $0.1-\Omega$ resistor and the 40-nF capacitor. The oscilloscope input is shunted with a 100-k $\Omega$ resistor. A11 attempts to construct the termination from standard components, i.e., 1/4-W resistors and leadless ceramic capacitors, have proved fruitless because of resonances in 


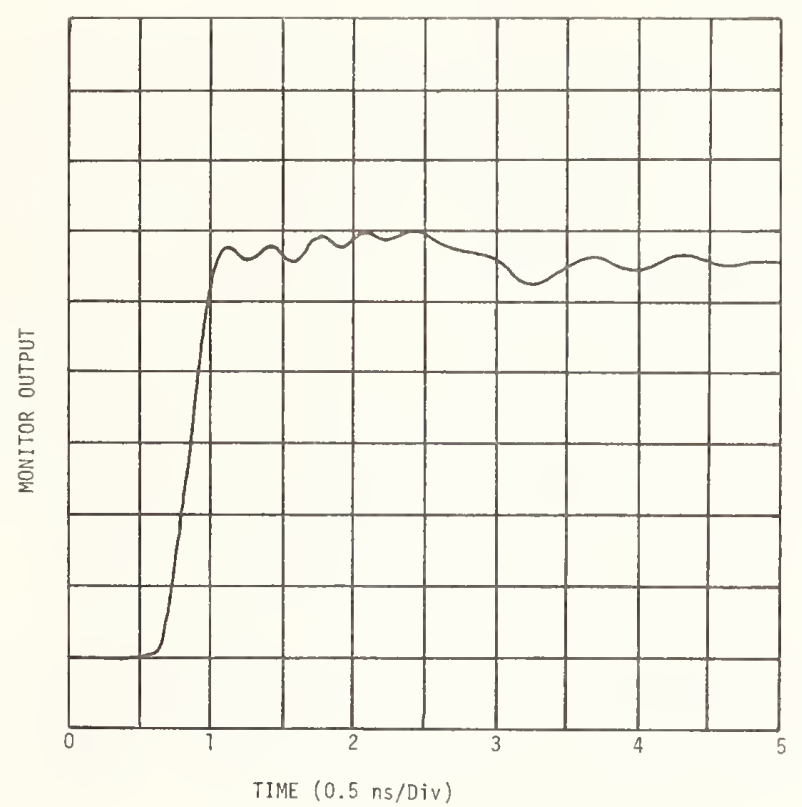

Figure 6. Transition cone monitor output response.

the 5 - to $30 \mathrm{MHz}$ range. The termination was then fabricated on a ceramic disk by the Los Alamos National Laboratory Hybrid Facility. The termination is made of 10 parallel arms radially soldered to a small circular ceramic substrate. The individual resistors are $1.0 \Omega$ each and the capacitors are $4 \mathrm{nF}$ each. No termination resonances below I GHz have been observed with the hybrid termination.

Having solved the ringing due to the termination, it was discovered that the probe input circuit was ringing because of the input capacity and the coupling inductance. Ferrite beads were put in series with the high-voltage section to damp the resonance, but this severely limited the risetime, as shown in Figure 7. It was finally necessary to add $150 \Omega$ of series resistance to the input of the probe to damp this resonance. Figure 8 shows a superposition of the input and output waveforms of the high-voltage probe. The risetime is about $1.5 \mathrm{~ns}$, which is the risetime of the Tektronix 7A16A vertical plug-in. Attempts to use a 900-MHz FET probe were unsuccessful because of severe ringing in the $100-$ to $300-\mathrm{MHz}$ range. The difficulty lies in coupling the hybrid to the probe input.

Some additional circuitry is required between the hybrid termination and the oscilloscope input. This circuit serves to flatten the first $100 \mathrm{~ns}$ of the output pulse and to flatten the 1- to 50-ms region in which the response switches from the ac probe to the dc probe. This circuit is shown in Figure 9.

When properly adjusted, the response is flat within $2 \%$, referenced to the dc level. The attenuation ratio is about 12,000 to 1 . The compensation network must be adjusted for a particular plug-in for optimaliy 


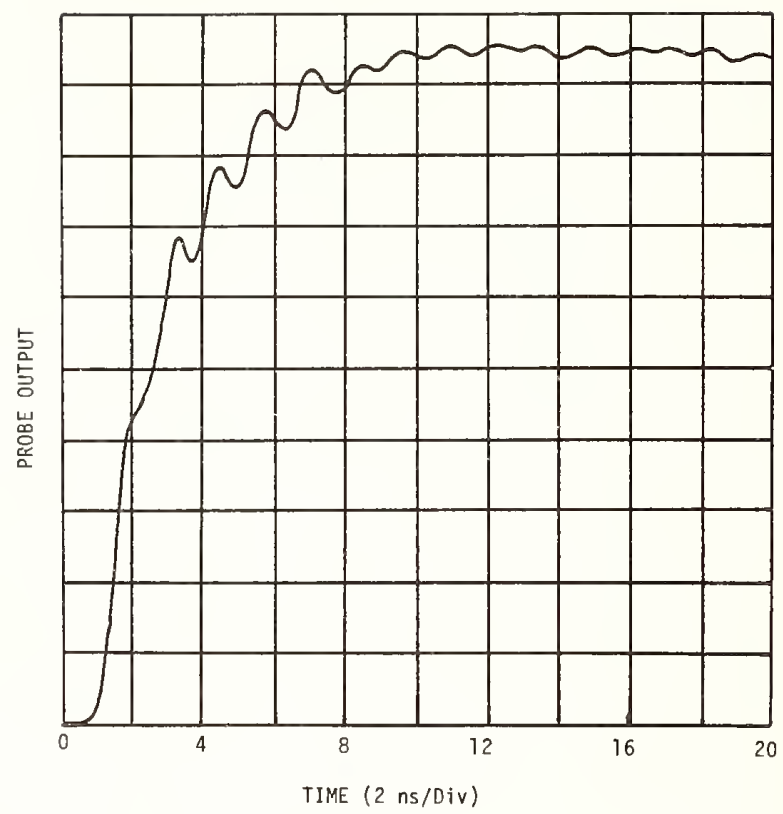

Figure 7. Probe response with ferrite damping.

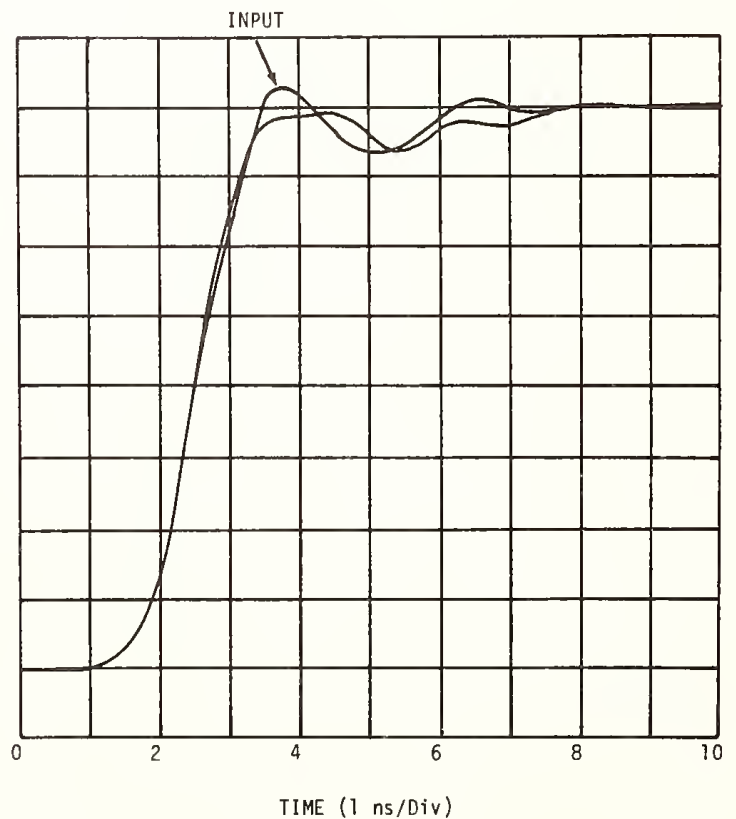

Figure 8. Probe input and output response. 


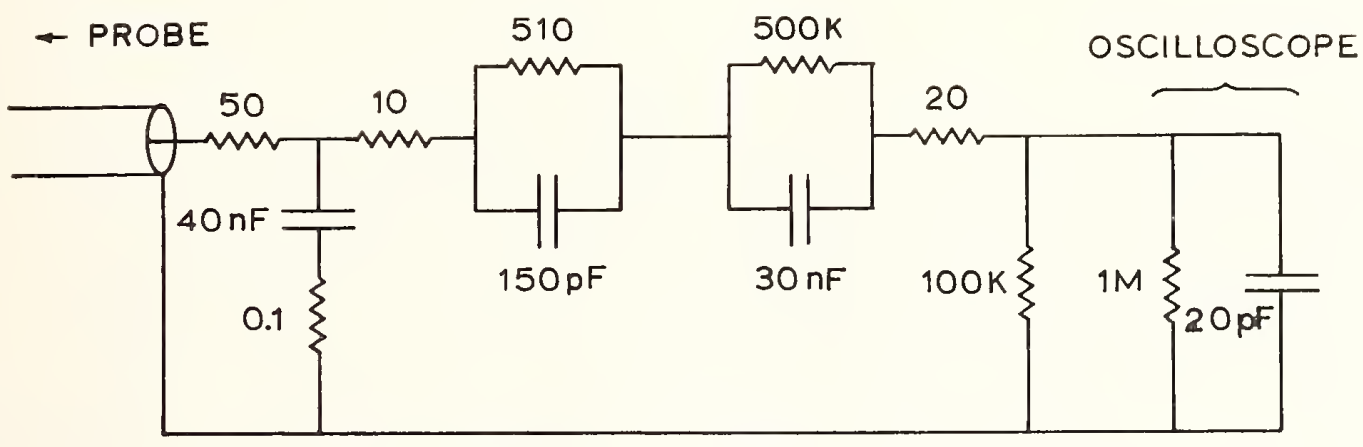

Figure 9. High-voltage probe termination and oscilloscope coupling network.

flat response. The overall accuracy is also limited by the oscilloscope and the plug-in combination, which have a typical accuracy of $4 \%$.

\subsection{High-Voltage Testing}

The probe was tested to $105-k V$ dc for $4 \mathrm{~h}$ to check for arcing and heating problems. The probe body was pressurized with 30 psig of nitrogen for this test. The input to the probe was covered with oil to prevent external insulator arcing, and no arcing was observed throughout the test. The temperature rise of the high-voltage divider was approximately $20^{\circ} \mathrm{C}$. This results in a dc response change of 3 to $4 \%$.

A high-voltage pulse was generated with a spark-gap-switched cable pulser that generated 60 - to $70-k V$ pulses. The pulse amplitude could not be accurately controlled in this pulser. The pulse risetime was limited to $3 \mathrm{~ns}$ because of the inductance of the spark gap. In observing the output pulse with the high-voltage probe, no frequency response changes were noted for the 20-ns pulse length.

\section{Conclusion}

A high-voltage probe has been developed for both dc and high-frequency voltage measurements at the 100-kV level. When properly compensated, the probe risetime is less than 2 ns. The probe must be connected to low-impedance, low-inductance systems for proper operation. For extended operation at high dc voltages, it is necessary to cool the oil insulating the high-voltage divider to eliminate temperature stability effects, which are about $0.16 \% /{ }^{\circ} \mathrm{C}$. The accuracy of the probe alone is approximately $2 \%$ neglecting temperature drift and amplifier accuracy. 


\section{Discussion Session 1}

Richard Malewski (IREQ). The first comment is associated with the presentation of $\mathrm{Mr}$. Harris who has shown design of a voltage probe used in a coaxial line with liquid dielectric. We had a similar problem in measuring fast transients in a coaxial bus filled with SF6. There are some common difficulties in these two applications. The schematic circuitry is shown in figure 1a. There is a high voltage bus and a probe which possesses some capacitance to the high voltage bus. This probe in the design of Mr. Harris' has a capacitance to the grounded envelope. If we look at the probe, we realize that there is a current flowing to ground through the capacitors which produces a magnetic flux. In a physical layout of this kind, the inductance of the low voltage section of the circuit is fairly high in comparison to that of the high voltage part with no damping whatsoever. So an equivalent schematic is like that shown in figure lb with an input high voltage, a capacitor, an integrating network and the coaxial cable. But, in the first loop, we have stray inductance and no damping if $R_{1}, R_{g}=0$. So obviously, the current in this loop will be of an oscillatory character with the frequency determined by capacitance $C_{1}, C_{g}$ and inductance $L_{1}, L_{g}$. $\mathrm{Mr}$. Harris proposes to simply smooth the response by inserting an RC circuit with a time constant which will smooth the oscillations. That's a fairly practical way out of it. A different approach is to fashion the low side capacitor from a plastic cylinder which has been painted with resistive paint. Now, the current which originally flowed through a straight inductance will have to circulate in this resistive layer. The damping will make this current aperiodic and eliminate the need for the RC element. Another approach was proposed by people from the University of Munich. They did not use the paint, but they put ferrite cores in the circuit introducing loss at high frequency. I believe it is better to control the current flowing through the divider and then to see the actual voltage drop across the low voltage arm.

I have another comment regarding the experiment described by Hebner in which he compared two dividers, one a large high voltage divider and the other one a small precise reference divider. A similar exercise in our lab used a bigger divider which was $20 \mathrm{~m}$ in length. The length of the lead to the small divider was $25 \mathrm{~m}$. When we compared the outputs from the two dividers, we obtained different results for different applied pulses, i.e., a square pulse or a front chopped triangular pulse. We realized that with pulses of duration of a few hundred nanoseconds, the frequency spectrum extends to fairly high frequencies and we are in a range where the connecting lead may be a quarter wavelength of some of the harmonic frequencies of these pulses. The lead is then long enough to be considered as an effective transmitting antenna with frequency selective features which can effectively radiate energy at a given wavelength. A simple calculation indicates that some pulses we are recording have a frequency spectrum 


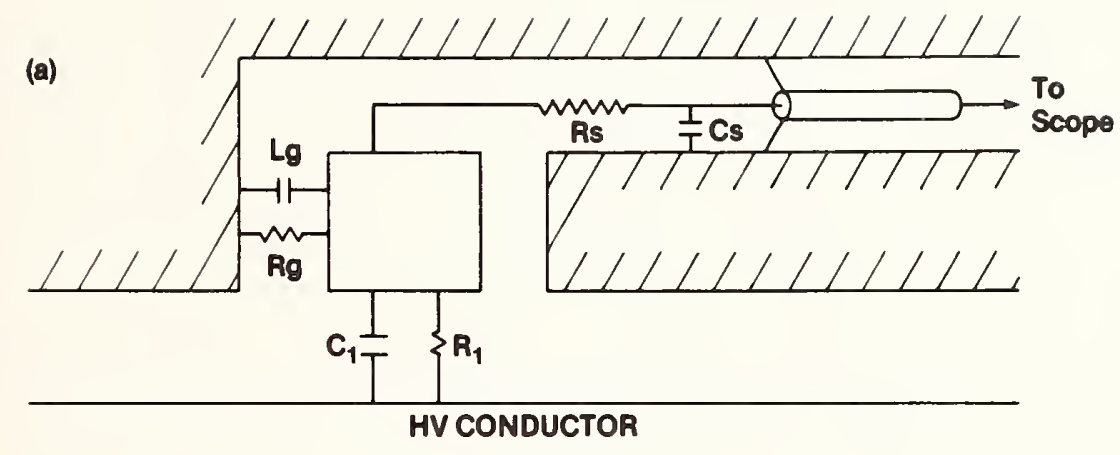

(b)

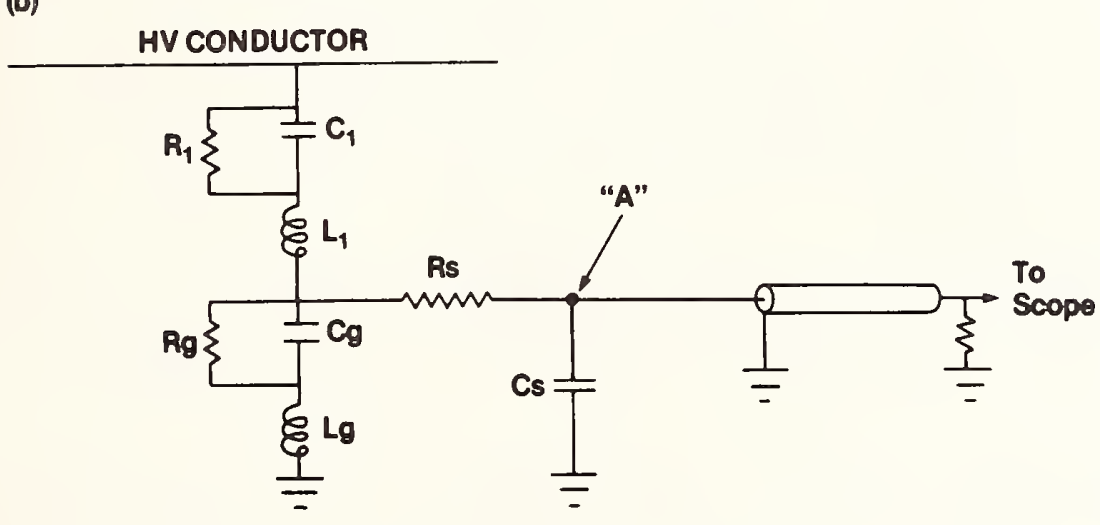

Figure 1. (a) Physical configuration of capacitive divider. (b) Lumped parameter equivalent circuit. 
rich enough to add significant contribution at the harmonic corresponding to this 1/4 wave transmission from the lead. The only approach we tried to eliminate this problem was to replace the copper wire by a resistive wire and vary the distribution of the resistance along the lead. This improved the performance a bit, but I should admit that at this stage we do not have a ready made answer for this problem. Perhaps the third comment is ahout what Dr. McComb said about the dividers used in their Physics Department. They are using some exotic materials such as liquids or carborundum-type resistors for the high voltage part of the device. A divider is a measuring device which is supposedly linear, both in voltage and in frequency. The dielectric constant of a liquid of the kind used for a divider varies with frequency. We can represent a divider which has a high voltage part in the form of a column filled with liquid as a parallel connection of resistance and capacitance. The capacitance varies with frequency and since the liquid has in general a high dielectric constant it is not negligible. In a resistor divider, the ground capacitance is usually predominant. This is not true in the case of a liquid type of divider because of the large capacitance of the high voltage element. Therefore, the variations of this capacitor with frequency results unvariably in a distorted response. Even a worse situation occurs when we use the type of resistors which are basically developed for lightning arrestors. These are very non-linear resistors and the response of such a resistor always indicates some small irregularities. Why? Because these resistors are composed of granules of carborundum enveloped in thin layers of silicone insulation and the conduction in such a divider is based on breakdown of these small layers of insulation which connects the conductive carborundum particles. These materials are non-linear and will always exhibit small variations of resistance as a function of time. So, to me, it is not a very promising idea to use the thyrite resistors or liquids for high voltage arms.

Neville Harris (Ion Physics). I am at a slight disadvantage since I have only made my probes for water lines. I would comment that Mr. Malewski has got the right diagram. However, the equivalent circuit is not right. He talks about a large inductance $\mathrm{L}_{\mathrm{g}}$ and a small one $L_{1}$. I think you will find this the other way around. The low voltage capacitor is rather like a coaxial line with a very low impedance and an inductance which is very very small. The inductance coming from the main gap is much larger. So in the divider circuit, the inductance $L_{1}$ is much larger than the inductance Lg. I agree that they form resonant circuits. However, I did not have any capacity at point $A$ (see figure $1 b$ ). There is stray capacity, but there was no integrating capacitor. The cable leading to the oscilloscope was matched and hence appears as a pure resistance. Where you get most of the trouble is in the resistor Rs. The inductance and the stray capacities of this resistor do, in fact, give most of the trouble. I did not try various materials. I used stainless steel which happens to be a fairly resistive material. The water losses, $R_{q}$, also serve to damp resonances. My opinion. is that any oscillations you get are more associated with the series resistor Rs. 
Malewski. Could you indicate the current flow through the divider, how the current flows from the high voltage bus to the ground through the divider?

Harris. Most of it flows through the capacitors $C_{1}$ and $C_{q}$ as shown in T(a). There is damping due to the water line since water has a loss tangent and also conductivity. As you increase the capacity $\mathrm{C}_{\mathrm{g}}$, then for the same bandwidth $\left(50+R_{S}\right) C_{g}$, you need only a low value for the resistor $R_{S}$. The impedance of this oscillating circuit is $\sqrt{\mathrm{Lg} / \mathrm{C}_{\mathrm{g}}}$ which should be compared with the damping resistance, $\left(R_{s}+50\right)$. Thus, if you make $C_{g}$ large the ratio of damping resistor to the circuit impedance increases as $\sqrt{C_{g}}$. Thus in practice, the circuit is damped by a parallel resistance rather than a series resistance as proposed by Mr. Malewski.

Bob Hebner (NBS). The second comment that Richard had to make was about my paper, and I want to thank him very much because what he has done is reinforce the point that I was trying to make. I had mentioned that we know the technique worked well for voltage of the order of a megavolt and for pulses longer than microseconds. We have some serious concerns that it may not work well outside of those regimes. I have shown how, under a specific condition, we extended that regime slightly. Richard has given a concrete example of how, when they attempted to extend that regime, the technique did not work. And it shows that there is definitely a need for a better measurement technique and it shows that it is naive to assume that the techniques that work in one range of frequencies and voltages will apply to all ranges.

Terry McComb (NRC, Canada). I think that a major point in regards to both the siticone carbide and the copper sulfate is that they are extremely cheap and rugged devices. We recognize that they are nothing like as good as wire-wound resistors as we showed in our results. However, they can be set up quickly, and we can overvolt them when we are playing in the lab. But, as Richard mentioned, they are certainly not ideal. We have a long way to go to extend and develop pulse measuring probes for very fast pulses.

Jim Sarjeant (Los Alamos). Richard, I would like to respond to your comments in a general way for a moment if I may. My impression here, listening to the talks given this morning, is that we are discussing two different categories or classes of voltage measurement needs. One is the extremely high accuracy calibration type of requirement such as the work that is going on at NRC, NBS, and various other places around the world and quite effectively over the last 20 or 25 years. The second class of application is where you have a device in the laboratory and you would like to measure its electrical parameters to some reasonable degree of accuracy and reproducibility. And, indeed, I feel that the bringing together of these two categories of diagnostics is one of the key points of this particular meeting. 
Copper sulfate dividers for very fast voltage measurements have been around now for nearly 50 or 60 years. Units have been built by people at Physics International with response times of under 100 picoseconds. They have been used at voltage levels of the order of 100 kilovolts per inch. The response times when using dc type or long pulse durations pulses were measured and none of the behavior that Richard has indicated was observed. I would like to point out that when we made our measurements at extremely high stress ievels in distributed systems we did indeed see the behavior that Richard referred to. So, in conclusion, we are talking about two classes of operation. One is something that is big and tall and the other is something that is short and small. In the laboratory, you may have a laser system operating as high as a megavolt. You would like to have a compact voltage monitoring device whose intrinsic response time and resonance in that system are much faster than any of the characteristic frequencies of your system. It was for such applications that Terry and I spent some time comparing the real devices that people have been using for the 1 ast 10 to 12 years to the highly accurate dividers used in the high voltage field. 


\author{
CALIBRATION OF CAPACITIVE VOLTAGE PROBES \\ IN WATER-DIELECTRIC, HIGH POWER PULSE GENERATORS* \\ M. Wilkinson, E. Chu \\ Maxwell Laboratories, Inc. \\ 8835 Balboa Avenue \\ San Diego, CA 92123
}

Capacitive voltage probes are frequently used in the water pulselines of high power pulse generators. These usually consist of small electrodes isolated from the outer conductor of the pulseline. The capacitance between the electrode and the inner conductor determines the output of the probe.

Due to finite resistivity of the water dielectric, a fast, high voltage pulser is usually required to calibrate these probes. We will discuss a method, which we call the "capacitance-current" method, that allows probe calibrations with a slow pulser independent of the resistivity of water. We will compare calibrations obtained using this method against those obtained using more conventional methods.

*Work supported by the Defense Nuclear Agency.

\title{
1. Introduction
}

Pulse power systems employing liquid dielectrics are characterized by time varying electric fields in the range of hundreds of kilovolts per centimeter. For the larger systems, this corresponds to megavolt potential differences, maintained over gap lengths of the order of ten centimeters. Voltage risetimes vary from $\leqslant 10$ ns to $z 1 \mu \mathrm{s}$. A probe is thus required which can measure these large voltages in a frequency range from $\leqslant 300 \mathrm{kHz}$ to $z 30 \mathrm{MHz}$.

A simple capacitive $\dot{E}$ probe can generally be built to satisfy these requirements. Figure 1 shows a diagram of the device in operation. The probe consists of a small circular portion of the pulseline outer conductor which has been isolated from the remainder by a $50 \Omega$ impedance. The capacitance, $c_{p}$, of the probe with respect to the 
pulselire inner conductor, together with the $50 \Omega$ impedance coristitutes an RC differentiation circuit. The resulting signal is thus proportional to $\mathrm{dV} / \mathrm{dt}$ and must be integrated, (in our case using a passive $R C$ integrator) to produce a signal proportional to $\mathrm{V}$.

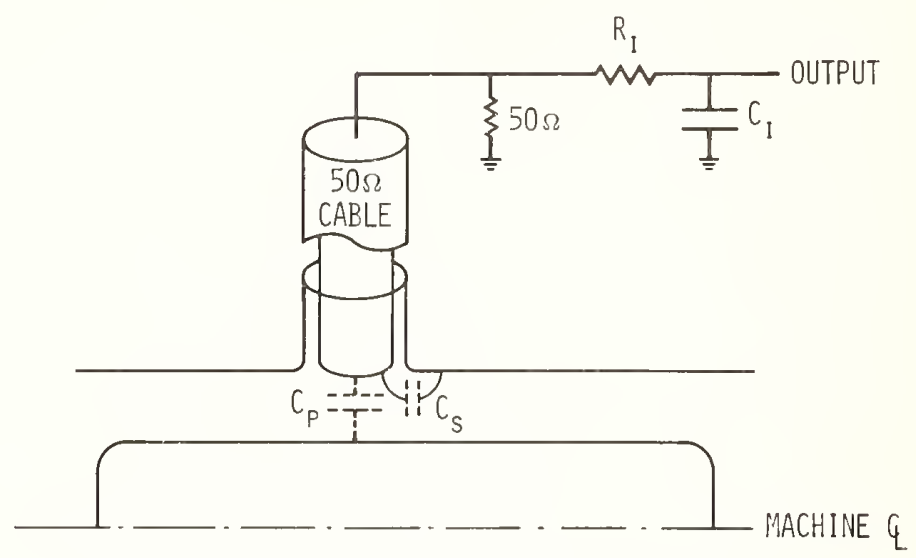

Figure 1. Diagram of $\dot{\mathrm{E}}$ probe.

The bandwidth of the resulting voltmeter is determined by the requirement that the passive differentiator and integrator truly differentiate and integrate. This places the following requirement on the angular frequency $\omega$, viz:

$$
\frac{1}{R_{i} C_{j}}<<\omega<\frac{1}{50 \Omega C_{p}}
$$

In reality, a more stringent limit exists on the high frequency response. This is caused by a small stray capacitance, (shown in Figure 1 as $C_{S}$ ), which exists at the cable-pulseline interface and shunts the $50 \Omega$ cable impedance to ground. The quantity $C_{S}$ is estimated from the probe design via electrostatic field plots. By careful probe construction, the value of $C_{\text {s }}$ may be pushed well below $25 \mathrm{pF}$. However, for most practical probe désigns $C_{S} \gtrsim C_{p}$ and $1 / 50 \Omega C_{s}$ represents the upper limit on $\omega$.

Figure 2 shows the calculated absolute value of probe output for unit eiwt pulseline voltage. The values for various elements are chosen to resemble those encountered in the BLACKJACK 5 probes at Maxwell Laboratories, viz:

$$
\begin{aligned}
& c_{p}=0.5 \mathrm{pF} \\
& R_{i}=5000 \Omega \\
& c_{i}=1 \mathrm{nF}
\end{aligned}
$$


Various values of $C_{S}$ are shown, in order to illustrate its effect upon the high frequency cutoff. The calculation also assumes that the dielectric is water, with a time constant $\rho \varepsilon=120 \mu \mathrm{s}$ (typical conditions of BLACKJACK 5 ). Thus, the water capacitances, $C_{p}$ and $C_{S}$, are shunted by resistances of $\rho \varepsilon / C_{p}$ and $\rho \varepsilon / C_{S}$ respectively. The latter quantity is shunted by $50 \Omega$ and is always negligible. The former quantity affects the probe response at very low frequencies.

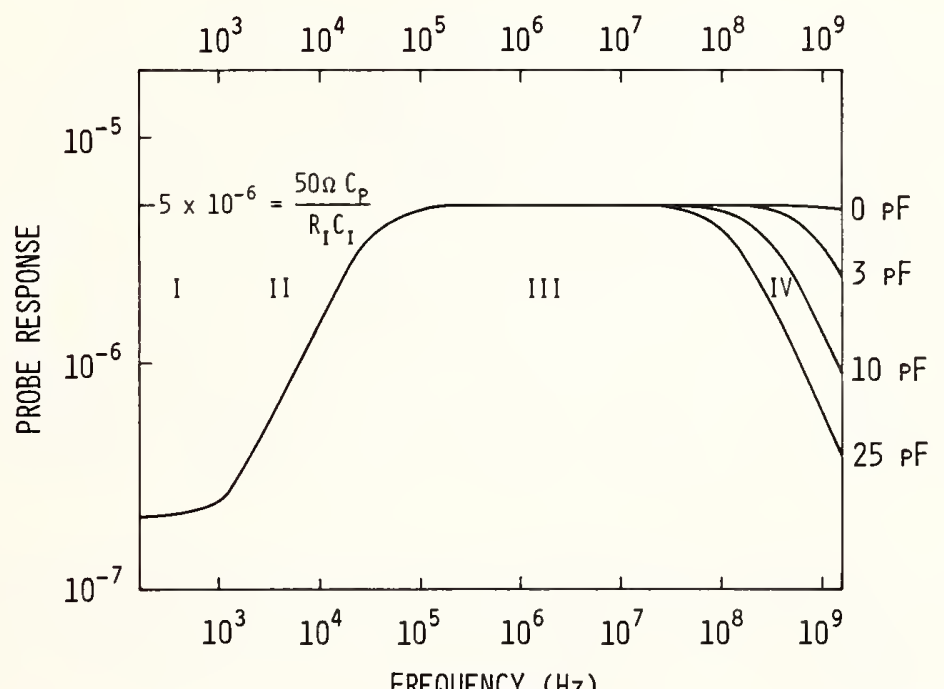

Figure 2. Response of $\dot{E}$ probe.

The curve in Figure 2 may be divided into four physically distinct regions. Region I, below $\sim 1 \mathrm{kHz}$, represents the quasi-DC limit, where the circuit is acting as a purely resistive voltage divider with response:

$$
\frac{V_{\text {probe }}}{V_{\text {pulseline }}}=\frac{50 \Omega C_{p}}{\rho \varepsilon}=2.08 \times 10^{-7}
$$

In Region II, roughly $10^{3} \mathrm{~Hz}$ to $10^{5} \mathrm{~Hz}$ the finite conductivity of the water is becoming less important, but we are still not satisfying the first of inequalities (1), i.e., the integrator droop remains substantial. Region III represents the dynamic range of the probe. Inequalities (1) are satisfied and the probe response is:

$$
\frac{V_{\text {probe }}}{V_{\text {pulseline }}}=\frac{50 \Omega C_{p}}{R_{i} C_{i}}=5 \times 10^{-6}
$$


Region IV comprises the high frequency limit, where either the effects of $C_{S}$ are being felt or the second of inequalities (1) is violated. If we define a five percent decline in probe response as our limit on bandwidth, we see that the upper limit is $230 \mathrm{MHz}$ for $C_{S}=25 \mathrm{pF}, 2100 \mathrm{MHz}$ for $C_{S}=10 \mathrm{pF}$ and several $\mathrm{GHz}$ for the ideal $C_{S}=0$.

\section{Probe Calibration - Direct Method}

The straightforward method of calibrating this or any voltage probe is to compare the probe's output with that of some "standard" voltmeter which measures the same voltage pulse. For a probe with the sensitivity of $\sim 5 \times 10^{-6}$ described above, we require a calibration pulse of $110 \mathrm{kV}$ in order to provide a substantial output signal $(\sim 50 \mathrm{mV})$. In addition, the pulse must have dominant frequency components which lie in Region III, the dynamic range of the $\dot{E}$ probe. On BLACKJACK 5, this is accomplished with a $20 \mathrm{nF}, 100 \mathrm{nH}$ pulser which injects a pulse on axis at the front of the machine (Figure 3 ). This results in a wave of frequency $\sim 3 \mathrm{MHz}$ which travels down the transmission line.

This traveling wave calibration pulse illustrates a general phenomenon encountered in large systems such as BLACKJACK 5, viz, that frequencies in Region III correspond to wavelengths shorter than the size of the system. This means that transit time effects dominate and it is necessary therefore to place the "comparison" voltage probes very close to the $\dot{E}$ probe in order to insure that both are viewing the same voltage. If we desire instead to have the convenience of treating the pulseline inner conductor as a lumped capacitance with uniform voltage $V(t)$, we must limit the bandwidth of $V(t)$ to regions I and II, and, hence, the output of the $\dot{E}$ probe wi11 not be directly comparable to that of a comparison voltmeter.

The BLACKJACK 5 machine has a Targe number of $\dot{E}$ probes along its length and for most of them it is not convenient to connect up a comparison voltage probe nearby. An exception is provided by the diode voltage probes at the output end. As shown in Figure 3 , these probes are located just beyond the plastic insulator which separates the water from the vacuum diode. Thus, by connecting a comparison probe on the vacuum side close to the insulator it is possible to sample nearly the same $V(t)$ which is seen by the E probes. We have used this method of calibration for the diode voltage probes, using a 1000:1 frequency compensated resistive voltage divider as our comparison probe. The results provide a useful benchmark for evaluating the less direct "capacitance-current" method described in Section 3. 


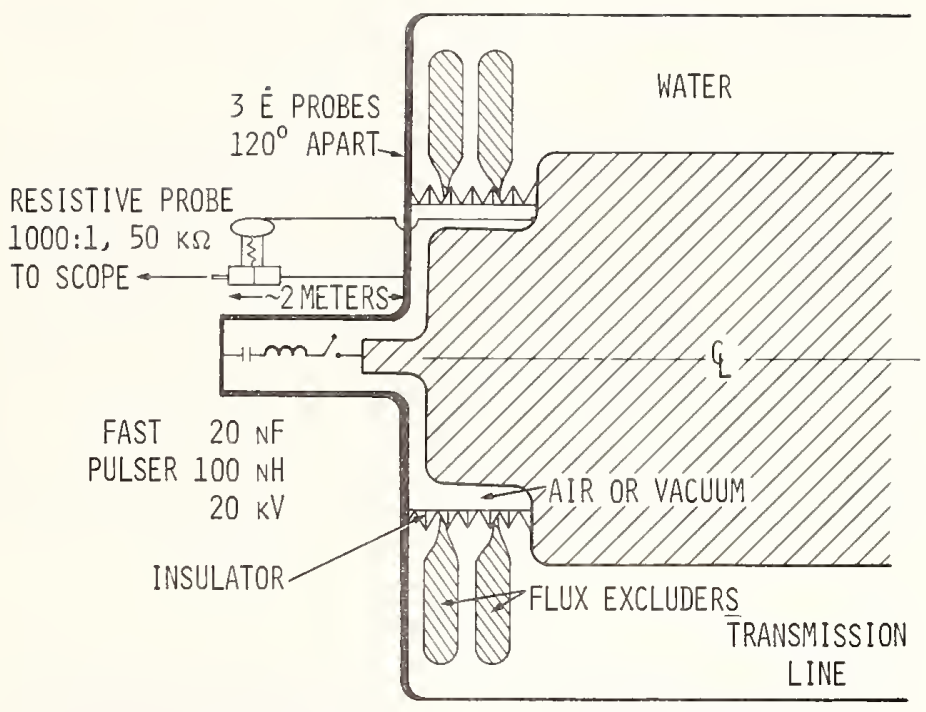

Figure 3. Diagram of direct calibration method.

The attenuation ratio of the resistive voltage probe was checked using the \pm 5 volt level sine wave output of the Tektronix SG503 function generator, recently calibrated and traceable to NBS. The probe output was measured using a Tektronix 485 oscilloscope, calibrated on the $5 \mathrm{mV} /$ div scale and also traceable to NBS. The probe was found to give nominal 1000:1 division up to frequencies beyond $100 \mathrm{MHz}$. It was connected across the diode insulator, taking care to minimize the area of the region bounded by the insulator and the probe leads. This was to avoid measuring any of the inductive voltage dropped across the diode and not seen by the $\dot{E}$ probe.

Figure 4 shows a comparison between the waveforms obtained from the $\dot{E}$ probe (with $5.06 \mu \mathrm{s}$ integrator) and the resistive probe. The small amplitude ringing on the resistive probe is probably due to excitation of waves in the resonant structure formed by the probe leads ( $\sim 2 \mathrm{~m}$ long), which are terminated by the $50 \mathrm{~K} \Omega$ probe impedance on one end and the $0.6 \Omega$ pulseline impedance on the other. The $0.6 \Omega$ end is almost a node for such a system, thus, no ringing is observed on the $E$ probe. The amplitudes of the ringing on the resistive probe does not constitute a severe noise problem, thus, we can obtain from Figure 4 the following calibrations for the three $\dot{E}$ probes: 
Table 1. Probe Calibrations: Birect Method

\begin{tabular}{ccccc}
\hline PROBE & ${\text { (sens })^{-1}=\frac{5.06}{50 \Omega \mathrm{CS}_{\mathrm{p}}}}$ & Derived $C_{\mathrm{p}}$ & Varue \\
\hline 1 & $1.903 \times 10^{5}$ & 0.532 & $\mathrm{pF}$ \\
2 & $1.873 \times 10^{5}$ & 0.540 & $\mathrm{pF}$ \\
3 & $1.786 \times 10^{5}$ & 0.567 & $\mathrm{pF}$ \\
\hline
\end{tabular}

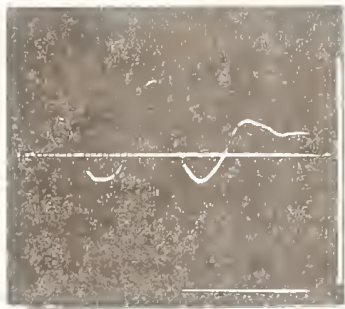

É PROBE NO. 1

$0.020 \mathrm{~V} / \mathrm{DIV} 100 \mathrm{NS} / \mathrm{DIV}$

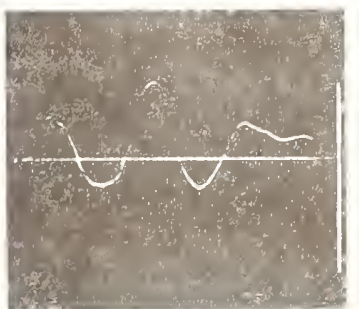

Ė PROBE NO, 2

$0.020 \mathrm{~V} / \mathrm{DIV} 100 \mathrm{NS} / \mathrm{DIV}$

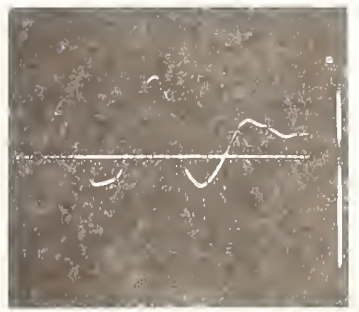

E PROBE NO, 3

$0.020 \mathrm{~V} / \mathrm{DIV} 100 \mathrm{NS} / \mathrm{DIV}$

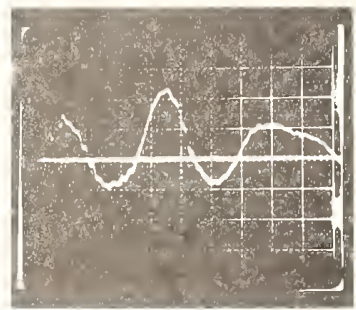

RESISTIVE DIVIDER

$2.0 \mathrm{~V} / \mathrm{DIV} 100 \mathrm{NS} / \mathrm{DIV}$

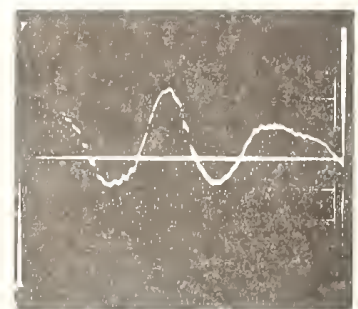

RESISTIVE DIVIDER

2.0 V/DIV 100 NS/DIV

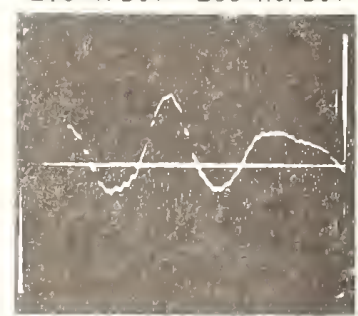

RESISTIVE DIVIDER

$2.0 \mathrm{~V} / \mathrm{DIV} 100 \mathrm{NS} / \mathrm{DIV}$

Figure 4, Comparison of $\dot{E}$ probes and resistive divider. 


\section{Capacitance - Current Method}

The $\dot{E}$ probe is a sufficiently simple and well understood device that we can state with confidence that its sensitivity (within its dynamic range) is given by Eq. 3. Thus, calibration of the probe rests upon measurement of two quantities: the integrator time constant, $\mathrm{R}_{j} \mathrm{C}_{j}$, and the probe capacitance $C_{p}$. Determination of the former quantity is a straightforward technical task. Some care must be taken in construction of the integrator in order to insure that it behaves like an ideal RC integrator out to $2100 \mathrm{MHz}$. Once this is accomplished, $R_{j} C_{j}$ may be measured, for example, by checking the signal attenuation as a function of frequency and fitting to a $\left[1+(\omega \mathrm{RC})^{2}\right]^{-\frac{1}{2}}$ expression.

The crucial task is thus measurement of $C_{p}$. Since this quantity can be estimated geometrically to be $20.5 \mathrm{pf}$ it is too small to be measured with an impedance bridge. To measure it, a method which we call the "capacitance current" method is adopted. This method uses a slow, $0.99 \mu \mathrm{F}$ capacitor to inject a current onto the central conductor of the pulseline. The injected current flows to ground (the outer conductor) largely as displacement current through the pulseline capacitance. The rest is shunted to ground due to the finite resistivity of water. A fraction of the displacement current as well as the shunting current is intercepted by the voltage probe and is responsible for the probe signal. A model of the actual circuit is shown in Figure 5. CTL and RTL are the capacitance and shunting resistance of the pulseline, (lumped circuit elements on this time scale). $C_{p}$ and $R_{p}$ are the capacitance and resistance between the probe and the central conductor of the pulseline. Noting that $C_{p} R_{p}=C_{T L} R_{T L}=\rho \varepsilon$, one can show that

$$
\frac{C_{p}}{C_{T L}}=\frac{V(t) / 50 \Omega}{I(t)}
$$

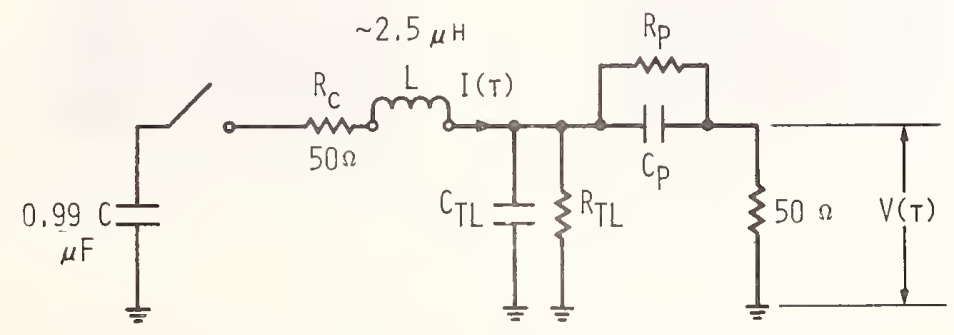

Figure 5. Model for the voltage calibration circuit. 
This relationship between $C_{p}, C_{T L}, V(t)$ and $I(t)$ is independent of the resistivity of water.

For BLACKJACK 5, $\mathrm{C}_{\mathrm{TL}}$ is approximately $280 \mathrm{nF}$ and, hence, can be measured accurately with an impedance bridge. I(t) is monitored with a model 1809 Pearson current transformer and $V(t)$ can in principle be measured directly with an oscilloscope. However, in practice a low pass filter is used to filter out high frequency noise on both $V(t)$ and. $I(t)$. The $5.06 \mu$ s integrator used in actual operation of the $\dot{E}$ probe turns out to be convenient for this purpose. As long as the same linear filter is used for both signals, the ratio of the two waveforms remains unchanged.

Figure 6 shows waveforms obtained for the three diode voltage probes and the Pearson current transformer. The latter is a selfintegrating Rowgowski coil with a $50 \Omega$ output impedance. The R/L droop rate for the device is quoted at $0.5 \% \mathrm{~ms}$, negligible for our purposes. The sensitivity of the probe is $0.1 \mathrm{~V} / \mathrm{A} \pm 0.1$ percent when unterminated. The same accuracy, and one-half of the sensitivity, is obtained provided one is careful to terminate in the characteristic impedance of $50 \Omega$.
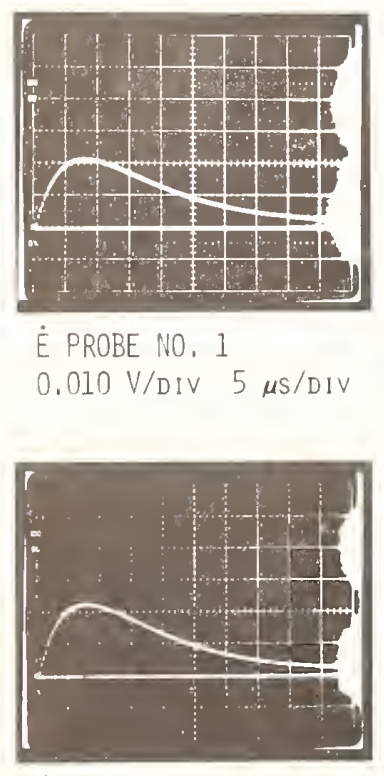

$\dot{E}$ PROBE NO, 3

0.010 V/DIV $5 \mu \mathrm{S} / \mathrm{DIV}$

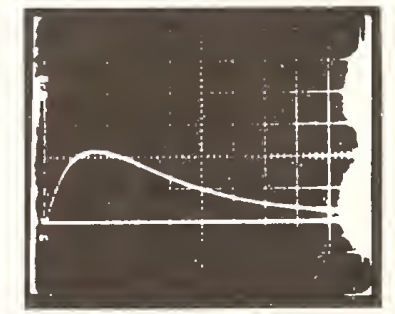

$\dot{E}$ PROBE NO. 2

?.010 V/DIV $5 \mu$ S/DIV

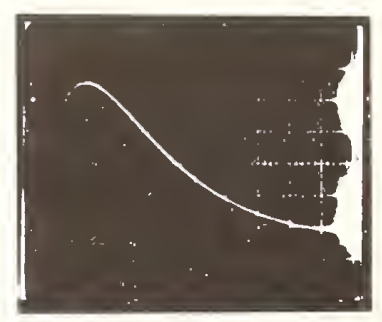

PEARSOII CURRENT PROBE $4.0 \mathrm{~V} / \mathrm{DIV} 5 \mu \mathrm{S} / \mathrm{DIV}$

Figure 6. Waveforms obtained in capacitance-current method. 
Since the same signal filter must be used on the $\dot{E}$ probe output and the Pearson output, it is necessary to rely on shot-to-shot reproducibility, taking repetitive shots at the same charging voltage.

For a given device, it was found that identical charging voltages gave indistinguishable waveforms, to the accuracy with which oscilloscope traces can be measured. The charge on the $0.99 \mu \mathrm{F}$ capacitor was

$23 \mathrm{kV}$ for these shots.

From the data in Figure 6 , together with a bridge measurement of $C_{T L}(280.5 \mathrm{nF})$, one can measure the following values of $C_{p}$ and
derive sensitivities based upon a $5.06 \mu$ s integrator:

Table 2. Probe Calibrations: CC Method

\begin{tabular}{ccc}
\hline PROBE & $C_{p}^{C_{p}}$ & $\begin{array}{c}\text { Derived } \\
\text { VALUE }\end{array}$ \\
\hline 1 & $0.546 \mathrm{pF}$ & $1.853 \times 10^{5}$ \\
2 & $0.556 \mathrm{pF}$ & $1.853 \times 10^{5}$ \\
3 & $0.571 \mathrm{pF}$ & $1.772 \times 10^{5}$ \\
\hline
\end{tabular}

4. Comparison of the Two Methods

The values of $C_{p}$ obtained from the direct and the "capacitancecurrent" methods are in good agreement as illustrated in the following table:

Table 3. Comparison of Calibrations

\begin{tabular}{cc}
\hline PROBE & $C_{p}\left(\right.$ "C $\left.-C^{\prime \prime}\right) / C_{p}($ "DIRECT") \\
\hline 1 & 1.026 \\
2 & 1.030 \\
3 & 1.007 \\
\hline
\end{tabular}


Thus, in this case, we find agreement to within three percent between the direct comparison method and the capacitance-current method in spite of the highly indirect nature of the latter. This gives us confidence in applying the capacitance-current method as a quick and easy means of calibrating the pulseline voltage probes, particularly for the less accessible ones.

The authors wish to thank John Shipman for helpful comments. Work supported by the Defense Vuclear Agency. 


\section{MEASUREMENT OF TRANSIENT POTENTIALS IN COAXIAL TRANSMISSION LINES USING COAXIAL DIVIDERS}

N. Fujimoto, S.A. Boggs and R.C. Madge

Research Division

Ontario Hydro

Toronto, Ontario, Canada

Techniques employing coaxial dividers, with flat response from the $\mathrm{kHz}$ region to $>1 \mathrm{GHz}$, have been developed for measurement of transient phenomena in coaxial transmission lines. These have been used to investigate partial discharge induced phenomena in gas-insulated transmission lines as part of a program to develop incipient fault detection and location techniques for gas-insulated switchgear. This paper describes the basic application, the theory and practice of coaxial couplers, and examples of partial discharge-induced transients.

Key Words: high voltage dividers, partial discharge; transient phenomena.

\section{Introduction}

A gas-insulated substation consists of a network of coaxial high voltage conductors, disconnect switches, ground switches, and circuit breakers all insulated with $\mathrm{SF}_{6}$. For 500-kV switchgear (300 kV normal conductor-to-sheath potential), the conductor is typically $17 \mathrm{~cm}$ and the sheath $55 \mathrm{~cm}$ in diameter. Typical gas pressures range from 350 to $450 \mathrm{kPa}$. Circuit breakers use $\mathrm{SF}_{6}$ as the breaking medium and are normally based on the "puffer" principle, whereby contact motion forces gas through the contact assembly to cool and extend the arc. Stations, including circuit breakers, rated to $100 \mathrm{kA}$ fault current are presently available.

A network of $17 \mathrm{~cm}$ aluminum conductors coaxially housed in aluminum enclosures with a lossless dielectric makes a superb high frequency distribution network, with an attenuation of less than $10 \mathrm{db} / \mathrm{km}$ at $1 \mathrm{GHz}$. These high frequency propagation characteristics facilitate the characterization and location of high frequency transients within the 
switchgear. Transients arise from two basic sources, dielectric breakdown and partial discharge.

Dielectric breakdown can occur either line-to-ground, as in the case of system failure (fault), or within the line, as in the operation of a disconnect switch. In either case, the transient magnitude is substantial and can cause severe overvoltages as a result of doubling at terminals or the superposition of reflections. System failures related to switching-induced transients have generated substantial interest in the subject. As well, these transients, which can have magnitudes in the MV range with about 7 ns risetimes, cause ground rises in the range of $100 \mathrm{kV}$ which can disrupt electronic control systems and present a danger to operating personnel [1,2]. Interest in measuring both internal transients and groundrise transients on external surfaces has stimulated research into high frequency high voltage measurement techniques.

Partial discharge can occur from several sources within a gasinsulated substation. Floating components (corona shields, spacer inserts, etc. Which are not bonded to the conductor or sheath) can cause substantial discharge [3]. Free conducting particles can be levitated in the field and bounce, causing small discharges each time they land [4]. Filled epoxy spacers are used to support the conductor. A manufacturing defect, such as a void or stress enhancement on an electrode can cause discharge within the spacer ("treeing"). Each of these sources of discharge is symptomatic of a defect within the system which can lead to breakdown. Thus, the ability to detect and locate partial discharge sources during commissioning tests and normal service is important to reliable operation. The transient magnitudes generated by these sources of partial discharge have a dynamic range of about $80 \mathrm{db}$. A small free-conducting particle generates a signal of about $0.1 \mathrm{~V}$ propayating in the coaxial transmission line, while a floating component can easily generate $1000 \mathrm{~V}$ transients. Discharge magnitude from "treeing" varies during the growth process. The transients from all sources of partial discharge are extremely "fast", with risetimes in the range of 350 ps (the limit of measurement) or less [3].

Under EPRI sponsorship, Ontario Hydro is developing methods for fault location and partial discharge location which depend on high resolution timing (fault location) or cross correlation (partial discharge location) of induced transients $[5,6]$. These techniques, as well as research into the dangers of switching-induced transients, depend on coupling the transients cleanly from the switchgear.

For measurement of external transients (groundrise), a transmission line divider technique developed by Newi [7] has been used with considerable success. For measurements of transients propagating within the coaxial switchgear, coaxial couplers have been used. These facilitate very clean measurement of transients, with measured risetimes limited only by the 1-GHz 'scope bandwidth over division ratios ranging from 3:1 to 2000:1. The low frequency response can extend to about $10 \mathrm{kHz}$ for a low division ratio coupler to less than $1 \mathrm{~Hz}$ for a high division ratio 
coupler. This excellent performance, extreme versitility, and unparaileled simplicity makes the coaxial divider very valuable for coaxial systems.

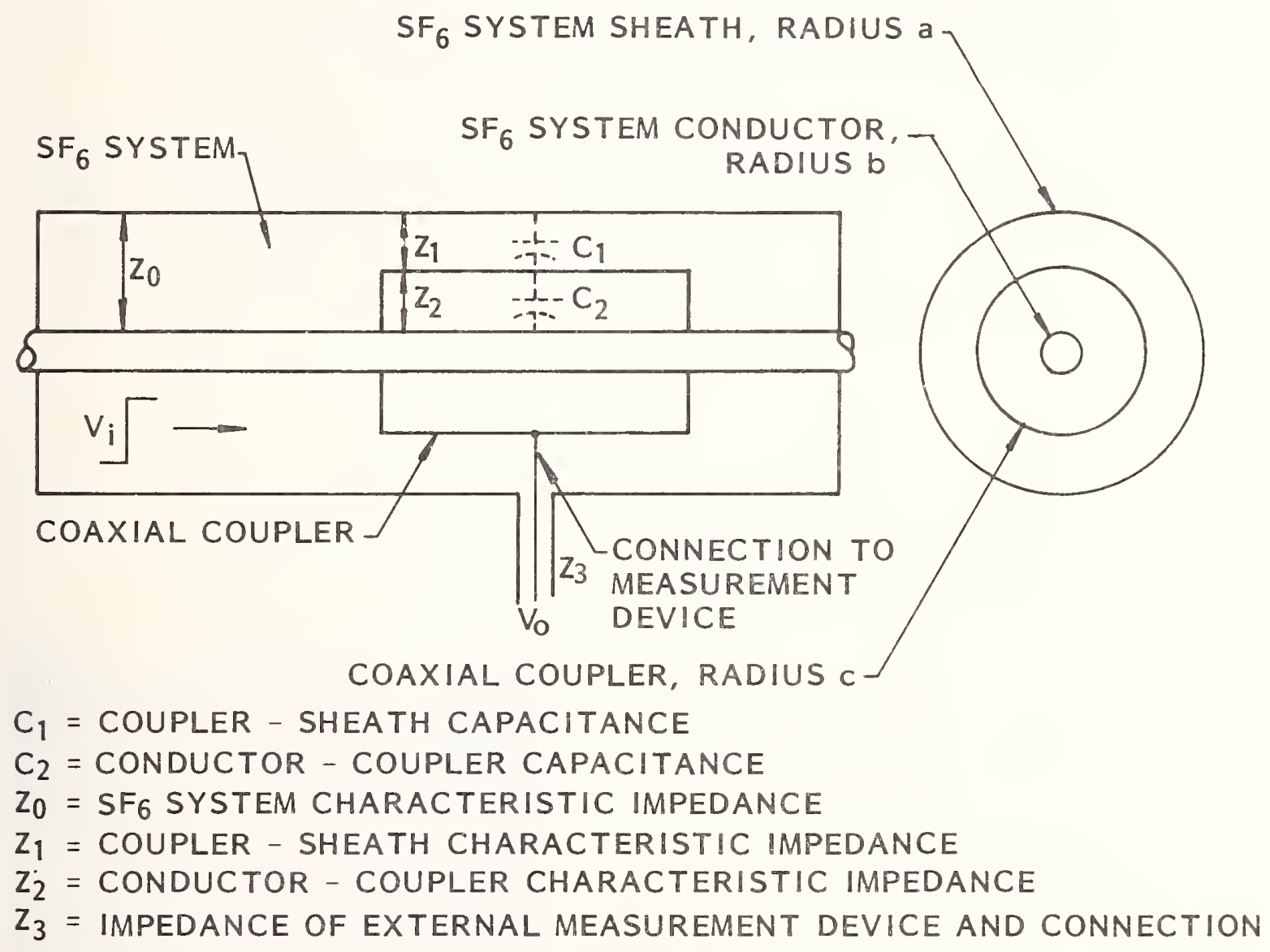

FIGURE 1

\section{BASIC COAXIAL COUPLER CONFIGURATION}

\section{The Theory and Practice of Coaxial Capacitive Couplers}

Couplers investigated for use in $\mathrm{SF}_{6}$ insulated coaxial systems are thin concentric metalic cylinders connected to an external potentialmeasuring device. An infinitely thin concentric cylinder lies on an equipotential surface and does not affect the propagation of signals in a coaxial transmission line. The potential of the cylinder, $V_{0}$, is described by (Figure 1):

$$
\frac{v_{0}}{v_{i}}=\frac{c_{2}}{c_{1}+c_{2}}=\frac{z_{1}}{z_{1}+z_{2}}=\frac{1 n_{b}^{c}}{1 n_{b}^{c}+\ln _{\frac{c}{c}}^{a}}=\frac{1 n_{b}^{c}}{1 n_{b}^{a}}
$$


The connection between the cylinder and the external measuring device substantially complicates the analysis of coupler response. The inevitably finite impedance, $Z_{3}$, causes a high pass characteristic and excitation of coupler resonances. Coupler eccentricity and thickness also cause deviations from the ideal case by introducing impedance mismatches which lead to signal scattering at both ends of the coupler.

In practice, less than 10\% impedance mismatch between the "open" transmission line and the transmission line in the region of the coupler is easily achieved through the use of relatively thin couplers, a high impedance connection from coupler to the measuring device, and reasonable attention to coupler concentricity. The last of these parameters is least critical and can be evaluated from the equation for the capacitance between two eccentric cylinders [8],

$$
c^{\prime}=\frac{2 \pi \varepsilon_{0}}{\ln \left(\frac{r_{0}\left(r_{i}^{2}-r_{0}^{2}+x^{2}\right)}{r_{i}\left(r_{i}^{2}-r_{0}^{2}-x^{2}\right)}\right)}
$$

where $r_{i}$ and $r_{0}$ are the radi $i$ of inner and outer cylinders, respective$1 y$, and $x$ is the distance between their axes. This equation can be used to calculate characteristic impedances for eccentric couplers, if one assumes that the inductance per unit length does not change appreciably for small eccentricities. For example, a system with conductor, coupler, and sheath radij of $1.9 \mathrm{~cm}, 3.8 \mathrm{~cm}$, and $5.1 \mathrm{~cm}$, respectively and a " $x$ " of $0.5 \mathrm{~cm}$ will have about $4 \%$ mismatch. For this same system (without eccentricity), a $0.3 \mathrm{~cm}$ coupler thickness will cause an $8 \%$ mismatch. Figure 2 shows computed step response as a function of the per cent impedance mismatch. Severe mismatches cause substantial degradation of coupler risetime.

If the measuring device appears purely resistive, a $50 \Omega$ terminated cable, for example, the coupler will exhibit a high pass characteristic with a response:

$$
\frac{V_{0}}{V_{i}}=\frac{j \omega R C_{2}}{1+j \omega R\left(C_{i}+C_{2}\right)}
$$

The response at infinite frequency is that of a "perfect" coupler, and the low frequency response is determined by $C_{1}+C_{2}$. By extending coupler length, low frequency response can be improved. For large coupler lengths, transmission line effects are superimposed on the lumped parameter high pass characteristic. Figure 3 shows that computed response 


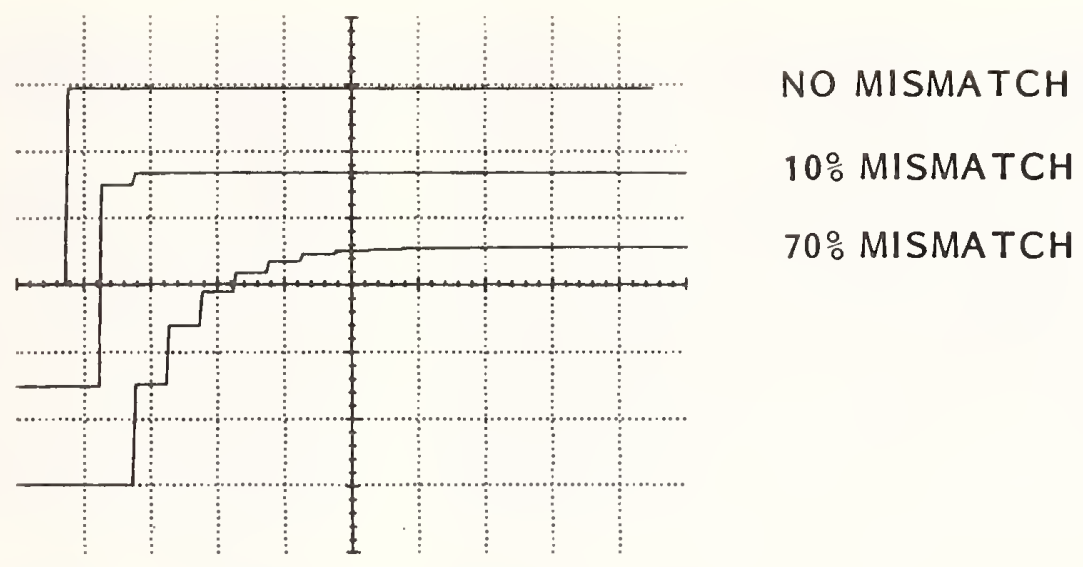

FIGURE 2

COMPUTER ANALYSIS OF IRREGULAR COUPLER RESPONSE

HORIZ SCALE: 2 COUPLER LENGTHS/DIV

VERT SCALE: $0.1 / D I V$, NORMALIZED TO INPUT

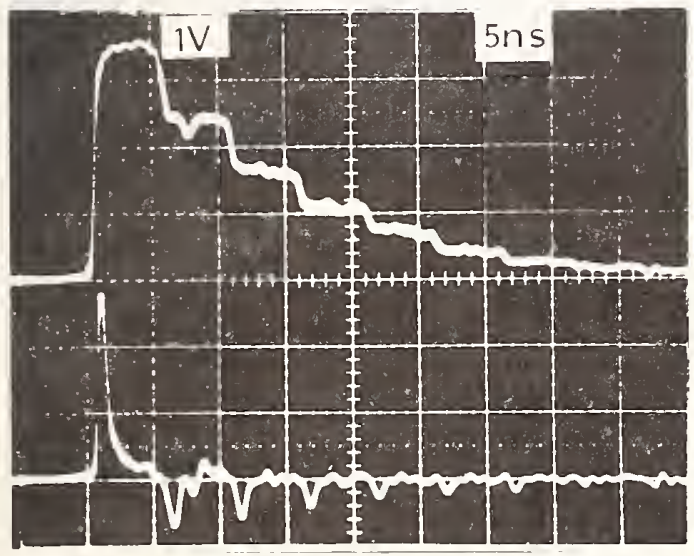

(a)

STEP \& PULSE RESPONSE OF $80 \mathrm{~cm}$ COUPLER WITH $50 \Omega$ PICK UP AT COUPLER'S LEADING END INPUT: $15 \mathrm{~V}$

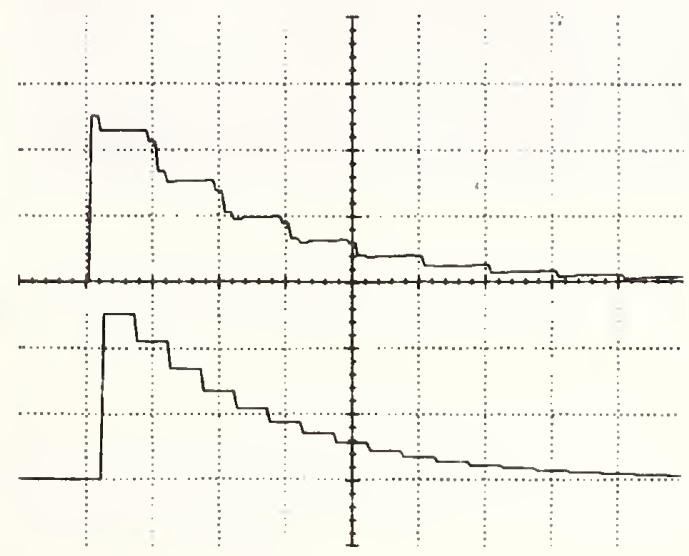

(b)

COMPUTER ANALYSIS OF COUPLER WITH $50 \Omega$ PICK UP TOP: PICK UP NEAR LEADING END

LOWER: PICK UP AT COUPLER'S CENTRE

HORIZ SCALE: 2 LENGTHS/DIV VERT SCALE : $0.1 / D I V$, NORMALIZED TO INPUT

FIGURE 3

COUPLER RESPONSE WITH $50 \Omega$ PICK UP 
based on transmission line parameters agrees well with measured response. The width of the "staircase" is determined by coupler length and position of the "pickup" along the coupler.

Low frequency response can be extended by many orders of magnitude through the use of a high impedance "pickup" from the coupler, e.g., a FET probe. The capacitances of such probes ( 1.5 to $3.5 \mathrm{pF}$ ) can cause resonances in coupler response which degrade measurement accuracy. The precise pattern of resonance-induced errors depends on coupler length and pickup position. Figure 4 shows the influence of residual coupler resonance and the effect of pickup position. Frequency doubling when

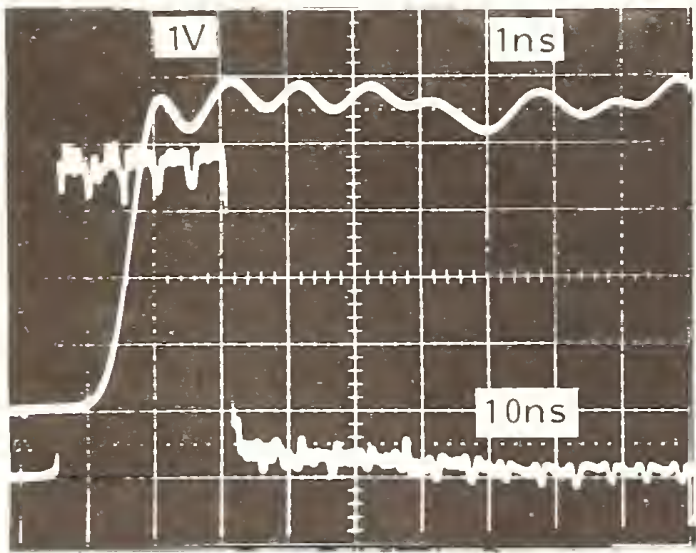

(a)

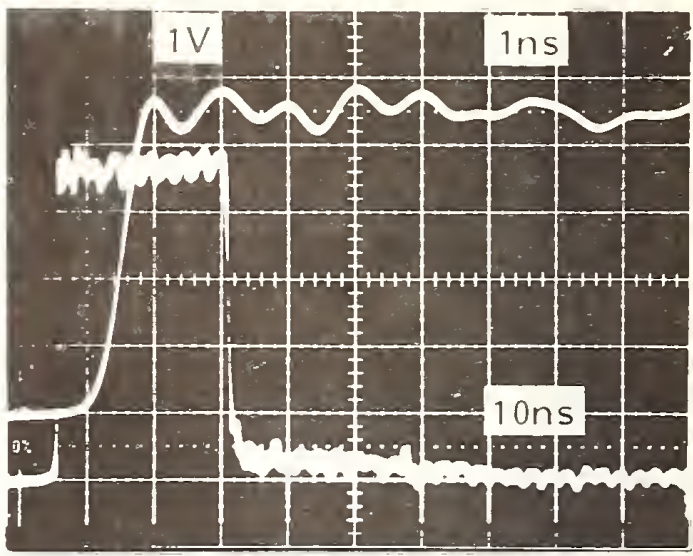

(b)

PROBE AT COUPLER CENTRE OF COUPLER

FIGURE 4

\author{
RESIDUAL RESONANCE IN COUPLER RESPONSE \\ ( PROBE CAPACITANCE $2 \mathrm{pF}$ )
}

the pickup is at the coupler center significantly reduces resonance amplitude, probably as a result of superposition-induced cancellation. Coupler resonant effects can be reduced by keeping the coupler transit time less than the risetime of the pulses to be measured. Figure 5 gives an indication of the quality of measurement possible under such conditions. In practice, coupler length must be based on a compromise between requirements for low and high frequency response. The coupler used to record Figure $5 \mathrm{a}$ has a $-3 \mathrm{db}$ high pass characteristic at about $1 \mathrm{kHz}$. The corresponding figure for Figure $5 \mathrm{~b}$ is about $10 \mathrm{kHz}$. Such cutoffs are not likely to impose serious restrictions for most high frequency measurements. 


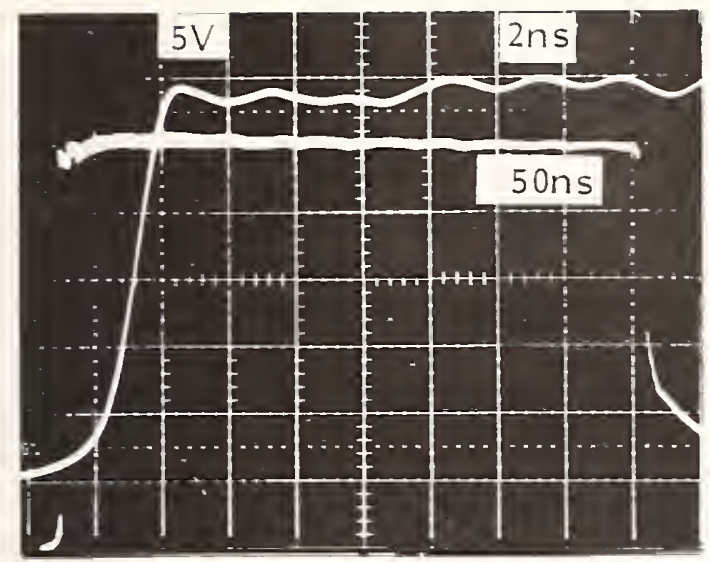

(a)

$80 \mathrm{~cm}$ LONG COUPLER INPUT: $100 \mathrm{~V}, 1.5 \mathrm{nS}$ RISE

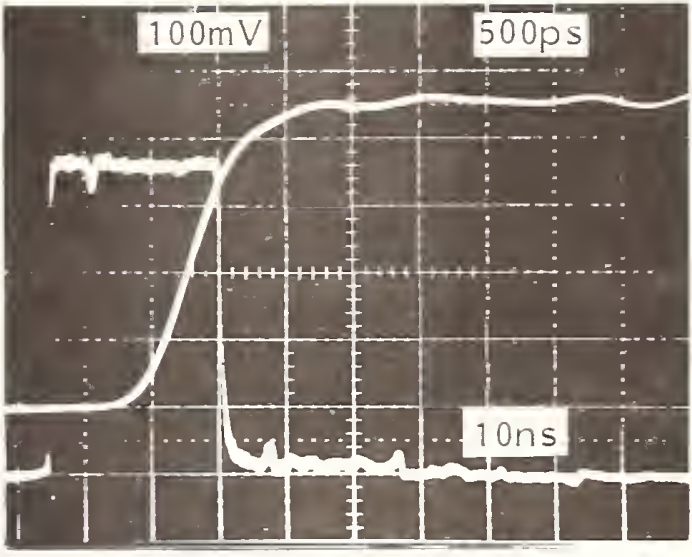

(b)

$7.6 \mathrm{~cm} \mathrm{LONG} \mathrm{COUPLER}$ INPUT: $1.5 \mathrm{~V}, 400 \mathrm{pS}$ RISE THE LARGE NEGATIVE SPIKE AT 10ns IS A REFLECTION FROM THE END OF THE TESTPIECE AND NOT ASSOCIATED WITH THE COUPLER

FIGURE 5

REDUCTION OF RESIDUAL RESONANCE

\section{Coupler Calibration}

Couplers with known geometries really need no calibration. The calculated response based on known geometry should be within a few per cent of the measured response. Couplers formed by the application of metallic foils over insulating tapes on the transmission line sheath require calibration, at least in the form of a low frequency foil-sheath capacitance measurement. In both cases, response characteristics can be determined by coupling fast risetime pulses into the transmission line through tapered conical terminations, which maintain the transmission line impedance while reducing the diameter to the point, where an appropriate cable connector can be used. If the transmission line impedance is greater than the generator and cable impedance, a resistor can be incorporated into the tapered coupler. This will cause a reflection back into the pulse generator, but will provide a matched termination to reduce ringing within the transmission line during calibration. Such a coupler, with a $10 \Omega$ resistor, was used to couple signals from a $50 \Omega$ pulse generator into the $60 \Omega$ transmission line used for experiments. For calibration of very high division ratio couplers, high voltage pulse generators are necessary. Generators which will produce 1000 $V$ pulses with 500 ps ristimes at 250 PRF or $200 \mathrm{~V}$ pulses with 1 ns risetimes at $10 \mathrm{kHz}$ PRF are commercially available. 
4. Measurements of Discharge-Induced Transients in Gas-Insulated Transmission Lines

During research related to the development of discharge location techniques for gas-insulated switchgear, measurements of transients typical of a number of discharge sources have been measured using a coaxial dividers shunted by $50 \Omega$ cable and a $1 \mathrm{GHz}$ bandwidth 'scope. A free conducting particle bouncing in the field generates a very smal1 transient, in the $100 \mathrm{mV}$ range. The rise time (Figure 6) is probably limited by the 350 ps ristime of the 'scope and signal degradation in the connecting cables. A floating component generates a similar transient, except with a magnitude in the $1000 \mathrm{~V}$ range (Figure 7 ).

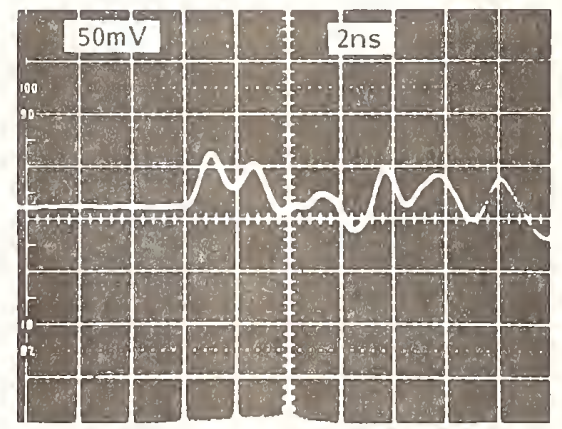

FIGURE 6

TRANSIENT GENERATED BY A FREE CONDUCTING PARTICLE BOUNCING IN THE COAXIAL TRANSMISSION LINE. THE MEASURED RISE TIME OF 500 PS IS PROBABLY LIMITED BY THE LENGTH OF CABLE EMPLOYED. THE DOUBLE PEAK AND REPETITION AT 6nS ARE THE RESULT OF REFELCTIONS WITHIN THE SYSTEM.

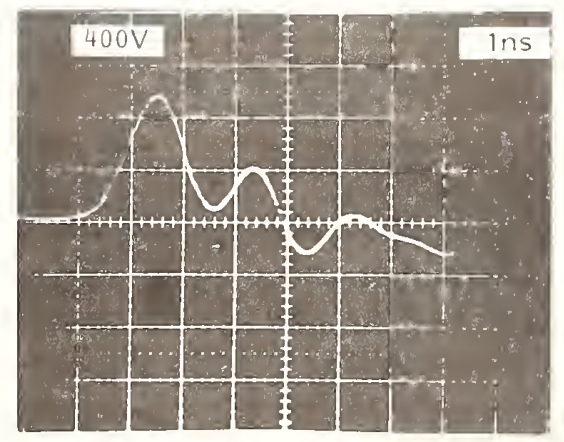

FIGURE 7

TRANSIENT GENERATED BY A FLOATING COMPONENT. THE AMPLITUDE CAN OFTEN BE PREDICTED ON THE BASIS OF EXPERIMENTAL GEOMETRY. 
Discharge in a solid dielectric ("treeing") generates very sharp transients (<350 ps risetime) whose duration and magnitude vary with the evolution of the tree (Figure 8). In these "treeing" measurements, the sample was placed at the end of a $60 \Omega$ gas-insulated transmission line with one electrode in contact with the conductor and the other with the sheath. Any discharges within the sample are "loaded" into the transmission line, thereby facilitating measurement with the coaxial divider. The 350 ps risetime measured is probably an order of magnitude less than can be measured by more conventional means and is limited only by the 'scope. The true risetime of such phenomena remains to be determined.

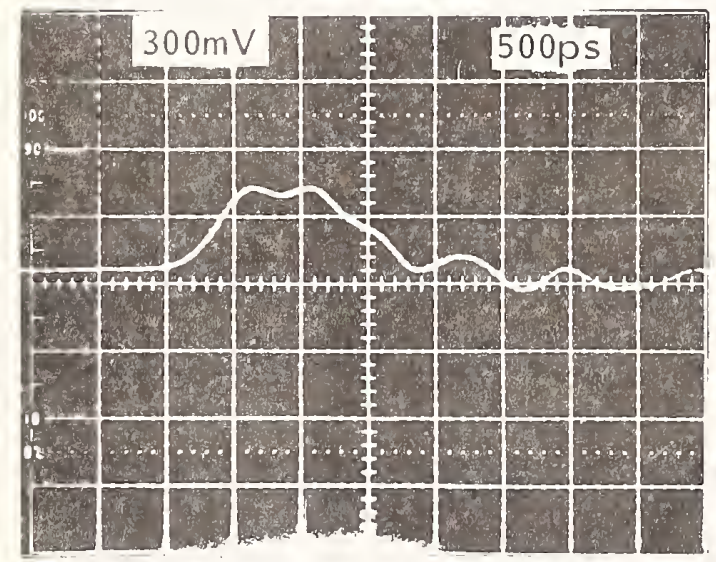

FIGURE 8

TRANSIENT GENERATED BY "TREEING" IN A SOLID DIELECTRIC SPACER LOCATED AT ONE END OF A GAS-INSULATED TRANSMISSION LINE. THE RISE TIME MEASURED IS LIMITED BY THE 1GHZ BANDWIDTH OF THE 'SCOPE.

Finally, a high division ratio coaxial divider was used in a study of the arc current necessary to sustain arcing in a transmission line with a gap in the conductor. The coupler was fabricated with very thin mylar tape and thin copper foil over the sheath. A division ratio of 2000:1 was achieved. In Figure 9, one can see the frequency doubling which occurred when the arc across the gap in the conductor extinguished. The oscillation is "ringing" of the entire testpiece prior to extinction and half the testpiece after extinction. Figure 10 shows the breakdown wavefront, which has the 7 ns risetime typical of $60 \Omega$ SF $_{6}$ insulated transmission line.

\section{Conclusion}

The measurements of breakdown and partial discharge induced transients described in this article and the relative ease with which they are obtained, indicate the usefulness of coaxial couplers for the measurement of high frequency phenomena in coaxial transmission lines. 


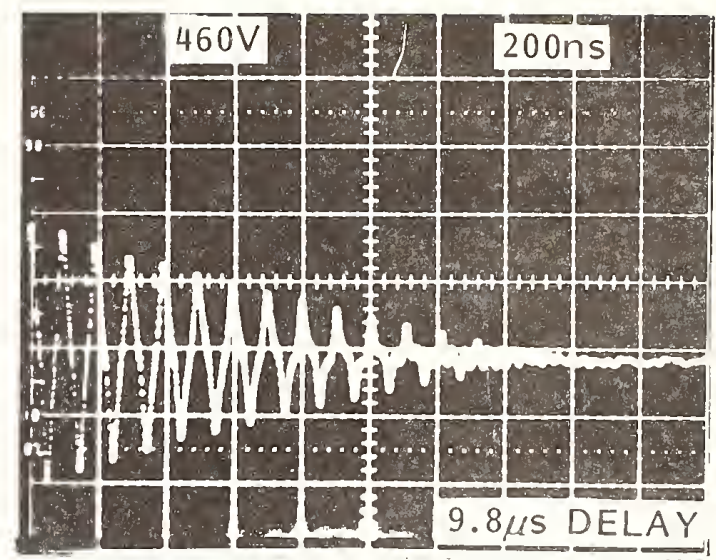

FIGURE 9

OSCILLATION OF A TRANSIENT THROUGH AN ARC. THE ARC ACROSS A GAP IN THE CONDUCTOR ACTS AS A SHORT CIRCUIT UNTIL NEAR MID PHOTOCRAPH WHERE A DELAY TO RE-IGNITION IS EVIDENT. THE ARC EXTINGUISHES ABOUT 1200ns INTO THE PHOTOGRAPH, WHERE THE OSCILLATION FREQUENCY DOUBLES.

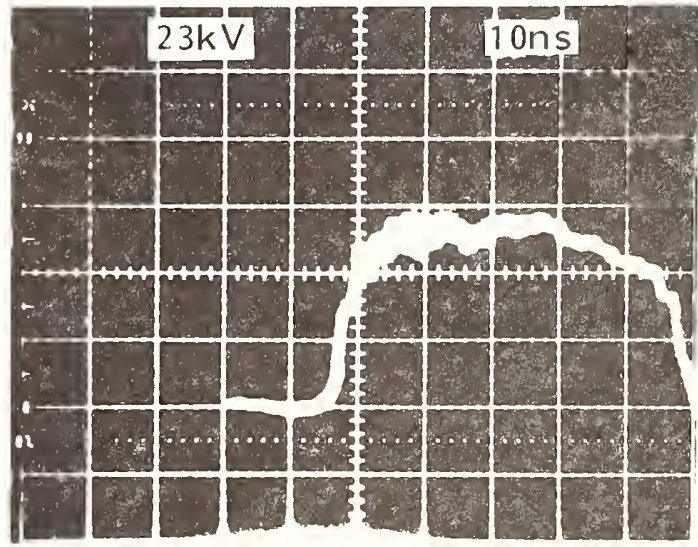

FIGURE 10

CONDUCTOR GAP BREAKDOWN-INDUCED TRANSIENT. NOTE THE RISE TIME OF ABOUT 5ns. THE 50ns PULSE WIDTH IS RELATED TO THE LENGTH OF THE TESTPIECE.

Through the careful design of couplers and ancillary apparatus, the measurement system can be matched to the specific needs of the experiment for division ratio, frequency response, and freedom from resonances. The use of commercially available FET probes as pickups greatly enhances low frequency response and facilitates a high pickup impedance with little attenuation. For many measurements, especially those of sharp transients or with large division ratios, $50 \Omega$ cable can be used directly from the coupler. Coaxial dividers have and will facilitate accurate measurements of phenomena which can be "staged" in a transmission line yeometry. 


\section{References}

[1] Ford, G.L., Boggs, S.A., and Chu, F.Y., "Transient Groundrise in $\mathrm{SF}_{6}$ Substations Investigated", Transmission and Distribution, August 1979.

[2] Ford, G.L., Boggs, S.A., and Dick, E.P., "Transient High Voltage on the Grounded Enclosures of Gas-Insulated Equipment", Proceedings of the Canadian Electrical Association Meeting, March 1980.

[3] Boggs, S.A., Ford, G.L., and Madge, R.C., "Coupling Devices for the Detection of Partial Discharges in Gas-Insulated Switchgear", Paper, IEEE PES Winter Meeting, February 1980.

[4] Wooton, R.E., "Investigation of High Voltage Particle-Initiated Breakdown in Gas-Insulated Systems", EPRI Research Report EL-1007, March 1979.

[5] Boggs, S.A., Ford, G.L., and Chu, F.Y., "Partial Discharge Location in Gas-Insulated Switchgear", Gaseous Discharges II, L. Christophorou, Ed. Pergamon Press, New York, 1980.

[6] Boggs, S.A., Chu, F.Y., and Ford, G.L., "Improved Fault Location for Gas-Insulated Switchgear", Transmission and Distribution, July 1980 .

[7] Newi, G., "A High-Impedance, Nanosecond Rise-Time Probe for Measuring High-Voltage Impulses", IEEE Transaction, PAS87, 9, 1779 (1968).

[8] Finkelman, M., "The Variation of the Capacitance of Cylindrical Electrodes Due to Loss of Concentricity", Ontario Hydro Research Division Report No. E66-43-K, September 1966. 


\title{
A VOLTAGE MONITOR FOR MAGNETICALLY INSULATED TRANSMISSION LINES *
}

\author{
Regan W. Stinnett \\ Sandia National Laboratories \\ Albuquerque, NM 87185
}

Magnetically insulated transmission lines pose special problems in voltage measurement because of the extremely high electrical stresses that are typical in them and because of the initial electron loss which is intrinsic to the establishment of magnetic insulation. This new voltage monitoring technigue uses negative ions which have been recent] lines. 10] These negative ions are produced in the cathode plasma and are accelerated by the gap voltage toward the anode. If two positively biased collectors are placed at different distances behind a hole in the anode, these negative ions may be collected to give time resolved signals which will provide the data necessary for a time of flight unfold of the gap voltage. A small magnetic field applied to the flight path can be used to deflect any electrons produced in the gap while not significantly deflecting the much heavier negative ion.

Although a complete voltage unfold may be difficult in practice, this technique may be used to obtain peak gap voltage very simply. If the collectors are located far enough away from the source, then the first negative ions to arrive will be those with the highest velocity, hence those produced at peak gap voltage. The velocity, and energy of these negative ions may be obtained to a few percent accuracy from arrival times at the two collectors.

1. Introduction

Magnetically insulated transmission lines (MITL's) are of interest in multiterrawat accelerators used in simulation and particle beam fusion. [1,2,3] These transmission

ॠThis work was supported by the U. S. Department of Energy, under Contract DE-AC04-76-DP00789. 
lines achieve power densities as high as $10^{13}$ watts per square centimeter because they operate at electric stress levels of over $10 \mathrm{mV} / \mathrm{cm}$. [4] This is possible because in a MITL electrons are prevented from crossing the vacuum gap between the cathode and the anode by a transverse magnetic field which causes them to move in cycloidal orbits. When the magnetic field is strong enough the electrons turn around before reaching the anode and magnetic insulation is achieved. This is shown schematically in Figure 1.

\section{MAGNETICALLY INSULATED FLOW}

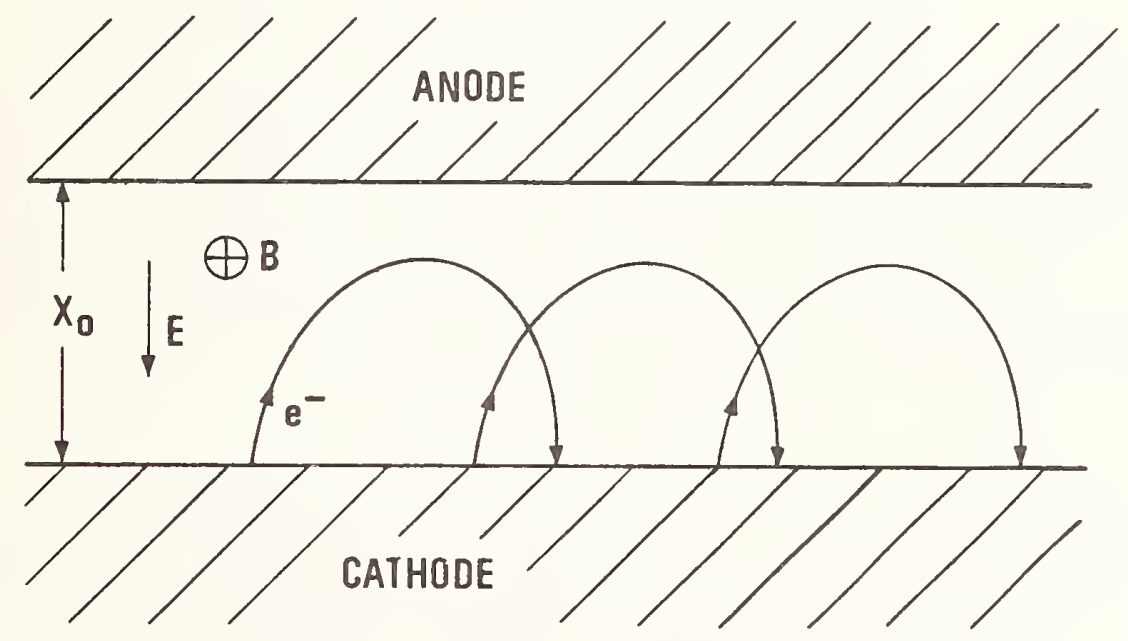

Figure 1. Magnetically insulated flow

Because of the high electric stress and the electron orbits in the vacuum gap between the anode and cathode it is difficult to directly measure the voltage in magnetically insulated transmission lines although such a measurement is extremely important. A method for measuring this voltage has been discovered which employs negative ions produced in the MITL's.

\section{Negative Ion Formation}

In MITL's a cathode plasma is formed by explosive emission $[5,6]$ when the electric field on the cathode rises 
above $250 \mathrm{kV} / \mathrm{cm}$. The constituents of this cathode plasma are not well known but seem to include both electrode material and hydrocarbon contaminants on the cathode surface [7] The electron density is in the range of $10^{17}$ $\mathrm{cm}^{-3}\{8\}$. with a plasma temperature of a few ev. 15,9$\}$ Explosive emission and desorption of gas molecules from the cathode could provide up to $10^{16}$ neutral atoms and molecules per square centimeter. These conditions, together with the excess of electrons in the cathode plasma resulting from the nature of magnetic insulation, provide an ideal situation for the creation of negative ions. An examination of the relevant cross sections and processes involved in negative low production in MITL's will be the subject of a later report.

Because the negative ions are produced in the cathode plasma they are accelerated to the full gap voltage as they move across the vacuum feed gap, so their energy accurately reflects the gap voltage at the time they crossed the gap. There are several atoms and molecules, such as $\mathrm{H}, \mathrm{C}, \mathrm{O}$, and $\mathrm{C}_{2}$, which have bound negative states. Different negative ion species produced in the cathode plasma would arrive at a collector at different times, producing distinct peaks. which could be used to cross check the gap voltage.

\section{The Experiment}

Van Devender, Stinnett, and Anderson[10] recently discovered the presence of negative ions in the long magnetically insulated transmission 1 ines of Mite and Hydramite, modular prototypes of Sandia National Laboratories Particle Beam Fusion Accelerator PBFA I. Both of these accelerators produce a $2 \mathrm{MV}, 400 \mathrm{kA}$ pulse lasting $35 \mathrm{~ns}$. This pulse travels down a 6 meter long, magnetically insulated triplate transmission line which is 20 centimeters wide and has a 1 centimeter gap. Both the anode and cathode surfaces are coated with a 25 micron thick graphite coating. These MITL's have been demonstrated to operate efficiently $\left.{ }_{11}\right]$ on both the MITE and Hydramite accelerators.

Further experiments on Hydramite have been performed to study negative ion production in greater detail. In these experiments a time of flight spectrometer (TOFS), shown schematically in Figure 2, was mounted behind a 0.635 $\mathrm{cm}$ hole in the anode and used to collect the negative ion current. The first collector was a $50 \%$ transmitting screen, allowing the second collector, mounted $40 \mathrm{~cm}$ behind the first, to collect negative ions along the same flight path. Both collectors were biased positively at 300 volts to recapture any secondary electrons emitted. This arrange- 


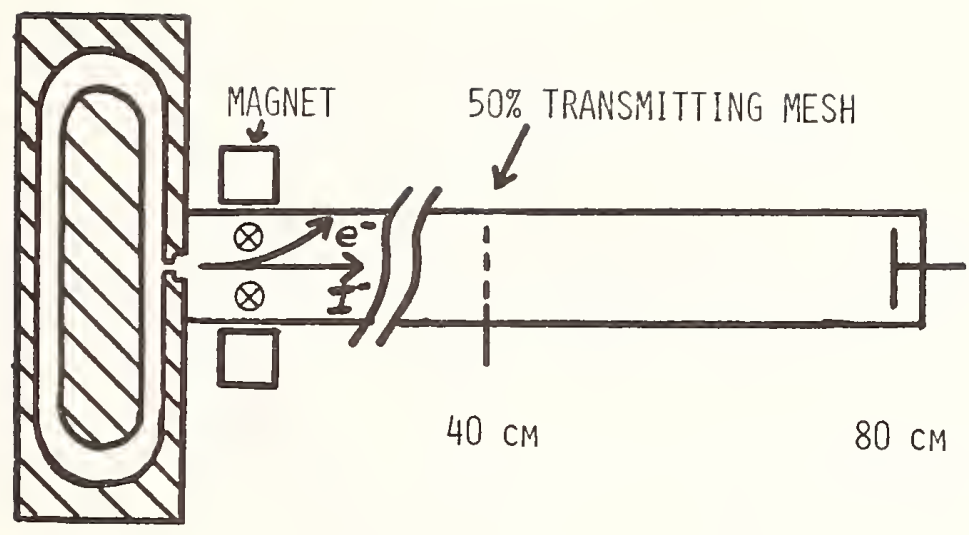

Figure 2. Time-of-flight spectrometer used in Hydramite negative ion experiments

ment made it possible to measure the negative ion velocities directly. The collectors were mounted at distances from 15 to 81 centimeters behind the gap.

Several techniques were used to confirm that the negative signals from the collectors were actually caused by negative ions. A magnetic deflector was mounted on the TOFS to sweep electrons out of the flight path. This magnetic deflector produced a transverse magnetic field of 500 Gauss over a distance of $10 \mathrm{~cm}$. When a $70 \mathrm{micron}$ thick aluminum foil (much less than one electron range but more than one ion range) was placed in the flight path betwen the MITL and the first collector, the collector signals were reduced by an order of magnitude, verifying that the signal was mainly due to negative ions rather than electrons.

\section{Experimental Results and Analysis}

The TOFS was used to measure voltage on the Hydramite MITL on several shots. Figure 3 shows the signals from collectors located 41 and $81 \mathrm{~cm}$ from the cathode on Hydramite shot 8936. The typical voltage at the insulator is shown in Figure 4. The dotted line shows the expected voltage waveform 5 meters down the MITL where the TOFS was located. The erosion and steepening of the leading edge of the pulse is caused by the lossy region at the front of a magnetic insulation wave and is typical.

Negative ions leaving the cathode plasma cross the vacuum feed gap in less than 3 ns so it is valid to assume a constant voltage to calculate their time of flight across the gap. The time of flight to the collectors is determined 


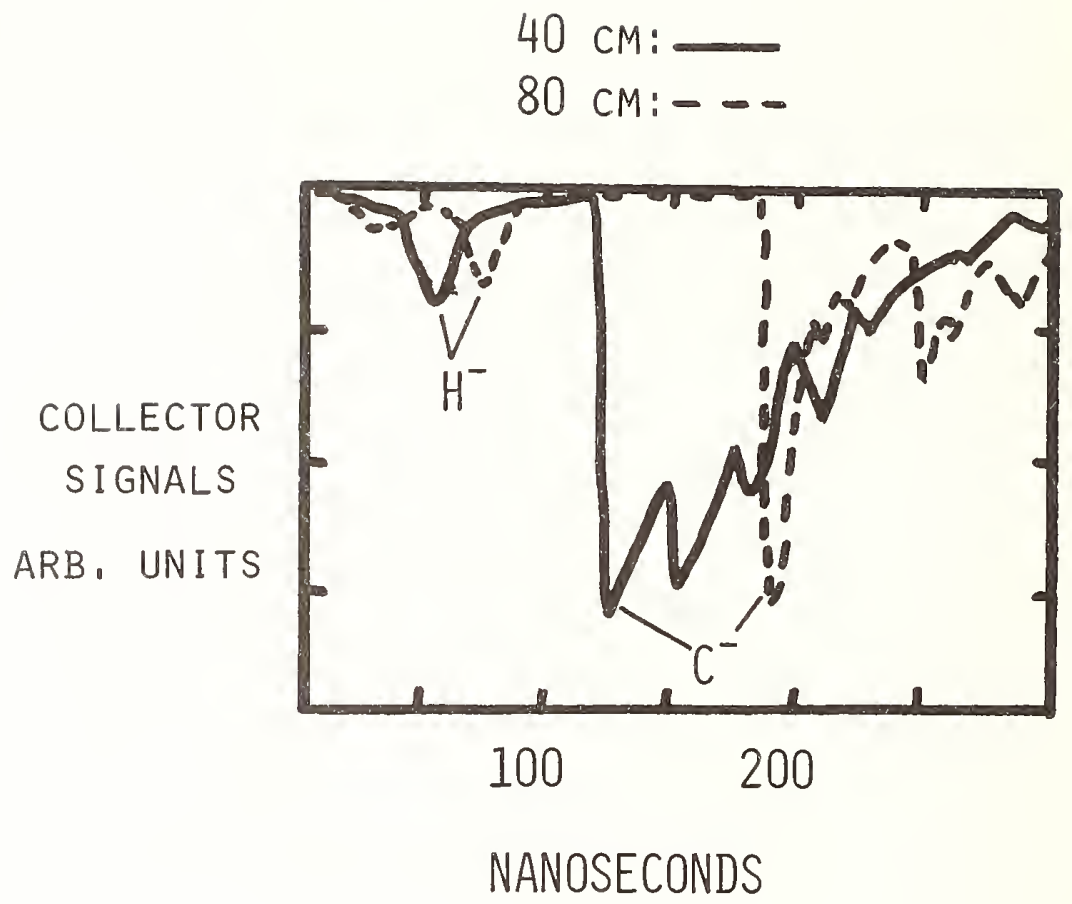

Figure 3. TOFS collector signals on Hydramite shot 8936

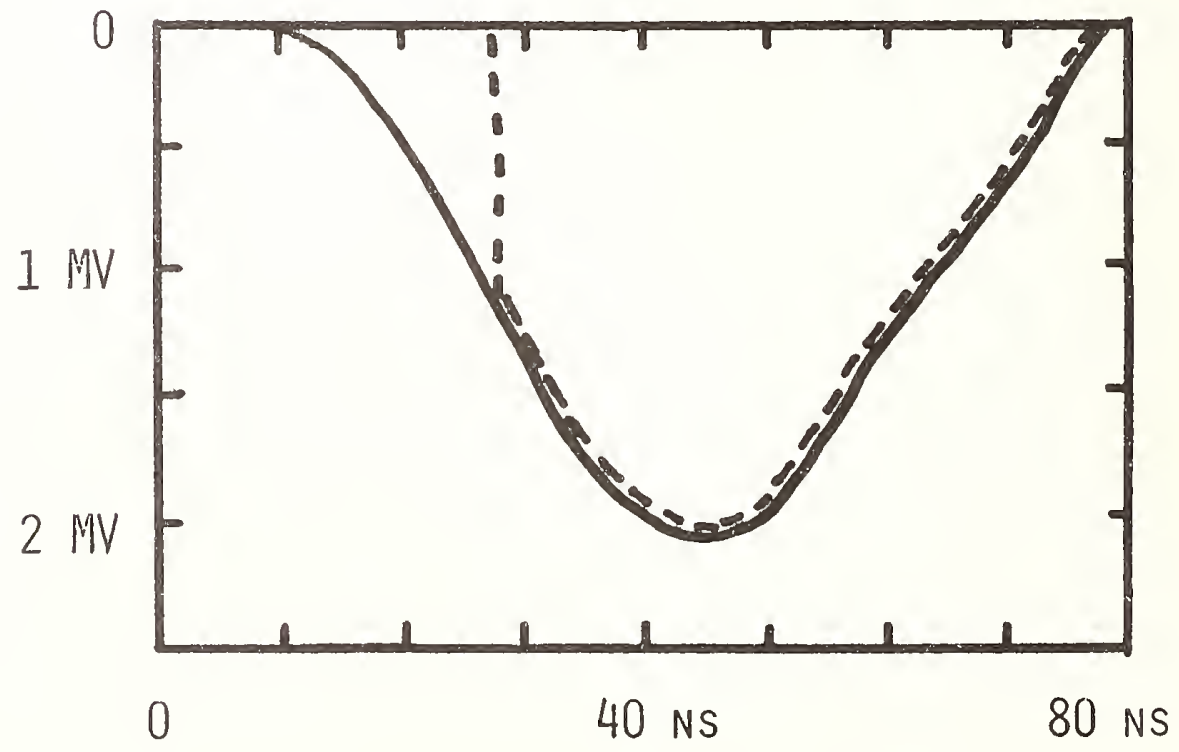

Figure 4. Typical voltage at the input of the Hydramite MITL. The dotted line indicates the expected waveform 5 meters into the MITL. 
by the same gap voltage. Negative ions formed on the rising portion of the voltage waveform will experience "bunching" as the higher energy ions catch up to those formed at lower voltage. If the flight path is sufficiently long the negative ions formed at peak voltage will arrive at the collectors first, making it simple to determine peak voltage. This occurs on Hydramite with the collectors located at 41 and $81 \mathrm{~cm}$ for negative ions heavier than hydrogen. This technique also works. for shorter flight paths if the approximate voltage waveshape is known so that small corrections can be made to the arrival times based on an estimate of the bunching. It should also be possible to determine $V(t)$ by locating collectors near the anode where bunching is a small effect. This technique has not yet been used on the Hydramite negative ion data.

The data in Figure 3 from shot 8936 can be used to illustrate the technique for determining peak gap voltage. The leading edges of the first peaks on the two collectors are separated by $24 \mathrm{~ns}$. These first peaks are presumably due to $\mathrm{H}^{-}$. Using the voltage waveform shown in Figure 4 , one finds that the $\mathrm{H}^{-}$ions created at peak voltage should arrive first of the collector located at $81 \mathrm{~cm}$, but would arrive about $4 \mathrm{~ns}$ later than the leading edge at the $41 \mathrm{~cm}$ collector. It is now possible to estimate the velocity of the $\mathrm{H}^{-}$ions to be $2 \mathrm{~cm} / \mathrm{ns}$ with an accuracy of about $+10 \%$. This implies a peak gap voltage of $2 \mathrm{MV} \pm .4 \mathrm{MV}$. Using this voltage one can then identify with reasonable confidence the second peak as being due to $C^{-}$. This is reasonable since both electrodes are coated with carbon. These second peaks are very sharp because of bunching. Again using the voltage waveform of Figure 4, one finds that the $C^{-}$ions created at peak voltage should arrive first at both collectors. The leading edges of the second peaks are separated by $68 \pm 2 \mathrm{~ns}$. THis gives a peak voltage of 2.I MV +.1 MV. The next significant peak in the collector s̄ignals can now be tentatively identified as $\mathrm{C}_{2}{ }^{-}$ based on this peak voltage.

\section{Conclusions}

These experiments provide the first detailed measurements of negative ions produced in magnetically insulated transmission lines. Several species including $\mathrm{H}^{-}, \mathrm{C}^{-}$, and $\mathrm{C}_{2}{ }^{-}$have been tentatively identified. These negative ions are accelerated to the full gap voltage in MITL's and provide an excellent, non-perturbing diagnostic of peak voltage which should be accurate to better than $5 \%$. 
These negative ions may also have important implications in MITL loss processes. In addition the negative ions produced in MITL's may have possible applications as a source of high energy neutral beams and in a negative ion diode.

These experiments have demonstrated the use of negative ions as a voltage monitor. The techniques employed are both simple and general. Confidence levels and simplicity can be further increased by a definite determination of the negative ion species. This determination was done by Stanley and stinnett and is being presented in another paper at this conference.

\section{References}

[1] T. H. Martin, Proceedings International Pulsed Power Conference, 1976, paper ID-1.

[2] E. I. Baranchikev, A. V. Gordeev, V. D. Korolev, and V. P. Smirnov, Sov. Phys. - Tech. Phys. 3, 42 (1977).

[3] S. Shope, J. W. Poukey, K. D. Bergeron, D. H. McDaniel, A. J. Toepfer, and J. P. VanDevender, J. Appl. Phys. 49 (7), July 1978.

[4] G. Dahlbacka, C. Gilman, C. Meins, H. Helava, I. Roth, R. Schneider, Bulletin of the Am. Phys. Soc. 25 (8), October 1980, paper 1E3.

[5] S. P. Bugaev, E. A. Litvinov, G. A. Mesyats, and

D. I. Preskurovskii, Sov. Phys. -Usp., Vol. 18, No. 1, p. 51 .

[6] G. N. Fursei and V. M. Zhukov, Sov. Phys. Tech. Phys., Vol. 21, No. 2, February 1976, p. 176.

[7] Baksht, Bugaer, Keshelev, Mesyats, Stas'ev, Sukhushin, and Timofeev, Sov. Tech. Phys. Lett. 3 (7), July 1977.

[8] D. W. Swain, S. A. Goldstein, L. P. Mix, J. G. Kelly, and G. R. Hadley, J. Appl. Phys. 48 (3), March 1977.

[9] R. B. Baksht, B. A. Kablambaev, G. T. Razdobarin, and N. A. Ratakhin, Sov. Phys. Tech. Phys. 24 (6), June 1979 , p.689.

[10] Negative Ion Losses in Magnetically Insulated Vacuum Gap, J. P. VanDevender, R. W. Stinnett, and R. J. Anderson, J. Appl. Phys to be published February 1980.

[11] J. P. VanDevender, J.Appl. Phys. 50 (6), June 1979. 
MEASUREMENT OF MAGNETICALLY INSULATED LINE VOLTAGE USING A THOMSON PARABOLA CHARGED PARTICLE ANALYSER

\author{
Timothy D. Stanley \\ Diagnostics Division 4243 \\ Regan W. Stinnett \\ High Density Plasma Source Division 4234 \\ Sandia National Laboratories \\ Albuquerque, NM 87185
}

\title{
Abstract
}

The absence of direct measurements of magnetically insulated line voltage necessitated reliance on inferred voltages based on theoretical calculation and current measurements. This paper presents some of the first direct measurements of magnetically insulated transmission line peak voltages. These measurements were made on the Sandia National Laboratories HydraMITE facility [1].

The peak voltage is measured by observing the energy of negative ions produced at the line cathode and accelerated through the line voltage. The ion energy and the-charge-to-mass ratio are measured using the Thomson Parabola mass spectrometry technique. This technique uses parallel $E$ and $B$ fields to deflect the ions. The deflected ions are detected using a microchannel plate coupled to a phosphor screen and photographic film.

The Thomson Parabola results are compared to Faraday Cup measurements and to calculated voltages based on current measurements. In addition, the significance of observed positive ions will be discussed.

Key Words: Magnetic Insulating Voltage Measurement, Negative Ions Thomson Parabola Charged Particle Analyser

\section{Introduction}

Magnetically Insulated Transmission Lines (MITL) [2] are an essential component of light ion beam fusion devices because they permit many radial pulse power lines to converge to give very large power densities. Voltage measurement of MITLs is critical to establishing 
energy transport and power available at diodes for particle beam fusion. Measuring the voltage on MITLs is difficult because conventional

monitors, i.e., capacitors or resistive voltage dividers, disrupt electron flow causing the magnetic insulation to break down. In the absence of direct. voltage measurements, a rather complex theoretical realationship between measurable currents and the line voltage was established by C. W. Mendel [3]. Because the sensitivity of the inferred voltage to small time shifts in the currents, a more direct voltage measurement was needed. This paper presents the theoretical basis and results for a MITL voltage measurement technique based on measurements made with a Thomson Parabola charged particle analyzer.

\section{Theoretical Basis}

R. W. Stinnett and J.P. Vandevender $\{4\}$ observed that during operation of a MITL, negative ions are produced at the cathode and are accelerated through the line potential to the anode. Measuring the peak energy of the ions exiting through a hole in the anode gives the peak l ine voltage. The Thomson Parabola charged particle analyser was used to determine the ion energy as well as the ion species.

\subsection{Thomson Parabola Charged Particle Analyser (TPCPA)}

The TPCPA operates by deflecting a collimated ion beam in parallel $E$ and $B$ fields [5]. The E field produces a vertical deflection proportional to the ion charge state divided by the ion kinetic energy while the B field produces a horizontal deflection proportional to the charge state divided by the ion momentum. Therefore, ions with an energy distribution trace out a parabola of the form yox ${ }^{2}$ with the peak energy determined by the point of least deflection on the parabolas (i.e., the point on the curve closest to the neutral particle spot formed by undeflected neutral particles and photons). Each unique ion specie and charge state produces a separate parabola. The deflected ion current is amplified by a microchannel plate (CEMA) and then activates a phosphor screen to expose a photographic film. Figure 1 gives the definitions and equations used in describing the TPCPA in this paper.

\subsection{TPCPA Accuracy}

The TPCPA used in these measurements is described in detail in Reference 5. The deflections in $\mathrm{mm}$ for this instrument are given by equations ( 1 ) and (2). The terms in these equations are defined in Figure 1 .

$$
\begin{aligned}
& Y=C_{E} \vee Z / E \\
& X=C_{B} B Z / \sqrt{E A}
\end{aligned}
$$




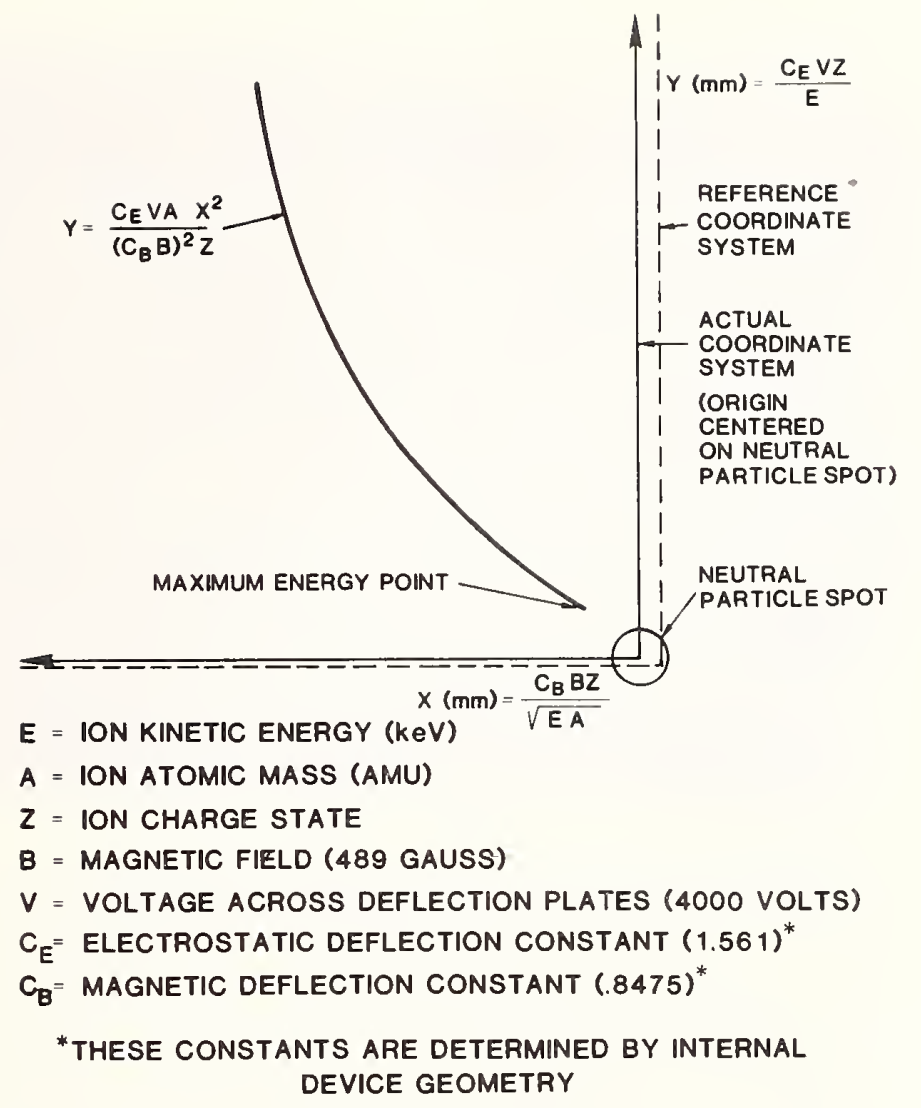

Figure 1. Thomson Parabola equations and definitions

For this instrument, the charge to mass ratio can be determined with an uncertainty of $\pm 11 \%$ while the velocity can be determined to within $\pm 7 \%$. Solving equation (1) for $E / Z$ yields:

$$
E / Z=C_{E} V / Y
$$

This quantity, $E / Z$, can be determined to within $\pm 3 \%[5]$.

\section{Experimental Setup}

Voltage measurements were made on the Sandia National Laboratories' (SNLA) HydraMITE facility. This facility has a nominal operating voltage of $1.9 \mathrm{MV}$ at $0.5 \mathrm{MA}$ current in a $40 \mathrm{nsec}$ pulse. The ions for voltage measurement were extracted through a port located midway along the MITL (see Figure 2). The $\dot{B}$ sensors for calculating the line voltage were located at the diode end of the MITL [6]. A Faraday cup to obtain line voltage from the time of flight of the ions was also lacated on the diode end of the MITL. Most of the data for this paper was obtained during a series of nine shots conducted on December 10-12, 1980. For these shots, the MITL was terminated with a razor blade diode, and the HydraMITE operation was nominally the same. 


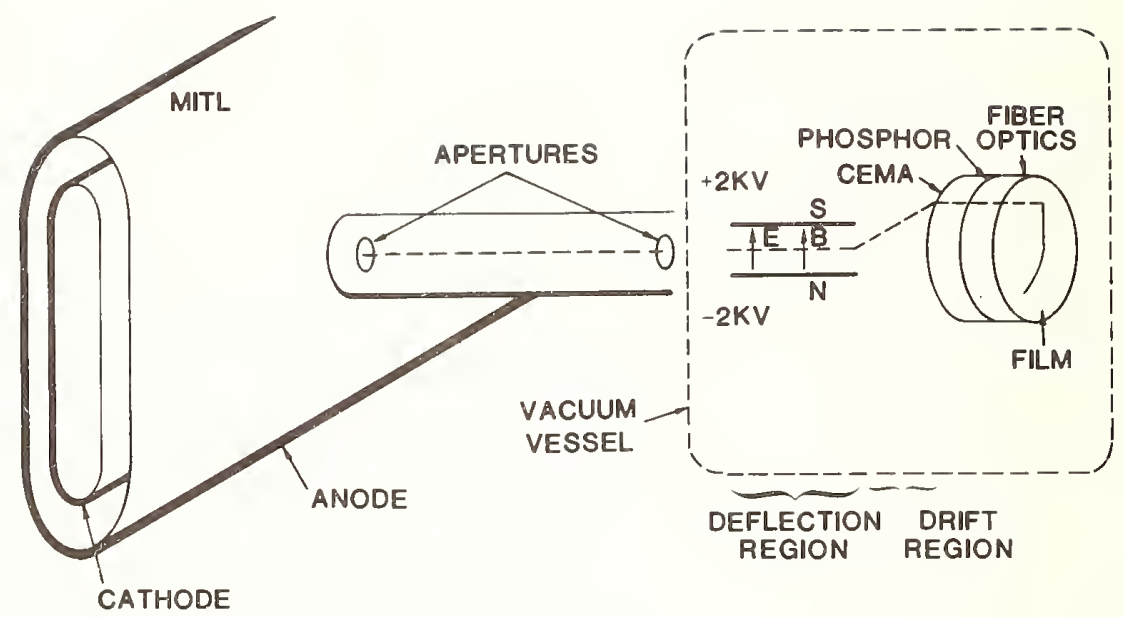

Figure 2. Experimental setup

4. Results

Figure 3 shows the negative ion parabolas for the first shot of this series. It is typical of the parabolas from this series. Figure 4 is a computer simulation of. parabolas for $\mathrm{H}^{-}, \mathrm{C}^{-}, \mathrm{O}^{-}$, and $\mathrm{C}_{2}{ }^{-}$ions with energies from $200 \mathrm{keV}$ to $2 \mathrm{MeV}$ using the equations (1) and (2). From comparison of the photo with the simulation, it is apparent that the left-most curve is $\mathrm{H}^{-}$. Some ambiguity exists for the remaining curves, for example, $1 \mathrm{MeV} \mathrm{O}^{-}$ions and $1 \mathrm{MeV} . \mathrm{CH}_{2}$ iōns would have the same $Z / E$ and Z/P. Also, the closeness of the curves makes resolution somewhat difficult. However, the other three distinct curves are most likely $\mathrm{C}^{-}, \mathrm{O}^{-}$and $\mathrm{C}_{2}{ }^{-}$. Note that the perpendicular lines on the photograph are for reference between photographs and not the coordinate system for measuring curves. The origin for the coordinate system is the center of the neutral particle or photon generated spot in the lower righthand corner of the photograph which is about $2 \mathrm{~mm}$ left and $1 \mathrm{~mm}$ above the intersection of the reference lines.

The voltages measured in these shots are summarized in Table 1. The Faraday cup voltage comes from a time-of-flight unfold of Faraday cup collected ion current to determine negative ion energy [6]. The current-derived voltage is obtained from boundary currents measured with small pick-up loops (B probes) and then applying Mendel's model [3]. Several current derived voltages are missing due to a loss of current data. Much of the fluctuation in these voltages results from difficulties in correcting for time shifts in the cathode and anode current measurements. The electrostatic voltage was measured from the vertical deflection of the maximum energy points on the Thomson Parabola photographs while the magnetic deflection measurement was determined by the horizontal deflection to the maximum energy point of the hydrogen curves. Shot 9068 was used to obtain a horizontal reference thus providing no voltage information while Shot 9074 -was used for a vertical reference and therefore only provided an electrostatic deflection measurement. 


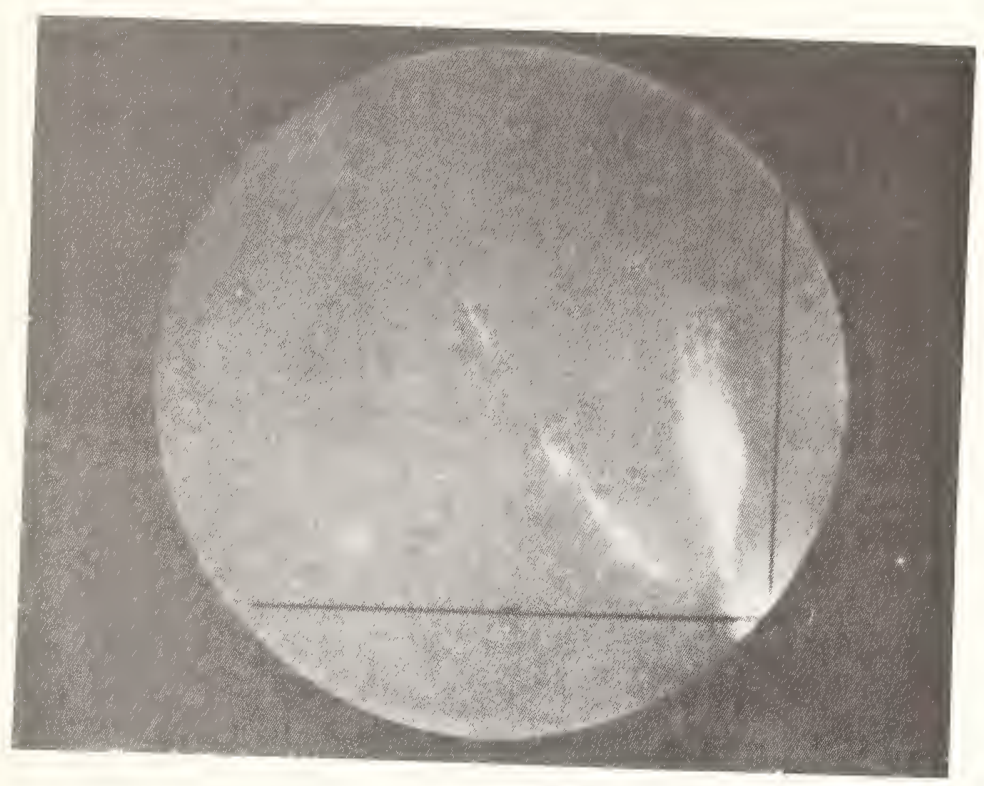

Figure 3. Negative ion parabolas from shot $9067^{*}$

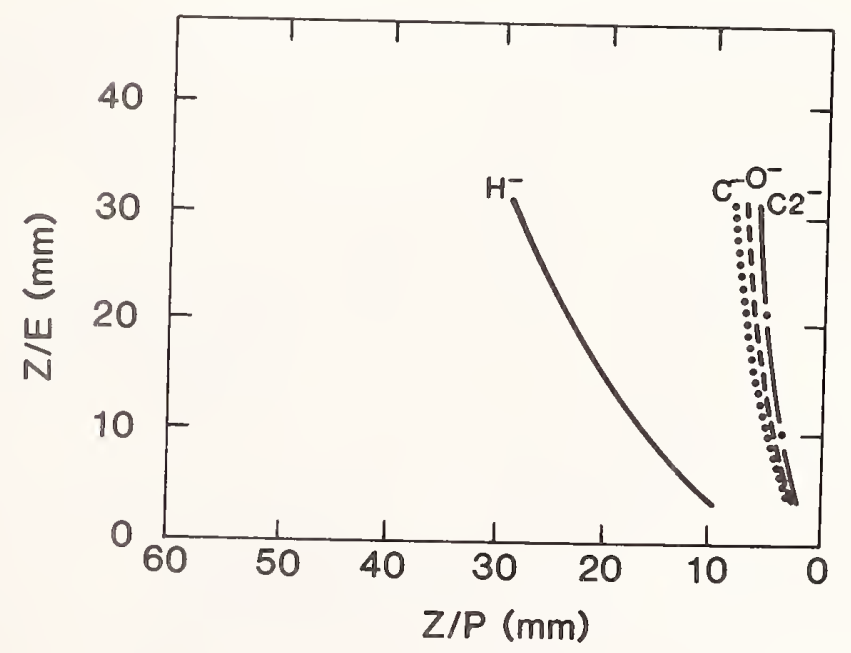

Figure 4. Computer simulation of Thomson Parabola curves*

* The scale sizes on the photo and computer simulation were changed in reproduction. The horizontal reference line in the photo was originally $57 \mathrm{~mm}$ long while the horizontal axis in the computer simulation was $60 \mathrm{~mm}$ long. 
Table 1. Peak voltage measurement summary

\begin{tabular}{|c|c|c|c|c|}
\hline $\begin{array}{c}\text { SHOT } \\
\text { NUMBER } \\
\end{array}$ & $\begin{array}{l}\text { ELECTROSTATIC* } \\
\text { VOLTAGE (MV) } \\
\end{array}$ & $\begin{array}{c}\text { MAGNETIC }+ \\
\text { VOLTAGE (MV) } \\
\end{array}$ & $\begin{array}{c}\text { CURRENT DER IVED } \\
\text { VOLTAGE } \\
\end{array}$ & $\begin{array}{l}\text { FARADAY CUP } \\
\text { VOLTAGE (MV) }\end{array}$ \\
\hline 9067 & 1.8 & 1.6 & 2.2 & 2.1 \\
\hline 9068 & - & - & - & - \\
\hline 9069 & 1.9 & 1.9 & - & - \\
\hline 9070 & 1.9 & 1.6 & - & 1.6 \\
\hline 9071 & 1.6 & 1.5 & 2.1 & 1.5 \\
\hline 9072 & 1.8 & 1.6 & - & - \\
\hline 9073 & 1.6 & 1.4 & 1.7 & - \\
\hline 9074 & 1.7 & - & 1.8 & 2.0 \\
\hline 9075 & 2.1 & 1.7 & 1.4 & 1.4 \\
\hline Ave & $1.8 \pm .2$ & $1.6 \pm .2$ & $1.8 \pm .3$ & $1.7 \pm 3$ \\
\hline
\end{tabular}

The small deflection in the photographs from these shots (approximately $4 \mathrm{~mm}$ in the vertical direction and $11 \mathrm{~mm}$ in the horizontal direction) compared to the width of the lines and the neutral particle reference. spot severely limits the voltage resolution on these tests. Since these tests, two improvements have been made to the system that increase the resolution by at least a factor of four. First aluminum wedges were placed over the deflection plates. These wedges increase the electrostatic field thereby more than doubling the ion deflection while not perturbing the $B$ field. By cross calibrating between electrostatic and magnetic deflections, the electrostatic deflection constant $\left(C_{E}\right)$ in equation (1) was found to increase from 1.56 to approximately 4.0 . Scheduled calibration on a Van de Graaff accelerator will identify this constant to within 3 percent.

The second improvement uses the observation that positive ions at peak line voltage also exist. We hypothesize the positive ions are produced from the negative ions through a stripping process during transit to the analyser which preserves the peak energy. This hypothesis is supported by the observation - that shortening the drift region before deflection reduces the intensity of the positive ion traces. Through centering the parabola display, both positive and negative ions can be observed simultaneously. Then the deflection can be measured between positive and negative ions of the same species, doubling the measured deflection and eliminating the need to use the large neutral particle spot as a reference.

Using the Pythagorean relationship and the parabolic relationship between $X$ and $Y$, a single equation giving the ion energy as a function of the radial distance from the neutral particle spot can be obtained. 
This relationship is:

$$
E=\frac{2 C_{E} V Z}{-k+k^{2}+4 r^{2}}
$$

where $r$ is the radial distance from the neutral particle spot to the maximum energy point on the parabola and

$$
k=\frac{\left(C_{B}\right)^{2} B^{2} Z}{C_{E} \vee A}
$$

Using this relationship, the peak voltage can be measured with approximately $30 \mathrm{~mm}$ deflection between the peak energy point of the $\mathrm{H}^{+}$ and $\mathrm{H}^{-}$ions with line widths less than one $\mathrm{mm}$, thus permitting relative voltage variations as small as $\pm 3 \%$ to be resolved. Figure 5 shows the centered display with both positive and negative ions (negative ions are deflected upward) and the increased vertical deflection resulting from the aluminum wedges over the deflection plates.

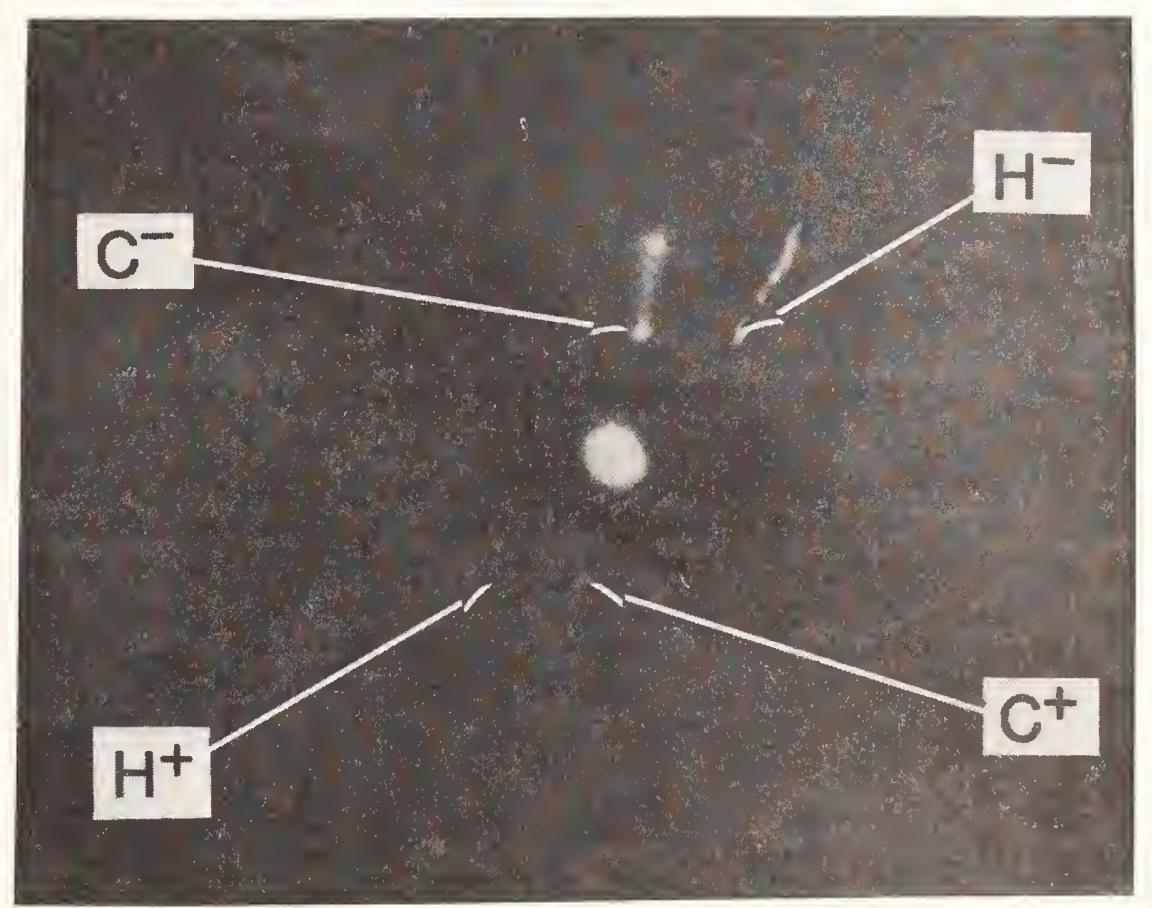

Figure 5. Parabolas from HydraMITE shot 9117 


\section{Conclusions}

An accurate technique has been developed to measure magnetically insulated line peak voltages with a Thomson parabola particle analyzer. Experimental results show that measured voltages are in reasonable agreement with calculated peak voltages deduced from boundary current measurements and time of flight measurements.

\section{Acknowledgement}

The authors thank Glenn W. Kuswa for his interest and suggestions, John Anthes for providing the Thomson Parabola, Carl Cianciabella for his technical support, James Chang for helpful suggestions, and the Sandia HydraMITE Operations staff.

\section{References}

[1] "Particle Beam Fusion Research Facilities at Sandia National Laboratories," Pulsed Energy Directorate, Sandia National Laboratories, 7/80.

[2] J.P. VanDevender, "Long Self-Magnetically Insulated Power Transport Experiments," J. Appl. Physics 50(6), June 1979.

[3] C.W. Mendel, Jr., J.P. VanDevender, and G. W. Kuswa, "Determination of Line Voltage in Self-Magnetically Insulated Flows." IEEE Second International Pulsed Power Conf., Lubbock, TX, June 12-14, 1979.

[4] J. P. VanDevender, R. W. Stinnett and R. J. Anderson, "Negative Ion Losses in Magnetically Insulated Vacuum Gaps," accepted for publication in Applied Physics Letters.

[5] M. A. Gusinow, M. M. Dillon, G. J. Lockwood, L. E. Ruggles, "The Thomson Parabola Ion Analyser, SAND78-0336, March 1978.

[6] R. W. Stinnett, "A Voltage Monitor for Magnetically Insulated Transmission Lines," Workshop on Measurement of Electrical Quantities in Pulsed Power Systems, Boulder, CO, March 2-4, 1981.

\section{Sponsor Acknowledgment}

This work supported by the U. S. Department of Energy under contract DEACO4-76-DP00789. 


\section{Discussion Session 2}

Terry McComb (NRC, Canada). If I may just consider our previous discussion where we were talking about the appropriateness of the two high voltage divider systems. Basically, these dividers are being used to calibrate the efficiency of lasers and the interest is to make voltage measurements to be combined with current measurements to look at the input power to a laser and compare this with the actual lasing power. The sort of accuracy that we have for the measurement of the power input from the laser is in the order of $10 \%$. So, basically, we are looking at 5\% for both the current and voltage accuracy. But, as I pointed out, the present accuracies are not at that level yet and we are still working toward those.

Marco Di Capua (Physics International). I would like to make a couple of remarks on voltage measurements with liquid dielectric resistor dividers. The first one addresses one of the comments of Richard Malewski. Richard's remarks about the dependence of the dielectric constant on frequency are correct. The way that Physics International has built dividers over the years is to incorporate the tap-off within a homogeneous column. In this way, the non-linear effects that exist in the dielectric resistive fluid and the variations of resistance and dielectric constants with temperature and frequency are compensated because the divider is a geometric divider. A second remark is that this type of divider will work in vacuum when the divider vessel is assembled from field grading rings and cone insulators. We have described such a monitor which operates up to two megavolts in a vacuum. We have used this to measure voltage directly in magnetically insulated transmission lines by connecting the divider to the inner conductor of the transmission line through a hole in the outer conductor. We measured voltages up to one megavolt with penetrations in the transmission line which are $11 / 2$ inches in diameter. A sketch of the voltage divider appears in figure 1 below. This type of measurement was described in the literature $[1]$.

Jack Katzenstein (Maxwell Laboratories). I have a comment on the paper by Dr. Stanley. Let me say I think that it is a very elegant application of what is also a very elegant technique, the Thompson parabola mass spectrometer. But, there is a more elegant, a more recent mass spectrographic technique which would be pertinent, namely the Bainbridge velocity selector. The deflection in the Bainbridge

1Two-Megavolt Divider for Pulsed High Voltages in Vacuum, D. Pellinen and M. S. Di Capua, Rev. Sci. Inst. 51, 1980, pp. 70-73. 


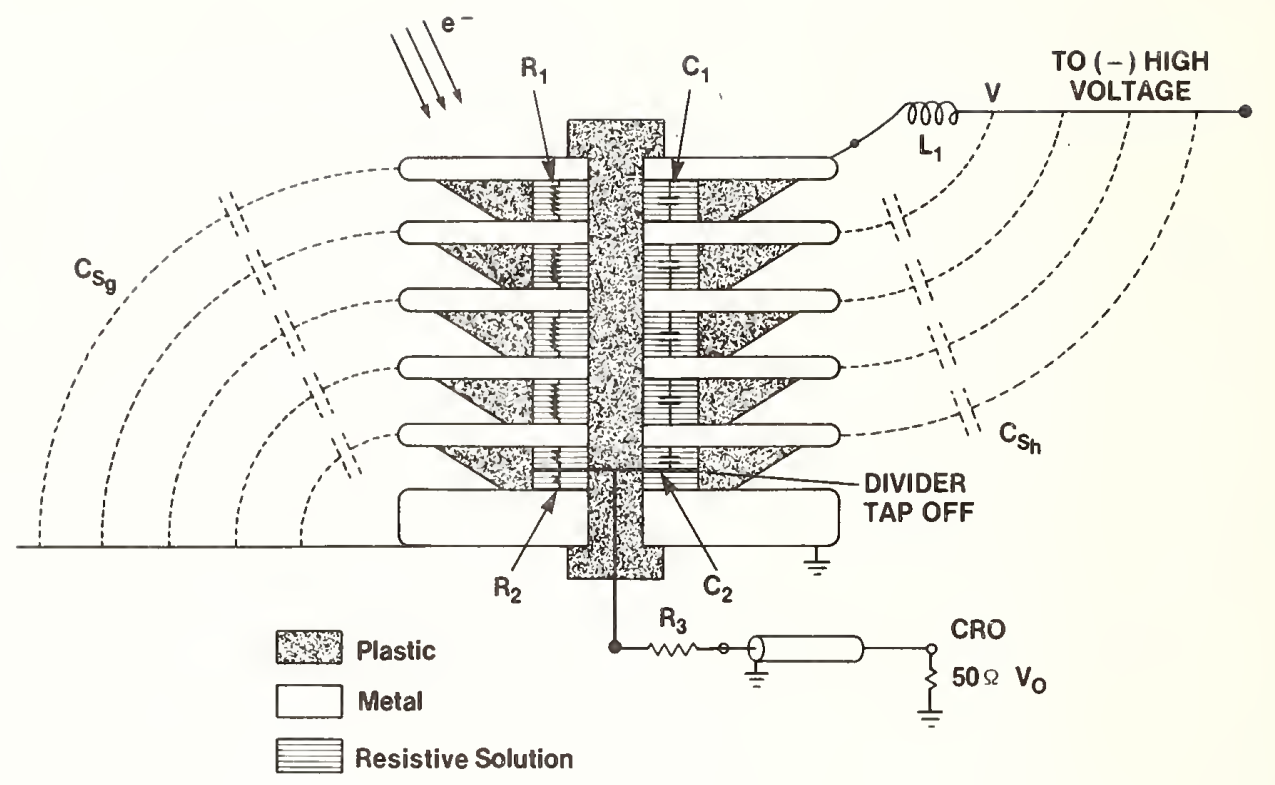

Figure 1. Sketch of vacuum voltage divider. Metal annuli and plastic cone frustra form containment vessel for resistive solution. Inductance of the measuring circuit $L_{1}$ places a lower bound on divider resistance. Stray electron current places upper bound on resistance. The strays $\mathrm{CS}_{\mathrm{g}}$ and $\mathrm{CS}_{\mathrm{h}}$ are much smaller than the interstage capacitances. Divider is geometric so $R_{1} C_{1}=R_{2} C_{2}$. 
velocity selector is proportional only to the velocity of the ion and independent of its species. It was used by Bainbridge as a device prior to magnetic deflection to sort the ions according to the charge mass ratios. It occurred to me that it would be easier to interpret the deflection produced by the Bainbridge velocity selector which uses crossed $E$. and $B$ fields instead of parallel fields then by using the parabolic method.

Tim Stanley (Sandia). I guess I have to plead ignorance on the Bainbridge velocity selector so I will have to look that up.

Jim Chang (Sandia). I think that an appropriate response to this question is that we do have multiple species and would like to make a survey of the species component of this negative ion beam at the same time as determining the energy (the peak energy in this case). We are working on a time resolution capability by sweeping the electric fields so that we do get essentially the time resolved voltage measurements of the magnetically-insulated transmission lines.

Mark Wilkinson (Maxwell Laboratories). While we are on the topic of this negative ion measurement, I would like to ask what current densities were observed for the negative ion beams.

Regan Stinnett (Sandia). It depends on the preparation of the lines. It seems to be typically one or two amps per square centimeter. We coat our lines with about 25 microns of carbon to form a lot of whiskers so that you have a uniform cathode plasma. We find that on the first shot, after coating the line with carbon, you get a much larger loss than that, but it then settles down to something on the order of a couple of amps per square centimeter. That is total current including all of the different species.

Di Capua. I want to know what the lag time is between the onset of voltage on the line onset of negative ion emission.

Stinnett. As far as I can tell it is immediate and there seem to be more negative ions produced at higher voltage or higher current. We are running matched so they occur at the same time.

Frank Young (NRL). I have a question for Tim Stanley. If you look at the parabolas produced by the Thompson parabola probe, you see some structure running from the high energy point further up the parabola to lower energy down along the parabola. This is particularly evident for the hydrogen line. What information can you extract from this structure?

Stanley. If you remember from the talk, there was a little bit of disagreement between the data and the computer simulation. If you look at the original of this photograph very carefully, you will see that there is a line which continues up past that discontinuity. Now, it turns out my apertures are a quarter-inch hole in front and 
then a small 20-mil pin hole in back. Because of my device geometry, I can have an eighth of an inch difference in my line positions. It turns out that my lines don't fill up that total area and, therefore, it appears that the cathode plasma is not continuous but that there is emission from discrete points. I think I am seeing discontinuities across the cathode.

Neville Harris (Ion Physics). I noted that you had positive ions and this is presumably due to the poor vacuum. If you can get reactions to actually remove two electrons from the ion, can you also not get a change of charge during flight, so that the velocity does not correspond to the complete voltage falling across the line? Do you get any aperture effects such as bombardment at the edge of the aperture with secondary stuff coming off the edges?

Stanley. I have a separate vacuum system where the deflection region is. The poor vacuum is in the region of the tube that connects my Thompson parabola apparatus to the magnetic insulated line. It is pumped from within the magnetic line through a quarter-inch hole. I don't think I am seeing any stripping or charge exchange after I get through my aperture and into my deflection regions. The current is fairly low at the second aperture. Something like $10^{11}-1012$ ions actually are exposing my film. I don't think that I am seeing any slit effects here. Other effects that may have been produced on the front aperture or space charge expansion are restricted by the second aperture.

Richard Malewski (IREQ). In the second part of the presentation, there was a description of the Ontario Hydro device for measuring voltage in a gas-insulated bus which is exactly the case as we referred to in the first part of this discussion. The difference is the dielectric. According to Neville Harris, there is some damping provided in the circuit which starts at high voltage bus and which ends at the ground envelop, due to the resistance of water. If we refer to a simple RLC circuit with this capacitance, which seems to be predominant with some inductance at, let's put it this way, a resistance, if I remember correctly, the critical damping resistance should be less than $1 / 2 \sqrt{L / C}$ which gives us about 200 ohms. I don't believe that the water used here will have resistance less than 200 ohms. So, I don't believe that water will act effectively as a damping element. I would rather stick to the first statement that this is an integrating element which simply does not pass the high frequency oscillation. The second comment is concerning the Ontario Hydro system, where there is a tapping device between the coaxial bus and ground. Depending on the length of the tapping sector, one can get more or less smooth response of such a divider. A suggestion is to not make it out of aluminum but make it out of a resistive material such as a resistive paint on some insulating substance. That will provide you with a regularly shaped response because you will damp internal oscillations in this sector. Try this, it should work. I agree with Mr. Di Capua 
that that is a very clever trick to tap the liquid divider column here, and it will certainly help to give the response that isn't oddly shaped. But that's the first approximation effect. If you really look at the equivalent circuit of such a divider, there will be a high voltage conductor, the resistive elements of this liquid, and the parallel capacitors. These elements do vary in the same way in the upper and lower parts of the divider. But, don't forget that we have also the ground here and we have stray capacitance to ground as well as stray capacitance to the high voltage bus. Ideally, the voltage distribution along the divider column should be such that the capacitive voltage distribution is the same as by resistive distribution. Unfortunately, this is very difficult to obtain because we cannot control the stray capacitance. It depends on the relative position of the divider relative to the bus high voltage. Since we have in the same circuit frequency and temperature variable elements, as well as stray capacitance which is not temperature nor frequency dependent, we might expect second order problems.

Di Capua. I agree that one has to consider the stray capacitance between the divider and ground and the divider and the high voltage conductor. We have built a device in which the interstage capacitance in the liquid dielectric is much larger than the strays to ground or the strays to the high voltage conductor. The high capacitance arises from the water dielectric. The strays are shown schematically in the voltage divider sketch (figure 1). Another remark is that the measurement gets difficult when transit times within the divider itself which are of the order of the duration of the pulse you are trying to measure. In this case, we try to position the divider so that the wave which is the voltage we are attempting to measure is normally incident to the divider column. Even then the going is sort of rough. The question then becomes really what are you measuring and I don't intend to enter into that discussion now.

Chat Cooke (MIT). One difference between the two capacitive dividers that Harris and Fujimoto presented is that the electric fields are oriented differently in the two. In one case, Harris', the low voltage capacitor is actually perpendicular to the main gap capacitance and field, while in the second case, Fujimoto's, the low voltage capacitor is in fact geometrically parallel to the main gap capacitance and hence the fields are in the same direction. My question is: besides convenience, is there any advantage or disadvantage between those two arrangements?

Harris. I will first deal with the point that Richard drew with regard to the damping of this circuit. I am saying that the series resistor which goes to ground at the oscilloscope termination forms a damping element for the oscillatory circuit in the divider. Now, back to the question raised by chat cooke. I have the low voltage capacitor perpendicular to the high voltage. I was working in a very high voltage line where the operation was near breakdown. If you have the capacitors parallel, then fundamentally the average field in 
the two capacitors must be the same. If, due to mechanical errors, the sensing element is at a slight angle, the field at the end is going to be greater. The field will also be increased by the insertion (of low dielectric constant) of the monitor cable. In addition, there is the field enhancement in the corners. However, if you recess the sensing electrode (see figure la of Discussion Session 1) you no longer get this trouble. By simpily making the area of this cylinder (corresponding to $\mathrm{C}_{g}$ ) greater than the area of the end of the plug (corresponding to $C_{1}$ ), you can lower the field in the recessed region. The other advantage is that of mechanics. When you wish to put it in a line, you can insert the complete assembly from the outside. If you have a mushroom shape, you are going to have to get to it from both sides. So there is mechanical convenience as well. That's the reason for the perpendicularity of the low voltage capacitor. Also note that because you have lowered the field in the low voltage region, you have lowered the requirements for mechanical tolerance with regard to your device. With regard to the monitor described by Mr. Fujimoto and the problems due to transit time between the ends of the sensing electrode, there is a very simple way to deal with that. You taper it. You don't have it the same length all the way around the circumference. Then transit time varies from location to location around the tube.

John Shipman (Sachs-Freeman, NRL). In a voltage divider circuit such as Neville Harris described, one can better understand the nature of the coupling to the divider by considering a short impulse wave traveling down a coaxial transmission line with the divider mounted on the outer conductor. The impulse wave will be accompanied by a thin disc of displacement current (and conduction current, if the medium has conductivity) flowing radially between the inner and outer conductor. Since there is no inductance associated with this thin disc, the coupling to the divider will be capacitance and resistance in parallel only and will occur as the disc passes over the divider.

In regard to ringing or resonance of such divider circuits, I did have a high frequency resonance at about $2500 \mathrm{MHz}$ with my dividers using Mylar between the center cylindrical plug and the outer grounded cylinder. This resonance occurs because the plug and outer cylinder form a low impedance, short transmission line essentially open circuited at both ends. The resonant frequency is that at which the line is a half wavelength. In Mr. Harris' case with water between the plug and the grounded wall, if the plug were 3 inches long, the resonant frequency would be $220 \mathrm{MHz}$ and would only be visible on very high frequency oscilloscopes if excited by very fast rising pulses.

N. Fujimoto (Ontario Hydro). Dr. C. Cooke raised a question regarding the orientation of the low voltage capacitor with respect to the high voltage capacitance in two designs of capacitive dividers. Initially, capacitances involved were considered to be lumped. However, presently our preference is to use a transmission. line analysis where the coupling cylinder defines two transmission line impedances $Z_{1}$ and $Z_{2}$ 
for a short length within a coaxial system of impedance $Z_{0}$ (figure 1 of our paper). This type of analysis illustrates the prime advantage of this geometric configuration. As described in the paper, if the sensing cylinder is perfectly thin and concentric, then no impedance mismatches are formed at either end of the coupler. As a result, any propagating wave (assumed to be TEM) incident onto the coupling system does not suffer any degradation in its high frequency characteristics and the division ratio is simply determined by the ratio of the transmission line impedances. Indeed we have been able to measure transients with risetimes about 350 ps (the limit of our ability to make single shot measurements) as shown in figure 8 of our paper. Degradations that do occur are felt to be caused by the connection to the external measurement device, which because of stray capacitance, may appear to have initially a low impedance. Admittedly, some disadvantages in this configuration may exist in high voltage applications. In practice, we would normally install a shunt inductor on the low voltage portion of the divider for $60 \mathrm{~Hz}$ protection and flare the coupler ends to prevent corona.

The suggestions made by Messrs. Malewski and Harris concerning the use of resistive couplers and the use of tapered ends on the coupler are interesting, but I will have to withhold comment until I have the time to examine these suggestions in more detail.

John Barth (Barth Electronics). When you drive the output into a 50 ohm Tine, you could have a directional coupler which would be the secondary transmission line B where the outputs would come out through the walls. As long as the secondary line (B) was terminated in its characteristic impedance, you would not have any resonances on it. You would have a short time response, of course, but you do get an exact division ratio, and you would have as fast a response as the loss factor of the insulating material would permit.

Bob Hebner (NBS). I think that in the last few minutes of the discussion, I am going to invoke my prerogative as Chairman and try to elicit a bit of discussion on a few of the other topics which were presented earlier, but which we have not had any discussion on so far. For example, we were treated, at the opening of this session, to a very eloquent discussion of electro-optic measurements of voltage by Jim Thompson. I was wondering if anyone had any questions or comments about that presentation or that field.

Harris. I noticed that you said that the number of fringes was proportional to the square of the voltage. You really mean the square of the field, and this is not just a nitpicking point because there are end effects in a Kerr cell. In other words, as the light goes through the cell, it goes through end regions as well, which have much lower fields and thus don't respond in the same way as the main field. Is that not true that you are going to have to compensate for that in a rather mathematical way? 
Jim Thompson (Univ. of South Carolina). Yes, that is true. But, usually what you assume though is the Kerr cell length is long compared to the path length through interaction of the fringing fields. That is the way one usually says that this is negligible. Now, I think that Bob Hebner has done some work and seen that this is a few percent or one percent or less for reasonably long Kerr cells. If one wanted to make a very short Kerr celi to reduce the capacitance, for example, then that would be a probiem.

Hebner. When we are developing high accuracy Kerr cells, we calibrate them by measuring what we call the cell constant. This is done by applying a known voltage and measuring the phase shift induced by that known voltage. This calibrates both the uniform field and the end field effects which range from maybe a half percent to two or three percent. We find that the end field effects are only geometry dependent and, therefore, having performed a voltage calibration, you have an end field effect which is a constant forever and you can use the cell for extended periods with very high accuracy. Over the past twenty years, techniques have been developed to account for this adequately so that measurements to about $0.1 \%$ precision can be made. We think that by the end of the year we will see about $1 / 4 \%$ accuracy.

Katzenstein. I don't think one ought to dismiss the Pockels effect out of hand for electro-optic voltage measurements for two important reasons. It is a longitudinal effect, and it is a linear effect. Since it is a longitudinal effect, it should be possible to eliminate a geometric factor in the calibration such that only the Pockels constant enters. The fact that it is linear is going to greatiy facilitate a numerical inversion. Of course, I must agree with Dr. Thompson that Pockels active materials are expensive and they have somewhat questionable dielectric strengths.

Malewski. I have had experience with an application of the Pockels effect to high voltage measurement. The problem was to measure voltage distribution along the column of a lightning arrestor under impulse conditions. We devised a simple gadget composed of a heliumneon laser whose beam was deflected by two roof prisms, passed through a Pockels cell, and was returned to the laser beam detector standing on the ground some distance from the high voltage object. We were able to measure the voltage applied to the Pockels cell which was installed in sequence at each particular shed of the lightning arrestor. The system worked fairly well but the main problem was ringing. Every electro-optic crystal is at the same time piezoelectric which means that when you apply a voltage to it, it is stressed and the mechanical response for such a rapid stress is an oscillation. We tried several tricks. Prestressing techniques used to eliminate this mechanical ringing were not too successful. A typical oscillogram showed a signal due to the high voltage impulse we applied perturbed 
by one due to the mechanical vibration of the slab of crystal which was excited by the electric field applied to the metallized transparent plates.

Katzenstein: It becomes a matter of time scale. The acoustical vibration of a crystal is going to be on a several microsecond time scale. If you are measuring voltages on nanoseconds or several nanoseconds time scale, that's important.

Gene Nolting (NSWC). I would like to ask a question of both Bob Hebner and Jim Thompson. I found the method of using the Taser to make high voltage measurements extremely interesting. However, I have some trouble relating how to use this in a real high voltage system and it appears perhaps that maybe the best way to incorporate this technique would be to calibrate monitors which are a little more mechanically easy to use on existing machines. Is it possible for you to, say, tap off the high voltage somehow and calibrate existing monitors with this technique? Perhaps you could have a traveling road show going around the country and making measurements so that everybody's measuring voltage with the same standard.

Hebner. I will comment first. Your point describes a process that we have used in the measurement of microsecond and longer pulses. One can use the Kerr effect devices to accurately transfer among laboratories. I think that a classic example is that for about ten years, when Sandia was having some interesting activities with a certain waveshape in the microsecond regime, accurate voltage measurements of those pulses were maintained among NBS, Sandia, and a variety of their subcontractors using transportable Kerr effect standards. That is an approach which has shown itself to be useful over a limited regime of voltages and time.

In addition, your suggestion to use a technique that has totally different sources of error from a conventional probe as a comparison to determine information about the accuracies of the existing probes is a good one. In our program at NBS, we have adopted exactly that philosophy.

Thompson. As far as calibration is concerned, I think that Bob's comments are correct. I think in the laboratory if you have to measure voltage, you reach for a capacitive or resistive voltage divider thinking that it is going to be easy. Then, you spend several months trying to get rid of your loops, noise, and various other things. It may be that we should begin to use electro-optical techniques more than we do if for nothing else than to see waveshapes. 
Session B - Power and Energy Measurements

ION CURRENT AND VOLTAGE DETERMINATIONS

BY NUCLEAR TECHNIQUES

Frank C. Young

Plasma Physics Division

Naval Research Laboratory

Washington, DC 20375

\begin{abstract}
Nuclear reactions induced by intense proton or deuteron beams from high-power pulsed generators can be used for current and voltage determinations. Average currents and voltages are determined from measurements of delayed radioactivity from nuclear reactions. This technique is illustrated for proton current using, the ${ }^{12} \mathrm{C}(\mathrm{p}, \gamma){ }^{13} \mathrm{~N}_{2}$ yeaction 2 nd for deuteron current and energy using the ${ }^{12} \mathrm{C}(\mathrm{d}, \mathrm{n}){ }^{13} \mathrm{~N}$ and ${ }^{27} \mathrm{Al}(\mathrm{d}, \mathrm{p}){ }^{28} \mathrm{Al}$ reactions. Time-resolved current and voltage determinations can be made from measurements of prompt nuclear radiations. Protop currents are determined by prompt-gamma measurements using the ${ }^{19} \mathrm{~F}(\mathrm{p}, \alpha \gamma){ }^{16} 0 \mathrm{re}-$ action. Neutron time-of-flight techniques are described to determine either the deuteron energy or current using the $d(d, n)^{3}$ He reaction.
\end{abstract}

\title{
1. Introduction
}

Pulsed power generators are being used to produce intense pulsed ion beams, and new techniques are required to determine the energy and current of such beams [1]. The ions are usually produced in these generators in a diode region at high vacuum where it is difficult to make electrical measurements. The current and voltage from the generator can be measured reliably across an insulator in an oil or water dielectric just before the vacuum region. The electrical characteristics in the vacuum diode may differ from those measured in the dielectric. An inductive correction ( $-\mathrm{L} d \mathrm{~d} / \mathrm{dt}$ ) is usually applied to the voltage where $\mathrm{L}$ is the inductance that the pulse "sees" in the vacuum region and I is the total diode current. Electron emission from surfaces in the vacuum other than the cathode causes the diode current to differ from the current measured in the dielectric. Also this electron emission makes it impossible to determine $L$ for the inductive voltage correction. Consequently, the electrical properties measured in the dielectric are not necessarily the same in the diode. In this presentation I shall review some new diagnostics based on nuclear techniques that have been 
developed to measure the energy (i.e. voltage) and current of proton or deuteron beams.

Many of the usual techniques for ion-beam measurements are inappropriate for the beams from pulsed power generators. These beams are frequently so intense that scintillators, for example, do not survive the burst of ions. Furthermore, these beams are generally space-charge neutralized so that charge collection and magnetic or electrostatic deflection are complicated and uncertain. In addition, a beam pulse may contain several different species (e.g. H, D, ${ }^{12} \mathrm{C}, \ldots .$. ).

The most unambiguous method for measuring a specific beam component in this situation is by selective nuclear reactions. Information concerning the energy and intensity of specific ion species can be inferred from measurements of nuclear reaction products. The number of nuclear reactions $N$ with cross section $\sigma$ produced by $M$ particles of energy $E$ incident on a target of thickness $\Delta x$ with $n$ target nuclei/ $\mathrm{cm}^{3}$ is given by $\mathrm{N}=\mathrm{Mn} \sigma \Delta \mathrm{x}$. The dependence of $\mathrm{N}$ on energy per particle is contained in $\sigma$ provided $\Delta \mathrm{x}$ is sufficiently small that $\sigma$ does not change appreciably as the particle traverses the target. The dependence of $N$ on intensity (i.e. current) is contained in M. In general these dependences on energy and intensity can not be separated readily to provide independent measurements of ion voltage and current. In a few specific cases this separation is possible. More often, an ion current and voltage deduced in some other way are used to calculate the expected number of nuclear-reaction products for comparison with experiment. Differences are then ascribed to an incorrect current or voltage. These may then be modified appropriately to bring the calculated number of nuclear-reaction products into agreement with experiment.

\section{Radioactivity Measurements}

The technique of nuclear activation has been used to provide information concerning the energy or intensity of proton or deuteron beams. The approach is to measure the delayed gamma-ray activity induced in a target by the beam. Target samples activated by the beam are removed and counted with a shielded gamma-ray detector. By using specific targets and measuring specific nuclear reactions, the energy or intensity of a specific ion species is determined.

The following steps are taken to optimize the sensitivity of the activation. First, thick targets are used to optimize the number of reactions. In this case, the reaction yield is given by the integral of the cross section over the range of the ions in the target. The yield may be written

$$
\mathrm{Y}=\mathrm{N} / \mathrm{M}=\int \sigma / \varepsilon \mathrm{dE}
$$

where $\varepsilon$ is the stopping cross section of the target. The stopping cross section is proportional to the stopping power $\left(\varepsilon=\mathrm{n}^{-1} \mathrm{dE} / \mathrm{dx}\right)$ and has 
been tabulated in the literature [2] for protons and deuterons. Second, short-lived activities are used. If a small number of radioactive nuclei (N) are produced, then a short lifetime $(\tau)$ is desired to increase the activity (i.e. $\mathrm{dN} / \mathrm{d} t=\mathrm{N} / \tau$ ). However, the lifetime must be long enough to allow the target sample to be removed for counting. Half-lives of the order of minutes are convenient. Third, good detection efficiency with adequate gamma-ray energy resolution is provided by NaI detectors. To achieve low background the detectors may be shielded with a few inches of lead. Also, for positron activities coincidence counting with two detectors at $180^{\circ}$ to each other is used to reduce the background by several orders-of-magnitude.

The intensity of MeV proton beams may be determined by using $(p, \gamma)$ resonance reactions [1]. The cross section for such a reaction peaks at the resonance energy $E_{R}$. For a resonance with a narrow width $\Gamma$, the reaction yield is nearly constant provided the proton energy $\mathrm{E}$ is well above the resonance energy $\left(i \cdot e \cdot\left(E-E_{3}\right)>\Gamma\right)$. This situation is illustrated in Figure 1 for the ${ }^{12} \mathrm{C}(\mathrm{p}, \mathrm{\gamma})^{13} \mathrm{~N}$ reaction [3]. This reaction has a resonance at $0.46 \mathrm{MeV}(\Gamma=37 \mathrm{keV})$ and a

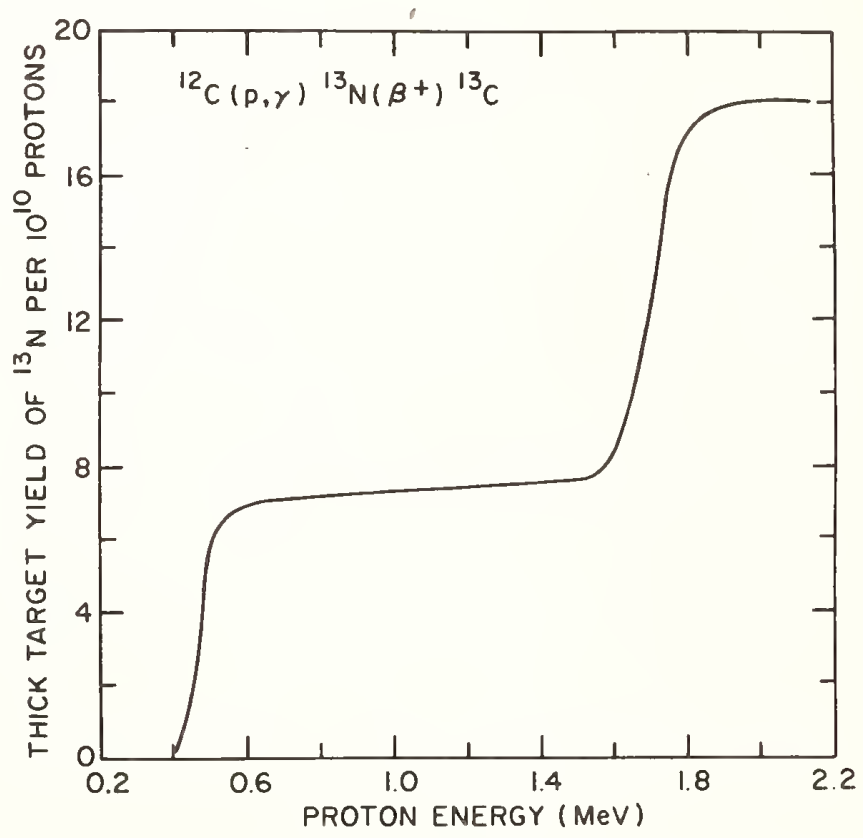

Figure 1. Thick-target yield for the ${ }^{12} \mathrm{C}(\mathrm{p}, \gamma)^{13} \mathrm{~N}$ reaction in a carbon target.

resonance at $1.70 \mathrm{MeV}(\Gamma=67 \mathrm{keY}){ }^{\circ}$ Between these resonances the yield is nearly constant ( $Y=7.5 \times 10^{-13} \mathrm{~N} /$ proton at $\left.1 \mathrm{MeV}\right)$. In this energy range the number of protons is given by $N=M / Y$ where $M$ is the number of radioactive ${ }^{13} \mathrm{~N}$ nuclei produced by the reaction. The $10-\mathrm{min}{ }^{13} \mathrm{~N}$ radioactivity can be measured efficiently with very little background by coincidence counting of the annihilation radiation from the positron decay of ${ }^{13} \mathrm{~N}$. In this way, as few as $2 \times 10^{11^{1}}$ protons of 0.6 to $1.5 \mathrm{MeV}$ energy can be detected. For an accurate determination of the intensity 
of protons greater than $0.8 \mathrm{MeV}, 1 \frac{1}{1} \mathrm{t}$ may be necessary to correct for the ${ }^{12} \mathrm{C}(\mathrm{d}, \mathrm{n}){ }^{13} \mathrm{~N}$ contribution to the ${ }^{13} \mathrm{~N}$ activity from the naturally-occurring isotopic abundance of deuterium in the ion source [1].

Nuclear activations can be used to determine the ion energy or voltage because the yields of most nuclear reactions are strongly dependent on the ion energy. For example, the yields of deuteron-induced reactions are small below the Coulomb barrier but increase rapidly with energy as illustrated in Fig. 2 for the ${ }^{12} \mathrm{C}(\mathrm{d}, \mathrm{n}){ }^{13} \mathrm{~N}$ and ${ }^{27} \mathrm{Al}(\mathrm{d}, \mathrm{p}){ }^{28} \mathrm{Al}$ reactions [4]. These reactions do not have narrow resonances, but they

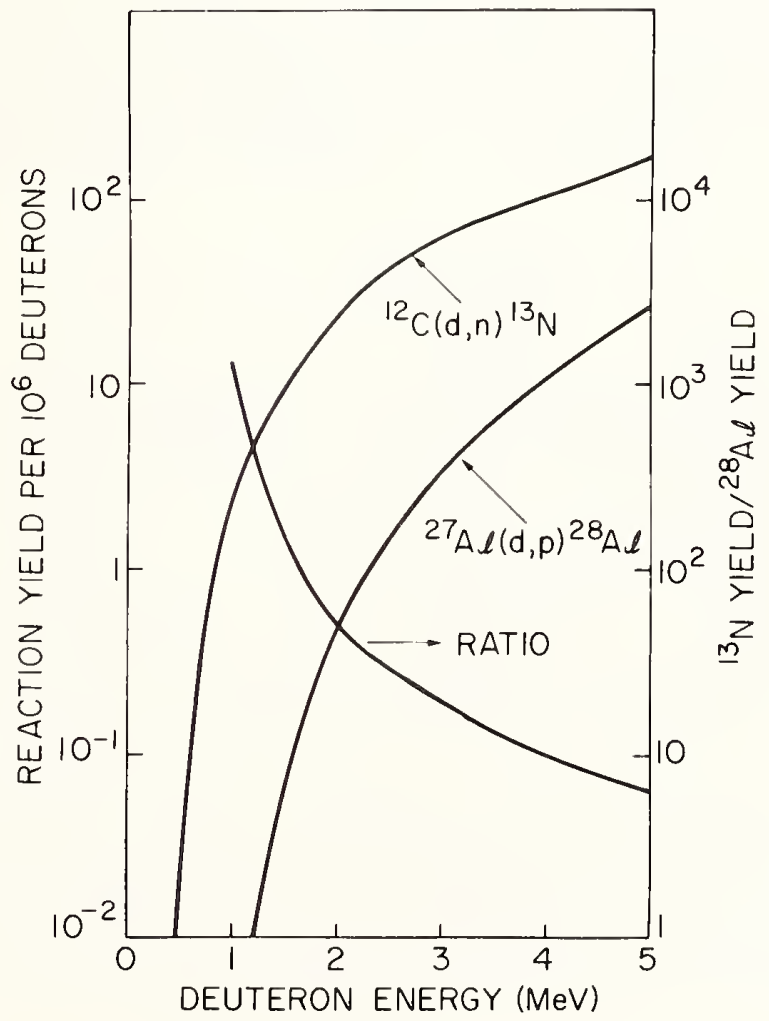

Figure 2. Thick-target yields $\mathrm{f}_{95}$ the ${ }^{12} \mathrm{C}(\mathrm{d}, \mathrm{n}){ }^{13} \mathrm{~N}$ reaction in a carbon target and for the ${ }^{27} \mathrm{Al}(\mathrm{d}, \mathrm{p}){ }^{28} \mathrm{Al}$ reaction in an aluminum target. Ratios of these two yields are also shown.

do reach yields about $10^{3}$ times larger than $(p, \gamma)$ reactions. These reactions produce short-lived delayed gamma-ray activities which are readily measured with a NaI detector [4]. Simultaneous activations of carbon and aluminum can be used to determine mean deuteron energies. The ratio of the yields of these two reactions is a sensitive function of deuteron energy (see Figure 2) so that a measured ${ }^{13} \mathrm{~N} /{ }^{28} \mathrm{Al}$ ratio can be used to determine the mean deuteron energy. Then the reaction yield at this energy is used to determine the number of deuterons. 
These nuclear activation techniques are not practical for ion beams of high current density. Ablation of the surface of the target being activated limits the technique to current densities of about $100 \mathrm{~A} / \mathrm{cm}^{2}$ depending on the target material, ion species and energy. This limit can be extended an order-of-magnitude by using attenuating screens to reduce the "beam intensity or a "pillbox" to confine the ablated radioactivity [5]. Even so, current and voltage determinations from radioactivity measurements correspond to values averaged over the duration of the pulse and can only be compared with average values of the electrically measured current or voltage.

\section{Time-Resolved Measurements}

The prompt-gamma and neutron time-of-flight (TOF) techniques provide time-resolved measurements which allow more detailed comparisons with the electrical measurements. Also, these measurements of prompt nuclear radiations are uneffected by ablation of the target.

\subsection{Prompt-Gamma Technique}

The prompt- $\gamma$ technique is used to measure proton current [6]. A nuclear reaction which produces prompt $\gamma$ rays of several MeV energy is selected. The ${ }^{19} \mathrm{~F}(\mathrm{p}, \alpha \gamma){ }^{16} 0$ reaction, which produces $\gamma$ rays of 6.1 to 7.1 MeV, has been used with Teflon targets. These $\gamma$ rays are measured with a scintillation-photomultiplier detector of good time resolution ( $\lesssim 5$ ns FWHM). The detector is located in a lead shield to attenuate the bremstrahlung $(\leqslant 1 \mathrm{MeV})$ from the generator and to convert the prompt $\gamma$ radiation into a secondary spectrum of lower energy quanta which can be detected efficiently with a small plastic scintillator.

The voltage output $V_{d}$ of the prompt- $\gamma$ detector (prompt- $\gamma$ response) depends on the proton current $I_{p}$ and the nuclear reaction yield $Y_{Y}$ which is a function of the proton energy $\mathrm{E}$. To determine the proton current from the measured prompt- $\gamma$ response, one must correct for the flight times of different energy protons from the diode to the Teflon target. Then the time variation of $V_{d}$ is related to the time variation of $I_{p}$ in the following way:

$$
V_{d}\left(t_{d}\right)=K \int_{0}^{\infty} I_{p}(t) Y_{\gamma}[E(t)] \delta\left[t_{d}-t-l / v(t)\right] d t .
$$

The distance $l$ is the proton flight path from the diode to the target, and $v$ is the velocity of protons of energy $E=1 / 2 m_{p} v^{2}$ emitted at $t$ ime $t$ from the diode. The thick-target yield $Y_{\gamma}(E)$ for this reaction has been determined [7]. The quantity $K$ is a constant specified by the detector geometry and target-to-detector distance, and it can be determined by calibration with a Van de Graaff accelerator [7]. In practice, the proton current is determined by iterating on Eq. 2 until the prompt- $Y$ response calculated with an assumed proton current agrees with the 
measured prompt-r response. An initial proton current may be determined from a Rogowski coil measurement [8] of the total ion current or from an analytic model [9] of ion generation.

An application [7] of the prompt- $\gamma$ diagnostic is illustrated in Figure 3. The prompt- $\gamma$ response measured from a Teflon target located $60 \mathrm{~cm}$ from an ion diode is shown in Figure 3a. The prompt- $\gamma$ signal is delayed in time due to the proton time-of-flight so that the diode brems strahlung precedes the prompt-r signal. The measured response is compared with the response calculated using the proton energy and current displayed in Figure $3 \mathrm{~b}$. The proton energy is given by the measured diode voltage corrected for inductive effects. The width of the prompt- $\gamma$ response is directly related to the width of the current pulse. The shape of the prompt-Y response reflects the shape of the proton current in the vicinity of its peak.
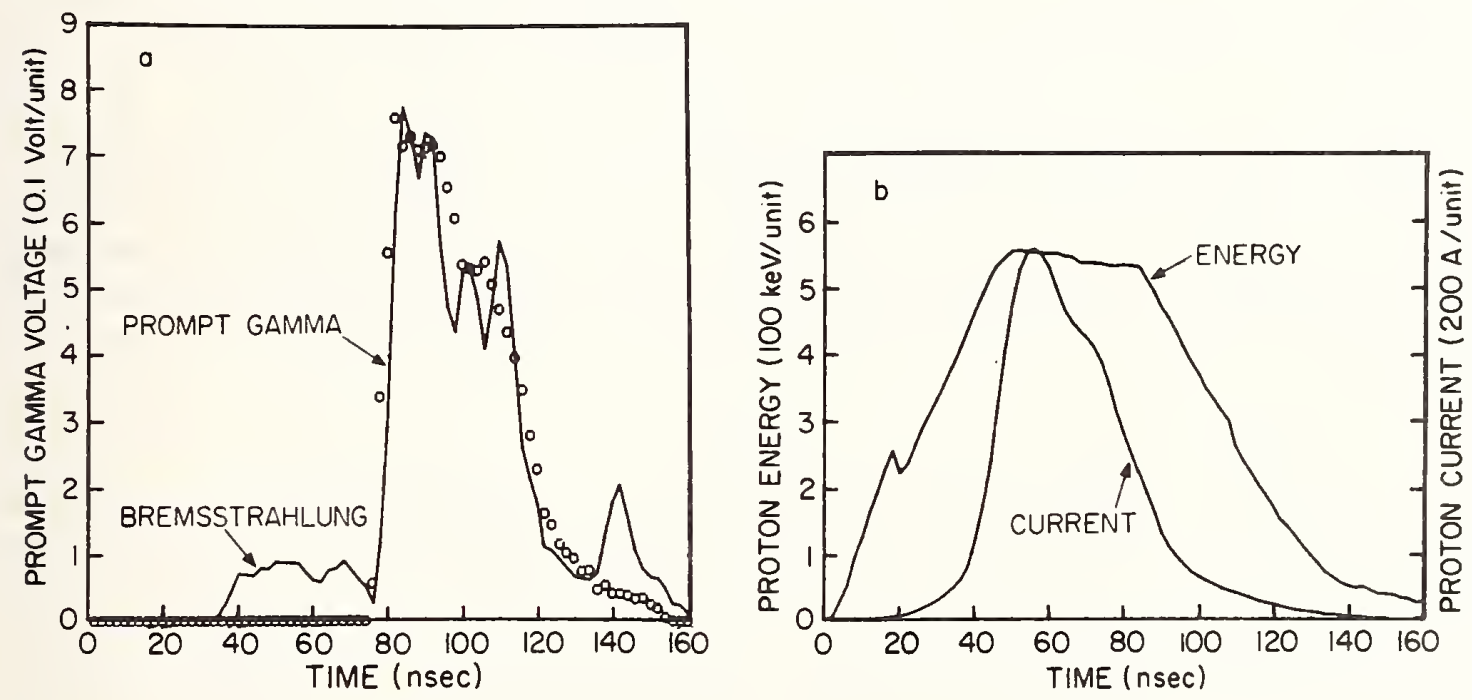

Figure 3. The measured prompt $\gamma$-ray detector response (solid line) in (a) is compared with the response (open points) calculated using the proton energy and current displayed in (b).

The proton current determined by the prompt- $\gamma$ measurement is compared with the proton intensity determined from the activation of carbon in the Teflon target. For this measurement, attenuating screens were used between the diode and the target to minimize target ablation. The ion energy is small enough that no correction for deuterons in the proton beam is necessary. A measurement of the positron decay of ${ }^{13} \mathrm{~N}$ indicates that $(5.9 \pm 0.6) \times 10^{4} \mathrm{~N}$ nuclei we re produced in the Teflon target. The number of $13 \mathrm{~N}$ nuclei produced by the proton current and voltage displayed in Figure $3 \mathrm{~b}$ is $(4.7 \pm 0.7) \times 10^{4}$. These independent determinations are in agreement within the experimental uncertanties. It should be noted that the prompt- $\gamma$ measurement only samples the current above $340 \mathrm{keV}$ and that the carbon activation only samples the current above $\sim 440 \mathrm{keV}$. These measurements are insensitive to the current profile for proton energies below these thresholds. 


\subsection{Neutron Time-of-Flight}

The pulsed nature of these ion beams makes the time-of-flight (TOF) rechnique ideal for measuring neutron energies. The neutron energy is determined from a measurement of the neutron flight time for a known flight path. Then the ion energy (i.e. voltage) is determined from the kinematics of the neutron-producing reaction. With fast scintillators and photomultipliers a detector resolution of about 5 ns can be achieved. For neutrons of $\mathrm{MeV}$ energies flight paths of several meters are used to separate the neutron pulse from the diode bremsstrahlung. As the flight path $(l=v t)$ is increased, the neutron energy resolution scales inversely with the flight path for a fixed time resolution

$\delta t(i . e) E / E=2 v \delta t / \ell)$, but the number of neutrons incident on a given detector decreases quadratically with distance. A compromise must be made between signal intensity and energy resolution.

The time spread of the neutron response in a TOF detector is composed of three contributions: 1)the detector response, 2)the energy dispersion of the neutrons and 3)the duration of emission of the neutrons. For many plasma sources the detector response time can be made much less than the other contributions. Ion-energy measurements require a long neutron flight path so that the time spread from energy dispersion dominates the duration of neutron emission. For ion-current determinations, a short flight path is required so that the time spread from energy dispersion is minimized relative to the duration of neutron emission.

These techniques are illustrated with some measurements [10] using the $d(d, n)^{3}$ He reaction. Kinematics [11] for this reaction are shown in Figure 4. The neutron energy depends most sensitively on the deuteron energy for neutrons emitted in the forward direction. Therefore TOF detectors should be deployed in this direction for ion-energy measurements. The neutron energy is almost insensitive to the deuteron energy near $90^{\circ}$, and this direction may be more useful for deuteron-current measurements.

A determination of the energy and emission time of the most energetic deuterons from an ion diode using the TOF technique is presented in Figure 5. The deuteron beam is directed onto a thick deuterated polyethylene $\left(\mathrm{CD}_{2}\right)$ target to produce neutrons by the $\mathrm{d}(\mathrm{d}, \mathrm{n})^{3} \mathrm{He}$ reaction. The responses of two neutron TOF detectors at $15^{\circ}$ and at two different flight paths are shown in Figure 5a. Both detectors record an initial pulse resulting from diode bremsstrahlung before the neutron responses are measured. The response corresponding to the longer flight path is delayed as expected. The energy and emission time of the most energetic neutrons are deduced from these traces. The most energetic neutrons have a flight time corresponding to the leading edge of the neutron response. (This time is measured at one-half of the signal maximum.) The neutron flight time for each detector is measured relative to the peak of the $x$-ray signal and corrected for the x-ray flight time. The difference of the neutron flight times implies a neutron energy of $3.41 \pm 0.15 \mathrm{MeV}$. The corre- 


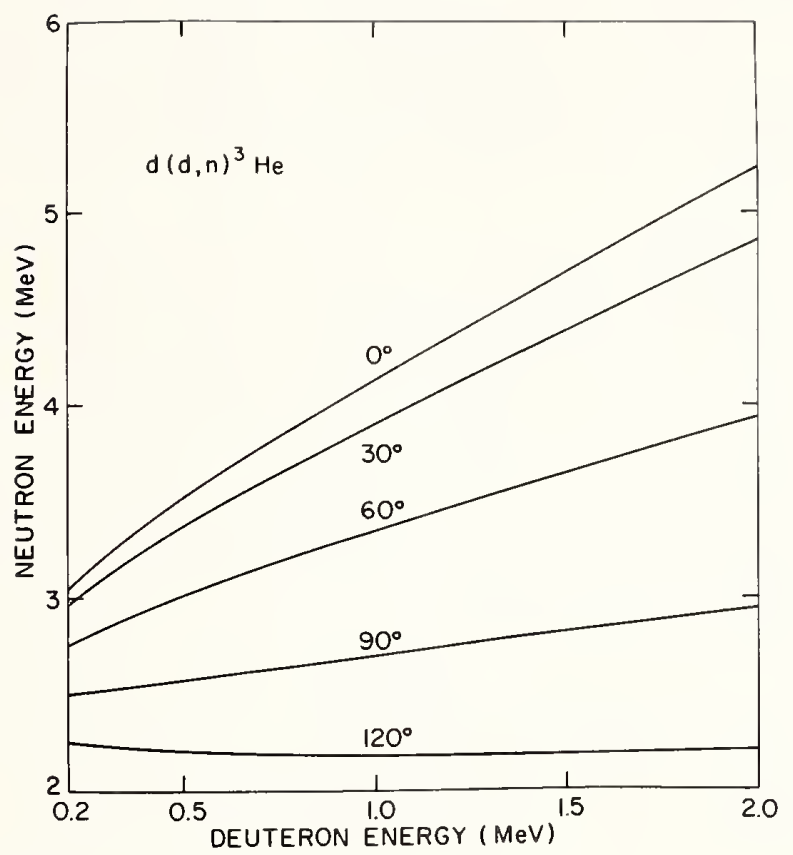

Figure 4. Dependence of the outgoing neutron energy at different angles on the incident deuteron energy for the $d(d, n)^{3}$ He reaction.
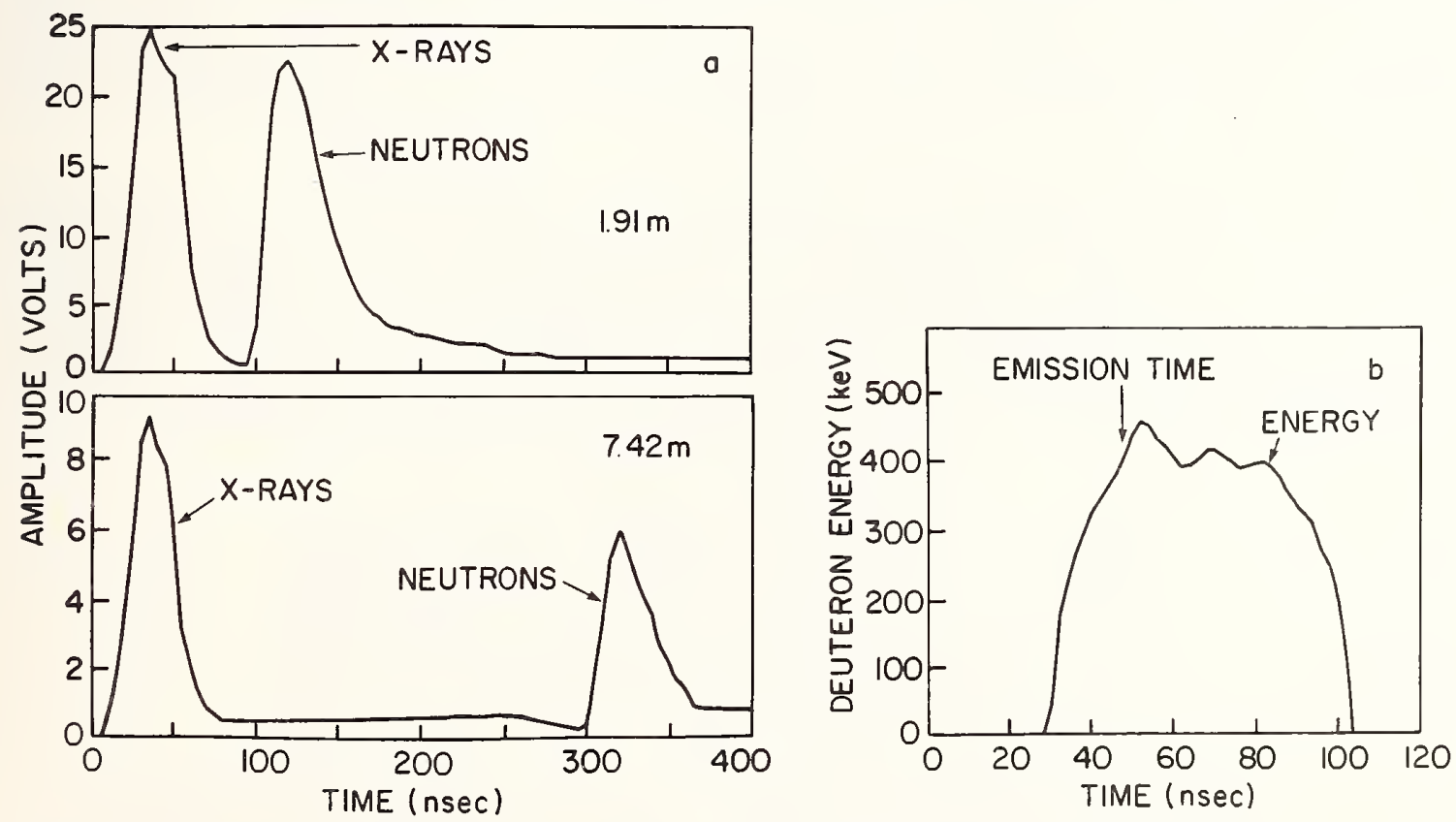

Figure 5. (a) Neutron TOF measurement for two detectors at $15^{\circ}$ to the deuteron beam direction and at flight paths of $1.91 \mathrm{~m}$ and $7.42 \mathrm{~m}$ respectively. The maximum deuteron energy corresponds to the most energetic neutrons which are detected at the leading edge of the TOF traces. (b) The emission time of these deuterons is compared with the deuteron energy trace. 
sponding deuteron energy is $0.45 \pm 0.10 \mathrm{MeV}$ based on the $\mathrm{d}(\mathrm{d}, \mathrm{n})^{3} \mathrm{He}$ reaction at $15^{\circ}$. The uncertainties in these values are a result of uncertainties in time and distance measurements. The neutron flight time versus distance is extrapolated to zero distance to determine the emission time for the most energetic neutrons.

The emission time and deuteron energy are compared with the time dependent accelerating voltage in Figure 5b. The ion energy trace in this figure corresponds to the measured diode voltage corrected for the diode and drift tube inductance and reduced by the energy loss of the deuterons in traversing a thin foil used for current neutralization [8]. The maximum deuteron energy for this trace is $0.46 \mathrm{MeV}$ in good agreement with the energy determination by TOF. The neutron emission time is detemined relative to this ion energy trace through a fiducial timing pulse. This corresponds to a time of $67 \mathrm{~ns}$ on the ion energy trace in Figure 5b. This time must be reduced by the flight time of deuterons from the diode to the target $(20 \mathrm{~ns})$ to give the emission time of deuterons from the diode of $47 \pm 7$ ns as shown in Figure 5b. The uncertainty in this value results from propagating uncertainties in time and distance measurements. The deuteron emission time corresponds to the maximum deuteron energy within experimental error.

In this example the neutron energy was measured to a precision of about $4 \%$ corresponding to a deuteron-energy precision of about $20 \%$. The deuteron-energy precision improves at higher deuteron energy because the deuteron energy is increasing more rapidly than the neutron energy (see Figure 4). For example, a $4 \%$ neutron energy precision corresponds to a deuteron-energy precision of about $10 \%$ for $1.5-\mathrm{MeV}$ deuterons.

The neutron response in a TOF detector provides a direct measure of the deuteron current if the width of the response results primarily from the duration of neutron emission. The measured neutron response can be compared with a calculated response to determine the deuteron current. For an instantaneous pulse of deuterons $N_{d}(t)$ of energy $E_{d}$ at $t$ ime $t_{n}$ incident on a thick $\mathrm{CD}_{2}$ target, the neutron response is given by

$$
R_{c}\left(t_{n}\right)=N_{d}(t) \int_{0}^{E} d(\sigma / \varepsilon) d E=N_{d}(t) Y_{n}\left(E_{d}\right)
$$

where

$$
\begin{aligned}
& t_{n}=t+\ell_{n} / v_{n}+\ell_{d} / v_{d} \\
& \ell_{n}=\text { neutron flight path } \\
& v_{n}=\text { neutron velocity } \\
& E_{d}=1 / 2 m_{d} v_{d}^{2}=\text { incident deuteron energy } \\
& \sigma=\text { cross section for the } d(d, n){ }^{3} \text { He reaction } \\
& \varepsilon=\text { stopping cross section for deuterons in polyethylene. }
\end{aligned}
$$

The neutron response is related to the deuteron current through the reaction cross section integrated over the target thickness. It is 
assumed that the target thickness is larger than the range of the deuterons. The time of arrival of a neutron at the TOF detector $\left(t_{n}\right)$ is different from the time that the deuteron was emitted from the diode by the flight time of the deuteron to the target $\left(\ell_{d} / v_{d}\right)$ and by the neutron flight time $(l / v)$. The slowing-down time of the deuterons in the target is negligible compared to the neutron flight time. Since the deuterons lose energy in the target, the outgoing neutrons have a velocity dispersion and hence a time dispersion. In addition, an angular dispersion of the deuterons incident on the target (e.g. in a focusing geometry) will produce a time dispersion through the reaction kinematics (see Figure 4). For this discussion we will assume a unidirectional beam on target. Complications arising from angular dispersion are discussed elsewhere [10].

Cross sections for the $\mathrm{d}(\mathrm{d}, \mathrm{n})^{3}$ He reaction have been well documented [11], and the dependence of the neutron yield $Y_{n}$ on the deuteron energy is shown in Figure 6 for two different angles. At all angles $Y_{n}$

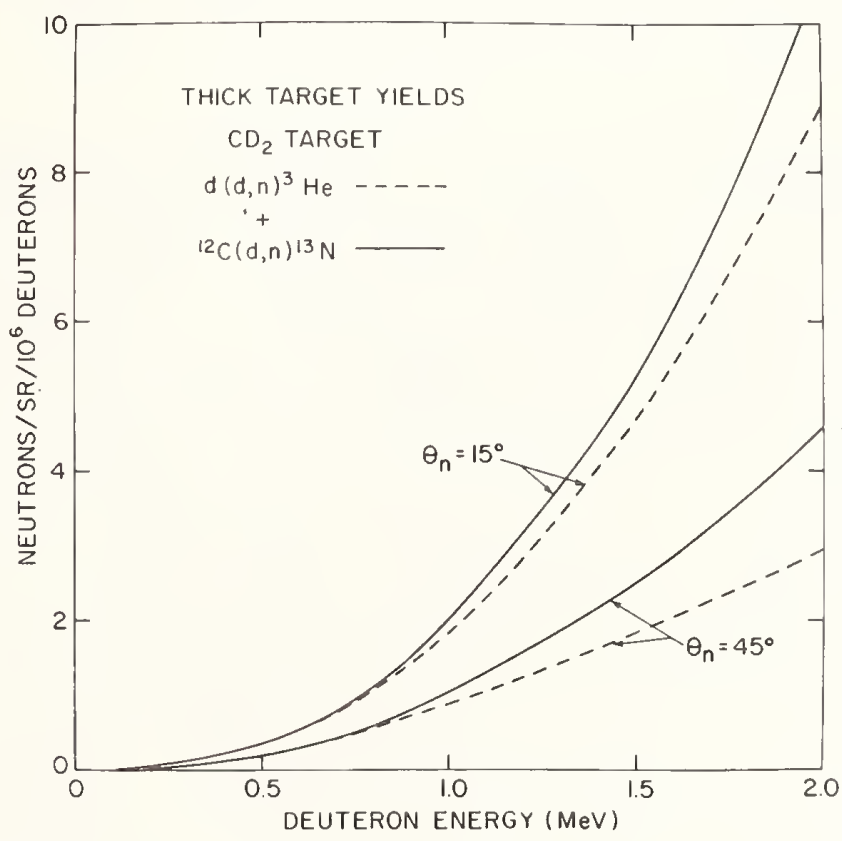

Figure 6. Thick-target yields for the neutron output from deuteron bombardment of a $\mathrm{CD}_{2}$ target. Yield curves at $15^{\circ}$ and $45^{\circ}$ are displayed for the $\mathrm{d}(\mathrm{d}, \mathrm{n})^{3}$ He reaction (dashed curves) and for the sum of the $\mathrm{d}(\mathrm{d}, \mathrm{n})^{3} \mathrm{He}$ and ${ }^{12} \mathrm{C}(\mathrm{d}, \mathrm{n})^{1{ }^{3} \mathrm{~N}}$ reactions (solid curves).

increases with energy. Consequently, the neutron response is largest for the most energetic neutrons and decreases in magnitude at later times due to deuteron energy loss in the target. Also shown in Figure 6 is the total yield including neutrons from the ${ }^{12} \mathrm{C}(\mathrm{d}, \mathrm{n})^{13} \mathrm{~N}$ reaction on carbon in $\mathrm{CD}_{2}$. These neutrons are separated in time from the $d-d$ neutrons, but they must be included in total yield measurements with neutron activation detectors [12]. Such total yield measurements can 
provide an absolute calibration of the deuteron current. Cross sections for the ${ }^{12} \mathrm{C}(\mathrm{d}, \mathrm{n})^{13} \mathrm{~N}$ reaction are taken from the work of Lefevre [13].

In an experiment the deuteron energy is a time-dependent pulse of finite duration. Also the neutron yield depends on the angle of the neutron detector $(\theta)$ relative to the deuteron-beam direction. Then Eq. 3 must be rewritten to include the effects:

$$
R_{n}\left(t_{n}, \theta_{n}\right)=\int_{0}^{E_{d}(t)} N_{d}(t) Y\left[E_{d}(t)\right] \delta\left(t_{n}-t-\ell_{n} / v_{n}-\ell_{d} / v_{d}\right) d t
$$

The neutron velocity is determined by the kinematics of the $d(d, n){ }^{3} \mathrm{He}$ reaction for given values of $E_{d}$ and $\theta_{n}$. A straightforward inversion of Eq. 4 for deuteron current $N_{d}(t)$ is not possible. Instead, the right side of Eq. 4 is calculated and compared with the measured neutron response. For this purpose, the corrected diode voltage trace is used for $E_{\mathrm{d}}(t)$ and the deuteron current predicted by a simple analytic model [9] is used for $\mathrm{N}_{d}(t)$.

Neutron TOF measurements to determine the deuteron current from an ion diode are presented in Figure 7. Detectors are located at $15^{\circ}$ and $85^{\circ}$ to the incident-beam direction, and short flight paths are used to minimize the neutron time spread from energy variations. However, the flight paths used are sufficiently long for the detectors to recover from the $x$-ray flash before recording neutrons. In this figure measured TOF responses are compared with yields calculated according to Eq. 4. The deuteron energy $E_{d}(t)$ and the deuteron current $N_{d}(t)$ for these calculations are given in Figure $7 \mathrm{a}$. Neutron yields are calculated in 2-ns time increments of $E_{d}(t)$ and $N_{d}(t)$ and then summed to give the curves which are plotted as open points in Figures $7 \mathrm{~b}$ and $7 \mathrm{c}$. The calculations have been normalized to the peaks of the measured responses. The widths of the calculated and measured responses are in good agreement, but there are some differences in shape. The doublehump in the calculated responses is due to this same structure in the energy. The energy profile can be modified to bring the calculated responses into better agreement with experiment. Finally, the energy and current profiles can be combined with the thick-target yield to calculate the time-integrated neutron output. The result is normalized to the neutron yield measured with an activation detector to set the absolute scale of the deuteron current in Figure $7 \mathrm{a}$.

To assess the importance of the duration of the deuteron current and energy traces on the neutron response, a calculation was carried out using a monoenergetic deuteron pulse of constant current of only 2-ns duration. Neutron responses calculated in this case are shown at the bottom of Figures $7 \mathrm{~b}$ and $7 \mathrm{c}$. Here the temporal dispersions at both angles are much less than the contribution from the duration of ion emission as given by the ion current and energy traces in Figure $7 \mathrm{a}$. 

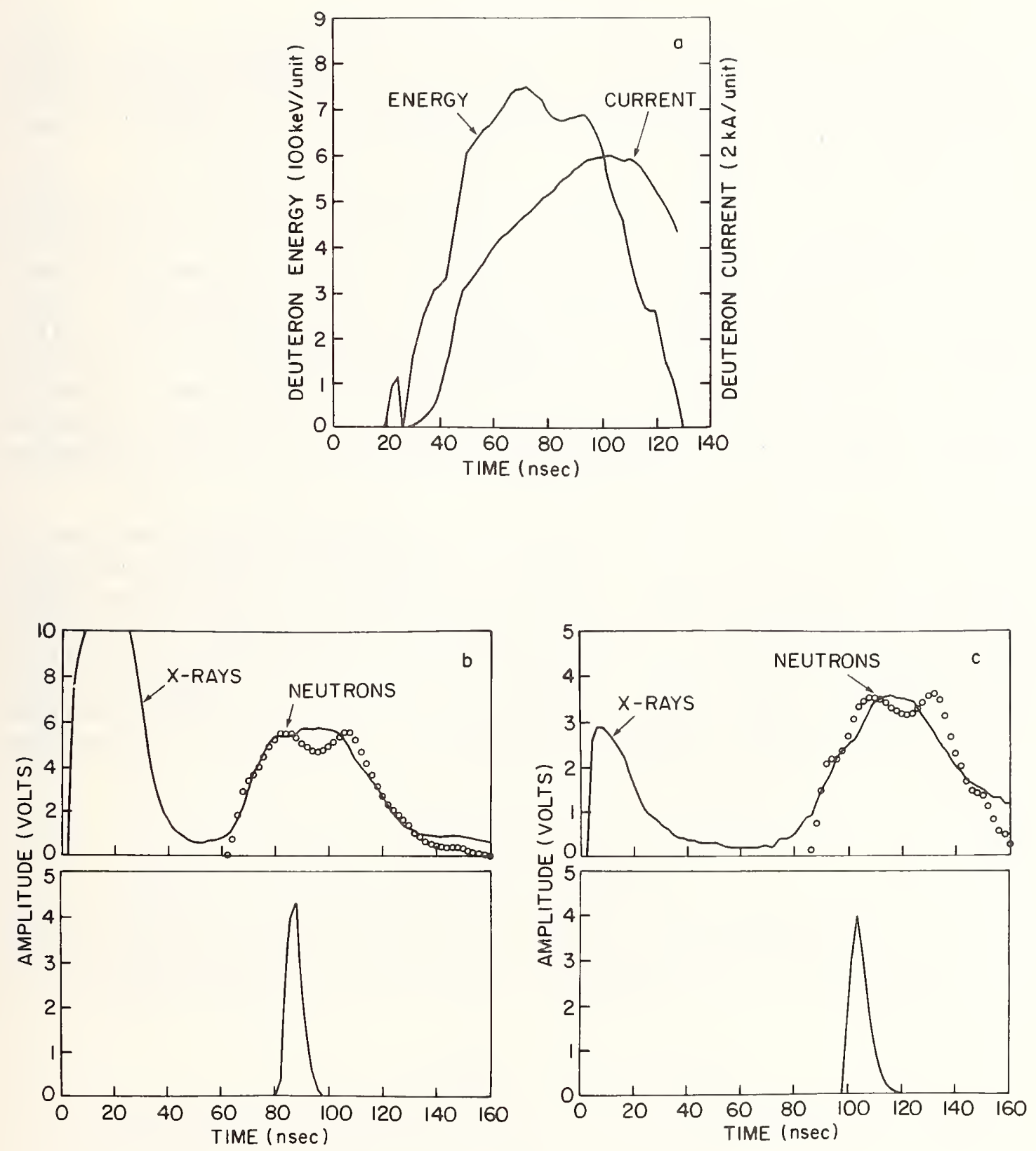

Figure 7. Comparison of neutron TOF traces with calculations using the energy and current traces displayed in (a). The measured TOF traces (solid curves) are compared with calculated yields (open points) for detectors at (b) $1.91 \mathrm{~m}$ and $15^{\circ}$ and at (c) $2.36 \mathrm{~m}$ and $85^{\circ}$. The results of calculations for a monoenergetic deuteron pulse of 2-ns duration are shown at the bottom of (b) and (c). 


\section{Summary}

Currents and voltages determined by nuclear techniques are consistent with electrical measurements. The generator voltage with inductive corrections and the current from a Rogowski coil can be used to reproduce measured prompt- $\gamma$ and neutron TOF responses. The precision of such measurements is about $20 \%$.

There are many nuclear reactions which can be used for current and voltage measurements. In this report only a few have been presented to illustrate different diagnostics techniques. Other reactions that have been used include: 1)delayed radioactivity from the ${ }^{14} \mathrm{~N}(p, \gamma){ }^{15} 0$ reaction to measure the current of protons greater than $280 \mathrm{keV}$ [1], 2) prompt- $\gamma$ measurements with the $\mathrm{Li}(p, \gamma)$ Be reaction to determine proton current [14], and 3) neutron TOF with the $\mathrm{Li}(\mathrm{p}, \mathrm{n}){ }^{7} \mathrm{Be}$ reaction to measure the energy of protons greater than $2 \mathrm{MeV}$ [15]. Many potential reactions remain to be exploited, particularly if reactions producing delayed B-radioactivity are considered.

New developments and improvements in these techniques can be expected as more attention is devoted to using nuclear measurements to understand the behavior of current and voltage in the vacuum diode region. The emphasis to date has been on characterizing the ion beam rather than relating the nuclear measurements to the electrical characteristics of the generator. Nuclear techniques will become even more attractive as pulsed-power technology moves toward higher voltage because the increased reaction yields will allow ever smaller ion currents to be measured.

\section{Acknowledgements}

The encouragement of D. Mosher, G. Cooperstein, S. A. Goldstein and $\mathrm{J}$. Golden in the development of these diagnostics is acknowledged. Also, discussions with J. Shipman were helpful in the preparation of this report. 


\section{References}

[1] F. C. Young, J. Golden and C. A. Kapetanakos, Rev. Sci. Instrum. 48, 432 (1977).

[2] H. H. Andersen and J. F. Ziegler, The stopping and Ranges of Ions in Matter, Vol. 3 (Pergamon, New York, 1977).

[3] J. D. Seagrave, Phys. Rev. 84, 1219 (1951)。

[4] F. C. Young and M. Friedman, J. App1. Phys. 46, 2001 (1975).

[5] A. E. Blaugrund and S. J. Stephanakis, Rev. Sci. Instrum 4 49, 866 (1978).

[6] J. Golden. R. A. Mahaffey, J. A. Pasour, F. C. Young and C. A. Kapetanakos, Rev. Sci. Instrum. 49, 1384 (1978).

[7] F. C. Young, W. F. Oliphant, S. J. Stephanakis and A. K. Knudson, IEEE Trans. P1asma Sci. PS-9, 24 (1981).

[8] G. Cooperstein, Shyke A. Goldstein, D. Mosher, R. J. Barker, J. R. Boller, D. G. Colombant, A. Drobot, R. A. Meger, W. F. Oliphant, P. F. Ottinger, F. L. Sande1, S. J. Stephanakis and F. C. Young, "NRL Light Ion Beam Research for Inertial Confinement Fusion," Fifth Workshop on Laser Interaction and Related Plasma Phenomena, Rochester, New York, Nov. 5-9, 1979.

[9] S. J. Stephanakis, D. Mosher, G. Cooperstein, J. R. Boller, J. Golden, and S. A. Goldstein, Phys., Rev. Lett. 37, 1543 (1976).

[10] F. C. Young, D. Mosher, S. J. Stephanakis, S. Goldstein and D. Hinshelwood, NRL Memorandum Report 3823 (Washington, DC, August 1978).

[11] H. Liskien and A. Paulsen, Nuclear Data Tables 11, 569 (1973).

[12] F. C. Young IEEE Trans. Nuc1. Sci. NS-22, $718(\overline{1975)}$.

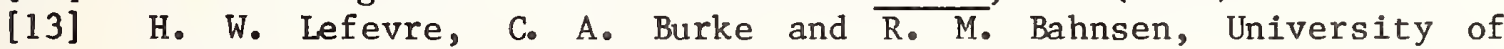
Oregon Report RLO-1925-74, 1971 (unpub1ished).

[14] R. J. Leeper, E. J. T. Burns, D. J. Johnson and W. M. McMurtry, Workshop on Measurement of Electrical Quantities in Pulsed Power Systems, Boulder, CO, March 2-4, 1981.

[15] R. A. Meger, F. C. Young, A. T. Drobot, G. Cooperstein, S. A. Goldstein, D. Mosher, S. E. Graybill, G. A. Huttlin, K. G. Kerris and A. G. Stewart, NRL Memorandum Report 4477 (Washington, DC, March 1981).

\section{Sponsor Acknowledgements}

This work was supported in part by the Defense Nuclear Agency and in part by the Department of Energy. 


\title{
ELECTRICAL MEASUREMENT TECHNIQUES USED AT THE CASINO FACILITY
}

\author{
E. NOLTING, R. MARTIN AND M. RUPPALT \\ Electronics Hardening Branch \\ Naval Surface Weapons Center \\ White Oak, Silver Spring, Maryland 20910
}

The Casino Facility is a Defense Nuclear Agency nuclear weapons effects simulator. The machine consists of four-one megavolt fieldemission diodes each capable of producing a one-half terawatt power pulse. The diodes are located on the end of four separate, 4 MV water-dielectric coaxial lines and can be energized singularly or in combination. Each diode has its own set of diagnostic monitors. Transient diagnostics include voltage monitors, on the pulse-forming lines adjacent to the vacuum diodes and current sensors, on each of the diodes. Normal shot-to-shot variation in the Casino output necessitates accurate measurement of voltage and current with computer aided data reduction to determine diode performance. This paper describes the relevant features of the hardware and software used in the Casino digital data acquisition system. Techniques used to calibrate the various diagnostic monitors are presented including a discussion of the procedure used to time phase the current and voltage signals. This is followed by a comparison of the calculated delivered energy with total absorbing electron beam calorimeter measurements.

\section{Introduction}

The Defense Nuclear Agency's Casino Facility was built to produce bremsstrahlung radiation for the study of its effects on military weapons systems and components. In order to produce this radiation the simulator generates intense, relativistic electron beams that are directed to a x-ray converter. Figure 1 is an electrical schematic of one-half of the simulator which shows that it is generically similar to other single-pulse, high-power machines used in inertial confinement research, material response studies, etc. Each of the two, 58-stage, $4 \mathrm{MV}, 400 \mathrm{~kJ}$, marx generators are connnected to two, $13 \mathrm{nF}$, waterdielectric, coaxial, pulse-forming lines (PFLs). The voltage on each PFL rises to a maximum of four megavolts in approximately $1.5 \mu \mathrm{sec}$, after which time the energy is transferred by water switches to matched impedance lines. These coaxial transmission lines are tapered to reduce their output impedance by one-half and this causes 


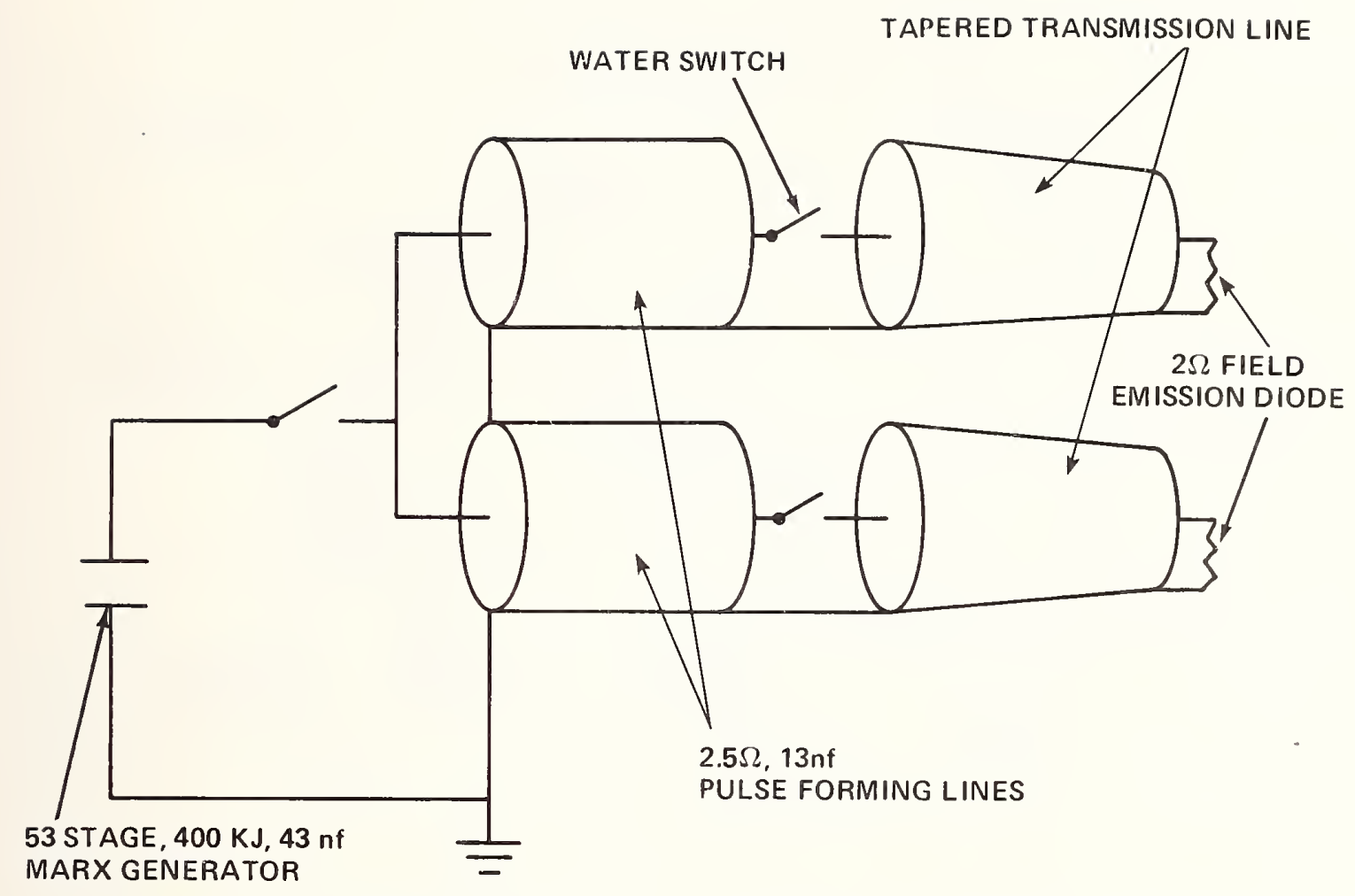

Figure 1. Schematic diagram of one-half of the Casino nuclear weapons effects simulator. The marx generator applies four million volts to the pulse forming lines which is reduced, after switching and transforming to one million volts at the diodes.

approximately one million volts to appear across the field-emission diodes. Each diode is capable of producing a $40 \mathrm{~kJ}$ l electron beam in a 70 ns pulse. A cross-sectional view of one line of the simulator illustrating the geometry is shown in Figure 2.

The Casino simulator is similar to many other large-.power, pulsed devices in that lack of shot-to-shot reproducibility makes accurate machine diagnostic essential. Since it is not possible to make direct measurements of delivered energy and beam conditions while the simulator is being used for nuclear weapons effects testing, electrical diagnostics must be used to indicate if machine performance is normal for a given shot. Without this information considerable operating time can be wasted.

Diagnostics used to monitor the simulator functions are of two general types, $D C$ and transient. The DC measurements include charge voltage and currents, water conductivity, vacuum, liquid levels, etc. Although only the transient diagnostic measurements made during the shot will be discussed in this paper, during simulator operation the DC 


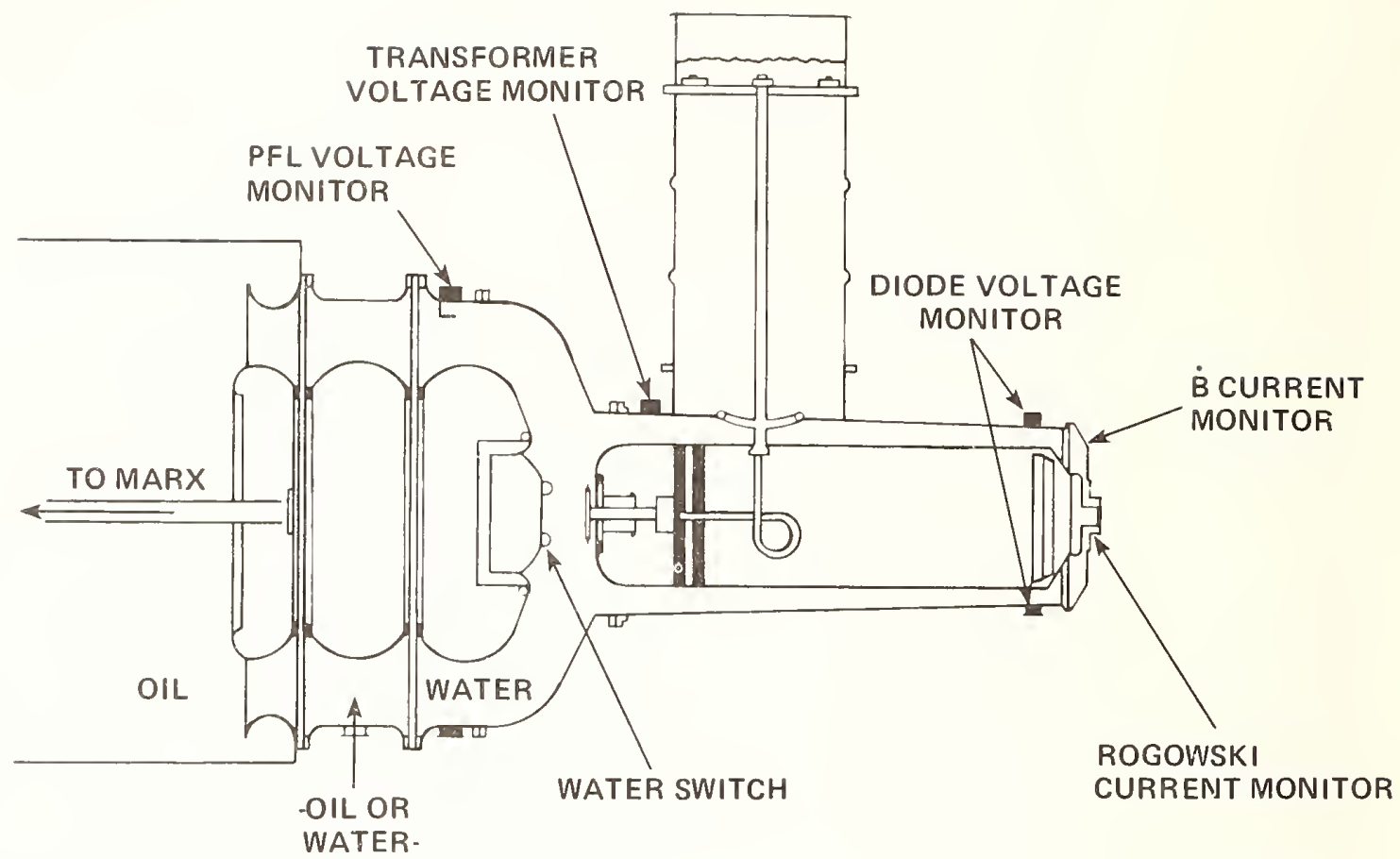

Figure 2. Diagram of a single, coaxial-transmission line of the Casino nuciear weapons effects simulator indicating relevant machine geometry and locations of diagnostic monitors discussed in text.

monitor records are frequently referred to in an attempt to explain an anomally in the pulse waveforms in terms of the machine's pre-shot status. This is particularly true when the transient diagnostics indicate a severe abnormality.

Transient voltage diagnostics are located at the PFLs, the input of the coaxial transformer and immediately in front of the vacuum diodes. Since the voltage monitor at the input to the transformer is only used as a timing signal for the purpose of synchronizing experiments to the output, it will not be discussed further. All these monitors are of the capacitive divider type similar to the one illustrated in Figure 3 (manufactured by Maxwel1 Laboratories, Inc., San Diego, CA 92123) and produce a signal up to $10^{5}$ smaller than the input waveform. Monitor response in times of about one nanosecond are achieved by compensation of the secondary resistive divider.

There are two types of transient current monitors located at the vacuum diode which measure the output current. Four, sma11, B-dot loops are equally spaced on the diode door; outputs from all these monitors are averaged by connecting their signal cables through power 


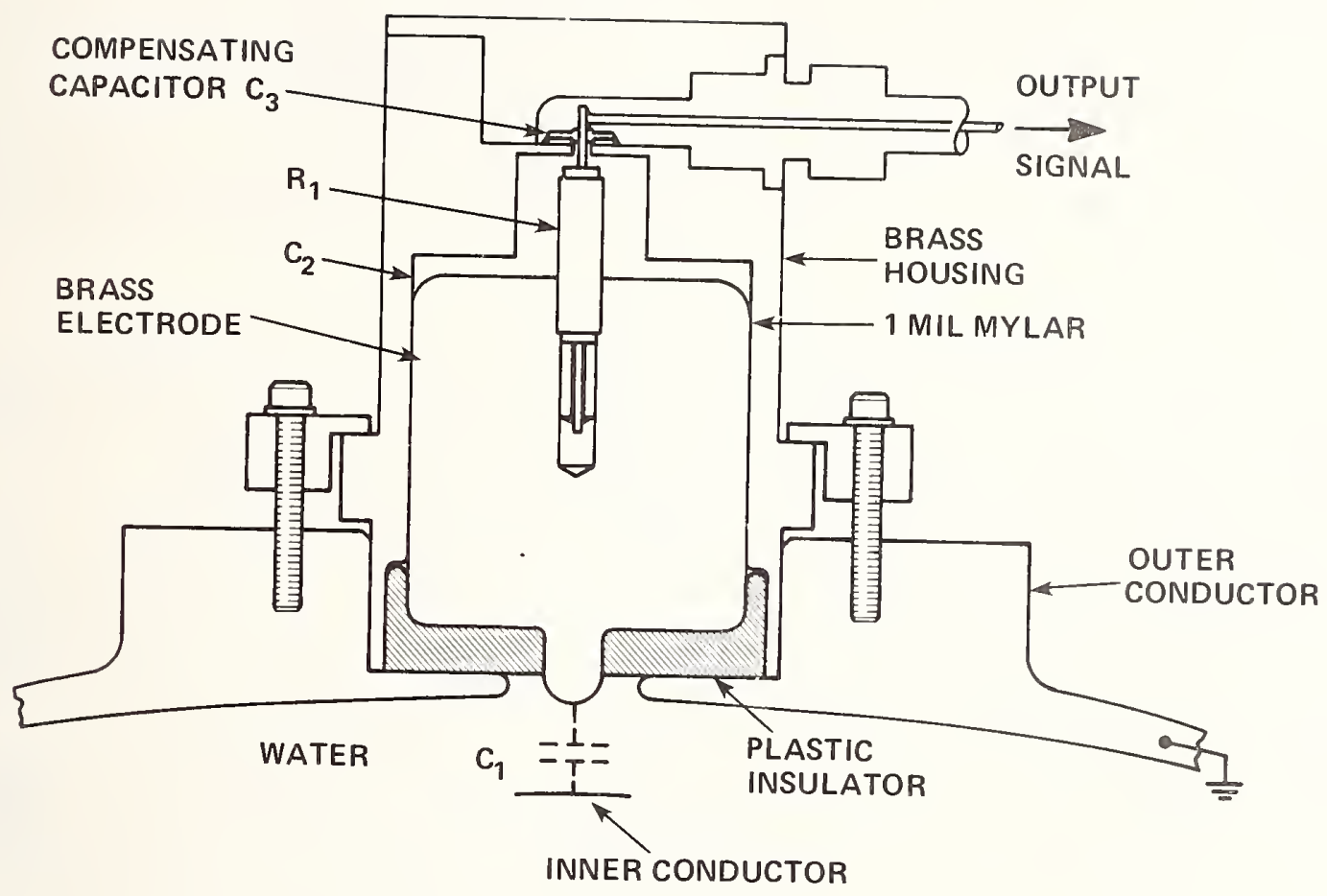

Figure 3. Capacitance divider used to measure voltage on the waterfilled, coaxial transmission line. The division ratio of this divider is on the order of $1: 10^{5}$.

tees to reduce the effects of current asymmetry on the measurement. A self-integrating Rogowski belt is positioned around the cathode shank approximately four centimeters from the anode-cathode gap. Comparison of the output of the two current monitors gives an indication of any spurious current paths by-passing the anode-cathode gap.

Transient digital analyzers are used to record the waveforms and a description of the data recovery and processing system is discussed in Section 2. Section 3 describes the procedures used to calibrate the voltage and current monitors, while Section 4 compares calculated delivered energy with measurements made by total absorbing electron beam calorimeters.

\section{Data Acquisition}

One of the most important parts of any data acquisition system is its grounding method and signal cable connection procedure. For the Casino Facility the primary grounding system provides conductance from the radiation test-cells electromagnetic shielding, Marx generators and facility data room -- where all transient simulator waveforms are 
processed -- to earth. A second ground system is used to earth a second instrumentation room and these two grounding systems can be connected or isolated as desired. Both grounding systems use 1/4-inch thick, 12-inch wide strips which have 5/8-inch diameter, copper-weld, ground rods driven into the earth at two-foot intervals. These grounding rods are placed underground, beneath the entire simulator area with numerous surface attachment sites. Provision is made to allow wetting of the earthing system for improvement of ground conduction, although this has not been found to be required to maintain an adequate signal reference.

Signals are transmitted from the diagnostic monitors to the facility data room via low dispersion type foam-flex cables which were carefully cut to specified lengths so that timing measurements could be made to within $0.3 \mathrm{~ns}$. These cables have a solid aluminum outer conductorswhich provide excellent noise attenuation; however, it was discovered during diagnostic calibration that the connectors attaching to the shield room were acting as a noise source which gave a consistent distortion of the transducer waveform. The explanation for this phenomena was found to be corrosion of the connectors mating threads; tightening the connector to over the specified torque was found to temporarily relieve the probilem, but risked permanently damaging the connector. A satisfactory solution was found by applying NOALOX (Ideal Industries, Inc., Sycamore, IL, Catalog No. 30-030) which is used in the electrical industry to prevent connector oxidation on aluminum wiring. It is important that all cable shield are tightly bonded to the shield room since difference in ground reference of spacially separated locations on large high-voltage machines can generate large shield currents. Approximately 400 amps net current have been measured on the foam flex cables which can cause sizable voltage drops across grounded connectors unless their resistance is very sma11.

Because of the noisy environment that exists when the simulator is fired, a good quality shield room is required to record diagnostic data. The facility data room has solid iron walls with welded seams to limit the penetration of magnetic as well as electric fields. This enclosure was tested and met the specified shielding requirements for both magnetic $(>35 \mathrm{db}$ at $1 \mathrm{kHz}$ to $\geq 90 \mathrm{db}$ at $\geq 10 \mathrm{kHz})$ and electric $(\geq 120 \mathrm{db}$ at $\geq 10 \mathrm{kHz}$ ) fields (Shield room mānufactured by Lectro-Magnetic, Inc., Los Angeles, CA). A11 60Hz power is brought into the enclosure through low pass filters that have an insertion loss of greater than $100 \mathrm{db}$ from $15 \mathrm{kHz}$ to $10 \mathrm{GHz}$; a 11 communication 1 ines entering the enclosure are similarly filtered.

Provision is made to electrically isolate the facility data room. The room is placed on two inch thick insulating pads and may be disconnected from the simulator ground system. Instrumentation power can be obtained either from a motor generator set, if electrical isolation is required, or standard utility power. It has been found that during normal operation satisfactory results are obtained using commercial power with the screen room connected to the facility earthing system. 
A11 pulsed data is recorded on Tektronix 7912 transient digital analyzers with type 7 A24 vertical plug-in units which gives the system an upper frequency response 1 imit of $400 \mathrm{MHz}$, far above the approximately three megahertz fundamental signal to be recorded. The transient digitizers record data in its internal, 4096-word memory and this information is numerically processed by a computer to obtain useful waveforms. At the Casino facility data processing is performed by a PDP $11 / 40$ using in-house developed software.

Waveform data processing can be described as being accomplished in five steps. Initially, the digitally recorded signals stored in the 7912 's internal memory are retrieved from the individual units and stored on disk. Each recorded data word represents a horizontal or vertical data point from the $512 \times 512$ diode matrix. Prior to storing waveform data, software must process the raw digitized data to correct for noise, remove points due to flaws in the diode matrix, and discriminate against multiple valued functions. Next, the units are triggered again with no input signal for the purpose of determining a zero amplitude reference, and this information is stored in computer memory. Then, each unit is triggered again to place an accurately timed marker pulse on the diode matrix and this information is also stored in memory. The purpose of the marker pulse is to time reference the data signal. Sensor sensitivities, cable delays, and offset voltages are used in conjunction with vertical and horizontal calibration factors to create a measured data waveform. A11 these operations are performed using assembly language to speed up data processing time. Once this is completed, the data is transformed into 32-bit real numbers so that the next step of data manipulation using standard Fortran can be employed. After the conversion routines are completed the raw data has been transformed to a waveform that is the equivalent of standard oscilloscope records, but with actual voltage and current scale factors, and time correlation.

The hard copy output, which is routinely obtained once all the data processing is complete, contains measured waveforms of the PFL voltage, diode voltage and current, plus calculated values of power and energy. Selected parameters for the waveforms are also printed on the display, such as maximum voltage, time maximum voltage occurs, etc. A typical hard copy display is shown in Figure 4. Besides the standard output package the data may be manipulated by any waveform operation; for example, dividing the diode voltage waveform by the current waveform to determine the time history of diode impedance. This capability was very useful during the calibration of the diode monitors.

\section{Monitor Calibration}

A typical problem that arises when calibrating monitors for large, high-voltage equipment is that often the device's sensitivity must be measured in-situ because of its geometrical dependence. This implies that considerable amounts of energy must be used to supply calibration voltage and current pulses at even modest levels. Therefore, transducers which have voltage or current coefficients are susceptible to error 

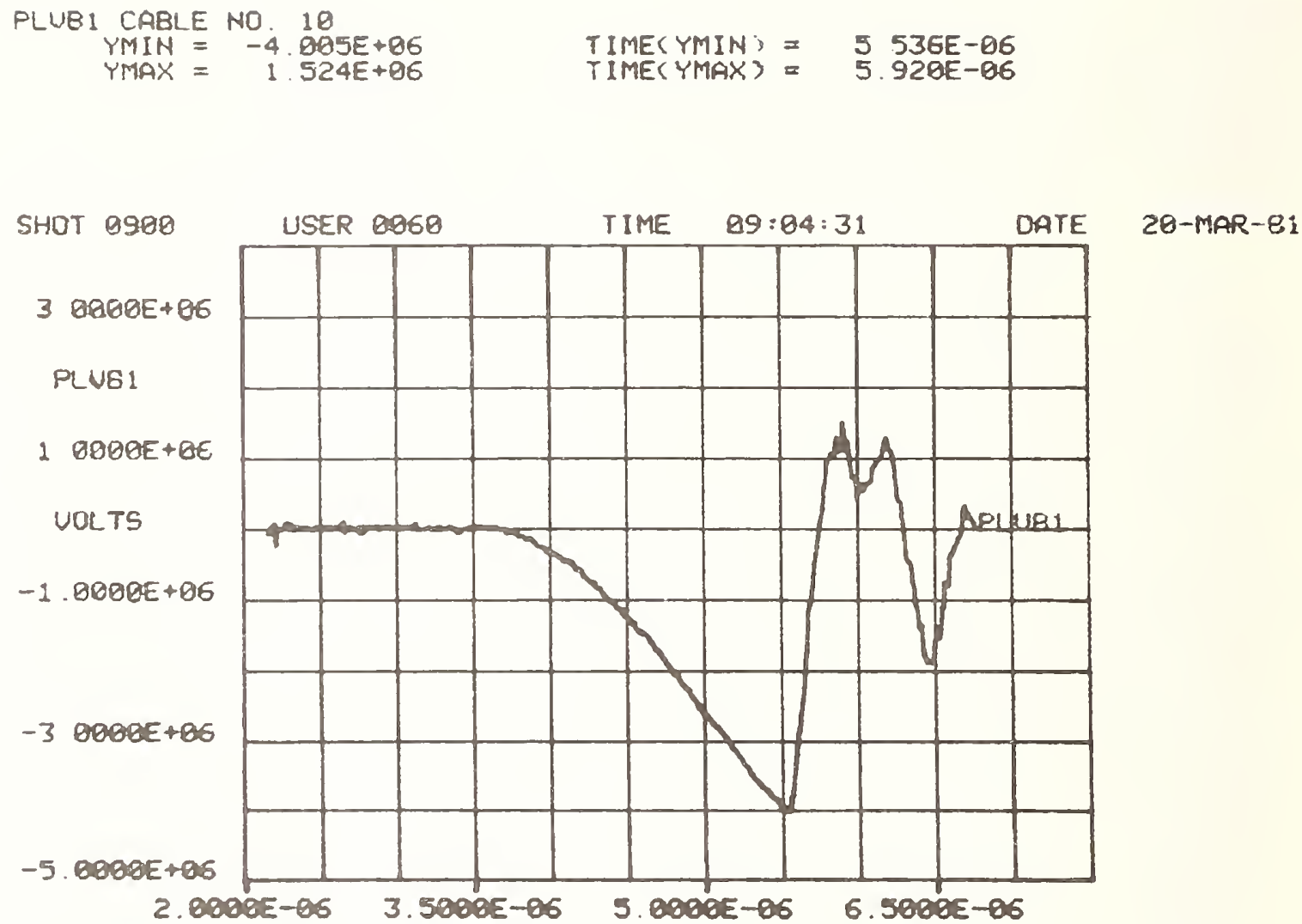

Figure 4. Typical hard copy display obtained from the voltage monitor located on the pulse forming line. Amplitude values contain al1 the calibration factors and correction terms. Print out above waveform indicates voltage maxima (both positive and negative) and the times when they occur.

since calibration levels are often 100 to 1000 times smaller than operating levels. Furthermore, monitor frequency response must be considered, since an increase in the calibration pulse amplitude often is followed by slower rise-times to the point that the calibration pulse is much slower than the event it is intended to simulate. When this is the case, it is difficult to correct for monitor droop and high frequency response.

Because of the difficulties in producing ideal voltage and current calibration pulses, calibration runs were made by using as many redundant checks as possible. These checks included calculations and independently calibrated monitors for use as cross-references. Accurate calculation of expected values was difficult since the calibration circuit values are not well defined; however, by careful comparison of both wave shape and amplitude good agreement between measurement and 


\section{calculation was reached.}

To calibrate one of the PFLs a single $(2.5 \mu \mathrm{F})$ capacitor was connected with a gas switch to the inner conductor by a length of high voltage cable. Since the capacitance of the PFL is only $13 \mathrm{nF}$ the maximum voltage on the PFL was approximately twice that of the capacitor charge voltage, and the resonant frequency was controlled by the PFL capacitance and high-voltage lead inductance. Consequently, it was possible to adjust the voltage rise-time to some extent by controlling the length and routing of the high voltage cable. This method was used to adjust the PFL charging time to be similar to the $1.2 \mu \mathrm{s}$ observed during operation.

In order to make a direct measurement of the PFL voltage during calibration a conducting rod was connected to the inner conductor and passed through the outer via an insulating fixture. The calibration voltage could then be measured with a standardized voltage probe and the PFL capacitive monitor was cross-referenced to it and also compared to the voltage predicted by calculation. A bench-calibrated Tektronix Pó015 high-voltage probe, capable of measuring transient voltages up to $40 \mathrm{kV}$, was used as the standard. A secondary copper-sulfate resistive divider (Manufactured by Physics International, Inc. San Leandro, CA 94577) was used as a cross check for higher PFL voltages (maximum output of calibrating pulse $\approx 60 \mathrm{kV}$ ). It should be noted that the $40 \mathrm{kV}$ limit of the standard is about 100 times smaller than the PFL operating voltage, although reference (1) indicates that the change in dielectric constant of water should be less than one percent at the peak expected stress.

A typical calibration waveform is shown in Figure 5 illustrating the damped-resonant oscillations and the longer term decrease in PFL voltage caused by the resistance of the water. By comparing the peak voltages a scale factor was determined for the capacitive divider while comparison of the capacitive monitor with the Tektronix probe during the long term exponential decay portion of the curve gives an indication of monitor "droop". The droop time-constant was determined by plotting the ratio of the capacitive monitor signal to that of the high-impedance Tektronix probe. This information was then used to correct the monitor output by usin? the following relationship,

$$
V_{c}(T)=V_{m}(T)+\frac{1}{\tau} \int_{0}^{T} V_{m}(t) d t
$$

where $\tau$ is the droop constant, $T$ is the time, and $V_{c}$ and $V_{m}$ are corrected and measured voltages respectively. This correction was incorporated into the Casino software.

Voltage pulses used to calibrate the diode monitors were introduced through the front of the simulator. Again, one of the major difficulties to overcome is to be able to produce a large enough voltage level to record on the capacitive voltage monitors and still have the necessary 


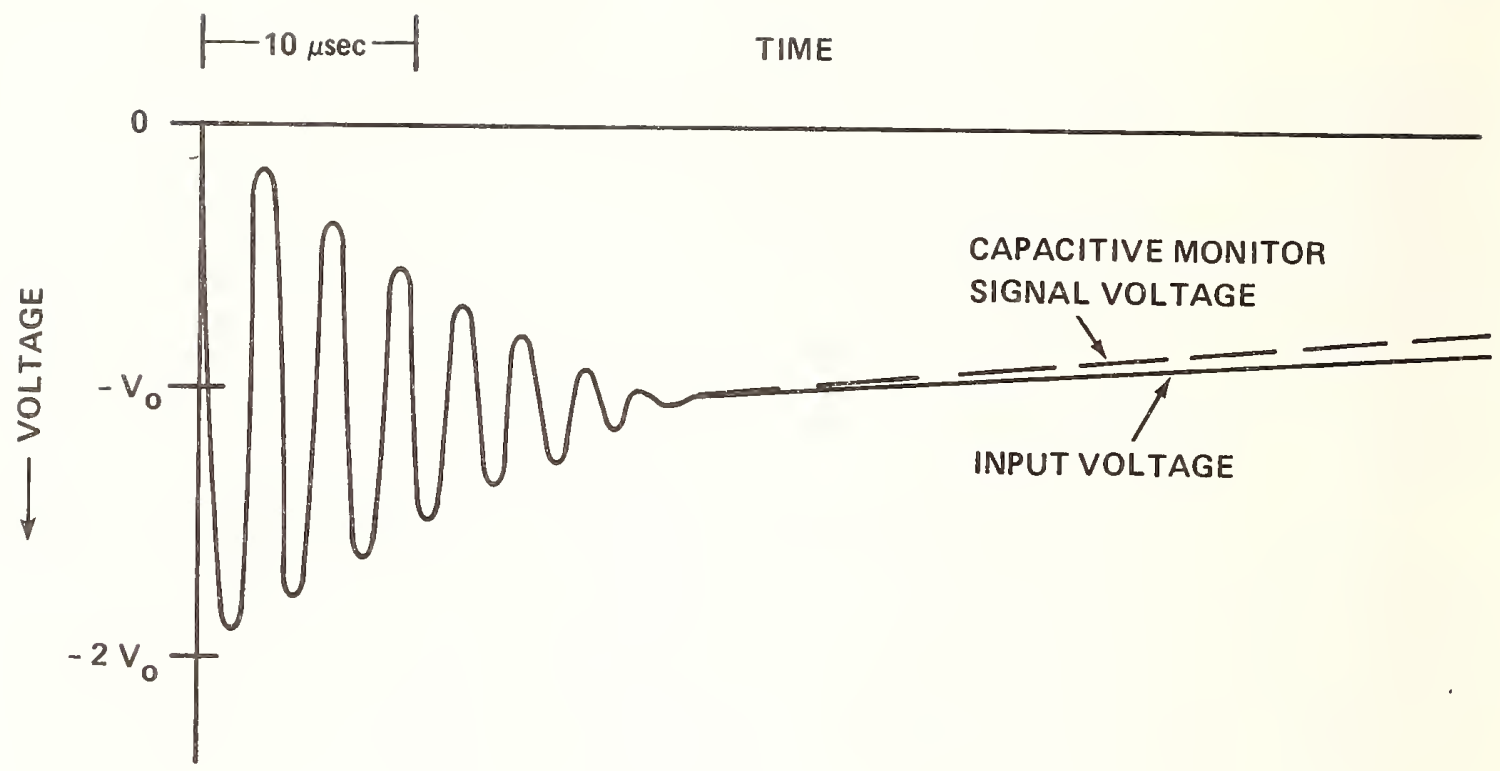

Figure 5. A drawing of the PFL calibration waveform illustrating both the damped oscillatory portion of the resonance charging of the water line and the long term voltage fall due to water conductivity. The difference between the rate of fall of the input signal and that of the monitor is due to capacitive droop which can be determined from this waveform and corrected numerically.

rise-time ( $50 \mathrm{~ns})$. In order to achieve a fast, large amplitude calibration pulse, a compact high-voltage pulser with a low-inductance connection was required. The connection was made by attaching the highvoltage terminal of the pulser to the cathode stalk and making a short, coaxial, ground connection to the anode-support structure. Even with this design, the maximum voltage signal generated was approximately $15 \mathrm{kV}$ with a 100 ns rise-time. A Tektronix P6015 was used again as reference and was connected by radial rods that were located in the same crosssectional plane as the two, diametrically-opposed capacitance monitors. Because of the faster rise-time of diode calibration pulse the standards were positioned as near as possible to the capacitive monitors without changing their coupling capacitances. Initially, each of the monitors were checked separately and their individual outputs were made equal by use of appropriate attenuators. Then both outputs were averaged together by means of a power-tee and calibrated for both amplitude and droop correction. The purpose of using two voltage monitors to measure the diode voltage is to help account for any asymetry in the voltage wavefront. 
The Rogowski coil, used to measure the diode current, was purchased specifically for use on the Casino diode and was supplied with a calibration factor (manufactured by Physics International, Inc., San Leandro, Ca;). This factor was obtained by using a special constant impedance $(50 \Omega)$ test fixture designed for the purpose of supplying a fast-rise. square waveform. These calibration factors were double-checked at the Casino Facility by using the same calibration pulser used to test the diode voltage monitors. The pulser was connected to the diode door and a Pearson $301 \times$ current monitor was mounted inside the door cavity; a shorting rod, connected to the outer edges of the door by wide conducting straps was passed coaxially through both current monitors. The supplied calibration-factor was confirmed (to within the stated $5 \%$ accuracy). The four B-dot current monitors were also calibrated while the diode door was in this configuration.

After the senitivities of the current and voltage monitors were determined, the final step in the diode calibration process was started. Figure 6 shows the schematic of an elementary circuit model of the vacuum diode. Since the voltage is measured at a location distant from the anode-cathode gap, this measurement must be corrected for inductive voltage drops to give the actuai electron acceleration potential. A standard procedure for making this connection is to determine the diode inductance by shorting the anode-cathode gap and ineasuring the voltage across the vacuum envelope. Once this diode inductance is determined then the diode voltage $\left(V_{D}\right)$ is related to the corrected measured voltage by

$$
V_{D}=V_{C}-L d i / d t \text {, }
$$

where $L$ is the diode inductance and di/dt is the derivative of the diode current.

Use of the digital data acquisition system greatly simplifies the diode inductance measurement. Since the self-integrating Rogowski coils supplied the diode short-circuit current directly and numerical differentiation of the current signal was quite noisy, the inductance was determined by integrating the corrected measured voltage about the current maximum,

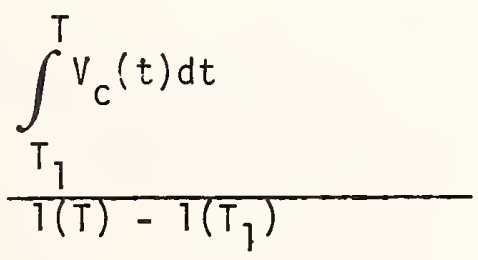




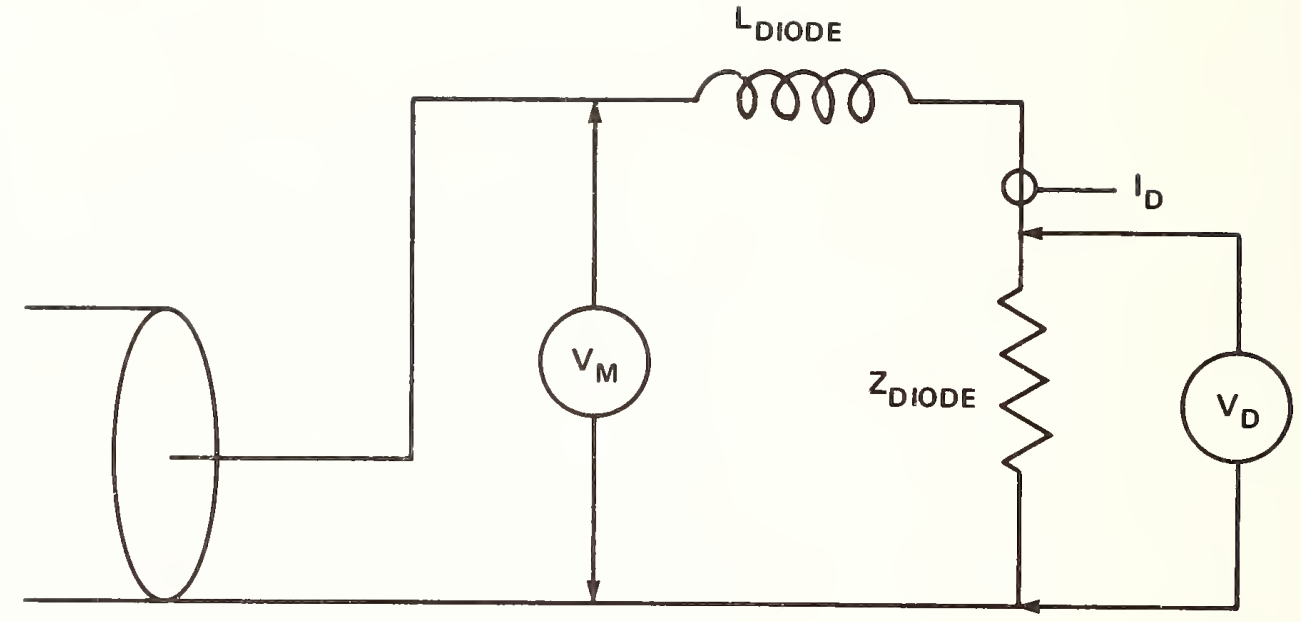

Figure 6. Simple lumped parameter circuit used to model vacuum diode. The measured voltage $\left(V_{m}\right)$ is corrected for the voltage drop across the anode-cathode gap.

In general, plotting the relationship gave a smooth, but non-constant value for the diode inductance. Values obtained for diode inductance could be changed by adjusting the time-phasing of the current relative to the diode voltage. The question of proper current and voltage phasing occurs because the current is measured at a different location from the voltage; the monitors are located approximately seven nanoseconds electromagnetic wave propagation time from each other. The simple lumped inductance model for the diode inherently assumes an infinite energy propagation velocity, i.e, all points on the series circuit pass the same instantaneous current. An alternative to this simple model is to attempt to describe the diode by transmission line analysis which becomes difficult because of the nonuniform geometry in the diode region (reference (2)).

Use of the lumped inductance model for the ideal short-circuit implies that (1) diode inductance is constant, (2) all measured voltage is due to the diode inductance, and (3) no net power or energy is delivered to the diode. Realistically it is not possible to make a a non-resistive short circuit because the large short circuit currents produced can cause several kilovolts to appear across even a few milliohm connection. Therefore, the approach chosen was that of adjusting the current and voltage phasing until an approximate constant inductance was calculated, see Figure 7. For each short circuit shot the time-average of the calculated inductance was made; five of these measured inductances were then averaged to obtain a value for the 


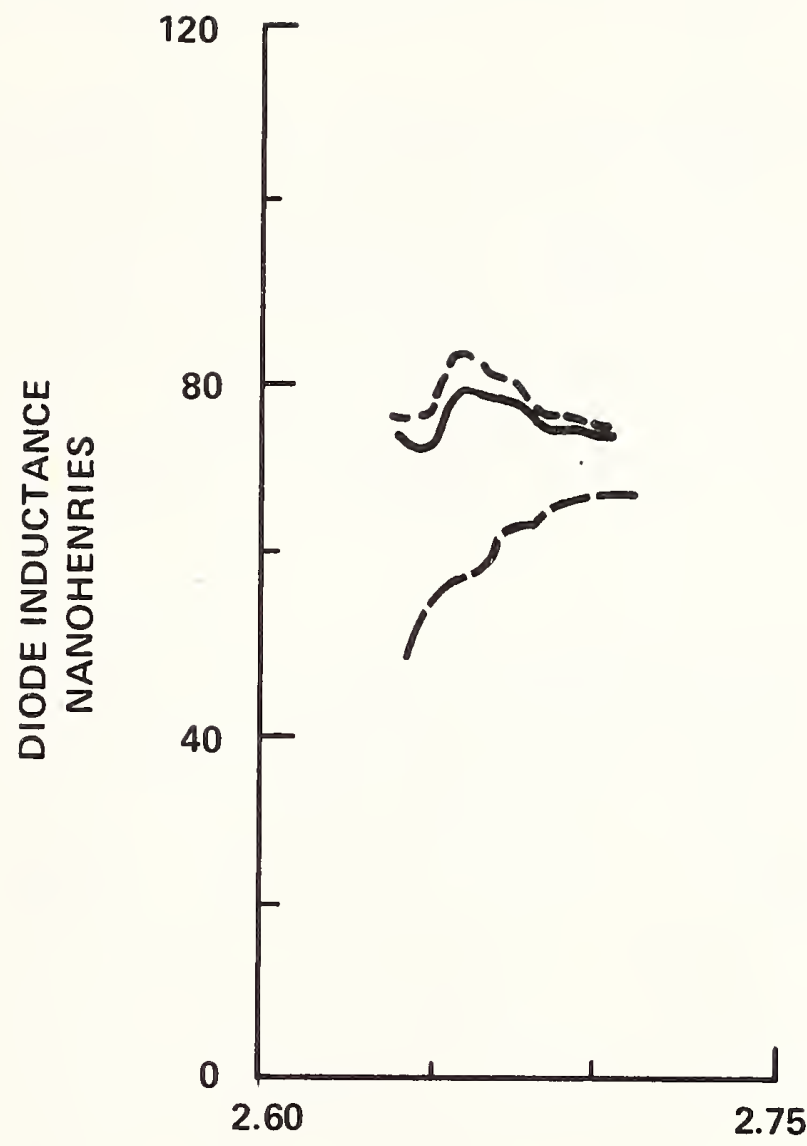

TIME ( $\mu \mathrm{sec})$

Figure 7. Variation of diode inductance with current and voltage phasing. Time shifts and associated time averaged inductances shown are no shift and $60.0 \mathrm{nH}$ (10ng dashes), $8 \mathrm{~ns}$ and $73.1 \mathrm{nH}$ (solid line), and $9 \mathrm{~ns}$ and $75.2 \mathrm{nH}$ (short dash line). The curve determined by the eight nanosecond shift was determined to be the nearest to a constant inductance valve during the short circuit pulse.

diode inductance. The diode inductance was found to be approximately $70 \mathrm{nH}$ with a voltage and current phasing delay of $8 \mathrm{~ns}$. Proper phasing is very important, because a shift of 1 ns changed the calculated energy (on non short-circuit shots) about $1 \mathrm{~kJ}$ ( $2 \%$ per ns). 


\section{Comparison of Calculated and Measured Energies}

As an independent check of the voltage and current calibrations, a series of experiments were conducted using total electron-beam absorbing calorimeters. These devices, which are used to determine the total energy of the electron beam by measuring the thermal energy delivered to the calorimeter, are subject to losses which lead to an under estimation of the beam energy. These losses arise from: (1) back scattered beam electrons that never enter the calorimeter, (2) radiative cooling of the calorimeter front surface before thermal equilibrium is reached, (3) heat loss to nearby bodies due to thermal conduction, and (4) front-surface spall and vaporization. Methods to reduce the effect of these mechanisms were used in setting up the energy correlation experiments.

The calorimeters were made from approximately $.5 \mathrm{~kg}$, cylindrical blocks of AXF-5Q Poco Graphite (Poco Graphite, Inc. Decatur, Tx, 76234.) for which the specific heat as a function of temperature was known. Although the calorimeter was electrically connected to the grounded, conducting drift-tube, a tortuous thermal conduction path was made to limit energy losses. Thermal couples used to measure the temperature rise of the carbon blocks were electrically isolated from the calorimeters by mica washers to prevent large electric transients from entering the high-impedance recording circuits and causing a permanent offset. A short drift tube was used to reduce the effect of backscattered-electrons. The electrons reflected from the carbon block were radially trapped by the magnetic field which forced them to undergo several oscillations between the calorimeter and the diode, thus increasing the probability of collection. In order to reduce the effect of radiative-cooling, spall and vaporization losses, the cross-sectional area of the electron beam was expanded almost a factor of two by reducing the magnetic guide field in the region of the calorimeter, and furthermore, the simulator was operated at half-power to reduce the delivered energy. Adjustment of the anode-cathode gap was made for the half-power shots in order to keep diode voltage at nominally one megavolt.

As a result of the precautions taken in making the calorimeter measurements, good agreement was obtained between the total diode energy measured by the carbon block and that calculated by integrating the product of the voltage and current. Table I gives the comparison between calorimeter measurements and the calculated diode energies and shows that the agreement is well within the experimental error estimated as being on the order of seven percent. Unfortunately, a similar set of experiments for full simulator power is not in as good agreement. From the description of calorimetry sources of error previously presented, it would be expected that the carbon block measurements should be smaller than the calculated values. This was not the case as can be seen by referring to Table I. A possible reason for this discrepancy appears to be that the Rogowski current monitor is ocassionally shorted during the power pulse by plasma bridging its electrostatic shield gap. The problem of plasma shorting arises because it is desirable to locate the current 
Table I. Comparison of calculated and measured diode output energy

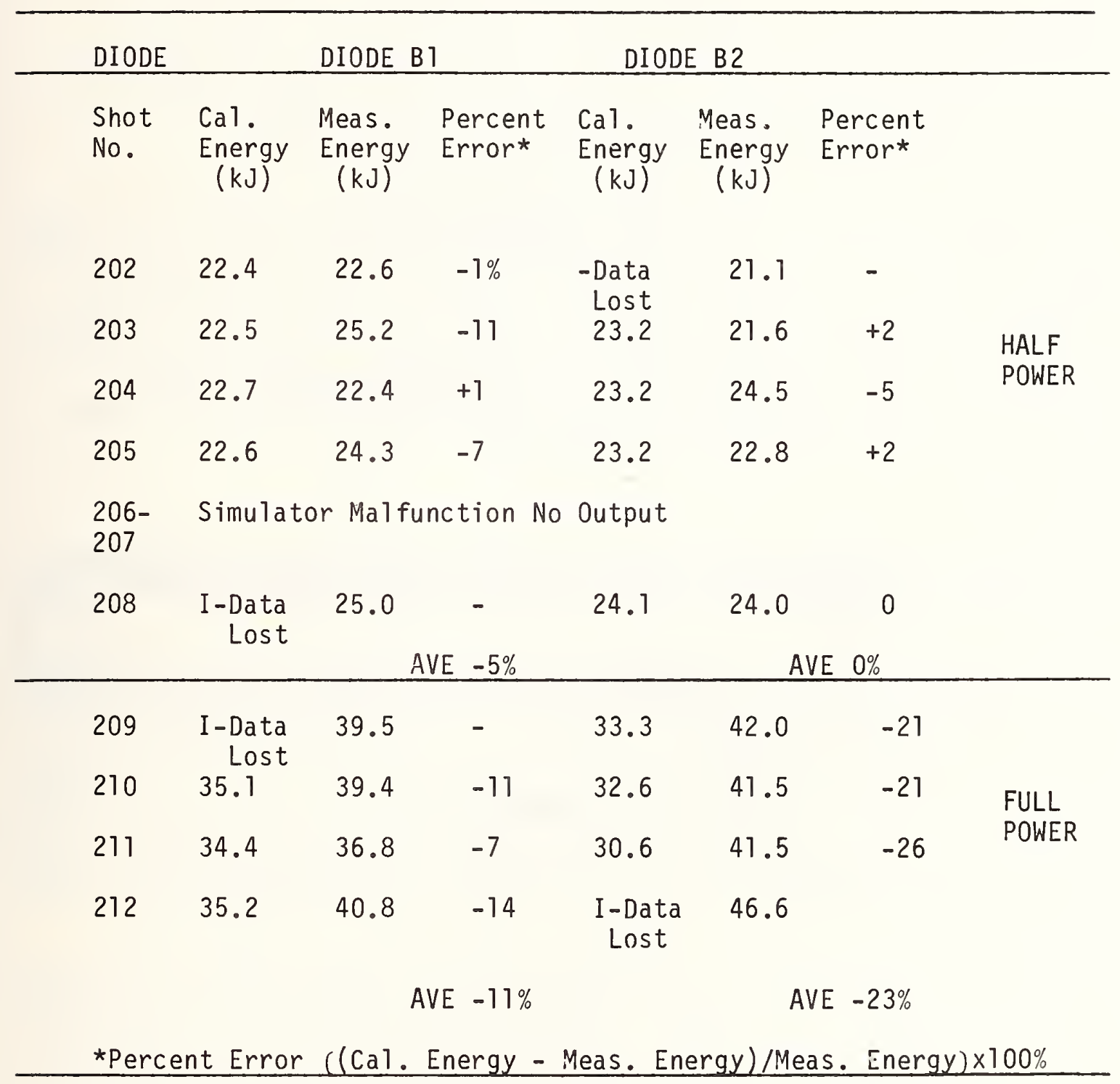

monitor monitor as close as possible to the diode anode-cathode gap. Therefore the Rogowski coil is in a high-stress, high-photon fluence environment which can lead to plasma formation and shorting of the monitor during the latter stages of the main power pulse. As seen in Table I, when current monitor is shorted, the uncertainty in the delivered energy can be on the order of $25 \%$.

\section{Summary}

Half-power measurements made at the Casino Facility have shown that it is possible to get good agreement between voltage and current diagnostics and total electron beam absorption calorimeters. This is 
a necessary, but not sufficient indication that the transient diagnostics are recording correct voltage and current values. Furthermore, it is possible to observe noticeable changes of the current waveform when computer values are suspect at full energy operation. Although this gives some confidence on the simulator waveforms recorded it does not imply that other possible correlation measurements, such as measured electron spectra, would necessarily give as good agreement.

The general philosophy used in developing the Casino diagnostic system was to incorporate wherever possible redundant measurements that are based on different physical principles and independently calibrated. Because it is not possible to directly relate any of these measured quantities to accepted international standards, the nolicy of redundant diagnostic monitors has been used so that at least consistency could be shown when they are in agreement. When agreement between monitors measuring the same parameter is poor, then at least some indication of the measurement uncertainty is obtained.

\section{References}

(1) Water: A Comprehensive Treatise, Volume 1 The Physics and Physical Chemistry of Water, Ed by Felix Franks, Plenum Press, New York, page $262(1972)$.

(2) G. A. Huttlin, "Interpretation of Remote Current and Voltage Data in a Pulsed System," HDL-R-22900-80-1, Harry Diamond Laboratories, Adelphi, MD (Oct 1980).

\section{Sponsors Acknowledgments}

This work was sponsored by the Defense Nuclear Agency MIPR No. 77-559 (1976). 


\title{
INDI RECT MEASUREMENT \\ BY \\ COMPUTER SIMULATION
}

\author{
ROBERT A. HILL \\ WESTINGHOUSE ELECTRIC CORP. \\ BALTIMORE, MD. 21203
}

\section{Enhanced instrumentation by means of computer} aided techniques is particularly useful for measurements of fast, high energy electrical transients. An overview presented in this paper offers a general approach to making indirect measurements by mathematical modeling. The illustrative examples are taken from case histories of simulating the dynamic behavior of pulse circuits. Various examples described herein were selected to indicate a wide range of usage. These examples are given without the intent of rigorous analysis or rigid procedure. The purpose is to give discussion in appropriate workshop style.

Mathematical modeling is particularly useful in design and development of high power, complex systems because of necessary time and resources that must be committed for actual construction. The model can serve a variety of purposes for analysis of the equipment. A single, all purpose model would be too unweidly for practical purposes; therefore, the model generally consists of a series of special purpose models, or related mathematical equations which describe a limited function. Solution to the descriptive equations may be determined by computer programs with suitable output display. Some models which may be adaptable for indirect measurements and estimating are discussed in the following sections. 


\section{TYPICAL PROBLEMS}

Several unique problems arise when making measurements of large electrical transients in pulse power systems. A few of the common difficulties are listed below.

a. Dynamic range of instrumentation presents a limitation of measuring extreme conditions.

b. Spurious interference affecting instrumentation, such as electromagnetic radiation.

c. Inaccessible point of measurement due to mechanical obstruction or high voltage hazards. In some cases, such as loop currents caused by stray reactance, a single point of measurement is non-existent.

d. Masking of quantitative measurement by extraneous sources and environmental noise.

e. Fault condition instrumentation where the fault would cause a dangerous situation.

\section{EXAMPLES OF SIMULATION}

Several illustrations of simulation may be taken from the line type pulser discharge circuit shown in Figure 1. The line type pulser was chosen since it is easily identified and offers many simple examples of modeling. Examples presented herein are reduced from complex problems which may exist in real situations. These examples indicate extensions of primary instrumentation performed by conventional means. In fact, the exsistence of a useful model depends upon its correlation to direct measurements.

Waveforms

A typical output waveform of current from a pulse forming network is shown in Figure 2. This waveform is the result of several currents which exist in the network loop elements as a function of time during the discharge period. In this case $i_{1}$ is the output 


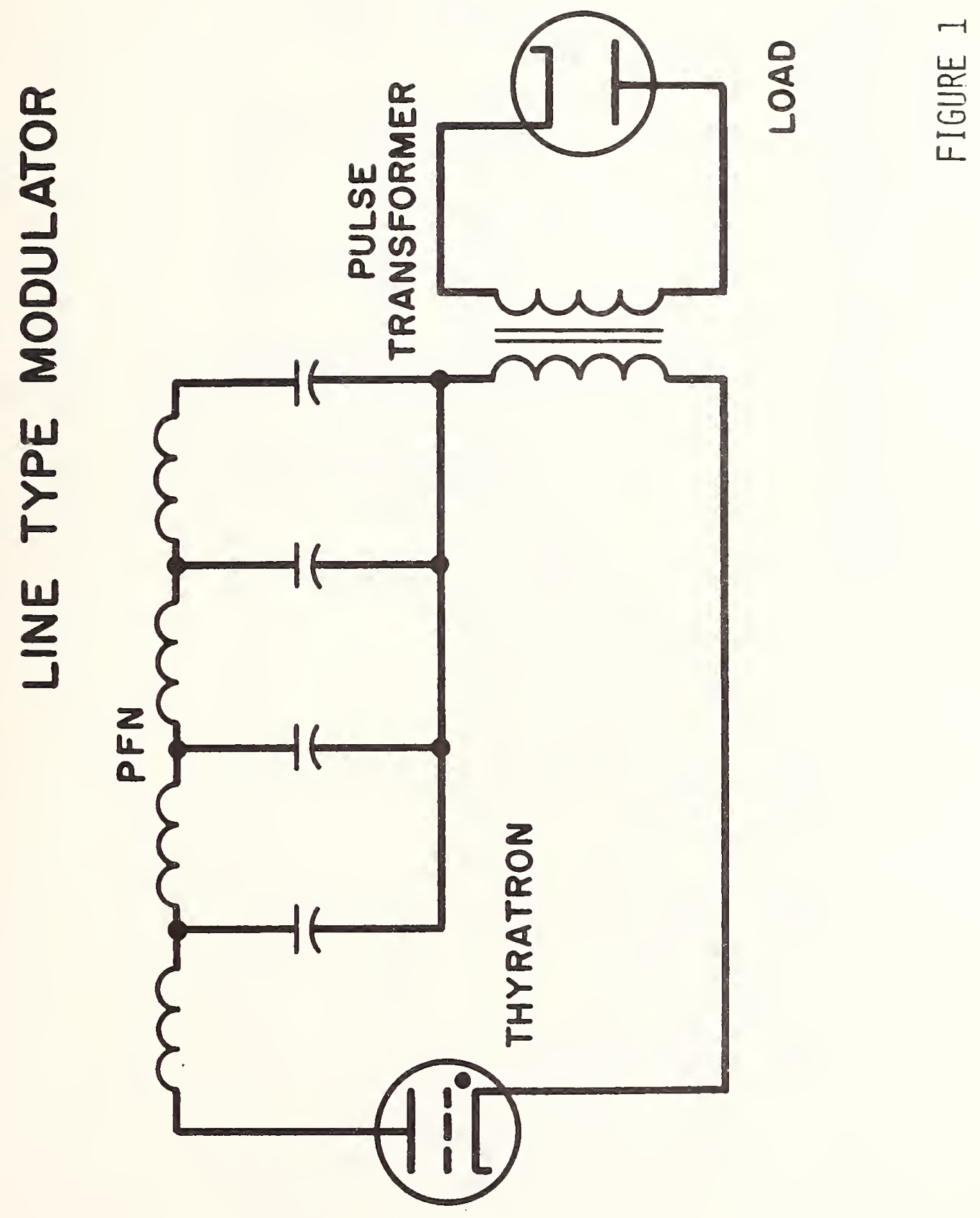



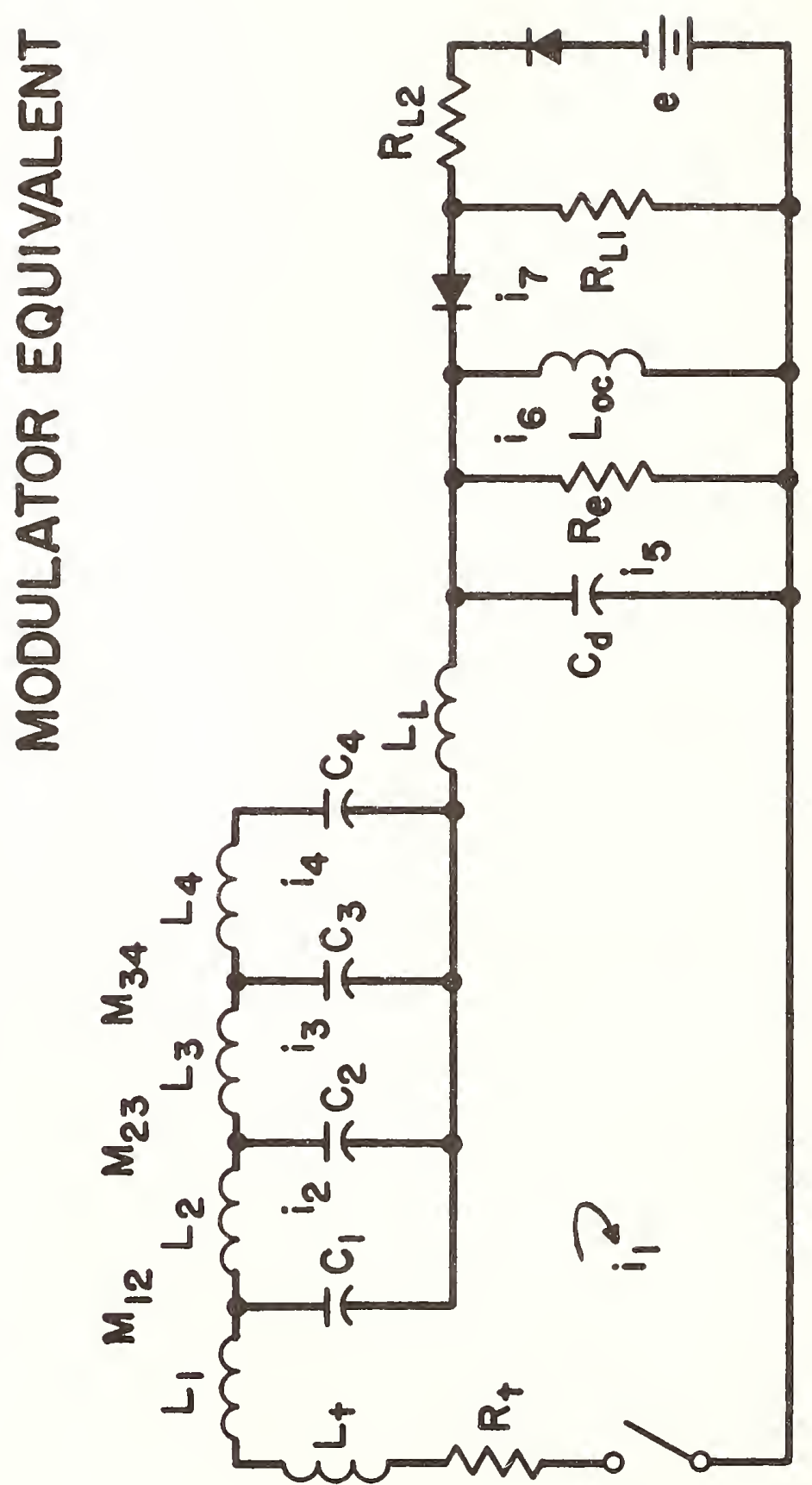

5
胫
点 


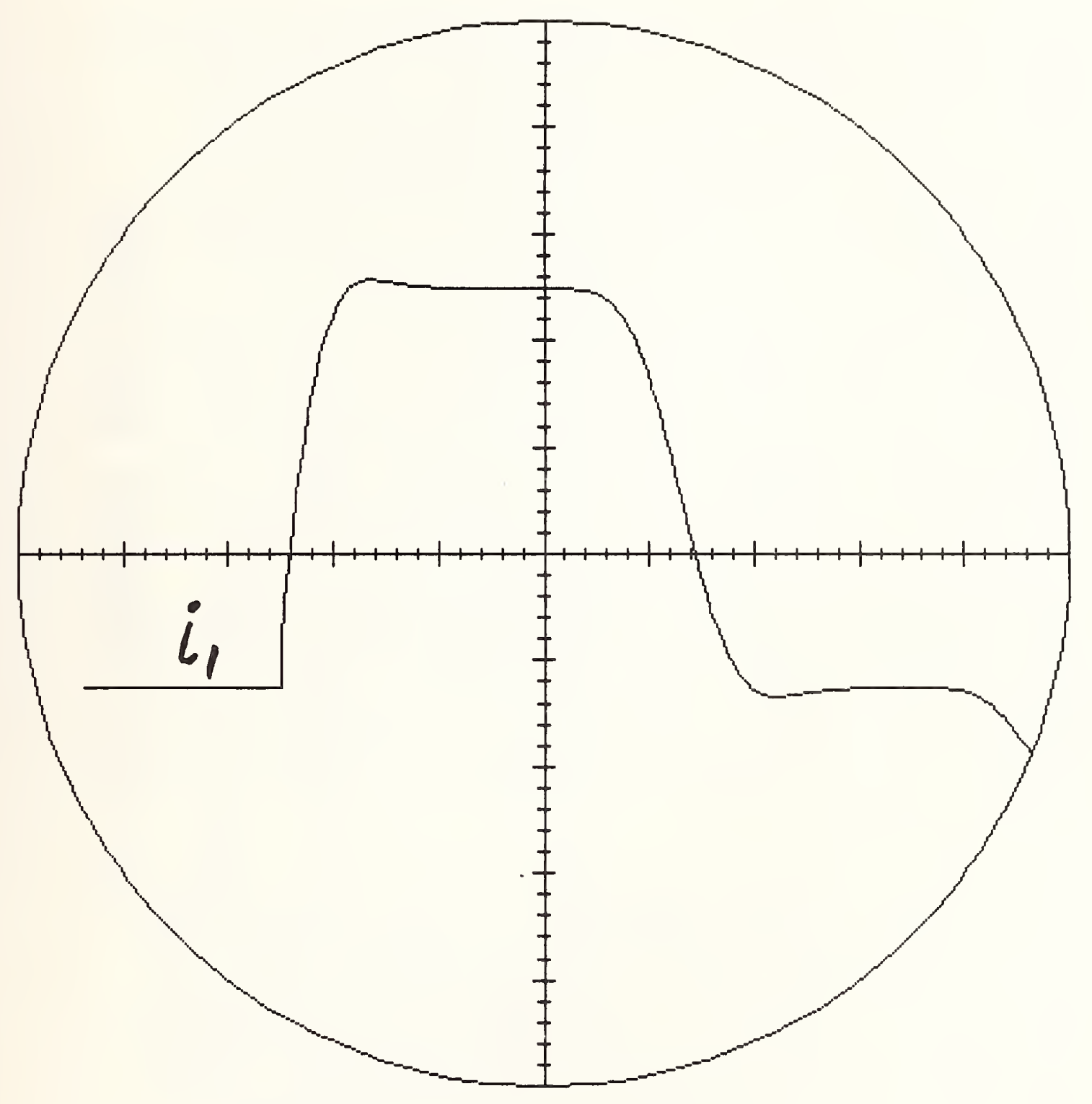

OUTPUT CURRENT

FIGIRE 2 
current in $R_{\mathrm{L}}$ which is readily available for direct measurement. However, the remaining currents in various other portions of the circuit may be of significant interest, yet locations of measurement may be inaccessible. If element values of the network are known, then the remaining current waveforms may be calculated by relating the one measured value to all others, such as is shown in Figure 3. An extension of this procedure can be taken another step. In this case, waveform of $i_{4}$ is in an inaccessible location.

Assuming that a load fault occurance was caused by a voltage arc or other shorted conditions, the current $i_{4}$ waveform may be simulated as shown in Figure 4 . In some cases, particularly high power pulse systems, the fault current cannot be easily measured nor is it advisable to attempt direct measurement. This in turn provides yet another step in indirect measurement by simulation. If a load fault current of large magnitude is to be prevented, then protective circuits such as a crowbar circuit must be used to divert the energy.

Other problems then arise with direct measurements such as the dynamic range of instrumentation required and masking effects caused by interference. Both of these problems are present during crowbar action of high power systems. Simulation of the circuit conditions offers a means of gaining insight into the quantitative values.

Stray Reactance

Quite a different approach of simulation may be used to measure stray reactance of a pulse transformer. In this case, the output pulse transformer of a line type modulator was found to cause excessive waveform distortion by ringing. Conventional means of directly measuring stray leakage inductance and stray winding capacitance failed to provide sufficient details. An equivalent circuit as shown in Figure 5 was used for analysis. Values of coupling between primary and secondary windings are shown as Mps . Stray capacitance values of the secondary windings are given as $C_{1}$ and $C_{2}$. Comparisons of actual observed waveform and simulated waveform were made as shown in Figure 6. Parameter values of the simulated circuit were adjusted until agreement was reached for magnitude and frequency of ringing. With this technique, the effective values of stray reactance could be 


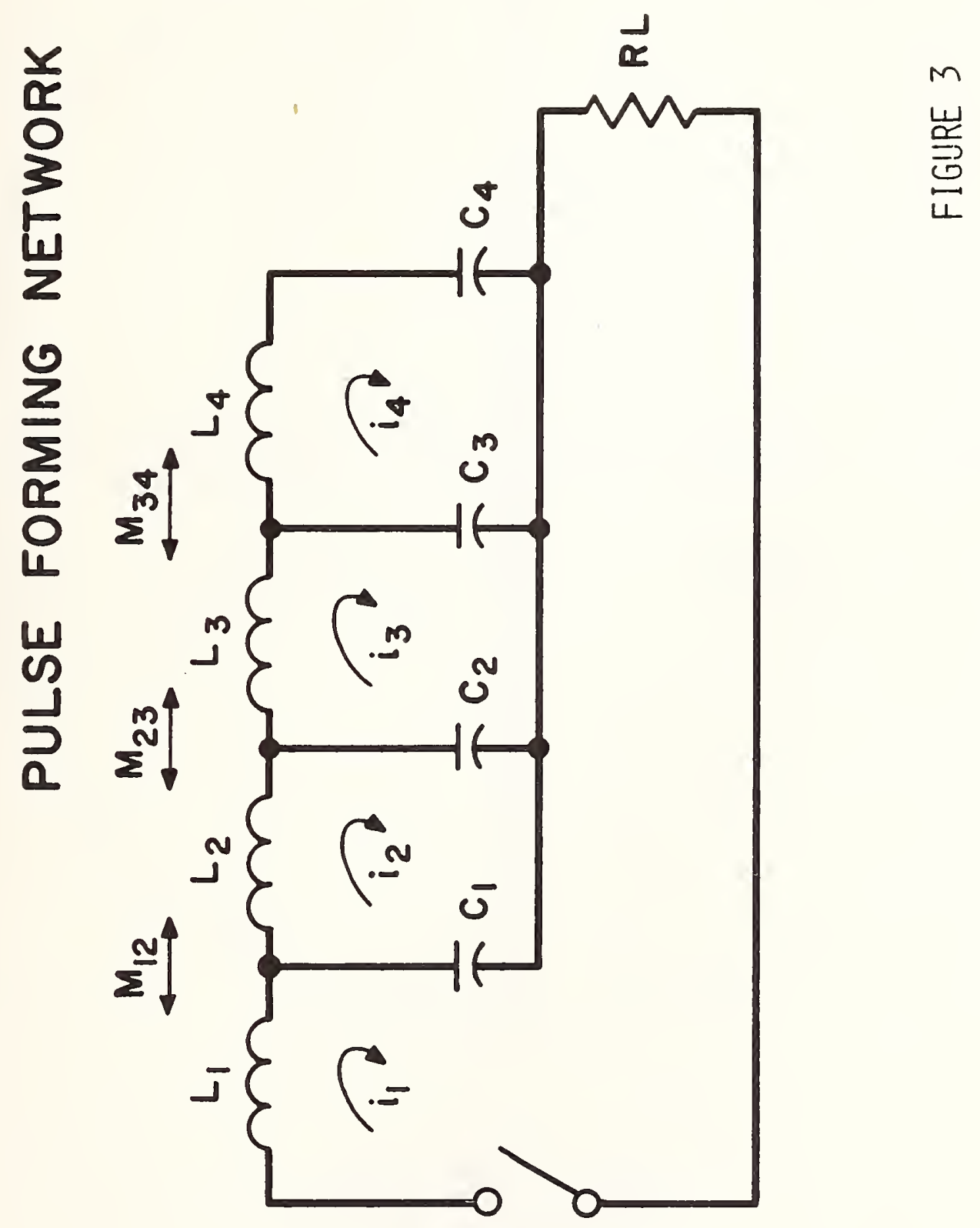




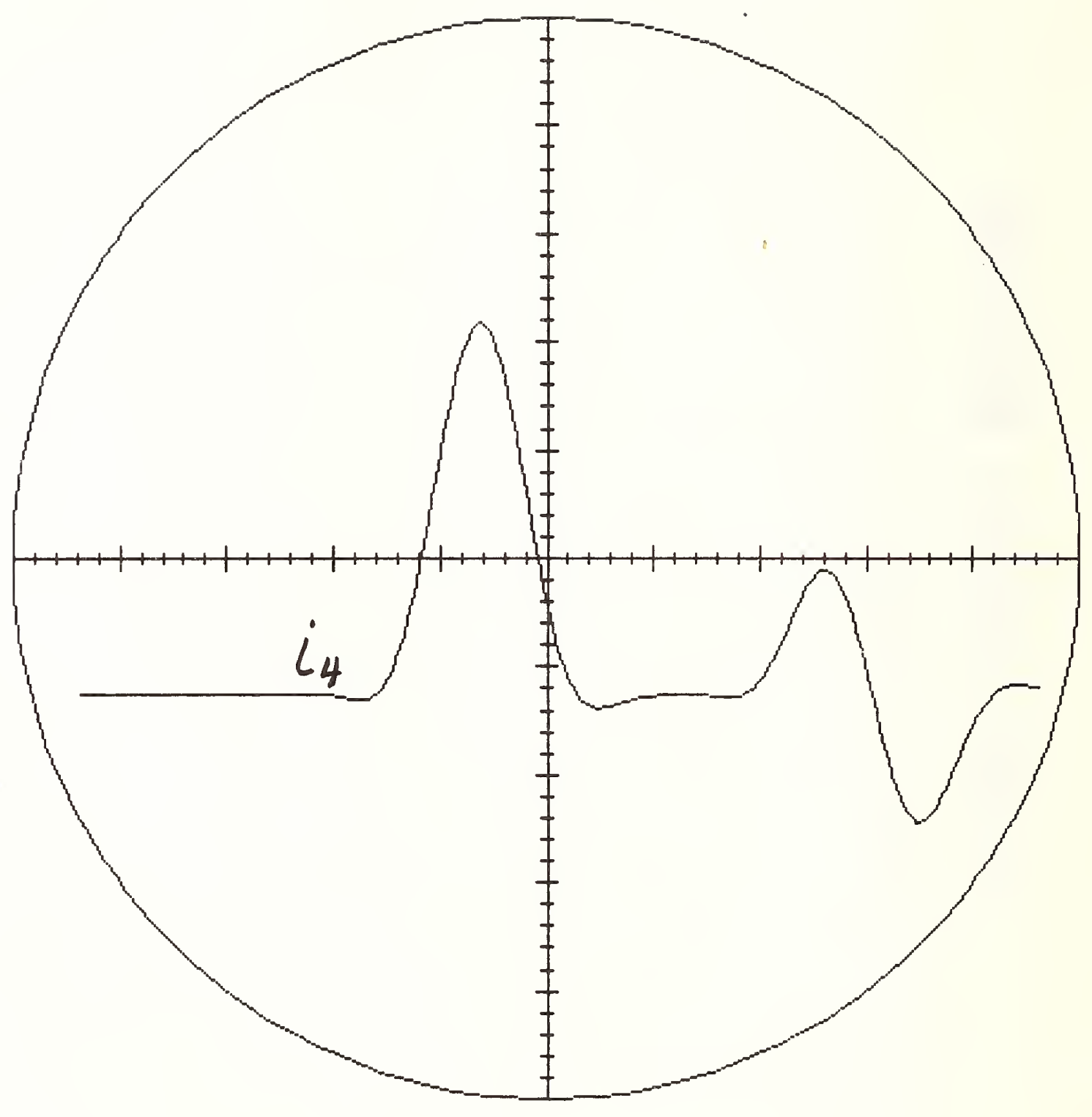

LOOP CURRENT I 4

FIGURE $3 A$ 


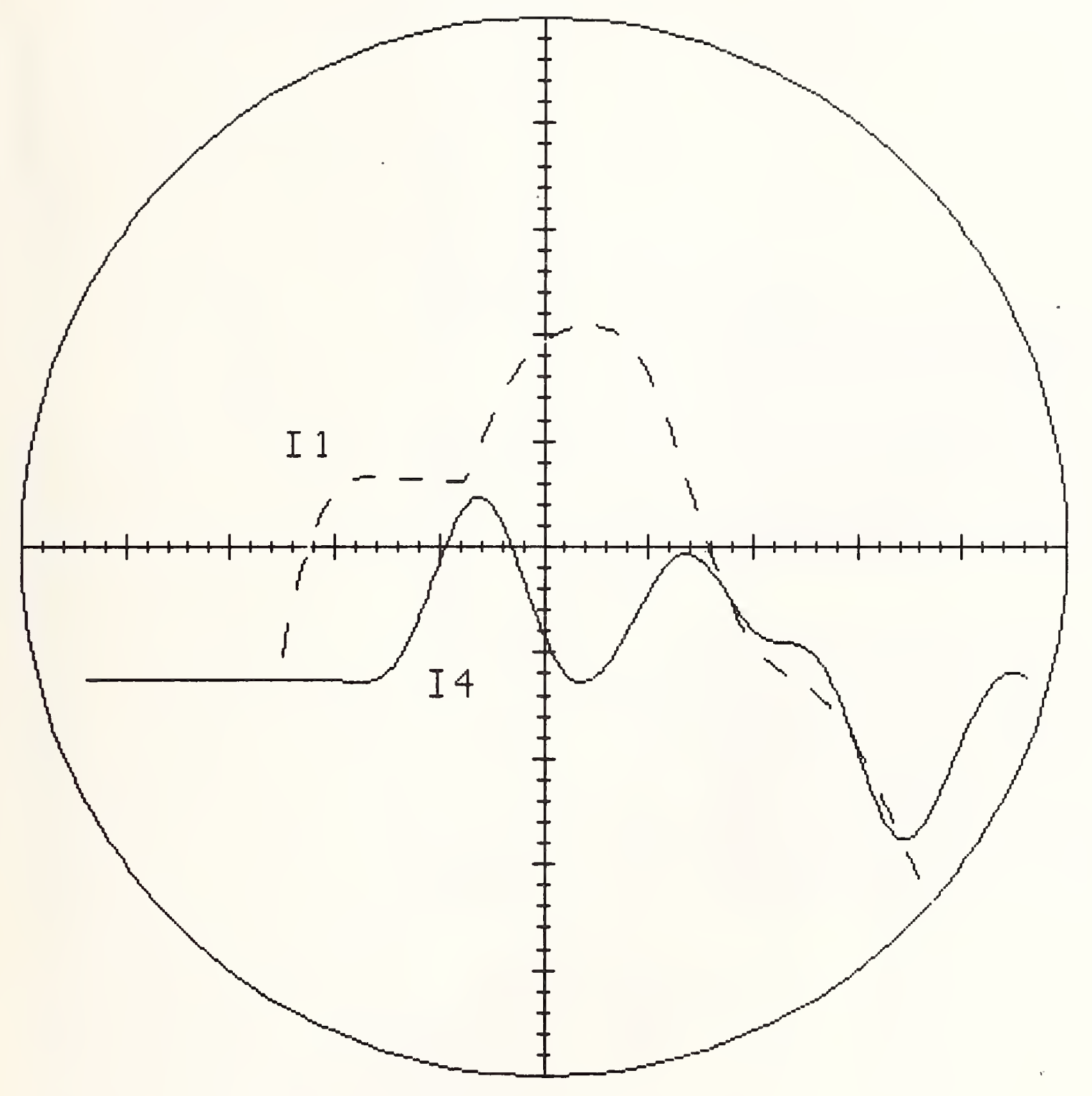

LOAD FAULT CURRENT

FIGIJRE 4 


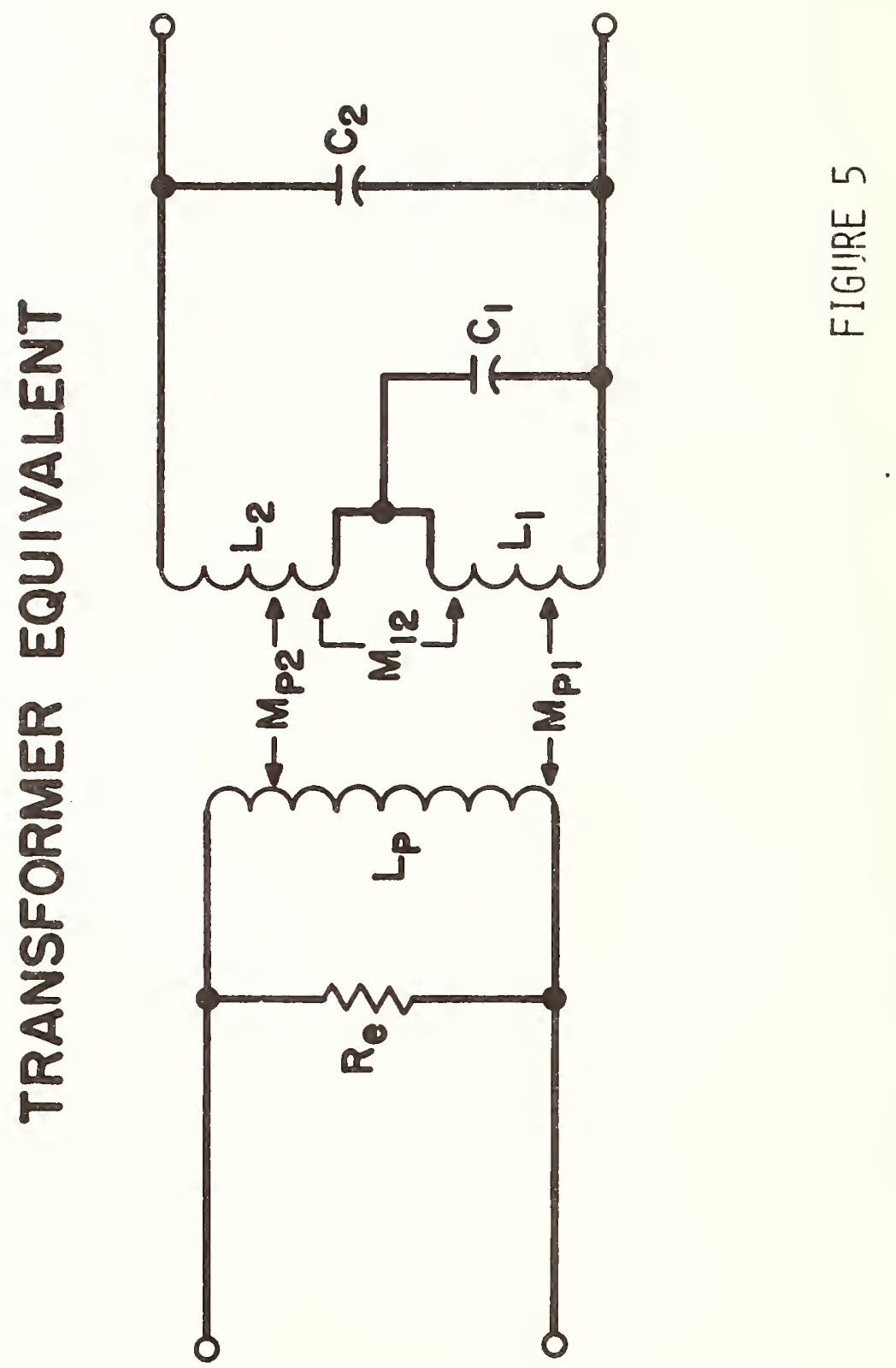




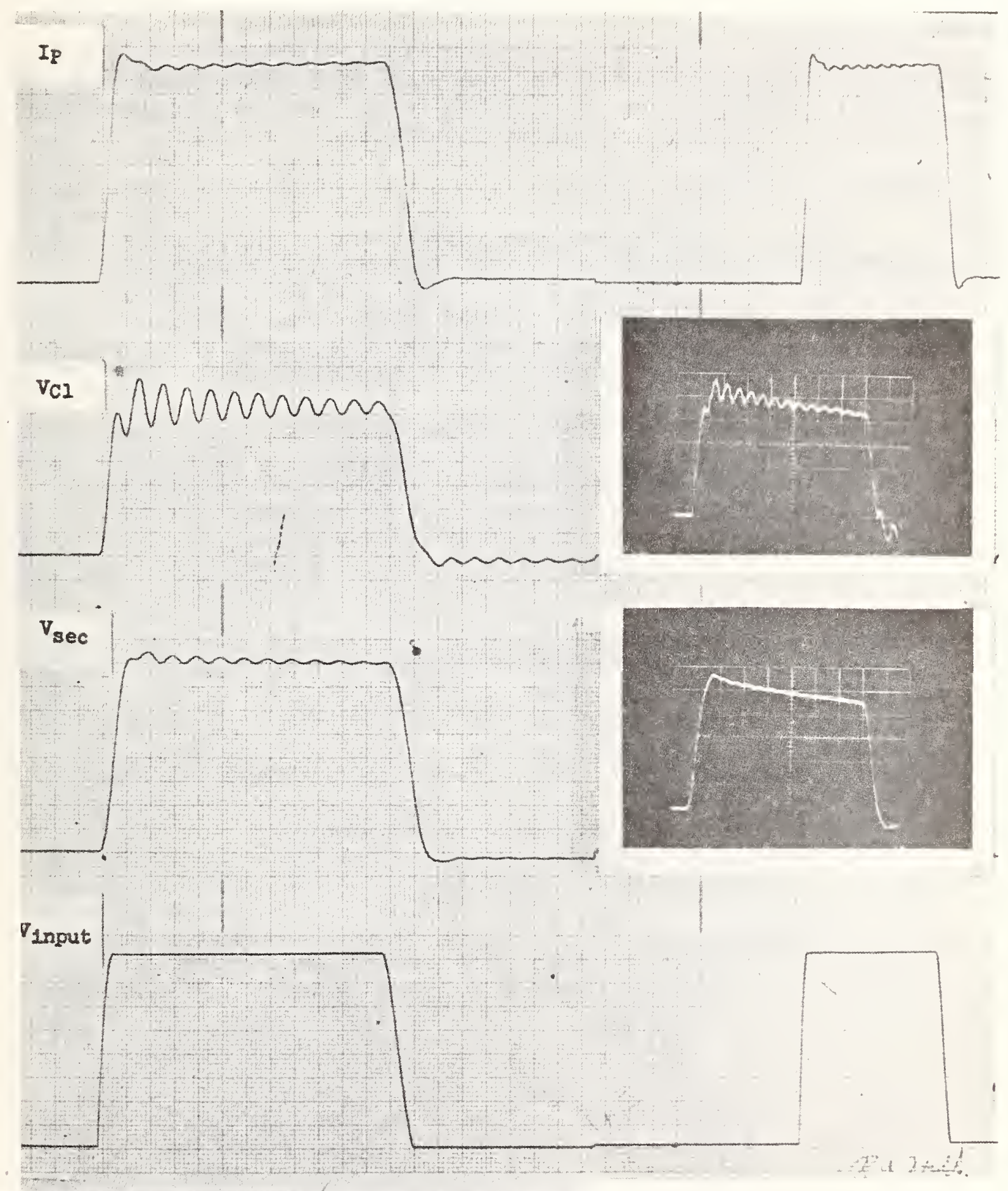

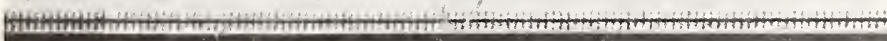

TRAISFORIER OUTPUT

FIGURE 6 
determined. This example illustrates a means of determining effective reactance values of an actual circuit by comparison with known model values which were derived by a trial and error procedure.

Magnetic Field

Consistency of scaling magnetic fields produced by current carrying conductors in a homogeneous medium provides a reliable means of modeling. Advantage of this fact may be used in several cases where direct measurements would be difficult or where the instrumentation would introduce extraneous errors.

Figure 7 shows a calculated magnetic field plot of two parallel, current carrying conductors. Each TIC mark on the $X-Y$ axes represents a unit of distance and the surrounding lines represent equal magnetic flux density. These lines also indicate the field gradient change as a function of distance. In this example, it was desired to produce a uniform field at distance $D_{y}$ from the conductors. As shown, a two conductor system produces a very non-uniform flux gradient. Because of dimensional limitations and interposing barriers in the actual system, direct measurement techniques were restricted. Field gradient at the desired points would also introduce instrumentation errors. With the aid of computer plotted data, it is possible to overcome some of the restrictions. First, an acceptable model of the conductor system was established by means of mathematical computation. This system has a go-return path of 8 conductors as shown in Figure 8 . It represents a multi-turn winding of 1200 amp-turns. Each group of conductors is shown as "*" on the graph, with spacing $1.8,1.533,1.266$, and 1 units of distance. The area of flux density lines shown are kounded by 300 and 500 gauss flux density lines which is the area of interest. The next step in deriving a workable system was the construction of a physical scale model. Scale modeling was used to provide information about field intensity variation and also provide a check on computational accuracy. The scale model was made to convenient dimensions for instrumentation. From measured parameters, the relationships of scale model and mathematical model could be determined. 


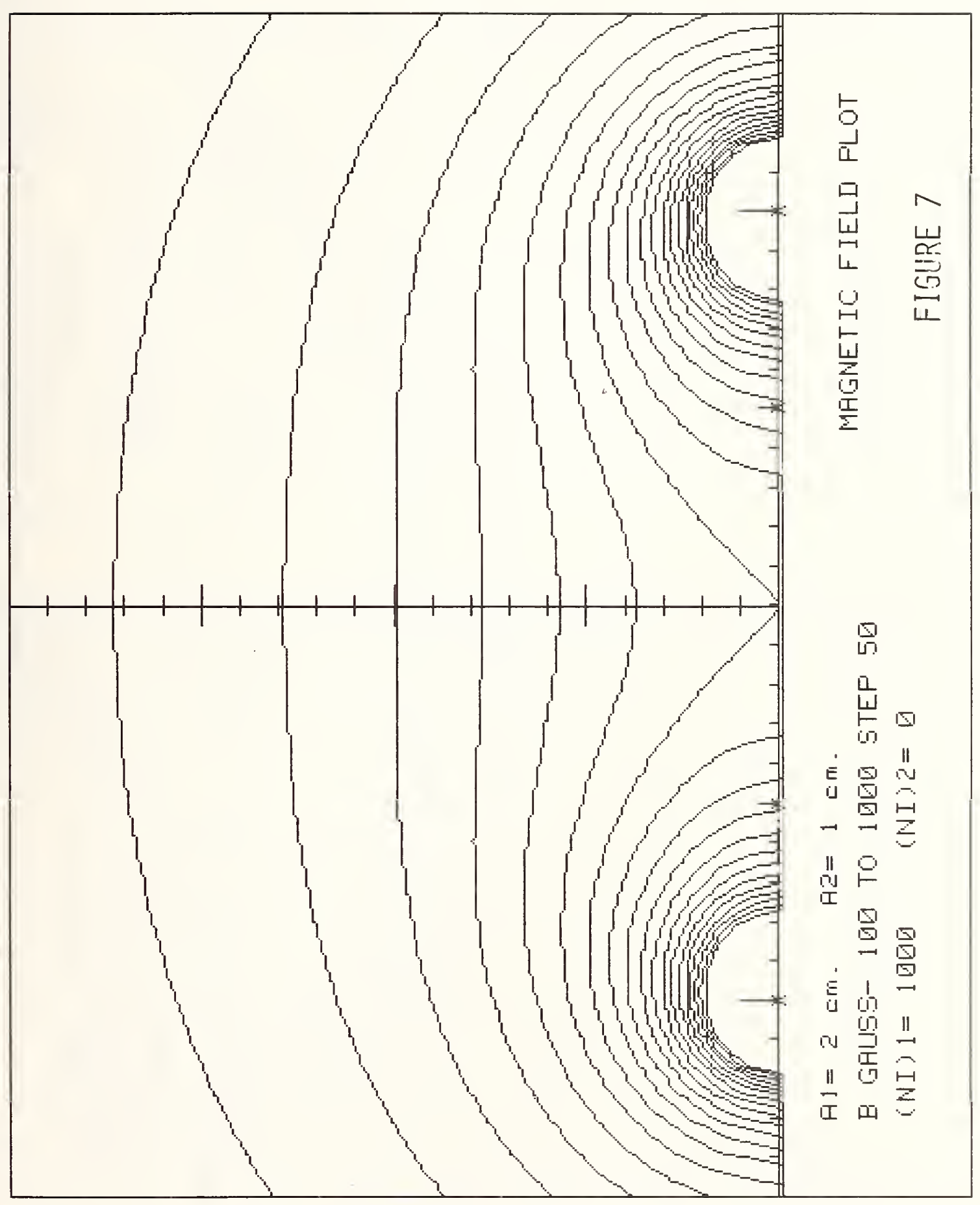




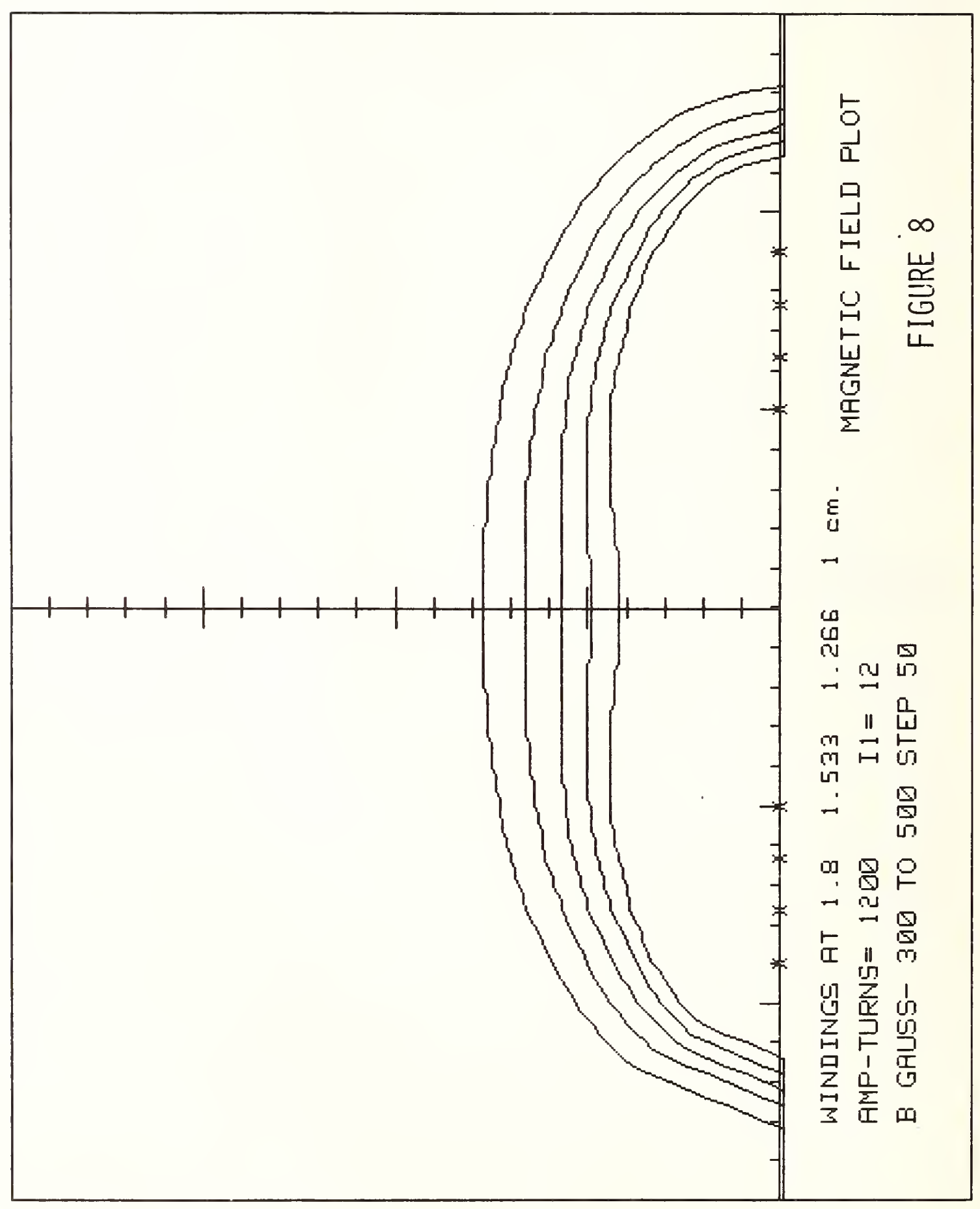


Measurements were made in areas of relatively low field gradients for correlation. After reasonable agreement was established between the two models, computer techniques were used to establish final design of the conductor system. In the final system, direct measurement of the magnetic field distribution are very difficult to measure. However, computed estimates provide sufficient data for practical purposes.

Dynamic behaviour of the magnetic field may be simulated. The magnetic system described above was designed for use in a repetitively pulsed application. Therefore, inherent inductance is a major consideration. Affects of inductance are implicit in the simulation. This can be seen by comparison of Figures 7 and 8 for flux line concentration. Although further elaboration of this technique is beyond scope of this paper, it can be seen that space fields may be modeled for illustration of a particular purpose.

Data Extrapolation

Beneficial information may be generated by extrapolation of limited measured data. This technique, although somewhat restricted by complexity of simulation, is useful over certain ranges for estimating purposes. Generally, it involves a "curve fit" program available on most computers. The system to be extrapolated does not necessarily have to be linear, but it does have to be consistent within the bounds of simulation. With a limited amount of data measurement points, certain mathematical functions can be generated for determining relationships. However, the number of points and the spread of data points are critical to the degree of approximation. If, on the other hand, data points are taken for a system having a known mathematical relationship, the extrapolation may be made with a fair degree of certainty. This is the case for component temperature rise over an extended period of operation. Temperature rise characteristics are related as shown in Figure 9. In most cases of uniform temperature rise, an estimate of the time taken to arrive at a final operating temperature can be made. Two temperature measurements taken at equally spaced intervals of time during initial temperature changes can be used to predict final operating temperature. With 


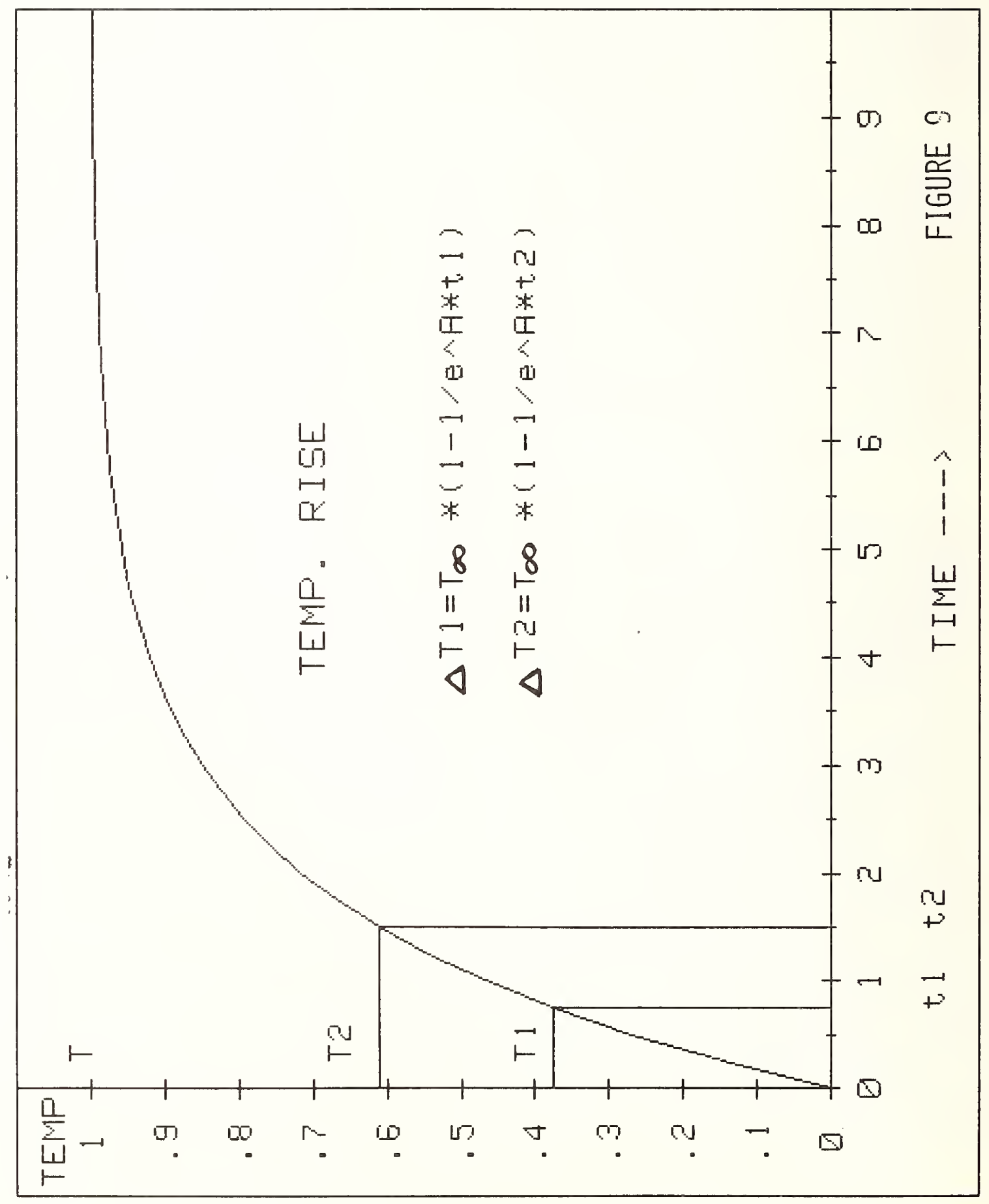


large components having time constants in the order of several hours, this technique provides a means of predicting final temperature in a time period less than one time constant.

\section{GENERAL SUMMARY}

Modeling is generally an abstract approximation of the actual working system. Perhaps, the most important consideration of modeling is "What and how much". Over simplification leads to gross errors in results. An overly complex simulation may be time consuming and expensive beyond value of the results. Deriving a reasonable compromise is usually based on past experience in simulation techniques. An overly ambitious program on first attempt is very difficult to "debug". One approach which seems to work best is a step by step model building: Starting with only the few essential elements as a crude approximation; then building and adding other considerations; and finally discarding those elements which have little significance.

An attempt at making meaningful measurements or even approximate estimates would seem to rely on how complete the model represent an actual system. Obviously, the best means of accomplishing this would be either reduction and simplification of the system or simulation of only limited aspects of the system. 


\title{
CALIBRATION OF THE BLACKJACK 5 PULSE GENERATOR OUTPUT POWER*
}

\author{
R. Richardson, E. Chu, W. Clark \\ J. Shannon and M. Wilkinson \\ Maxwel1 Laboratories, Inc. \\ 8835 Balboa Avenue \\ San Diego CA 92123
}

A discussion will be presented of the calibration techniques routinely used to ensure precise measurement of output power from the BLACKJACK 5 pulse generator. Because of the high voltages ( 3 MV) and high currents ( 25 MA) which must be measured diring normal operation, probes with very low absolute sensitivity are used. Calibration procedures will be described which consist of applying voltages and currents of sufficient levels to overcome the low sensitivity and provide good signal-to-noise ratios. The calibration pulsers are also designed with outputs having dominant frequency components close to the measure signals. Cavity type B current monitors are calibrated in in situ and corrected for wall effects. These monitors are also checked against $\dot{B}$ loops. $\dot{E}$ type diode voltage monitors are also calibrated in situ by measuring the probe capacitance and by direct comparison with a voltage divider. Careful attention is paid to the frequency response of passive RC integrators used in the calibrations and final measurements. A1l cable lengths are trimmed to ensure precise phasing of the signals, and FFT techniques are used to correct for cable attenuation.

*Work supported by the Defense Nuclear Agency.

\section{Introduction and Background}

This paper presents the results of a set of experiments designed to measure the output power on the BLACKJACK 5 generator at Maxwe 11 Laboratories. BLACKJACK 5 (shown in Figure 1) is a water insulated transmission line structure with four time compression stages. The first stage (transfer capacitor) is charged with a Marx generator and sequential closing of water switches 
transfers the energy in successively shorter times to the output (diode). The output (diode) is a water-vacuum insulator and vacuum transmission line which converges the energy to the load. The machine is used to produce dense plasmas by imploding wire arrays, foils and gas jets. The nominal output parameters are:

$\begin{array}{lr}\text { Voltage } & 3 \mathrm{MV} \\ \text { Current } & 5 \mathrm{MA} \\ \text { Power into the diode } & 10 \mathrm{TW} \\ \text { Energy into the diode } & 500 \mathrm{~kJ} \\ \text { Pulse width } & 50 \mathrm{~ns}\end{array}$

Other pulse widths can be achieved by switch variations but the $50 \mathrm{~ns}$ mode is the usual one.

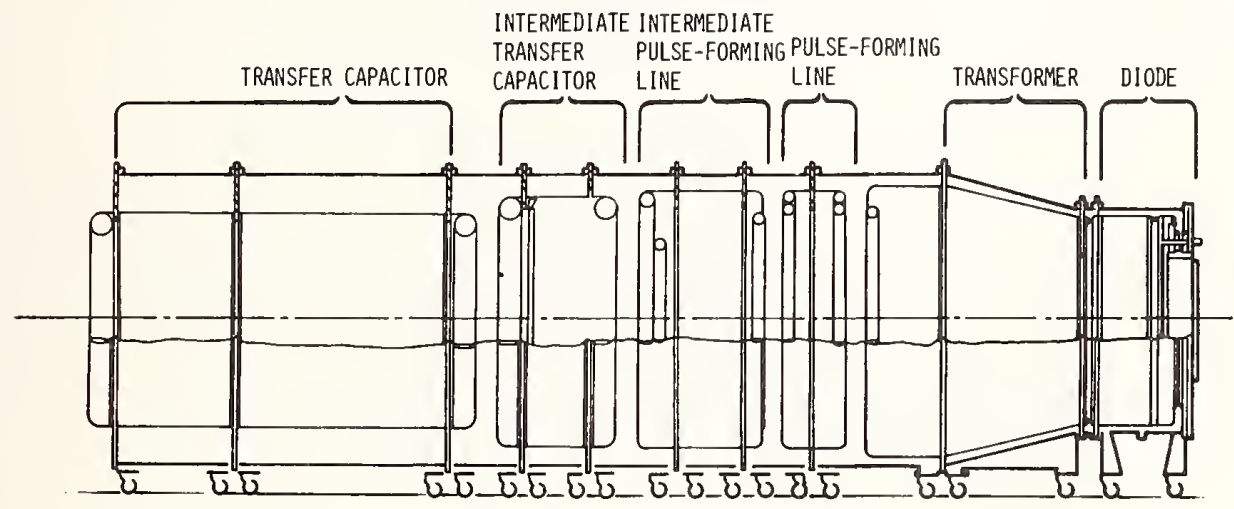

Figure 1. BLACKJACK 5 pulseline -cross section of structure.

An accurate determination of the power is important for two reasons. First, some of the plasma parameters (such as radiated energy) appear to depend strongly on the diode input power. For this reason, an accurate power measurement helps verify scaling laws which are used to determine future machine requirements. Second, there are certain design goals (such as $10 \mathrm{TW}$ ). It is necessary to know the power to sufficient accuracy to demonstrate that the goals of the program have been achieved.

In January 1980, the opinion was voiced that the Maxwell BLACKJACK 5 system did not achieve the 10 TW that had been advertised. Because of this we began an intensive review of all the diagnostics to determine the actual power of the system into the diode load. The effort included the calibration of the pertinent data channels and careful scrutiny of all effects (such as risetimes) that might influence the power measurements. The result of this effort is presented below. 


\section{Factors Affecting Diode Power Measurement}

Along with the usual calibration accuracy, there are additional factors that have to be considered in determining the power into the diode. Examples of these are the relative phasing on the current and voltage waveforms and such effects as high and low frequency degradation. Since the present diode voltage has a large inductive component, the $V$ and I waveforms are out of phase. This exaggerates the effect of phasing erors and frequency cutoffs.

\subsection{Calibration Accuracy}

The accuracy with which the voltage and current probes can be calibrated is the most critical factor in the power measurements. It is clear that to get a \pm 10 percent power measurement a \pm 5 percent calibration must be done. This calibration is discussed below and in other papers presented at this conference [1].

\subsection{Voltage and Current Phasing}

Because the diode is largely an inductive load during the early part of the pulse, $d I / d t$ is near maximum at peak voltage. This means that an error in the phase (relative timing) of the voltage and current waveforms has maximum the effect. For an inductive load, the effect of a time shift in the peak power is given by

$$
\frac{p}{\hat{P}} \simeq 1+\frac{\Delta T}{T_{0}}
$$

where $P / \hat{P}$ is the normalized power $\Delta T$ is the time shift of the voltage with respect to the current and $T_{0}$ is the width of the power pulse (FWHM). The effect of a time shift on the BLACKJACK 5 waveforms is shown in Figure 2.

At first it appears that the only issue here is the correct trimming of cable lengths to the desired accuracy. There are, however, some subtleties that must be considered. In this machine, as well as others, the voltage measurement is made by a capacitive probe upstream of the diode insulator 22.5 ns away from the current probe which is in the vacuum. This raises the question as to whether voltage waveform should be delayed by this time difference. This question can be answered by considering the circuit of Figure 3. This shows the relation of the diode voltage probe with respect to the current probe in the BLACKJACK 5 generator. 


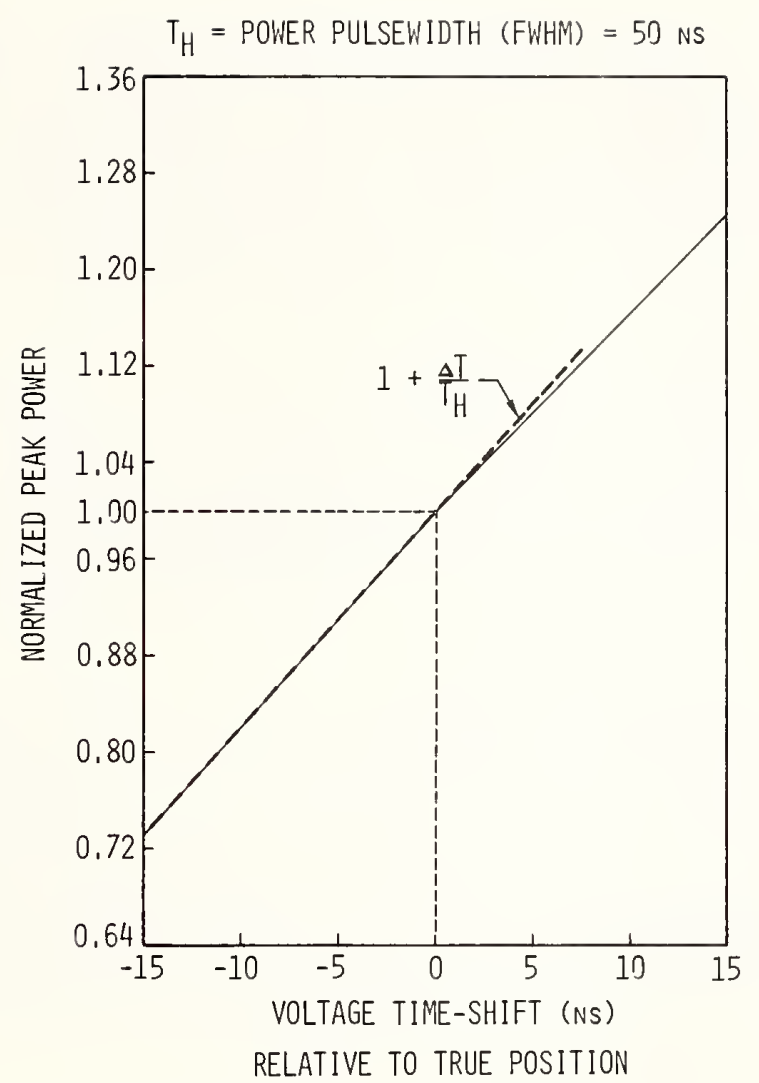

Figure 2. Plot showing the sensitivity of the power to an error in voltage and current position.

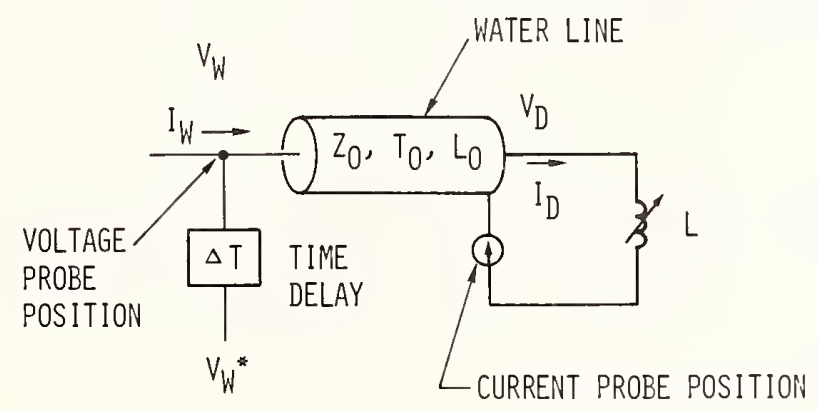

$$
\begin{aligned}
V_{W}, I_{W} & =\text { VOLTAGE, CURRENT AT VOLTAGE PROBE POSITION. } \\
V_{W}{ }^{*} & =\text { VOLTAGE AFTER TIME DELAY } T . \\
V_{D}, I_{D} & =\text { VOLTAGE, CURRENT IN DIODE. }
\end{aligned}
$$$$
Z_{0}, T_{0}, L_{0}=\text { IMPEDANCE, ELECTRICAL LENGTH AND INDUCTANCE OF }
$$$$
\text { TRANSMISSION LINE BETWEEN DIODE AND VOLTAGE PROBE. }
$$$$
Z_{0}=0.6 \Omega, T_{0}=2.5 \mathrm{Ns}, L_{0}=1.5 \mathrm{NH}
$$

Figure 3. Relation of voltage and current probes. 
This circuit can be analyzed in the frequency domain. For times long compared to characteristic electrical lengths $\left(\Delta T, T_{0}\right)$ the solution can be expanded for small values of frequency and the coefficients of various derivatives can be identified. The voltage at the oscilloscope $V_{W}^{*}$ is then related to the diode voltage $V_{D}$ by

$$
\begin{aligned}
V_{W}^{*} & \simeq V_{D}+z_{0} T_{0} \frac{d I_{p}}{d t}-\Delta T \frac{d V_{D}}{d t} \\
& -z_{0} T_{0} \Delta T \frac{d^{2} I_{D}}{d t^{2}}+\frac{\Delta T^{2}+T_{0}^{2}}{2} \frac{d^{2} V_{D}}{d t^{2}}+\frac{z_{0} T_{0}^{3}}{6} \frac{d^{3} I_{D}}{d t^{3}}+\ldots
\end{aligned}
$$

It can be seen that adding a time delay $\Delta T$ in the voltage channel to account for the probe position adds terms proportional to $d V_{D} / d t$ and $d^{2} I_{D} / d t^{2}$. The term added by the transmission 1 ine is proportional to $\mathrm{dI}_{D} / \mathrm{dt}$ in this 1 imit and cannot be cancelled by the $d V_{D} / d t$ term. For this reason, it is better to make $\Delta T=0$, in which case there is a first derivative correction term in the current and the next corrections are second derivative in voltage or higher. In this case, the voltage at the probe is related to the voltage at the diode by

$$
V_{w}=V_{p}+L_{0} \frac{d I_{D}}{d t}+\frac{T_{0}^{2}}{2} \frac{d^{2} V_{D}}{d t^{2}}+\frac{z_{0} T_{0}^{3}}{6} \frac{d^{3} I_{D}}{d t^{3}}+\ldots
$$

where we have put $L_{0}=Z_{0} T_{0}$. The power defined by the probe voltage and diode current is then

$$
\begin{aligned}
P & =V_{W} I_{D} \\
& =V_{D} I_{D}+\frac{d}{d t}\left(\frac{1}{2} L_{0} I_{D}^{2}\right)+\ldots
\end{aligned}
$$

From this we see that the power defined in this fashion is the true diode power plus the time derivative of the magnetic energy stored in the transmission line between the probe and the diode. This is all consistant if the diode is considered to be the volume downstream of the voltage probe. Typically, the second term in Eq. 4 is $\sim 5$ percent of the diode power. Hence, the power measured in this fashion is that which flows past the voltage probe. 


\subsection{Frequency Roll-0ffs}

Another factor which affects the power measurement is the frequency response of the diagnostivs used in measuring the voltage and current. These effects can be characterized in the high and low frequency limits by frequency cutoffs. There are three separate effects, one at low frequency and two at high frequency.

The low frequency roll-off is caused by finite integration time of capacitive probes or Rogowski coils or the finite time constant of a passive integrator used with differential probes. In the time domain these manifest themselves as a droop in the later parts of the waveform. In the frequency domain the low frequency roll-off is described by a transfer function

$$
T_{L}(w)=\frac{j\left(\frac{f}{f_{L}}\right)}{1+j\left(\frac{f}{f_{L}}\right)}
$$

where $f_{L}$ is the cutoff frequency. For a passive integrator, $f_{L}$ is given by

$$
f_{L}=\frac{1}{2 \pi R C}
$$

where $R C$ is the time constant of the integrator. A typical value of $f_{L}$ is $\sim 3 \times 10^{4} \mathrm{~Hz}(R C=5 \mu \mathrm{s})$. The degradation of the peak power due to the low frequency roll-off is shown in Figure 4 vs the cutoff frequency.

The first of the high frequency effects is a simple $1 / f$ type degradation at high frequencies. This could be due to stray capacitance or inductance at the probe or in the passive integrator. In the frequency domain it is described by the transfer runction

$$
T_{H}=\frac{1}{1+j\left(\frac{f}{f_{H}}\right)}
$$

where $f_{H}$ is the cutoff frequency.

In the time domain this would appear as a risetime degradation. The effect of the high frequency cutoff on the power is shown in Figure 4. A typical value of $f_{H}$ is $210^{8} \mathrm{~Hz}$. 


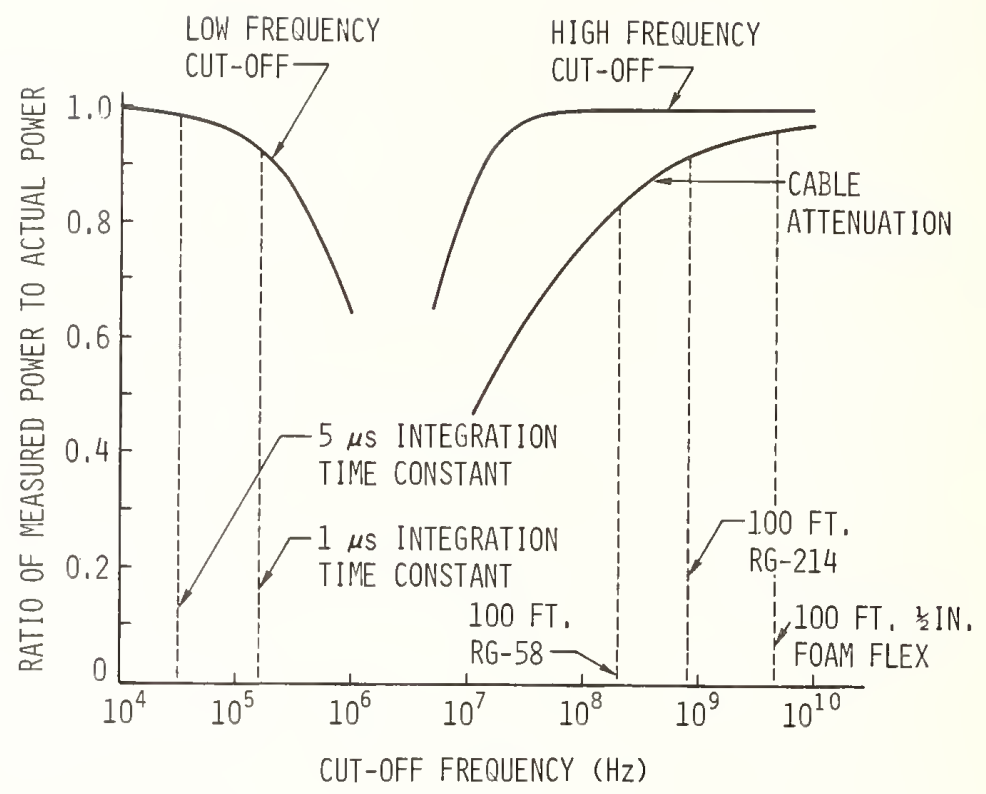

Figure 4. The effect on the BLACKJACK 5 power measurement of high and low frequency degradation.

The second type of high frequency attenuation is that due to cable 1osses. In the frequency domain, the transfer function has the form

$$
T_{C}=\exp \left(-\sqrt{j \frac{f}{f_{C}}}\right)
$$

where $f_{c}$ is given by

$$
f_{c}=\frac{1}{2 \pi}\left(\frac{2 z_{0}}{\alpha x}\right)^{2}
$$

Here, $z_{0}$ is the cable impedance, $x$ the length of the cable and $\alpha$ a constant describing the high frequency attenuation. Typical values of $f_{c}$ are $10^{9}-10^{10} \mathrm{~Hz}$. These are very large but since the frequency appears as $\sqrt{f}$, the effect persists at frequencies in the range of interest. The effect of the cable cutoff on the power is shown in Figure 4. 
A11 of the above frequency roll-offs can be compensated. In our case, the compensation is done in the computer software. This will be discussed at more length below.

\section{Diagnostic Calibration}

In the BLACKJACK 5 diode, the probes used to infer the power measurement are a capacitive voltage probe in the water near of the diode interface and a cavity type current probe on the vacuum side of the insulator. These are shown schematically in Figure 5 . The voltage is measured at three positions and the current at six positions. This was done to average out the non-transmission line modes of the water PFL and irregularities in the current waveform due to losses. The individual probes are connected together and a single cable is run to the screen room for each diagnostic.

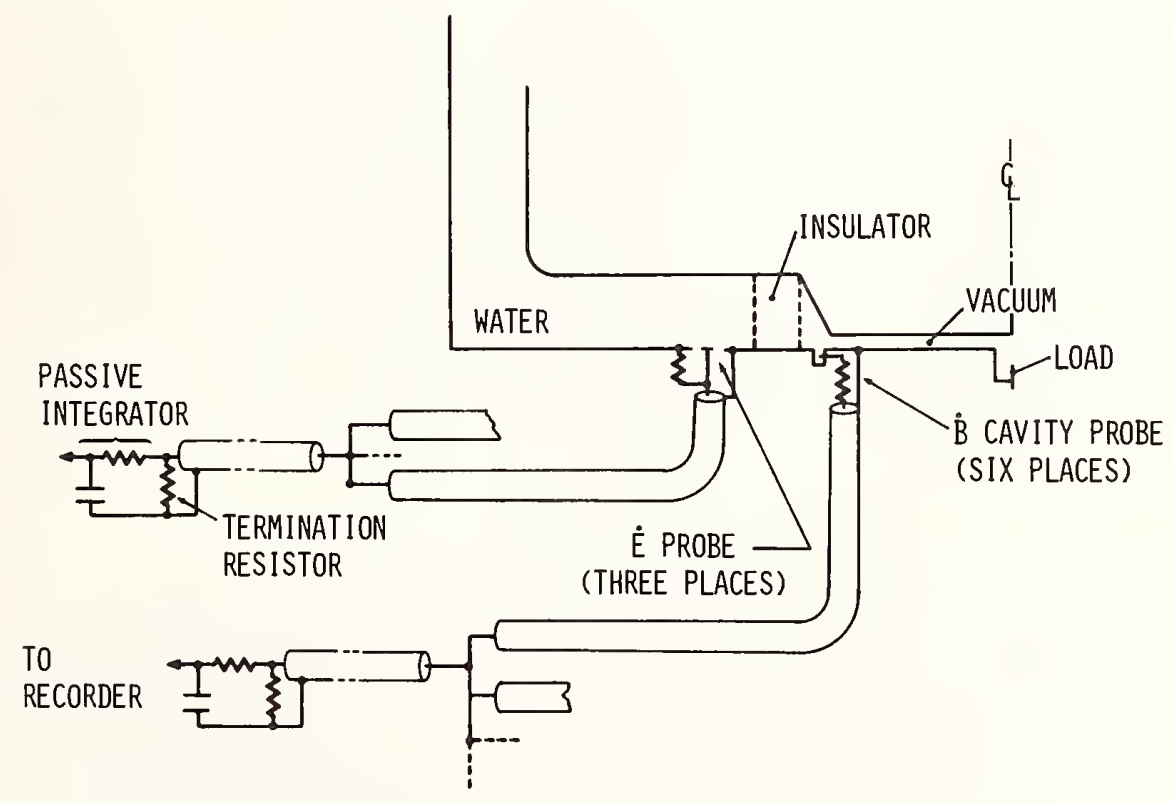

Figure 5. BLACKJACK 5 diode diagnostics.

\subsection{Voltage Probe}

The diode voltage probe basically consists of a small capacitance with respect to the pulseline central conductor, connected via a $50 \Omega$ cable to a passive integrator. Two methods were used to calibrate this probe. The first was a direct comparison with the output of a 1000:1 resistive voltage divider obtained from Los Alamos Scientific Laboratory. The divider had previously been 
checked out and found to give nominal 1000:1 division over a wide band of frequencies centered on $\sim 3 \mathrm{MHz}$. A fast-ringing discharge of approximately that frequency was injected at the diode axis using a $20 \mathrm{nF}, 2100 \mathrm{nH}, 30 \mathrm{kV}$ capacitor bank. The resistive divider ( $250 \mathrm{k} \Omega$ impedance) was connected at the diode periphery as near as possible to the voltage probe location. The outputs of the two probes were compared directly using Tektronix 485 oscilloscopes.

The second method of voltage probe calibration, dubbed the "capacitance-current" (CC) method involves measuring the coupling capacitance from the high voltage terminal to the E-field probe. It can be shown [1] that ratio of the probe current to the input current is proportional to the ratio of the probe capacitance to the pulseline capacitance. Thus, the essence of the capacitancecurrent method is to determine precisely this ratio since the pulseline capacitance can be accurately measured. This is accomplished by monitoring the charging current injected by a capacitor into the cental conductor, which flows to ground largely as displacement current through the pulseline capacitance. A portion of this displacement current is intercepted by the voltage probe and is responsible for the probe signal. Comparison of the probe signal with the signal provided by a Pearson current transformer monitoring the charging current gives the desired capacitance ration. This is discussed in detail in reference [1].

The two methods of voltage probe calibration were in excellent agreement with each other, giving estimates of probe sensitivity which differed by two percent. The average of the two methods was the sensitivity used in power calculations.

\subsection{Current Probe}

The injected current used for the $\mathrm{CC}$ voltage calibration was also used in calibration of the current monitor. The latter consists of a precisely machined circular groove $\left(0.20 \mathrm{~cm}^{2}\right.$ area) just inside the insulator. A voltage proportional to $\mathrm{dI} / \mathrm{dt}$ develops across the groove inductance and is integrated with a passive integrator to produce a signal proportional to I . The calibration used the 23 $\mathrm{MHz}, 20 \mathrm{nF}, 2100 \mathrm{nH}$ pulser described above and consisted of a direct comparison between the integrated output of the diode current monitors and the signal from the Pearson transformer, which monitored the injected current.

Since the wall material of the diode current monitors (aluminum) has nonzero resistivity, a small frequency-dependent correction must be made in order to eliminate a contribution to the unintegrated sianal due to finite wall resistance [2]. Fortunately, this problem is well understood and since data acquisition is via Tektronix 7912 transient digitizers, the correction may be made using computer software, as will be discussed below. 


\subsection{Synchronization}

Synchronization of the current and voltage was accomplished by precise measurement of all cable lengths using time-domain reflectometry. The instrument used was the Tektronix 1502 TDR. A $210 \mathrm{~ns}$ wide pulse is generated in the screen room during each shot and is added to all diode waveforms. This pulse, triggered by the output switch of the pulseline, provides a fiducial timing mark for purposes of synchronization.

\section{Compensation Procedures}

Because the data is acquired on transient digitizers, the compensation for the various effects mentioned above is easily done. The software required is relatively straightforward and has been checked against test waveforms to determine its accuracy.

\subsection{High and Low Frequency Compensation}

In our case, the high and low frequency cutoffs arise from the same circuit element, the passive integrators used in both the current and voltage measurement. Since they are so far apart in frequency $\left(3 \times 10^{4}\right.$ vs $\left.10^{8} \mathrm{~Hz}\right)$, they can be treated as two independent effects. The correction routine has the form,

$$
V_{\text {True }}=V+2 \pi f L \int V d t+\frac{1}{2 \pi f_{H}} \frac{d V}{d t}
$$

typically. The two frequencies $f_{L}$ and $f_{H}$ are determined from a transfer function versus frequency plot of the passive integrator. The second term in Eq. 10 is due to the low frequency cutoff and usually makes the largest correction. The third term, due to the high frequency roll-off, is usually smal1. However, it makes the waveform appear to have a time shift. This can cause an apparent discrepancy between current/voltage relation determined from the measured cable lengths and the actual position (a few ns difference). For this reason, this correction must be done.

\subsection{Cable Correction}

A method of compensating for the coaxial cable attenuation/ dispersion has been developed [3] that works well in our parameter range. The correction has the form 


$$
V_{\text {True }}=V+\int_{0}^{t} K(t-\tau) \frac{d V}{d \tau} d \tau
$$

where $K(t)$ is given by

$$
K(t)=\frac{\alpha x}{2 Z_{0}} \frac{1}{\sqrt{\pi t}} t>0 .
$$

Here, $z_{0}$ is the impedance of the cable, $x$ is the length of the cable and $\alpha$ is constant of the particular cable and is a measure of the high frequency attenuation. The constant $\alpha$ can be determined from published data, but it is more convenient to adjust $\alpha$ by correcting a test waveform as described in reference [3] .

\subsection{Cavity Monitor Wa11 Resistivity Correction}

As seen in reference [2], the finite resistance of the cavity current monitor leads to an error in the latter parts of the pulse. It was shown in the reference that an approximate compensation could be done by adjusting the integrator decay time. However, an exact compensation can be done with the computer software.

Following the analysis in reference [2], the impulse response of a cavity current monitor in Laplace transform space is

$$
H(s)=1+\frac{1}{\sqrt{T_{C}^{s}}}
$$

where $T_{C}$ is a cavity time constant given by,

$$
T_{C}=\mu_{0} \sigma\left(\frac{A}{D}\right)^{2}
$$

Here, $\sigma$ the conductivity, $A$ the area of the cavity and $D$ is the perimeter of the cavity. For the current monitor in BLACKJACK 5, $1 / \sigma=4 \times 10^{-6} \Omega-\mathrm{cm}, A=0.2 \mathrm{~cm}^{2}, D=2.85 \mathrm{~cm}$, giving $T_{C}=15.5 \mu \mathrm{s}$. The second term in Eq. 13 leads to an over estimate of the current in late time. For the present monitor the error is $\sim 5$ percent at peak current. 
Fortunately, this impulse response has an inverse which allows us to write

$$
\tilde{\mathrm{I}}_{\text {True }}=\tilde{\mathrm{I}}\left[1-\frac{1}{\sqrt{1+\mathrm{T}_{c} \mathrm{~s}}}\right] \text {. }
$$

In the time domain, this becomes

$$
I_{\text {True }}(t)=I(t)-\int_{0}^{t} I(\tau) G(t-\tau) d \tau
$$

where

$$
\begin{gathered}
G(t)=\frac{1}{\sqrt{\pi T_{C} t}}-\frac{1}{T_{C}} \exp \left(\frac{t}{T_{C}}\right) \operatorname{erfc} \sqrt{\frac{t}{T_{C}}} \\
t>0
\end{gathered}
$$

This removes the wall resistivity error from the current pulse. It should be noted in passing that cavity monitors are only practical with high conductivity metals (copper, aluminum, ...). In metals like stainless steel, the cavity time constant becomes comparable to the pulse and large distortions occur.

\section{Output Power Measurements}

Power measurements have been made with short circuits and plasma loads. The voltage, current and power are shown in Figure 6 for a titanium plasma load. The pulse shape shown is typical of the plasma load shots. In this mode, the power pulse is between 50-60 ns wide. At the highest level of operation, 10-11 TW are generated at the diode envelope and 500-600 kJ are delivered to the diode. 


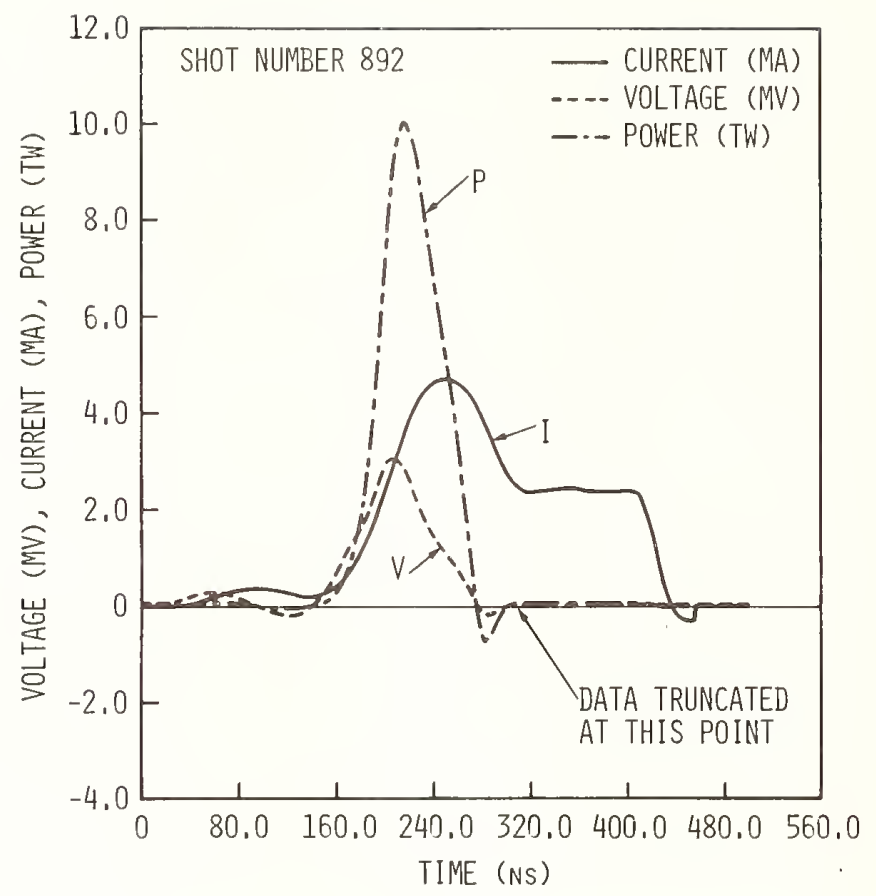

Figure 6. BLACKJACK 5 waveforms with titanium plasma load.

Two checks have been made on the consistency of the diagnostics. One check in the linearity of the diagnostics is to calculate $\int V d t$ and compare it to the current I . For a short circuit, these should be identical in shape with the proportionality constant being the inductance. These quantities are shown in Figure 7 for a short circuit shot. The fit is very good during the main pulse. During the prepulse, the resolution of the transient digitizer makes the fit less accurate. After $280 \mathrm{~ns}$ in the figure, the input voltage waveform is truncated by the end of the trace so the waveform is not realistic after this time.

The output power scaling is shown in Figure 8 as a function of Marx generator charging voltage. The straight line shown is proportional to $V^{2}$. This would be the case if the machine electrical parameters stay the same at different levels and if there are no nonlinearities in the diagnostics. The probable error on the power measurement is about \pm 11 percent due to the estimated accuracy of the calibration. The highest power achieved was $11 \mathrm{TW}$, confirming that BLACKJACK 5 can achieve powers in excess of $10 \mathrm{TW}$. 


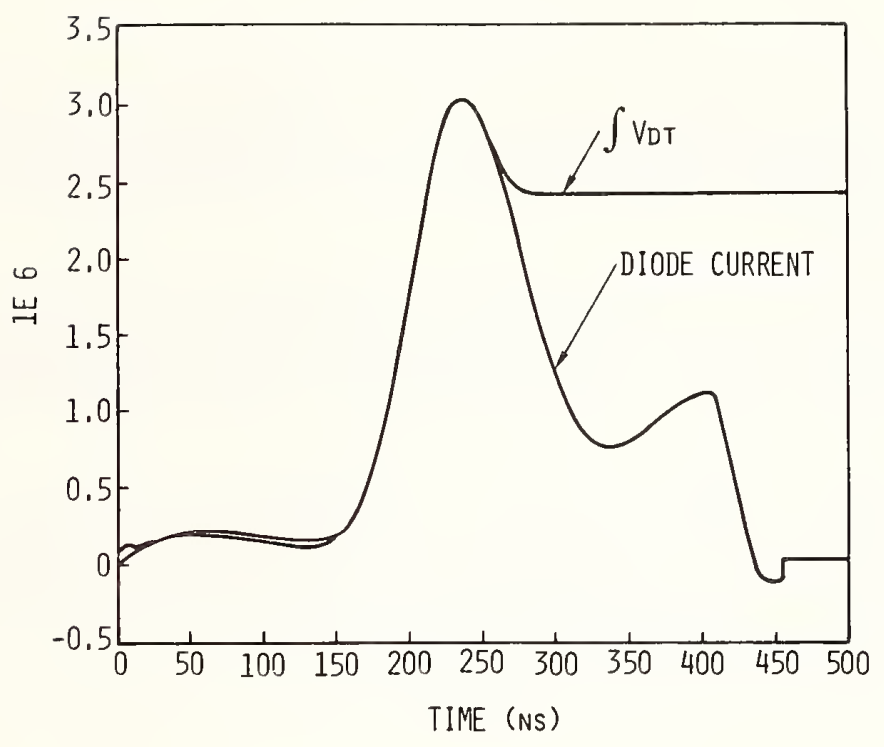

Figure 7. Plot showing the comparison of I and SVdt for a short circuit shot.

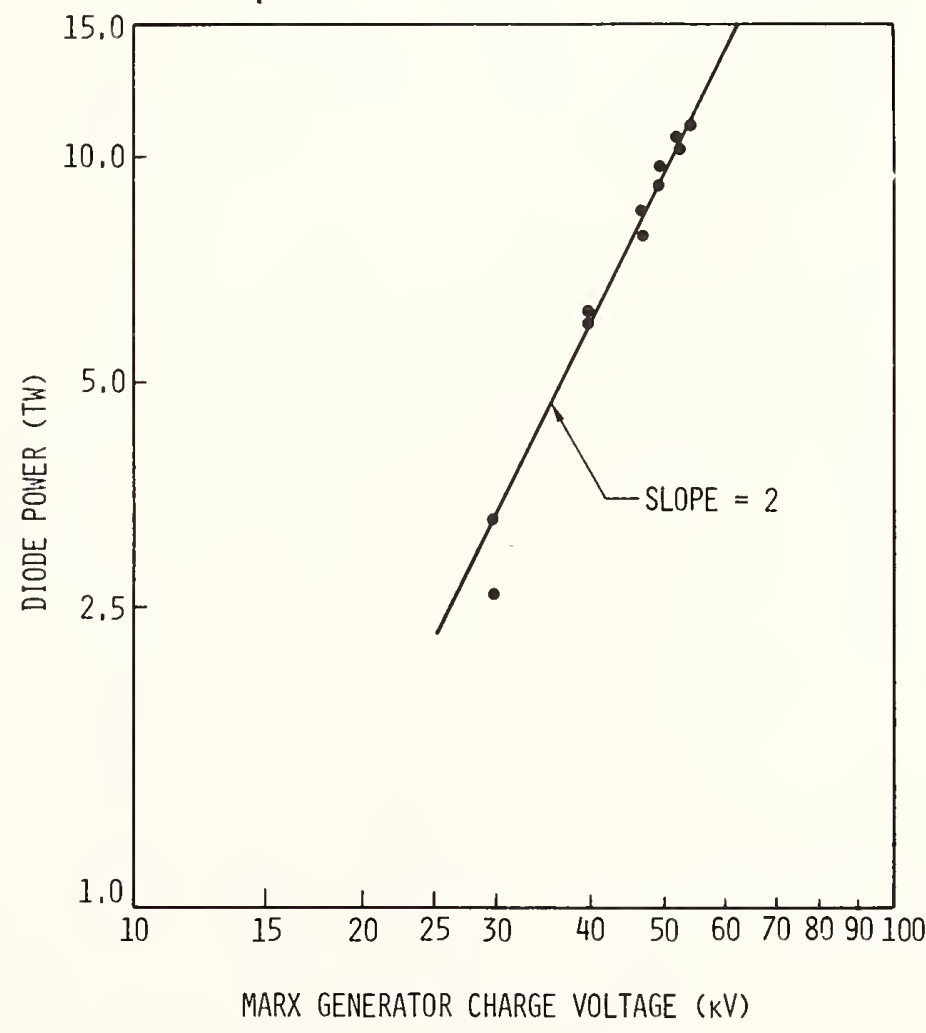

Figure 8. Scaling of BLACKJACK 5 output power with Marx charge voltage. 


\section{References}

1 M. Wilkinson, E. Chu, "Calibration of Capacitive Voltage Probes in Water-Dielectric, High Power Pulse Generators", NBS Workshop on Measurement of Electrical Quantities in Pulse Power Systems, 1981.

2 J. Shannon, E. Chu, R. Richardson, M. Wilkinson and C. Trivelpiece, "Cavity Current Monitors", NBS Workshop on Measurement of Electrical Quantities in Pulse Power Systems, 1981.

3 C. Trivelpiece, R. Richardson, J. Shannon, J. B. Smith, "Digital Correction of Cable Attenuation Losses", NBS Workshop on Measurement of Electrical Quantities in Pulse Power Systems, 1981. 


\section{Discussion Session 3}

Gene Nolting (Naval Surface Weapons Center). Now we come to the group therapy part of the session in which everybody gets to participate. Are there any questions?

Bill Boyer (Sandia). I have a question for John Shannon. I notice that you use hardware integrators. It seems if you were using waveform digitizers that you could software integrate and eliminate the corrections for low frequency droop.

John Shannon (Maxwell Laboratories). That is correct. But, you have to think how historically we got into this. We are relatively new at the transient digitizer business and the initial problems that we had with them was that because of the base line shift, we used to have errors in the integrations. The feeling at that time was since we had these integrators with reasonably good frequency domain properties, we would use them. Now, I think that we would replace them.

Neville Harris (Ion Physics). You were referring to the phase shift between the current and the voltage caused by the voltage monitor being some distance from the diode. We11, that is fair enough, providing that you don't have any reflected waves from the diode. If the diode is mismatched from the line, then the reflected wave from the diode is "after" in time rather than "forward" in time. If you try to extend the machine by working into a mismatched diode, you can run into very severe problems.

Shannon. These diodes are always a mismatch because they are essentially inductances. The analysis that I gave to you in the talk, where I make the frequency expansion, includes that effect. That was derived by doing the transmission 1 ine theory correctly, expanding in small powers of $s$ and identifying the various powers of the derivatives. As far as I can see when you are talking about a few nanosecond transit times, it doesn't make any difference. It is not as if you are looking for a wave going past and then waiting sometime later and getting a bounce. That would be too high a frequency effect for that analysis to deal with. Our monitors are close enough to the diodes so we feel 2 nanoseconds doesn't cause any problems on that line.

Nolting. I would like to make one comment. We use the foam flex cable referred to by John Shannon. The cable is connected to the machine at various points and then comes back to a screen room where all of the cables are connected to approximately the same point on the screen room. During the calibration exercises that we went through we found a distortion of the waveform, by comparing our calibration pulse measured with the Tektronix probe to the calibration pulse as witnessed by the capacitive dividers. Upon investigation, we discovered that there were sizeable currents flowing in the solid 
aluminum shield of the foam flex which generated noise at the cable connector and when we tightened down on the connection to the screen room the phase shift went away. Unfortunately after a period of time, the problem would return and one never knew in any particular portion of the data cycle when this phase shift would reappear because of the oxidation of the aluminum connector. We solved the problem by using a substance called Noalox. I think that the use of Noalox is a standard operating procedure for most electricians who work with aluminum wiring. Since that time, we have bypassed the problem. I don't know if you ever saw anything like that or not, John, but I thought I would bring that out as something you might want to look for.

Ray Leeper (Sandia). Frank (Young), when you are measuring the deuteron voltage by looking at the neutron yield in the forward direction and the deuteron current by looking at $90^{\circ}$, I think you are assuming that the beam is normal to the target. I wondered how sensitive is the measurement to this assumed alignment.

Frank Young (Naval Research Laboratory). For the calculations in figure 7 of my talk, it is assumed that the deuterons are emitted from a flat anode foil (flat geometry) and proceed in straight lines toward a $\mathrm{CD}_{2}$ target which is located inside a hollow cylindrical cathode. In a focusing geometry (e.g., a diode with a curved anode), the ions are projected normally from the anode into the region within a hollow cathode. For a target placed at this location, the ions are incident on target with a broad spread of angles. In fact, the half angle in our experiments is $35^{\circ}$, and if the detector is located at $15^{\circ}$ to the diode axis, angles between the deuteron and neutron directions range from $0^{\circ}$ to $50^{\circ}$. Results in focusing geometry are presented in figure 1 of this section which are analogous to those in figure 7 of my paper in a flat geometry. If you look in the forward direction $\left(15^{\circ}\right)$, the energy resolution or time resolution is somewhat larger than it is for the flat geometry case (compare bottom of figures $7 \mathrm{~b}$ and $1 \mathrm{~b}$ ) and at $85^{\circ}$ it is much larger (compare bottom of figures $7 \mathrm{c}$ and $1 \mathrm{c}$ ). But even for this focusing geometry, the time resolutions are small compared to the total widths of the neutron signals. The widths of the neutron signals are dominated by the duration of the deuteron current where it overlaps the voltage pulse (see figure 1a).

John Shipman (Sachs Freeman/Naval Research Laboratory). I would like to ask John Shannon about the probes he showed feeding a junction. He put a resistor from the outer conductor to the case of his machine to absorb the reflection. Somehow, I feel like I have asked a sucker question, but there must be something tricky here.

Shannon. At each probe there is a $50 \mathrm{ohm}$ termination. It is in paraliel with the current sensors since they are voltage probes and it is in series with the E-field sensors. So the reflection going back from this junction goes back into the cable and gets absorbed. If a signal from one of the probes comes down the cable, it sees this 

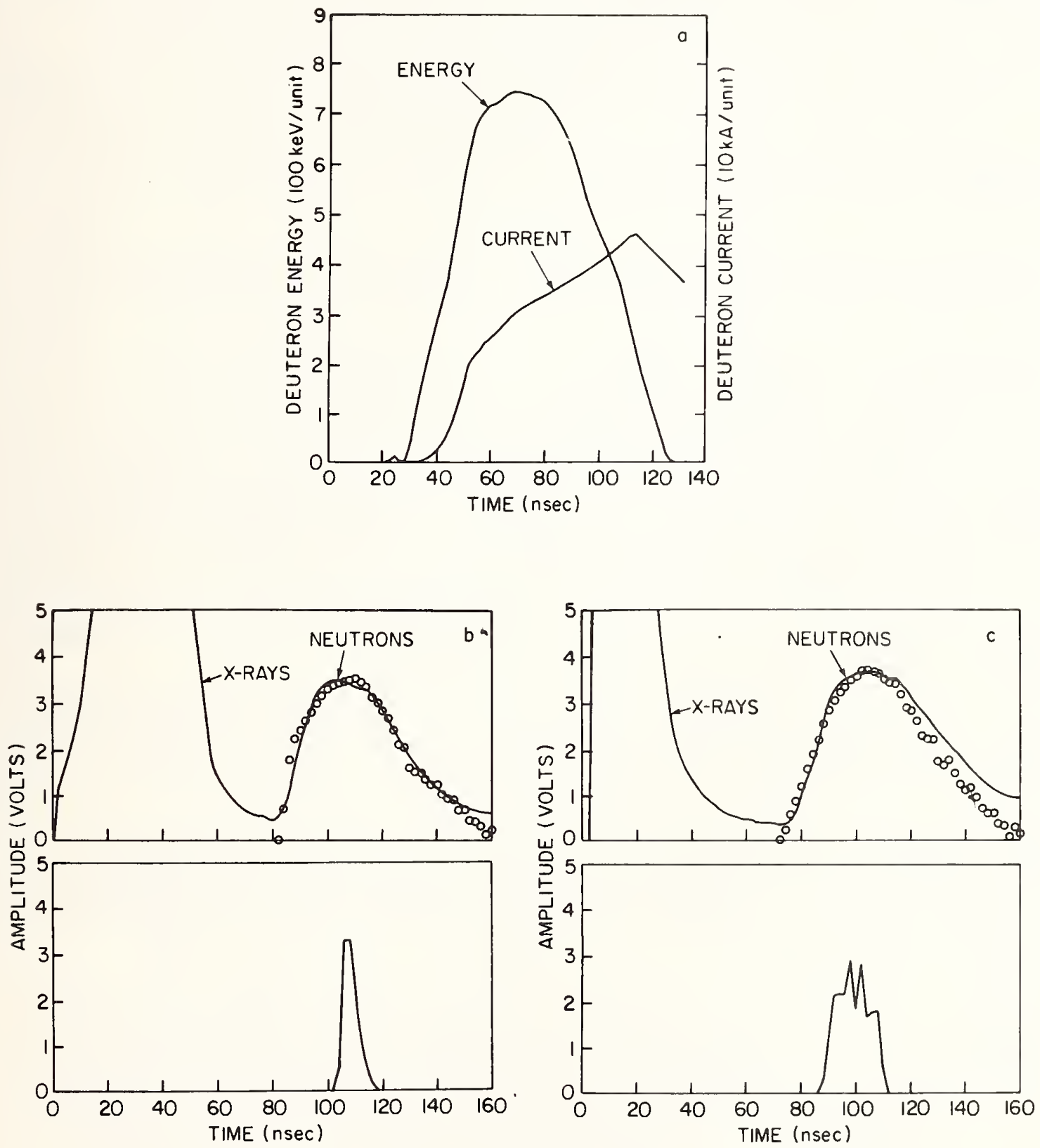

Figure 1. Comparison of neutron TOF traces obtained using a focusing diode geometry with calculations using the energy and current traces displayed in (a). The measured TOF traces (solid curves) are compared with calculated yields (open points) for detectors at (b) $1.91 \mathrm{~m}$ and $15^{\circ}$ and at (c) $2.36 \mathrm{~m}$ and $85^{\circ}$. The results of calculations for a mono-energetic deuteron pulse of 2-ns duration are shown at the bottom of (b) and (c). 
mismatch in impedance. Part of it gets reflected back the same cable and part of it gets transmitted down each of the other five or six cables. When these reflected currents get back to the diode, they get absorbed by the resistor back there. So there is not a multiplicity of reflections that give you sort of a noisy waveform.

Shipman. It looks like the resistor would have to be the $V$ probe to the outer conductor to be determinant.

Shannon. OK. The problem is an error in the viewgraph. The resistor should be from the probe end to ground. (There was an error in figure 5.)

Marco Di Capua (Physics International). I have a question for Frank Young. I remember vaguely from my old days of electron beam focusing that one could use a gamma reaction with a very sharp threshold for calibration of voltage monitors. It was a $1.4 \mathrm{MeV}$ gamma that had a very sharp threshold and you could actually determine whether you had at least 1.4 megavolts or not. You could check the peak voltage against this reaction. I was wondering whether you know of any reaction with a threshold below 2 MV.

Young. The lowest gamma-ray induced threshold I can think of is the photo-disintegration fo the deuteron at $2.2 \mathrm{MeV}$.

Leeper. There is a $\gamma-n$ reaction on $\mathrm{Be}$ at $1.7 \mathrm{MeV}$ and a $\gamma-\mathrm{n}$

reaction on deuterium at $2.2 \mathrm{MeV}$. Then, all the rest of the photonuclear reactions have thresholds above about $6 \mathrm{MeV}$.

Dan Schweickart (Wright-Aeronautical Lab). I have two questionsg. First, for Gene Nolting concerning the attenuation for your high voltage dividers. If I understand you correctly, you are doing all of your attenuation for your high voltage dividers quite close to the output of the generator.

Nolting. That is true. The Physics International probes, which are resistive dividers, are divided down to about 200 volts for about a 4 megavolt signal and then we attenuate those signals further inside the screen room. The capacitive dividers provided by Maxwe11, however, have low voltage outputs and vary from 1 to 10 volts depending upon the particular divider. We typically read them with Tektronix 7A24 scope preamplifier which means that we have to further attenuate down to one volt.

Schweickart. Have you done any work in trying to couple a larger signal voltage into the screen room and then attenuating inside? You may find that this will help to eliminate some of your problems in the phase shift that you were talking about.

Nolting. I have mentioned two phase shifts associated with the diagnostic waveforms which are totally unrelated. First, the problem 
which I spoke of in this discussion session which was due to a poor ground connection. Second, there was the phase shift that I discussed in the presentation which was due to the relative phasing of voltage and current. I believe that you are talking about the connector phase shift problem. Certainly, in general, I would say that one would be wise to bring a larger signal into the screen room and then attenuate it there. However, we have found that the signals have not been very noisy even with the low voltage levels at which we operate. I think that that is attributable to the foam flex cable itself.

Schweickart. One last question concerning the use of the Tektronix probe. I assume that if you are using the standard high voltage probe, your screen room facility is relatively close to the measuring point. Is that correct?

Nolting. Actually not. The Tektronix probe was measured on an osciTToscope that was located next to the machine and the signal that was sent into the screen was read on a Tektronix 7912 transient digitizer. These two waveforms were then compared both in waveshape and in amplitude, but the signal from the Tektronix probe was not transmitted further than its standard $10 \mathrm{ft}$ cable. It would have been nice to run them together and do a point by point analysis with the 7912 , but that was not feasible.

Jack Katzenstein (Physics International). I was wondering if you couldn't eliminate some of the errors in your beam calorimetry measurement by putting the carbon target inside of a "Hohlraum."

Nolting. I guess that I don't understand your term "Hohlraum."

Katzenstein. What you would have would be a hollow space, with a small hole as an inlet which may be one problem because the beam is pretty big.

Nolting. Our beam diameter, without expansion, is only about 2 inches.

Katzenstein. OK. Well then, make the enclosure 6 to 12 inches in diameter and put the target inside of that. Make it of low heat capacity, such that when you measure the temperature rise of the whole thing, you essentially first measure the target absorption plus any energy losses from radiation, or from spalation, or back scattered electrons which get included in the sum.

Nolting. We11, that is an interesting suggestion. As you recall, I discussed the calorimeter loss mechanisms which I expected would limit accuracy and how we tried to correct for these. In low energy operating mode, we did get good agreement with the techniques we used. At full energy, the error.was in the opposite direction anticipated, that is, our calculated values were lower than our 
measured values. So I don't think your suggestion would help in that regard. Our errors appear to be due primarily to current cutoff. The Rogowski coils are in a shielded enclosure with a cavity opening to allow the flux to enter. These are located about 3 centimeters from the actual AK gap. I believe that the gap is being shorted after a certain period of time and therefore the current hangs up.

Shannon. I would like to make a comment on the differential probe vs. the integrating probe. We used differential probes so we could beat this noise problem. You can get into trouble with integrating probes because you are handling low voltage in a rather noisy environment. So we always went the opposite route and brought hundreds of volts into the screen room allowing the integrator to take it down to the volt level. This is great, except you have to be careful that in some cases you can start mixing slow signals with very fast signals. on breakdown, for example, you can generate kilovolt spikes that scopes and other instruments don't like very well. If you are not very careful, your differential measurements can blow things out. But they do have the advantage that they are very much noise immune to problems like leaky connectors.

Jim Fockler (Physics International). A question for Gene Nolting. You attributed the fact that your Rogowski coil had a funny waveshape to clamping at the end due to flashing the gap. How were you able to determine that was the clamping mechanism as opposed to the feed flashing somewhere which would also give you constant current around that monitor?

Nolting. To tell the truth, I cannot distinguish between the two strictly from the waveshapes. However, if the feed were flashing over, and shorting the diode at some point in time, that should short the energy that you are delivering into the diode from the transmission line. Recall that the energy measured by the carbon block was 30 percent or so higher than what we had calculated and there is not enough energy inductively stored in the diode when it shorts to account for this large of a difference.

Jim Sarjeant (Los Alamos). Gene (Nolting), as I understood it a moment or two ago, you were using one of the Tektronix probes as one of your measurement probes. We have had the experience of working with the P6015 probe for several years and have developed a version of it that is capable of operation to $60 \mathrm{kV}$. Our system utilizes a hybrid amplifier that can drive up to $100 \mathrm{~m}$ of cable so that you no longer have to worry about having the device close to your experiment. These modified devices now have a reproducibility of about half a percent and a long-term drift characteristic of 1 to $2 \%$. The only problem with them is the fact that you have a line amplifier device that requires battery power and copper box screening. We have executed a number of intercomparisons between these probes and the probes that John Power has developed and found that the Tektronix probe performs 


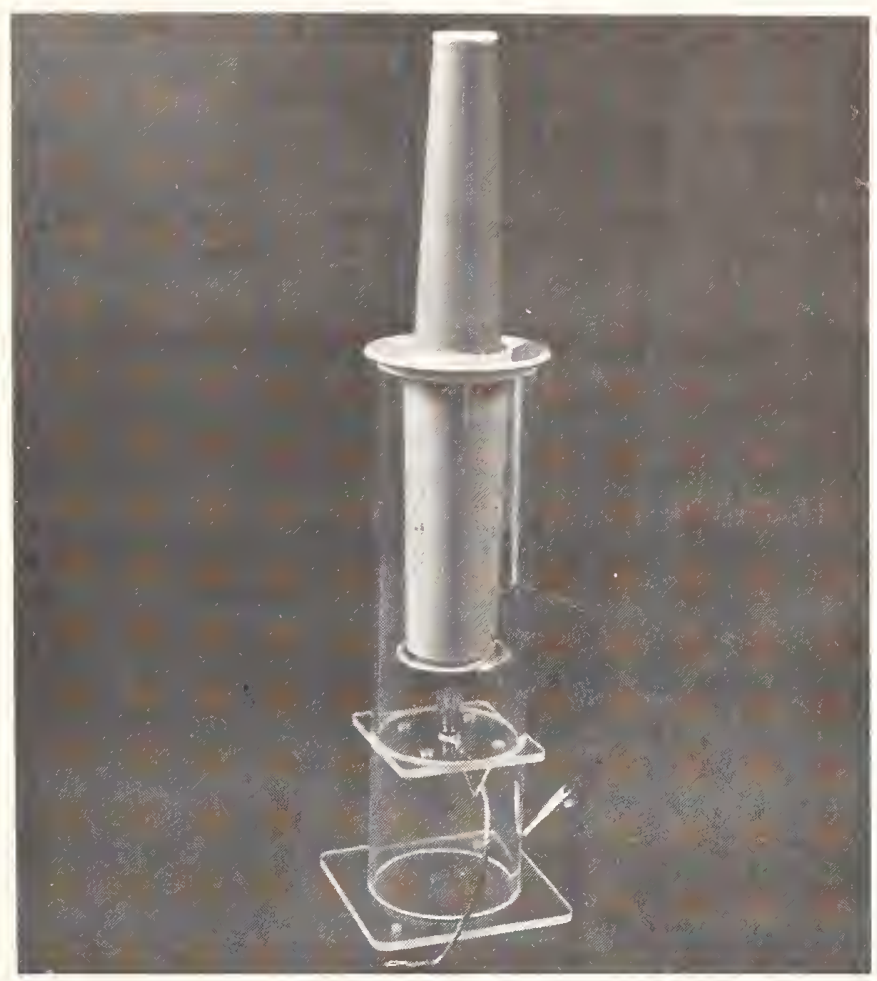

(a)

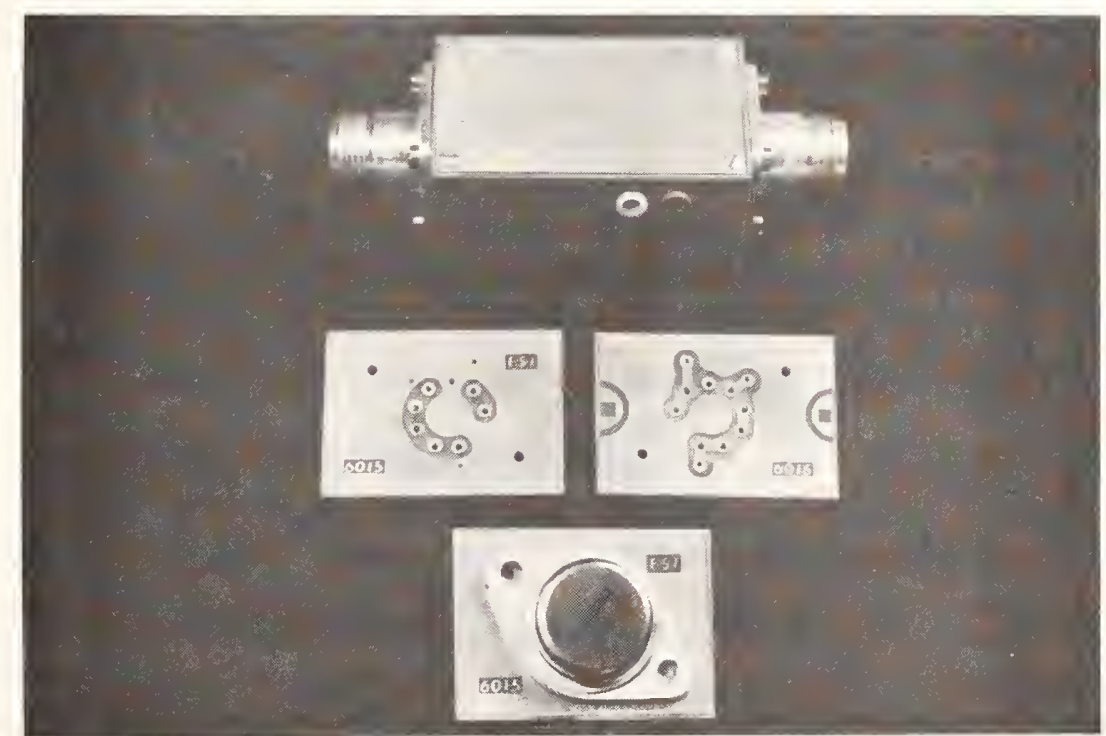

(b)

Figure 2. (a) Modified high voltage probe.

(b) Circuit board and assembled line driver. 
best used nose down (see figure $2 \mathrm{a}$ ).

The 60151 ine driver (see figure 2b) developed at the Los Alamos National Laboratory is specifically designed to be used in conjunction with the Tektronix P6015 high-voltage probe. Due to the short length of the P6015 probe cable and its termination/compensation box, it is necessary to have the diagnostics physically close to the point of measurement. Using the 6015 line driver makes possible the remote location of diagnostics with minimum degradation of response while retaining simultaneous LDC mensuration capability. Typical risetimes of the 60151 ine driver into a 50- $\Omega$ load (measured at the output) are $4 \mathrm{~ns}$. When driving a long length of coaxial cable, say $70 \mathrm{~m}$, the risetime at the cable end measured typically 8 ns.

Connections to the 60151 ine driver are straightforward, but for optimum response characteristics a few items should be mentioned. A special printed circuit board was designed to minimize stray capacitance, component lead length, and thus ringing in the LH0063. The circuit board fits inside a Model 2411 Pomona Box and solders directly to the Type $N$ connectors mounted on the box. Type $N$ connectors are used for best pulse performance. A schematic (see fig. 3a) and parts lists (3b) are attached. Compensation of the P6015 probe and line driver is nontrivial. With a fast risetime signal connected to the P6015 probe, the Tektronix instructions for compensation can be followed except that C2, the 4-15-pF variable capacitor, adjusts only the droop of the pulse, not the flatness. The compensator box on the P6015 probe adjusts the risetime and the flatness of the calibration pulse.

The maximum output of the 60151 ine driver is $10 \mathrm{~V}$. An overall attenuation factor of the $P 6015$ probe and 6015 line driver will be in the range of $10,000-12,000$, and the precise value is determined when compensation is completed.

Ron McKnight (NBS). How much does the modification cost?

Sarjeant. The modifications to the Tektronix P6015 probe (see figure 2 a) are straightforward and involve removing the internal plastic ring and adding a 3/8-inch radius corona ring around the end of the metal shield at the probe nose. The probe is used nose down, placing the high voltage on the bottom, and the grading ring is placed around this nose. The company will make a system with a cable that is $24 \mathrm{ft}$ long. The probe output is sent into a very high speed hybrid operational amplifier, giving about a 10-V output signal with a dynamic range of about 500-1. If anyone is really interested in it, we would be pleased to help.

Mcknight. I have a general question to the audience. In assembling the abstracts and sending them out for evaluation and putting the agenda book together, I was struck by the lack of papers in this 


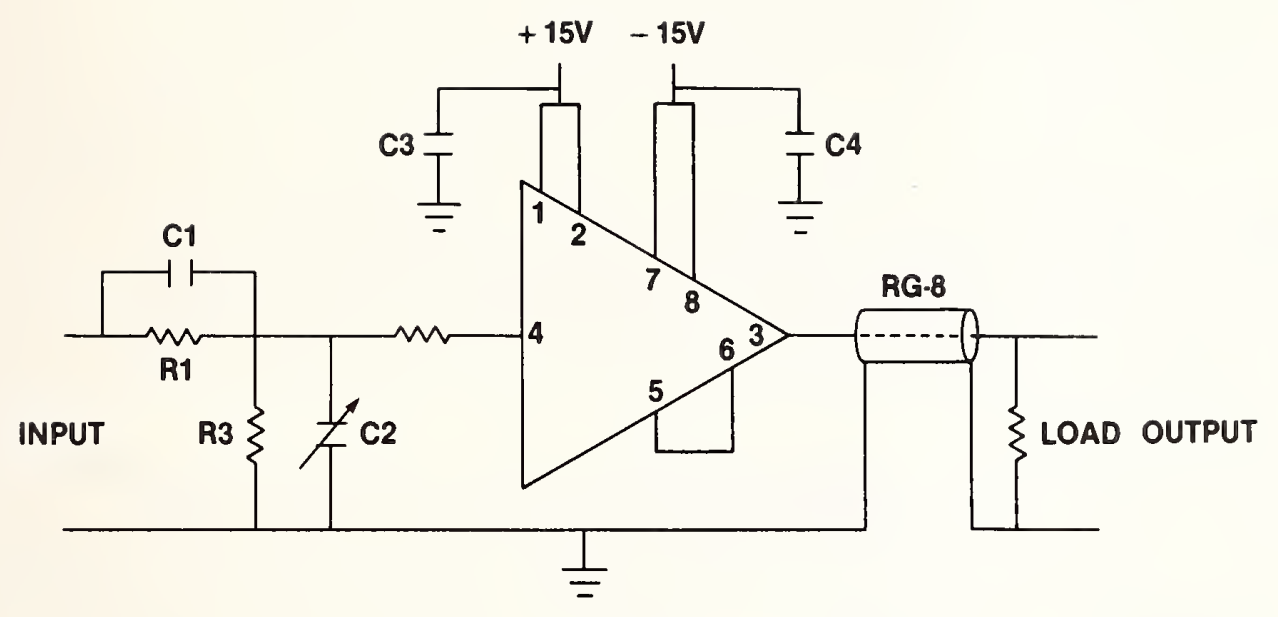

Parts List

C1 $\quad 3$ pfd $50 \mathrm{~V}$ ceramic

C2 4-15 pfd $50 \mathrm{~V}$ ceramic variable

C3,C4 $\quad 4.7 \mu f d \quad 35 \mathrm{~V}$ tantalum

R1 909 kohin carbon

R2 51 ohm carbon

R3 97 kohm carbon

IC National Semiconductor LH0063K

Buffer Amplifier

Special ground plane printed circuit board is recommended to obtain best performance.

Figure 3. Schematic and parts list for high voltage probe line driver. 
general area of power and energy measurements. I was curious why. Is it a matter of because no one is really interested or because it is felt to be trivial or because it is so hard? Is it generally felt that it is good enough to measure V\&I somewhere and that will tell you the-power output? Or is it that you take what you can get?

Di Capua. I think that, in a sense, the difficulties lie within some of the problems that Frank Young mentioned. In these superpower generators, the energy densities are so large that the ability to obtain a total energy measurement before the detector disintegrates is very very limited. Calorimeters blow up, and activated materials get lodged in every corner of the apparatus.

Local power density measurements become difficult because of the magnitude of the electric and magnetic fields associated with high power densities. Voltages except for particle energy measurements cannot be made directly and magnetic field measurements are exceedingly difficult. In addition, non-symmetric power flow limits the accuracy of extrapolation from local to total measurements. 


\title{
Session $C$ - Current Measurements
}

\author{
ROGOWSKI COILS, FLUXMETERS, AND RESISTORS \\ FOR PULSED CURRENT MEASUREMENTS
}

\author{
Marco S. Di Capua \\ Physics International Company \\ 2700 Merced Street \\ San Leandro, CA 94577
}

This paper discusses the frequency response of current sensors for pulsed current measurements and sources of error measurement in these sensors. We examine the operating principles of Rogowski coils and fluxmeters from a lumped parameter as well as a transmission line viewpoint. This second, less conventional approach, is necessary when transit times in the sensor are comparable to the time resolution of the measuring circuit. Our discussion of current viewing resistors (shunts) emphasizes the transient behavior associated with diffusion of magnetic field in the sensor, and the limitations on the sensor imposed by Joule heating.

The choice of measurement method, based on the above analysis, will depend on the frequency, amplitude, distribution of current, as well as on the sensitivity and bandwidth of the recording device.

\section{Introduction}

Fluxmeters [1], Rogowski coils [2], current transformers [3], and current viewing resistors (CVR) [4] have been used extensively in the past [5] to measure currents in pulsed power systems and surge currents in electric distribution systems. The purpose of this paper is to:

(1) describe these devices; (2) summarize their theory of operation;

(3) explore sources of measurement error; (4) indicate design features which minimize error; (5) describe the methods of calibration; and (6) display some representative measurements. The limited space allotted to this task requires that I rely on published literature for many of the detailed derivations.

\section{Field Coupled Current Sensors}

A field coupled current sensor utilizes the magnitude of either currents or voltages induced in the sensor as a measurement of the pri- 
mary current. Depending on the geometry of the windings, a field coupled sensor may be a fluxmeter, a current transformer, or a Rogowski coil.

A fluxmeter is simply a single or multiple turn loop. The flux linked by the loop is $\phi=N \int \vec{B} \cdot d \vec{A}$, where $N$ is the number of turns. When the field is homogenous over a scale comparable to the dimensions of the loop, then the flux linked by the loop is a measure of the local magnetic field normal to the loop. To measure a current with such a loop we require either (1) an a priori knowledge of the current density distribution or, (2) a distribution for calibration, which is identical to the distribution being measured. Despite these limitations, the fluxmeter is still a very useful device for pulsed power measurements.

A high permeability core in a current transformer couples the magnetic flux of the primary current $I_{p}$ into the current sensing (secondary) windings. The electrical measurement literature treats such transformers thoroughly [6].

The Rogowski coil is a current transformer of deceptively simple geometry. The shape of the curve, c (formed by the coil) and the current distribution within it do not affect measurement when simple conditions are met. The flux linked by the coil is

$$
\phi=\int_{C} \int_{A} n(\vec{B} \cdot d \vec{A}) d \ell
$$

where $\mathrm{n}$ is the number of turns per unit length, $\vec{B}$ is the local magnetic field, $d \vec{A}$ is the area element of the turn, and $d \ell$ is the element of length along the coil. This equation becomes

$$
\phi=\mathrm{nA} \underset{\mathrm{C}}{\int \vec{B}} \cdot \overrightarrow{\mathrm{d} l}
$$

under the following assumptions: (1) that the cross section $A$ of the coil is everywhere the same; (2) that the number of turns per unit length $\mathrm{n}$ is constant; ( 3 ) that the $\vec{B}$ field is homogeneous over a scale comparable with the dimension of a turn; (4) that the individual areas $A$ of each turn are oriented perpendicular to the curve described by the coil. Hence, when $c$ is a closed curve and when $c$ itself links no flux, the flux linked by the windings of the sensor is $\phi=n A \mu I$ enclosed independently of $\mathrm{c}$ and the distribution of current density within it. Thus, the enormous advantage of this geometry is due to its transportable calibration. An example of a Rogowski coil design appears in Figure 1. The purpose of the housing is to guarantee that c encloses no flux.

Rogowski coils in general have air or plastic cores such that $\mu=\mu_{0}^{\prime}$ the permeability of free space. The permeability of core materials drops with increasing frequencies [3], and for high frequency applications an air core is adequate. 


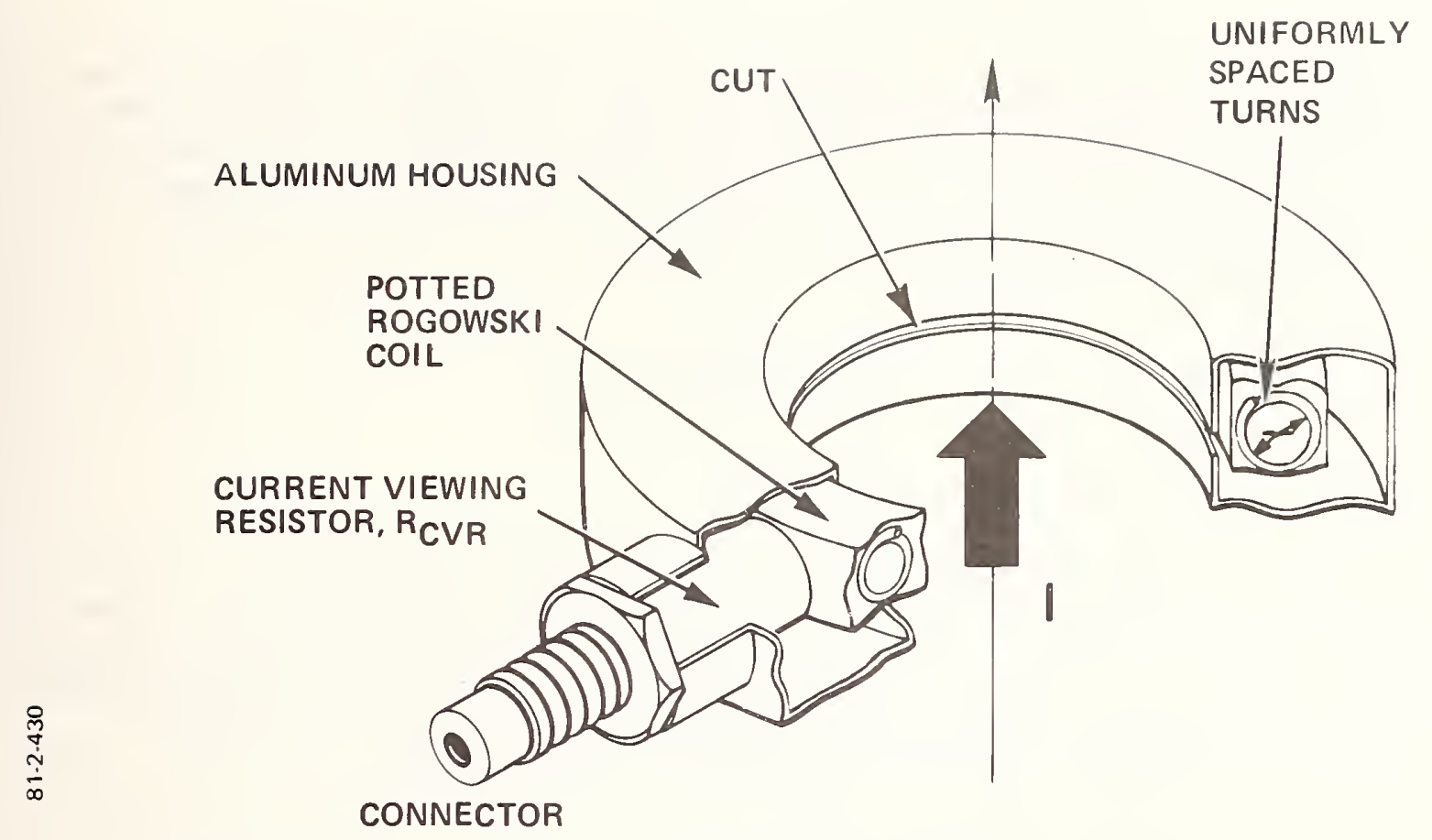

Figure 1. A typical Rogowski coil. Aluminum housing shortcircuits major turn. Current viewing resistor is built into output connector. Housing is cut to allow flux to penetrate. Turns are uniformly spaced

\section{1 Lumped Parameter Model}

A lumped parameter approach $[7,8]$ can be used to describe both types of sensors when the transit time in the sensors is small compared to the resolution time of the metering circuit. Figure 2 shows the

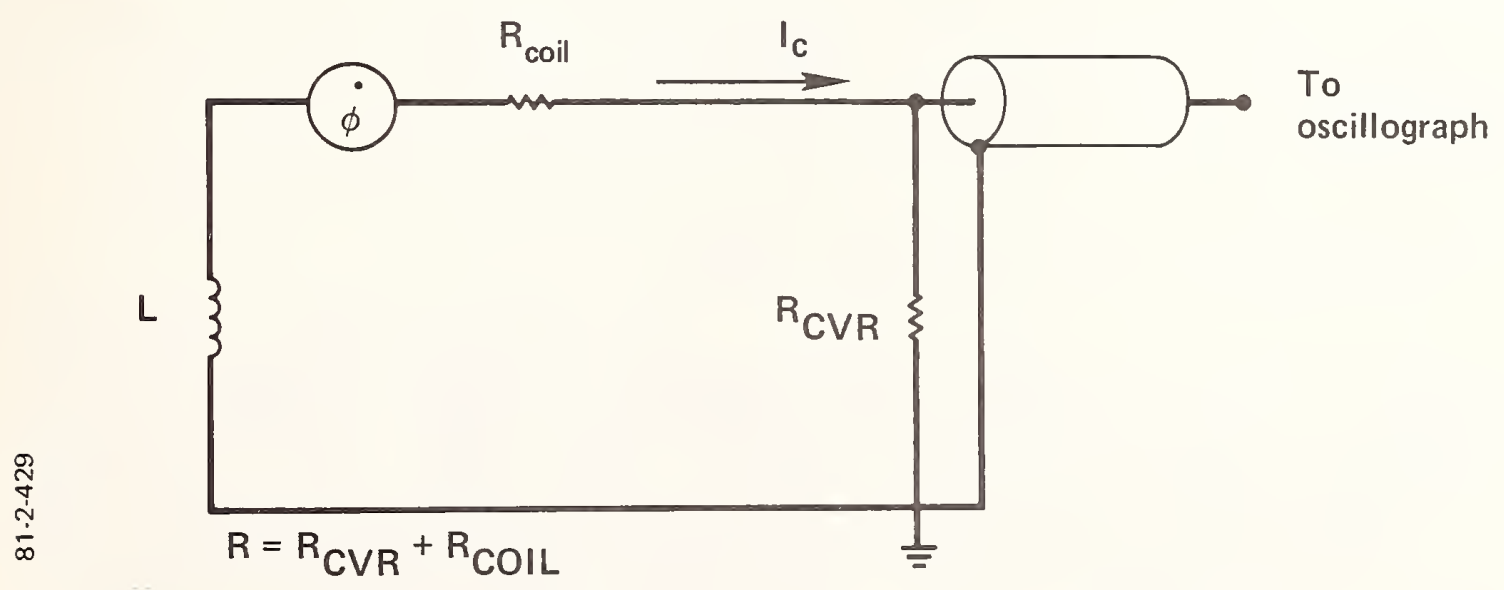

Figure 2. Equivalent circuit for field-coupled current sensor. Sensor differentiates when $t_{C} \gg L^{-1}$ and integrates when $t_{C} \ll L_{R}^{-1}$ 
circuit diagram of a field coupled sensor. Assuming no relative motion between the current density distribution and the sensor, $\phi$ is the time rate of change of flux, linked by the sensor, due to changes of current in the primary circuit. $R=R_{C}+R_{C V R}$ is the sum of coil resistance $R_{C}$ and metering resistor $R_{C V R}{ }^{*} L$ is the self-inductance of the sensor.

For this circuit,

$$
\frac{1}{R} \frac{d \phi}{d t}=\frac{L}{R} \frac{d I_{C}}{d t}+I_{C}
$$

where $I_{C}$ is the current in the sensor.

The three terms in the equation suggest two time domains. In the first one the characteristic time $t_{C}$ for changes in the primary current is $t_{C} \gg L / R$. In this domain, $L / R \mathrm{dI}_{C} / d t<<I_{C}$ and the current in the sensor is $I_{C} \simeq 1 / R \mathrm{~d} \phi / d t$. In this domain, the sensor is differentiating one since its output $U=I_{C} R$ is equal to the $\phi$ arising from current changes in the primary circuit. The other time domain is $t_{C} \ll L / R$. In this domain, $\mathrm{L} / \mathrm{R}$ dI $I_{C} / \mathrm{d} t>I_{C}$. The current in the sensor is $I_{C} \approx \phi I^{-1}=$ ${ }^{I} p^{N}$ and the output voltage is $U=R_{C V R} I_{N^{N}}{ }^{-1}$ when $I_{p}$ is the primary
current and $N$ is the number of turns. This self-integrating sensor is preferable to the differentiating one for reasons which will become clear when we consider transit time effects in the coil itself. The design of the self-integrating Rogowski coil is discussed in detail in [7]. (The use of ferromagnetic cores in Rogowski geometry coils is discussed by Anderson [3] •)

\subsection{Transmission Line Model}

The lumped parameter circuit description of the field coupled current sensor is no longer adequate when the transit time in the sensor is longer than the resolution time of the metering circuit. Large, fast risetime pulsed power systems and the improved time resolution of metering circuits often require transmission line treatment of the field coupled sensor (see Figure 3 ). The voltages induced at different locations along the sensor reach the metering impedance with different delays. Transmission line treatment assures that transit time effects are properly accounted for. In a Rogowski geometry, the two ends of a coil are joined so the coil forms a torus.

In $1963 \mathrm{~J}$. Cooper [9] developed a transmission line model, shown in Figure 4, for field coupled current sensors. His model includes the distributed capacitance per unit length between the coil and the electrostatic shield, the distributed inductance per unit length, and the distributed excitation voltage $u(x)$ per unit length along the coil. He applies a Laplace transform technique to solve the partial differential equations that model the coil. The boundary conditions, which may be applied to the sensor, include short circuit, resistive, inductive, and capacitive terminations at the ends of the coil. 


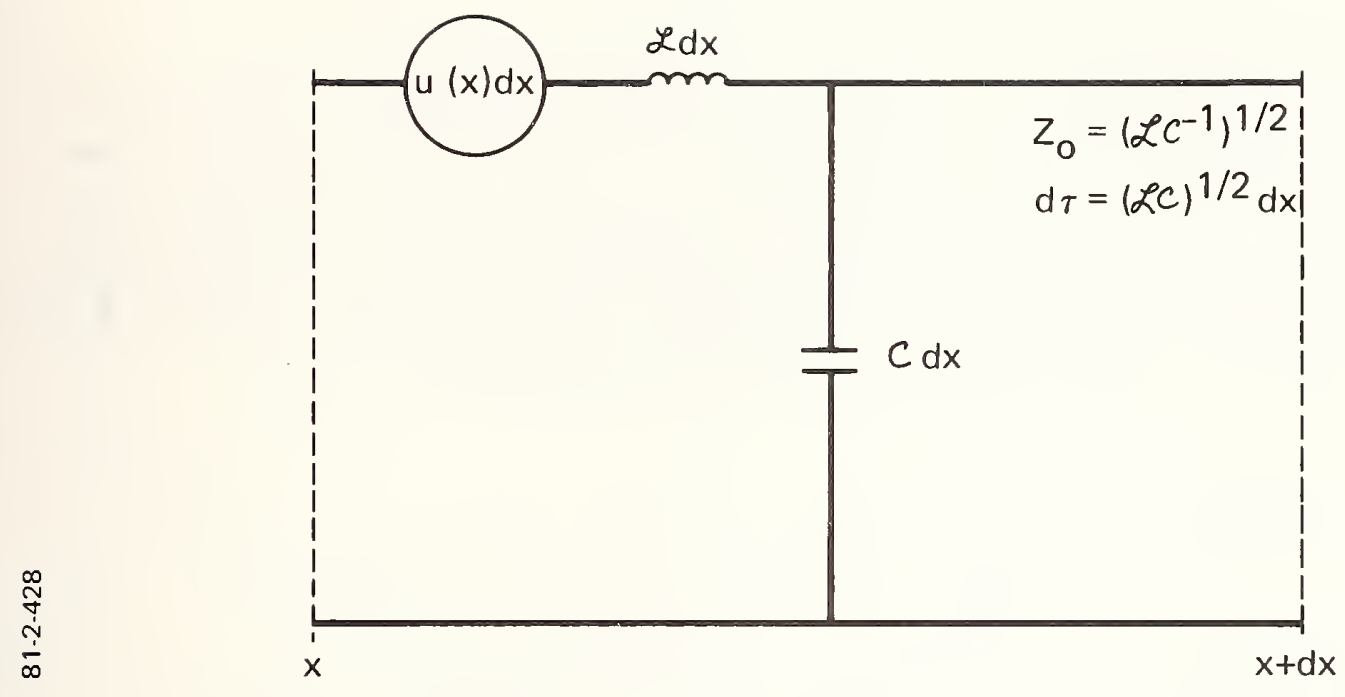

Figure 3. Differential section of transmission line geometry current sensor $(u(x)$ is excitation voltage per unit length)

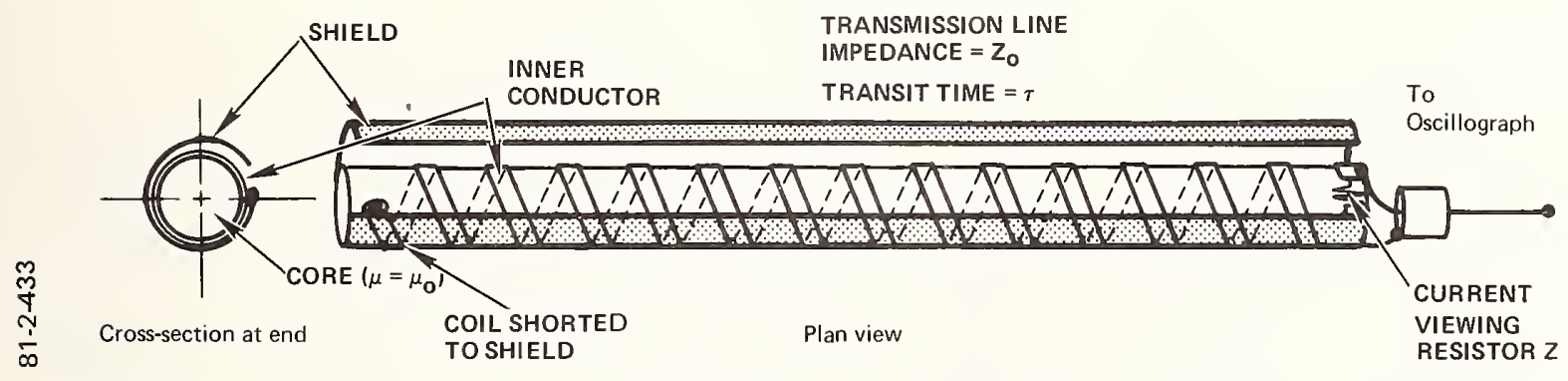

Figure 4. Transmission line geometry current sensor. Gap in shield allows flux to penetrate coil. Wave impedance of coil is $z_{0}$ and its one-way transit time is $\tau$. Coil is terminated with current viewing resistor $z$ at one end and shorted at other end. coil forms closed curve for Rogowski geometry

We assume a delta function excitation to illustrate the effect of transit time upon the sensor output. We assume $I_{p}=\delta(t-0) I$ such that the primary current excitation is $I_{p}=0, t<0 ; I_{p}=I, t>0$. The excitation of the sensor is uniform, such that $u(x)$ is a constant in the Rogowski geometry sensor. The transmission-line-like sensor of impedance $z_{0}$, one-way transit time $\tau$, length $\ell$, with total number of turns $N$, is terminated by a short circuit at one end and with a metering impedance $Z$ at the other end. We measure the coil output as a voltage across $\mathrm{z}$. The $\delta$ function input and output for terminations $\mathrm{z} \gg \mathrm{z}_{0}$ ' $\mathrm{Z}=\mathrm{z}_{\mathrm{o}}$ and $\mathrm{z} \ll \mathrm{z}_{0^{\prime}}$ appear in Figure 5. Cases $(\mathrm{a})$ and $(\mathrm{b})$ are the transmission line model of the differentiating sensor. Indeed, for a differentiating coil $\int U_{\text {coil }} d t=\phi=\mu_{0} \operatorname{In} A$. Assuming no flux leakage between the coil and the primary circuit $I=\mu_{\mathrm{O}}$ AnN so that $\phi=\mathrm{ILN}^{-1}$. 
We integrate the waveforms (a) and (b) of Figure 5 and using $\ell(\mathscr{L} C)^{1 / 2}=\tau$ and $\left(\mathcal{L C}^{-1}\right)^{1 / 2}=z_{\text {o }}$ we obtain the waveforms $(a)$ and $(b)$ of Figure 6. The mean value of $\int_{\text {Coil }} d t=I_{I N}{ }^{-1}$ for $z \gg z_{0}$ and the final value $I_{L N}^{-1}$ for $z=z_{0}$ for $t \gg{ }^{2}$ agree with the lumped parameter description. The sensor has, however, introduced a time structure that did not exist in the current waveform under measurement. (The output of a differentiating transmission line sensor is illustrated in Figure 5 of our contribution to Diagnostics for Fusion Experiments [10].

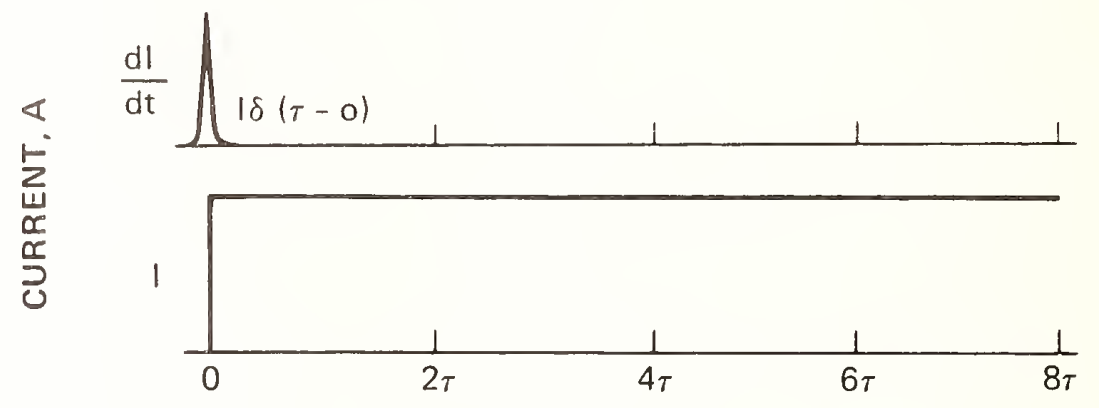

TIME $\tau, \mathrm{s}$

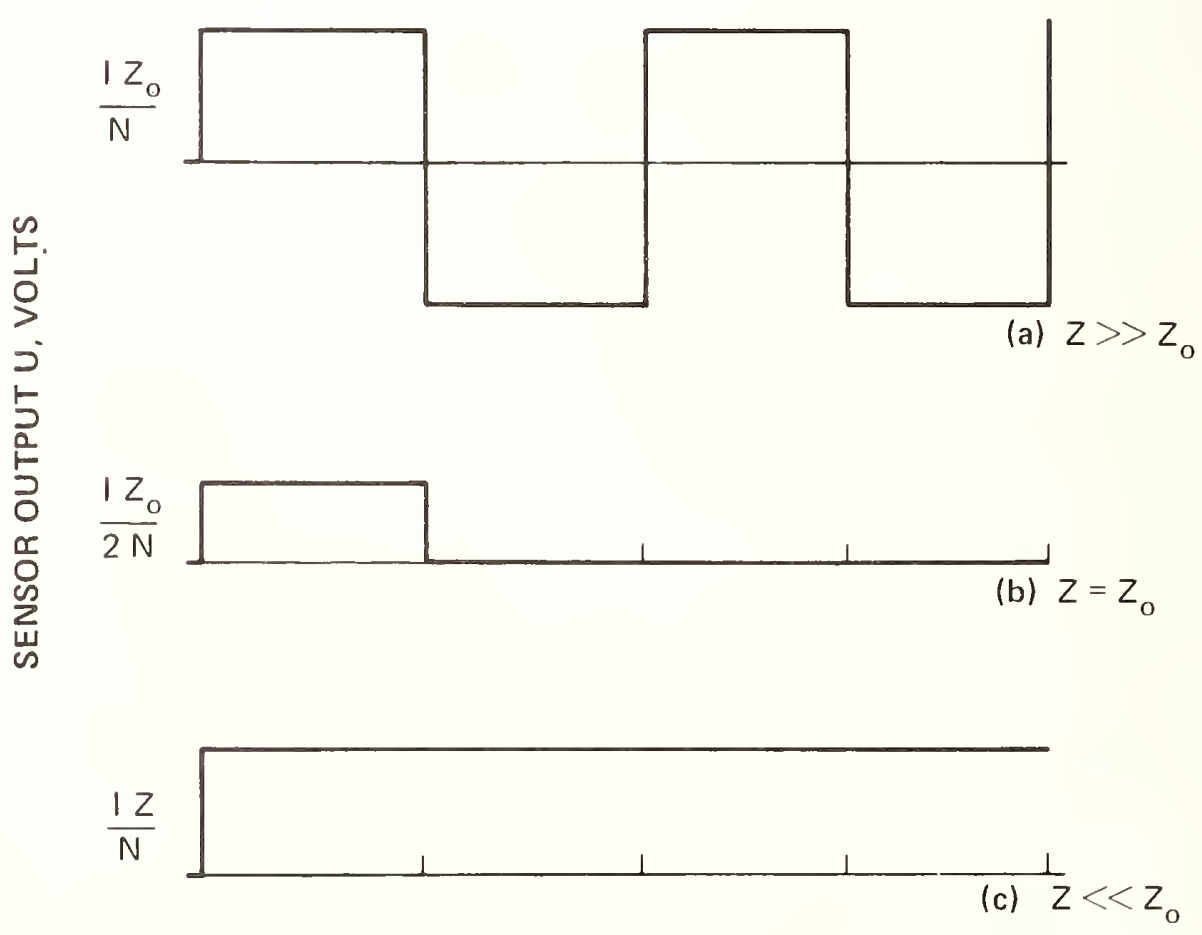

Figure 5. Response of transmission line coil to step input. Waveforms (a), (b) and (c) correspond to a current viewing resistor impedance $\mathrm{z} \gg \mathrm{z}_{0}, \mathrm{z}=\mathrm{z}_{\mathrm{O}}$, and $\mathrm{z} \ll \mathrm{z}_{\mathrm{o}}$ respectively 


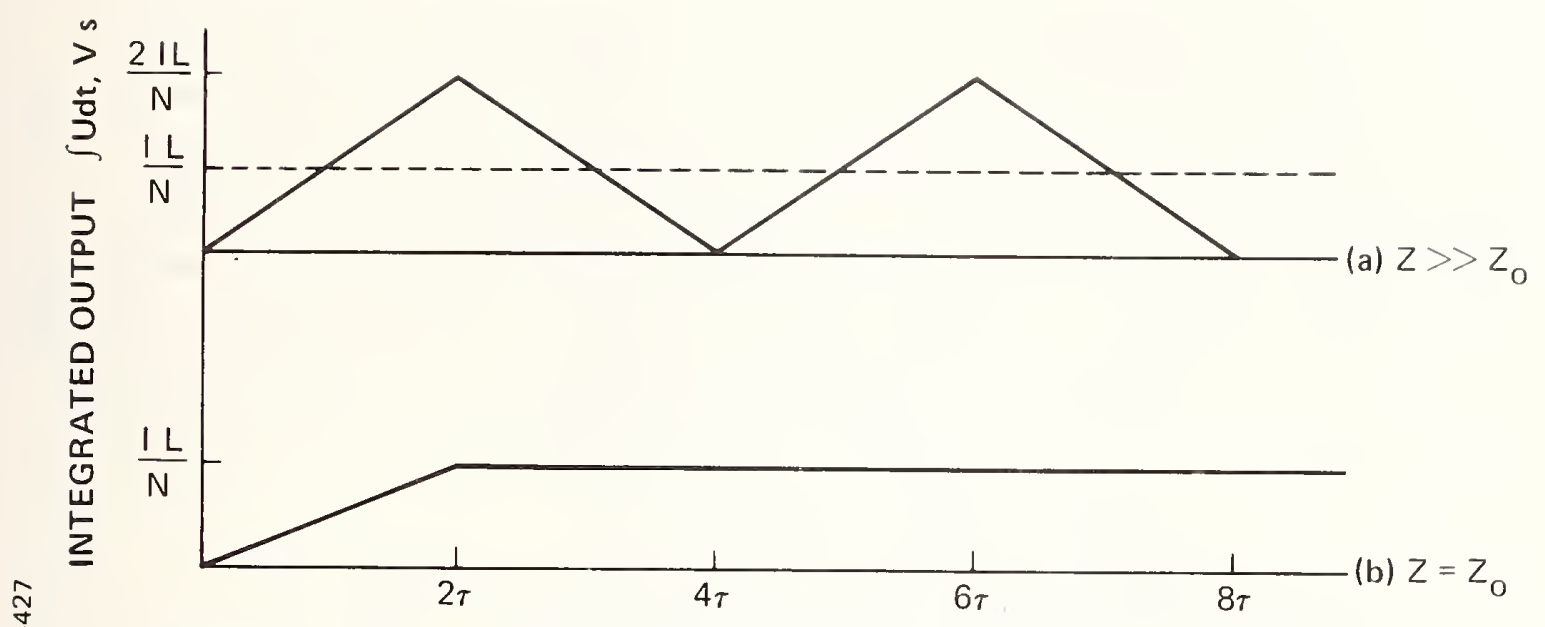

Figure 6. Integral of transmission line sensor waveforms. Waveforms (a) and (b) correspond to (a) and (b) of Figure $5\left(z \gg z_{0}\right.$ and $\left.z=z_{0}\right)$. Response for $t \gg \tau$ agrees with lumped parameter description ( $L=$ lL $\tau=\sqrt{\mathscr{2 C})}$

The advantages of the self-integrating sensor are clear when we observe waveform (c) of Figure 5. This waveform exactly reproduces the primary current, and the frequency components of its output are no higher than the components of the primary current. Indeed, it is possible to construct a subnanosecond risetime sensor whose physical size results in $\tau \geqslant 10 \mathrm{~ns}$ transit time. Another advantage of this sensor is that its output is insensitive to electron input or photon induced currents. In constructing such a sensor, it is critical that the turns are wound uniformly, that the major turn is shorted with the electrostatic shield, and a sturdy, nonreactive current viewing resistor built. Solutions to these and other technical details appear in the literature $[6,11]$. Sample waveforms from a large, self-integrating Rogowski coil, which demonstrate fast risetime, are illustrated by Pellinen [7].

\subsection{Sources of Error in Field Coupled Current Sensors}

The low frequency cut-off of a self-integrating current sensor is produced by penetration of magnetic flux into the "core" of the coil. This penetration is characterized by a time constant $\tau_{d}=L R^{-1}$. While a high permeability core will increase this time constant, the risk of saturation is always present in a coil with such a core. Therefore, an unknown current should not be measured with a ferromagnetic core coil whose ampere-second rating is unknown.

The time varying resistance of the winding due to skin effects and to heating the current viewing resistor may introduce some error in the determination of $\tau_{d}$. This quantity is required for a numerical compensation of the decay obtained with Equation (9). 
Nonuniform excitation $(u=u(x, t))$ is the Achilles' heel of the self-integrating Rogowski coil. It causes severe oscillations in the output whose period is the two-way transit time in the coil and which damp very slowly due to the high $Q$ of the sensor. When a measurement of total current is required, a terminated differentiating sensor (Figures $5 \mathrm{~b}$ and $6 \mathrm{~b}$ ) is preferable even though the risetime information is lost. This information can always be retrieved from a smaller selfintegrating fluxmeter. In practice, we measure current in large devices $(r>0.2 \mathrm{~m}$ ) with three or four sensors positioned symmetrically in the same axial location. This also measures the degree of symmetry in the current distribution. Damping resistors can also reduce oscillation [3].

Another limitation and source of error is the temperature rise due to power dissipated in $R_{C V R}$. The risetime in this resistor is $\tau=0.25 \mu \rho^{-1} \delta^{2}=0.25 t_{C}^{\prime}$, where $\rho$ is the resistivity and $\delta$ is the thickness of the CVR material. The temperature rise $\Delta T$ of the CVR is

$$
C_{p} D \Delta T=0.25 \mu \rho \frac{t}{t_{c}}\left(\frac{I_{0}}{N w}\right)^{2}
$$

$D$ is the density of the CVR material, and $w$ is its width.

We conclude this discussion of field coupled sensors with a brief overview of differentiating sensors. In general, they are easy to build and provide a high output voltage per given area. Their disadvantage is the large bandwidth their data acquisition requires (short cable runs), and their susceptibility to arcing due to fast transients. Trivelpiece [12] describes a numerical technique for compensating cable aberrations, while McDaniel discusses fast-transient limitations [13]. The high frequency response of these sensors is governed by $L R^{-1}$, which must be smaller than the timescale characterizing the current being measured. Since the pulse begins at $t=0$, there is always an interval in which $t<L R^{-1}$. During this interval, a differentiating monitor delivers the integral of the waveform. The task of the designer, therefore, is to minimize the interval. Random errors in measurement with these devices are caused by stray charges, photon-induced currents, and capacitive coupling.

The voltage drop developed at the junction between two flanges is a source of systematic error when differentiating sensors are mounted at such a location. One example of this application appears in Figure 7 $[14,15]$. The voltage drop is added to and integrated with the signal of the sensor. If the sensor is a groove of area, $A$ at a radius, $a$ and the contact resistance between the flanges is $R$, then the error, $\Delta$ is

$$
\Delta=\frac{\phi}{I_{\text {contact }}}=\frac{2 \pi \mathrm{Rt}}{\mu_{\mathrm{O}} \mathrm{A}}
$$



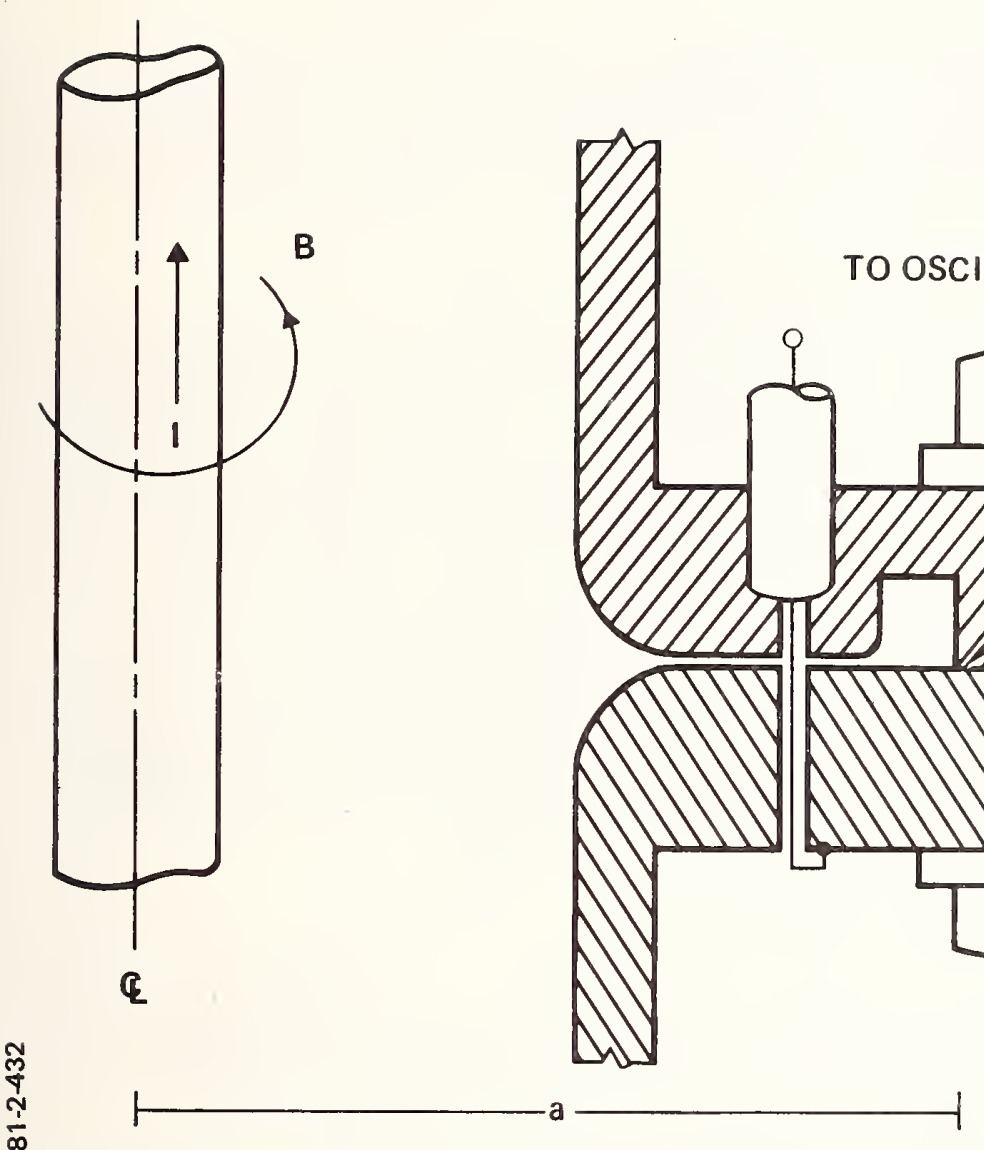

Figure 7. Flange mounted differentiating fluxmeter

For the sensor shown in the figure $\left(a=10^{-1} \mathrm{~m}, t=10^{-6} \mathrm{~s}, A=0.6 \mathrm{x}\right.$ $\left.10^{-4} \mathrm{~m}^{2}\right), R<10^{-5} \Omega$ for $\Delta<0.05$.

The finite resistivity of the loop material is another source of error in this arrangement. This source is present even when the loop is simply a groove. Error is introduced by a voltage drop along the loop due to the primary current, and a change in loop area caused by the penetration of the magretic field in the loop material.

The error due to the voltage drop in the loop material is

$$
\Delta=\frac{\phi}{I_{\text {loop }}}=\frac{\delta}{b}
$$

where $\delta=2 \sqrt{t \mu^{-1} \rho}$, and $b$ is the side dimension of a square groove. We obtain the above result by assuming that current penetrates a depth $\delta$ at time $t$. Lastly, the error due to the increase in area of the loop is

$$
\Delta=\frac{\Delta \mathrm{A}}{\mathrm{A}}=\frac{4 \delta}{\mathrm{b}}
$$


These three additive errors, which become more pronounced for longer pulses, are larger for more resistive materials. The last two errors are considered in more detail by Chu [16], who also presents a technique for their numerical correction.

\section{Current Viewing Resistors (CVRs)}

The widespread use of flux-coupled current sensors has been justified by their ruggedness, reliability, accuracy, and excellent frequency response. Recently, however, the application of these devices to magnetically insulated vacuum transmission lines (MITL) has become somewhat limited. The large electrical stresses associated with high power densities and fast risetimes, and the presence of space charge clouds in the gaps, break down either the sensor or its shielding groove. Moreover, the groove introduces a substantial inductance penalty in accelerators with a large rate of current rise. These difficulties have led to the development of alternate current measuring techniques, such as the CVRs described in this section or piezoelectric transducers developed at other laboratories [17].

\section{1 Modeling a CVR}

The frequency response of a CVR is limited by: (1) diffusion of the magnetic field in the CVR material; (2) the insertion inductance of the CVR in the circuit; and (3) the transit time of electromagnetic waves in the CVR itself. Magnetic diffusion is the most important limitation; thin CVRs of small dimensions have little insertion inductance. A small dimension also circumvents the problem of electromagnetic wave transit time.

The time response of a CVR has been treated in detail in the literature [4]. Consider a tubular CVR with internal radius $r$, external radius $a$, length $\ell$, CVR material thickness $r-a=\delta$, resistivity $\rho$, permeability $\mu$, and ratio of radii $\varepsilon=\mathrm{ra}^{-1}$. We introduce a characteristic time $t_{C}=\mu \rho^{-1} \delta^{2}$, characteristic voltage $U_{C}=I_{p} \rho \ell(2 \pi a \delta)^{-1}$ where $I_{p}$ is the peak current in the circuit. We further assume a CVR with a thickness $\sigma \ll a$. Then the output voltage $U$ of such a CVR to a step function input of magnitude $I_{p}$ is approximately

$$
U^{*}=2\left(\pi t^{*}\right)^{-1 / 2} e^{-\frac{1}{4 t^{*}}}
$$

where $t^{*}=t t_{C}^{-1}=t \rho\left(\mu \delta^{2}\right)^{-1}$ and $U^{*}=U U_{C}^{-1}=2 U \pi a \delta(I p \rho l)^{-1}$. Equation 8 describes a CVR response that is indistinguishable from the exact solution in the interval $0<U^{*}<0.95$. Equation 8 appears as the solid line in Figure 8. 


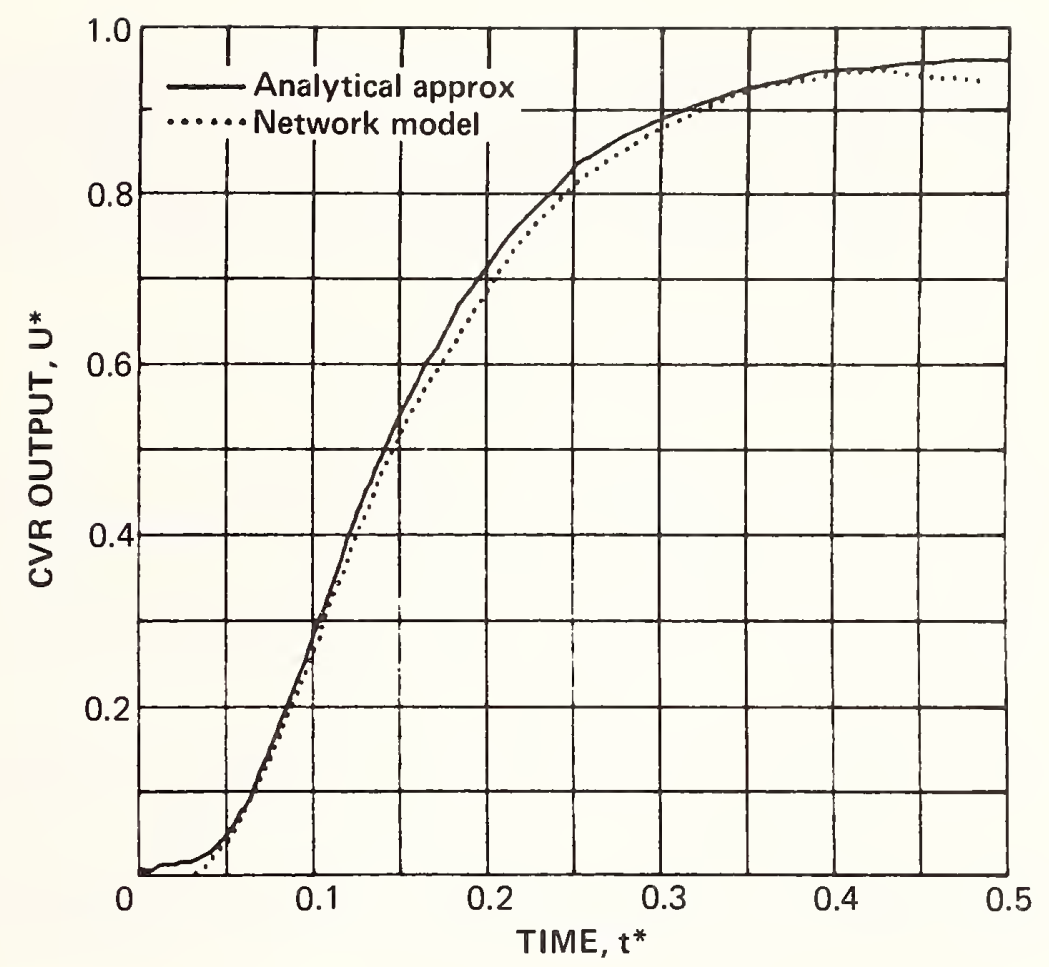

Figure 8. CVR output as a function of time for a step current input. Voltage and time are nondimensional

The diffusion process may also be modeled with an equivalent network of inductors and resistors [18]. The circuit diagram of such a 10-section network appears in Figure 9. The inductance of each section $L_{n}$ is normalized with respect to the total inductance of the foil: $L=$ $\mu \ell \delta w^{-1}$ such that $L_{n}{ }^{*}=0.1=L_{n} L^{-1}$ where $L_{n}$ is the thickness of each section and $w=2 \pi a$. The resistance of each section is normalized with respect to the total resistance of the foil: $R=\rho \ell(w \delta)^{-1}$ such that $R^{*}=10=L L_{n}^{-1}$. The time is normalized with respect to $t_{C}=L R^{-1}=$ $\mu \delta^{2} p^{-1}$. As before, the output is normalized with respect to $U_{C}$.

0.1

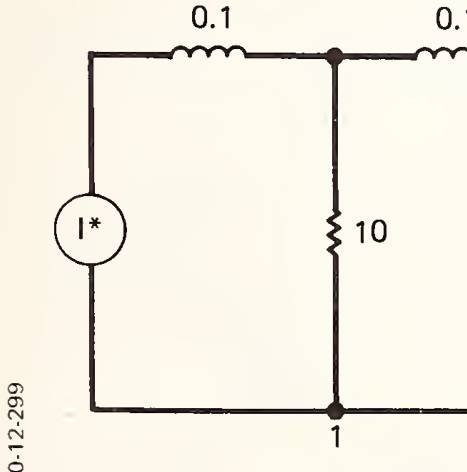

0.1

0.1

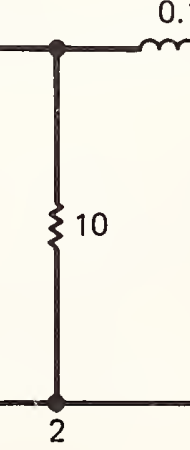

0.1

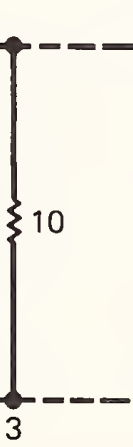

0.1

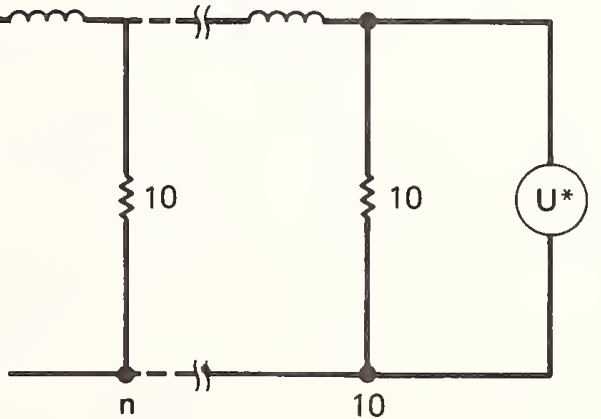

Figure 9. Equivalent network model for current diffusion. The input current is $I^{*}=I_{p}{ }^{-1}$; the output voltage is $U^{*}$ 
The result of a simulation using a current waveform with a risetime of $5.2 \times 10^{-2} t_{C}\left(t^{*}=5.2 \times 10^{-2}\right)$ appears as the dotted waveform in Figure 8. This simulation waveform is indistinguishable from the analytic approximation represented by Equation (1). Both the analytic approximation and the circuit analysis simulation show a 10 to 90 percent risetime $t^{*}=0.25$.

\subsection{Sources of Measurement Error}

There are several techniques to compensate for the diffusion of current in the CVR. One technique $[4,19]$ relies on a loop that links some of the flux within the resistive material. Another taps the output midway in the resistive material [20] by using a shunt made with two parallel foils. A third technique relies on compensation with a passive network $[18,21]$.

To reduce error, the CVR should be a four-terminal network so that the metering contacts are independent of the current-carrying contacts. We also require a sturdy support for the foil. Figure 10 schematically illustrates a CVR construction that accomplishes both objectives. The foil (a) is stretched over a metal yoke (b). The metering contacts are connected to the foil at (c).

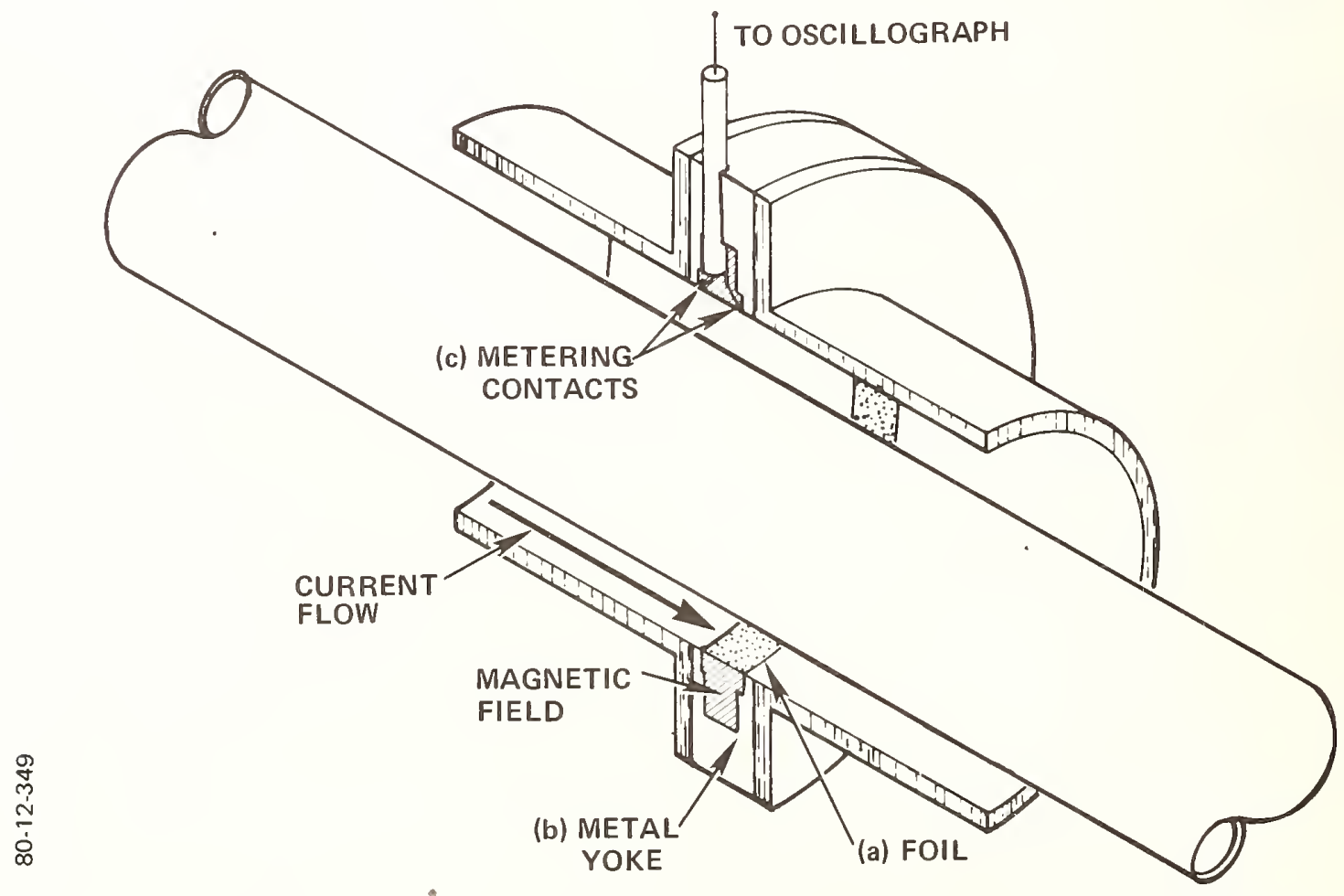

Figure 10. Cross-section of CVR. Foil (a) is stretched across metal yoke (b); metering contacts are at (c). Current flows in direction of arrows 
The yoke carries the current for pulse durations, which are long compared to $t_{d}=L_{y} R^{-1}$, where $I_{y}$ is the inductance of the region between the foil and the yoke, which is crosshatched in Figure 10. A decay time of $t_{d}=10^{-6} \mathrm{~s}$ is easily obtained since foil resistances are typically $10^{-3} \mathrm{~d}_{\Omega}$, and inductances of $10^{-9} \mathrm{H}$ result from reasonable geometries. The decay associated with $t_{d}$ is compensated with the algorithm

$$
u_{m}=U+\frac{1}{t_{d}} \int_{0}^{t} u d t
$$

where $U_{m}$ is the compensated value of the measurement.

There are three other possible sources of error when measuring currents with CVRs. The first source is spurious signals that arise from the direct coupling of the measuring instrument (oscilloscope or digitizer) and the circuit carrying the current. We reduce the error by introducing either a decoupling transformer or a large inductance between the CVR and the oscillograph.

The second source of error is the time-varying flux coupled in the connection to the CVR. The coupling arises from the CVR construction illustrated in Figure 10. It is easy to show that the ratio of resistive voltage, $U_{C}$ to inductive voltage $\phi_{\mathrm{m}}$ is:

$$
\frac{\mathrm{U}_{\mathrm{C}}}{\dot{\phi}_{\mathrm{m}}}=\frac{\mathrm{A}}{\mathrm{A}_{\mathrm{m}}}
$$

where $A_{y}$ is the area between the yoke and the foil, and $A_{m}$ is the area subtended by the metering connections. Therefore, we must construct the CVR, minimizing the area subtended by the connections to the foil. A Park shunt minimizes this error [22].

Joule heating of the foil is the third source of error. This affects the CVR response by changing foil resistance during the pulse. Heating takes place in two phases. In the first phase, heating occurs in the foil as the magnetic field diffuses in the conductor. In the second phase, the current has fully diffused and heating takes place throughout the foil.

We now discuss foil heating during the diffusion phase. Assume a step current $I_{p}$ impressed on the foil at time $t=0.0$ The penetration of the current in the foil at time $t$ is $\delta(t)=\left(2 \rho t \mu^{-1}\right)^{1 / 2}$. The factor 2 arises from full penetration at $t t_{C}{ }^{-1}=t * / 2$. The resistance of the foil is $R(t)=\rho \ell(w \delta)^{-1}$. The energy input at time $t$ is

$$
E(t)=I_{p}^{2} \int_{0}^{t} R(t) d t=2 I_{0}^{2} \frac{\rho l}{w} \sqrt{\frac{\mu}{2 \rho}} t^{1 / 2} .
$$


This heat input occurs in a volume $V=\delta l w$. Equating the thermal energy of the foil with the energy dissipated in it, we obtain

$$
C_{p} D \Delta T=\mu_{0} I_{0}^{2} w^{-2}=2 B^{2} / 2 \mu_{0}
$$

where $\Delta T$ is the temperature rise of the foil, $B=\mu_{0} I^{-1}$, and $D$ is the density of the material. This result is well known and originated from work with pulsed magnetic fields [23]. It was found during the diffusion phase that the thermal energy per unit volume of the material is equal to the energy density of the magnetic field.

Once current diffusion is complete, the thickness of the foil carrying the current is constant. A parallel analysis to the one above yields

$$
C_{p} D \Delta t=\frac{2 t}{t_{c}} \frac{B^{2}}{2 \mu_{0}} .
$$

Both equations have a similar form. Making the foil thicker does not affect the temperature rise in the diffusion phase. However, when diffusion is complete, the thicker foil will have a smaller temperature rise. Unfortunately, this is achieved at the cost of CVR response.

\subsection{A Practical CVR Design}

The CVR for the Macrotor REB drive experiment [24] appears in Figure 11. The schematic of this CVR appears in Figure 10. Its dimensions are: $a=1.34 \times 10^{-2} \mathrm{~m} ; \quad l=7.6 \times 10^{-3} \mathrm{~m} ; \delta=7.6 \times 10^{-5} \mathrm{~m}$. Therefore, $t_{C}=9.6 \times 10^{-9} \mathrm{~s}, U_{C} / I_{p}=9.01 \times 10^{-4} \mathrm{VAA}^{-1}$ and the risetime $\left(t / t_{C}=0.25\right)$ is $2.5 \mathrm{~ns}$.

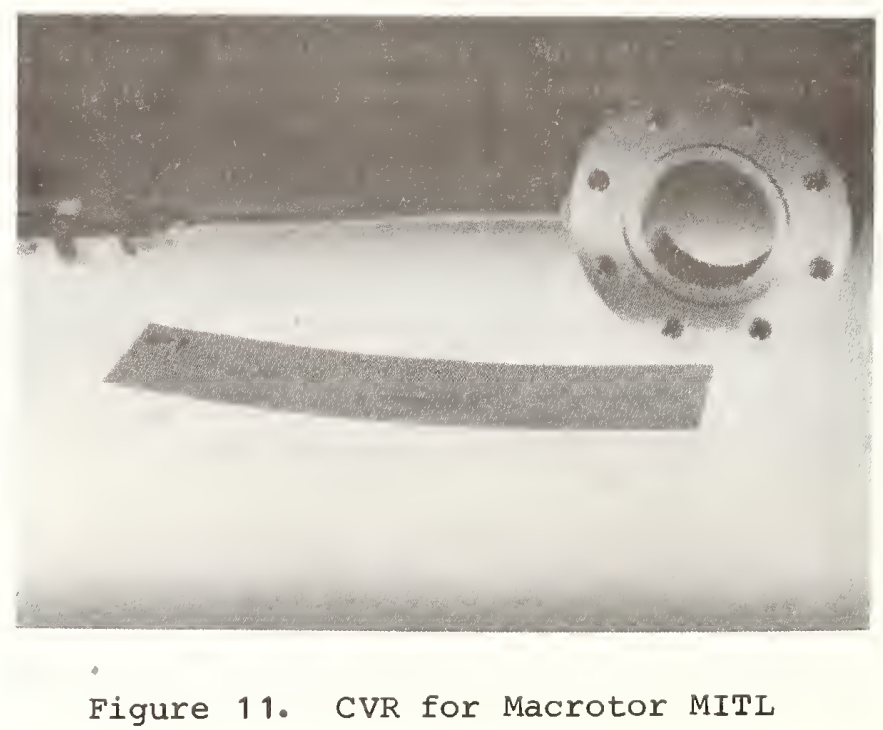


We measured the response of this CVR to a 500 ps risetime current drive of $20 \mathrm{~A}$ with a sampling scope at a $500 \mathrm{ps} / \mathrm{div}$ sweep speed. The response normalized with respect to $t_{C}$ and $U_{C}$ appears as the solid line in Figure 12. If we compare the response to the simulation results in Figure 8 , there is significant oscillation $(\sim 1 \mathrm{GHz})$ in the leading edge of the CVR output, not visible on a $250 \mathrm{MHz}$ bandwidth oscilloscope. Otherwise, the CVR output risetime of $2.5 \mathrm{~ns}$ compares quite favorably with the numerical simulation.

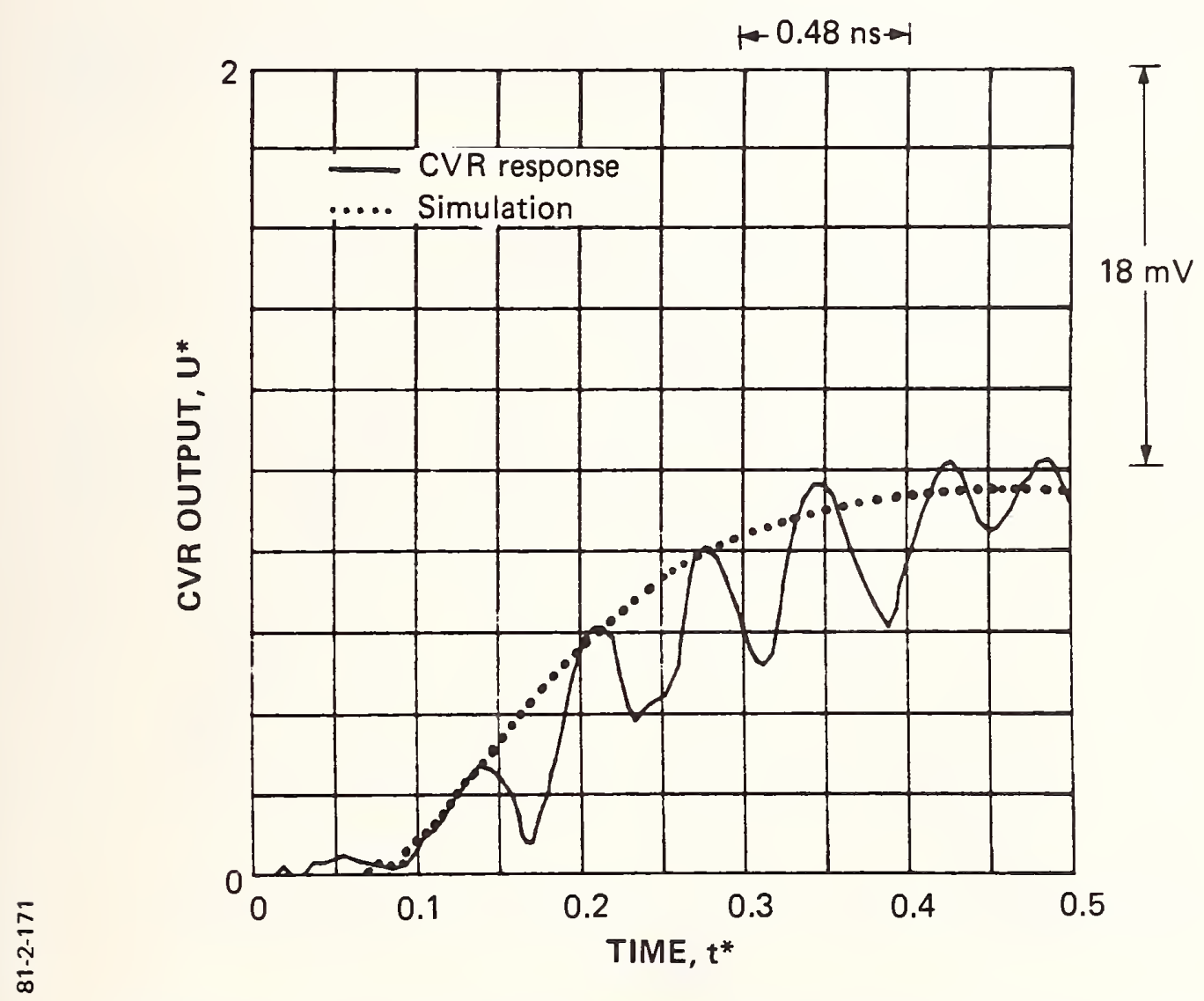

Figure 12. CVR output as a function of time for a step current input. Voltage $U^{*}$ and time $t^{*}$ are nondimensional

Figure 13 shows a measurement obtained with this CVR on a $0.95-m-$ long MITL. The solid line is the MITL input current, while the curve annotated with diamonds is the MITL output current. The input current was measured with a conventional Rogowski coil; the output current was 
measured with the CVR described above. The measurements were obtained with a short circuit at the output end of the MITL. The two waveforms have been displaced by the one-way transit time of light in the 0.95-mlong structure. The current measured by the CVR faithfully reproduces the input current measured by the Rogowski coil, except for a slight expected erosion of the leading edge of the pulse. The waveforms are almost identical in the vicinity of peak current.

The maximum linear current density in this CVR has been as high as $200 \mathrm{kA} / 8.4 \mathrm{~cm}=24 \mathrm{kA} \mathrm{cm}^{-1}$, which implies a peak magnetic field of $3.0 \mathrm{~Wb}-\mathrm{m}^{-2}$ at the surface of the foil. A temperature rise of about $5^{\circ} \mathrm{C}$ accompanied by less than 0.5 percent change in resistance is implied for this particular CVR at peak current.

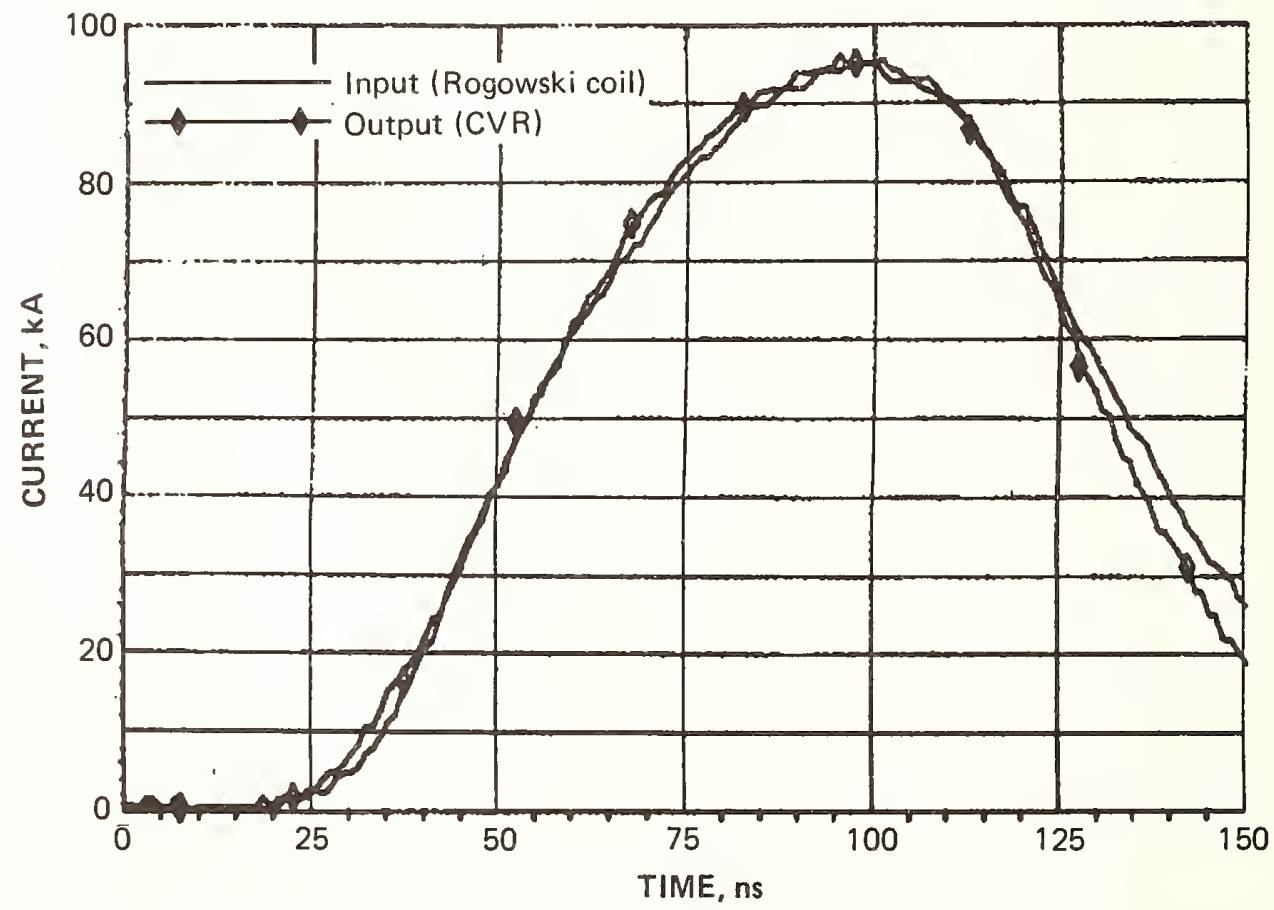

Figure 13. Current measurements in a 0.95-m-long MITL. Input

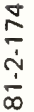
current was measured with a Rogowski coil. Output current was measured with the CVR shown in Figure 3

4. Calibrating Rogowski Coils, Fluxmeters, and CVRs

The calibration of current sensors is very difficult in the $>100 \mathrm{kA}$ current range. The difficulty arises from lack of sources with a precisely known current. In this section, we discuss the relative merits of various calibration methods.

From the standpoint of high frequency response, a cable pulser with a reed switch [25] is the best source. Its advantages are: exactly known output level, < 500 ps risetime, and reproducible repetitive 
output. Its main disadvantage is its low level output ( $20 \mathrm{~A}$ into a short circuit), which limits its application to bench calibration of high sensitivity sensors ( $100 \mathrm{~V} / \mathrm{MA}$ ). In situ calibration with this pulser is even more difficult due to its low level output.

Another current source is a high voltage cable pulser. A spark gap (air or oil, self-breaking, triggered, or mechanically actuated) connects the charge line to the load under calibration. This kind of pulser can drive as much as $400 \mathrm{~A}$ per cable. Unless it operates in the relaxation mode or with a triggered spark gap, it is nonrepetitive. The switching losses, which are somewhat difficult to quantify, and the switch inductance become more important as we increase the number of cables.

A third source applicable in the microsecond timescale is the ringdown of a capacitor in an RLC circuit. The current is easily obtained with some simple calculations and with measurements of voltage, period and decay rate.

A useful device for calibration is a thin foil shunt [26] which is well characterized in the laboratory under dc and pulsed conditions, and is used for calibrations in situ. A coaxial device of this type has been used standardly in our laboratory for several years. 


\section{Summary}

The design of current sensors depends heavily on the environment and time domain of operation. There is no current sensor ideal for every application. This paper points out a rationale for proper choice of current sensor.

\section{Acknowl edgements}

I am grateful to Don Pellinen--friend, former colleague and mentor. This paper reflects many of his ideas and designs. I also thank S. Stillwachs for the generous hospitality at Carnelian Bay, California, where I wrote this paper. Thanks also to Anne and little Enily for their patience, and to Margaret Thomas and Bronya Feldmann for their skillful editing.

\section{References}

[1] S. Glasstone and R. Lovberg, Controlled Thermonuclear Reactors, D. van Nostrand, Princeton, NJ (1960).

[2] W. Rogowski and W. Steinhaus, Arch. Elektrotech • 1, 141 (1912).

[3] J. M. Anderson, Rev. Sci. Instr., 42,915-926 (1971).

[4] R. Malewski, Rev. Sci. Instr., 39, 90-4 (1968).

[5] A. Ebinger, Archiv fur technisches Messen (ATM), 320-F4, 467, 211-214, DK 621.317 .31 (1974).

[6] D. Bartholomew, Electrical Measurements and Instrumentation, Allyn and Bacon, Boston (1963).

[7] D. G. Pellinen, et al., Rev. Sci. Instr. 51, 1535-46 (1980).

[8] F. Labuhn and K. Weinhardt, DK 621.317 .31 .014 .33 (1971).

[9] J. Cooper, Plasma Physics, $\underline{5}, 285-9$ (1963).

[10] M. Di Capua and D. G. Pellinen, in Diagnostics for Fusion Experiments, E. Sindoni and C. Wharton, eds., Pergamon Press, Oxford (1979).

[11] D. G. Pellinen, Rev. Sci. Instr., 42, 667-70 (1971).

[12] C. Trivelpiece, paper read at Workshop on Measurement of Electrical Quantities in Pulsed Power Systems, NBS, Boulder, CO., March 2-4, 1981 (unpublished).

[13] D. H. McDaniel, ibid. 
[14] C. A. Ekdahl, Rev. Sci. Instr., 51, pp. 1645-48 (1980).

[15] ibid., 1649-51 (1980).

[16] E. Chu, paper read at Workshop on Measurement of Electrical quantities in Pulsed Power Systems, NBS, Boulder, Co., March 2-4, 1981 (unpublished).

[17] R. R. Williams et al., IEEE Conf. Record, 14th Pulsed Power Modulator Symposium, 8OCH1573-5, ED, NY (1980).

[18] A. J. Schwab, IEEE Trans, on Power App. and Syst., PAS-90, 5, 225-7 (1971).

[19] R. Malewski, IEEE PES Summer Power Meeting, Minneapolis, MN, July 13-18, 1980; also IEEE Trans. on Power App. and Syst., PAS, (to be published in May 1981). , ibid., PAS-96, 2, 579-85 (1977).

[21] G. Hortopan, V. Hortopan, Rev. Rum. Sci. Tech., Electrochn. et Energie, 19, 1, 41-47 (1974).

[22] J. Park, J. Res. Natl. Bu. Std. 39, 191 (1947).

[23] H. Knoepfel, Pulsed High Magnetic Fields, American Elsevier Publishing Co., Inc., New York (1970).

[24] V. Bailey et al., Japan-U.S.A. Compact Toroid Workshop A9, Osaka, Japan, February 17-19, 1981; also Physics of Fluids (to be published).

[25] R. L. Garwin, Rev. Sci. Instr. 21, 903 (1950).

[26] D. G. Pellinen, ibid. 41, 1347-8 (1970).

\section{Section 8. Sponsor Acknowledgements}

This work was supported partially by DNA, U.S. DOE and Physics International internal funds. 


\author{
PRECISE MEASUREMENT OF CURRENT IN PULSED POWER SYSTEMS \\ Michael E. Wilmer and Paul A. Pearson \\ Pearson Electronics, Inc. \\ 4007 Transport Street \\ Palo A1to, CA 94303
}

In order to precisely measure currents in a pulsed power system, the capabilities and limitations of the instrumentation used must be well characterized. In this presentation, we shall examine the theoretical and empirical properties of internally terminated current monitors in pulsed power applications. The advantages of current monitors over current viewing resistors will be discussed. The factors affecting the inherent accuracy of current monitors will be analyzed and their temperature dependence reported. The effect of the current monitor on the circuit being measured will be examined and the insertion inductance and insertion resistance will be calculated as a function of the key current monitor design parameters. The voltage standoff characteristic of these monitors will be derived and the results tabulated for several clearance hole sizes both in air and in transformer oil. Core saturation will be related to the design parameters of the individual current monitors and its effect on the output waveform will be examined. The talk will conclude with an explanation of the usefulness of dc biasing in extending the range of current monitors, particularly those employing high permeability cores.

Key Words: Current measurement; current monitor; current transformer.

\title{
1. Introduction
}

The accurate measurement of currents in pulsed power systems is often crucial for the successful operation of the system. In applying current transformers to this task, it is important that the limitations imposed by the design constraints of the transformer be observed. In this paper we shall review the theory of operation of terminated current monitors and relate their performance characteristics to the key design parameters. In particular we shall carefully investigate the various factors which can lead to discrepancy between the actual primary current waveshape and the secondary output voltage waveshape. 


\section{Competing Approaches}

Current viewing resistors or resistive shunts are presently the most practical alternative to the use of current monitors in measuring pulsed currents. While the resistive technique is frequently adequate, current monitors have several distinctive advantages which offer significant improvements in accuracy and overall performance.

Since current monitors are transformers, the primary and secondary are electrically isolated and interference and noise problems associated with ground loops can be avoided. A further advantage of this isolation is in the improved protection of personnel and equipment which it provides. There are two further disadvantages in the use of resistive shunts for measuring pulse currents. First, resistive shunts are subject to an increase in resistance with frequency due to skin effect resistance $[1,4]$ causing significant error in the measurement of pulses with short rise and fall times. Second, resistive shunts present a relatively high resistance to the primary circuit. For a particular sensitivity $S$, a resistive shunt presents a load of $S$ ohms to the primary circuit while a current monitor presents a load of only S/N2 ohms where $\mathrm{N}_{2}$ is the number of secondary turns. When the currents to be measured are in the thousands of amps, this can be a very significant factor.

\section{Simplified Theory of Operation}

In the simplest case, a terminated current monitor consists of a magnetic core (typically toroidal in shape), a number of primary turns $N_{1}$, a number of secondary turns $N_{2}$, and a terminating resistor $R_{T}$.

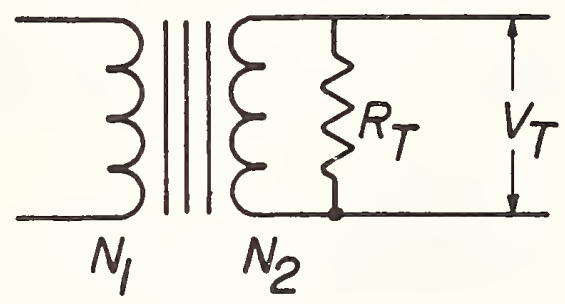

Figure 1. Simplified Current Monitor

Normally, there is but a single primary turn and therefore the output voltage for an alternating primary current is:

$$
V_{T}=i_{2} R_{T}=i_{1} \frac{R T}{N_{2}}
$$


The ratio of $R_{T}$ to $N_{2}$ is called the sensitivity of the monitor and is expressed in units of volts/amp. The equivalent circuit consists of a pulse current source shunted by the secondary inductance, shunted by the winding resistance in series with the terminating resistance. The voltage which is generated across the terminating resistance in response to a stepfunction current in the primary is given in equation (2).

$$
\begin{aligned}
& V_{T}=\frac{I 1 R_{1}}{N_{2}} e^{-t / \tau} \\
& \text { where } \tau=L_{2} /\left(R_{W}+R_{T}\right)
\end{aligned}
$$

The decay of this exponential term causes the output to droop. The initial rate of droop (in percent per microsecond) is a parameter which is commonly used in specifying current monitors.

The total voltage per turn is given by the sum of the voltage dropped across the winding resistance $\left(V_{W}\right)$ and the voltage dropped across the terminating resistance $\left(V_{T}\right)$. The magnetic induction $B$ of the core is equal to the integral of voltage per turn per unit area as shown in equation (3).

$$
B(t)=\int_{0}^{t} \frac{\left(V_{T}+V_{W}\right)}{N_{2} A} d \tau
$$

For a step function excitation, we can substitute equation (2) into equation (3), yielding equation (4).

$$
B=\frac{I_{1}\left(R_{T}+R_{W}\right) \tau\left(1-e^{-t / \tau}\right)}{A N_{2}{ }^{2}}
$$

For times which are short relative to $\tau$, the exponential may be approximated by the first term of its power series expansion and $B$ becomes:

$$
B=I_{1} \frac{(R T+R W)}{A N_{2}^{2}} t
$$

This equation shows that $B$ increases linearly with time for times short relative to the droop time constant, and after some time, B will increase to the point at which the core is effectively saturated. The product of primary current and time for which this saturation occurs is another common parameter of current monitors and is called the currenttime product.

$$
I_{1} t_{\max }=\operatorname{Bmax} \frac{A_{N_{2}}^{2}}{\left(\frac{R_{T}+R W}{R}\right)}
$$




\section{Factors Affecting Accuracy}

When a current monitor is used to measure pulsed currents, there are several factors which contribute to the potential error. If an ideal square pulse with an amplitude $\mathrm{I}_{0}$ is assumed as the primary current, then the actual output of a current monitor with sensitivity $S$ would have the general shape shown in Figure 2.

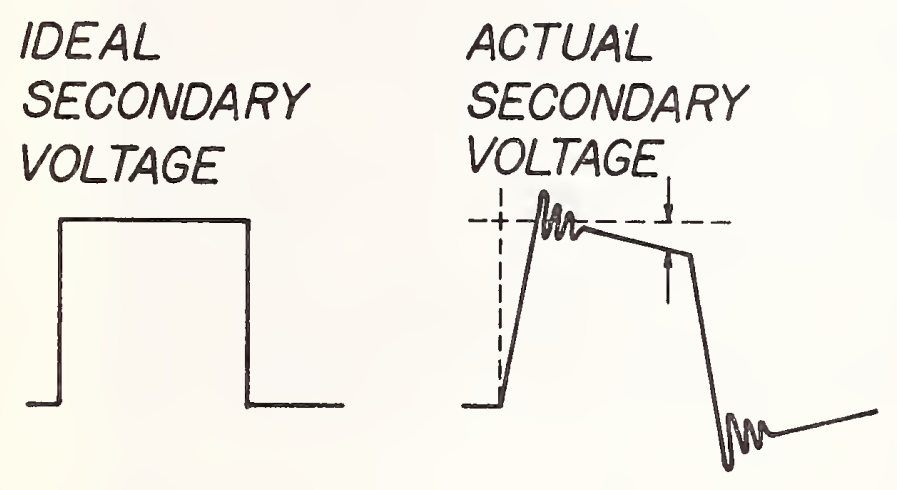

Figure 2. Ideal and Actual Waveform

There are four ways in which the actual output differs from an idealized output. These are (1), the rise time and fall time are not zero; (2), there is some overshoot and ringing at the leading edge; (3), there is some droop of the flat top with time; and (4), the output voltage may not equal the ideal value of $\mathrm{SI}_{0}$ because of sensitivity error in the current monitor. We shall examine these errors one at a time in order to estimate their potential impact and to understand how to minimize or compensate for each.

\subsection{Rise Time Limitations}

When an ideal step function of current amplitude $I_{1}$ is passed through the center of a toroidal current transformer having $\mathrm{N}_{2}$ secondary turns, a current $\mathrm{I}_{1} / \mathrm{N}_{2}$ is induced in the secondary winding. Several authors have developed the high frequency equivalent circuit for terminated current monitiors $[2,3]$ and there is general agreement that the leakage inductance is negligible and that the rise time of the output voltage waveform is 1 imited by the distributed capacitance in parallel with the terminating resistance. There is also a small inductance associated with the termination network which can give rise to ringing. Because of the excellent high frequency response of terminated current monitors, increases in observed rise time when the monitor is placed in the circuit are usually found to be due to the inductance of the primary connection. 


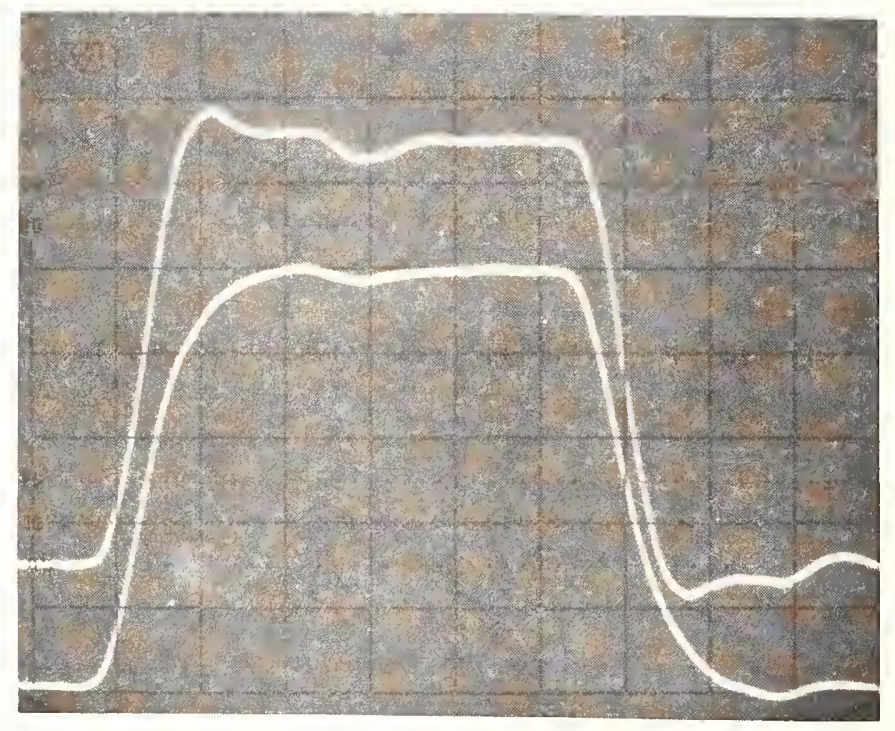

Figure 3. Current Monitor input (Tower trace) and output waveforms with an 8 inch primary wire connection. Time scale TOns/div.

In Figure 3, we see the waveshape which results when a 0.1 amp current pulse with a rise time of 5 nanoseconds is passed through a Pearson Model 4100 current monitor using approximately 8 inches of wire. The rise time of the resulting output waveform is approximately 7.5 nanoseconds.

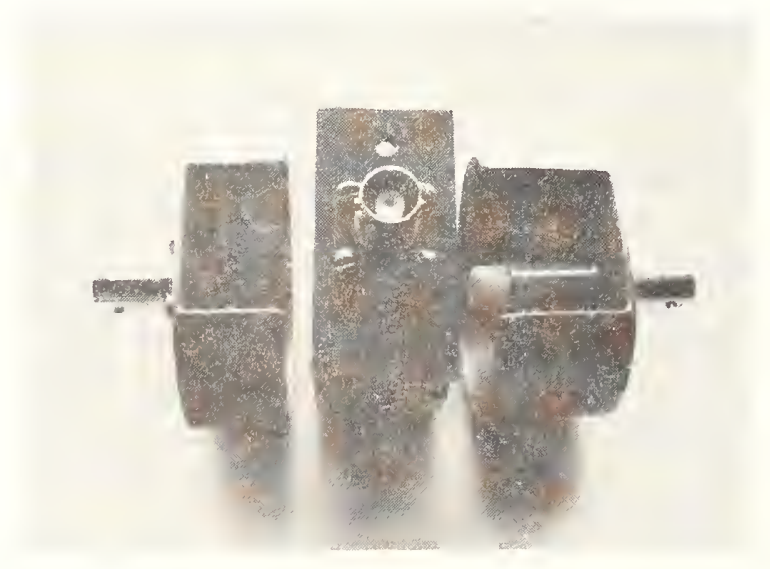

Figure 4. Coaxial Current Monitor test apparatus with a Pearson Model 4100 Current Monitor. 
In an effort to minimize the effects of primary circuit inductance, the coaxial current monitoring cavity shown in Figure 4 was constructed to hold the model 4100. The response of the Model 4100, enclosed in this device, to the same excitation which was used in Figure 3 , is shown in Figure 5. The rise time is approximately five nanoseconds. Thus, the inherent rise time of this system is limited by the geometry of the primary circuit.

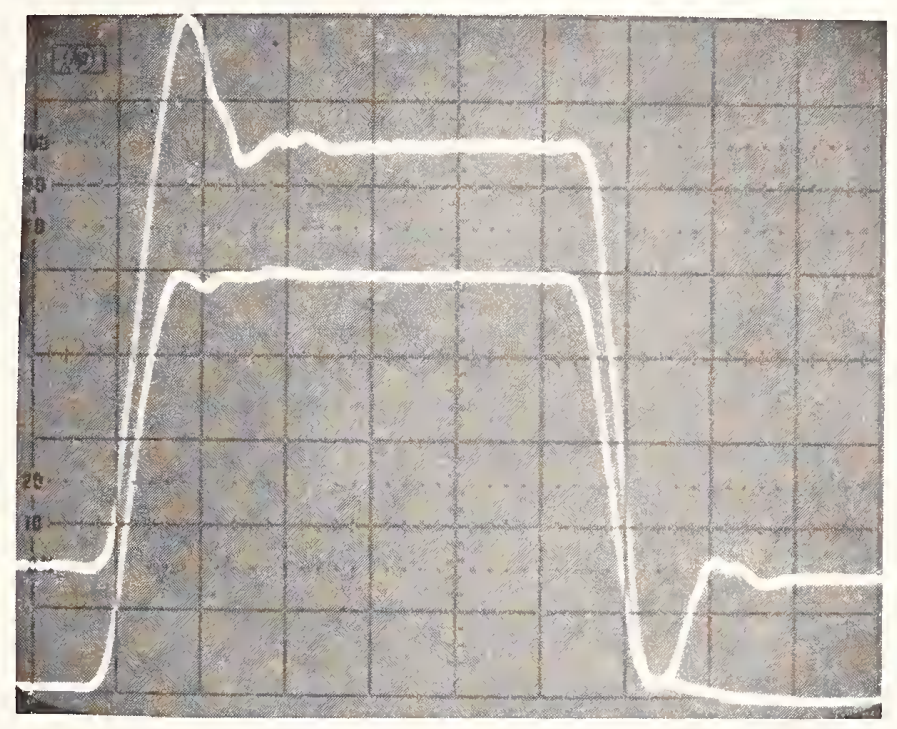

Figure 5. Current Monitor input (lower trace) and output waveforms using the coaxial structure. Time scale $10 \mathrm{~ns} / \mathrm{div}$.

\subsection{Overshoot and Ringing}

Referring once again to Figure 5, we observe an overshoot of about $30 \%$ and several cycles of damped oscillation having a period of about 8 nanoseconds.

This phenomenon is due to a very high frequency complex pole in the secondary circuit which arises from the distributed capacitance of the secondary winding and the inductance of the resistive termination. Because this pole is of such a high frequency in this particular current transformer, its resonance is excited only when the rise time of the primary current is short relative to the resonance period. It is generally true that large capacity current transformers have more secondary turns and larger overall size and therefore have more distributed capacitance. For this reason, the resonance tends to occur at lower frequencies for larger units with lower sensitivity.

The usable rise time specification which is given for all standard Pearson current monitors is that rise time for which the secondary resonance will be only negligibly excited when the primary current is passed through the center of the transformer. It is also true that the rise time of the primary current can be accurately measured if it is greater than the quoted usable rise time. 


\subsection{Droop}

As explained earlier, the droop of a current transformer is a direct result of the fact that it is a high pass device and when the droop rate is known, pulse measurements can be corrected for droop. This is most easily done when the total amount of the droop is small (less than $10 \%$ ) so that the exponential decay can be accurately estimated by a linear decay. Thus, for a transformer with a droop rate of $\alpha \% / \mu$ second, the corrected output voltage at a time $T$ following the onset of the pulse is given in equation (7).

$$
\operatorname{VC}(T)=\operatorname{VOUT}(T)\left(\frac{100}{100-\alpha T}\right)
$$

The correction given in equation (7) is most accurate for times short relative to the droop time constant and for ideal square puised currents, but it also can be used to reduce error due to droop for pulses having other shapes.

\subsection{Sensitivity Error}

Sensitivity error in current transformers is most readi?y observed when the current pulse being measured has insignificant rise time, overshoot, or droop error. It has two causes which can be readily understood upon examination of equation (1). There can be a difference between the specified number of secondary turns and there can be a difference between the specified value of the terminating resistance and its effective value.

The number of turns can be very carefully controlled in manufacture and therefore only the resistance error is significant. This effective resistance is actually composed of the physical terminating resistor network shunted by a resistance representing the hysterisis and eddy current losses of the transformer core. These losses are typically small and therefore their equivalent shunt resistance is fairly large. For most practical devices, this resistance contributes an effect which is between $0 \%$ and $0.5 \%$ and therefore, even though it exhibits some frequency dependence, its impact on overall accuracy as a function of frequency is quite smal1.

The conclusion which can be drawn is that the sensitivity error is primarily attributable to the secondary termination resistance. The sensitivity of the device can be determined by comparing its output to the voltage dropped across a wideband precision resistance standard driven by a pulse current source. The resistances used in Pearson current monitors are constructed of extremely stable Manganin wire with a very low temperature coefficient of resistance. This results in current monitors whose error is essentially independent of temperature over the operating range. 


\section{Effects on Measured Circuit}

The effects of a current monitor on the circuit it is monitoring can be divided into indirect effects and direct effects. The indirect effects are changes to the circuit layout necessary to make the connection through the aperture of the transformer. The most common and most important phenomenon is an increase in inductance which leads to increased rise time in the primary circuit. This effect can best be avoided by minimizing the length of the conductors and otherwise choosing a geometry which minimizes inductance.

The direct effect of a current monitor can readily be calculated upon consideration of the equivalent circuit in Figure 1 . The impedance added to a single turn primary is essentially all due to the terminating resistance reflected into the primary. The relationship governing this transformation is given in equation (8).

$$
R_{I N}=\frac{(R T+R W)}{N_{2}^{2}}
$$

For reasonably designed current monitors, this value lies between $10^{-8}$ and $2 \times 10^{-2}$ ohms. The winding capacitance and inductance of the terminating network are extremely low and therefore have negligible effect on the primary circuit within the operational bandwidth of the current monitor.

\section{Voltage Isolation}

A current monitor enclosing a primary conductor forms a coaxial configuration. In order to estimate the voltage at which breakdown occurs between the primary and the current transformer shield, we will calculate the breakdown voltage for an ideal, endless coaxial line.

The electric field within a coaxial line is given by equation (9). The voltage is the integral of the electric field between the conductors and is given by (10). In this equation, $a$ is the radius of the inner conductor, $b$ is the radius of the outer conductor and $\varepsilon$ is the dielectric constant of the medium.

$$
\begin{aligned}
& E=\rho / 2 \pi \varepsilon \\
& V=\rho \ln \left(\frac{b}{a}\right) 2 \pi \varepsilon
\end{aligned}
$$

The maximum value of $E$ occurs at $r=a$ and therefore the voltage as a function of inner conductor radius, outer conductor radius, and maximum field strength is given by (11). 


$$
V=E_{\text {MAX }} a \ln \frac{b}{a}
$$

Maximizing $V$ as a function of $a$, one finds that the maximum voltage occurs when $\ln (b / a)=1$ or when $a=b / 2.73$, and for this case, the maximum voltage is given in (12).

$$
V=a E_{M A X}
$$

In Table 1, we show optimum values of inner conductor diameter and resulting calculated breakdown voltages in air and in oil for several current monitor hole sizes. These assume breakdown strengths of $80 \mathrm{kV} /$ inch for air and $400 \mathrm{kV} /$ inch for oil. Experimental work has shown these numbers to be quite conservative.

Table 1. Voltage Breakdown

$\begin{array}{llll}\text { Current } & \text { Approx. Optimum } & \text { Approx. Breakdown } & \text { Approx. Breakdown } \\ \text { Monitor } & \text { Primary Conductor } & \text { Voltage } & \text { Voltage } \\ \text { Hole Diameter } & \text { Diameter } & \text { In 0i1 } & \text { In Air }\end{array}$

(Inches) (Inches) (kV)

$\begin{array}{llrr}2 & .75 & 150 & 30 \\ 3.5 & 1.25 & 250 & 50 \\ 10.75 & 4 & 1000 & 200\end{array}$

\section{Core Saturation Effects}

Due to the limiting values of saturation magnetization in ferromagnetic materials, all transformers built around ferromagnetic cores are subject to saturation. Saturation can be defined in several ways, but perhaps the most meaningful observation is that the effective permeability of the material rapidly decreases with the onset of saturation. This in turn results in a dramatic increase in the droop rate for pulses.

For terminated current monitors, saturation can be predicted for pulses short with respect to the droop time constant by computing the current-time product. This product is actually the integral of the primary current pulse with respect to time and for rectangular pulses, it is simply the product of pulse width and amplitude.

In the situation in which a current monitor is being operated near saturation, the increased droop can cause error in measurements at or near the trailing edge of the output pulse. Since saturation is actually a voltage effect, reduction of the terminating resistance of the current transformer effectively increases its current-time product. This can be accomplished by externally terminating the current monitor with a $50 \mathrm{ohm}$ 
termination (note that this reduces the output to half of nominal value.)

If you are using a device somewhere close to its saturation ratings, it can be quite convenient to quickly decide whether or not the pulse current is causing saturation. One way to do this is by looking at the waveform with the current monitor terminated in a high impedance and then looking at it with the device terminated in 50 ohms. A significant difference in the latter part of the pulse waveshape with the $50 \mathrm{ohm}$ termination versus the high impedance termination is an indication that the device is being operated at or near saturation.

The effective current-time product for a given device can be more than doubled through the use of DC biasing. When a DC current of polarity opposite to the pulse is passed through the secondary of a current transformer, the effect is to move the operating point of the magnetic core to the opposite side of the hysterisis loop, thereby providing a much larger change in magnetization before saturation.

\section{References}

[1] F.D. Bennett, and J.W. Marvin, "Current Measurement and Transient Skin Effects in Exploding Wire Circuits," The Review of Scientific Instruments, Vol. 33, pp. 1218 - 1226 (1962)

[2] F.J.U. Ritson and J. Wood, "Wide Band Current Transformers for the Examination of Current Waveforms," Electronic Engineering, pp. 483 - 485 (JuTy 1964)

[3] J. Cooper, "On the High Frequency Response of a Rogowski Coil," Plasma Physics (Journal of Energy, Part C), Vol. 5, pp. $285-289$ (1963)

[4] John H. Park, "Shunts and Inductors for Surge Current Measurements," Journal of Research, National Bureau of Standards, Vol. 39, pp. 191 212 (1947) 


\title{
LOW-INDUCTANCE SHUNTS FOR MEASURING LARGE PULSED CURRENTS
}

\author{
Walter F. Praeg \\ Accelerator Research Facilities Division \\ Argonne National Laboratory \\ Argonne, IL 60439
}

Precision shunts have been developed at Argonne National Laboratory (ANL) to measure pulsed currents. Direct-coupled air-or water-cooled coaxial shunts have been built for a frequency response up to $100 \mathrm{kHz}$ and with an rms current rating of up to $10 \mathrm{kA}$. Smaller shunts were designed to terminate current transformers for measuring $\leq 300 \mathrm{kA}$ pulses of 50 to $3000 \mu \mathrm{s}$ duration. Some of our applications require the shunt resistance to be repeatable within $\pm 0.005 \%$. This made it necessary for us to determine the resistance-stress coefficients of manganin and to design the shunts for minimum stress. Stress in the manganin is caused by thermal expansion, by cooling-water pressure, by mounting arrangements, and by magnetic fields. Design details and performance data are presented.

\section{Introduction}

Pulsed magnets used with the beam lines of the Zero Gradient Synchrotron at ANL have peak currents ranging from a few KA to several hundred KA. The pulse width varies from $50 \mu \mathrm{s}$ to $3000 \mu \mathrm{s}$. The required stability and repeatability of current pulses was $\leq 1 \%$ for pion focusing horns, $\leq 0.1 \%$ for quadrupole magnets and $\leq 0.005 \%$ for dipole switching magnets. These current pulses were measured with low inductance shunts which were either directly in the current path or coupled to it via current transformers (CT's). No suitable shunts were available commercially, we, therefore, developed precision coaxial shunts.

The EMF between shunt potential leads may be written as

where

$$
E=I(R+j \omega L)+d \phi / d t
$$

$$
\begin{aligned}
& I=\text { shunt current } \\
& \omega=2 \pi f, \\
& R=\text { effective resistance of shunt, }
\end{aligned}
$$


$L=$ four-terminal inductance of the potential circuit, linked with flux set up by shunt current embraced by potential circuit, $\mathrm{d} \phi / \mathrm{dt}=$ voltages induced in potential circuit due to flux of currents flowing in circuits other than the potential circuit.

The effective shunt resistance will change as a function of

frequency of shunt current,

temperature,

time,

chemical reactions with surroundings, and

stress.

For our applications, coaxial shunts were chosen because

- the four-terminal impedance can be computed from the dimensions,

- uniform stray fields will not affect the potential circuit,

- there is a nearly uniform temperature distribution throughout the resistance element,

- they are simple to water-cool.

A coaxial shunt consists of a pair of concentric cylinders. One cylinder, or part of it, is made from resistance material, the other is an external or internal copper cylinder. These cylinders are joined on one end by an annular conductor; the other ends connect to current terminals.

\section{Effective Impedance of Coaxial Shunt As A Function of Frequency}

The impedance of a coaxial shunt has been computed by Silsbee [1]. His formula accounts for the current distribution over the thickness of the coaxial conductors due to skin effects. It applies as long as the potential leads are connected to the outside of the outer cylinder or to the inside of the inner cylinder. Simplifying the exact solution to cover frequencies above $1 \mathrm{kHz}$ and using the unprimed dimensions of Figure 1 , the impedance of the inner cylinder is

$$
Z=R_{d c} \delta \frac{(\sin \delta \cosh \delta+\cos \delta \sinh \delta)+j(\cos \delta \sinh \delta-\sin \delta \cosh \delta)}{(\sin \delta \cosh \delta)^{2}+(\cos \delta \sinh \delta)^{2}} .
$$

For low frequencies, the ac resistance of the shunt is [2]

$$
R_{a c}=R_{d c}\left(1-\frac{7}{90} \delta^{4}\right)
$$

and the inductance [3] is abhenries per $\mathrm{cm}$ is 


$$
L=-\frac{d}{3 b}-\frac{d^{2}}{6 b^{2}} \text {, }
$$

where

$$
\begin{aligned}
\delta & =2 \pi d \sqrt{f / \rho}, \\
d & =b-a=\text { thickness of shunt cylinder wall, in } \mathrm{cm}, \\
f & =\text { frequency of current through shunt, } \\
R_{d c} & =d c \text { resistance of cylinder in abohm, and } \\
\rho & =\text { resistivity of shunt material, in abohm-cm. }
\end{aligned}
$$

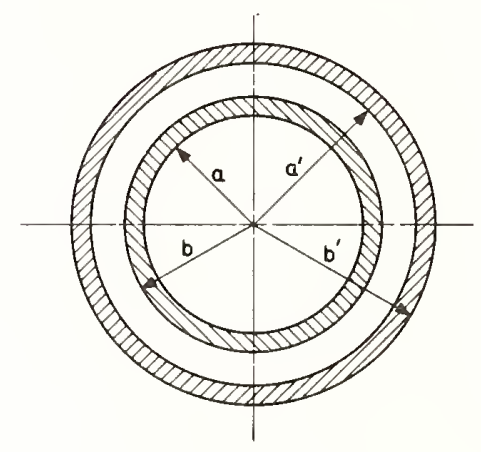

Figure 1. Horizontal cross section of coaxial shunt

Equations (2), (3), and (4) also apply when the resistance material is in the outer cylinder. In this case, the primed dimensions of Figure 1 must be used.

\section{Choice of Design and Resistance Material}

\subsection{General}

Manganin was chosen because of its stability with time and its low temperature coefficient of resistance. General information on this alloy is given in Table I.

Manganin cylinders are usually not commercially available. A nearly homogeneous cylinder can be made by bending sheet material into a circle and butt-welding the joint, using tungsten inert-gas or electronbeam welding techniques. After assembly and before the potential leads are attached, the manganin has to be artificially aged to avoid a slow decrease in resistance with time. Commonly this is done by baking the shunt at $150^{\circ} \mathrm{C}$ for 60 hours. Within two or three months after baking, the shunt resistance wi11 ordinarily increase by about $0.01 \%$ and wil1 then be stable and ready for calibration [4].

Change of resistance versus temperature of commercial shunt manganin is illustrated in Figure 2. The temperature at which the maximum resistance occurs is a function of the thermal and mechanical treatment of the manganin as well as of its composition. 
Table I. Physical Characteristics of Manganin

Nomina 1 analysis

Coefficient of 1 inear expansions between $-200^{\circ}-100^{\circ} \mathrm{C}$

Resistivity at $20^{\circ} \mathrm{C}$

Temperature coefficient of resistance between $15^{\circ}-35^{\circ} \mathrm{C}$ for wire between $37^{\circ}-57^{\circ} \mathrm{C}$ for shunt strip

Thermal EMF against copper between $00-100^{\circ} \mathrm{C}$ Approximate melting point

Tensile strength at $20^{\circ} \mathrm{C}$
$84 \mathrm{Cu} 12 \mathrm{Mn} 4 \mathrm{Ni}$

$0.0000187^{\circ} \mathrm{C}^{-1}$

wire $48.2 \mu \Omega \mathrm{cm}$ shunt strip $38.1 \mu \Omega \mathrm{cm}$

$$
\begin{aligned}
& \pm 0.000008{ }^{\circ} \mathrm{C}^{-1} \\
& \pm .000004{ }^{\circ} \mathrm{C}^{-1} \\
& <0.003 \mathrm{mV}{ }^{\circ} \mathrm{C}^{-1} \\
& 1020^{\circ} \mathrm{C} \\
& \geq 2800 \mathrm{~kg} \mathrm{~cm}^{-2}
\end{aligned}
$$

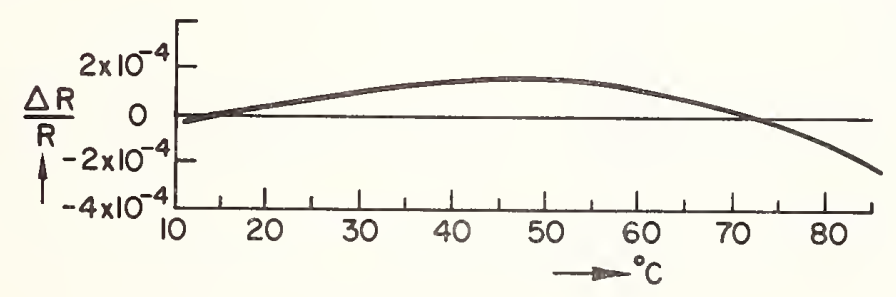

Figure 2. Change of resistance versus temperature of shunt manganin

Figure 3 is an assembly view of a coaxial shunt. The upper current terminal, which is silver-brazed to the inner copper cylinder, supports the weight of the lower current terminal and the upper extension cylinder through spring assemblies. An 0-ring seals the cylinders. The shunt is cooled with distilled water, which is de-ionized, de-oxygenated, and filtered. Corrosion of the manganin due to cooling water is retarded by an epoxy-phenolic coat of about $0.005 \mathrm{~cm}$ thickness. A fiberglass jacket $0.8 \mathrm{~cm}$ thick, which is separated from the manganin tube by a layer of silicon grease, protects the manganin.

\subsection{Potential Leads}

The effects of uniform magnetic stray fields on the potential circuit are reduced by placing four pairs of potential leads symmetrically around the manganin cylinder and connecting them in parallel. Since these leads cannot be attached on an equipotential line, a circulating current will flow through them. This current is kept small by making these leads from flat-rolled manganin wire which gives each lead a resistance of a few tenths of an ohm. The leads are insulated from and taped to the manganin cylinder. 


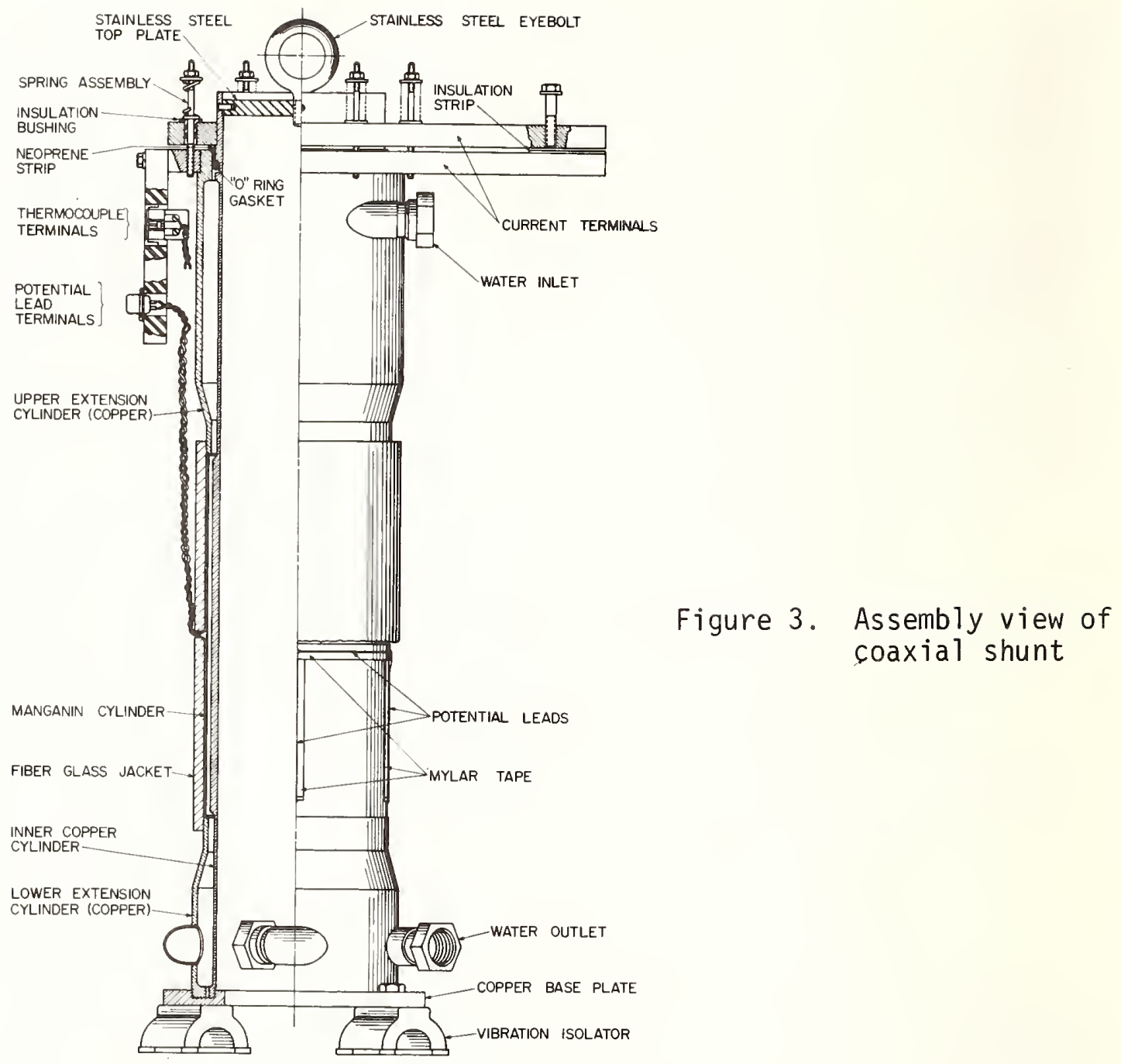

\subsection{End Effects}

There are no end effects in a coaxial shunt if the current in the cylinders is distributed uniformly around the axis and if the magnetic field is not affected by the current in the current terminals.

These conditions might be realized by extending the current carrying cylinders, which connect to the current terminals, several diameters beyond the location of the potential leads. In our case, this would have resulted in very tall shunts. To keep the height down, we forced uniform current distribution by cutting slots in the current terminals, as shown in Figure 4.

The separation of the current terminals and their current distribution (eddy current effects) affects the voltage drop across a pair of potential leads. These induced voltages have been computed [5]; 
they cause an error of $\leq 0.001 \%$ in our application.

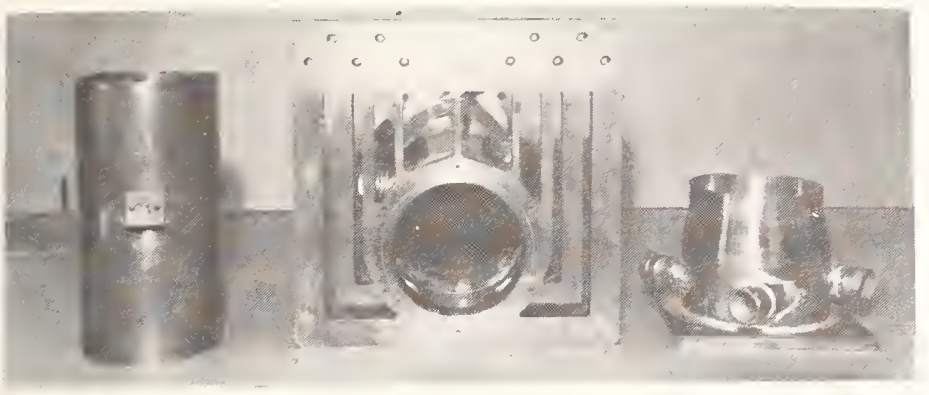

Figure 4 .

View of manganin cylinder, current terminal and lower extension cylinder.

Figure 5 gives $|Z| / R_{d c}$ and $R_{a c} / R_{d c}$ computed from Eq. (2) for a $200 \mu \Omega$ shunt rated $10 \mathrm{kA}$ (shunt $A$ ), and for a $175 \mu \Omega$ shunt rated $6 \mathrm{kA}$ (shunt $B$ ). Some features of these shunts are listed in Table II.

Table II. Data on Water-Cooled Coaxial Shunts

Shunt Type

A $\quad B$

\begin{tabular}{l|l|l|l}
\hline Current rating & 10000 & 6000 & $\mathrm{~A}$ \\
Resistance between potential leads & 200 & 175 & $\mu \Omega$ \\
Length of manganin cylinder & 30.48 & 30.48 & $\mathrm{~cm}$ \\
Length of manganin cylinder between & & & \\
potential leads & 25.3 & 24.5 & $\mathrm{~cm}$ \\
Diameter of manganin cylinder (ID) & 24.15 & 14.8 & $\mathrm{~cm}$ \\
Thickness of manganin cylinder & 0.0635 & 0.1143 & $\mathrm{~cm}$ \\
Heat flow in manganin cylinder & 10.5 & 5.54 & $W \mathrm{Cm}^{-2}$ \\
Water flow & 3.8 & 5.2 & $\mathrm{Liter} \mathrm{s}-1$ \\
Radial water pressure & $<0.36$ & $<0.47$ & $\mathrm{~kg} \mathrm{~cm} \mathrm{~cm}^{-2}$ \\
Temperature of manganin above water & 40 & 17 & $\mathrm{OC}$ \\
temperature (200) & 98 & 76 & $\mathrm{~cm}$ \\
Overall heights of shunt & & \\
\hline
\end{tabular}

\subsection{Effects of Transverse Magnetic Fields}

The electrical resistance of metals changes in magnetic fields. For the transverse magnetic field caused by the shunt current, the increase of electrical resistance of manganin is given by [6], [7]

$$
\frac{\Delta R}{R}=K \frac{B^{2}}{3 B_{C}}
$$


where

$$
\begin{aligned}
\Delta R & =\text { change in resistance, } \\
R & =\text { resistance at zero magnetic field, } \\
K & =1.16 \times 10^{-5} \text { gauss } \\
B & =\text { transverse magnetic field, in gauss, and } \\
B_{C} & =1.4 \times 10^{5} \text { gauss. }
\end{aligned}
$$

The peak transverse magnetic field in the cylinder of shunts $A$ and $B$ at $10 \mathrm{kA}$ are $166 \mathrm{G}$ and $270 \mathrm{G}$ respectively. These fields cause a negligible resistance increase.

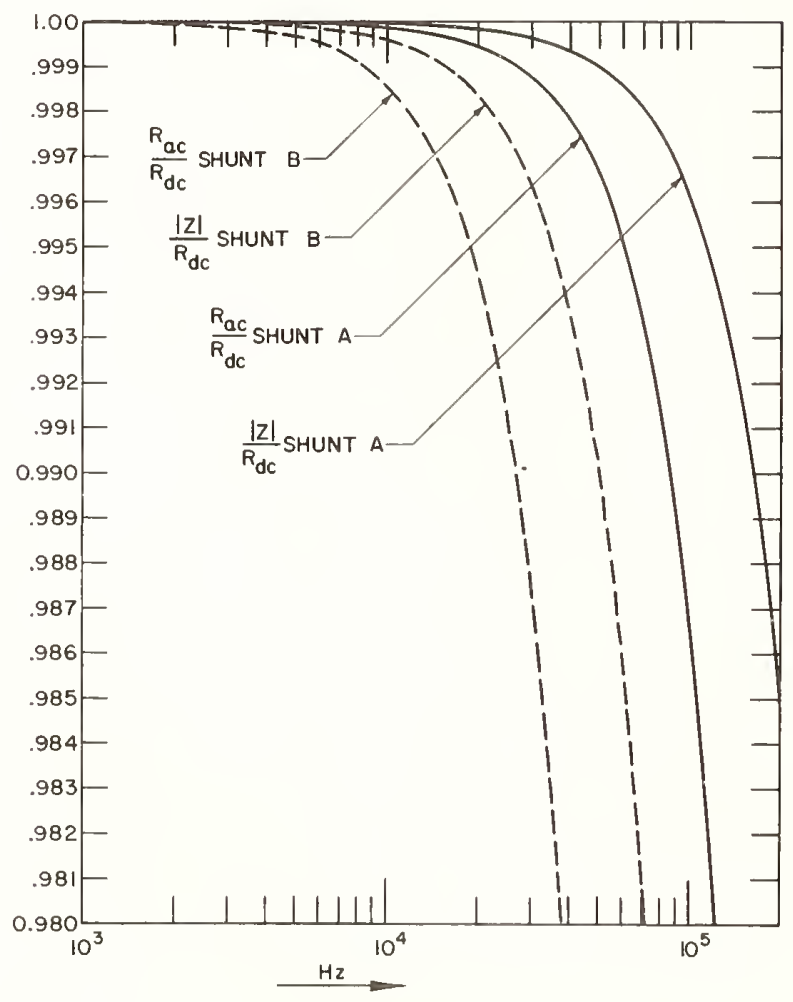

Figure 5. Ratios of ac resistance $R_{a c}$ and impedance $Z$, to $d c$ resistance $R_{d c}$ vs frequency

\section{Changes in Resistance Due to Stress}

Stress in manganin can change the shunt resistance beyond tolerable limits. Unfortunately, manufacturers of manganin do not include resistance-stress coefficients in their general information nor could they give such information upon request. A subsequent literature search revealed that several investigators had measured resistance-stress coefficients of thin manganin wires. In these measurements, stress was applied in the direction of current flow, which is not always the case with shunts.

Below the proportional limit, which we measured as $1050 \mathrm{~kg} \mathrm{~cm}^{-2}$ on anneals strip samples, the resistance changes linearly with stress. 
The dependence of resistance upon stress is

$$
R_{\sigma}=R_{0}(1+a \sigma)
$$

where

$$
\begin{aligned}
R_{\sigma} & =\text { resistance under stress and ambient temperature, } \\
R_{0} & =\text { resistance under zero stress and ambient temperature, } \\
a & =(1 / R)(\mathrm{dR} / \mathrm{d} \sigma)=\text { resistance-stress coefficient, } \\
\sigma & =\varepsilon E=\text { stress, } \\
\varepsilon & =\text { strain, and } \\
E & =\text { Young's modulus of elasticity. }
\end{aligned}
$$

4.1 Resistance Changes Due to Stress in the Direction of Current Flow

From the literature [8], [9], and [10] a resistance-stress coefficient for tension in the direction of current flow was obtained as alf $=0.59 \times 10^{-6} \mathrm{~cm}^{2} \mathrm{~kg}^{-1}$. For pressure in the direction of current flow the coefficient is $a_{p}^{\prime l}=2.31 \times 10^{-6} \mathrm{~cm}^{2} \mathrm{~kg}^{-1}$.

From Eq. (6) we obtain the relative change in resistance $\Delta R / R_{0}=a \sigma_{0}$ With $a_{t}^{\prime \prime}=0.59 \times 10^{-6} \mathrm{~cm}^{2} \mathrm{~kg}^{-1}$ and $a_{0}^{\prime \prime}=2.31 \times 10^{-6} \mathrm{~cm}^{2} \mathrm{~kg}^{-1}$, the stress that causes a $0.01 \%$ change in resistance is

for tension

$$
\begin{array}{ll}
\text { for tension } & \sigma_{t}=10^{-4} /\left(0.59 \times 10^{-6} \mathrm{~cm}^{2} \mathrm{~kg}^{-1}\right)=170 \mathrm{~kg} \mathrm{~cm}-2, \\
\text { for compression, } & \sigma_{p}=10^{-4} /\left(2.31 \times 10^{-6} \mathrm{~cm}^{2} \mathrm{~kg}^{-1}\right)=43.3 \mathrm{~kg} \mathrm{~cm}^{-2} .
\end{array}
$$

\subsection{Resistance Changes Due to Stress Transverse to the Current Flow}

\subsubsection{Tension}

Radial pressure is exerted on the manganin cylinder of Figure 3 by the cooling water causing tension in a direction transverse to the current flow. Resistance coefficients for transverse stress could not be found in the literature and were, therefore, measured on the shunt of Figure 3 with the circuit of Figure 6 . In this circuit, after standardization of potentiometer $P_{1}$, the voltage drops across the reference resistor $R_{S}$ and the coaxial shunt $R_{x}$ were compensated to zero. The value of $R_{x}$ is then computed from the potentiometer readings and the known value of $R_{S}$. Corrections for variations in current between the successive measurements of $R_{S}$ and $R_{x}$ with $P_{1}$ were made by monitoring these fluctuations with potentiometer $P_{2}$. Measurement accuracy was $0.02 \%$.

The relation of resistance to radial pressure in the manganin cylinder of the $200 \mu \Omega$ coaxial shunt was measured three times, and the resistance found to be a linear function of stress within an experimental error of $\pm 4 \%$. From this the resistance-stress coefficient for tension, transverse to the current flow, was calculated as 
$a_{t}^{1}=-0.78 \times 10^{-6} \mathrm{~cm}^{2} \mathrm{~kg}^{-1}$. The water pressures for shunts $A$ and $B$ are $\leq 0.36$ and $\leq 0.47 \mathrm{~kg} \mathrm{~cm}^{-2}$, causing a change in resistance of $\geq-0.0053$ and $\geq-0.0023 \%$, respectively.
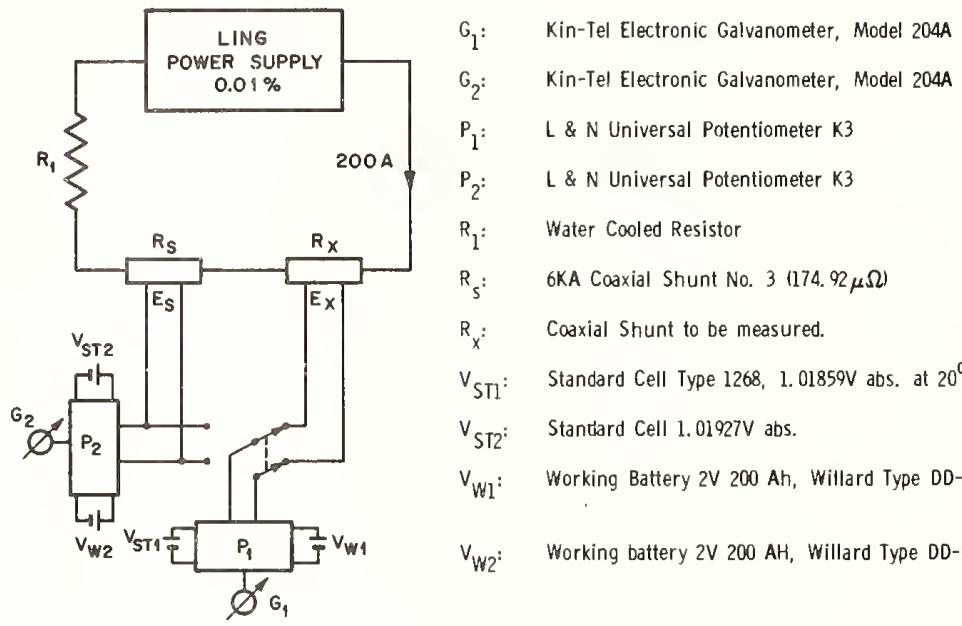

$R_{s}: \quad$ 6KA Coaxial Shunt No. $3(174.92 \mu \Omega)$

$R_{x}: \quad$ Coaxial Shunt to be measured.

$\mathrm{V}_{\mathrm{STI}}: \quad$ Standard Cell Type $1268,1.01859 \mathrm{~V}$ abs. at $20^{\circ} \mathrm{C}$

Figure 6. Resistance measuring circuit

$\mathrm{V}_{\mathrm{ST} 2}: \quad$ Standard Cell $1.01927 \mathrm{~V}$ abs

$\mathrm{V}_{\text {Wl }}$ : Working Battery $2 \mathrm{~V} 200 \mathrm{Ah}$, Willard Type DD-5-1.

$\mathrm{V}_{\mathrm{W} 2} \quad$ Working battery $2 \mathrm{~V} 200 \mathrm{AH}$, Willard Type DD-5-1

\subsubsection{Compression}

Circumferential pressure is exerted on the manganin cylinder of Figure 7 by the coolant causing compression transverse to the current flow. The resistance-stress coefficient for transverse compressive stress was measured in the circuit of Figure 6 as a $\frac{1}{C}=1.73 \times 10^{-6}$ $\mathrm{cm}^{2} \mathrm{~kg}^{-1}$.

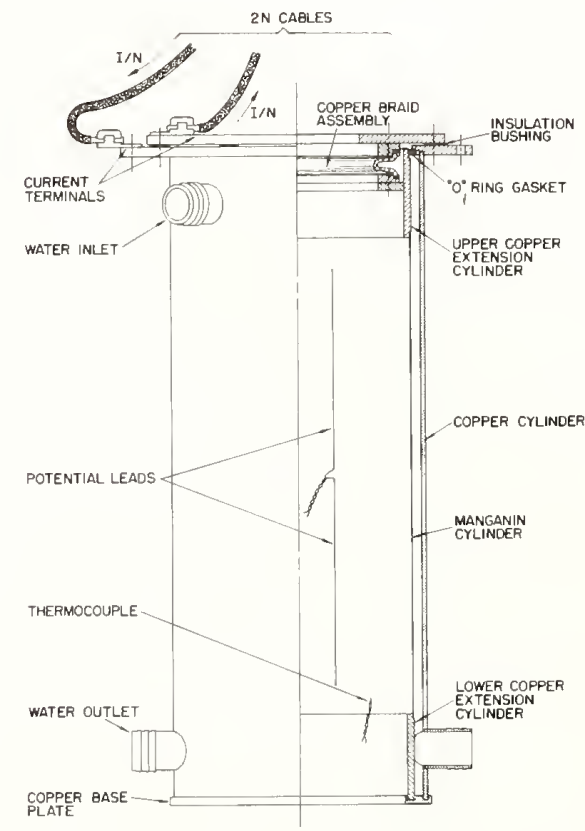

Figure 7. Water-cooled shunt

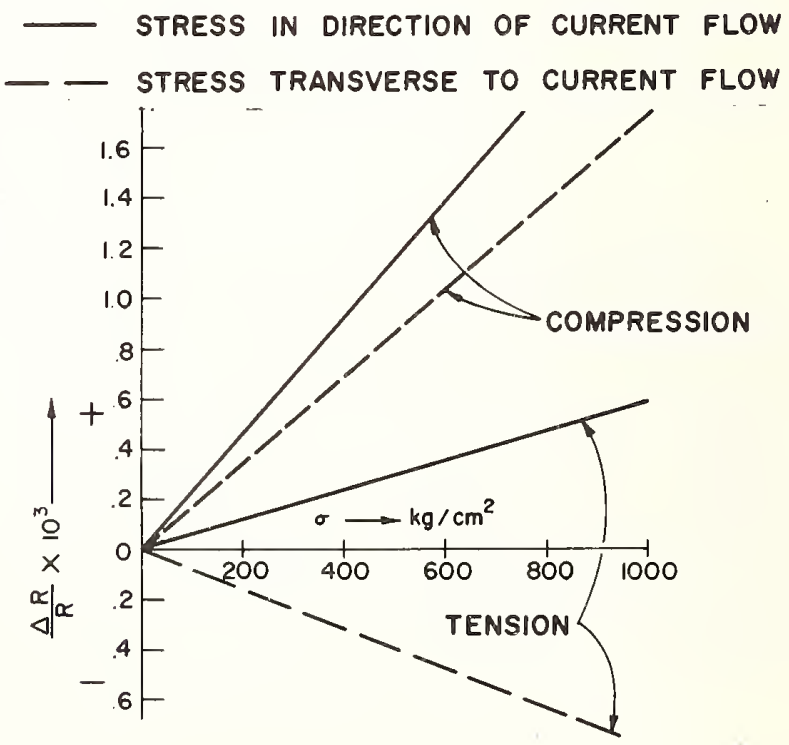

Figure 8. Resistance-stress sensitivity of manganin 
The resistance stress coefficients of manganin in $\mathrm{cm}^{2} \mathrm{~kg}^{-1} \times 10^{-6}$ are:

For tension in the direction of current

For tension transverse to the direction of current

For compression in the direction of current

For compression transverse to the direction of current

$$
\begin{aligned}
& a_{t}^{\prime \prime}=0.59, \\
& a_{t}^{1}=-0.78, \\
& a_{c}^{\prime \prime}=2.31, \\
& a_{c}^{\frac{1}{c}}=1.73,
\end{aligned}
$$

Figure 8 illustrates the resistance-stress sensitivity of manganin.

\section{Sources of Stress}

\subsection{Longitudinal Thermal Stress}

The thermal stress $\sigma$ induced in a restrained bar by a temperature change $\Delta T$ is

$$
\sigma=E \alpha(\Delta T)
$$

where

$\alpha=$ coefficient of linear expansion $=18.7 \times 10^{-6}{ }^{\circ} \mathrm{C}^{-1}$ for manganin

$E=$ Young's modulus of elasticity $=1.39 \times 10^{6} \mathrm{~kg} \mathrm{~cm}^{-2}$

A resistance change of $0.01 \%$ due to compressive stress of $\sigma_{p}=43.3 \mathrm{~kg}$ $\mathrm{cm}^{-2}$, is caused if we increase the temperature of a rigidly mounted strip of manganin by

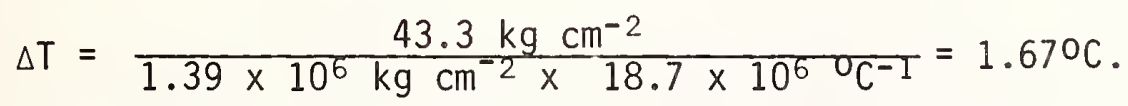

The resistance change due to thermally induced compressive stress is 15 times larger than the resistance change due to the temperature coefficient of resistance. It is apparent that the resistance material of a precision shunt must be free to dilate. The part of the manganin cylinder of Figure 3 that is embraced by potential leads can expand radially, and the 0-ring allows vertical movement.

One coaxial shunt of nominally $200 \mu \Omega$ (Type A) and four of nominally $175 \mu \Omega$ (Type B) were fabricated at ANL. Without cooling water, their resistances were measured at the NBS at $30^{\circ} \mathrm{C}$ and $40^{\circ} \mathrm{C}$ with direct current. Their change in resistance averaged $32 \mathrm{ppm}$ or $3.2 \mathrm{ppm}{ }^{\circ} \mathrm{C}^{-1}$ which can be fully explained by the temperature coefficient of resistance of manganin.

For comparison, two commercial shunts were also measured. Their resistance element consists of a number of spaced, parallel manganin strips secured at each end in massive copper terminal blocks. Their limit of error is stated as $0.04 \%$ at $3 \mathrm{kA}$. The change in resistance of these shunts over a temperature range from $35^{\circ} \mathrm{C}(1 \mathrm{kA})$ to $49^{\circ} \mathrm{C}(2 \mathrm{kA})$ 
averaged $702 \mathrm{ppm}$ or $50 \mathrm{ppm}{ }^{\circ} \mathrm{C}^{-1}$. This is 16 times larger than what could be explained by the temperature coefficient of resistance. This additional change is due to thermally induced compressive stress in the manganin strips.

\subsection{Pressure of Coolant}

Radial pressure is exerted on the manganin cylinder by the coolant (forced air, cooling liquid, etc.). The water pressures for the shunts of Table I cause a change in resistance of $\leq-0.0053 \%$ for shunt $A$ and $\leq-0.0023 \%$ for shunt $B$.

\subsection{Magnetic Forces}

The magnetic field produced by the current flowing through the coaxial cylinders will act upon the current itself. These magnetic forces will exert pressure on the outside of the inner cylinder, tending to shrink it, and pressure on the inside of the outer cylinder, tending to expand it. Expressed in $\mathrm{kg} \mathrm{cm}^{-2}$, these pressures are [2]

$$
\begin{aligned}
& P_{\text {in }}=0.2162 \times 10^{-3} I^{2} \frac{2 a+b}{b(a+b)^{2}} \\
& P_{\text {out }}=0.2162 \times 10^{-3} I^{2} \frac{a^{\prime}+2 b^{\prime}}{a^{\prime}\left(a^{\prime}+b^{\prime}\right)^{2}}
\end{aligned}
$$

where

$$
\begin{aligned}
I & =\text { shunt current in amperes, 'and } \\
a, b, a .^{\prime}, b^{\prime} & =c m .
\end{aligned}
$$

For the manganin cylinders of shunts Type $A$ and $B$, the radial magnetic pressures at $10 \mathrm{kA}$ are $0.001 \mathrm{~kg} \mathrm{~cm}^{-2}$ and $0.0029 \mathrm{~kg} \mathrm{~cm}^{-2}$, respectively.

\section{Alternate Coaxial Shunt Designs}

\subsection{Manganin Cylinder Cooled on Both Sides}

Shunts are often used in conjunction with water-cooled power supplies. Usually the water is at static pressures too high for onesided cooling. To relieve the manganin from static pressure both sides must be exposed to the coolant. Figure 9 illustrates a design with both sides of the manganin equally cooled. In the design of Figure 10 a few holes have been provided in the extension cylinder and in the disk soldered to the top of the manganin to relieve static pressure; here cooling is done essentially on the outside of the manganin. The allimportant freedom of vertical expansion for the manganin is assured by a copper braid assembly that connects the second current terminal to the manganin via an upper extension cylinder (Figure 9) or via a copper disk (Figure 10). 


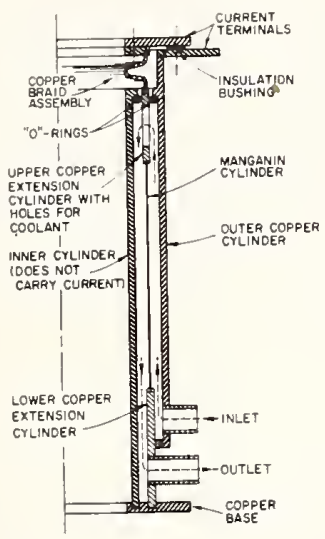

Figure 9. Shunt with manganin cooled on both sides.

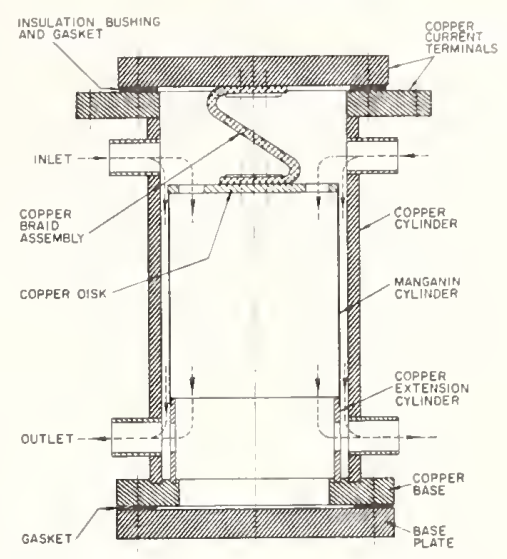

Figure 10. Shunt cooled on outside but without static pressure on manganin

\subsection{Air Cooled Shunts}

Convection cooling can dissipate approximately $0.04 \mathrm{~W} \mathrm{~cm}^{-2}$ at a temperature rise of about $15^{\circ} \mathrm{C}$ above a $20^{\circ} \mathrm{C}$ ambient. Forced air cooling will increase the dissipation by up to an order of magnitude. An inexpensive design is illustrated by Figure 11. End effects are reduced by arranging cables, to connect the shunt to the external buswork, symmetrically around the axis of the shunt as shown in Figures 7 and 12 .

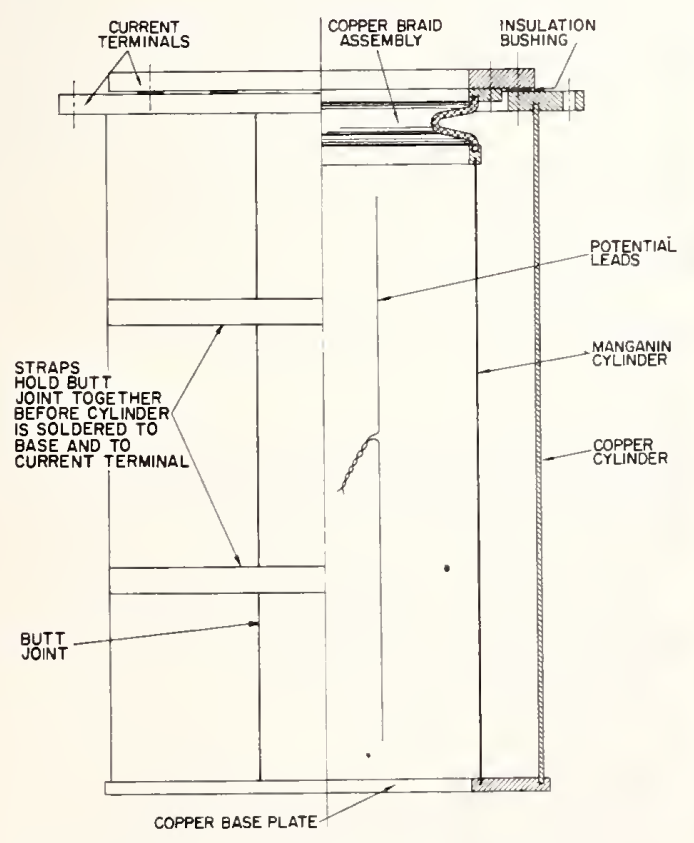

Figure 11. Assembly view of air-cooled shunt

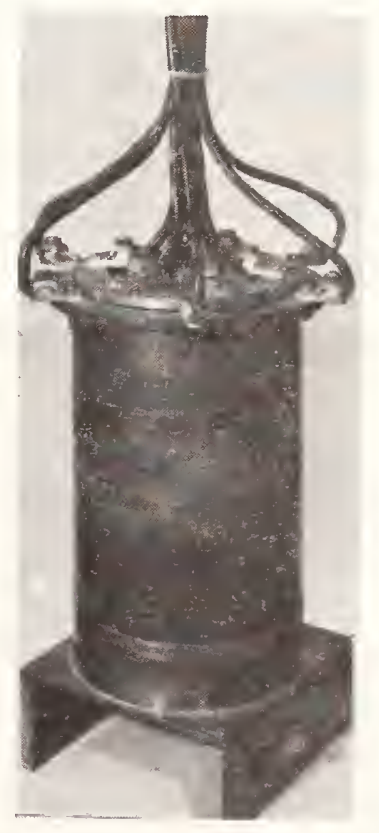

Figure 12

External connections

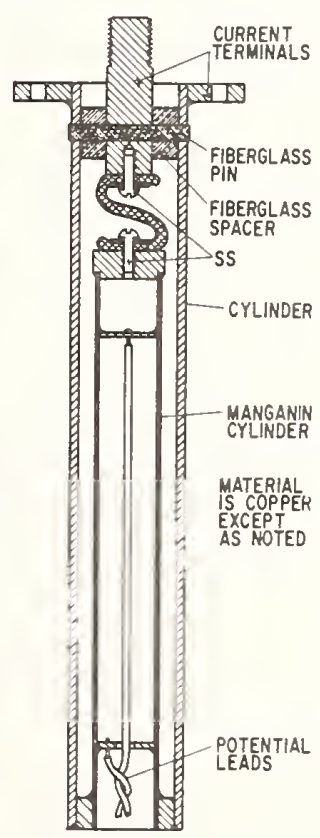

Figure 13. $60 \mathrm{~A}$ shunt 


\section{Measuring Very Large Currents Via Current Transformers}

Single sinewave current pulses of $\leq 300 \mathrm{kA}$ and $167 \mathrm{~Hz}$ or $3.5 \mathrm{kHz}$, into pion focusing horns, were measured by connecting two current transformers (CT's) in series and terminating them with a coaxial shunt. The focusing horn is the one-turn primary threading through the $92 \mathrm{~cm}$ ID of the toroidal core of a CT with a 1:25 turns ratio. This CT connects to a $1: 200$ CT which is terminated by the $500 \mu \Omega, 60 \mathrm{~A}$ shunt shown in Figure 13.

\section{References}

[1] F. B. Silsbee, "Notes on the Design of Four-Terminal Resistance Standards for Alternating Current," NBS Research Paper RP. 133, Vo1. 4, January 1930.

[2] J. H. Park, "Shunts and Inductors for Surge-Current Measurements," National Bureau of Standards, Washington, D.C., NBS Research Paper RP 1823, Vol. 30, September 1947.

[3] "A Study of the Inductance of Four-Terminal Resistance Standards," Bulletin of the Bureau of Standards, Vol. 13, pp. 375-421, June 1916.

[4] J. L. Thomas, "Precision Resistors and Their Measurements," NBS Circular 470, October 1948.

[5] W. O'Nei11, "Error Voltages Induced in Potential Circuit of Coaxial Shunt by Spatial Disparties in Current Terminal," Argonne National Laboratory, Argonne, IL, Particle Accelerator Internal Report WD0-33, July 18, 1966.

[6] P. Kapitza, "The Change of Electrical Conductivity in Strong Magnetic Fields," Proc Roy. Soc. (London), Vol. 123, pp. 292372, March 1929.

[7] Smithsonian Physical Tables. Washington, D.C.: Smithsonian Inst., p. $463,1959$.

[8] P. W. Bridgman, "The Effect of Tension on the Electrical Resistance of Certain Abnormal Metals," Am Acad. Proc., Vol. 57, pp. 41-66, April 1922.

[9] J. 0. Linde, A. von Heijne, and E. von Sabsay, "A Study of the Effect of Tension on the Electrical Resistance of Alloys," Arkiv fur Fysik, Bd. 2, No. 10, pp. 81-87, 1950-51.

[10] I. Vigness, "Investigation of Stress-Strain Relations of Meta Wires by Electrical Resistance Changes," J. Appl. Phys., Vol. 23, pp. 43-47, January 1952.

Work supported by the U. S. Department of Energy 


\title{
MEASURING FAST PULSE CURRENT USING LOU \\ INDUCTANCE CURRENT VIEUING RESISTORS AND di/dt PROBES
}

\author{
C. A. Muehzenweg \\ Engineering Manager \\ T \& II Research Products, Inc. \\ Azbuquerque, New Rexico 87108 \\ G. McDuff BSEE \\ Los Alamos Scientific Laboratory \\ Los Alamos, New Mexico 87545
}

\section{Introduction}

The ever increasing use of thyratrons and stripline pulse forming networks (PFN) in pulsed circuits has created a need for an accurate, zow noise, extremely low inductance, wide bandpass method of current measurement. Recently designed Current Viewing Resistors (CVR) meet and surpass most of the requirements. Quality diagnostics are essential in the development of high efficiency, low zoss, pulsed accelerators and a variety of other pulsed powered circuits.

Research and development in the area of current measurements for specialized requirements has been in progress for at least two (2) years. The purpose of these efforts were two-fold. to develop a low inductance CUR that could easily be integrated into fast pulsed thyratron circuits and develop a method of directly measuring current rate of change (di/dt) at the high bandwidth.

This report contains the results of these efforts and a simplified description of how the CUR and di/dt probes were designed and fabricated.

\section{CUR Characteristics}

Typicalzy three (3) characteristics of CUR's are of particuler interest to the user: (1) input inductance, (2) frequency response and (3) puzse energy capacity. Good estimates of the input inductance of coaxiaz CUR's can generalzy be obtained from a summation of series coaxial cylinder of inductance by:

$$
L(n \mathrm{H} / \mathrm{m})=2 \times 10^{-7} \times \text { nat } \log \frac{\text { outer diameter }}{\text { inner diameter }}
$$

Similarly the inductance of a flat plat CUR (stripline) may be computed from: 


$$
L(n H)=2 P i x \frac{\text { separation between plates }(i n)}{\text { width of plates }(i n)} \times \text { zength }(i n) \times 2.54
$$

and, typicalzy, ranges from a few to less than one nanohenry.

CUR frequency response is primarizy determined by skin effect current distribution in the resistance foiz. This mechanism was described in detail by J. H. Park in an analysis attributed to F. B. Sizsbee.

The important results of this analysis presented graphically in the Park article in an impedance versus a scaled frequency parameter functionalzy dependant upon foiz thickness and resistivity.

Pulse testing some years ago experimentalzy confirmed the theoretical analysis and all subsequent CUR design has been based upon this theory.

CUR frequency response generally ranges between $2000 \mathrm{mHz}_{\mathrm{z}}$ and a few 10 's of $\mathrm{KHz}$ and is typically coupled to the CUR energy requirements. This latter parameter, as would be expected, is primarizy dependant upon the resistance foil mass.

It folzows then, that the three (3) prime factors in CUR design (1) inductance, (2) bandpass, and (3) energy capacity are interdependent and, therefore, often necessitating design compromise.

\section{Thyatron CUR}

Resent advances in hydrogen thyratron technology have made these devices avaizable for a variety of applications. Uith the increasing use of the thyratron in R \& D experiments, the need for accurate diagnostics becomes a necessity.

Current Viewing Resistors are the most economical solution to the problem of accurate current measurements. CVR's are easizy custom tailored to the wide range of pulsed currents of thyratron circuits. The output signal of the CUR is clean and relatively noise free over several orders of magnitude. Due to the extremely low source impedance, noise due to EMI is reduced and the CUR is conveniently terminated into 50 ohms.

Several generations of CUR configurations were evolved in the program and two (2) basic designs were eventually developed.

The standard version has an inductance of 35 to $40 \mathrm{nH}$ and for circuits where inductance is critical, a low inductance unit of 3 to $5 \mathrm{nH}$ was developed. Both versions serve as the mounting flange for the respective thyratron. The standard cVR mounting flange is shown in Figure 1 and the low inductance version is shown in Figure 2. A closer look at both figures reveal

several important features.

Note the removable di/dt probes wrapped around the base of the CUR. These probes are discussed in detaiz later in this report.

Proper cooling is also an important feature to thyratron zife and two 
methods of cooling are provided on each CUR.

Thyratrons that are operated in oiz can utizize the open system of cooling. This method directs aiz onto the base of the thyratron and returns it to the environment through an exit port.

The inlet port (seen in Figure 1) is the lower hose brad and the exit port is the large square hole. For thyratrons operated at high power where more cooling is required, a closedsystem for chilled water is also provided. The closed system should be used for thyratrons operated in air to maintain the cathode at a safe operating temperature. The closed system cooling ring is directly beneath the cathode mounting flange as seen in Figure 1.

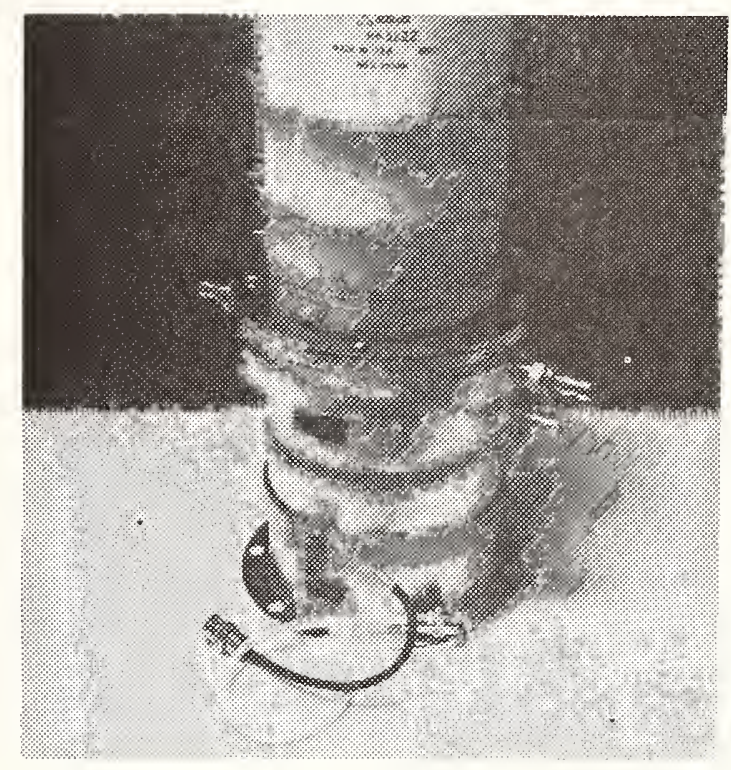

Figure 1. Standard thyratron CUR mounting flange

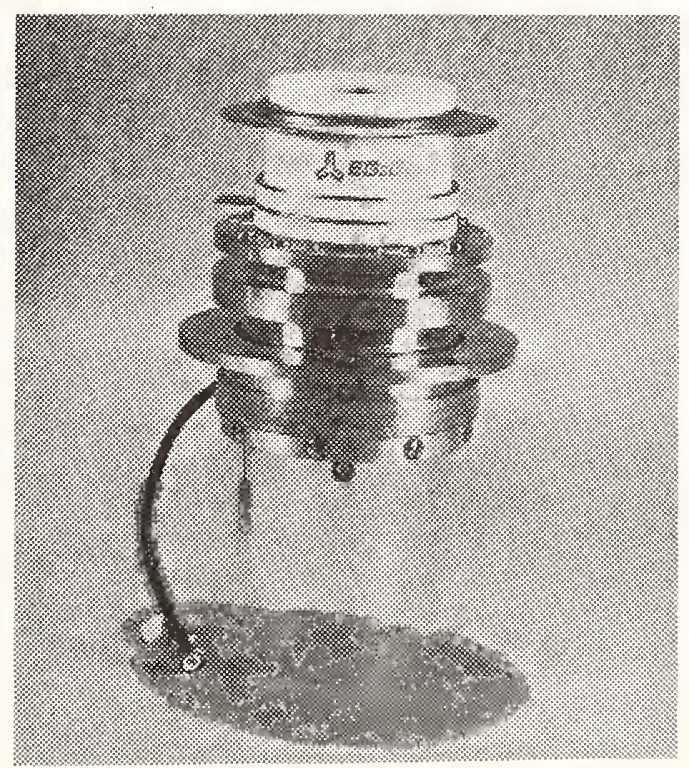

Figure 2. Low inductance thyratron CUR mounting flange 
The standard version CUR was tested in the circuit shoun in Figure 3.

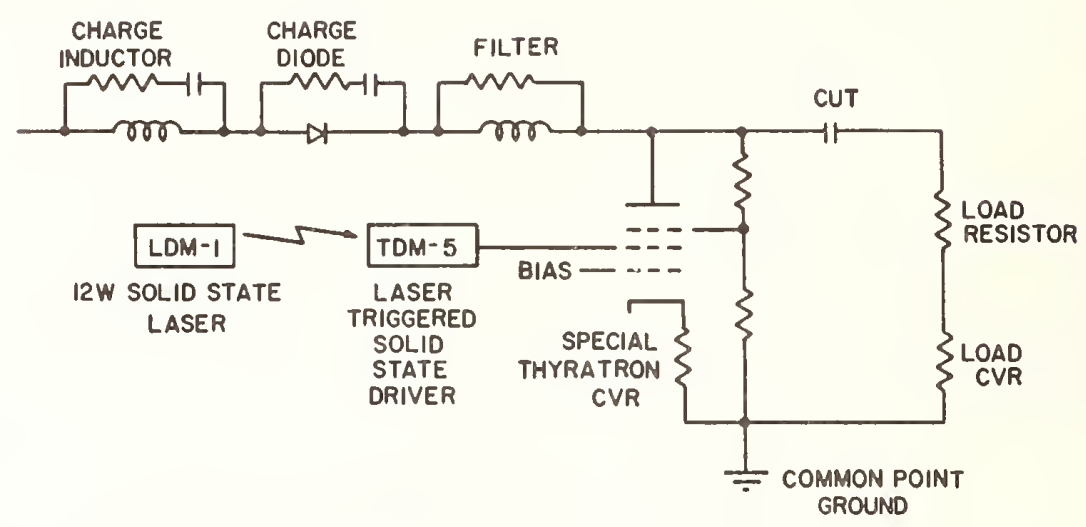

Figure 3. CUR test ciruit

Maximum test parameters for the CUR test circuit in Figure 3 are shown in Table 1.

Table 1. Test parameters for CUR test circuit

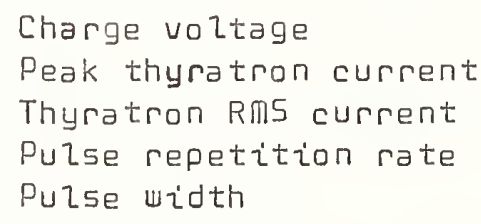

Charge voltage

Peak thyratron current

Pulse repetition rate

Pulse width

Sample output signals of the standard thyratron CUR are shown in Figure $4 A$ and Figure $4 B$.

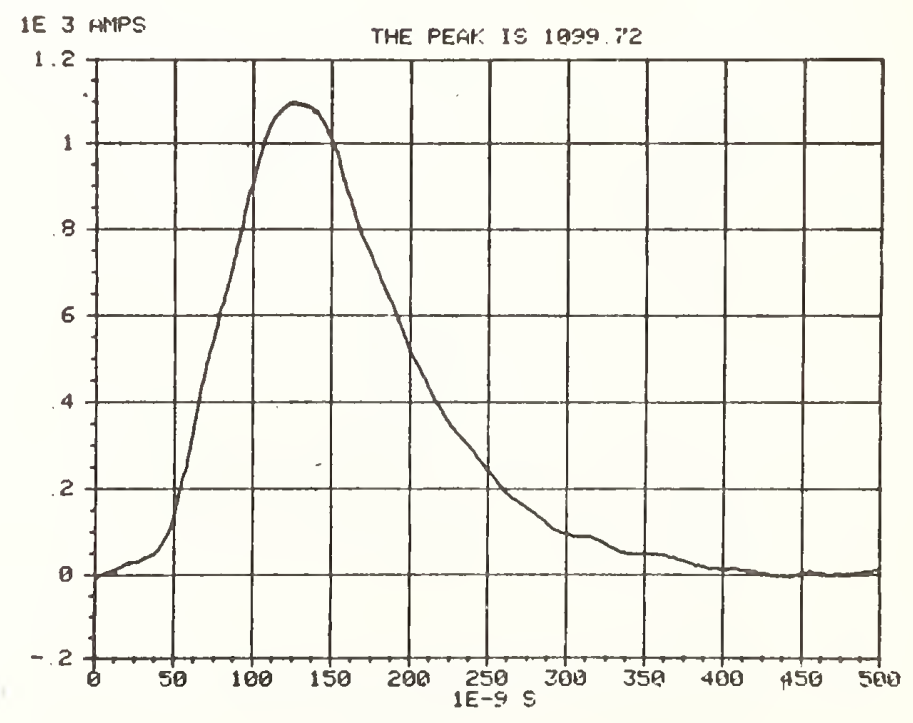

Figure $4 \mathrm{~A}$ 


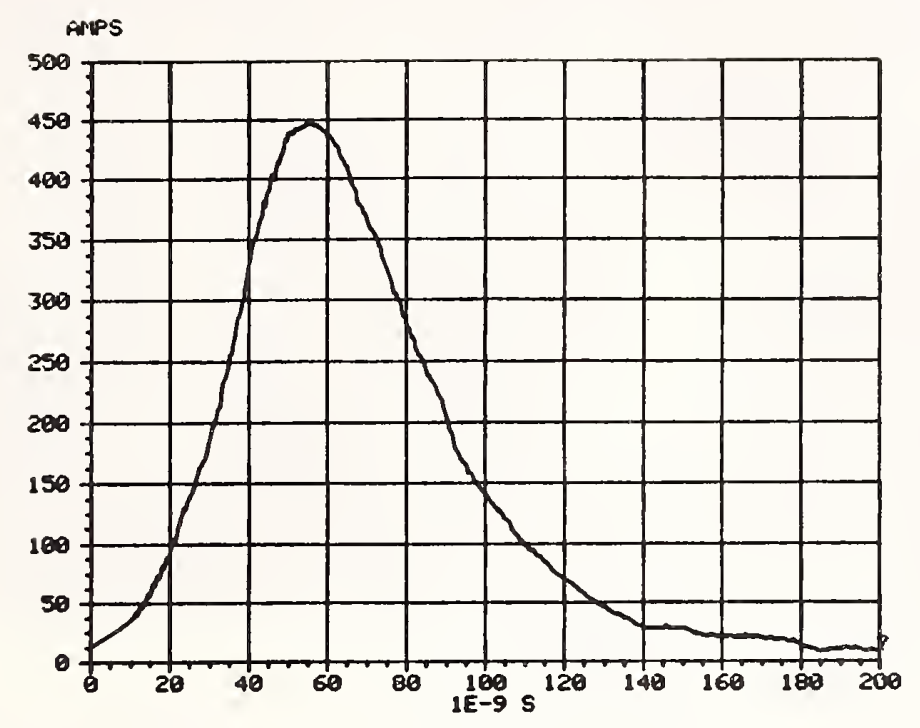

Figure 48.

The risetime of the standard CUR in a 50 ohm circuit with a mercury switch is equal to or less than 1ns. It is important to note that the noise free signal of the CUR at the low current level shown in Figure 4B.

Figure $4 \mathrm{~A}$ is the thyratron current seitching a 7.5nf capacitor at $25 \mathrm{KV}$. The discharge circuit is almost critically clamped to simulate the conditions at which the capacitor will be used in a laser application. Much lower current level, 450 amp peak, is shown in Figure $4 B$ as the thyratron current from a discharge of a 500pf capacitor at $25 \mathrm{kV}$. The risetime of the waveform is $35 n s$ which is also the switching time of the Hy5322 in this particular circuit.

Response of the low inductive version of these thyratron CUR's has been measured in a 50 ohm mercury switched circuit to be equal to or less than 300ps. This particular design provides an extremely low noise output. In fact, the output signal is noise free over three orders of magnitude from 10 amps to 1000 amps peak. Even though the CVR has not been tested at a 10KA level, there is no reason why it should not perform with comparable quality.

Two (2) of these CUR's are presentzy in use in paralzel thyratron arrangement seen in Figure 5.

The output signal of the low inductance CVR at a pulsed current of 15 amps is shown in Figure 6. The risetime of the current pulse in Figure $6 A$ is inductance zimited by the PFN. The output is noise free and the measured pulse current matches the calculated current exactly. 


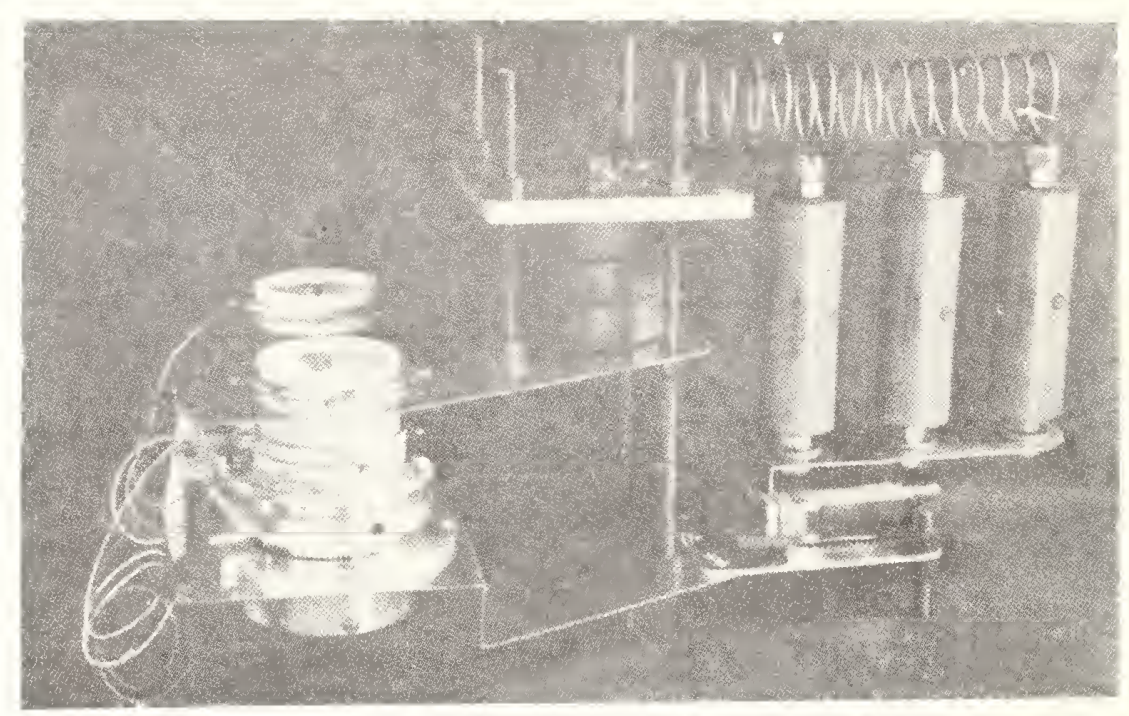

Figure 5.

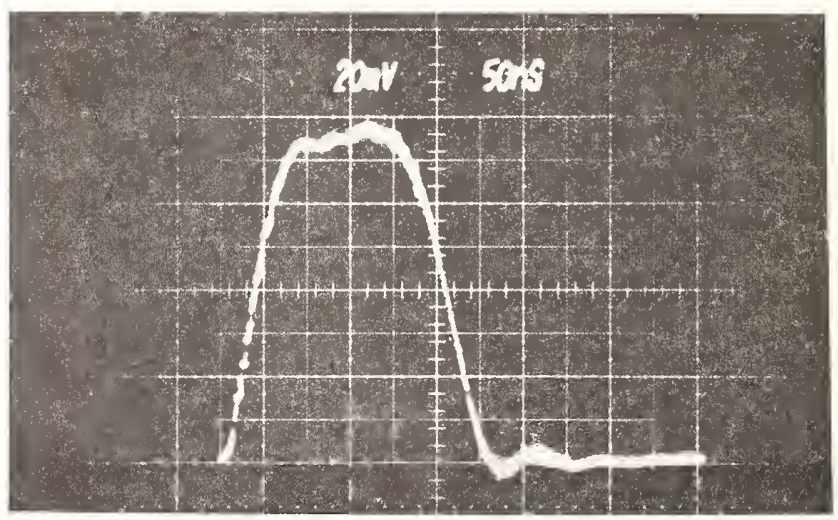

Figure 6.

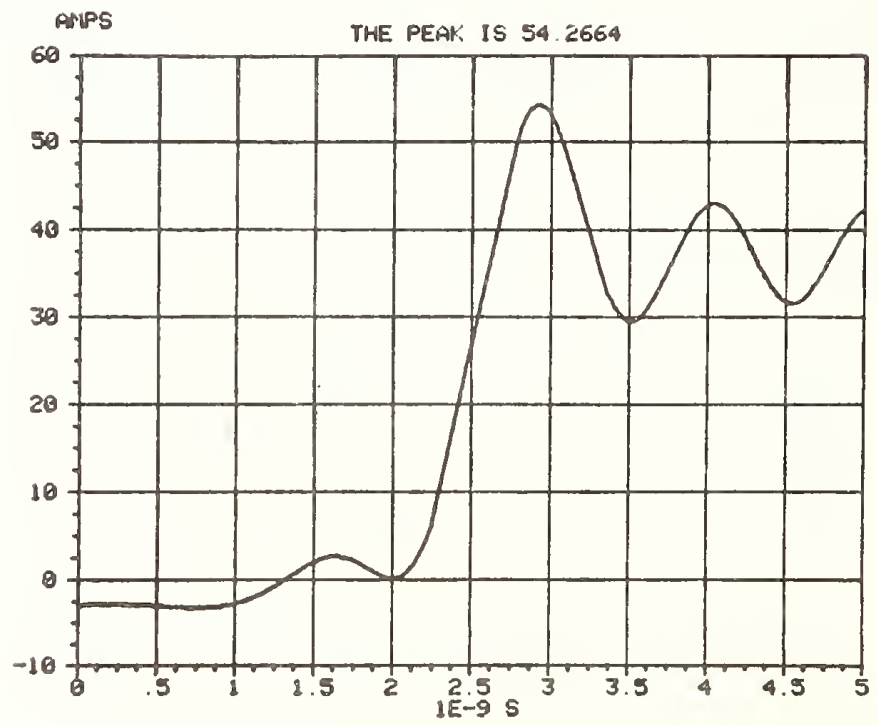

Figure 6A. 


\section{Stripline CUR - U Series}

The stripline type of energy storage network has become very common due to the faster risetime and shorter pulse requirements of many lasers. The stripline type CUR (Series $\mathbb{U}$ ) is the best method of monitoring the puzse currents in these fast networks and is shown in Figure 7. This type cuR have risetimes of 1 ns or less. These stripline CUR's range from a few to several feet in width and can be mounted directly in the current return on zaser heads of considerable uidth. Circuits using plate conductors (to minimize inductance) similar to the one shoun in Figure 8, are common applications of this flat cUR. Dutput signal of the flat CUR is shoun in Figure 9.

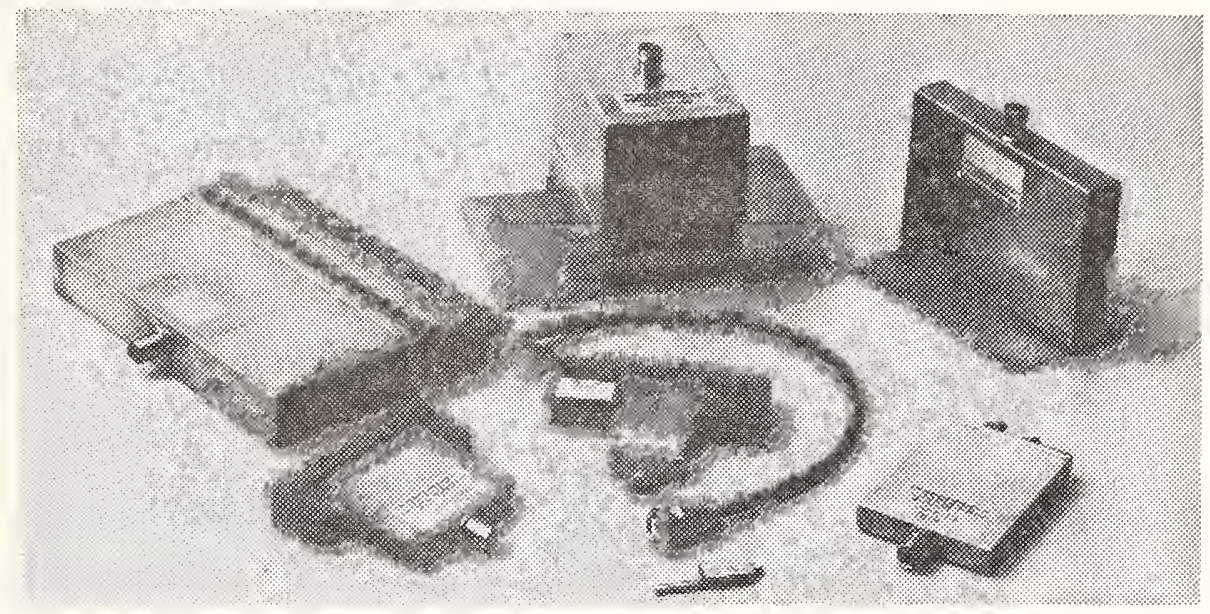

Figure 7.

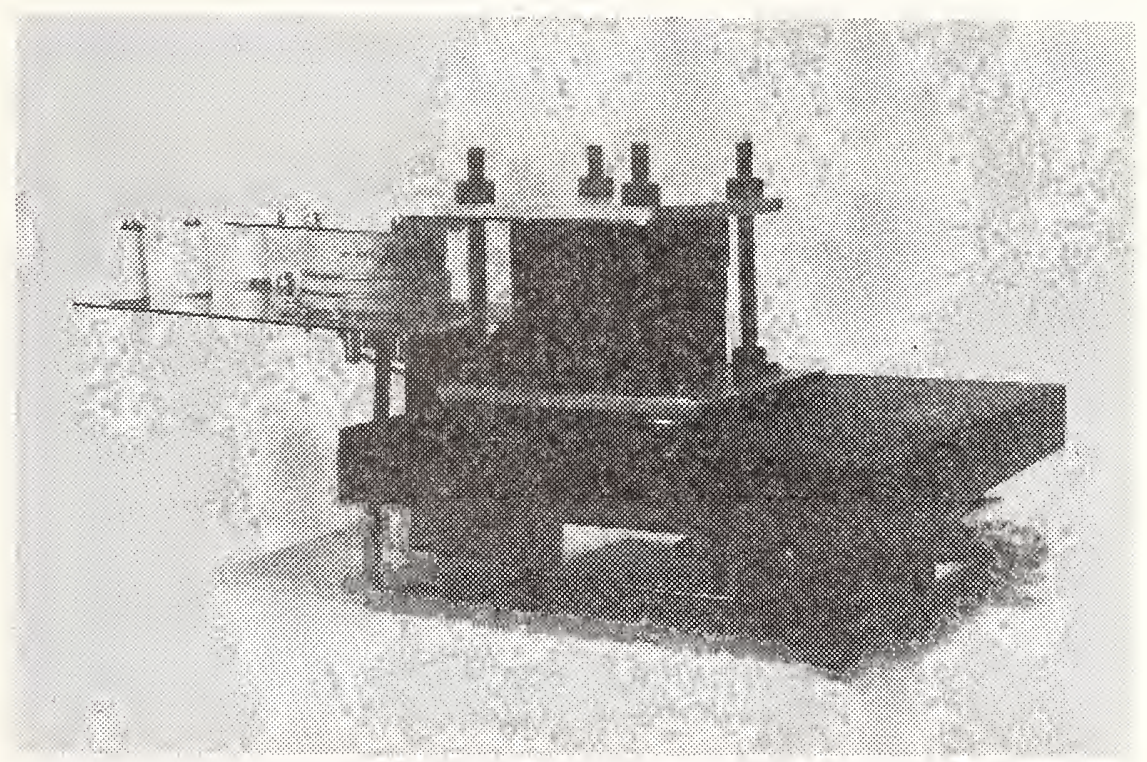

Figure 8. 


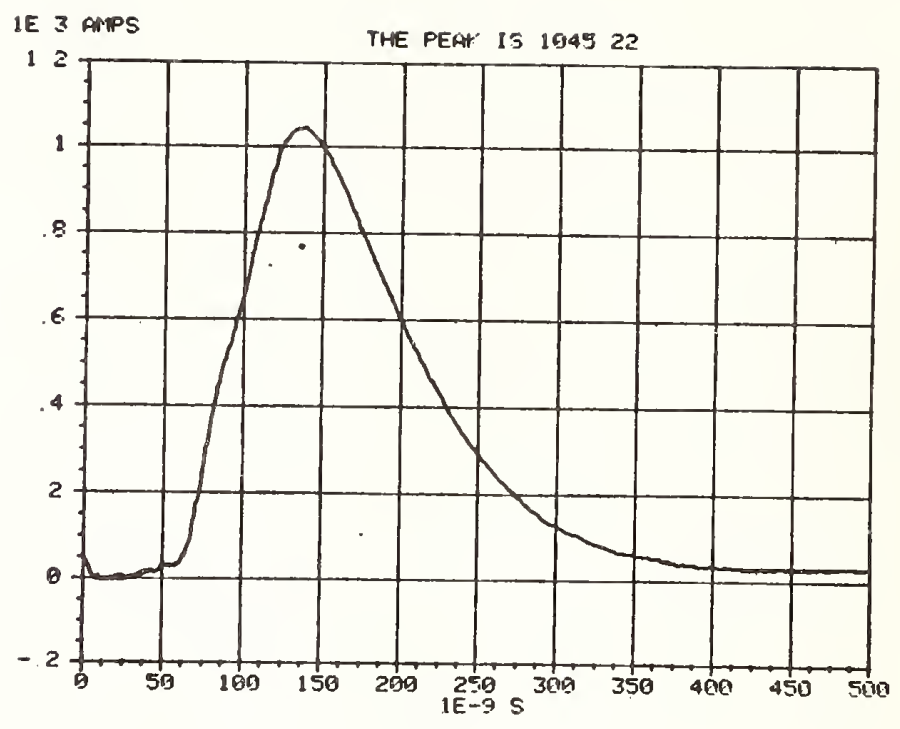

Figure 9.

This waveform is the discharge current pulse of a 7.5 nf capacitor at 25KV. It should be noted that this is the same current puzse as is shown in Figure $4 A$ and that was measured with the thyratron CUR. Since the thyratron CUR and the flat CUR were used in the same loop as seen in Figure 3 , the currents shouzd be identical. Superimposing the output 5 ignals of the load CVR and the thyratron CVR in the discharge loop of a 500pf capacitor at $25 \mathrm{kV}$, we get the waveforms shown in Figure 10.

The flat CUR's have a measured bandwidth of OC to $300 \mathrm{mHz}$ in a $50 \mathrm{ohm}$ circuit with a mercury switch. Figure 6 A shows the risetime of the mercury pulser and is less than 1ns and the risetime of the CVR to be faster than the PFN. .

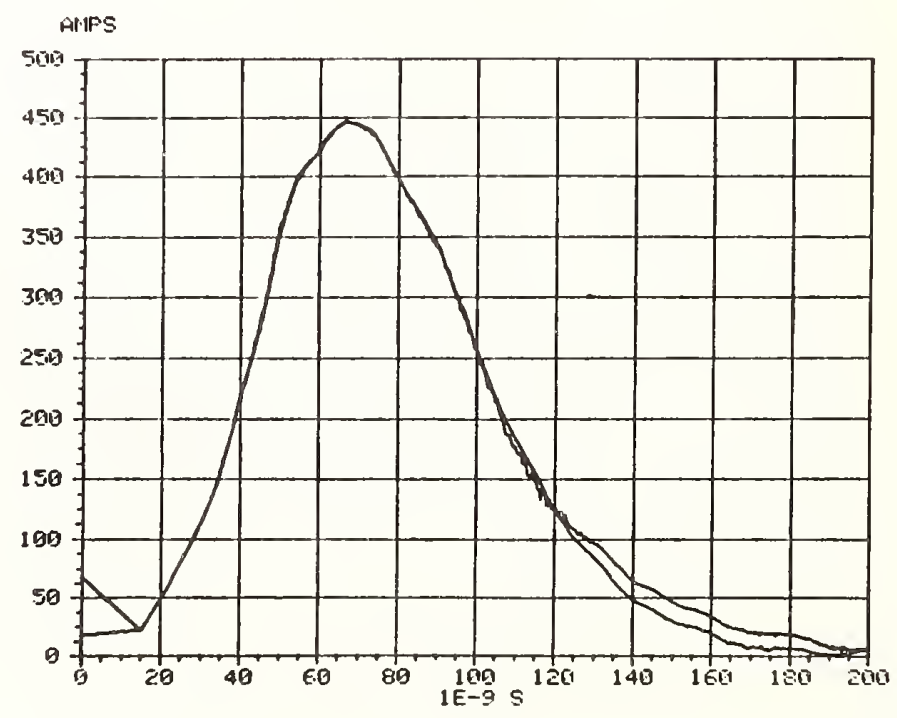

Figure 10. 


\section{5. di/dt Probes}

Many experimental systems where isolation requirements may require the use of urap around Rogouski coils. Two specifications are generally required for probe selection, they are sensitivity and bandpass. Keeping within the operational frequency range, the probe output voltage is given by the formula:

$$
\text { Output voltage }=\frac{\text { Ampsper microsecond (di/dt) }}{\text { Probe's sensitivity }(5)}
$$

The probe's sensitivity is depentent upon the probe's mutual inductance, resistance of the probe, and load impedance as shown below:

Sensitivity $(5)=\frac{\text { Impedance }(z)+\text { Resistance(ohms) }}{\text { Impedance( }(z)} \times$ mutuaz Inductance (m)

mutuaz Inductance $(m)=$ Inductance $(n h) \times$ i (Amps per microsecond)

Bandpass can be similarly shown to be primarizy zimited by the potential drop across the probess self-inductance. Neglecting resonance effects the bandpass (3db down point) is given by:

$$
\text { Bandwidth }(H z)=\frac{\text { Resistance (ohms) }+ \text { Impedance }(\mathrm{Z})}{\text { Inductance }(\mathrm{L})}
$$

However, it should be noted that probe oscizlations may dominate over this simple relationship.

In'the development of many pulsed power devices, "risetime" and "rate of rise" of currents becomes important quantities. Direct measurement of risetime was simplified with the use of computers and waveform digitizers and the rate of rise is measured directly by di/dt probes like the one shown in Figure 11A.

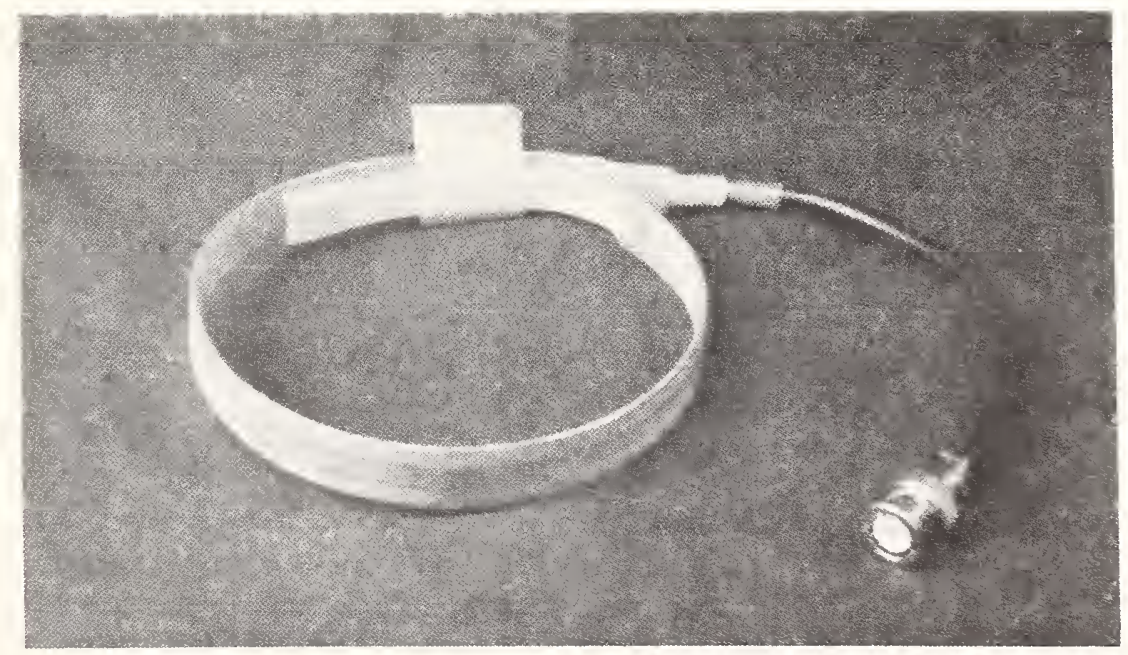

Figure 11A. 
The di/dt probe is placed around the conductor of the discharge loop. When used in high peak power pulsed circuits, insertion is negligible.

Another type of probe is simply placed on a conducting surface. This type probe, shoun Figure 11B, is particularly useful in stripline circuits. An important feature of this type probe is that it does not have to encircle the conductor. This is especialzy advantagous in extremely high voltage circuits. Both types of these flat di/dt probes must be terminated into 50 ohms and then read by the test equipment or integrated by a passive integrator.

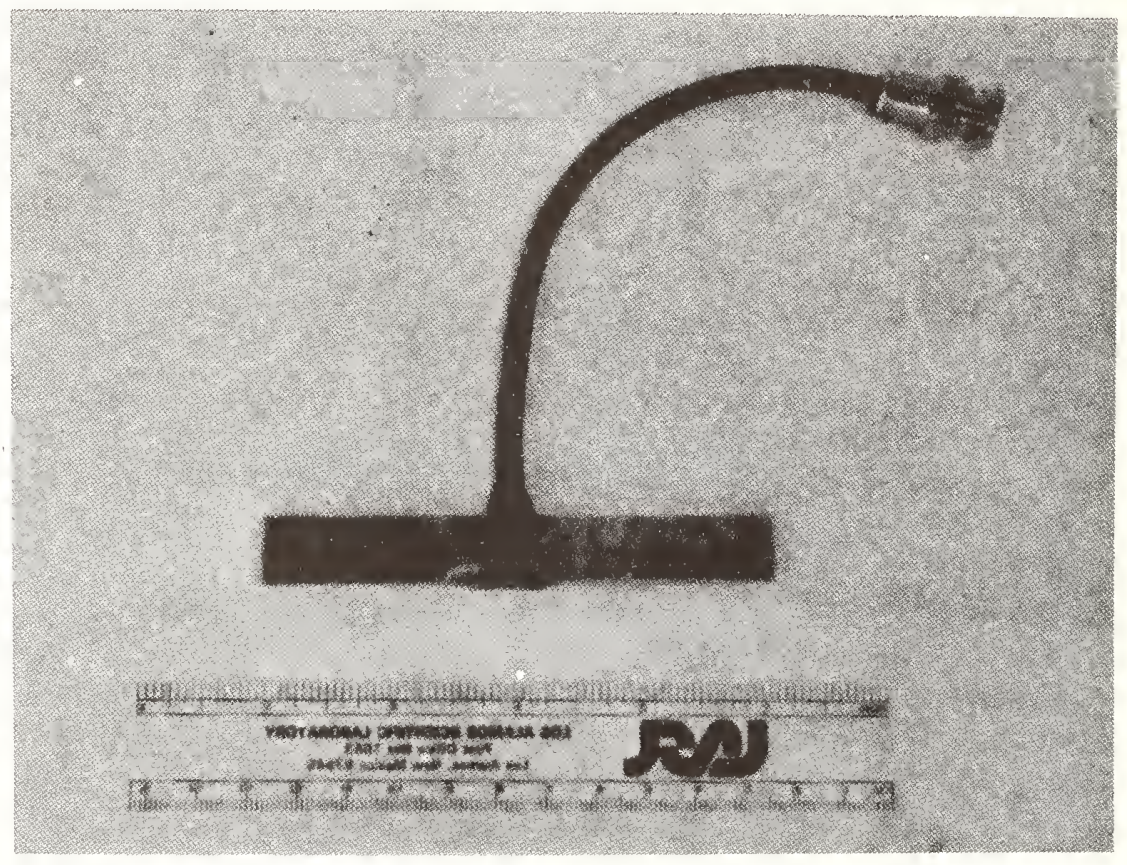

Figure 11B.

Figure $12 \mathrm{~A}$ shows the output of the di/dt probe supplied with the CVR for a Hy-5 Thyratron.

Figure 12B shows the output of the passive integrator when fed by the output of the di/dt probe. Thyratron.

Figure 12C shows the output of the CVR itself when attached to a HY-5

This integration of the di/dt signal gives a true current waveform output.

When computor controlzed digitizers are being used then the probe output can be integrated by a computor program. A sample program using a Tektronic Word Processor Transient Digitizer is shown in Appendix A. This program prints a graph of the di/dt signa2, maximum di/dt rate, and a graph of the integrated di/dt signaz. 


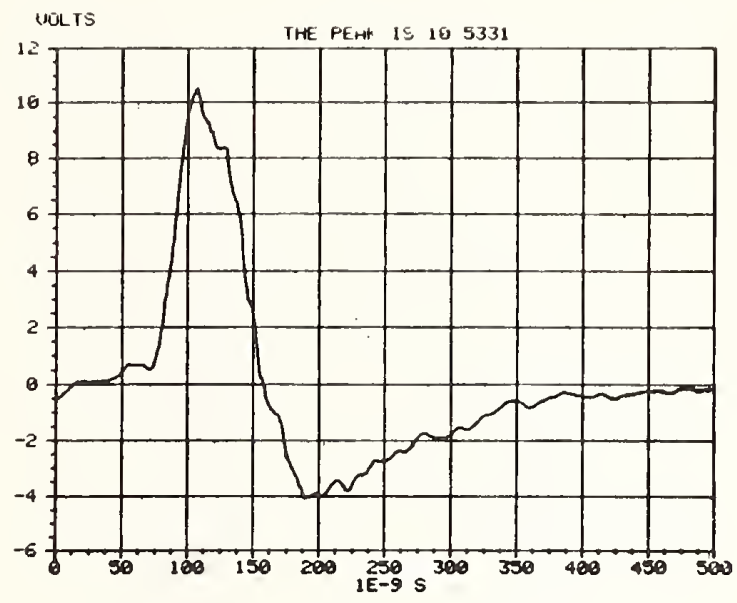

Figure $12 \mathrm{~A}$.

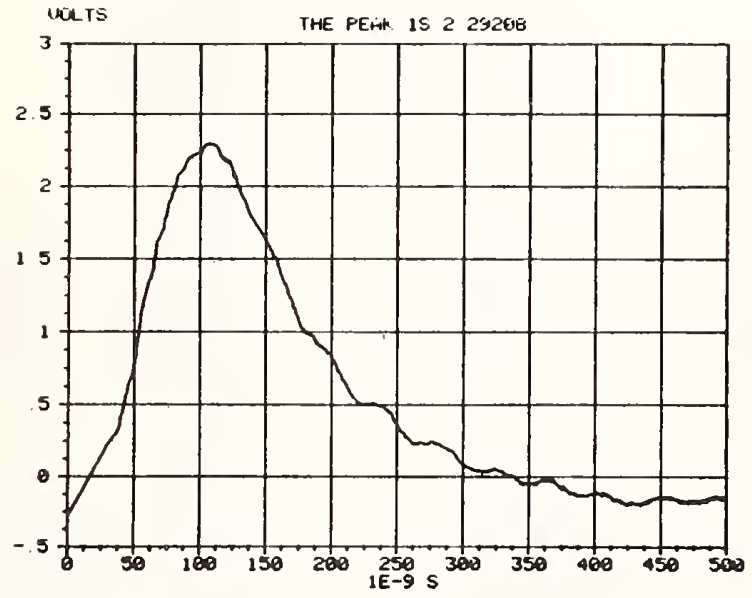

Figure $12 \mathrm{~B}$.

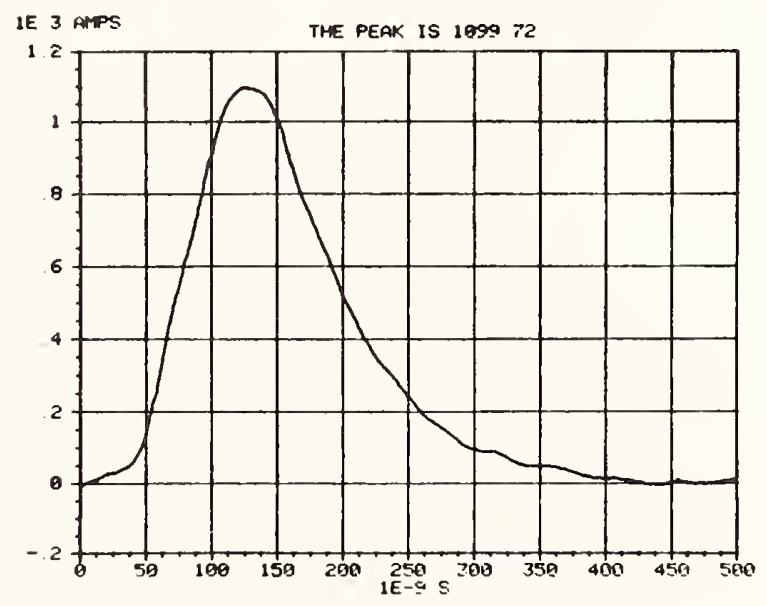

Figure $12 C$. 


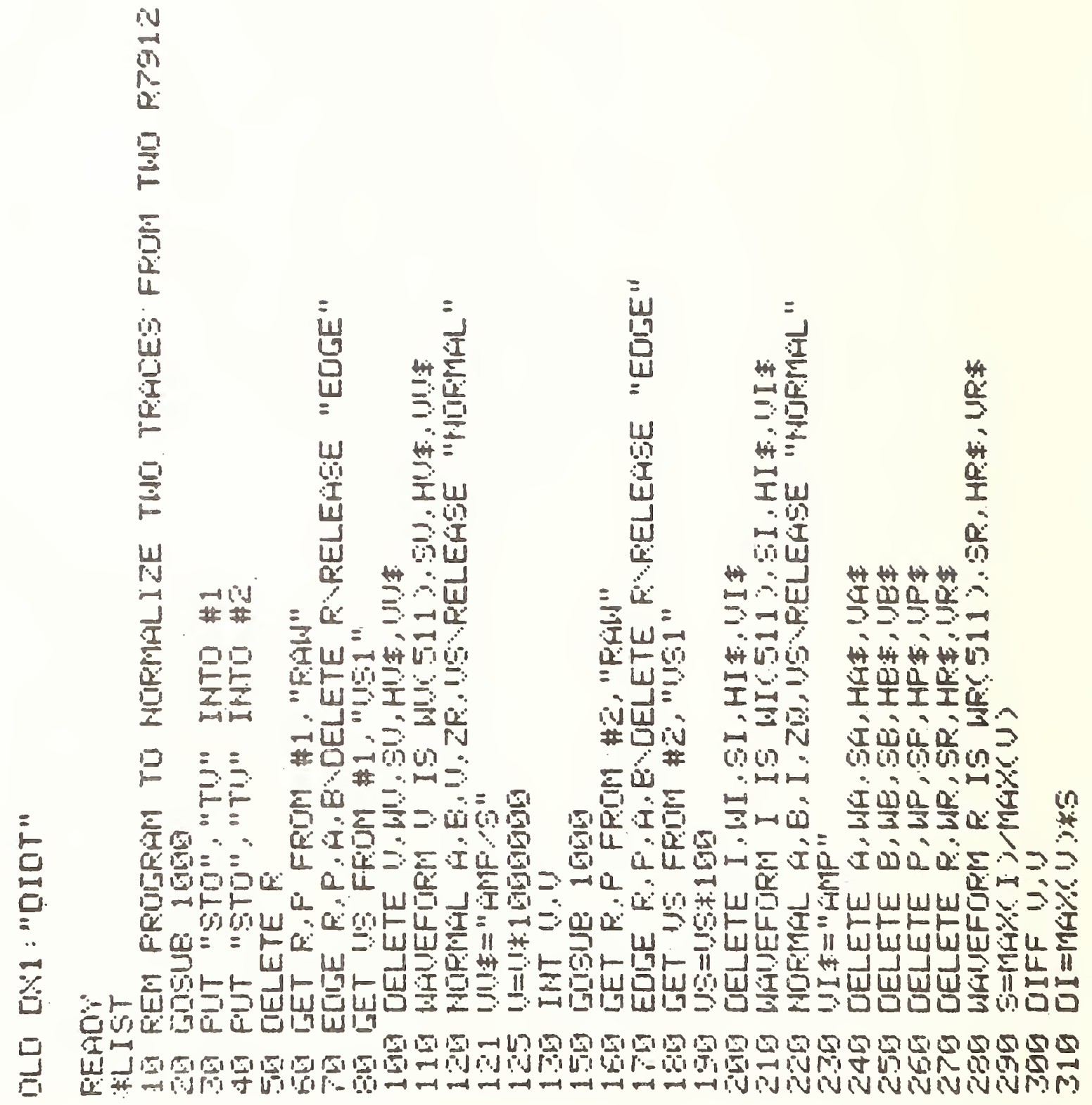




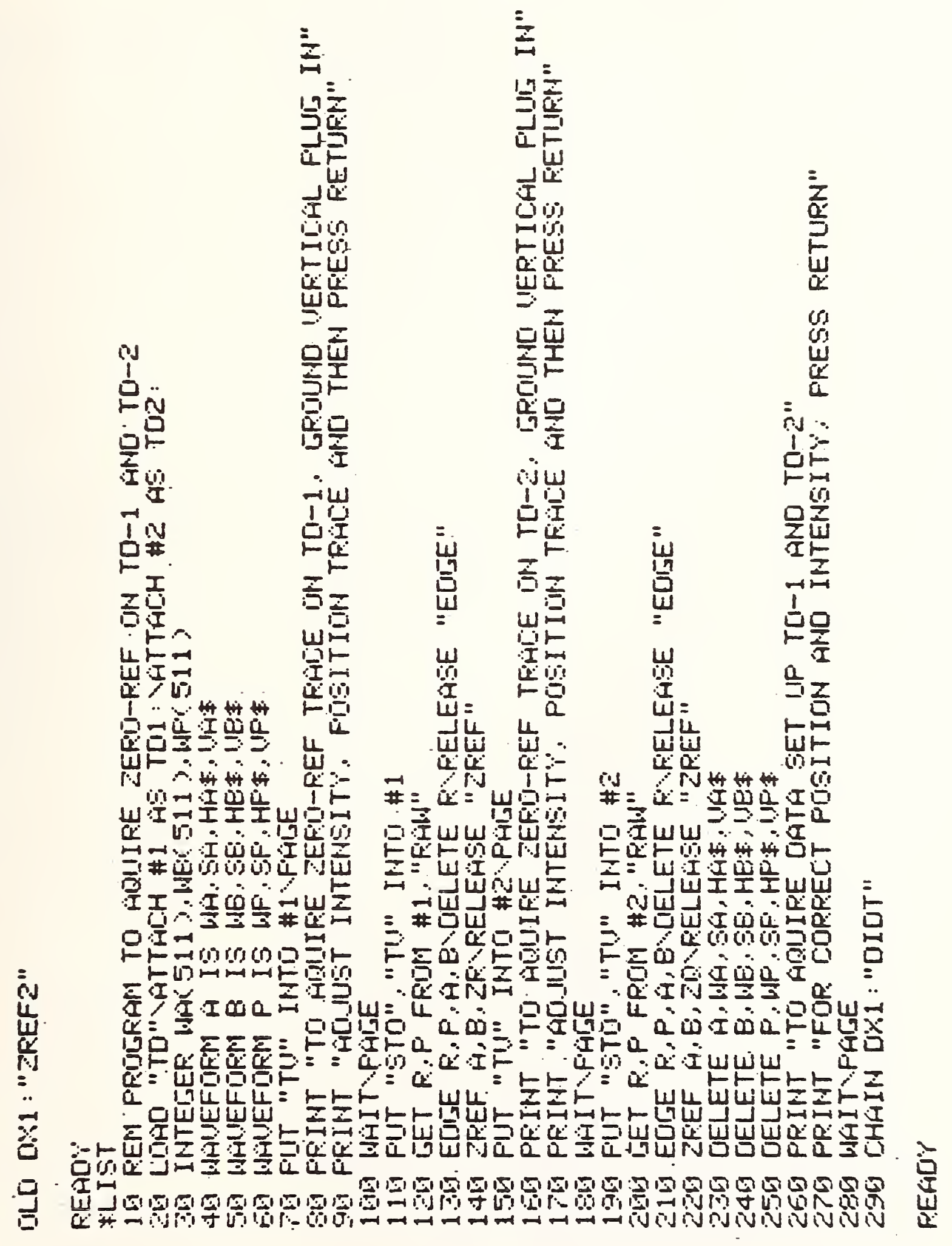




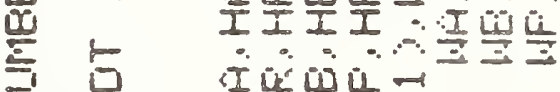

is

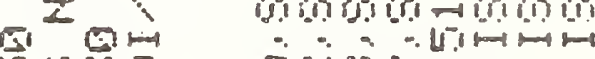
$\vec{r}(6) \sin$ P:I $\frac{\alpha}{3} \frac{\omega}{3}=I$ I -

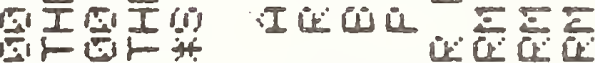

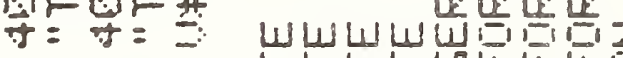

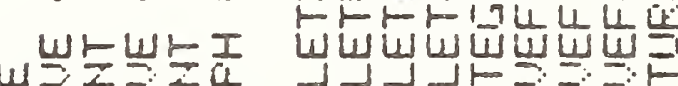

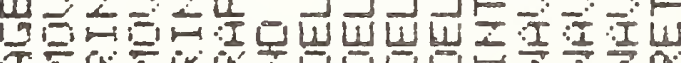

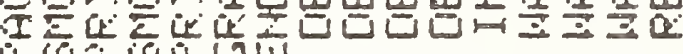

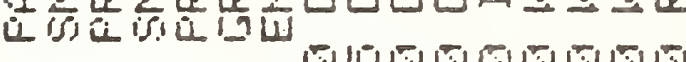

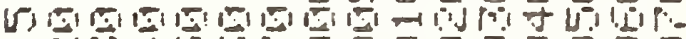

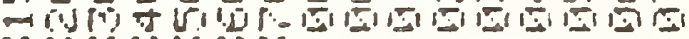

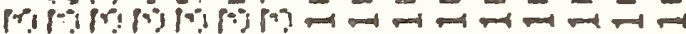


Actual Output

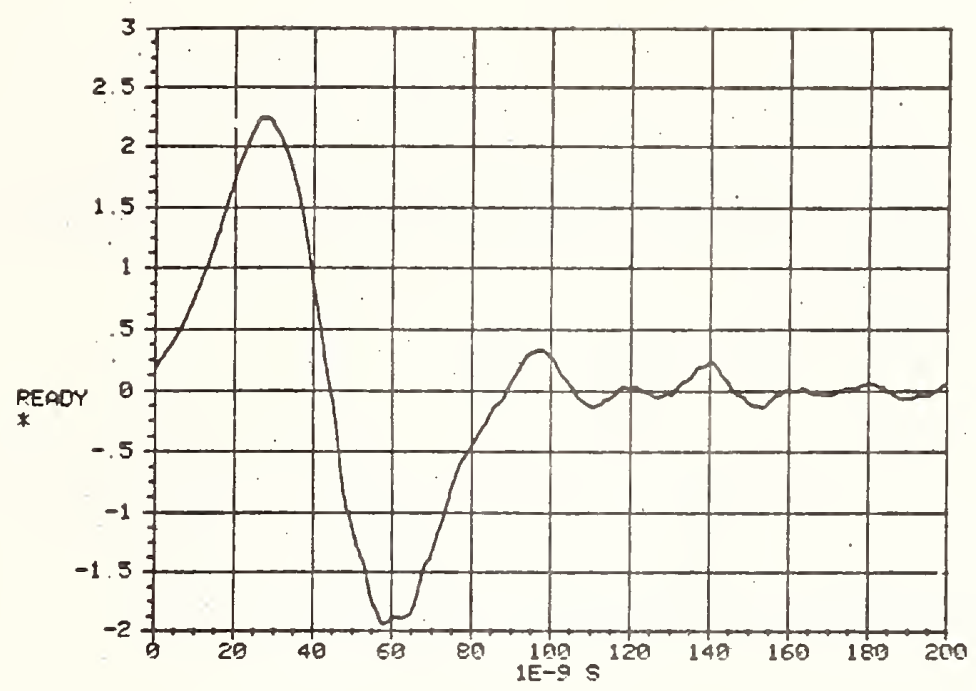

Real time plot of $d i / d t$

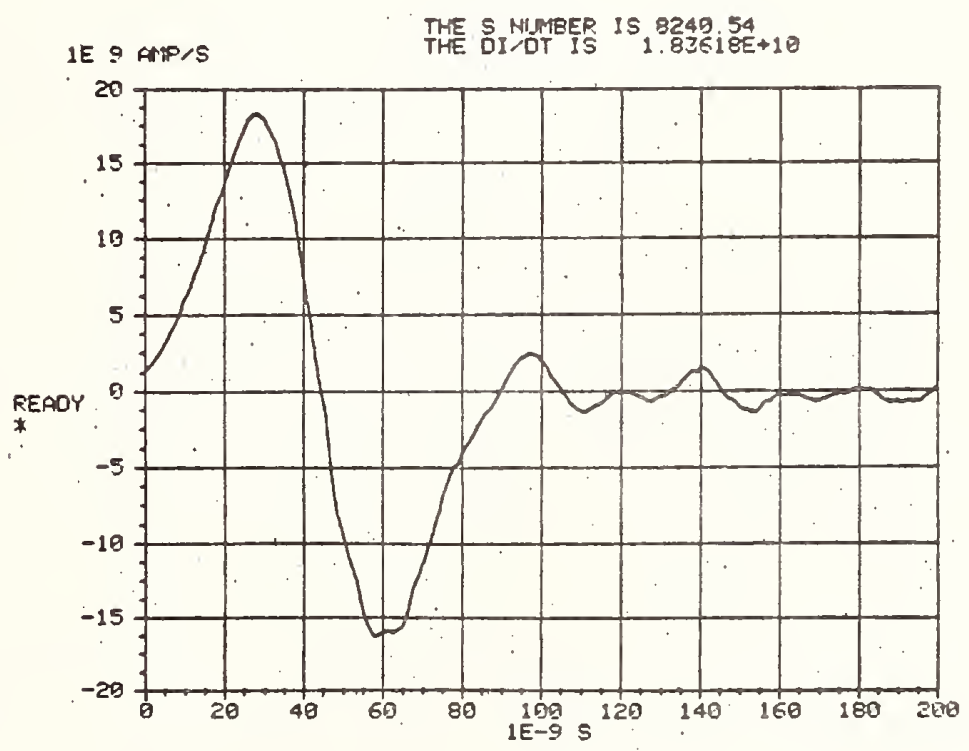

Integrated

Dutput

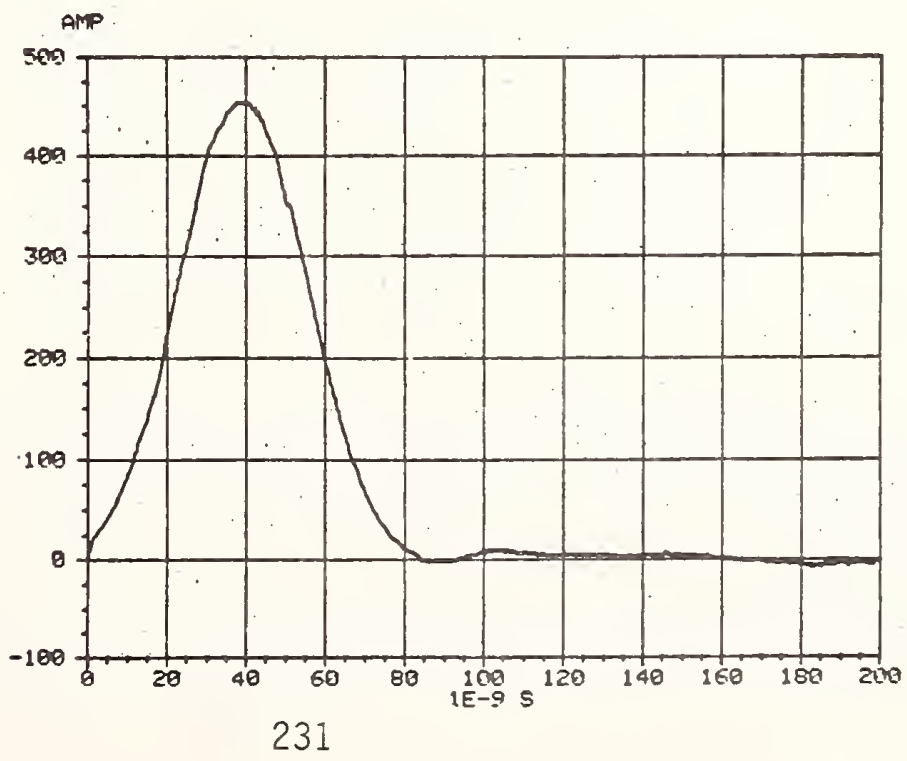


References

1. Park, John H., "Shunts and Inductors for Surge-Current Measurements", Journal of Research of the National Bureau of 5 tandards, Volume 39 , September 1947. 


\author{
WIDE-FREQUENCY-RANGE CURRENT TRANSFORMERS \\ AND APPLICATION TO PULSED POWER SYSTEMS \\ John M. Anderson \\ Electrical Systems and Technology Laboratory \\ Electronic Power Systems Branch \\ Corporate Research \& Development \\ General Electric Company \\ Schenectady, New York 12301
}

Current transformers, constructed as a uniform toroid, can have excellent high frequency response into the microwave region. Their natural isolation against ground $100 \mathrm{ps}$ and ability to feed standard 50 ohm coaxial 1 ines make them ideal for current sensing in high-power pulsed systems. Transformers having response from $4 \times 10^{-2} \mathrm{~Hz}$ to $2300 \mathrm{MHz}$, ixt product of 72 Amp-sec (250 kA for $290 \mu \mathrm{s}$ ) and droop of $0.025 \% / \mathrm{ms}$ have been constructed with al1-ferrite cores.

These transformers have been applied to measurement of current near current-zero in the testing of high-power vacuum interrupters. Currents $210 \mathrm{kA}$ were commutated to zero in 10 s and post arc currents lasting 2 s with 500 amperes peak were detected with current transformers. The ability to introduce opposing currents in the window of the transformer allows cancellation of capacitive rurrent driven by the large recovery voltage.

Key Words: Current transformer; wide-band transformer

\title{
1. Introduction
}

Properly-constructed current transformers (CT's) can be made to have better frequency response, load the primary circuit less, and give greater output voltage than shunts. Better high frequency response is possible because the value of burden resistance can be larger than the equivalent shunt and therefore suffer less from skin depth or self inductance. Also the burden can by physically smalier because it absorbs less continuous watts or impulse Joules. Resis- 
tance reflected into the primary circuit may be less because it is transferred according to the turns ratio squared. Current transformers do have a low frequency rol1-off, although this may be made $3 \mathrm{~dB}$ down at a very small fraction of a Hertz.

In previous work [1] we have described the construction of small CT's which showed uniform response to several Gigahertz. However, their small size precludes use in high power pulse circuits. A somewhat larger CT with pass band from less than one Hertz to about one Gigahertz and ixt product of 7 ampere-seconds has found general use at our laboratory. More recently a larger transformer, also with a ferrite core, has been used for transient measurements where its ixt product of 72 ampere-seconds with a 0.01 ohm burden made it more applicable for high current pulses. Presetting the core $f 1 u x$ so that $\triangle B$ can be at least 4000 Gauss permits observation of a half cycle at $60 \mathrm{~Hz}$ up to $13.5 \mathrm{kA}$ peak. A window diameter of $6.3 \mathrm{~cm}$ permits use at very high voltages when properly insulated.

In the following a very brief description of wide frequency range CT's will be followed by more specific construction details. The application of this transformer to observe post arc currents in high power vacuum interrupters will be described to show at least one case where its unique properties are helpful.

\section{General Discussion}

A toroidal secondary winding is excited uniformly by the magnetic field from a single turn primary if the primary wire is coaxial with the principal torus axis. Independence from circumferential variation means that the current is induced to flow with near equal phase and amplitude at all parts of the toroidal winding, and sensing this current by a small-value resistance gives an overall time delay of response which approaches that imposed by the retarded potential. This structure is thus capable of a very rapid rise time or high frequency response.

Figure 1 shows the elementary physical structure and the secondary current which flows in response to the primary current. For a moment one can imagine the secondary to be a continuous conducting box in toroidal shape. The surface current which flows is exactly that necessary to exclude the magnetic field from the interior of the box, and its integrated magnitude is simply ip ampere turns, where ip is the current in the primary wire: If the box is slit into $n_{S}$ helical turns the current in each turn is $i_{p} / n_{s}$ in agreement with ideal transformer conditions. A conducting shield with appropriate slit around the toroid has currents induced on 
its surface which serve to reproduce the external magnetic field in the vicinity of the toroidal secondary as if the shield was not present. The above is true only on a

transient and quasistatic basis. Current in the secondary is sensed by interrupting the winding and connecting, by a tightly-twisted pair or coaxial cable, a burden resistor outside the shield.

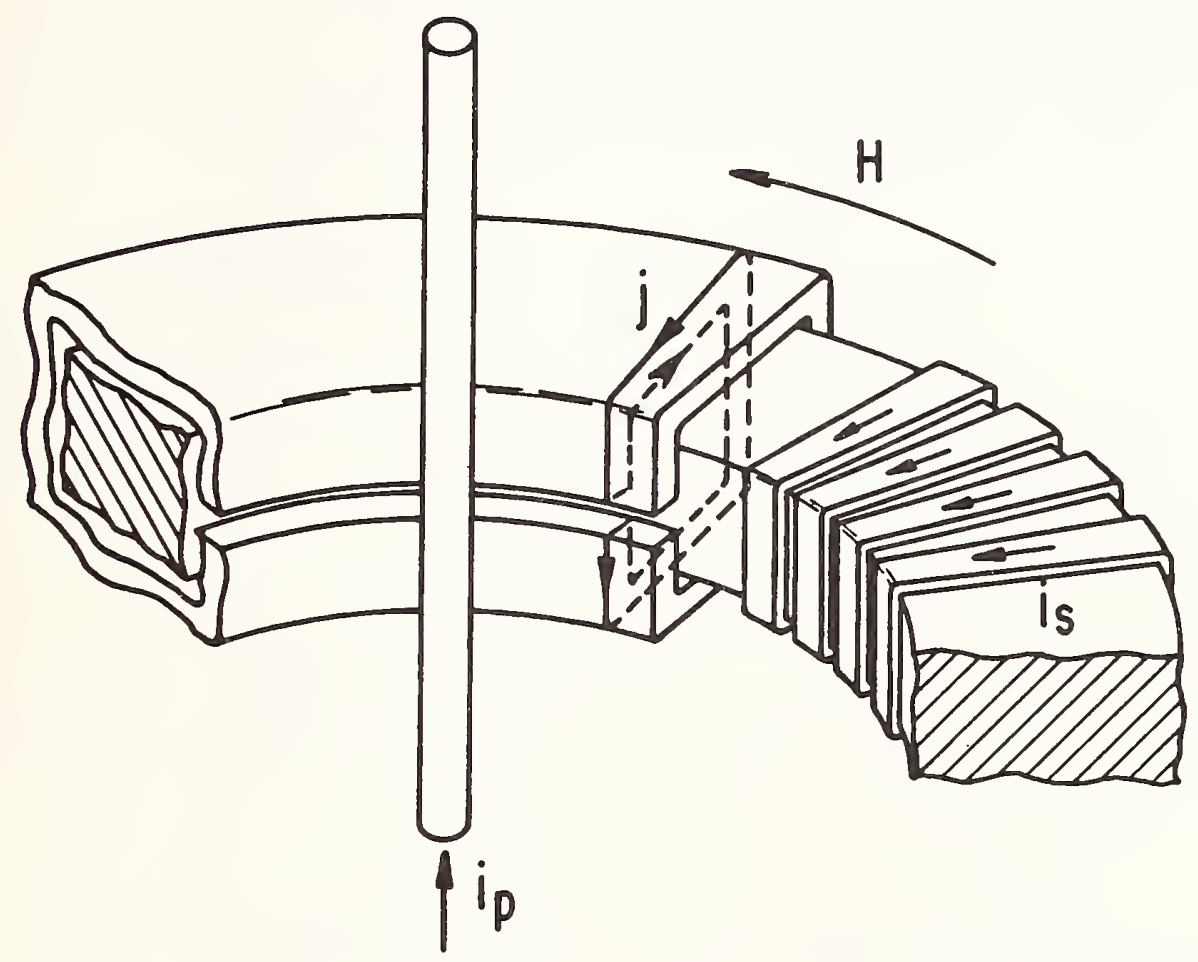

Figure 1. Cut-away sketch of current transformer primary and secondary orientation

The transformer described so far should show little dispersion and should have a response delay of only a few tens of picoseconds, even for a large structure 30 or so centimeters in extent. Where, then, are the limits to frequency response? They are primarily uniformity of structure and internal resonances. The first prominent resonance in the few megahertz region is a coil resonance where the coil inductance resonates with stray capacity to core and shield. Characteristic of this resonance is a variation of fields azimuthally on the toroidal winding. We have found [1] that a network of resistances around the secondary as shown on Figure 2 damps these spurious responses without affecting the desired signal current. The next resonance of consequence is due to the shield and 
secondary acting as a re-entrant cavity. This can be damped by placing a low value of resistance uniformly across the slit in the shield. The correct value, generally 1 to 3 ohms, critically damps the resonance before eventual capacitive roll-off at higher frequencies. Feeding the CT from a coaxial cable where the outer conductor is interrupted by the slit in the shield removes currents from the outside of the shield and extends the frequency response as well as assures uniform azimuthal excitation, but application at high voltage points in a non-coaxial circuit is precluded. A notable exception might be a coaxial high voltage trans mission line as used in gas-insulated substations.

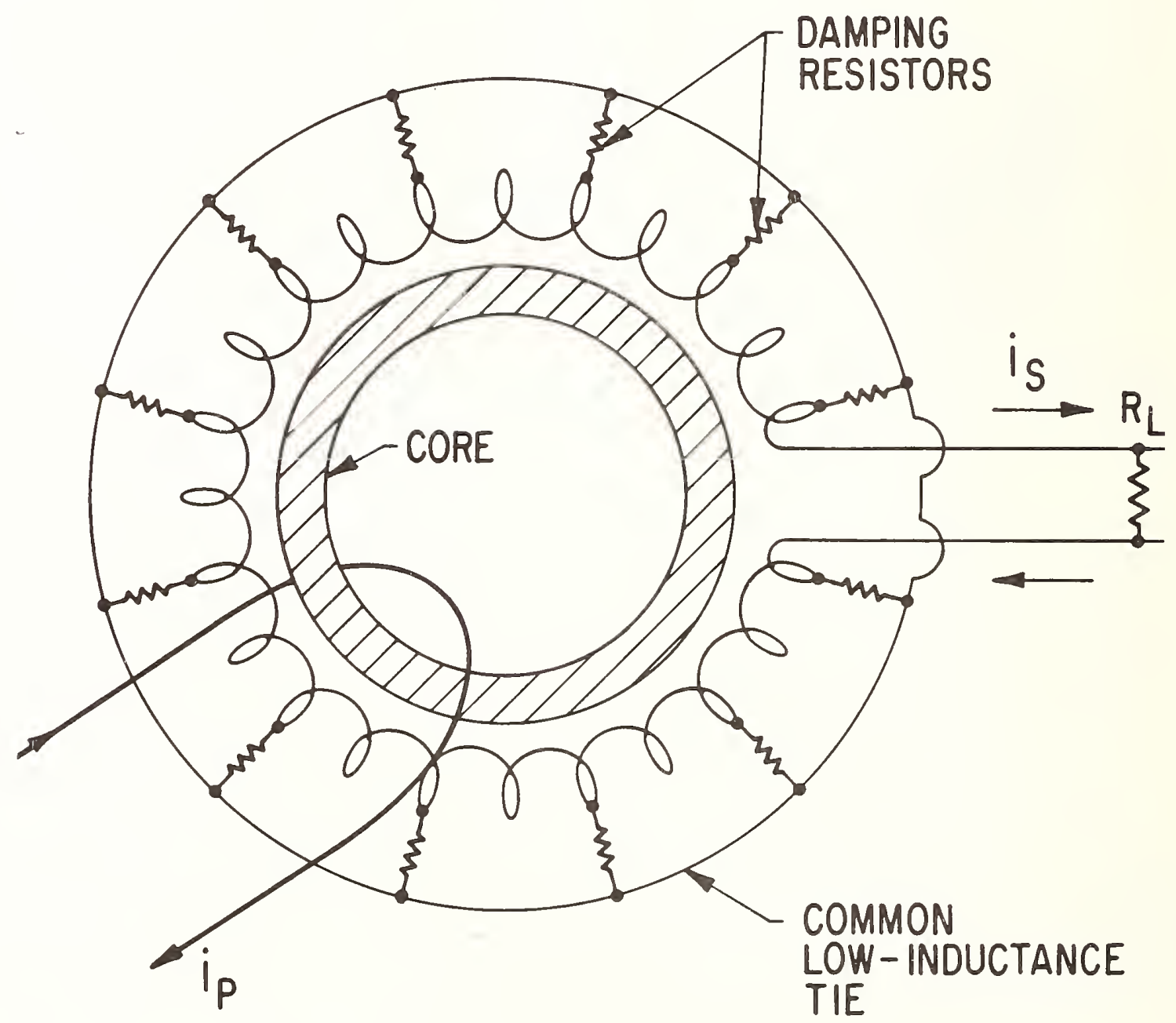

Figure 2. Arrangement of resonance-damping resistances placed around the secondary winding (taken from Ref. [1]) 
Mid-band and $10 w-f r e q u e n c y$ responses are straightforward and easily calculated.[1] The magnetic core acts to maintain a gradient of tangential $H$ across the secondary until it saturates, maintaining a "surface" current in the secondary, which is the desired signal. At very high frequencies the core material does not respond magnetically and approaches a dielectric, possibly lossy. Transition between low and high frequencies is smooth with constant CT sensitivity. At very low frequency, flux buildup and decay in the core leads to a phase shift of output voltage for a.c. primary currents, 45 degrees at the $3 \mathrm{~dB}$ point, and to a small negative nyerswing following a primarv current Dulse, ?..3 mv if the fulf ixt product is used ( 0.01 ohm burden).

\section{Current Transformer Construction and Testing}

The transformer secondary should be in close proximity to the core and wound with a wire size which leaves little space between turns at the inner radius. We have used round magnet wire, 10 gauge, although one might argue that rectangular wire would be better. Because the CT is a very 10 w impedance device, there are no places where large voltages develop and insulation need be only for a few hundred volts. The inside of the shield must be a uniform distance from the winding to keep a constant magnitude of magnetic field at the winding surface. Wires from the secondary are tightly twisted and go immediately into a small channel to the outside of the shield to minimize induced voltage. The burden is attached coaxially for best frequency response.

The density of damping resistors around the secondary can be increased by experiment until coil resonances disappear. We have found that $1.3 \mathrm{kilohm} 10 \mathrm{w}$-inductance film resistors attached every 4 turns on a 100 turn secondary suffice. They are commonly tied to a thin copper washer which is insulated from the windings and the shield as shown on Figure 3. If the transformer is worked directly into a terminated 50 ohm cable, i.e. a 50 ohm burden, the loading from these resistors may reduce the output voltage at midband a few percent. Generally the burden for pulsed high current observation is 0.1 ohm or less and loading is negligible. Connection to the oscilloscope is by 50 ohm terminated coaxial line which alters the burden resistance by a very small and known amount.

The ferrite core was obtained by special order from Ceramic Magnetics Company and had dimensions $0.0 .17 .4 \mathrm{~cm}$, I.D. $9.5 \mathrm{~cm}$, with thickness $2.8 \mathrm{~cm}$. The material was MN60 which has an initial permeability of 6500 and saturation flux of 4500 Gauss. Remanent flux is cataloged at 1200 Gauss. Overall dimensions of the CT shown on Figure 3 are $20.3 \mathrm{~cm}$ square by $4.76 \mathrm{~cm}$ thick. Internal cavity dimensions 


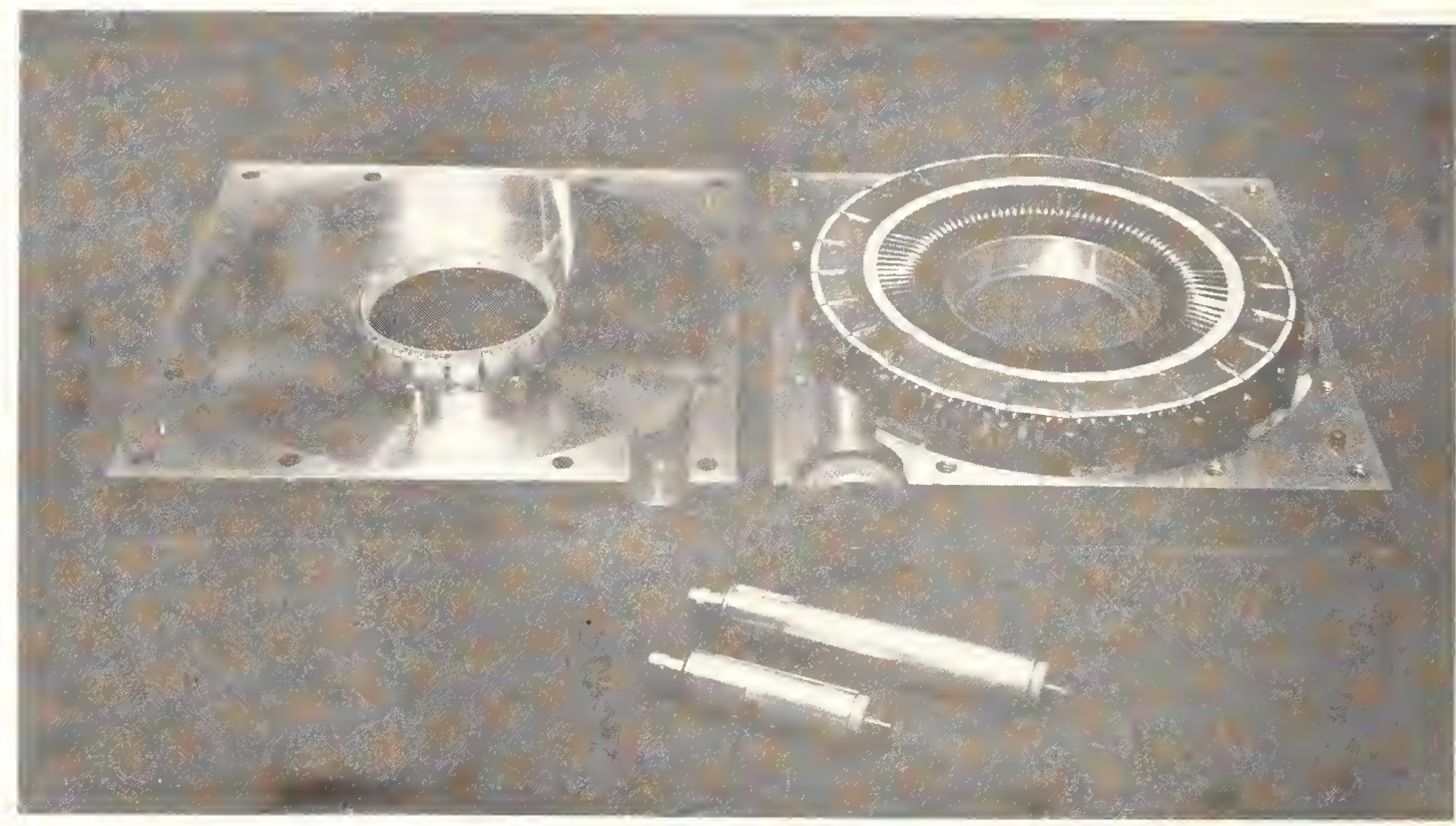

Figure 3. CT disassembled to show construction

of 0.D. $19.4 \mathrm{~cm}$, I.D. $6.67 \mathrm{~cm}$ and height $4.13 \mathrm{~cm}$ allowed ample space for the core and winding. Burdens of 0.1 and $0.01 \mathrm{ohm}$ were obtained from $T$ and $M$ Research Products. Catalog numbers were SBNC-1-1 (700 MHz, 40 Joules) and SBNC-2-01 (300 MHz, 16 Joules), respectively. Their d.c. resistances were spec'd to within $1 \%$, but values are of course measurable as received to greater accuracy. We have observed no internal resonances, and rise times for the burdens alone are in reasonable agreement with the cut-off frequencies given above. Some calculated performance data for the CT described above are given on Table 1.

\section{Measurement of CT Response}

Mid-band sensitivity (transfer resistance) of the current transformer can be rather simply tested in the audio frequency range. We have used a General Radio Type 1450 TB Precision Decade Attenuator followed by a Hewlett-Packard $400 \mathrm{~F}$ voltmeter as a constant voltage indicator only. A resistance, generally one ohm, was alternately used as a burden or directly fed from the same constant current source used to feed the CT. The difference in attenuator settings as it was connected to measure the voltage across the resistance in the two cases gave the current ratio of the CT. Precision of visual reading was well within one percent. 
Table 1

Load (Burden) Resistance

Voltage out for $250 \mathrm{kA}$

primary current

Pulse length at $250 \mathrm{kA}$ for ixt 1 isted

Impulse energy in the burden

for above pulse

Secondary inductance measured at $1 \mathrm{kHz}$

L.F. cutoff* (small signal)

Droop* (small signal)

Resistance reflected

to primary*

0.1

250

117

73.1

239 ixt product for $\triangle B=4000$ Gauss 29.3

\author{
0.098 \\ 0.062
}

$1.5 \times 10^{-5}$

*Includes secondary winding resistance of $0.051 \mathrm{ohm}$.
0.01

25

72.5

290

18.3

239
$\mathrm{mH}$

ohm

volt

Amp-sec

uS

Joules

$\mathrm{Hz}$

$\% / \mathrm{mS}$

ohm

The attenuator was 1 imited by internal resistor accuracy of $\pm 0.25 \%( \pm 0.02 \mathrm{~dB} \pm .25 \%$ of indicated $\mathrm{dB})$. By $200 \mathrm{kHz}$ this possible error increases to $0.1 \mathrm{db} \pm 7 \%$. At $400 \mathrm{~Hz}$ and 100 $\mathrm{kHz}$ the turns ratio of the CT agreed with attenuator readings so that much less than $1 \%$ voltage change was seen.

This result should be expected if the permeability of the core is adequate and losses are small. CT accuracy then depends principally upon the burden which can fortunately be wide band and accurately measured at d.c.

The CT, unless damaged, is mechanically and electrically stable, which leaves oscilloscope techniques and physical placement in the circuit as the most probable sources of inaccuracy and non-reproducibility. For the most uniform response at frequencies above about $10 \mathrm{MHz}$, the primary wire should be centrally placed in the CT window. This reduces. non-uniform excitation of the secondary. Where coaxial excitation is possible, the requirement is immediately met. In other cases the primary wire should be coaxial for 3 to 4 diameters of the secondary to minimize error, although this was not quantified.

Testing the transformer for highest frequency response is most easily done by a fast step of current. To generate the step, we have used an air line, discharged through mercury-wetted contacts. The current is limited by the contacts to wone-half ampere, that is a 50 volt line dis- 
charged into $50 \mathrm{ohm}$. The current step so obtained is shown as the top trace on Figure 4. Its rise time is 2200 ps. The output of the CT with 10 ohm burden is shown as the lower trace. At first glance the response looks to be a disaster, but it is mostly explainable and correctable for our purposes. Noise on the trace is due to a high gain setting on the sampling oscilloscope. The oscillations at 2 GHz are largely cavity resonances and were not fully damped by 3.2 ohn placed across the slit in the shield. They could have been further reduced but were not. The possibility of some spurious responses due to winding irregularities should not be discounted. The CT response does not step to the same relative amplitude as the current source and, in addition, rises more slowly thereafter. This is caused by a too-large burden, $10 \mathrm{ohm}$, necessary because of equipment limitations, and tests on other transformers [1] shows that the effect will reduce proportionately when the burdens of 0.1 and 0.01 ohm are used.

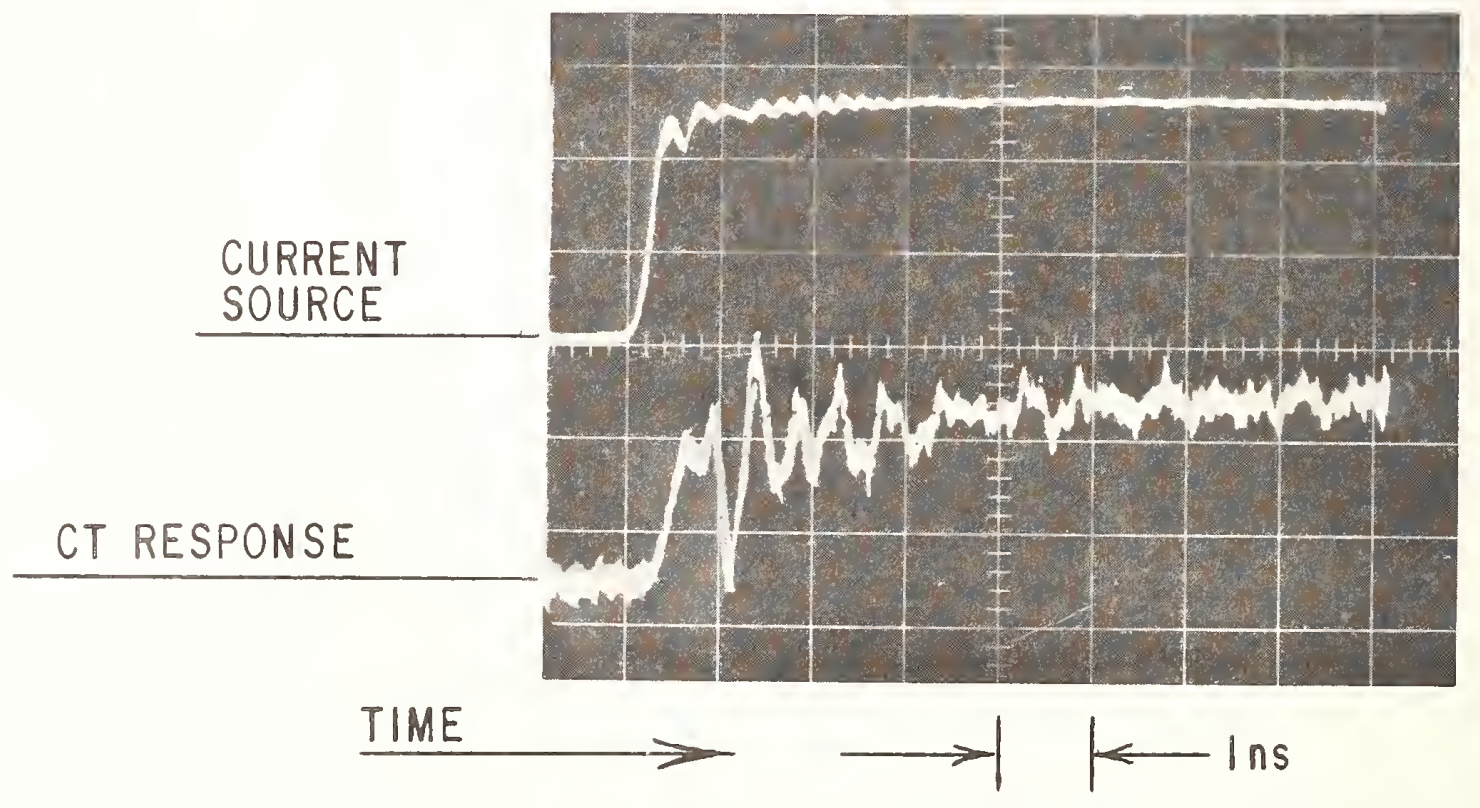

Figure 4. Response, lower trace, of the CT to a step current, upper trace, showing residual cavity resonances.

We expect to use this CT with oscilloscopes responsive to $2100 \mathrm{MHz}$ and therefore a $100 \mathrm{MHz}$ roll-off was imposed on the source step current, see the top trace on Figure 5. The CT response is shown on the lower trace. Rise time with the $100 \mathrm{MHz}$ rol1-off was $3.5 \mathrm{~ns}$ and with the CT in addition it was 4 ns. Noise on the trace does not permit accurate measurements. However, using the approximation that the 
cascaded rise time is the root of the sum of the squares of individual rise times, the CT in responding to a cut-off 2 $770 \mathrm{MHz}$. This will improve if a lower resistance burden is used as noted above.

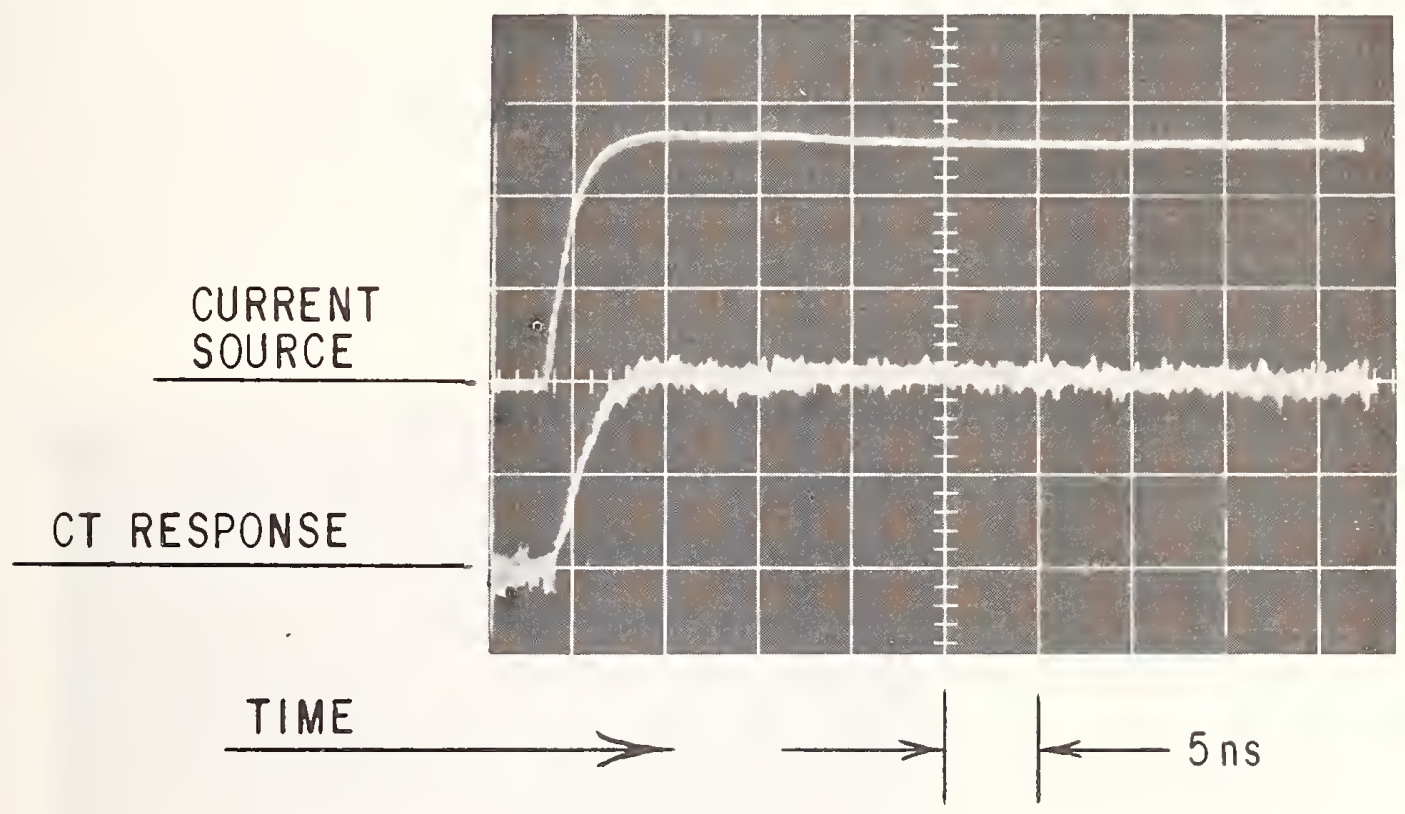

Figure 5. Response, lower trace of the CT to a current step with high frequency response limited by a L-R roll-off at $100 \mathrm{MHz}$.

\section{Applications}

In the past few years we have used CT's of this design in various sizes for a variety of applications. Coaxially fed, they have been used for example to observe sub-nanosecond-rise-time pulses of current to light emitting diodes, current pulses in plasma display panels, and microwave currents to antennas. Larger size transformers match nicely in frequency response to laboratory oscilloscopes good to $>100 \mathrm{MHz}$. The CT described in this report was used to observe post arc currents in vacuum interrupters during synthetic testing of their interrupting capabilities. [2] A large capacitor bank was switched through an air inductor to reproduce a one-half cycle current pulse at $60 \mathrm{~Hz}$. A second, lower capacity bank, charged to $100 \mathrm{kV}$, was switched to create a counter-pulse in the vacuum interrupter and bring net current to zero in $270 \mu \mathrm{s}$. If the vacuum interrupter does in fact interrupt at current-zero, it faces up to $200 \mathrm{kV}$ of reverse "recovery" voltage and residual plasma 
is swept from the vacuum discharge space. Figure 6 shows the post arc current, in this case about 200 amperes. Recovery voltage was here about $150 \mathrm{kV}$. Time scale was one microsecond per cm on the scope face. The available frequency response of the CT is helpful to observe noise on the post arc current and exact times of failure to interrupt as well as the nature of the failure. Reference [2] gives examples of failure which will not be reproduced here. In addition to a divorce from direct connection to the circuit necessary with a shunt, the window of the CT is available to pass bucking currents for cancellation of stray capacitive currents to or compare currents differentially in different parts of the circuit.

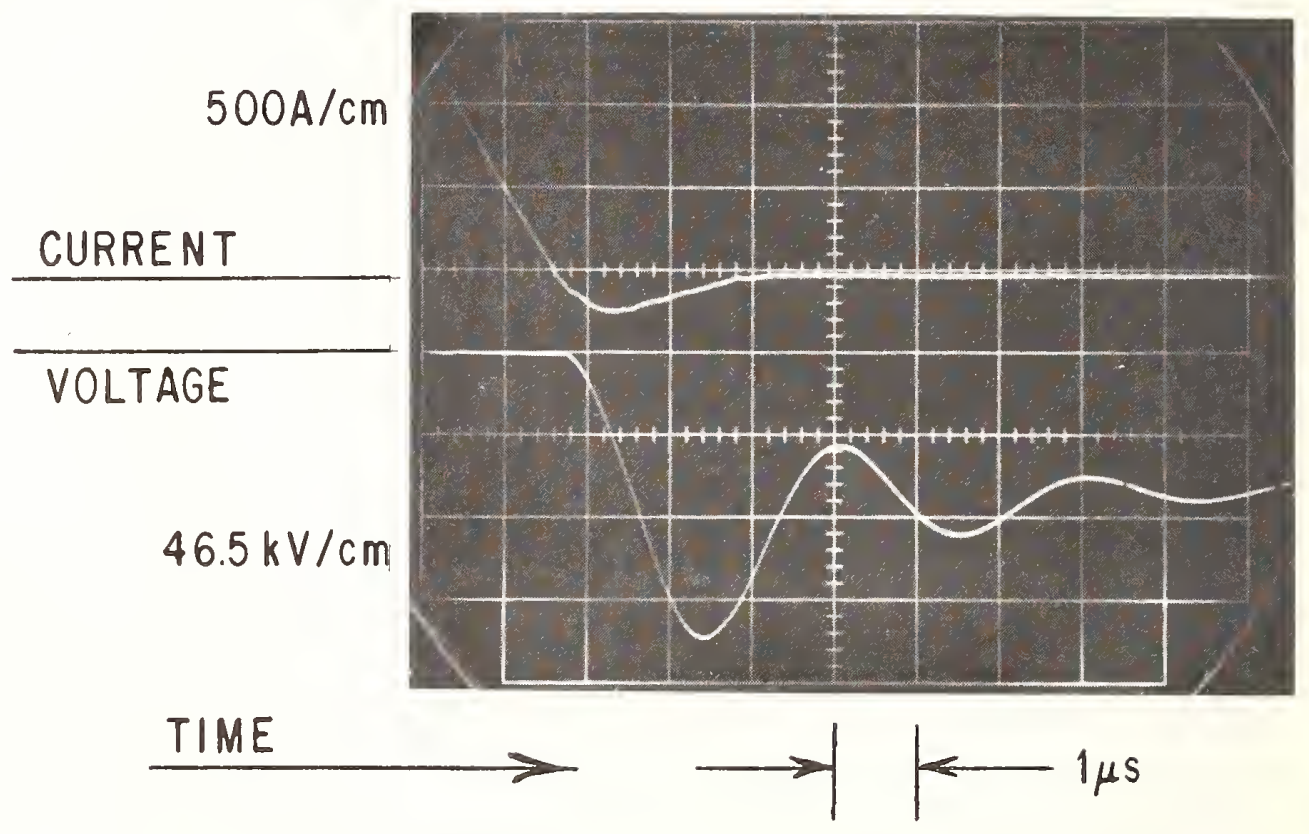

Figure 6. Waveform of current near currentzero, upper trace, and recovery voltage during synthetic testing of a vacuum interrupter. (Taken from Ref. [2])

\section{Acknowledments}

It is a pleasure to acknowledge the assistance of C.P. Goody in the construction and testing of these transformers. J.J. Carroll collaborated with the author in the testing of vacuum interrupters and A.N. DeTommasi assisted in set up and testing. 


\section{References}

[1] J.M. Anderson, "Wide Frequency Range Current Transformers", Rev. Sci. Instrum., 42, pp. 915-26, (1971).

[2] J.M. Anderson and J.J. Carroll, "Applicability of a Vacuum Interrupter as the Basic Switch Element in HVDC Breakers", IEEE Trans. PAS, PAS-97, pp. 1893-1900, (1978).

\section{Sponsor Acknowledgment}

Testing of vacuum interrupters was supported by a contract with the Energy Research and Development Administration, now the Department of Energy. 


\section{Discussion Session 4}

Richard Malewski (IREQ). My experiences with current measurements were different. There are several common points in measuring techniques used by physicists who are here and power engineers. I feel that probably power engineers know more about shunts whereas the physicists involved in the measurements of very fast pulses know more about current transformers or Rogowski coils. I believe that we have made a contribution in the area of shunts. Shunts must meet the two contradictory conditions. One is to provide sufficiently large volume to absorb the energy dissipated during the measurement of current pulse, while the second is to have sufficiently fast response to reproduce the measurement pulse. Unfortunately, the voluminous shunt has fairly thick walls and, therefore, the skin effect slows down the response. The classical tubular shunt such as devised by Francis Silsbee is a tubular conductor having certain thickness of the tube wall. If the shunt is subjected to a unit step of current, the current density initially is very high at the outer wall of the shunt and is practically zero at certain depths. The current density will gradually penetrate into the shunt until a steady state is obtained. If our measurement circuit is attached to the inner wall of the shunt, as proposed by Silsbee and Park, the voltage which builds up will have a shape representing the gradual increase of current density of the inner wall of the shunt. The increase of the response is dictated by the rate of penetration of current density into the material. We would like to shorten the response of the shunt. This can be done in two ways. The first is to take the probe and introduce it inside the wall of the shunt at a given radius. There is an optimum position between the inner and outer wall for the probe. Technologically, one can laminate this shunt out of two tubes and introduce this probe in between. The second other improvement is technologically more complicated. Assume that we cut the shunt tube with a longitudinal cut. By introducing the probe into the shunt wall and running it along a parabolic profile, the response will be an exact replica of the applied pulse. Therefore, we do eliminate error caused by skin effect in the shunt and also conserve the large volume of the material necessary to prevent thermal drift of resistors due to heating. This shunt has been described in the literature. 1 This shunt could be incorporated into some of the systems I have seen here shown during the discussion. In the area of current transformers, I have a question which I would like to address mainly to two authors who presented their design of this transformer. If I am right, the main problem

\footnotetext{
TR. Malewski, et al. "Elimination of Skin Effect Error in hieavy Current Shunts." IEEE Transactions, Vo1. PAS-100, No. 3, March 1981, p. 1333-1340.
} 
with current transformers comes from non-axial excitation. If we assume that the current flows through a conductor which is put asymmetrically into a current transformer we see immediately that the magnetic field generated by this current will be symmetrical along the axis of the conductor. This means that different parts of the coil will see very different field intensities. The current induced into secondary winding around the donut will have a tendency to cancel the primary flux but the current recirculating in this winding will produce a flux of opposite direction but of the same value all around the coil. Under transient conditions we will have some net flux inside the coil which results because the primary flux is stronger than the secondary at some locations (figure 1). Such a situation cannot exist for a long time and energy stored in the magnetic field of this coil will be dissipated showing up as ringing due to some stray capacitance and inductance in the coil. This is the reason why people designing these coils added a burden in the form of distributed resistance attached to some sections of the coil. In this way, current flows in the small local loops in addition to the total currents circulating in the coil (figure 2). And this will allow the flux due to the current in the coil to bond better, perhaps stepwise the primary flux. I once opened a transformer made by Pearson and found inside the transformer a winding made in such a way that there were taps from the coil bonded to a resistive wire with the total resistance corresponding to the burden resistance here. There were also some parallel high ohmic resistors probably for damping resonances in the winding. Each of them was in the range of 500 ohms. The resistive wire was, I think, about 50 ohms. There was also an apparently controlled capacitance of the winding elements to the grounding reference slab in order to build the line of a known impedance $Z=\sqrt{L / C}$. I believe that by changing the inductance by using for instance tape or ribbon, instead of wire, and controlling this capacitance one can reduce the impedance of the line and, therefore, this would project on values of the resistors. I don't know exactly how the transformer works as we have in parallel a fraction of an ohm resistive wire section and several ohms of the radius-type resistors, and the two somehow perform their roles despite the obvious fact that one seems to be short circuited by the other. Perhaps Mr. Pearson could explain this if it is not infringing with the proprietary information of the company. 

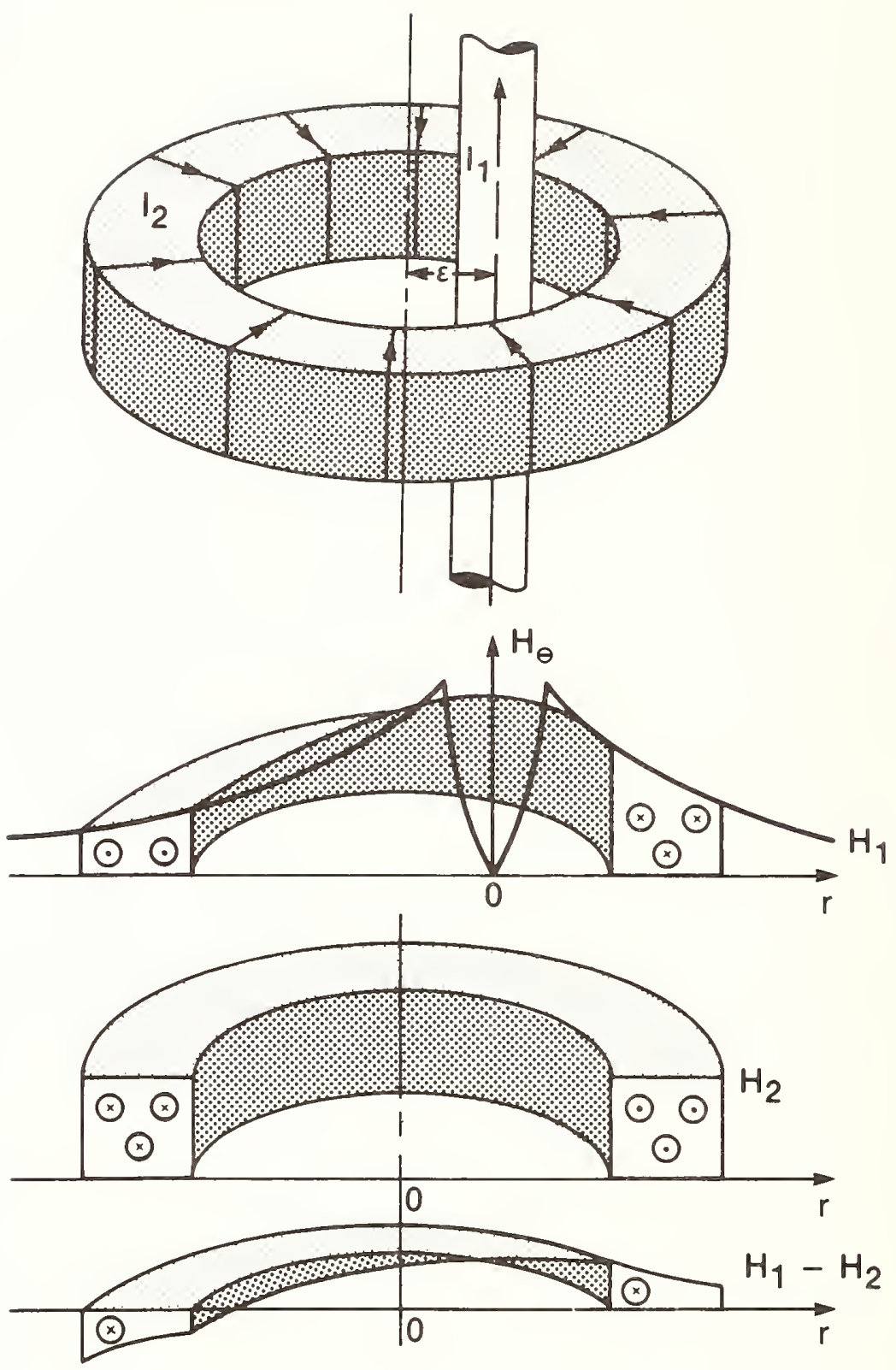

Figure 1. Schematic representation of flux in a current transformer with off-axis excitation. 

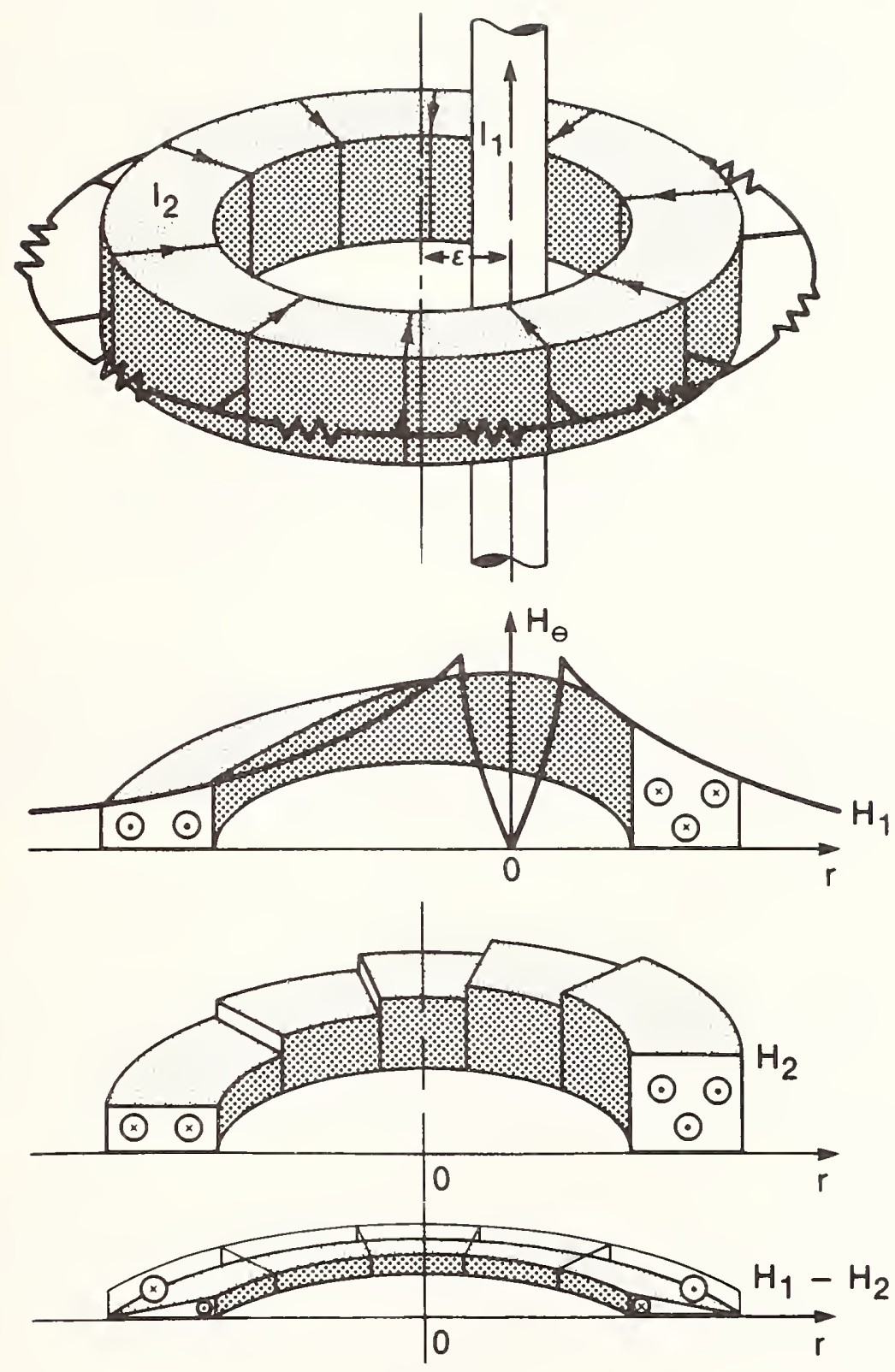

Figure 2. Schematic representation of flux in a current transformer with off-axis excitation and distributed load for $i_{2}$. 


\title{
DATA ACQUISITION AND PROCESSING TECHNIQUES
}

\author{
R. E. Kolibas \\ P. A. Corbiere \\ J. J. Moriarty \\ Raytheon Company \\ Missile Systems Division \\ Bedford, Mass. 01730
}

\begin{abstract}
Current measurements, signal transmission, and system fault isolation of a line type modulator have been accomplished in the presence of pulse power noise. This paper presents techniques successfully utilized to achieve valid results.
\end{abstract}

\section{Introduction}

This paper describes techniques of data acquisition and processing in the high noise environment of a pulse power system. These techniques, which are considered to be generally valid, are illustrated by means of an example taken from the design of a hardware system which has been built, tested and delivered.

The objective in this example was to monitor the operation of an array of high power thyratrons and to provide fault detection, fault isolation and system shutdown commands to a high-voltage, line-type modulation. Manual test procedures by trained personnel were unacceptable in this system both because of the hazards involved and the large number of thyratrons to be monitored.

The parameter monitored and measured to determine valid operation was the thyratron cathode current. Both the timing and the amplitude of the thyratron current were analyzed to determine that all switches fired concurrently within the limits required for proper operation of the line-type modulator. In the event of a thyratron malfunction, the detection, processing and storage of the failure information as well as the generation of a system shutdown signal, under predetermined fault conditions, were provided by the thyratron monitor. 
The key problem was to achieve valid operation despite the large electromagnetic radiation, electrostatic coupling, and ground current transients. The following discussions will explain the analog noise suppression techniques as well as the operation of the monitored and transmitted thyratron cathode current signal. The isolation of large ground voltage transients between subsystems will be addressed. Finally, the diagnostic methods utilized to perform the more complex tasks of fault isolation, including digital techniques that can achieve noise suppression via judicious logic architecture combinations, will be presented.

\section{Measuring the Cathode Current}

The operating principle of the Rogowski current transformer is the use of a toroidal core transformer as the secondary winding enclosing a one-turn high-pulse current source. The pulse current-carrying conductor in this case is the plasma formed within a thyratron switching a peak current in the range of 1000 to 10,000 amperes with a plate potential of $45 \mathrm{kV}$.

The design premise of such a transformer is based on its reflecting zero insertion loss into the high pulse current circuit being monitored. In practice, the secondary circuit impedance reflected into the single turn primary is made to be from 0.01 to 0.10 percent of the source impedance by means of matching output coaxial impedances of 50 to 100 ohms.

Thus the equivalent operating circuit of Figure 1 may be used for transformer and signal circuit test and evaluation. By means of a

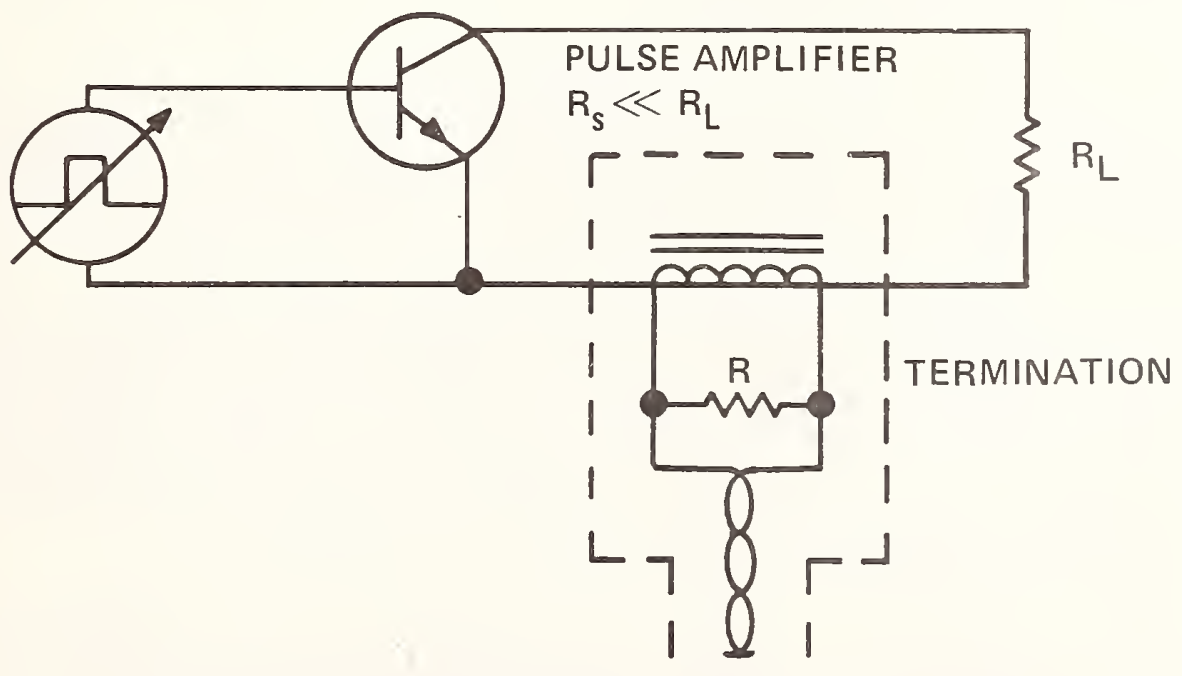

Figure 1. Pulse current transformer equivalent operating/test circuit 
three-place digital or analog visual comparator (oscilloscope), the test and signal circuits may both be calibrated well within the maximum desired accuracy of 1 percent.

From a design point of view the current transformer leakage inductance is part of the equivalent impedance reflected into the primary circuit and therefore will not attenuate output circuit risetime. Current transformer core loss and magnetizing current must be maintained sufficiently low in order to be within the transformer linearity (or accuracy) requirements over the operating current range specified. Response to fault current levels may be permitted to degrade, generally to the point of avoiding core saturation effects, and is specified as the transformer I capability, where $I$ is the peak pulse current and $\tau$ is the equivalent square wave pulse duration.

Output pulse droop requirements are met by appropriate output winding inductance. In cases where nickel-iron core materials are required for unidirectional input pulse currents, an isolated dc bias core reset current is continuously applied.

Output waveform fidelity is maintained by distributing the built-in terminating resistance symmetrically around the transformer such that an upper cutoff frequency of $10 \mathrm{MHz}$ is readily obtained. In this case a I-microsecond pulse current risetime is faithfully reproduced and the internal waveform ringing is damped by the load resistor distribution technique.

\section{Protecting the Monitored Signal}

Shielding the monitored signal against electromagnetic interference at the pulse current transformer includes a variety of techniques as shown schematically in Figure 2.

First and foremost is the principle of using a uniformly wound toroidal core for the transformer. As a consequence only the net axial current or magnetic flux through the inner diameter will induce output current, all others being cancelled by the core and coil geometry.

Secondly the monitored signal is enclosed in a Faraday cage which does not carry signal current. In the transformer this takes the form of the low impedance case structure electrically connected to the shell of the output twinax coaxial cable connector. A gap in the case inner circumference is used to pick up current and to prevent a shorted turn around the core. This Faraday cage is electrically grounded at a point remote from the high pulse currents being monitored.

Lastly an internal electrostatic shield covers the output winding in the same manner as the case. This shield is electrically tied to the low end of the unbalanced current transformer output winding to short potential signals electrostatically induced in the winding. Additional electrostatic and magnetic shields may be used to line the case as required. 


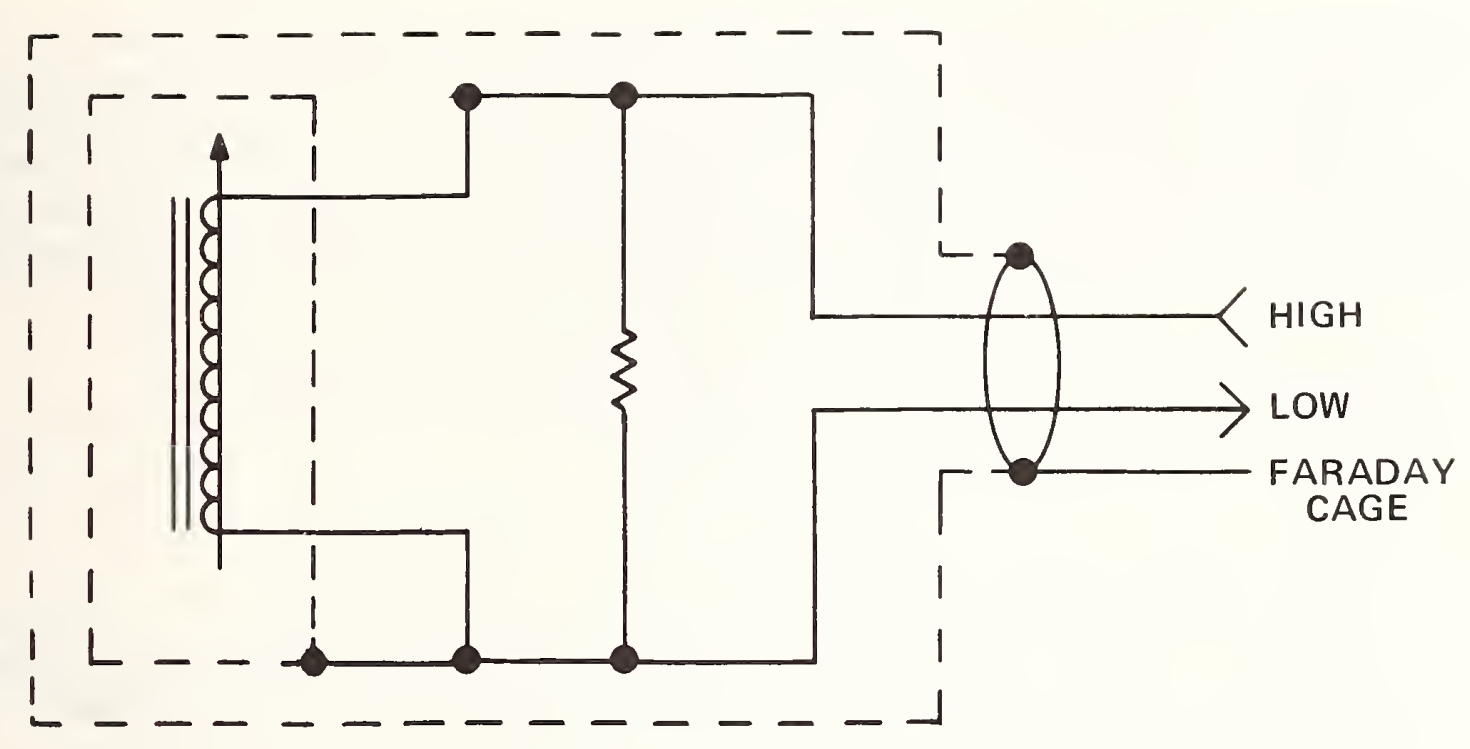

Figure 2. Pulse current transformer electrical schematic

4. Protecting the Transmitted Signal

A balanced line transformer is used to couple the unbalanced signals through the balanced windings as shown in Figure 3. Here,

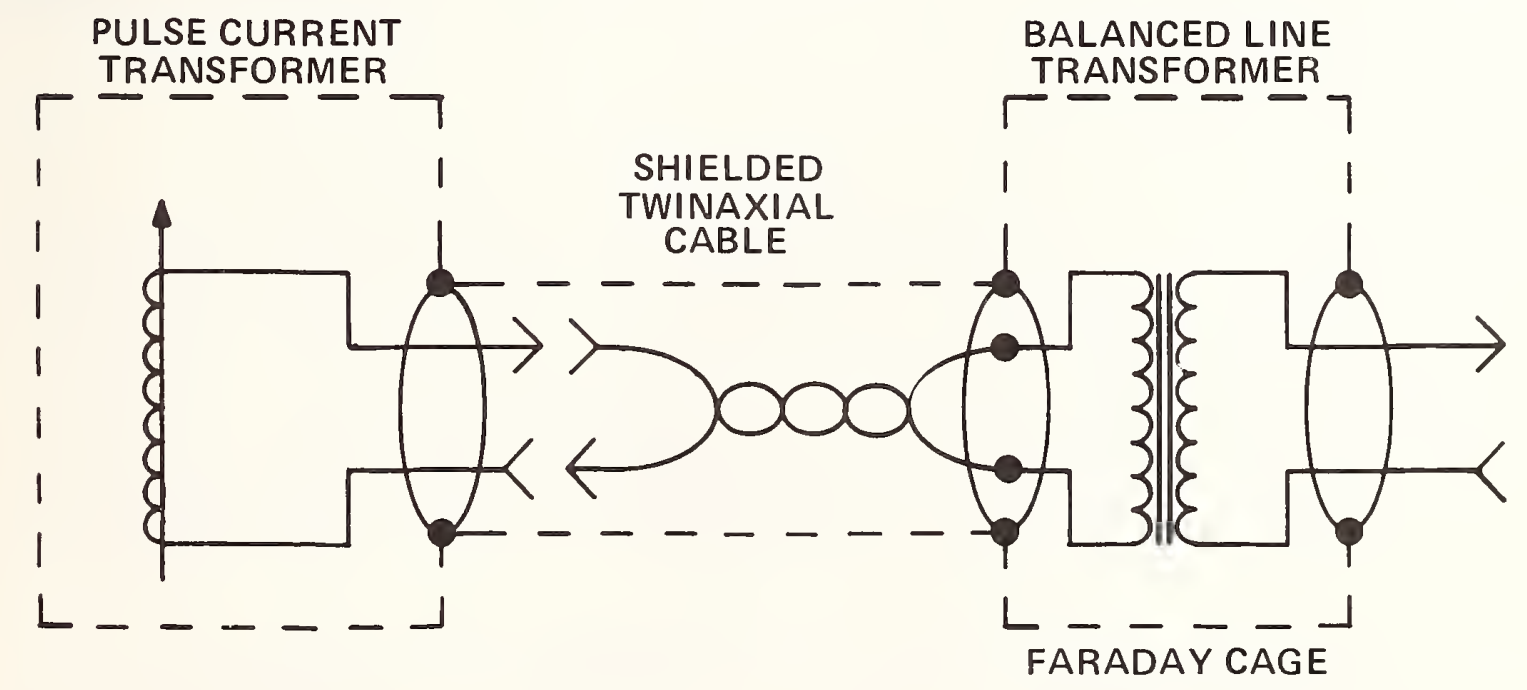

Figure 3. Pulse current signal transmission 
unwanted common mode signals are rejected. This configuration uses a twinaxial, twisted-pair, shielded cable. Protection against low fre quency electromagnetic fields is achieved two ways. First, the twisted wire cancels the induced $\mathrm{H}$ fields, and secondly, the balanced line transformer common mode rejection cancels the induced signal remaining. Protection against capacitive electrostatic coupling is achieved using a Faraday cage technique with a nonsignal-carrying shield. Lastly, isolation from multiple subsystem grounds is accomplished by this floating pulse current transformer and balanced line transformer configuration, thus enabling the monitored signal to be effectively referenced at the data processing section.

The balanced line transformer design used in this application to couple unbalanced signals through the balanced windings has a $50 \mathrm{~dB}$ common mode noise rejection between input and output signals. Proper design for common mode noise rejection across the frequency passband requires that the input and output winding halves be balanced within several percent for all winding characteristics such as resistance, leakage inductance, and distributed capacity. This balance is obtained by fully symmetric coil geometry implemented with attention to mechanical tolerances. Winding resistances are sufficiently balanced by using wire from the same mill run and wound on the same machine, with similar winding tensions, speed, etc. The winding turns ratio is 1. 0 to 1.0 and is strictly maintained between winding halves for the necessary balance, i.e., the winding center tap must be exactly held.

Flexibility has been maintained in this design to permit any electrical hookup desired for case, shields, and windings. Thus multiple use of this one design is not only possible but has proved effective depending on the application. As shown in Figure 4, each transformer winding is

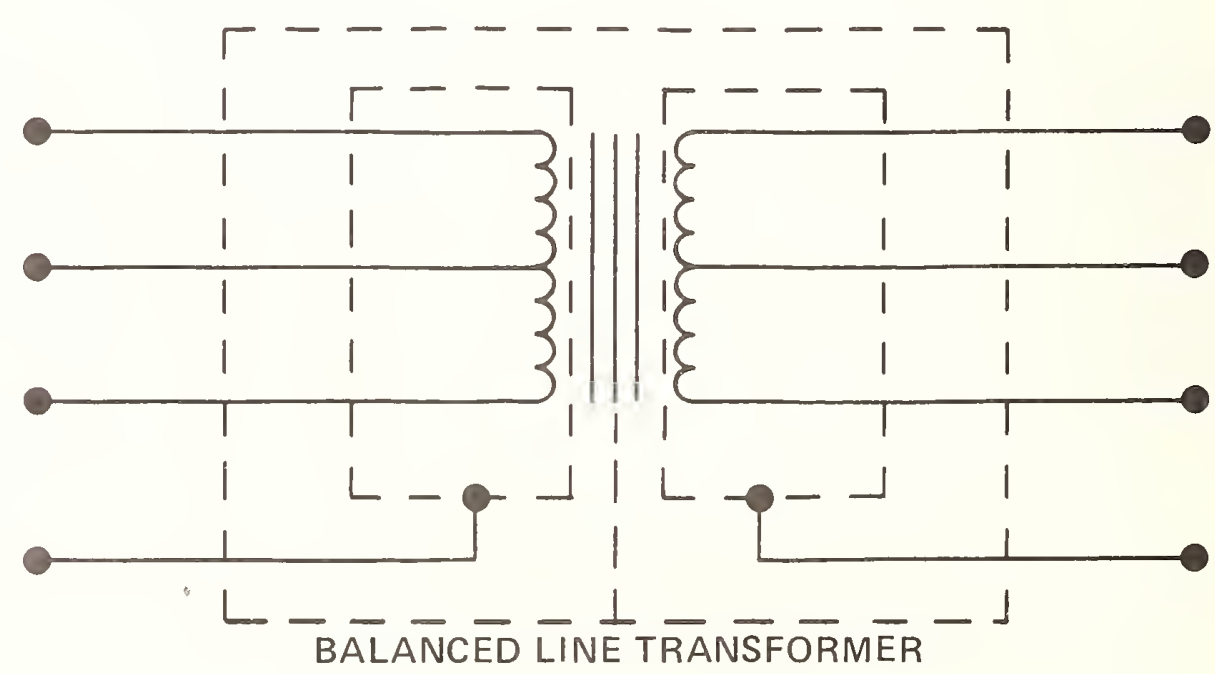

Figure 4. Balanced line transformer 
individually screened. Electrostatic shielding of $50 \mathrm{~dB}$ can be attained by connecting these shields to the nonsignal-carrying Faraday cage shown in the signal transmission schematic of Figure 3. Although additional electrostatic and magnetic shields could have been effectively employed in this transformer, it was deliberately located in a relatively low noise environment such that they were not required in the present example.

The transformer design has upper and lower cutoff frequencies approximately a factor of 10 beyond those of the transmitted pulse, so that waveform fidelity is assured.

\section{Processing the Data}

The thyratron current cathode signals monitored and transmitted are now processed to determine valid operation. This analog signal is terminated into its characteristic impedance and measured for a minimum level using a comparator. This signal is now time-processed and analyzed by the logic circuitry. The achievement of valid circuitry operation despite the large electromagnetic, electrostatic, and ground current transients is addressed in the following paragraphs.

Problems encountered designing with the logic remain. The unavoidable noise, regardless of grounding philosophy, signal distribution and isolation techniques still requires the logic circuitry to have a respectable amount of noise immunity. Transistor-transistor logic $\left(\mathrm{T}^{2} \mathrm{~L}\right)$ with only a $400 \mathrm{mV}$ noise margin is not an adequate choice. However, a logic series with both adequate noise immunity and complex large scale integration (LSI) capabilities is not available.

The decision to use two different specialty logic series in combination is the solution. A high noise immunity (HINIL) series with limited logic capabilities but with noise immunity nine times greater than $\mathrm{T}^{2} \mathrm{~L}$ is used as the protective interface logic. Sandwiched inside is a buffered complementary metal oxide semiconductor (CMOS) logic series which performs the decision and memory storage functions. These logic series are operated at a $12 \mathrm{~V}$ level which is more than twice the standard $5 \mathrm{~V}$ logic levels. This results in a CMOS logic noise immunity six times greater than standard $\mathrm{T}^{2} \mathrm{~L}$. Thus the judicious combination of these two logic architectures results in a logic system that survives the electrostatic, electromagnetic, and ground transients with adequate noise immunity to reliably perform even the most complex decision functions.

Processing of the detected thyratron cathode current begins with the timing and control logic (Figure 5) which develops a prefire zone, a valid fire zone, and a misfire zone. This information controls the time sequenced gating logic which then compares in real-time the detected output to these zones. When a failure occurs, the fault decision and computation logic defines and isolates the type of problem (for example, 


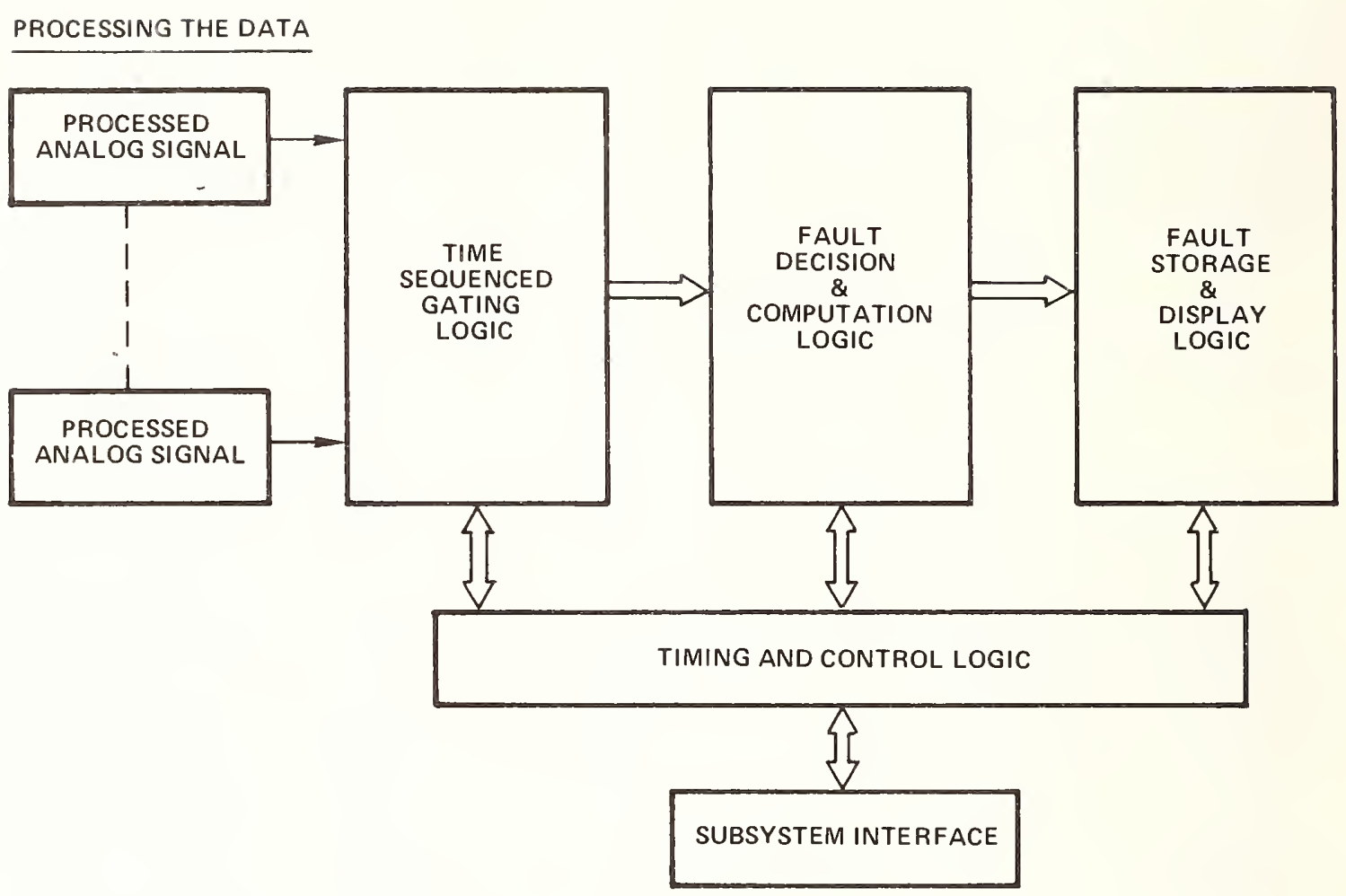

Figure 5. Data processing

thyratron number 14 prefired). This information is stored and displayed. This is accomplished by having separate detectors for each thyratron tube.

Furthermore, after each line modulator's output an additional scan occurs which checks each thratron's output for a misfire failure. This type of failure could be either a thyratron that did not fire or fired late. Thus after additional fault encoding is performed this information and the pulse number during which the problem occurred is stored in a random access memory (RAM). This enables one to detect and store failures of multiple thyratrons on the same pulse or multiple failures of the same thyratron on different pulses. Finally the system shutdown control signal is generated during this scan sequence. When the failure meets a predetermined set of conditions the logic automatically powers down the system, preventing serious damage from occurring.

\section{Conclusions}

The capability of automatically powering down the system to a safe level, especially during initial test and integration is significantly useful. The failure data stored is retrievable for display on the front panel. Therefore during troubleshooting, this data, properly analyzed, can reduce the complexity and time required to isolate the line-type modulator problem areas. 
Thus the complex tasks of automatic fault detection, fault isolation, data storage and system shutdown can be achieved through the judicious use of analog and digital noise suppression techniques. The techniques of data acquisition and processing described above have enhanced the value of fault protection for the integration, operation, control and maintenance of pulse power systems.

\section{Acknowledgements}

The authors wish to acknowledge the many contributions received during the performance of the work described in this paper. Thanks are due to $\mathrm{Mr}$. J. R. Arsenault and Mr. G. K. Simcox for sharing their technology and much appreciated experience. 


\title{
A TECHNIQUE FOR MEASURING BEAM CURRENT DENSITY \\ IN THE ANTARES ELECTRON GUN
}

\author{
W. Robert Scarlett \\ University of California \\ Los Alamos Scientific Laboratory \\ Los Alamos, NM 87545
}

The Antares Electron Gun produces a radial beam of high energy electrons for controlling the discharge in a large laser power amplifier. The gun delivers a $5 \mu \mathrm{sec}$ pulse of 450-550 kev electrons with a current density of $50-100 \mathrm{~mA} / \mathrm{cm}^{2}$. The $9.3 \mathrm{~m}^{2}$ beam is delivered through 48 foil windows.

Because of the low electron flux, traditional means of making high resolution measurements of the time integrated beam uniformity have not proven very useful. We have developed a technique by which Cerenkov light produced in a plastic radiator placed above a foil window is recorded photographically and then measured with a densitometer. This data is then reduced to provide the integrated current density.

The design, calibration and performance of this technique will be presented. An extension of the technique to provide time resolved measurements will also be discussed. 


\title{
THOMSON SPECTROMETER MEASUREMENT OF HEAVY ION BEAMS PRODUCED BY A PULSE POWERED PLASMA FOCUS DEVICE*
}

\author{
M. J. Rhee
}

\section{Electrical Engineering Department University of Maryland \\ College Park, Maryland 20742}

A compact Thomson spectrometer is constructed for the purpose of observing helium, nitrogen and argon ions accelerated in a pulse powered plasma focus. The spectrometer employs a single aperture system and CR-39 plastic as a detector. The energy, charge state and mass of the ions are determined by analyzing the Thomson parabolas. Also methods of obtaining the energy spectrum and the energy-resolved emittance from the same Thomson parabolas are discussed.

Key Words: charge to mass ratio; energy resolved emittance; energy spectrum; Thomson spectrometer.

\section{Introduction}

The Thomson: spectrometer is a versatile device which has the ability to analyze simultaneously the energy distribution of ions having a wide range of charge to mass ratios. Since this spectrometer does not have focusing property like many other types of spectrometers, a well collimated beam is required for higher resolution. Accordingly, a high sensitivity detector system is required because collimation reduces beam intensity. Kuswa [1] and others [2] have reported successful use of the Channeltron Electron Multiplier Array (CEMA) of high gain ( $10^{3}$ ) as a detector in Thomson spectrometer. This high gain detector permitted the use of highly collimated beam to increase resolution and recording of single shot event.

The nuclear track detector in general has $100 \%$ detection efficiency i.e., individual ions can be recorded within certain range of energy for given species. Recently the CR-39 plastic plate is known as an excellent material for track detector [3] and has been used as a detector in a Thomson spectrometer [4,5]. The main advantage of this detector is not only its sensitivity which enable use of a highly collimated beam, but also its spatial resolution of ion tracks. Since individual ion tracks are a few microns in diameter, the accuracy of measurement of 
ion deflections may be an order of magnitude higher than other systems, thus one can build a relatively compact device with such a detector without sacrificing its resolution.

The two aperture system is commonly used to produce a collimated beam. In the case of an approximately point source or parrallel beam, the single aperture system has the advantage of simplifying the structure and the operation of the system. The aperture plate(s) is commonly placed in the upstream of the deflecting field region. It was found, however, that placing the aperture plate in downstream has some advantage if a single aperture system is employed. In the latter case, the measured deflection includes only the portion of the ion path where there are no fields and the path is straight whereas in the former case, the deflection also includes the portion of the ion path where the electric and magnetic fields are present and the path is curved. Thus fringing fields in the transition region introduce errors in the ion deflection in former case but not the latter. Furthermore, for the same target deflection, the beam cross-section size is smaller for the latter case because the ions spend less time after the aperture in the latter case than the former, allowing less time for beam spreading due to space charge and emittance effects.

The ion beam investigated using the Thomson spectrometer is produced by a new kind of plasma focus device which is powered by a H.V. pulse as reported earlier [6]. It is apparent that since the ions are generated in tightly pinched plasma column, the ion beam is generated from analmost perfect point source and good emittance is expected.

The following two sections describe ion beam source and the Thomson spectrometer. The remaining sections are concerned with experimental results, their analysis and discussions.

\section{Ton Beam Source}

A Marx generator charged Blumlein pulse system [7], which was designed for the injector to the University of Maryland Electron Ring

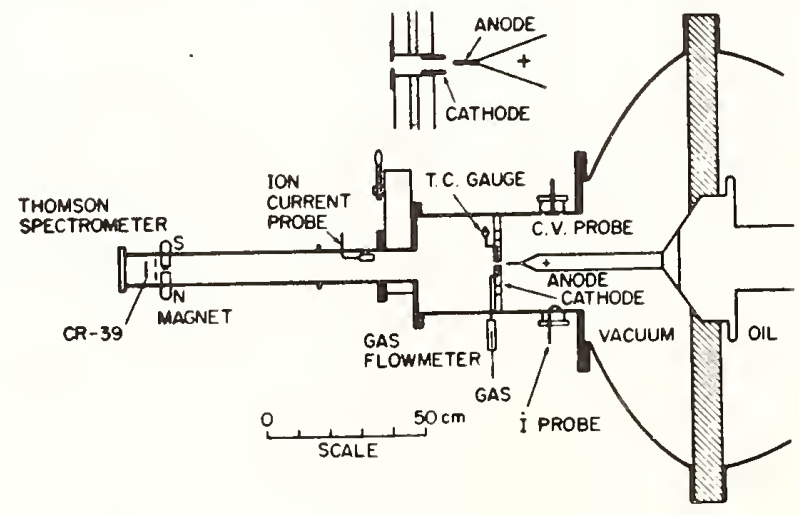

Figure 1. Schematic of experimental configuration. 
Accelerator, was modified to produce a pulse with positive output polarity. The output is coupled to a small plasma gun which consists of an anode (inner electrode) of approximately $5 \mathrm{~mm}$ diameter metallic rod and a planar cathode (outer electrode) with a $1.25 \mathrm{~cm}$ diameter hole on axis as shown in Figure 1. The upstream side of the hole is plugged with $1 \mathrm{~cm}$ long molybdenum or stainless steel rod which as a $6 \mathrm{~mm}$ diameter hole on axis. The downstream side is covered with $3 \mathrm{~mm}$ thick stainless steel plate with a $3 \mathrm{~mm}$ diameter hole on axis. After passing through a flowmeter and a needle valve, various gases are continuously injected into the plenum in the cathode where its pressure is monitored by a thermocouple gauge. The gas is diffused into the main vacuum tank mainly through the upstream hole in the plug rsesulting in formation of a volume of localized gas density in the anode-cathode area. When a pulse arrives in the gun area, a heavy discharge current flows through the gas which quickly pinches itself off due to the $m=0$ MHD instability. As a result of the pinching off of the current carring plasma column, a very high voltage is induced in the gap of the broken plasma column. Thus the ions and the electrons in the plasma column are accelerated to an energy equal to the product of the charge stage and the induced voltage and are moving in the positive and negative axial directions, respectively. Hence the ions and electron accelerated by this induced voltage are expected to have a broad and continuous spectrum. The magnitude of the induced voltage is highly transient, because the current carried by the earliest accelerated electrons and ions acts to reduce that voltage.

\section{Thomson Spectrometer}

A compact spectrometer as shown in Figure 2 is comprised of deflection sector, an aperture plate, and CR-39 detector plate.

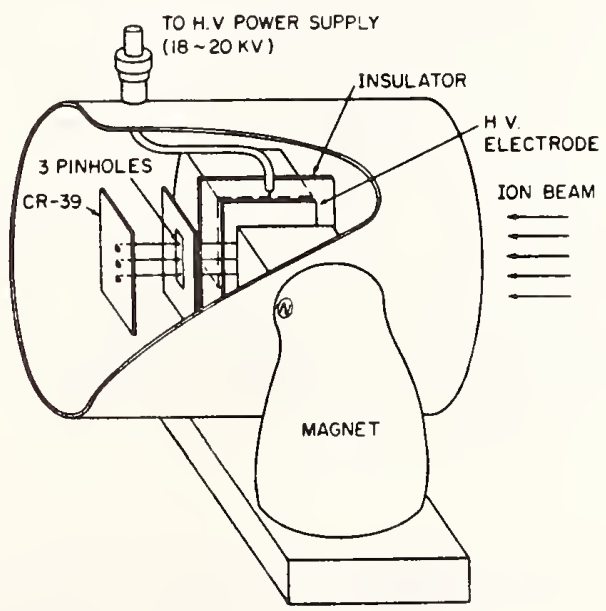

Figure 2. Thomson spectrometer. 
The deflection sector consists of two soft ion pole pieces of $3 \mathrm{~cm}$ $x 3 \mathrm{~cm}$ cross section, which are held by two brass rings (not shown) to maintain pole gap of $1 \mathrm{~cm}$ inside the $10 \mathrm{~cm}$ diameter aluminum vacuum chamber. Placed outside the vacuum chamber is a permanent magnet which produces a field of $2.6 \mathrm{kG}$ in the gap. The electric field parallel to the magnetic field is obtained by biasing a stainless steel electrode to high voltage. This electrode is mounted on one of the magnet poles and is separated from it by an insulating lucite plate. The aperture plate with single or multi-pinholes is placed in the downstream of the deflection sector. The pinhole is made by using a sharpened needle to drill a hole through a $0.05 \sim 0.15 \mathrm{~mm}$ thick copper foil. The size of pinhole is readily adjusted from $25 \mu \mathrm{m}-250 \mu \mathrm{m}$.

A CR-39 plate is placed $1 \sim 2 \mathrm{~cm}$ further downstream of the aperture plate. If the region of electric and magnetic fields has a cross sectional area large enough, more than one parabola may be formed on any given shot through the use of multiple apertures. For example, Figure 2 shows the use of a three pinhole system in which parabola at three different verticle positions are formed simultaneously. Because of high spatial resolution of the detector, the size of the parabola track pattern can be as much as two orders of magnitude smaller than similar patterns on conventional types of detectors.

The deflection of ions in the Thompson spectrometer is well known; ions of a given charge state and a distribution of energies appear as a parabola on the detector screen. Consider a charged particle passing through and at a right angle to a region of uniform, parallel and horizontal magnetic and electric fields of length $\mathrm{L}$. The particle experiences two different types of forces, one in vertical direction due to the magnetic field and the other in horizontal direction due to electric field. Since, as was stated before, the deflection paths are straight lines, one may consider deflection angle (normalized deflection) rather than absolute deflection. The calculation of the deflection angles are elementary under the assumptions of uniform fields and small deflection angles. The magnetic deflection angle is given by

$$
\Theta_{b}=\frac{Z e B L}{\sqrt{2 A M T}}
$$

where $\theta_{b}$ is the magnetic deflection angle in radians, $Z$ is the charge state of the ion, $e$ is the electronic charge, $B$ is the magnetic field in Tesla, $L$ the length of uniform field region, $A$ is the ion mass number, $M$ is the unit nucleon mass based on $C^{12}$, and $T$ is the kinetic energy of the particle in Joules. Similarly the electric deflection angle is found to be

$$
\theta_{\mathrm{e}}=\frac{\mathrm{ZeEL}}{\mathrm{AMT}} \text {, }
$$

where $E$ is the uniform electric field in $\mathrm{V} / \mathrm{m}$. 
- Combining Equations (1) and (2), eliminating kinetic energy $\mathrm{T}$, we obtain the well-known equation of parabola as

$$
\Theta_{\mathrm{e}}=\frac{\text { AMEL }}{\mathrm{Ze}(\mathrm{BL})^{2}} \theta_{\mathrm{b}}^{2} .
$$

\section{Experiment}

The pulser system is operated at relatively low voltage producing typical output of $+300 \mathrm{kV}$. This output voltage is monitored by two probes, a $\mathrm{CuSO}_{4}$ resistive divider immersed in oil section and capacitive divider mounted on the vacuum tank wall as shown in Figure 1 . Also the current is monitored by a dI/dt probe in the vacuum tank. Three gases, helium, nitrogen and argon, are used as the source of ions in this experiment. The gas flow is adjusted by a needle valve in the range of $3 \sim 10 \mathrm{cc} / \mathrm{min}$. Which gives pressure range at the plenum of $5 \sim 25 \mathrm{~m}$ Torr. The anode is $5 \mathrm{~mm}$ diameter molybdenum rod and the cathode is a stainless steel plug with a $6 \mathrm{~mm}$ diameter hole on axis. The ion beam is monitored by a Faraday cup which is immersed in a transverse magnetic field ( $1 \mathrm{kG}$ ) so as to suppress the possible secondary electron emission. The Thomson spectrometer is placed at the end of drift chamber, $100 \mathrm{~cm}$ from the cathode. The aperture plate has three $65 \mu \mathrm{m}$ pinholes spaced $5 \mathrm{~mm}$ in the vertical direction and is placed $10 \mathrm{~mm}$ upstream of the CR-39 target plate. Three shots are made to complete the exposure of each detector plate. One shot with both electric and magnetic fields forms the parabolas; the next with magnetic field only forms the vertical axis; and the last with electric field only forms the horizontal axis. The exposed CR-39 plate is then etched for $120 \mathrm{~min}$. in $6.25 \mathrm{~N} \mathrm{NaOH}$ solution at $70^{\circ} \mathrm{C}$. Each plate shows three sets of parabolas corresponding $r=0, r= \pm 5 \mathrm{~mm}$ pinhole locations and the parabolas are found to be very similar to each other. One of the parabolas for the nitrogen ion case is enlarged by using a microscope and is shown in Figure 3(a). The term BL in Equations (1) and ( 3 ) is replaced by more realistic value $\int \mathrm{Bd} 1=0.0133 \mathrm{TM}$; i.e., numerical integration of measured magnetic field distribution along the axis and approximate particle path. Unlike the magnetic field, the electric field is very difficult to measure; the field of ideal parallel plate capacitor multiplied by the lengths of electrode is used in place of the term EL in Equations (2) and (3). EL $\equiv(18 \mathrm{kV} / \mathrm{cm}) \times 3 \mathrm{~cm}=$ $5.4 \times 10^{4} \mathrm{~V}$. With these measured values the Equation (3) is rewritten as

$$
\theta_{\mathrm{e}}=3.164 \frac{\mathrm{A}}{\mathrm{Z}} \theta_{\mathrm{b}}^{2}
$$

where the angles are measured in radians. From these equations the kinetic energy can be deduced from the parabola by measuring either electric deflection or magnetic deflection. A family of parabolas and possible values of charge to mass ratios for the nitrogen case are displaced in Figure 3(b). 


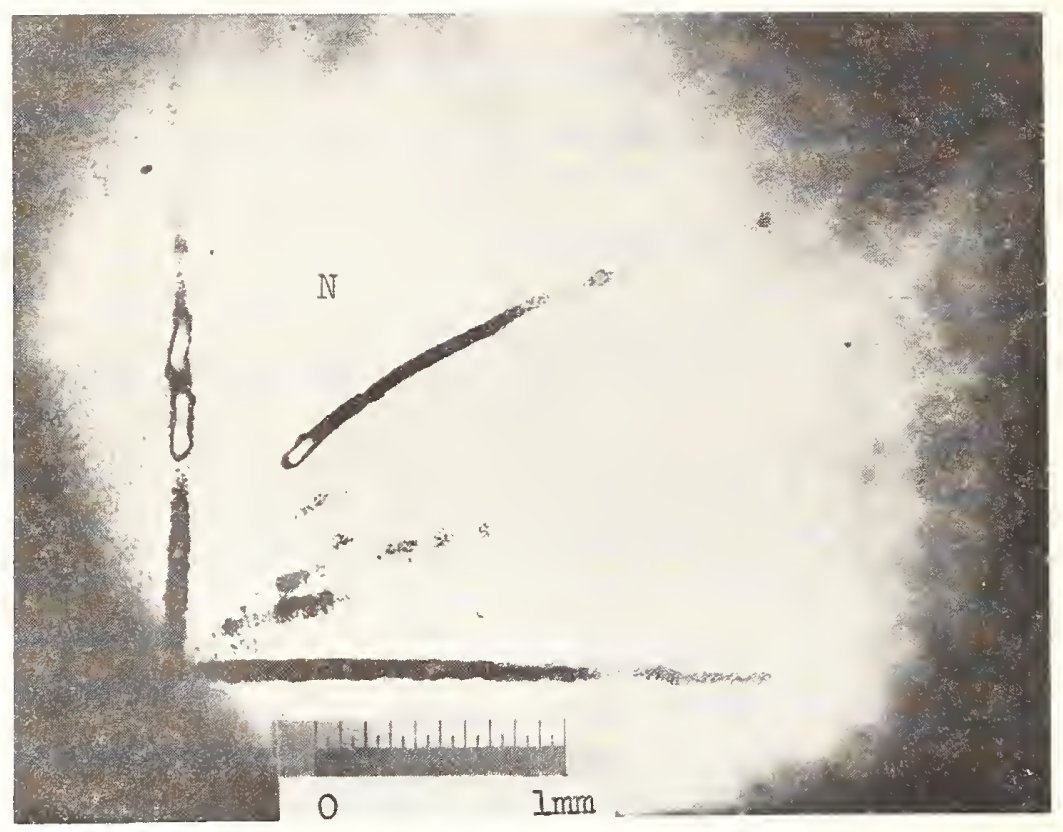

Figure 3(a). Thomson parabola of nitrogen ions enlarged from CR-39 detector plate.

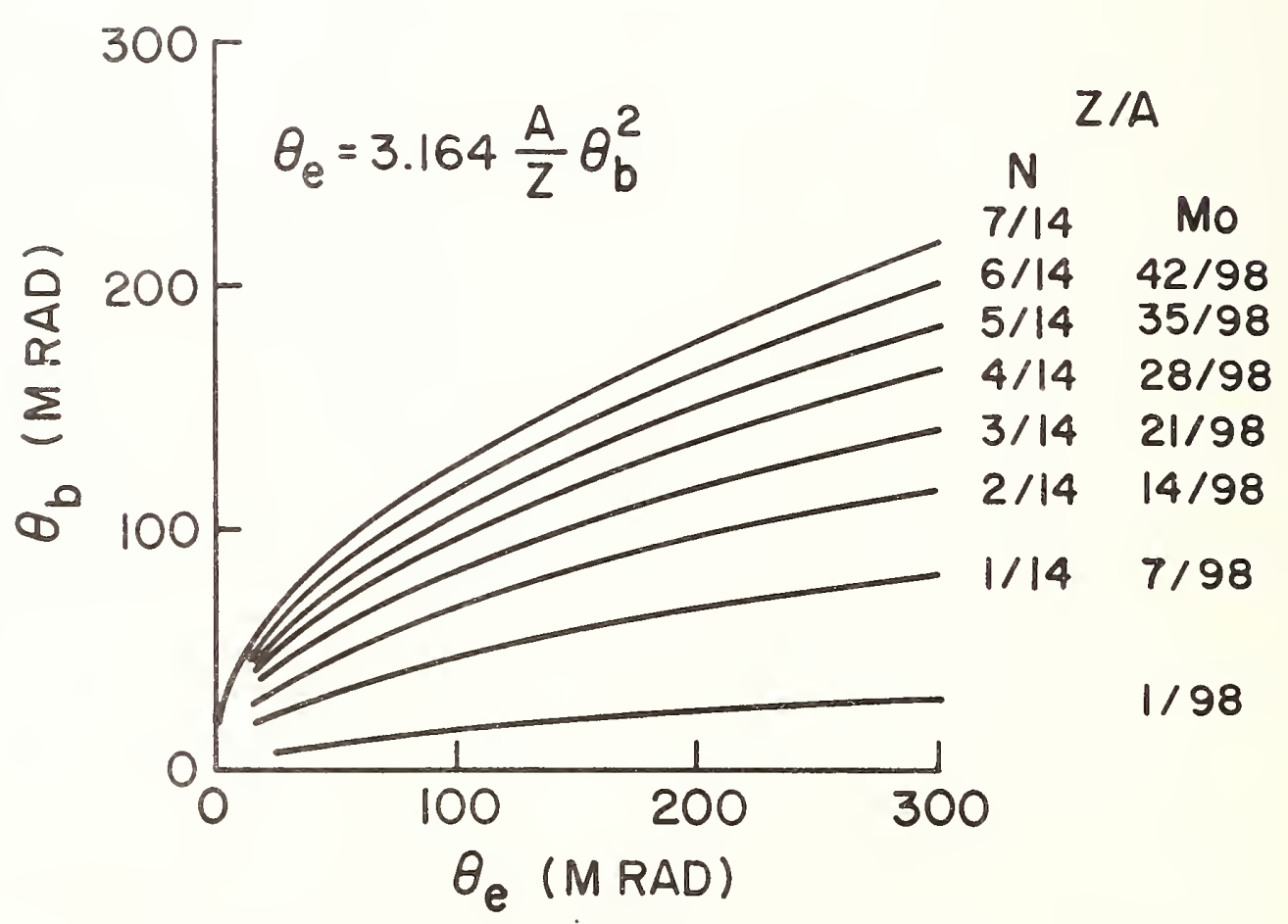

Figure 3(b). Calculated Thomson parabolas. 
Also Equations (1) and (2) are rewritten with the measured constants and in terms of convenient laboratory units for the kinetic energy

$$
\begin{aligned}
& \mathrm{T}_{[\mathrm{MeV}]}=8.54 \times 10^{3} \frac{\mathrm{Z}^{2}}{\mathrm{~A} \Theta_{\mathrm{b}[\mathrm{m} \mathrm{rad}]}^{2}}, \\
& \mathrm{~T}_{[\mathrm{MeV}]}=\frac{27 \mathrm{Z}}{\theta_{\mathrm{e}[\mathrm{m} \mathrm{rad}]}} .
\end{aligned}
$$

Despite the fact that the electric field in the spectrometer is approximated by an ideal parallel plate capacitor, the calculated and measured parabolas are in excellent agreement. The ions shown on the upper most parabola corresponds to charge to mass ratio of $7 / 14$ for nitrogen which are fully stripped nitrogen ions, whereas the ions below that parabola do not match the calculated ones for lower charge states of nitrogen ions. Similar patterns of parabolas are found in the cases of helium and argon gases as well. Comparison of these track patterns with that of the theoretical curves for singly and fully ionized molybdenum ions supports the hypothesis that these tracks were made by various charge states of molybdenum ions, molybdenum being the material making the anode. Since there are so many (42) charge states available to molybdenum, the exact charge states of the detected molybdenum ions could not be resolved with the present spectrometer.

\section{Results and Discussions}

As seen in Figure 3(a) virtually all of the ions from the gas are in the fully stripped state. Also the energies of ions detected have definite upper and lower limits. The lower value of energy is that determined by the acceptance angle in the horizontal direction of the spectrometer system. The Thoms on parabolas for the other gases, helium and argon, were obtained under the same operating conditions as that for the nitrogen. The peak energies of those ions are easily found by measuring either electric or magnetic deflections and using Equations (5) and (6). The results is plotted in Figure 4 as a function of charge when the ions are fully stripped, i.e., atomic number. It is interesting to note that the peak energy is proportional to charge of the ion, which is consistent with the prediction of the plasma diode model [8] whose detailed description will be treated elsewhere [9].

An important characteristic of the pulsed ion source for its possible application is its energy spectrum. In principle one can construct an energy spectrum by counting the ion track density along a parabola, provided that the ion tracks are well separated from each other. The track density along a given parabola as a function of either magnetic or electric deflection angle, which is a function of energy, 


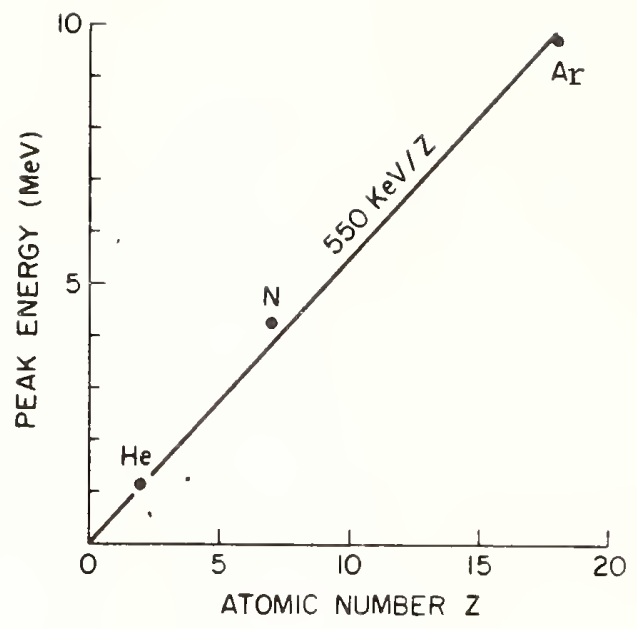

Figure 4. Peak energies of fully stripped helium nitrogen, and argon ions as measured by the Thompson spectrometer.

can be found experimentally by using a optical microscope. Then the energy spectrum is constructed by using the following relation

$$
\frac{\mathrm{dN}(\mathrm{T})}{\mathrm{dT}}=\mathrm{K} \frac{\mathrm{dn}}{\mathrm{d} \Theta} \frac{\mathrm{d} \Theta}{\mathrm{dT}}
$$

where $d n / d \Theta$ is the measured track density in $\Theta$ direction, (either $\theta_{e}$ or $\theta_{\mathrm{b}}$ direction) $\mathrm{K}$ is the ratio of total cross sectional area to the pinhole cross sectional area of the spectrometer. Unfortunately, the track density of the parabola displayed in Figure 3(a) is too large for individual tracks to be distinguished, thus not allowing the construction of an energy spectrum. A simple remedy for this problem is to either use a smaller pinhole or move the detector plate further from the aperture such that ion tracks are well separated.

Another application of such a Thomson spectrometer is an energy resolved emittance measurement. Assuming a cylindrically symmetric beam and no aberration, the normalized emittance is approximated [10] as

$$
\varepsilon_{\mathrm{n}}=\beta \gamma \mathrm{ar}_{\mathrm{o}}^{\prime}
$$

where $a$ is the radius of the beam, $r_{0}^{\prime}$ is the diverging angle, $d r / d z$ at $r=0, \beta=v / c$, and $\gamma$ is ratio of relativistic to rest mass. The emittance of helium ion beam produced using this principle was estimated and reported earlier [6]. 
The same parabolas can be used to determine the energy resolved emittance. Consider an idealized case in which the size of pinhole of the spectrometer is infinitesimally small. Then a half of the line width of parabola divided by the distance between pinhole and detector plate is the diverging angle. In the case when the size of pinhole is finite, the diverging angle may be approximately found by taking $r^{\prime}=$ $\frac{1}{2}$ (the full line width - dia. of pinhole)/separation distance. Thus the energy resolved emittance can be calculated from the measured line width along the parabola as a function of deflection angle which is again a function of energy. The line width of the parabola for fully stripped ions found in Figure 3 (a) is shown to be nearly constant over the entire length and measured to be $66 \pm \mu \mathrm{m}$. Since the diameter of the pinhole measured by a microscope is $\overline{6} 5 \pm 1 \mu \mathrm{m}$, the diverging angle is too small to be analyzed for emittance calculation with reasonable accuracy. Again smaller pinhole or larger separation distance or combination of both may improve the data reduction for such beam of low emittance.

\section{Acknowledgments}

The author would like to thank L. Floyd for his assistance in the experiment and for valuable discussions. J. Pyle also is to be thanked for his technical assistance.

\section{References}

1. G. W. Kuswa, et al, IEEE Trans. Nuc. Sci. 20, 305 (1973).

2. J. N. O1sen, et a1, J. App1. Phys. 44, 2275 (1973).

3. W. Prior, et al, Bull. Am. Phys. Soc. 24, 1039 (1979).

4. M. J. Rhee, Bull. Am. Phys. 25, 958 (1980).

5. F. Begay, Bu11. Am. Phys. 25, 1010 (1980).

6. M. J. Rhee, App1. Phys. Lett. 37(10), 906 (1980).

7. J. K. Burton, et al, IEEE Trans. Nuc. Sci. 20, No. 3, p. 321 (1973).

8. R. L. Gullickson, IEEE Conf. Record-Abstracts, 1980 IEEE, Int. Conf. Plasma Sci., p. 75.

9. M. J. Rhee, to be published.

10. J. Fasolo, ERDA Summer Study of Heavy Ions for Inertial Fusion, LBL-5543, 47 (1976).

8. Sponsor Acknowledgments

* Work supported by NSF and AFOSR. 


\title{
CURRENT MEASURING DIAGNOSTIC TECHNIQUES FOR HIGH DI/DT PARTICLE BEAM ACCELERATOR*
}

\author{
D. H. McDaniel \\ Sandia National Laboratories \\ ATbuquerque, NM 87185
}

Sandia National Laboraatories has developed the 10 TW Proto-II and 30 TW PBFA-I accelerator. The Proto-II accelerator has a dI/dt of $2.5 \times 10^{14} \mathrm{~A} / \mathrm{s}$ and has delivered $5 \times 10^{6} \mathrm{~A}$ to an imploding plasma load. PBFA-I has a dI/dt of $1.5 \times 10^{15} \mathrm{~A} / \mathrm{s}$ and has delivered $1.5 \times 10^{6} \mathrm{~A}$ to an electron beam diode load. At these values of $\mathrm{dI} / \mathrm{dt}$ and current we have surpassed the limits at which current viewing resistors (CVR's), Rogowski coils, cavity current monitors and B-dot probes are capable of giving accurate measurements when closer than $15 \mathrm{~cm}$ to the load.

The accuracy of CVR's are affected when heating of the material changes its resistivity during the current pulse. Rogowski coils are primarily limited 1 ) when the heating of the integrating resistor causes it to change resistivity, and 2) when the voltage due to $\mathrm{dI} / \mathrm{dt}$ across the coil housing causes breakdown. The cavity B-dot monitor fails due to breakdown over the cavity opening at high $\mathrm{dI} / \mathrm{dt}$ 's. B-dot probes fail when induced voltage causes breakdown of insulation in which they are mounted.

The need to measure high currents at high dI/dt's has led us to use piezoelectric gauges to measure the pressure induced in the electrode walls by the currents in the accelerator. Situations in which this technique is applicable are when the actual impedance at the current monitoring position is much less than the geometric free space impedance. This ensures that the electrostatic pressure effects are negligible. These gauges have subnanosecond response when properly mounted. The maximum pressure that these gauges can view, without resorting to complicated code analysis of the signal, is presently $10^{10}$ pascals. This corresponds to a level of $1.3 \times 10^{8} \mathrm{~A} / \mathrm{m}$ of 1 iner current density. The accuracy of the current measurements depends on the machining tolerances of the gauge and the accuracy at which material shock impedances can be determined. This technique has been used successfully on Sandia's Proto II accelerator and the Python accelerator at Physics International. Piezoelectric gauges are currently being fielded on the PBFA-I accelerator at Sandia National Laboratories.

*This work supported by the U. S. Department of Energy. 


\title{
PROTON CURRENT MEASUREMENTS USING \\ THE PROMPT GAMMA RAY DIAGNOSTIC TECHNIQUE
}

\author{
R. J. Leeper, E.J.T. Burns, D. J. Johnson, \& W. M. McMurtry \\ Particle Beam Fusion Research Department 4240 \\ Sandia National Laboratories \\ Al buquerque, New Mexico 87185
}

\begin{abstract}
Prompt gamma ray signals from the nuclear reaction $7_{\mathrm{Li}}(p, \gamma)^{8} \mathrm{Be}$ have been used to make time resolved proton current measurements. In these measurements, the proton beam was allowed to strike cylindrical thick lithium metal targets. The time integrated proton çurrent was measured using gamma activation of copper via the reaction ${ }^{63} \mathrm{Cu}(\gamma, n)^{62} \mathrm{Cu}(\beta+)$. The positron activity of the copper sample was easily measured using coincidence counting techniques. The number of ${ }^{62} \mathrm{Cu}$ atoms produced per proton incident on a thick Li metal target was determined with separate calibration runs performed on the Sandia $2.5 \mathrm{MeV}$ Van de Graaff accelerator. The time history of the prompt gamma production was measured using six EGG NPM-54 scintillator photomultiplier combinations shielded by $96.5 \mathrm{~cm}$ of concrete and $5.1 \mathrm{~cm}$ of $\mathrm{Pb}$. The use of six scintillator photomultiplier combinations was necessary to increase the statistical precision of the data. The normalization of the prompt gamma time history data with the total time integrated proton current measurement yielded the absolute time resolved proton current on target. Data from runs performed on the Sandia Proto I accelerator will be presented.
\end{abstract}

\section{Introduction}

Intense, pulsed ion beams with current densities $\geq 25 \mathrm{kA} / \mathrm{cm}^{2}$ have been generated by reflex triode, reflex pinched and magnetically insulated ion diodes $[1,2,3]$. The determination of the ion beam current and current density for these diodes is difficult. In part, this is due to the fact that intense ion beams are partially space-charge and current neutralized. These traits preclude or make difficult many electrical measurements involving charge collection or the measurement of self-magnetic fields. In addition, the use of conventional nuclear activation analysis 14 l at these high current densities is precluded because of beam induced target ablation. Furthermore, any screen used to prevent target ablation will be vaporized and the holes in the screen thereby closed off. This subsequently prevents most of the beam from reaching the activation sample. 
In an effort to overcome many of these difficulties, this paper describes a diagnostic method to measure the time resolved current of integse proton beams by detecting the prompt gamma rays emitted from the $\mathrm{Li}(p, y)^{8} \mathrm{Be}$ nuclear reaction induced in a lithium metal target by the beam [5]. The paper will also describe the procedure used to calibrate the diagnostic. Finally, an experimental demonstration of its use will be presented.

\section{Diagnostic Method}

When an intense proton beam pulse strikes a thick lithium metal target, all protons above $440 \mathrm{keV}$ in energy may induce the nuclear re-

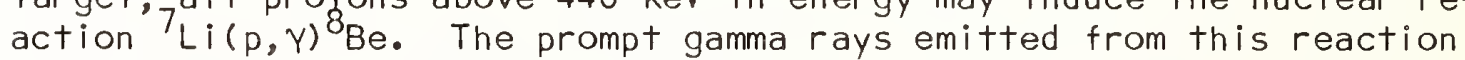
are produced in two energy groups of $14.7-15.2 \mathrm{MeV}$ and $17.6-18.2$ MeV. By measuring the time history of the prompt gamma ray production with fast scintillator photomultiplier detectors, the proton current on target can be determined provided the $7_{\mathrm{Li}(p, y)}^{8} \mathrm{Be}$ thick target crosssection is known. In principle, it is possible to calibrate the scintillator photomultipliers detectors directly on a Van de Graaff accelerator. In practice, however, due to the intense Bremsstrahlung background associated with the ion beam production, the scintillator photomltipliers require thick shields of some $76.2 \mathrm{~cm}-96.5 \mathrm{~cm}$ of concrete and $5.1 \mathrm{~cm}$ of lead for the preferential detection of the prompt gamma rays. The use of these massive shields would require proton beam sources beyond the capabilities of conventional accelerators. In order to achieve absolute calibration, we have ysed the photonuclear activation of copper via the reaction ${ }^{63} \mathrm{Cu}(\gamma, n){ }^{62} \mathrm{Cu}$ to measure the total number of prompt gamma rays produced. Then the proton current is determined by normalizing the prompt gamma ray time history signal with the total number of prompt gamma rays produced. A correction is made for dependence of the thick target yield on proton energy. The time history of the prompt gamma ray production required the use of an array of six scintillator photomultipliers to insure data of good statistical quality because of the rather low $7_{\mathrm{Li}}(p, \gamma){ }^{8} \mathrm{Be}$ production cross-section and the gamma ray attenuation in the massive shield.

\subsection{Prompt Gamma Ray Time History}

The six detector array used to measure the prompt gamma ray time history is shown in Figure 1. The scintillator photomultiplier combinations each consist of a $5.6 \mathrm{~cm}$ diameter $\times 6.7 \mathrm{~cm}$ thick cylinder of NE-111 plastic scintillator viewed by an Amperex XP2020 photomultiplier tube [6]. The FWHM response of the detectors is 4.5 ns when used to observe a single instantaneous event such as a cosmic ray. Each detector is capable of linear currents to 0.5 amp and is fully saturated at 1.0 amp of current, The detectors are operated at a sensitivity of 0.2 amps $/(\mathrm{rad} / \mathrm{sec})$ to ${ }^{60} \mathrm{Co}$ gamma rays. The scintillators are coated with white paint except for the photomultiplier tube viewing port. The gains of each detector are cross calibrated using the Compton edge technique. In addition, the gain of each detector is periodically monitored for any shift throughout an experimental run. Typically the gain shifts observed were less than $1 \%$. 


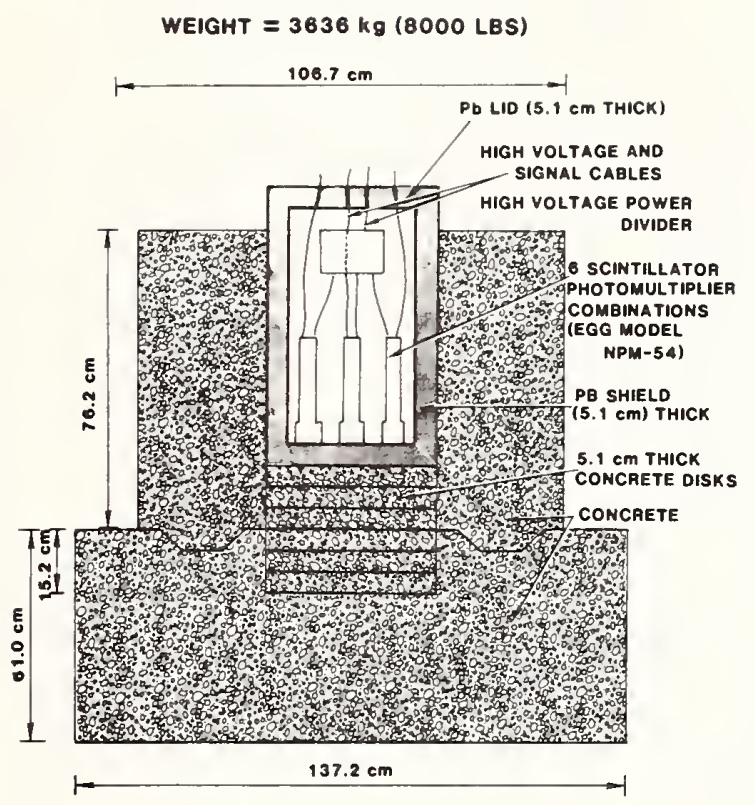

Figure 1. Schematic of prompt gamma ray time history detector

The six detector array is required in present experiments to insure data of good statistical quality. It is found that on an individual shot (at an average signal level of 6 volts) there are variations of $\pm 80 \%$ between individual detectors. By simply averaging the six detectors together we produce a signal that is statistically better by a factor of the $\sqrt{6}$ resulting in a net signal good to $\pm 33 \%$. It should be noted that a secondary benefit of a multiple detector array is that it allows the statistical precision of the data to be measured experimentally, whereas with a single detector, this is more difficult to determine.

The massive concrete shield shown in Figure 1 is necessary to attenuate the large $x$-ray bremsstrahlung background that is generally produced by electron loss in an ion diode. Whereas an ion diode may be 80-90\% efficient in producing ions, there is an accompanying electron current which can amount to some $100 \mathrm{kA}$ or more $(10-20 \%$ of the total diode current) at voltages exceeding $1.5 \mathrm{MV}$. Experimentally, it is found that $76.2 \mathrm{~cm}-96.5 \mathrm{~cm}$ of concrete and $5.1 \mathrm{~cm} \mathrm{~Pb}$ are enough shielding to reduce this bremsstrahlung background to less than $35 \%$ of the prompt gamma ray signal for the $1.8 \mathrm{MV}$, I TW Proto I accelerator. The reason concrete is chosen as the primary shielding material is because its gamma ray attenuation coefficient is a factor 2.1 smaller at $15 \mathrm{MeV}$ - $18 \mathrm{MeV}$ than it is at $2.0 \mathrm{MeV}$ 171. Consequently, the concrete shielding will preferentially attenuate the lower energy $x$-ray bremsstrahlung background while passing the higher energy prompt gamma rays with the overall effect of increasing the prompt gamma ray signal to noise ratio. The extension of this diagnostic method to higher energy bremsstrahlung backgrounds, is not examined in this paper and therefore use of this technique on other accelerators of higher output voltages must be carefulty investigated. 


\subsection{Total Prompt Gamma Ray Yield}

A schematic of the experimental arrangement that is employed to measure the total number of prompt gamma rays using photonuclear activation of copper is shown in Figure 2. The energetic gamma rays from the ${ }^{7} \mathrm{Li}(p, Y)^{8} \mathrm{Be}$ reaction are allowed to strike a $7.6 \mathrm{~cm}$ diameter by $1.0 \mathrm{~cm}$ thick copper disk located $19.1 \mathrm{~cm}$ from the lithium target. These gamma rays then induce the photonuclear reaction ${ }^{63} \mathrm{Cu}(\gamma, n)$ ${ }^{62} \mathrm{Cu}(\beta+)$. This reaction has on the average $952.3 \mathrm{mb}$ cross section for the $14.7-15.2 \mathrm{MeV}$ and $17.6-18.2 \mathrm{MeV}{ }^{2} \mathrm{Li}(p, Y)^{8} \mathrm{Be}$ gamma rays, and has a threshold of $10.9 \mathrm{MeV}$. The $10.2 \mathrm{~cm}$ thick polyethylene moderator that encapsulates the copper disk is used to partially moderate and to attenuate any fast neutrons that might be produced in the reaction ${ }^{7} \mathrm{Li}(d, n)^{8} \mathrm{Be}$ by the natural abundance of deuterium in the proton beam. This is important since neutrons above $11.0 \mathrm{MeV}$ may produce ${ }^{62} \mathrm{Cu}$ via the reaction ${ }^{63} \mathrm{Cu}(n, 2 n)^{62} \mathrm{Cu}(\beta+)$. This thickness of moderator will attenuate these fast neutrons by a factor of approximately 18.5 and make their contribution to the activation negligible [8]. Note that photonuclear neutron production in the moderator due to the bremsstrahlung background does not occur since the bremsstrahlung end point energy is below any photonuclear reaction threshold.
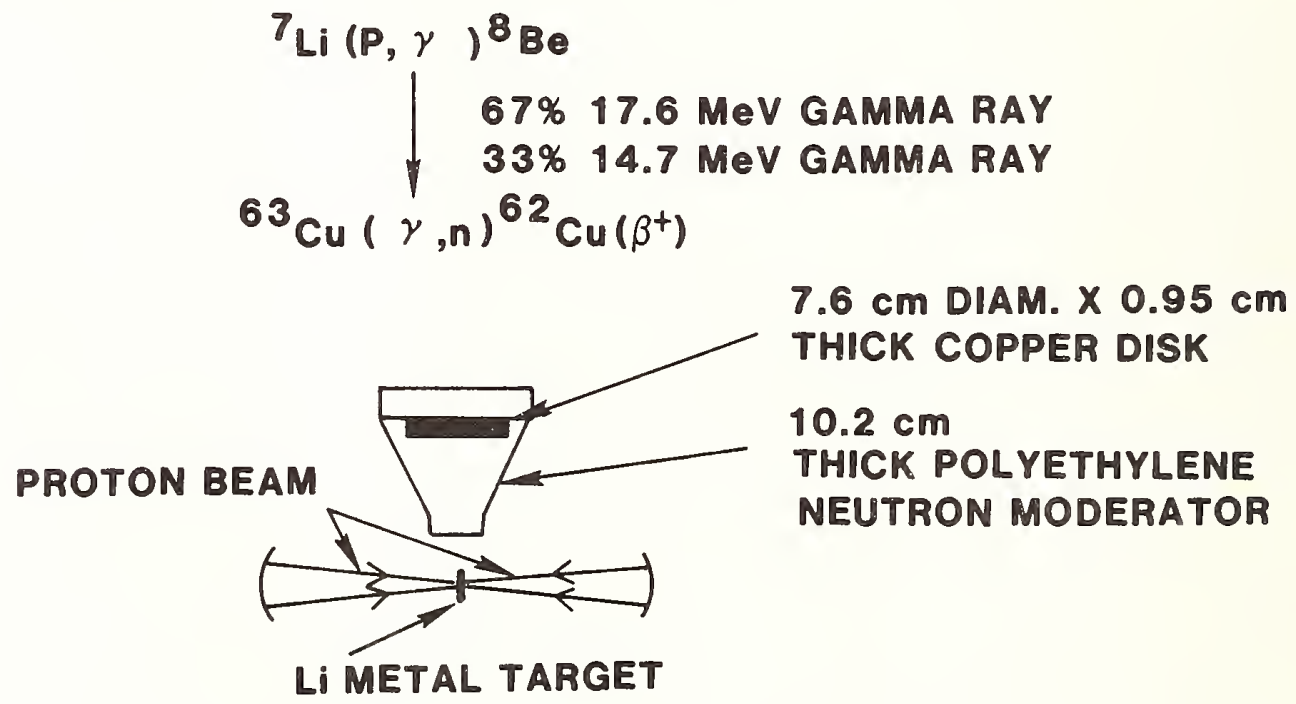

Figure 2. Experimental arrangement for measuring the total number of prompt gamma rays using the photonuclear activation of copper

The ${ }^{62} \mathrm{Cu}$ produced is a positron emitter with a $9.8 \mathrm{~min}$ half-l ife. After activation, the copper disk is removed from the accelerator vacuum chamber and placed between two $12.7 \mathrm{~cm}$ diameter by $7.6 \mathrm{~cm}$ thick $\mathrm{Nal}(\mathrm{Tl}$ ) scintillator photomultiplier detectors. The two $0.511 \mathrm{MeV}$ annihilation gamma rays from the ${ }^{62} \mathrm{Cu}$ decay are then energy selected and counted in a standard coincidence counting arrangement. In this way a highly sensitive and low background counting arrangement for positron annihilation is realized. The background count rates are generally between 3 and 4 counts per minute. 


\section{Diagnostic Calibration}

The absolute calibration of the copper disk was carried out on the Sandia 2.5 MeV Van de Graaff accelerator. A thick lithium metal target was irradiated by protons ranging in energy from $0.5 \mathrm{MeV}$ to $1.5 \mathrm{MeV}$. Target currents were generally $500 \mathrm{nA}$ to $750 \mathrm{nA}$ with target spot sizes of $9 \mathrm{~mm}^{2}$. Beam runs of 30 minutes were used throughout the calibration. The $7.6 \mathrm{~cm}$ diameter by $1 \mathrm{~cm}$ thick copper disk was encapsulated in a $10.2 \mathrm{~cm}$ thick polyethyene moderator and located at an angle of $90^{\circ}$ to the beam axis. After a beam run was completed, the copper disk was removed and counted for a period of 20 minutes in the coincidence counting arrangement described above.

Figure 3 shows the results of the calibration. Plotted are the (background corrected) counts per gm Cu per incident proton on target versus proton energy. The absolute detection efficiency of $\approx 5 \%$ for detecting positron decays from the copper disk has not been unfolded from the data. It is clear from Figure 3 that the calibration factor has a rather slow dependence on proton energy. The open circle data shown is the thick lithium target yield data from Reference 9 normalized to our data at $0.9 \mathrm{MeV}$. The solid curve is obtained by connecting the open circle data between $0.5 \mathrm{MeV}$ and $1.1 \mathrm{MeV}$ and then extrapolating the curve through our data to $1.5 \mathrm{MeV}$. As can be readily seen, there is good agreement between the present data and the data of Reference 9.

$17.6 \mathrm{~cm}$ DIAM. $\times 0.95 \mathrm{~cm}$ THICK CU DISK AT $14.9 \mathrm{~cm}$ IN $10.2 \mathrm{~cm}$ THICK MODERATOR)

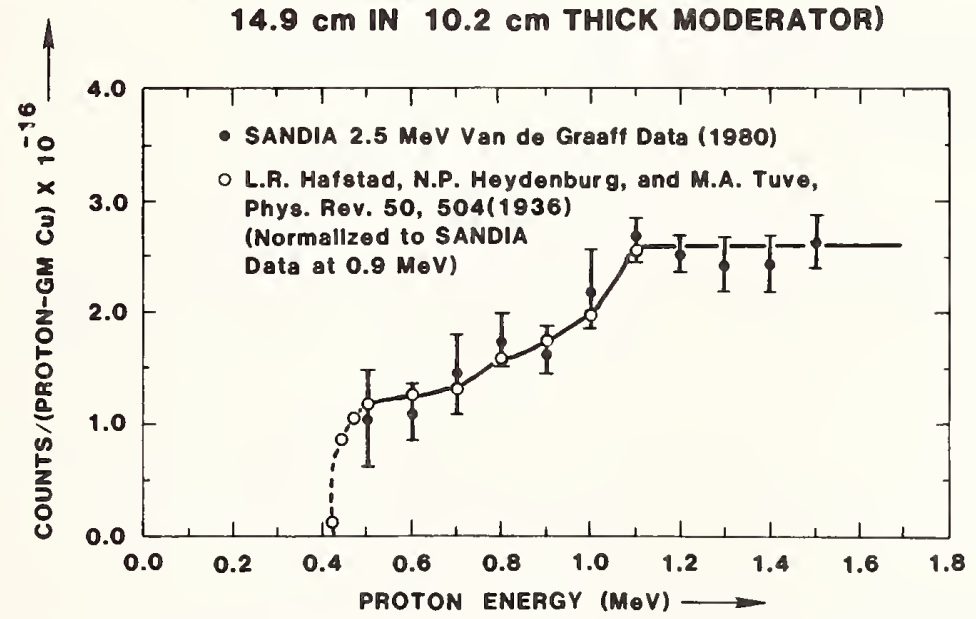

Figure 3. Copper calibration factor ${ }^{\mathrm{Y}} \mathrm{CU}$ versus proton energy

\section{Experimental Demonstration}

The current of a proton beam onto a $19 \mathrm{~mm}$ diameter by $12.7 \mathrm{~mm}$ tall thick lithium metal target has been measured as an experimental demonstration of this diagnostic method. The proton beam is generated by a radial magnetically insulated ion diode powered by the 
Sandia Proto I generator. A schematic of this diode is shown in Figure 4. The copper activation sample is located $19.1 \mathrm{~cm}$ from diode center and along the vertical axis of the diode. The six detector array is positioned along the vertical axis at a distance of $1.8 \mathrm{~m}$ from diode center. A description of the operation of the diode is given in Reference 3 .

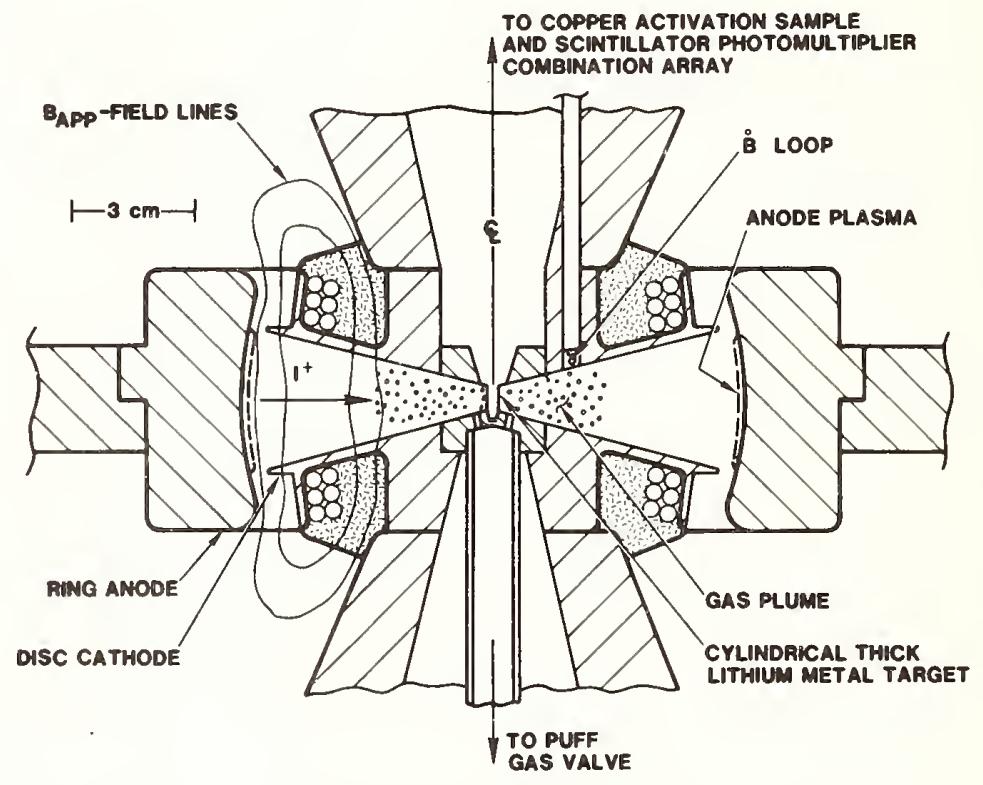

Figure 4. Schematic of the Proto I radial magnetically insulated ion diode

The larger of the two waveforms shown in Figure 5 is the signal obtained upon averaging the signal from each detector of the six detector array for the case of the $19 \mathrm{~mm}$ diameter lithium target shot. The copper disk activity on this shot was 128 counts/min measured starting $115 \mathrm{sec}$ after the shot. The smaller waveform in Figure 5 shows the average signal from an identical shot for the case of a $17 \mathrm{~mm}$ diam. aluminum target. This signal. is due to bremsstrahlung production from electron loss in the anode cathode gap of the diode. The lower production cross section and lower energy gamma rays from proton induced prompt gamma emission from Al combine to make their contribution to the signal neglible $[10,11]$. The copper disk activity for this shot was 8 counts/min at $130 \mathrm{sec}$ after the shot. As can be seen, the peak of the signal from the aluminum target comes earlier than the lithium target signal and has an amplitude equal to $35 \%$ of the lithium target signal. The delay between the signals is due to time-of-flight of the protons transversing the $6 \mathrm{~cm}$ distance from the anode to the lithium target. Consequently the smaller aluminum target signal, the delay between the aluminum and lithium signals, and the absence of any counts above background on the copper disk for the case of the aluminum target, when taken together, serves as positive evidence that we are indeed observing ${ }_{L i}(p, Y)^{8}$ Be prompt gamma rays. 


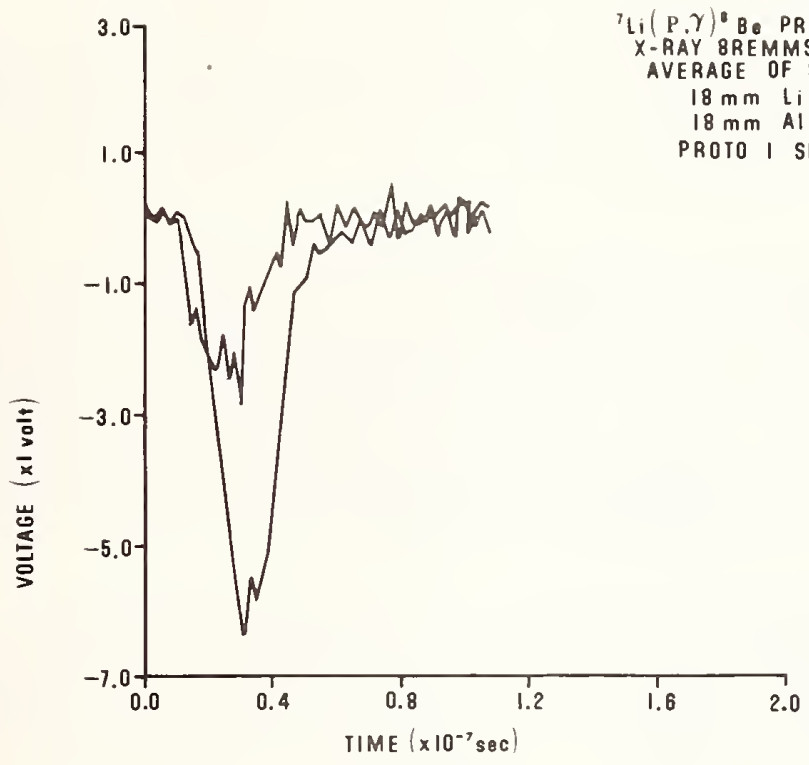

Figure 5. Average ${ }^{7} \mathrm{Li}(p, \gamma)^{8}$ Be prompt gamma ray signal and $x$-ray bremsstrahlung background signal

To determine the proton current from the normalization of the measured prompt gamma time history and copper activation count, a correction must be made for the flight times of different energy (voltage) protons from the diode to the lithium target. Then the time variation of the prompt gamma ray detector vo!tage $V_{\text {Det }}(t)$ is related to the variation of the proton current $I_{p}(t)$ in the following way

$$
v_{\text {Det }}(t)=k \quad \int_{0}^{\infty} Y\left(V\left(t^{1}\right)\right) I_{p}\left(t^{1}\right) \delta\left(t-t^{1}-\frac{l}{V\left(t^{\prime}\right)}\right) d t^{1}
$$

where $K$ is a constant specified by the detector geometry, sensitivity, and target-to-detector distance; $V\left(t^{\prime}\right)$ is the inductively corrected diode voltage at time $t^{\prime} ; Y\left(V\left(t^{\prime}\right)\right)$ is a quantity proportional to the thick lithium target yield for protons of energy $V$ (number of prompt gamma rays per proton); $I_{p}\left(t^{\prime}\right)$ is the proton current at time $t^{\prime}$ (number of protons per second'); $l$ is the proton flight path from the anode to the target and $v$ is the velocity of protons of energy $v=1 / 2 \mathrm{Mpv}^{2}$ emitted at time $t^{i}$ from the anode $[121$. The calculated copper activation counts above background $(\mathrm{C}-\mathrm{B}) \mathrm{Ca}$ is obtained by adapting to our case the standard activation formula of Reference 13. This relationship is 


$$
(C-B)_{C a l}=M_{C u}\left(e^{-\lambda t_{1}}-e^{-\lambda t_{2}}\right) \int_{t_{1}}^{t_{F}} \int_{0}^{\infty} Y_{C u}\left(v\left(t^{\prime}\right)\right) I_{p}\left(t^{i}\right) \delta\left(t-t^{i}-\frac{l}{v\left(t^{\prime}\right)}\right) d t^{i} d t
$$

Here $\mathrm{M}_{\mathrm{Cu}}$ is the mass of the copper sample, $\lambda$ is the copper decay constant, t, is the time interval between the end of irradiation to the start of counting, $t_{2}$ is the time interval from the end of irradiation to the end of counting, $t_{1}$ is the time the voltage pulse starts, ${ }_{F}$ is the time at the end of the voltage pulse, $Y_{C u}\left(V\left(t^{\prime}\right)\right)$ is the absolute copper activation yield curve given in Figure 3 , and all other variables are the same as those defined for Eq. (1).

The first step in the data reduction routine we have adopted is to determine the temporal shape of the proton current $i_{p}(t)$ from the prompt gamma ray time history signal $V_{\text {Det }}(t)$. In practice, this is determined by iterating on Eq. (1) until the prompt gamma ray response calculated with an assumed proton current agrees in temporal shape with the measured prompt gamma ray response. We use the absolute copper activation yield $Y_{C u}\left(V\left(t^{\prime}\right)\right)$ of Figure 3 for $Y\left(V\left(t^{\prime}\right)\right)$ since it is a quantity directly proportional to the lithium thick target yield. The initial proton current assumed is the total diode current corrected for electron lost. Often this initial guess is sufficient to give good agreement between the measured and calculated prompt gamma responses. Once the temporal shape of $I_{p}(t)$ has been determined, it is substituted into Eq. (2) which is then integrated for the duration of the voltage pulse. The net result of the calculation of Eq. (2) is the total number of copper activation counts above background, $(\mathrm{C}-\mathrm{B})_{\mathrm{Cal}}$, for the counting interval $t_{1}$ to $t_{2}$ for the initial assumed proton current $1_{p}(t)$. The calculated copper activity $(\mathrm{C}-\mathrm{B}) \mathrm{Cal}$ is then normalized to the experimentally observed copper activity, $(C-B)_{\text {Exp, to yield a final proton }}$ current given by

$$
\left.\right|_{p} ^{F i n a \mid}(t)=\left.\left[\frac{(C-B)}{(C-B) C a l}\right]\right|_{p}(t)
$$

Applying the above data reduction procedure to the present data yields the results presented in Figure 6. Shown in Figure 6a are the final proton current and energy (diode voltage) used in Eq. (1) to calculate the prompt gamma ray signal presented in Figure $6 \mathrm{~b}$. The experimental signal in Figure $6 b$ is obtained by subtracting the aluminum target signal from the lithium target signal in Figure 5. In this way, the Bremsstrahlung contribution to the lithium signal is subtracted away. The small prepulse in front of the large prompt gamma ray signal in Figure $6 \mathrm{~b}$ is due to this subtraction not being perfect. This prepulse being neglected, it is observed that there is excellent agreement 
between the calculated and experimental prompt gamma ray pulses and that there is $353 \pm 54 \mathrm{Ka}$ peak current on the $19 \mathrm{~mm}$ diameter lithium target. The error quoted is the addition in quadrature of a $\pm 5.4 \%$ statistical error from the copper activity used to obtain (C-B) Exp and an estimated error of $\pm 14.2 \%$ in the value of the quantity $(C-B)$ Cal. This latter error is estimated from a calculation of ${ }^{(C-B)} \mathrm{Cal}$ in which values of $Y_{C u}(V(t))$ in Eq. (2) were allowed to deviate one standard deviation from the mean. We note, however, that this rather modest error in $(C-B)$ is due in part to the unique behavior of the voltage and current in this experiment that just happens to emphasize the higher precision $Y_{C u}\left(V\left(t^{\prime}\right)\right.$ data points of Figure 3. In an experiment that has high currents at substained low voltage this error will be somewhat larger. In yet another parameter sensitivity calculation, it is found that varying the voltage by $\pm 10 \%$ resulted in a variance of $\pm 7.6 \%$ in the quantity $(\mathrm{C}-\mathrm{B}) \mathrm{Ca}$. No error in the quantity $(\mathrm{C}-\mathrm{B}) \mathrm{Cal}$ due to voltage variations was included in the present error analysis. Finally, it should be pointed out that the prompt gamma ray measurements only sample the proton current above $440 \mathrm{keV}$. Therefore, the measurement is insensitive to the current profile for proton energies below this threshold.

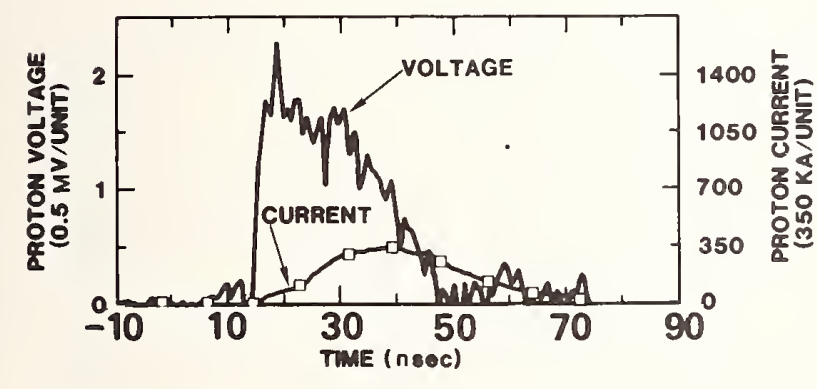

Fig. $6 a$

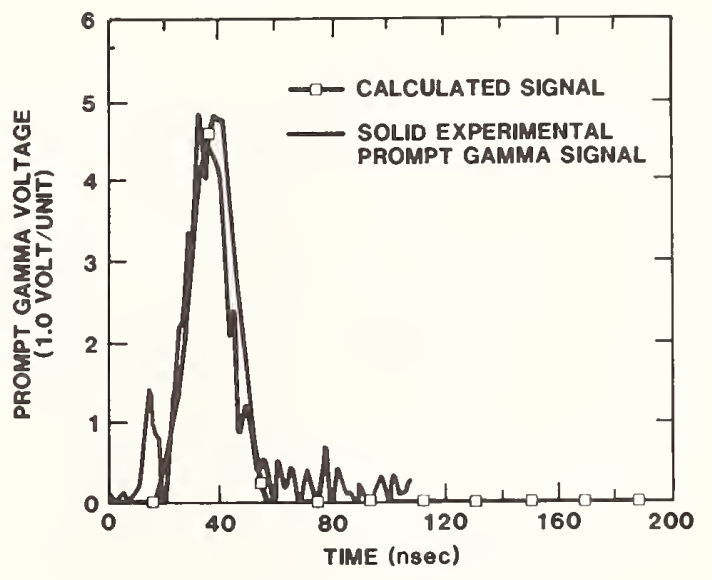

Fig. 6b

Figure 6. The measured prompt gamma ray detector response (solid line) in Figure $6 \mathrm{~b}$ is compared with the response (open square points) calculated using the proton voltage and current displayed in Figure 6a.

\section{Conclusions}

A diagnostic technique for measuring the current of intense pulses of protons with a time resolution of $\sim 4.5 \mathrm{~ns}$ has been described. The absolute calibration of a $7.6 \mathrm{~cm}$ diameter by $1.0 \mathrm{~cm}$ thick copper disk used to measure total prompt gamma yield has been calibrated using the Sandia 2.5 MeV Van de Graaff accelerator. Finally, as an experimental demonstration of the technique, the absolute proton current onto a $19 \mathrm{~mm}$ diameter lithium metal target in a pulsed beam experiment was determined. 


\section{Acknowledgments}

We wish to acknowledge the help and cooperation of R. A. Burchard and $W$. R. Wampler in helping us perform the copper calibrations on the Van de Graaff accelerator. Also we wish to thank J. Heise, O. Marten, and D. Tanner for their able operation of the Proto I accelerator.

\section{References}

[1] S. A. Goldstein, G. Cooperstein, R. Lee, D. Mosher, and S. J. Stephanakis, Phys. Rev. Let†. $\underline{40}, 1504$ (1978).

(2) J. Golden, C. A. Kapetanakis, S. J. Marsh, and S. J. Stephanakis, Phys. Rev. Lett. 38, 130 (1977).

[3] D. J. Johnson, G. W. Kuswa, A. V. Farnsworth, Jr., J. P. Quintenz, R. J. Leeper, E. J. T. Burns, and S. Humphries, Jr., Phys. Rev. Lett. 42,610 (1979).

(4) F. C. Young, J. Golden, and C. A. Kapetanakis, Rev. Sci. Instrum. $48,432(1977)$.

[5] J. Golden, R. A. Mahaffey, J. A. Pasour, F. C. Young, and C. A. Kapetanakos, Rev. Sci. Instrum. 49, 1384 (1978).

[6] Available from EGG, Inc., Las Vegas, Nevada as Model NPM-54.

17] R. E. Lapp and H. L. Andrews, Nuclear Radiation Physics, PrenticeHall, Inc., Englewood Cliffs, N.J., p. 424 (1972).

[8] S. S. Nargolwalla and E. P. Przybylowicz, Activation Analys is with Neutron Generators, John Wiley and Sons, New York, p. 30 (1973).

[9] L. R. Hafstad, N. P. Heydenburg, and M. A. Tuve, Phys. Rev. 50, 504 (1936).

[10] K. J. Brostrom and T. Huus, Phys. Rev. 71, 66I (1947).

[1I] G. P. Plain, R. G. Herb, C. M. Hudson, and R. E. Warren, Phys. Rev. 57,187 (1940).

[12] F. C. Young, F. Oliphant, and S. J. Stephanakis, NRL Memorandum Report 4171 (1980).

[13] M. Barbier, Induced Radioactivity, John Wiley and Sons, New York, pp. 13-17 (1969).

8. Sponsor Acknowledgment

This work supported by the U.S. Department of Energy under contract DEAC04-76-DP00789. 


\section{THE MEASUREMENTS OF PULSED ELECTRIC CURRENTS BY THE FARADAY EFFECT}

J. Katzenstein, W. Caton and G. M. Witkinson

Maxwe 11 Laboratories

8835 Balboa Avenue

San Diego, CA 92123

\section{Introduction}

The Faraday effect has been known for over one hundred years and has been applied extensively to the measurement of magnetic fields. Its use for the measurements of electric current is, however, less common because the magnetic field arising from the current flow in an arbitrary circuit in general has a spatial variation. Hence, the calibration of a current probe employing the Faraday effect involves geometric factors such as the dimensions of the rotative medium and its position relative to the circuit [1] . An obvious way out of this difficulty is to design the optical path in the rotative medium so that it encircles the current. Since the Faraday rotation angle is proportional to the line integral of the magnetic induction $\vec{B}$ along the path $\bar{l}$, we can write for a closed light path.

$$
\theta=V \oint \vec{B} \cdot d \vec{l}
$$

where $V$, the constant of proportionality, the so-called Verdet constant, is characteristic of the rotative medium and has the units of minutes of arc per gauss-centimeter.

From the circuital statement of Amperes law we may replace the circuital integral $\$ \vec{B} \cdot d \vec{l}$ by $4 \pi / 10 \mathrm{I}$ where $\mathrm{I}$ is the current in amperes that is enclosed by the path of integration. The resulting Faraday rotation is thus 


$$
\theta=\frac{4 \pi}{10} V I
$$

The Faraday rotation produced in such a current probe depends only on the Verdet constant of the rotative medium; geometric factors do not enter. Current monitors based on this principle have been developed [2,3], using optical fibers as the rotative medium. Unfortunately, optical fibers which rigorously preserve the state of polarization of light traversing them are presently beyond the start of the art. As the Verdet constant of the fiber core material (essentially fused quartz) is sma11, a large number of turns of the fiber around the conductor is needed to obtain a measurable rotation which greatly increases the stray birefringenece due to fiber strain and curvature. To minimize the latter effect the radius of curvature is made large which increases the overall size of the probe. Nevertheless, Faraday-effect current monitors have been developed for use on high voltage utility transmission lines and offer a cost-effective alternative to conventional current transformers $[4,5]$

The light entering the Faraday probe is rendered plane polarized by means of a polarizer (unless a laser emitting plane polarized light is used as a source) and the light emerging passes through a second polarizer or analyzer before entering the detector. The intensity of Tight $\mathscr{I}$ reaching the detector for an incident intensity of $\mathscr{I}_{0}$ is given by

$$
\dot{I}=\mathscr{I}_{0} \cos ^{2}(\Theta+\chi)
$$

where $x$ is the angle between the axes of transmission of the polarizer and analyzer.

Two different methods of handling the Faraday effect signals can be used depending upon whether the value of $\Theta(I)$ at peak current is very much greater or very must less than $\pi$.

In the latter case, the usual practice is to make use of a differential analyzer such as a Wollaston prism which transmits two output beams of intensity $\mathscr{I}_{1}$ and $\mathscr{I}_{2}$ which are polarized orthogonally to each other [4,5]? The analyzer is oriented at $45^{\circ}$ with respect to the transmission axis of the polarizer. The resulting transmitted intensities are:

$$
\begin{aligned}
& \mathscr{I}_{1}=\mathscr{I}_{0} \cos ^{2}\left(\theta-45^{\circ}\right), \\
& \mathscr{I}_{2}=\mathscr{J}_{0} \cos ^{2}\left(\theta+45^{\circ}\right) .
\end{aligned}
$$


The sum and difference of these two quantities and their quotient are computed. The resulting signal $s$ is

$$
\mathrm{s}=\frac{\mathscr{I}_{1}-\mathscr{I}_{2}}{\mathscr{I}_{1}+\frac{2}{\mathscr{I}_{2}}}=\sin 2 \theta
$$

or for $\Theta \ll \pi$ using Eq. 2

$$
s=k I
$$

where $k$ is a proportionally constant determined by choice of units.

The resulting signal is then linearly proportional to I . The accuracy of the measurement depends on the accuracy which the computations of Eq. 5 can be carried out. Since these involve the determination of the small difference of two large quantities the accuracy is critically dependent on the system signal-to-noise ratio.

An alternative measurement technique useful when $\theta\left(I_{\max }\right)>\pi$ is to use crossed analyzers $(x=\pi / 2)$. The signal is then

$$
\begin{aligned}
\mathscr{I}(t) & =\mathscr{I}_{0} \sin ^{2} \Theta(t) \\
& =\mathscr{I}_{0} \frac{1-\cos 2 \Theta(t)}{2}
\end{aligned}
$$

or using Eq. 2

$$
\mathscr{I}(t)=\mathscr{I}_{0}\left[\frac{1-\cos \left(\frac{8 \pi}{10} V I(t)\right)}{2}\right]
$$

The signal given by Eq. 8 can be inverted numerically to obtain I as a function of $t$. Figure 1 shows the Faraday signal resulting from a current waveform and its subsequent numerical inversion to recover the original waveform. 

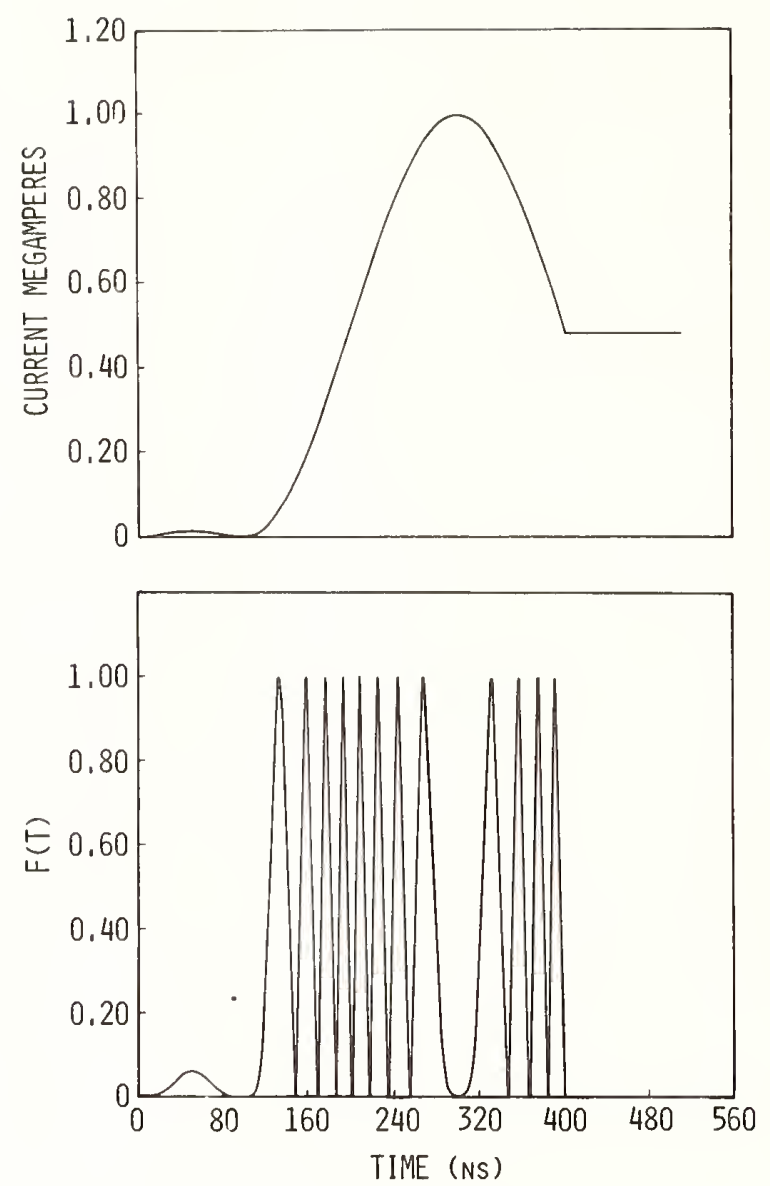

Figure 1. Sample current waveform and corresponding Faraday rotation signal.

The accuracy of measurement is determined by the precision with which the phase of $\cos \theta$ can be determined from the record of $I(t)$ which in turn depends upon the signal-to-noise ratio. If we call this phase uncertainty $\Delta \phi$ then the relative error $\xi$ is

$$
\xi=\frac{\Delta \phi}{\pi N+\phi} \approx \frac{\Delta \phi}{\pi N}
$$

where $N$ is the total number of cycles of Faraday rotation. Thus, even though a relatively large value of $\Delta \phi$ is obtained due to a poor-signal-to-noise ratio, the error in the current measurement can be made small by making $N$ large. It is assumed, however, that $\Delta \phi$ is not so large that a charge of sign of the time derivative of the phase cannot be detected. Such a reversal in $d \phi / d t$ occurs when I attains an extremum. 
In our particular application, the current I necessary to change the phase of $\theta$ by $\pi$ is $2105 \mathrm{~A}$. For the currents we wish to measure $N$ is 20-40. Thus, our maximum relative error is 2.5 percent. With a favorable signal-to-noise ratio this can be improved by an order of magnitude (a resolution of 10 percent in signal amplitude).

\section{Detailed Design of a Faraday Current Monitor for Large Pulse Currents}

We have designed a Faraday-effect current monitor to measure pulsed currents of the order of 4 MA max whose duration is typically $100 \mathrm{~ns}$. The immediate application of the monitor is the calibration of the magnetic pickup loops heretofore used for current measurements on the BLACKJACK 5 pulse generator. The tests will initially be undertaken at reduced powers and short circuit loads in order to accomplish this calibration. Should the Faraday-effect monitor survive at higher power levels used for operational shots with plasma loads, it will be incorporated into the apparatus as a regular diagnostic for routine measurements. As discussed above such a Faraday effect monitor is an absolute instrument whose calibration depends upon a fundamental property of the rotative medium. Furthermore, the limiting time resolution of such a monitor is given by the transit time of light around the closed optical path.

We initially considered the use of an optical fiber as the rotative medium as was done in references [2] and [3], but rejected this approach because of the fiber birefrigence difficulty discussed above as well as the low value of the Verdet constant of the fiber core material. Such a low value of Verdet constant would limit the number of cycles of Faraday rotation and hence the accuracy of the measurement as discussed above. The use of optical fibers to transmit light to the Faraday probe from the source and back from the probe to the detector is, however, very convenient. Such an arrangement provides the necessary electrical insulation of the probe from ground and eliminates the need for optical alignment during installation. The light source, detector and recording equipment could be placed in a screen room at some distance from the pulse generator, thus, minimizing electrical noise. Since the monitor is provided with polarizers at the entry and exit of the measurement light path, the birefringence and residual Faraday rotation in the fiber due to stray fields play no role in the final result.

We selected as the Faraday rotative medium a high density flint glass, Schott type SF-58. This glass has a Verdet constant of 0.082 and an index of refraction of 1.907 at the wavelength of the helium neon laser, $6328 \AA$. The glass has a very low residual birefrigence and a high transmission for the He-Ne wavelength. An alternative choice might have been one of the Terbium-doped glasses manufactured 
by the Hoya Company. These glasses have Verdet constants of the order of 0.2. We rejected the Hoya glasses both because they are very expensive and because their Verdet constants are temperature sensitive since the glasses are paramagnetic. While the diamagnetic glasses such as SF-58 have lower Verdet constants, their values are essentially constant independent of temperature variations. Since the current required to produce a rotation of 1800 is $\sim 10^{5} \mathrm{~A}$ for SF-58, the number of cycles of Faraday rotation at the operating peak currents of 2-4 MA will be 20 or more. Thus, a larger value of Verdet constant, while useful for reducing the relative error of the measurement is really not needed.

The closed light path within the rotative medium is realized by multiple total internal reflections (TIRs) within the rotative medium. As is well known a TIR introduces a phase shift between the components of polarized perpendicular and parallel to the plane incidence. Hence, if the incident light is plane polarized the reflected light becomes elliptically polarized. This ellipticity in the incident light would lead to a Faraday signal in which the amplitude as well as the phase varied with time unlike the simple expression (Eq. 8). The computer inversion of the Faraday signal would thus be very complex.

A very simple solution to the problem posed by the ellipticity in polarization induced by TIR was found by Kard [6]. He showed that a coating on a TIR interface one quarter wavelength thick (corrected for angle of incidence) whose index of refraction was the geometric mean of that of the substrate and the external medium (usually air or vacuum) would compensate the phase shift between the parallel and perpendicular components of polarization. Thus, plane polarized light of arbitrary azimuth incident on a TIR surface equipped with such a coating would be reflected as plane polarized light and could be completely extinguished with an analyzer. This solution is applicable only to cases for which the angle of incidence on the substrate-film interface is less than the critical angle, i.e., part of the light must enter the coating and be totally reflected from the coating-vacuum interface. For the SF-58 glass of refractive index 1.907, magnesium flouride of index 1.38 is almost a perfect match, besides being a very satisfactory material for making durable optical coatings of precisely controlled thickness. The critical angle at the coating-substrate interface is arc sin $(1.38)^{-1}$ or $46.4^{\circ}$. Thus, a coating of MgF will effectively compensate the ellipticity for an angle of incidence of $45^{\circ}$. We have tested such coatings at $45^{\circ}$ incidence and found that a very good null of the reflected light can be obtained with an analyzer for any arbitrary azimuth of the incident polarization.

We constructed a Faraday probe in which the light path is along the sides of a square. The light path was realized by TIR from three surfaces at a $45^{\circ}$ angle of incidence, the reflecting surfaces being equipped with $\mathrm{MgF}_{2}$ coatings to compensate ellipticity. In 
order to accurately align the surfaces they were formed by planeconvex lenses which were cemented to a ring of SF-58 glass $6 \frac{1}{4} \mathrm{in}$. OD, $3 \frac{1}{2}$ in. ID, and $5 \mathrm{~mm}$ thick.

The light was conducted to and from the ring by optical fibers equipped with lenses to provide a parallel beam of $\sim 5 \mathrm{~mm}$ aperture. The light was coupled into the ring by a crown glass prism cemented to a plane convex lens similar to those used for the reflecting surfaces. Total attenuation introduced by the ring and fibers was less than $25 \mathrm{db}$. Figure 2 is a diagram of the device.

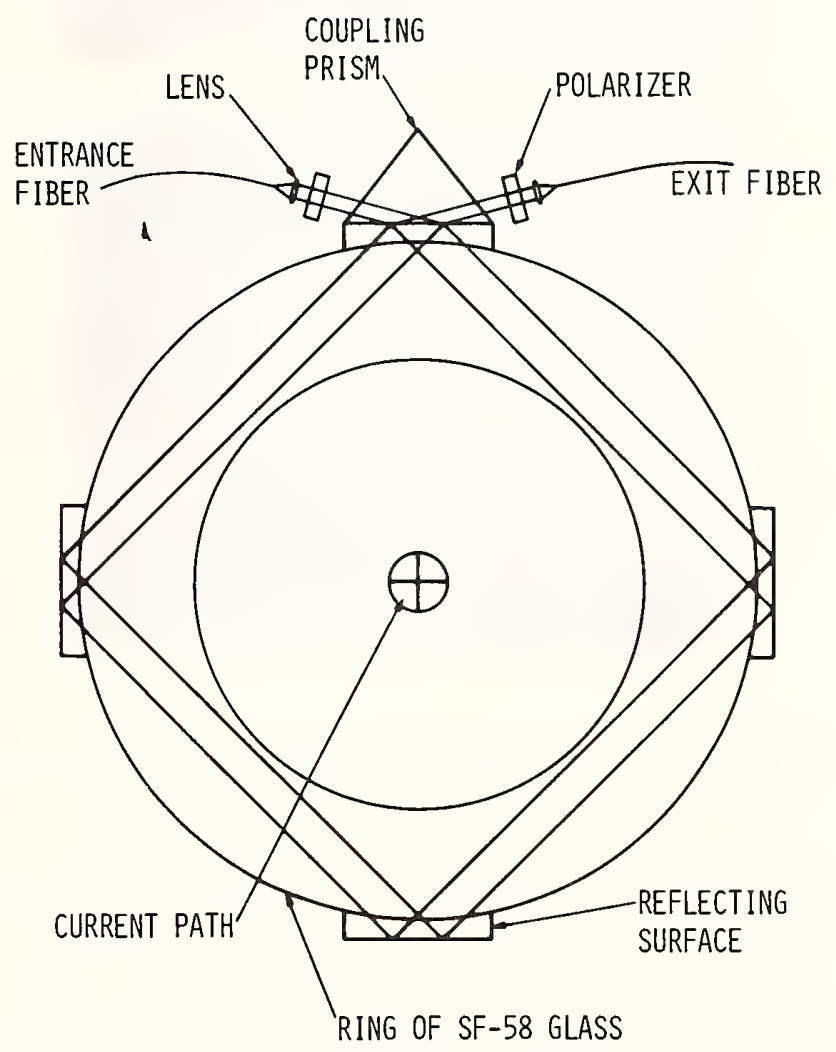

Figure 2. Diagram of Faraday effect current monitor.

Figure 3a shows a photograph of the ring in its plastic protective housing with mounts for the optical fibers and polarizers. Figure $3 b$ is a photograph of the housing with the cover removed to show details of the ring itself. 

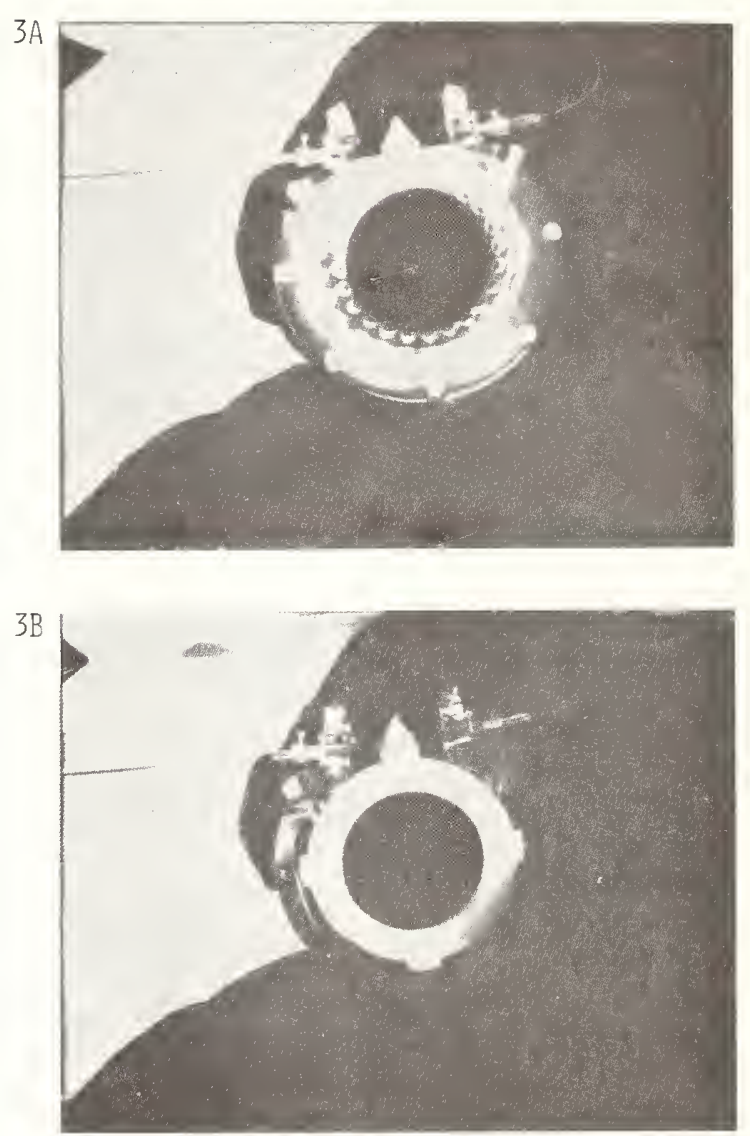

Figure 3. Photograph of Faraday effect current monitor with (upper) and without protective housing (10wer).

The light source was a $5 \mathrm{MW}$ He-Ne 1aser. The laser beam was focused into the end of the fiber with a microscope objective. The detector was a TIED55 avalanche photodiode followed by a transimpedance amplifier with a $10 \mathrm{k} \Omega$ feedback resistor. The light from the fiber was rendered parallel by a lens and passed through an interference filter of $60 \mathrm{~A}$ bandwidth and then focused by a second lens on a photodiode. The interference filter served to eliminate stray light from the spark gaps that switch the test current from a condenser bank.

\section{Results -- The Problem of Mirror Reversion}

The previously described Faraday monitor was tested with the pulse current supplied the discharge of a $40 \mathrm{kV}-37.5 \mu \mathrm{fd}$ condenser bank. The peak current of the first half cycle was $326 \mathrm{kA}$. No Faraday rotation was detected at all despite a peak current over three times that required for a full cycle of Faraday rotation based on published values of the Verdet constant. 
This decisively negative result lead us to reexamine the entire concept and led to the realization of a fundamental defect in the existing monitor which is correctable; the effect of mirror reversion. The easiest way to understand the effect is by the use of the Jones calculus [7]. This calculus treats the effect of various polarization components such as rotators or retarders on polarized light by representing the polarized light by a two-row column vector which is operated on by $2 \times 2$ matrices representing the optical components in the light path. The effect of a number of components is obtained by multiplication of the series of matrices representing the optical train. We need only two of these matrices for the present discussion; a rotator matrix $S(\alpha)$ representing a rotation of the plane of polarization through an angle $\alpha$ and a retardation matrix $G(\gamma)$ representing a retardation by a phase angle $\gamma$ of one orthogonal component relative to the other. These matrices can be written as

$$
\begin{aligned}
& S(\alpha)=\cos \alpha-i \sigma_{2} \sin \alpha \\
& G(\gamma)=\cos \gamma / 2+i_{3} \sin \gamma / 2
\end{aligned}
$$

where $\sigma_{2}$ and $\sigma_{3}$ are two of the well known Pauli spin matrices.

$$
\begin{aligned}
& \sigma_{1}=\left(\begin{array}{ll}
0 & 1 \\
1 & 0
\end{array}\right) \\
& \sigma_{2}=\left(\begin{array}{cc}
0 & -i \\
i & 0
\end{array}\right) \\
& \sigma_{c}=\left(\begin{array}{rr}
1 & 0 \\
0 & -1
\end{array}\right)
\end{aligned}
$$

The effect of mirror reversion can be taken into account by ascribing the matrix of a half-wave retarder to a mirror; i.e., a mirror is represented by $G(\pi)$. The net effect of a number of Faraday rotations are interspersed by mirror reflections is given by

$$
S\left(\alpha_{1}\right) G(\pi) S\left(\alpha_{2}\right) G(\pi) S\left(\alpha_{3}\right) G(\pi) \ldots
$$

Now the following identity follows from the anti-commutive property of the Pauli Matrices

$$
S(\alpha) G(\pi)=G(\pi) S(-\alpha) \text {. }
$$


Using this identity and noting that $G(2 \pi)$ is the unit matrix we have for expression (Eq. 11)

$$
S\left(\alpha_{1}\right) S\left(-\alpha_{2}\right) S\left(\alpha_{3}\right)\left(-\alpha_{4}\right) \ldots
$$

Thus, if the total light paths in the Faraday medium be decomposed into segments between mirror reflections and the segments be numbered sequentially the sign of the Faraday rotation changes with the parity of this number. We can thus write for the Faraday rotation instead of Eq. 1

$$
\begin{aligned}
& \Theta=V\left[\int_{\text {odd }} \overrightarrow{\mathrm{B}} \cdot \mathrm{d} \vec{\ell}-\int_{\text {even }} \overrightarrow{\mathrm{B}} \cdot \mathrm{d} \vec{\ell}\right] \\
& \text { segments segments }
\end{aligned}
$$

The reason for our negative result is now obvious. Since the even and odd numbered segments are of equal length in a square path formed by three reflections the net Faraday rotation is zero.

The solution to the problem of mirror reversion lies in first making the length of the even numbered segments of the light path as short as possible and second arranging the direction of these even numbered segments as nearly as possible at right angles to the local field direction so that the integrand of the second integral of Eq. 13 tends to vanish. Figure 4 shows such a light path and the glass configuration which will satisfy these requirements. The odd numbered segments form a closed square in the plane normal to the current direction and the Faraday rotation due to these should be just that of Eq. 2. The even numbered segments are then parallel to the direction of the current so the magnetic field will be normal to the direction of the light path and hence these segments should make a negligible contribution to the net Faraday rotation.

If the plane of the ring be tipped at an angle $\beta$ with respect to the current direction the relative error $\xi$ will be

$$
\xi=(1-\cos \beta)+\frac{l_{\text {even }}}{l_{\text {odd }}} \sin \beta
$$

As leven is the order of 5 percent of lodd or less for a typical construction a substantial tilt can be tolerated without impairing the accuracy of the measurement. 


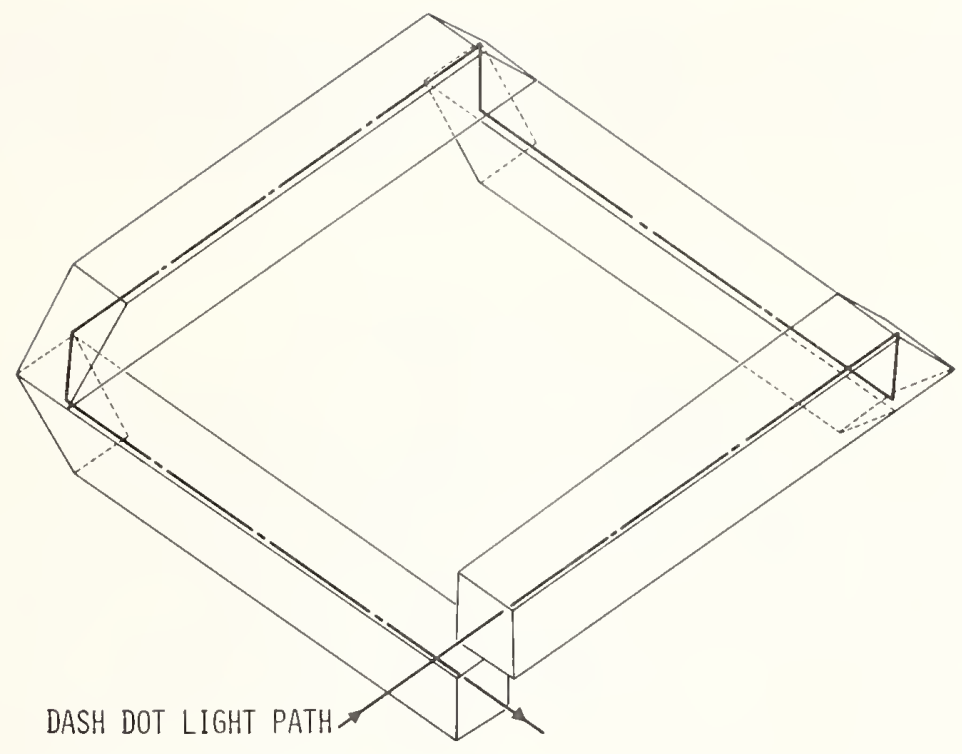

Figure 4. Proposed Faraday effect current monitor modified to eliminate mirror reversion effects.

The existing Faraday ring could be modified by cementing Amici or roof prisms ( $45^{\circ}-90^{\circ}$ isoceles prisms) to the reflecting faces with the roof direction in the plane of the ring. Unfortunately, the single quarter wave coating of Kard cannot be used to compensate the ellipticity for now the angle of incidence on the prism is 600 greater than the critical angle. In principle, the ellipticity can be compensated for any angle of incidence and any index of refraction of the substrate by use of Heperin coatings, i.e., symmetrical three layer coatings of low and high index of refraction [8]. The specification of the Heperin coating for a given angle of incidence and substrate index requires, however, a rather complex numerical calculation.

\section{Future Work}

A Faraday rotation of the configuration of Figure 4 will be constructed of prisms of SF-58 glass and either cemented together or clamped in optical contact by a frame of nonconducting material. The latter procedure would have the advantage that the ring could be temporarily split for installation around a conductor with inaccessible ends. The large apertures and normal incidence would simply the use of full-transmission polarizers such as calcite rhombs which will greatly improve the transmission over that of polarizing films. This improved transmission may be necessary for very rapid measurements to insure an acceptable signal-to-noise ratio. The large clear apertures would facilitate the use of mutiple series light paths to increase the sensitivity of the monitor. 
An attempt will be undertaken also to compute the required Heperin coatings for the ellipticity compensation of a roof prism using the procedure of reference [8]. The existing monitor can then be made functional by the addition of these prisms to the reflecting surfaces.

\section{Acknowledgements}

We wish to acknowledge many valuable discussions with John Shannon and Norman Rostoker of Maxwell Laboratories, Inc. The skill and dedication of Leslie Vargady of Metrooptics Co., Glendora $C A$, in fabrication of the optical components is greatfully acknowledged. We also acknowledge the devoted services of Lawrence Houghton and David Lischer of Maxwell for their assistance with the experimental work and to Clifford Fowler also of Maxwell for developing the algorithm for the numerical inversion of the Faraday signa7. This work was carried out under Contract No. DNA001-79-C-0019 of the Defense Nuclear Agency.

\section{References}

[1] G. A. Massey, D. C. Erickson and R. A. Kadlec, Applied Optics, 14 2712, (1975).

[2] A. M. Smith, Applied Optics 17, 52, (1978).

[3] A. Papp and H. Harms, Applied Optics 19, 3729 (1980).

[4] H. Aulich, W. Beck, N. Douklias, H. Harms, A. Papp and H. Schneider, Applied Optics 19, 3735, (1980).

[5] H. Harms and A. Papp, Applied Optics 19, 3741, (1980).

[6] P. G. Kard, Optics and Spectroscopy 6, 339, (1959).

[7] R. C. Jones, J. Opt. Soc. Am. 31, 488, (1941).

18 ] P. Mauer, J. Opt. Soc. Am. 56, 1219, (1966). 


\section{CAVITY CURRENT MONITORS*}

J. Shannon, E. Chu, R. Richardson

M. Wilkinson and C. Trivelpiece

Maxwe11 Laboratories, Inc.

8835 Baiboa Avenue

San Diego CA 92123

In measuring short ( 10 ns risetimes), high ( $M M A)$ current puises, a single turn $B$ probe is useful only when the current path is precisely known. On the other hand, self-integrating resonance-free Rogowski coils are relatively difficult to build.

We present a new type of current monitor that is predictable, simple in construction and immune to capacitive pickups. These monitors are basically cavities machined in the current carrying conductors. We will discuss the design criteria and the high frequency response of these cavity current monitors along with the late time effects due to resistive losses in the wall of the cavity.

*Work supported by the Defense Nuclear Agency.

\section{Introduction}

The development of high power pulse generators has led to the need for a current probe that is capable of measuring fast ( 10 ns risetimes) mega-ampere current pulses. Although these current pulses are often constrained to flow in current carrying conductors of known geometries, the actuai current distribution in the conductors is not necessarily known. The cavity current monitors we are reporting allow one to precisely measure sub-microsecond, mega-ampere current pulses with high insensitivity to the current distribution. These monitors are mechanicaliy more rigid, more immune to capacitive pickup than conventional $\dot{B}$ probes. Also, they are much easier to construct than self-integrating, resonance-free Rogowski coils. Moreover, the sensitivities of these monitors can also be calculated fairly accurately once the physical dimensions are known.

Basically, the cavity current monitors are re-entrant cavities machined in the current carrying conductors as shown in Figure 1. 
For short pulses, the current in the conductor essentially flows as a surface current. As a result, the re-entrant cavity develops a voltage drop across itself corresponding to the rate of change of flux in the cavity. For longer pulses, the response of the monitor will change due to the diffusion of magnetic flux into the cavity walls.

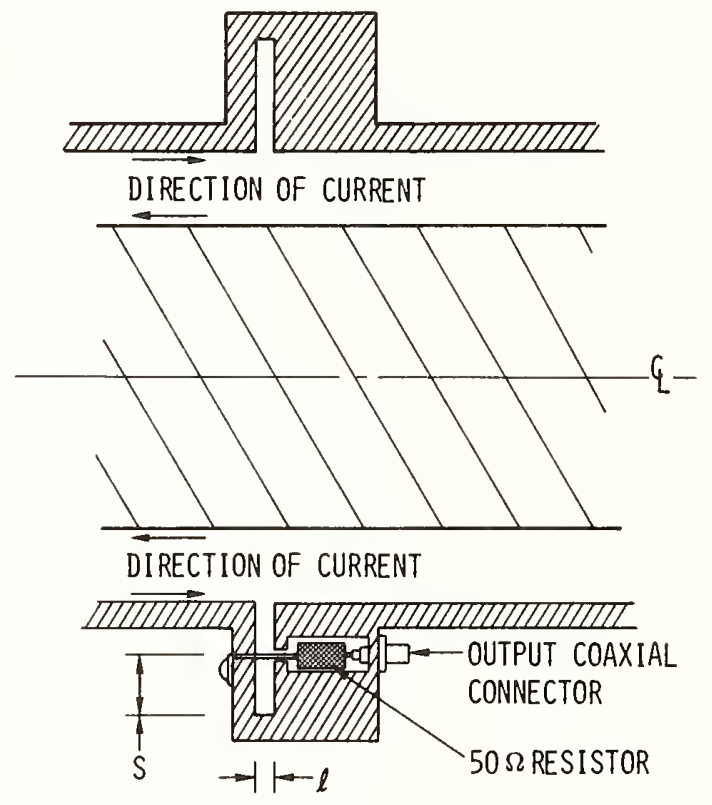

Figure 1. Sectional view of cavity current monitor.

In the following sections, we will discuss the theory of operation, the high frequency response, the low frequency response, and the design considerations of the cavity current monitors.

\section{Theory of Operation}

As shown in Figure 2, the cavity walls along with the connecting lead of the $50 \Omega$ resistor defines a magnetic pickup loop. An array of magnetic pickup loops is generally used to sample the flux change in the cavity at different points around the current path. The signals generated by these pickup loops are combined using cables of the same length at a summing circuit as shown in Figure 3 . The combined output is then integrated using a passive RC integration network to yield a signal proportional to the current. 


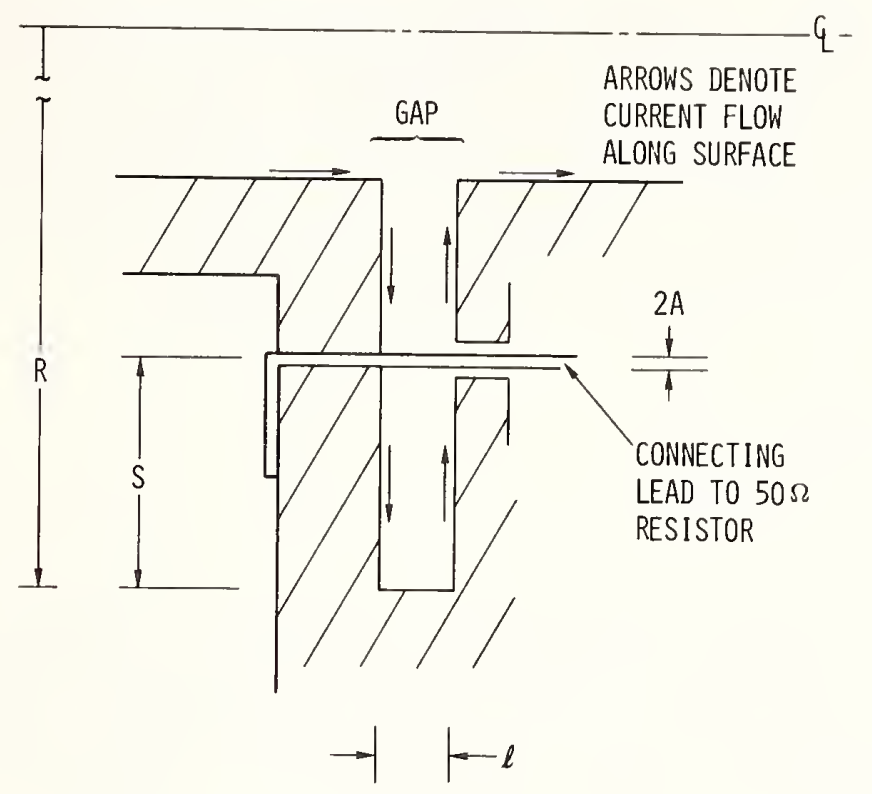

Figure 2. Expanded view of cavity.

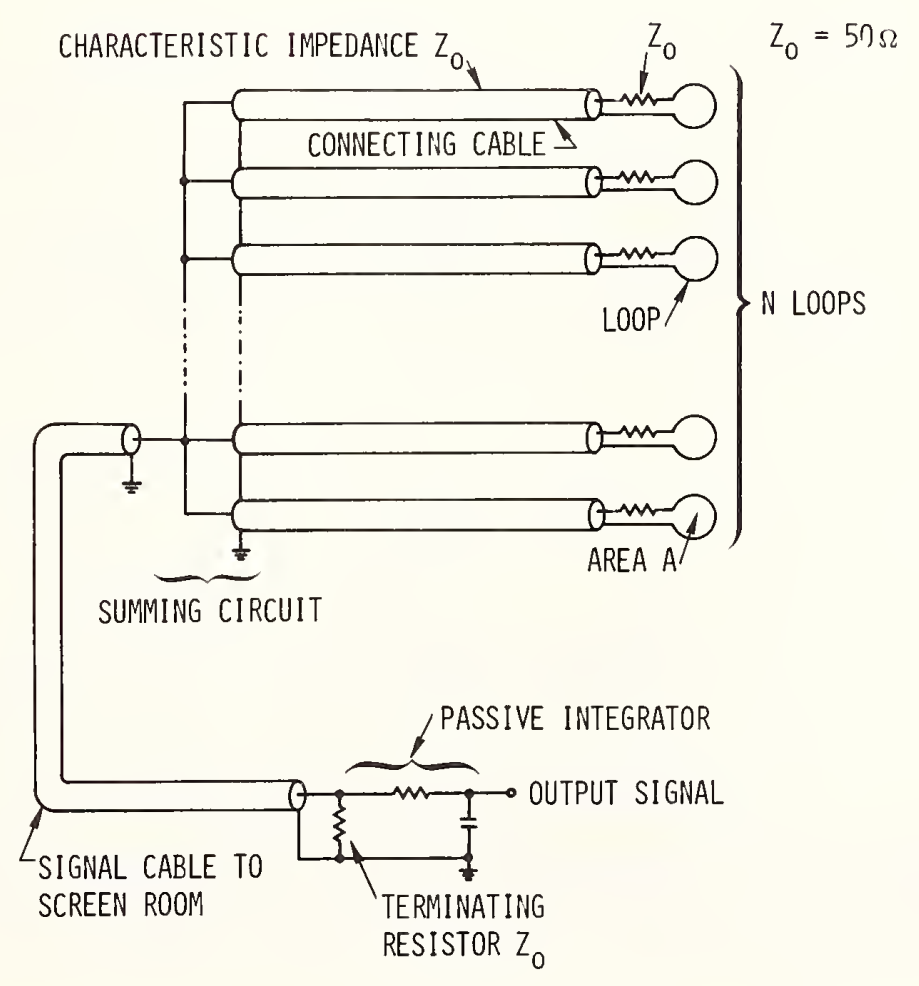

Figure 3. Equivalent circuit of current sensor. 
In the circuit diagram shown in Figure 3 , it is seen that each loop is connected to a cable through a $50 \Omega$ resistor and the cables are connected in parallel in the summing circuit. The purpose of the resistor at the loop is to absorb signals coming back on the cable from the summing circuit thereby preventing oscillations. Because of this termination we can separate the connecting cable into two equivalent circuits as shown in Figure 4. In Figure $4 a$, the wave launched onto the cable is $V_{1}$ and is nominally given by

$$
V_{1}=\frac{1}{2} A \frac{d B}{d t}
$$

where $A$ is the area of the pickup loop and $B$ the magnetic induction at the 1oop. At the other end of the cable the remaining $\mathrm{N}-1$

connecting cables and the signal cable constitute a load of $Z / N$ as shown in Figure $4 \mathrm{~b}$. From this equivalent circuit the voltage in the signal cable, $V_{2}$, as a result of $V_{1}$ is given by

$$
V_{2}=\frac{1}{N+1} A \frac{d B}{d t}
$$

(A)

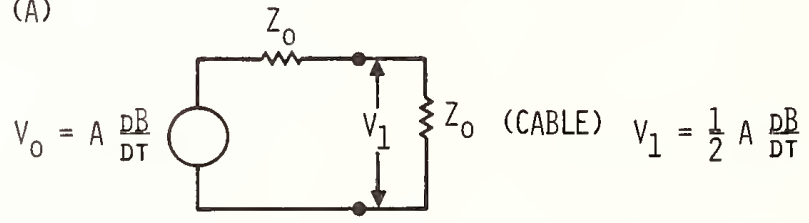

(B)

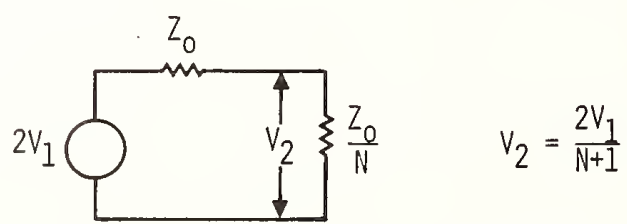

Figure 4. Equivalent circuits of 1oop/adder circuit link.

By superposition, the signals from all the loops can be combined to give on the signal cable

$$
V_{S}=\frac{1}{N+1} A \sum_{i=1}^{N} \frac{d B_{i}}{d t}
$$


where $B_{i}$ is the field at the $i^{\text {th }}$ position.

If all the loops are spacea a distance $\Delta l$ apart, then Eq. 3 can be written as

$$
V_{S}=\frac{1}{N+1} \frac{A}{\Delta l} \frac{d}{d t} \sum_{i=1}^{N} B_{i} \Delta l
$$

The sum can be approximated by

$$
\sum_{i=1}^{N} B_{i} \Delta l \quad \oint B d l=\mu_{0} I
$$

when the variation $B$ over distance $\Delta l$ is small. Eq. 5 can now be rewritten as

$$
V_{S}=\frac{\mu_{0} A}{(N+1) \Delta l} \frac{d I}{d t}
$$

The signal cable output is integrated and the voltage out of the passive integrator is given by

$$
V=\frac{1}{R C} \frac{\mu_{0} A}{(N+1) \Delta l} I
$$

where $R C$ is the time constant of the integrator.

\section{High Frequency Response}

For a magnetic probe operated in the differentiative mode, the risetime, $t_{R}$, is roughly the self integration time of the probe

$$
t_{R} \sim \frac{L_{P}}{Z_{0}}
$$


where $L_{p}$ is the inductance of each loop. The inductance each loop sees depends on the geometry of the cavity for the particular case shown in Figure 1.

$$
L_{p} \simeq \frac{\mu_{0} l s}{2 \pi \frac{R}{N}}+\frac{\mu_{0} l}{2 \pi} \ln \frac{R}{N a}
$$

where $R$ is inner radius of the outer conductor, a the radius of the connecting lead to the $50 \Omega$ resistor (see Figure 2).

Consider an example when $R=0.5 \mathrm{~m}, a=0.05 \mathrm{~cm}$, $l=0.1 \mathrm{~cm}, \mathrm{~s}=1.0 \mathrm{~cm}$, and $\mathrm{N}=6$. The probe inductance is approximately $126 \mathrm{pH}$ giving a risetime of $2.5 \mathrm{ps}$.

As illustrated in the above example, the high frequency response of the cavity current monitor for fast, mega-ampere current pulses should not, in general, be a limitation in its applications.

\section{Low Frequency Response}

As mentioned previously, the response of the cavity current monitor to longer current pulses will deviate from that of the high frequency response. To calculate the low frequency response, consider the model shown in Figure 5. In the cavity walls, which are assumed to have a finite conductivity, the electric and magnetic fields satisfy:

$$
\begin{aligned}
& \frac{\partial E_{W}}{\partial x}=\frac{\partial B}{\partial t} \\
& \frac{\partial B}{\partial x}=-\mu_{0} \sigma E_{W}
\end{aligned}
$$

where $\sigma$ is the conductivity and $x$ is the distance into the wall. In writing these equations, we have made the problem one dimensional. This is a good assumption if the thickness of the current sheath $\delta$ is small compared to the cavity dimensions. This thickness $\delta$ is roughly

$$
\sqrt{\frac{t}{\mu_{0} \sigma}}
$$

which is the distance the field can diffuse in a time $t$. 


$$
\begin{array}{ll}
\text { For aluminum } & \frac{1}{\sigma}=4 \times 10^{-6} \Omega-\mathrm{cm}, \\
\text { and if } & t=0.1 \mu \mathrm{s}, \\
\text { then } & \delta \sim 5 \times 10^{-2} \mathrm{~mm} .
\end{array}
$$

Hence, this assumption can easily be satisfied.

Using Laplace transforms $B$ and $E_{W}$ are given by

$$
\left.\begin{array}{l}
B=\mu_{0} I_{0}(s) e^{-\alpha x} \\
E_{W}=\frac{\alpha I_{0}(s)}{\sigma} e^{-\alpha x}
\end{array}\right\}
$$

where

$$
\alpha=\sqrt{\mu_{0} \sigma S}
$$

and $I_{0}(s)$ is the current flowing around the cavity per unit width. To find the response of the monitor we use Faraday's law

$$
\oint \vec{E} \cdot d \vec{l}=-\frac{\partial \Phi}{\partial t}
$$

integrating around the boundary $(x=0)$ of the cavity. At the walls we pick up a contribution from $E_{W}(0)$. The gap voltage is (ignoring signs)

$$
V_{g}=\frac{I_{0} \alpha}{\sigma} D+\mu_{0} I_{0} A S
$$

$D$ being the distance around the loop and $A$ being the area. We can write this as 


$$
V_{g}=\mu_{0} I_{0} A s\left[1+\frac{D}{A} \frac{1}{\sqrt{\mu_{0} \sigma s}}\right]
$$

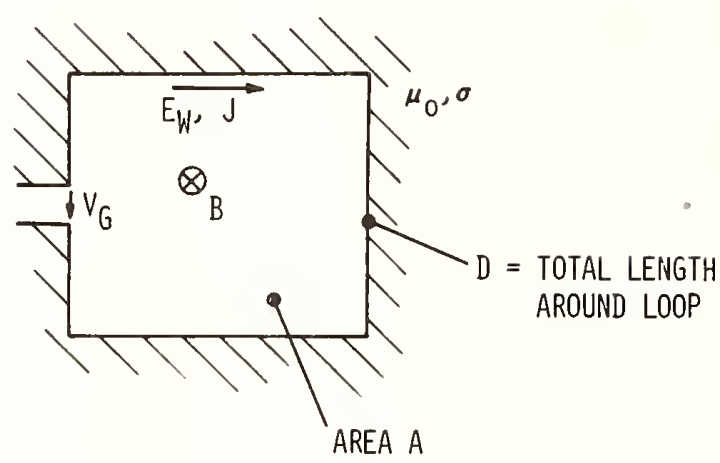

$$
\begin{aligned}
E_{W} & =\text { ELECTRIC FIELD IN THE WALL } \\
J & =\text { CURRENT DENSITY IN THE WALL } \\
B & =\text { MAGNETIC INDUCTION } \\
V_{G} & =\text { VOLTAGE ACROSS THE GAP }
\end{aligned}
$$

Figure 5. Model for low frequency calculations.

Let us now assume the current is a unit step function $\left(I_{0}=1 / \mathrm{s}\right.$ ) and that we have a perfect integrator (multiply by $1 / \mathrm{s}$ ) and let us normalize to the high frequency response (divide by $\mu_{0} A$ ). We then get for a normalized output signal (from the integrator)

$$
V(s)=\frac{1}{s}\left[1+\left(\frac{D^{2}}{\mu_{0} \sigma A^{2}}\right)^{\frac{1}{2}} \frac{1}{\sqrt{s}}\right]
$$

In the time domain, this becomes

$$
V(t)=1+\left(\frac{4 D^{2} t}{\pi A^{2} \mu_{0} \sigma}\right)^{\frac{1}{2}}
$$

or

$$
V(t)=1+\left(\frac{t}{t_{D}}\right)^{\frac{1}{2}}
$$


where

$$
t_{D}=\frac{\pi^{2} A^{2} \mu_{0} \sigma}{4 D^{2}}
$$

Here, $t_{D}$ plays the role of an effective cavity diffusion time.

The term $\left(t / t_{D}\right)^{\frac{1}{2}}$ in Eq. 14 represents an error term in that it is a deviation from an ideal step function. The error can be quite significant for late times. In passing, it can be noted that $t_{D}$ is a minimum for fixed $A$ if the cavity is a square.

\section{Wall Resistivity Compensation}

Since the error increase with time, the droop caused by the finite time constant of the integrator tends to compensate. The effect of the finite integration time constant of integrator can be determined by replacing $1 / s$ with $1 /(s+1 / R C)$ in the front of Eq. 12. This gives

$$
V(s)=\frac{1}{s+\frac{1}{R C}}\left[1+\left(\frac{D^{2}}{\mu_{0} \sigma A^{2}}\right)^{\frac{1}{2}} \frac{1}{\sqrt{s}}\right]
$$

where $R C$ is the time constant of the integrator. In the time domain,

$$
V(t)=e^{-\frac{t}{R C}}\left[1+\sqrt{\frac{R C}{t_{D}}} \int_{0}^{\sqrt{\frac{t}{R C}}} e^{\lambda^{2}} d \lambda\right]
$$

for $t<R C$, the integral can be expressed as

$$
V(t)=e^{-\frac{t}{R C}}\left[1+\sqrt{\frac{t}{t_{D}}}\left(1+\frac{1}{3}\left(\frac{t}{R C}\right)+\frac{1}{20}\left(\frac{t}{R C}\right)^{2}+\ldots\right)\right]
$$


from Eq. 17, we see that the exponential term tends to compensate for the diffusion term. For an aluminum cavity current monitor with $A=0.1 \mathrm{~cm}^{2}$ and $D=2 \mathrm{~cm}, t_{D}$ is approximately $8.7 \mu \mathrm{s}$. The percentage deviation from a step response for various values of RC is shown in Figure 6.

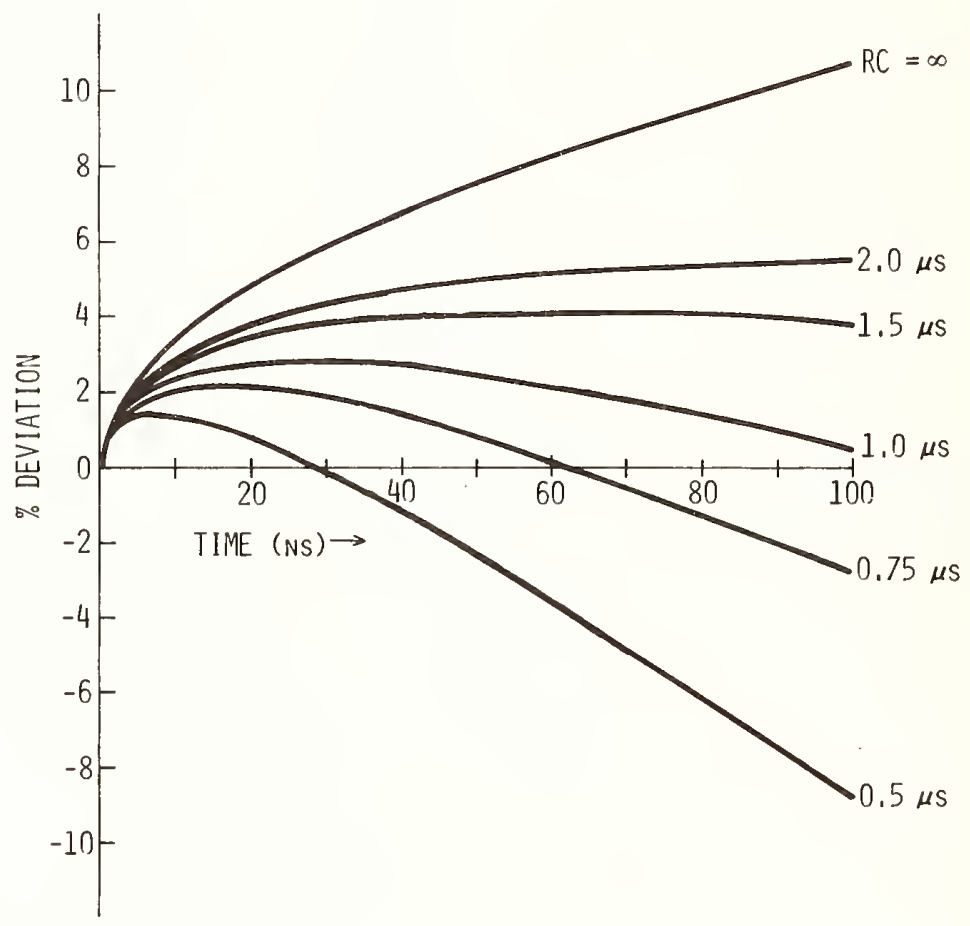

Figure 6. Deviation from step response of rectangular beam Faraday cup for various integrator time constraints.

\section{Conclusion}

We have described the characteristics of a current probe that is capable of measuring mega-ampere current pulses with sub-nanosecond risetimes. The construction of the cavity. current monitor is simple. Although the response of the probe changes with low frequency signals, the effect is well understood and can be minimized by using materials with high electrical conductivities along with a square cavity. This effect can be further minimized by choosing integrators with appropriate RC time constants for each application. Moreover, this effect can be totally corrected using digital computer techniques [1]. 
7. References

1 R. Richardson, et. al., "Calibration of the BLACKJACK 5 Pulse Generator Output Power", this conference. 


\section{Discussion Session 5}

Richard Malewski (IREQ). To clarify my question which ended the previous Discussion session, I think that it is more practical to make a drawing on the blackboard (see figure 1). The question was to the design of an impulse current transformer such as manufactured by the Pearson Company. If that stands for a section of the winding of the transformer, we can distinguish some elements in it. There is a line of resistors which are in the kilo ohm range. There are taps of the winding connected to the resistive wire which probably constitutes the distributed burden and there are extensions of this, legs separated by a thin milar foil from a metal copper band and this increases capacitance between these legs and the ground foil. On top of this, the whole winding was terminated by a fairly complex resistor network which I should admit was difficult for me to understand why. So, if this question can be answered, I would appreciate it. It would cast some light on the particularities of behavior of the Pearson transformer.

There is perhaps another element in the case of coils made without magnetic core. I feel that one can improve the bandpass of the coil by using tape rather than wire to produce the winding. The idea is to reduce the characteristic impedance of a line formed by the helix of the winding and the external metal shield. Such lines can be matched by an appropriate resistor at the end if it is kept within a few hundred ohms. I don't know whether this idea is viable and to what other comments people manufacturing these coils might make.

Marco Di Capua (Physics International). We11, our experiences have been with rather simple devices like the transmission lines that I described. We have not made any attempt either to introduce damping or to build a complicated structure. In general, Physics International's design philosophy has been to keep the device as simple as possible so it can be easily modeled. The transformer under discussion could perhaps be described as a parallel connection of two transmission 1ines. (One is associated with the shield and the other is associated with the damping network.) The transformer could be modeled with one of the finite element network analysis codes.

Jon Barth (Barth Electronics). I think that you will find that something like this would be more amenable to a delay line type of analysis. The analysis is not quite as simple as has been implied. It is much more complex when you have the other conductors right next to each other.

John Shannon (Maxwell Laboratories). OK. Can we Teave Rogowski coils and go on to some of the other topics? Are there any more questions on the papers in the morning? 

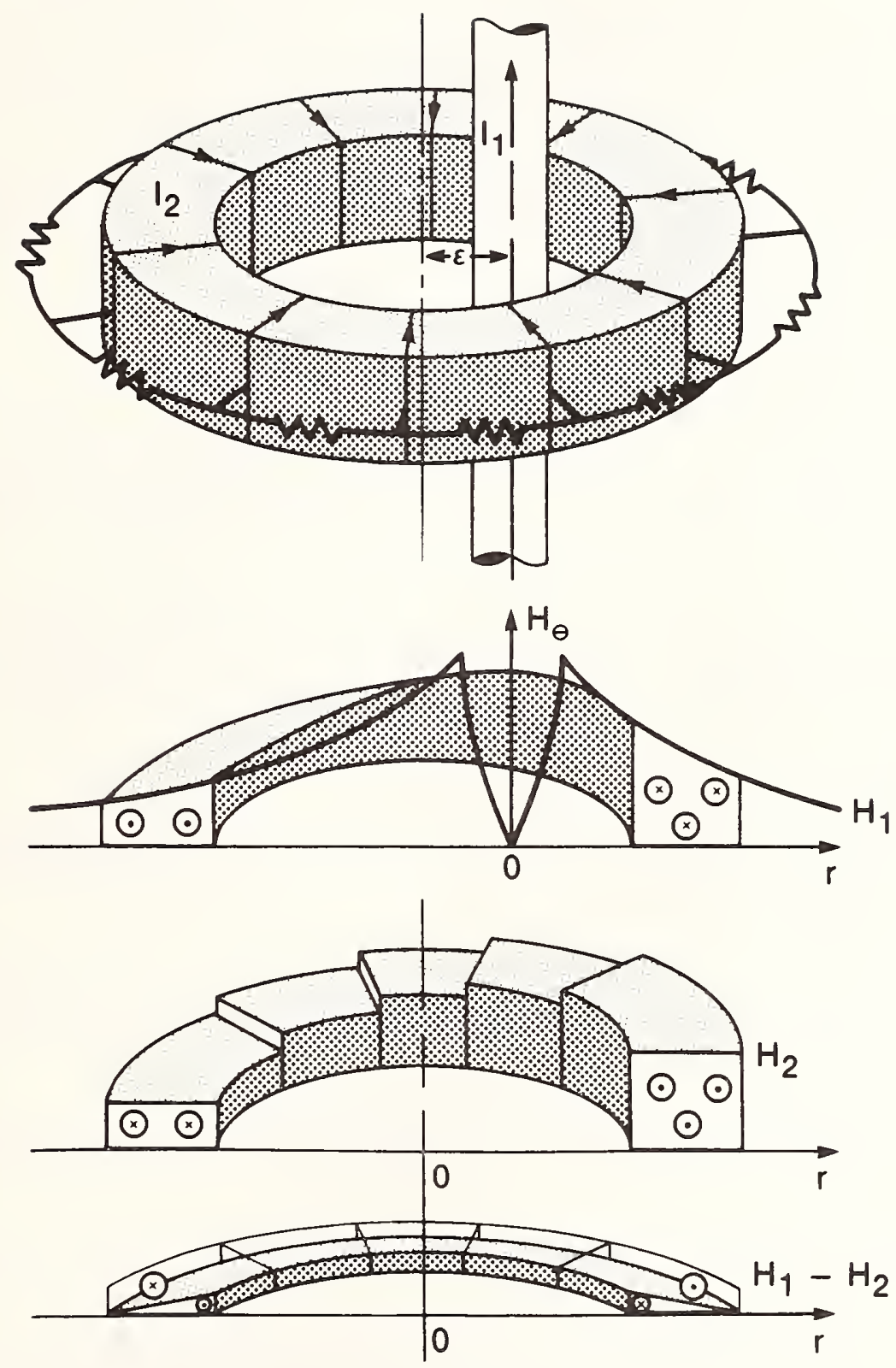

Figure 1. Schematic representation of flux in a current transformer with off-axis excitation and distributed load for i2. 
Steve Levy (U.S. Army Electronics \& Technology Laboratory).

I would like to ask a question of the Raytheon paper on the data acquisition system. I noticed in the data acquisition system that they used a current transformer as a method of picking up the signals which means that they were not dc coupled into their modulator. When you have multiple switches that are operating parallel, how do you detect the continuous conduction mode or that mode where the tube fails to recover without using a voltage divider or some sort of dc current sensors? That is my question.

Bob Kolibas (Raytheon). It's a good question and the answer is you don't unless you do have a dc coupled method available.

Levy. The answer I guess is that they do not detect continuous conduction. We have a paper we will be reporting on at the pulse power conference in Albuquerque where we do treat the problem of continuous conduction. So, I will wait until that meeting to discuss this topic.

Di Capua. I have one question about the monitoring of current densities with a scintillator. Does scintillator material dendrite at all when you deposit a large number of electrons in it?

Bob Scarlett (Los Alamos). It does after a number of shots. At these current densities $\left(50-100 \mathrm{~mA} / \mathrm{cm}^{2}\right.$ for $\left.5 \mu \mathrm{s}\right)$, we can get twenty or thirty shots before it dendrites or starts to turn yellow due to radiation damage. We have also noticed that there is an electret effect in the plastic.

Di Capua. What provisions do you make to remove the large amounts of charge from the back of it? Do you have a collector behind it?

Scarlett. I think that most of the charge is removed by the plasma itself. The beam plasma in the gas tends to discharge the surface charge. The deep charge doesn't come out except from a slow diffusion.

Di Capua. How many joules per square centimeter are incident on the firm that you are using?

Scarlett. There is about 50 milliamps per square centimeter and the pulse Tength that was actively contributing the light was probably on the order of a microsecond or so and these are $350 \mathrm{kev}$ electrons.

Shannon. I think Uglam and Cooperstein did this for total current monitors in about 1970 or 1972 and one of the comments that was made to me was that in order to get away from the scintillation problem, you had to use vacuum UV grade fused silica. All plastics were claimed to have scintillation light that was not Cerenkov light. Would you make a comment on that? 
Scarlett. I used to do research which involved the detection of relativistic heavy ions. One of the things that we did at that time was to look at the scintillation properties of various glasses and plastics using relativistic heavy ions at the Bevalac. We determined that the scintillation properties of plexiglass were very low, not more than a percent or so of the Cerenkov signal.

Neville Harris (Ion Physics). I would like to ask the reproducibility of the lucite. For example, was the Cerenkov radiation dependent on samples, impurities, or degree of polymerization? I would like to compare your method with the use of blue cellophane.

Scarlett. The Cerenkov effect is a classical, collective optical effect. It doesn't depend on any of the microstructure or the molecular physics of the material. It depends purely on the index of refraction of the material. Measurements that I made when I was doing particle detection work indicated that it usually was very consistent.

Jack Katzenstein (Maxwell Laboratories). I have a question for Dr. McDaniel about his very interesting piezoelectric pressure probes for measuring current. If the probe is placed too close to the plasma source, isn't there going to be an appreciable pressure contribution from the ablation from soft $x$-rays?

Dillion McDaniel (Sandia). The answer to that is yes there is. The way we run our plasma implosion source, the plasma does not implode until long after current peak. So, we use the probe to verify the total current delivered to the load and there is no problem because of the separation time between current peak and radiation from the plasma in our experience. That may be different for other people who implode on peak current though.

Katzenstein. Well, the only comment I would make to that though is how do you optimize coupling or is this a problem with you?

McDaniel. For a low inductance diode which is like inductances of Tnanohenry or less, imploding after peak current is the optimal for coupling kinetic energy. Other labs may not have seen this since no other labs have accelerators with as low inductance diodes. So I will be glad to discuss that with you after the meeting. Since this meeting is not on plasma implosion, I think I would not want to take the time here.

Shannon. This is a sensitive subject and let's not get too far into this implosion business. We will all get into trouble. Are there any other questions?

Ed Chiu (Maxwell Laboratories). Yes, I guess I would like to ask a question to Ditlion. You mentioned that the pressure transducers are very good for high magnetic pressure. Can you comment on the other 
end of the application? At how low a current should I stop using that probe and how long a pulse would be really convenient for the application of these transducers?

McDaniel. It depends on the situation. If you are in a strip transmission line where the $B$ is uniform, then you can get more signal out of the probe by going to a larger diameter area since the probe output goes up as area. I feel that you can probably use this type of probe down to where you have magnetic pressures on the order of 50 or 100 bars provided you can make a large enough area probe that you are over your signal-to-noise ratio from EMP. So, you have to look at each specific case. The paper will have piezoelectric crystal constants in it for quartz. There are other piezoelectric materials that have higher sensitivities and we are investigating lithium niobate at the moment although for some reason it doesn't seem to work right.

Di Capua. I would like to make a comment about that. In 1978, we performed a magnetic insulation experiment at PI ( $I=1 \mathrm{MA}$, $r=6 \times 10^{-2} \mathrm{~m}, \mathrm{~B}=3.3 \mathrm{~T}, p=44 \mathrm{bar}$ ) and Vern Bailey, a colleague at PI, suggested I use quartz gauges for current measurements. I turned down the suggestion because of the low signals we would obtain. This is the reason we used the shunt approach instead.

John Shipman (Sachs Freeman/NRL). I would like to ask Dillion what the indications were that the $B$ probe holes were flashing over?

McDaniel. If you look closely at the probe holes, you can see "crow feet" tracks in the radial direction of the outside of the hole to the inside of the hole. Of course, we also lost signal. The B signal would go up, come down and all of a sudden would drop to zero which indicates that something is flashing. Inspection of the hole showed "crow feet" tracks that started on the outer radius of the hole going in towards the radial direction and simultaneously there would be a burn mark within a centimeter radius of where that hole was located. But, I can't explain why it happened because I can't really calculate the voltage drop across the hole between the quarter-inch diameter sitting out at a radius of five centimeters even though there are those enormous $d I / d t$ 's.

Di Capua. I would like to share some data on the effect of penetrations for diagnostics on magnetically-insulated vacuum transmission lines. After a few pulses (20-30) we noticed a very severe erosion, in the vicinity of the penetration, downstream from it. This erosion, which gave the penetration the appearance of a keyhole is shown schematically in figure 2 below and photographs appear in figures 3 and 4 . The erosion is probably caused by perturbation of the space charge electron flow due to the absence of return currents in the vicinity of the hole. The figures contain all the experimental details. 
(a)

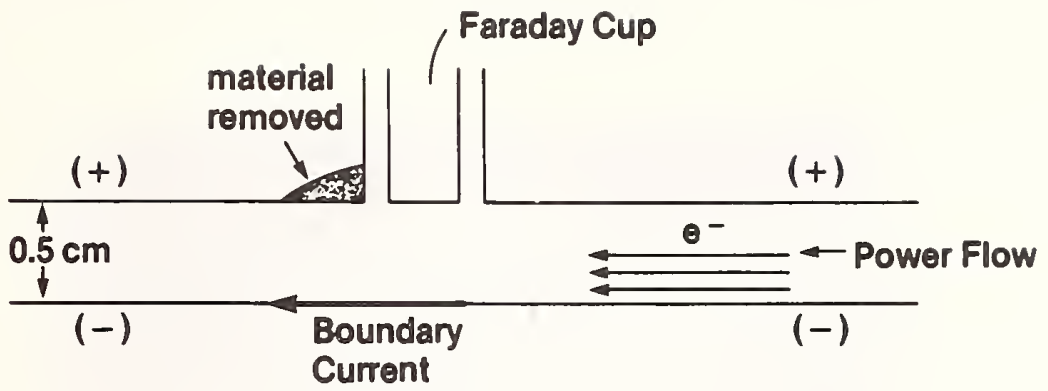

(b)

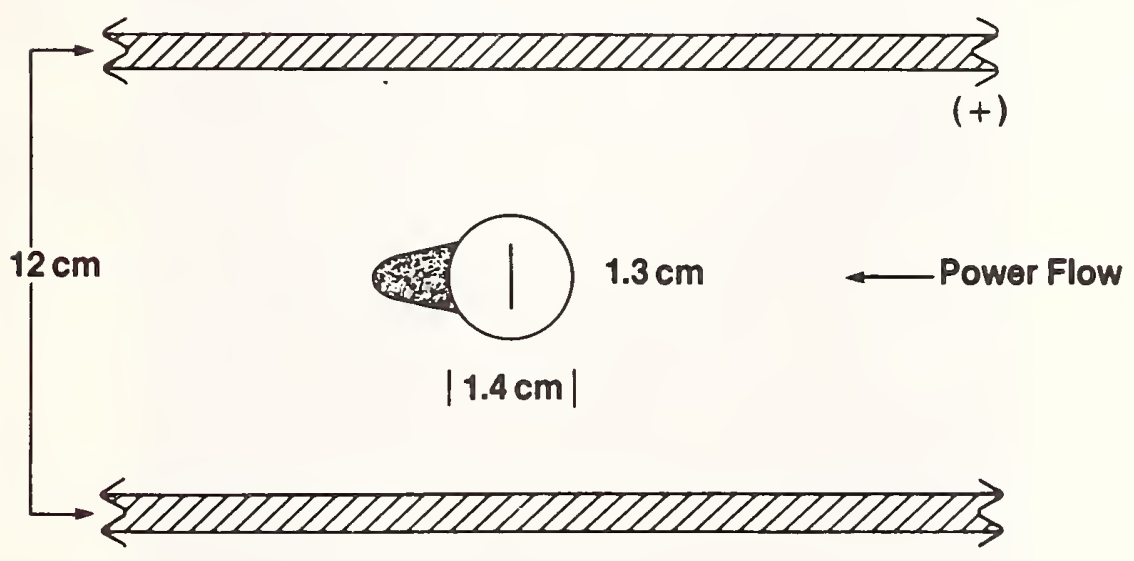

Figure 2. Schematic of anode penetration for magnetically insulated transmission line diagnostics (a) side view (b) view from cathode. 

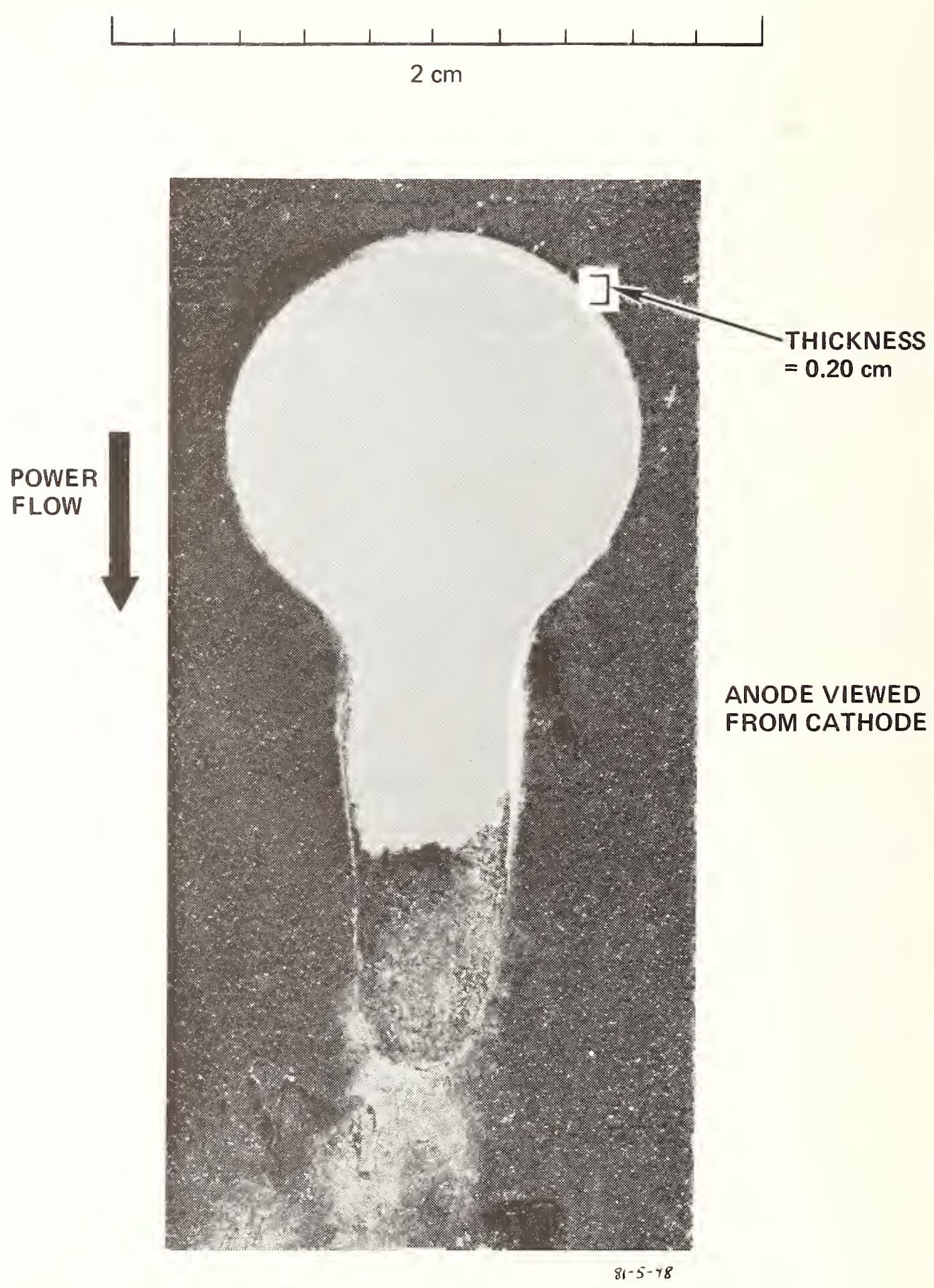

Figure 3. Anode erosion caused by penetration in anode wall. View is from cathode, inside transmission line. Hole was circular at first. Erosion was caused by 30 shots. (M. DiCapua and T. S. Sullivan, Proc. 2nd IEEE International Pulsed Power Conference, Lubbock, TX, IEEE 79CH 1505-7, 1979). 

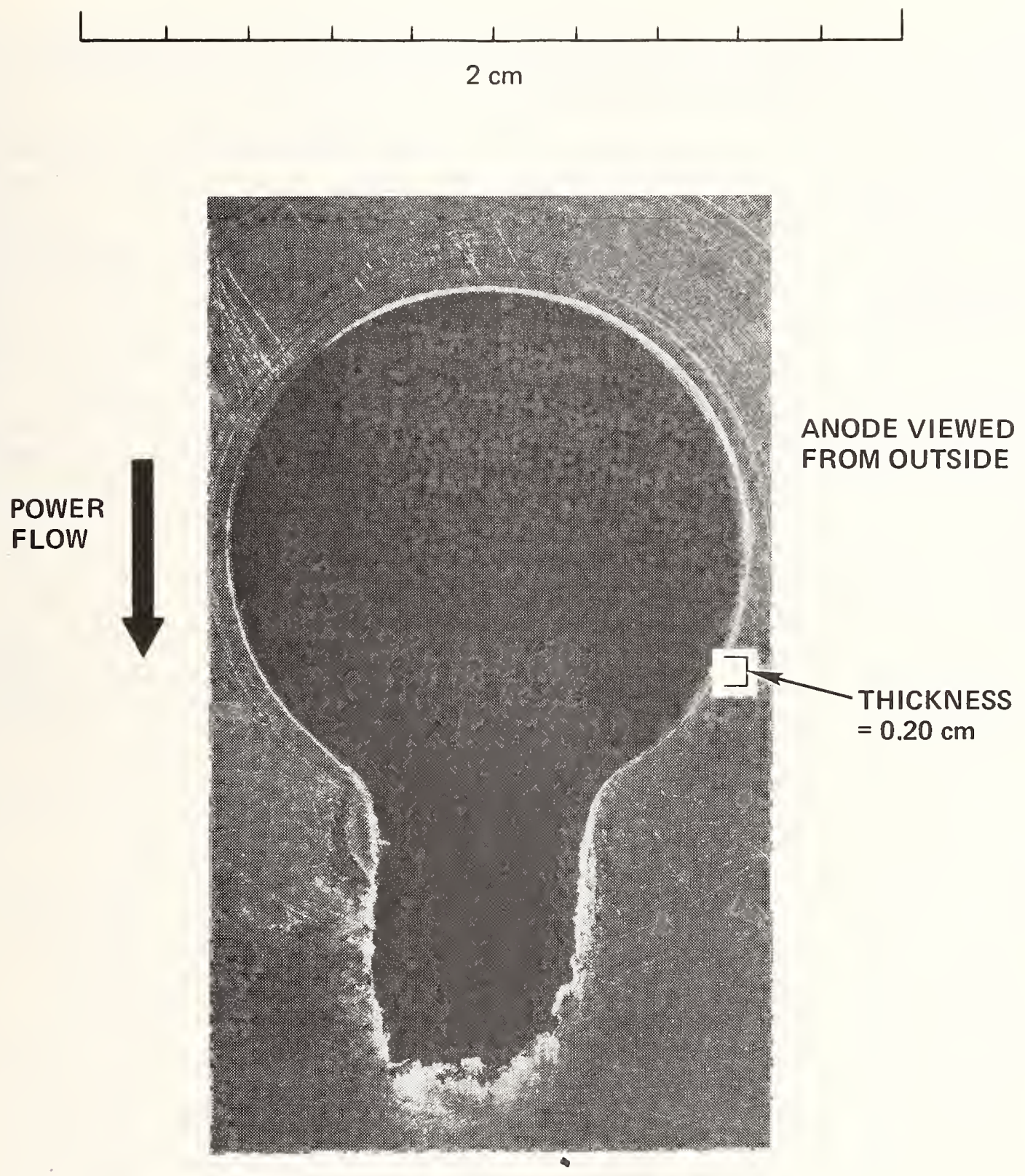

Figure 4. Anode erosion caused by penetration in anode wall. View is from outside the transmission line. Hole was circular at first. Erosion was caused by 30 shots. (M. DiCapua and T. S. Sullivan, Proc. 2nd IEEE International Pulsed Power Conference, Lubbock, TX, IEEE 79CH 1505-7, 1979). 
Harris. Was that arrow you drew in the direction of the magnetic field? The direction of the magnetic field rules out a multipactor mechanism.

Bob Hebner (NBS). I would like to know how soon Maxwell anticipates having a magneto-optic current monitoring device operational?

Katienstein. We have ordered the glass, and if the optician doesn't think that it is too difficult a job to do it, we ought to be testing it within three to four months.

Malewski. We have been playing with magneto-optic current transformers for some time. And there were finally two elements which decided that we abandon these particular techniques. Perhaps it is wise to show where the particular problem was found. The idea was to monitor current in high voltage transmission lines at high potential and what we did was to have a slab of lead glass placed in the vicinity of the current carrying conductor in such a way that the magnetic field of the current was along the axis of the glass slab. A polarized light beam was transmitted through the slab and deflected down to ground potential to monitor the current as expressed as the rotation of the polarization plane of polarized light. The glass slab was approximately 10 inches long and roughly 1/4-inch diameter. Unfortunately the heat generated by the current circulating in the conductor produced some local temperature gradients in the glass probe in turn producing mechanical stress. The consequence was that the piece of glass became birefringent. The linearly polarized light beam which came out of the slab was not only twisted by a certain angle but was also elliptically polarized due to the residual birefringence induced by thermal shock. Recently, I heard some encouraging news. People have abandoned the idea of using massive glass slabs which are naturally prone to this effect, and are thinking in terms of using optical fiber wound around the current carrying conductor. The beauty of this solution is evident. By increasing the number of turns, one can increase the sensitivity of such a modulator because the twist of the polarization plane due to the measured current would be proportional to the path of the light passing in the several turns of this coil and, therefore, several revolutions of the polarization plane can be achieved even with a moderate current. The problem here is that when one starts to twist fiber optics, birefringence will be produced by the act of bending. This apparently can be compensated by twisting the fiber in the process of winding the coil and it has been reported that such coils were built which are free of birefringence. If it is all true, this type of coil fixed in some sort of epoxy block could be an excellent magneto-optic modulator. Even if there was some residual thermally induced birefringence it will be much smaller than before for two reasons. First of all, fiber is thin, therefore the stress due to the thermal effects would be smaller and, secondly, the signal, due to the number of revolutions of the twisted polarization plane would be much stronger in comparison to the effect of birefringence. 
Katzenstein. First I would like to thank Dr. Malewski for bringing out or reiterating the points that I made in the first few minutes of my paper. We have looked at this. One of my co-authors, Bill Caton, is a fiber optics expert and it is his opinion that a polarization preserving fiber is beyond the present state-of-the-art. Now, this can work, if you are prepared to make measurements of very small polarization rotation angles based on differential intensity measurements. I think you made a bad choice of glass, incidentally, because there are the SF58 and certain other Schott glasses which have an extremely low strain birefringence. Furthermore, they al so have extremely low loss tangents. I think also that another disadvantage of this scheme is that it does not use a closed light path which means it is prone to changes of position or orientation of the rotating medium with respect to the conductor. If you have something dangling from a transmission line, this is apt to happen. 


$$
\text { Session D - Data Acquisition }
$$

HIGH-VOLTAGE MONITORING AND CONTROL THROUGH FIBER OPTICS

John G. Stewart, Jr.

William A. Petty

Harry Diamond Laboratories

Abstract

Recent advances in the field of fiber optics have enhanced our ability to monitor and control high voltage in a hostile electromagnetic pulse (EMP) environment. Harry Diamond Laboratories has utilized these advances to pursue the development of a Remote Command Data Link (RCDL) for the Army's threat-level EMP simulator. This simulator utilizes twin Marx generators which can produce output voltages in excess of \pm 3 MV. Through the use of the RCDL, this simulator can be remotely fired and real-time oscillograph records made that represent the \pm 3 MV voltage waveforms that are impressed across the simulator peaking capacitors.

The RCDL was designed to allow normal pulser diagnostic maintenance, Marx output waveform monitoring, and adjustment of the Marx timing signals from a command and control center located approximately $30 \mathrm{~m}$ from the pulser. Specifically, the RCDL features synchronized command firing of the bilateral Marx generators, performance check of the pulser high-voltage trigger system, Marx generator erection waveform, advance or delay of a single Marx trigger for optimum simulator performance, and interlock with the simulator console for the arm and abort function.

The RCDL has recently been made an integral part of the Army's threat-level EMP Simulation Facility, located at Woodbridge, VA. This system is presently being evaluated for long-term-performance. Preliminary data reveal that the RCDL provides the desired design features with reliable performance.

This paper presents an overview of the RCDL with emphasis placed on its usage in a particular EMP test facility. A follow-up paper by the Physics International Company presents a detailed circuit design of this system. 


\section{Introduction}

Recent advances in the field of fiber optics have enhanced our ability to monitor and control high voltage in a hostile electromanetic pulse (EMP) environment. Harry Diamond Laboratories has utilized these advances to pursue the development of a Remote Command Data Link (RCDL) for the Army's threat-level EMP simulator. Before discussing in detail the unique characteristics of the RCDL, the need for developing this type of instrumentation will be established. This introduction will focus on the historical development that led to this effort, with an explanation of how the RCDL relates to the high-voltage system that it supports. Some general background information will be given on the complex high-voltage electronics used by the specific EMP simulator that interfaces with the RCDL.

The need for EMP simulators and, hence, the RCDL can be understood by focusing on the electromagnetic environment that is generated when a nuclear device is detonated in the exoatmosphere. This detonation generates an EMP which propagates over a vast geographical area. The nature of this pulse makes it potentially destructive to many electronic systems, including a significant number of defense related systems. The present restriction on testing of nuclear devices in the atmosphere created an urgent need for simulating this electromagnetic environment in order to empirically investigate the susceptibility of certain military electronic hardware.

\section{Approach}

The Army has filled the need for generating a simulated EMP environment through the development of an EMP simulator that hereafter is referred to as AESOP. Army EMP Simulator Operations. This facility uses state-of-the-art high-voltage components, electronics instrumentation, and calibration techniques to achieve the high field level experienced by the real EMP threat. Because of the very high voltages developed in this simulator, reliable and efficient monitor and control methods for the AESOP pulser are constantly being pursued. For example, the AESOP pulser can generate a voltage in excess of $6.5 \mathrm{MV}$ accompanied by an energy of over $60 \mathrm{~kJ}$ across a single high-voltage output switch. With voltages and energy of such large magnitudes, a critical requirement is timing synchronization between command pulses. Two generators are required to erect to their full potential, and the energy is delivered to a single output switch within a time window of approximately $70 \mathrm{~ns}$. This event must be accompanied by a trigger system timing jitter of \pm 10 ns. The \pm 3.5 MV impressed across the pulser output switch determines the radiated output of the simulator antenna.

It is the need to measure and monitor each polarity of this voltage in terms of erection time amplitude and timing jitter that prompted the development of the RCDL. 
In determining the specifications to be established for the RCDL, the pulser's operational characteristics were examined closely. Two areas that are perhaps the most critical to the simulator's operation are the synchronization of high-voltage triggers and individual Marx generator erection characteristics. Factors that influence synchronization are Marx erection times, Marx erection jitter, and level of voltage delivered by each Marx generator. Marx erection, which proceeds very rapidly, is the process by which the Marx generator's spark gaps are overvolted, usually sequentially, and broken down. Marx erection time, the time for all Marx switch gaps to close so that load current begins to flow, is on the order of 150 ns for the 35-stage Marx generators in the AESOP. During erection, the Marx generators pulse charge their respective peaking capacitors in about 65 ns to the required high voltage (1 to $3.5 \mathrm{MV}$ of opposite polarity for each generator). After the generators erect, the output switch breaks down, discharging the peaking capacitors and Marx generators into the antenna. Fig. 1 shows actual Marx generator waveforms and illustrates the electrical parameters of the bilateral Marx generator configuration.

Trigger synchronization is another important feature considered when establishing design characteristics for the RCDL. To insure that the two Marx generators will erect simultaneously, the individual Marx generator stages for each side of the pulser must be triggered with a very low timing jitter. During this erection time, the pulser peaking capacitors are charged to the peak voltage of the Marx output. This voltage is simultaneously delivered to the pulser output switch. This output switch is self-breaking and is designed to close at $90 \%$ to $100 \%$ of the peak output voltage from the individual Marx generators. With this closure, the peaking capacitors supply the initial fast-rising pulsed voltage across the closed output switch to the antenna. This voltage can be used to analyze the performance of the pulser. However, to acquire knowledge of this voltage waveform and output amplitude, we must have the capability to sample voltages in excess of 3 MV with rise times in the nanosecond range. Thus, we have established a real need for the subject RCDL.

Through the use of the RCDL, it was anticipated that one could monitor the real-time critical operation characteristics of the AESOP simulator. This information on the pulser output voltage could help troubleshoot pulser operational problems. The voltage waveforms could reveal the operational characteristics of the individual Marx generators. This information would provide insight into any problems that may have occurred during pulser firing, as well as establishing a record of the pulser's normal operational performance normality.

\section{Significance of RCDL Data on Operations and Maintenance}

Because of the critical timing and synchronized erection requirement for the Marx generators, diagnostic maintenance is required for any 
Side 2

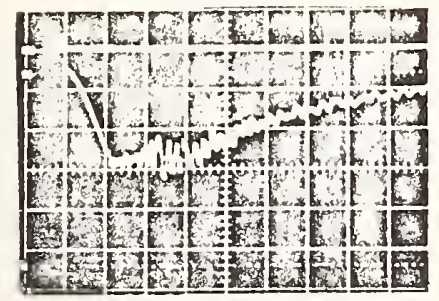

Side 1

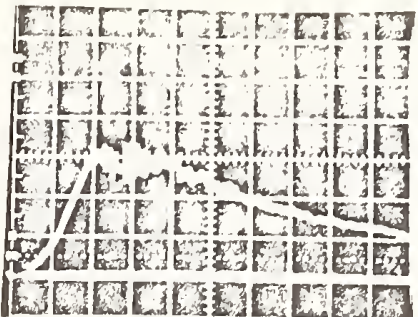

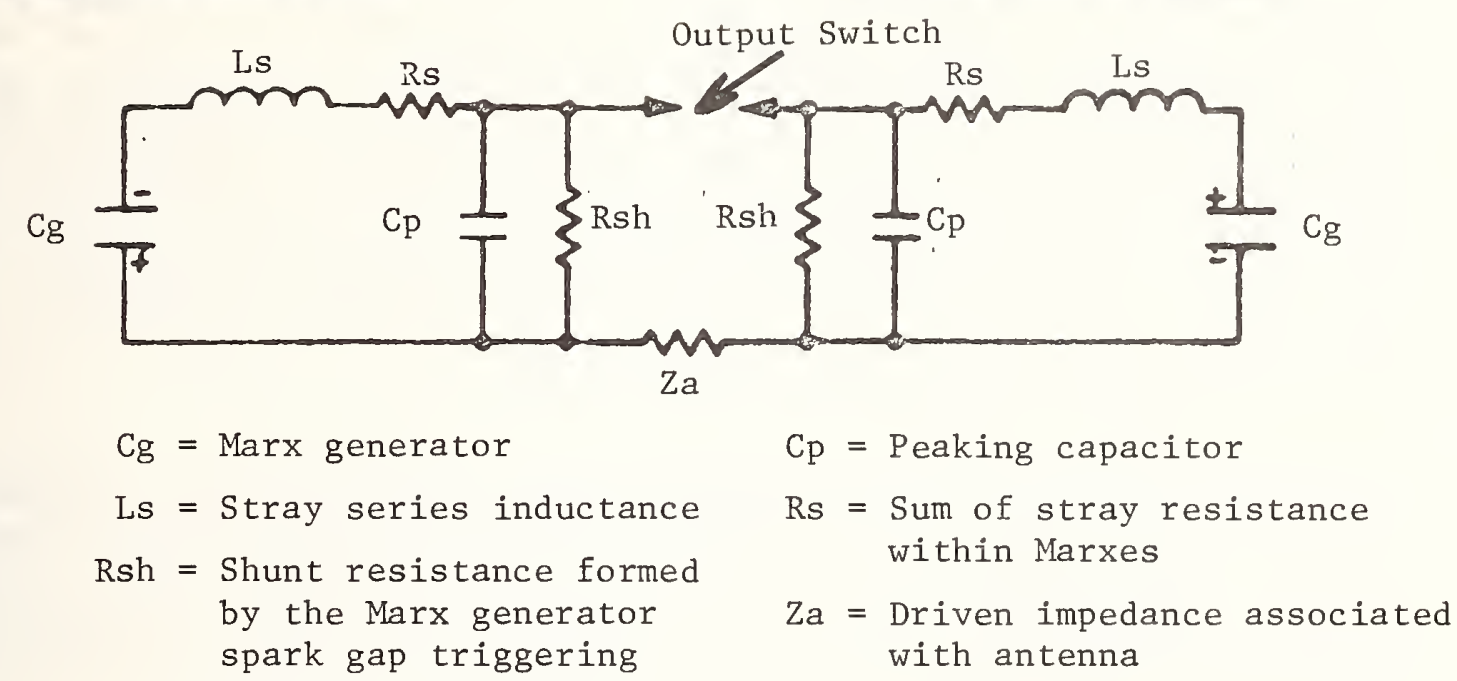
resistors.
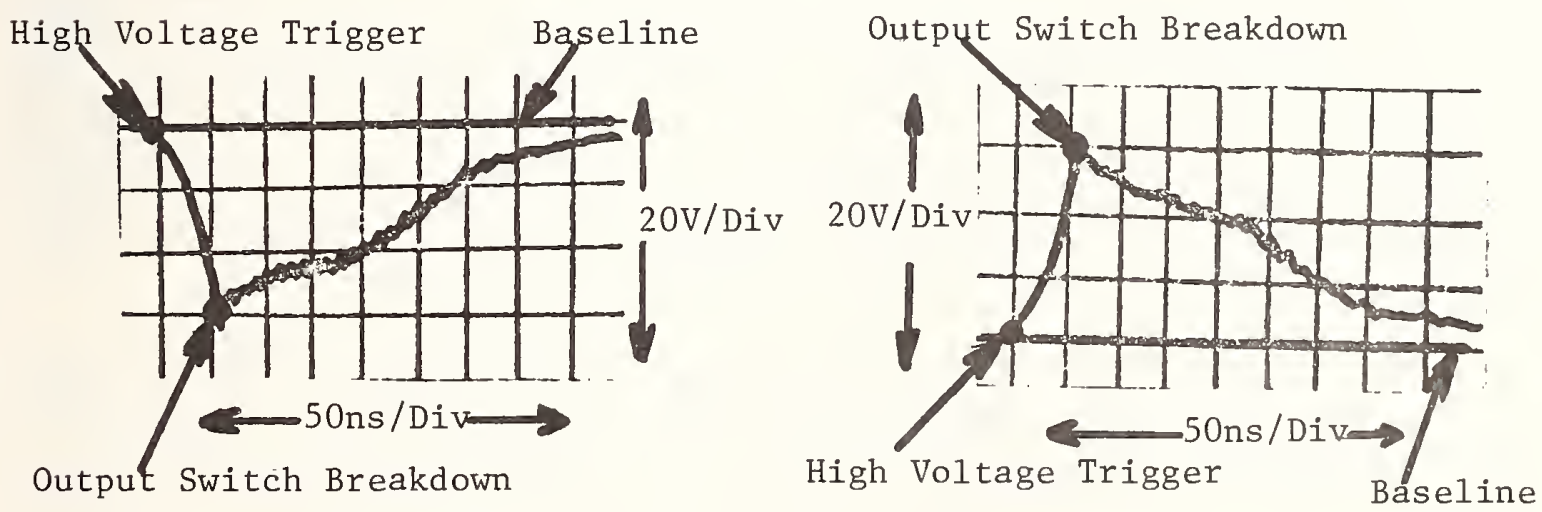

Figure 1. Bilateral Marx pulser simplified schematic and Marx generator output waveforms 
change in output level or replacement of critical components. The RCDL has helped save time and simplified diagnostic maintenance and analysis of the Marx waveform erection times. The Marx waveforms are recorded as oscillographic data output from the RCDL fiber-optic down-link system each time the EMP is launched. This visual aid, along with the delay control mode located in the command and control center, adjusts the erection times of the Marx generators by controlling the trigger timing synchronization. Nonsynchronized operation can lead to voltage breakdown across one set of pulser peaking capacitors if allowed to exceed certain bounds. The RCDL down link checks the performance of the synchronized trigger system. The initial check or coincidence mode insures that the total trigger system is operational prior to actually firing the antenna and also checks the simultaneity of the Marx triggers. This mode is interlocked with the Marx dc charging, and unless the trigger pulse timing is acceptable within a +10 ns tolerance, dc charging of the Marxes cannot take place.

The up link or command trigger initiation is located in the command and control tower. These command pulses are activated through a variable advance or delay control to insure that the proper synchronization requirement exists between the command pulses. The modular construction and the light-emitting diode (LED) monitor indications of several events in the timing system have enhanced the troubleshooting techniques and decreased out-of-service time.

The coarse or initial synchronization takes place by monitoring the high-voltage triggers. The monitor points, one for each pulser trigger side, measure the instantaneous current representing the $80-\mathrm{kV}$ highvoltage trigger required to erect the Marx generators. This monitoring is done with a dual input oscilloscope for synchronizing the two trigger pulses. Once the trigger pulses have been physically synchronized within the \pm 10 ns specification by cable selection, onboard or Marx waveform synchronization is done as described previously.

The technique for measuring these waveforms required time-consuming tests before the integration of the RCDL to the system. Shield boxes and data acquisition hardware were placed on board each side of the pulser. Here, the real-time waveforms could be monitored by onboard technicians. By observing the waveform erection and breakdown criteria, the high-voltage triggers could be synchronized through adjusting the trigger signal delay to one generator relative to the other. This technique is considered the fine tuning synchronization method.

The RCDL eliminates onboard hardware and personnel needed for Marx waveform monitoring. The monitoring is done by using electronic hardware and fiber-optic data links to observe these trigger pulses and Marx erection waveforms. By built-in adjustable delay lines, the Marx generator synchronization or fine tuning is simplified. 
The calibration and the accuracy of these measurements are governed by Army calibration standards for oscilloscopes and the initial verification of vendor specifications of the fiber-optic systems. To insure amplitude verification, an external pulse is injected into the fiberoptic data link, and a bias control adjustment is made on the fiberoptic receivers.

\section{Reliability}

No single design objective, except for the actual simulated pulse itself, is as important as the reliability of the pulser/antenna simulator facility. Current EMP vulnerability data-acquisition methods require the production of a large number of identical EMP's. Deviations from this concept extend data processing and interpretation. System reliability is enhanced by the initial pulser design and development emphasizing reliable high-voltage techniques.

Prior to interfacing the RCDL with the AESOP pulser, consideration was given to the hostile EMP enviroment as radiated from the AESOP antenna. Sound shielding practices were applied to the RCDL commandmodule interconnecting cable that is interfaced with the console used in the command and control function for the AESOP simulator. Grounds, where necessary, were bonded to form common grounds. Onboard and RCDL console modules were contained in shielded enclosures with interface connections filtered for protection from the electromagnetic environment. 
REMOTE COMMAND DATA LINK PROVIDES ENHANCED SIMULATOR PERFORMANCE IN HIGH EMP ENVIRONMENT

\author{
Stan Lyons \\ Physics International Company \\ 2700 Merced street \\ San Leandro, California 94577
}

The Remote Command Data Link (RCDL) system was designed to provide an advanced method of determining EMP simulator performance on a shot-to-shot basis.

\title{
1. Overview of the RCDL sytem
}

The purpose of the RCDL is to command trigger each half of the AESOP EMP simulator with a low jitter (< 1 ns) pulse and with an adjustable delay of 0 to \pm 25 ns between the two halves. A signal generated by the output of the onboard trigger systems is then transmitted to the RCDL, and the timing is compared for coincidence ( $\pm 5 \mathrm{~ns}$ ). Delay compensation is necessary to provide time-equalized pulses regardless of the delay time setting. The pulses are compared for conincidence. If they are within the \pm 5 ns window, an interlock is activated. This allows de charging of the AESOP Marx generators in preparation for the discharge of an EMP pulse. The self-check of the triggers is necessary to assure proper trigger operation because of the twin Marx generator configuration of AESOP. The Marx generators must be triggered almost simultaneously to avoid overvolting various components of the simulator. Since the Marx erection time varies slightly with charge voltage and polarity, an adjustable delay is provided to correct for this variation. The delay adjustment is determined by observing the Marx generator erection waveforms monitored by E-field plates on the simulator. These waveforms are then transmitted to the RCDL and to the operator. Overall performance of the simulator is evaluated from observation of the waveforms, and any timing corrections are made.

\section{Design Approaches - Optical Link}

The above conditions required several novel design approaches. The high EMP environment of the AESOP simulator and the necessity of total electrical isolation limited methods of data transmission to optical means. The requirement of transmitting wide bandwidth analog signals and digital timing pulses with low jitter introduced several difficult design problems. 
The main problem was the transmission of Marx erection waveforms. If a totally digital system were used, analogto-digital conversion would be necessary. But this raised the problem of converting wide bandwidth video signals with good resolution. On the other hand, the one advantage of digital transmission is that data can be stored on board and retrieved at the operator's convenience. However, waveform fidelity was believed to be more important than absolute voltage value. Therefore, analog real-time data transmission was selected. To accommodate the digital timing pulses, a video bandwidth in excess of that needed for the Marx output waveforms was required. For low jitter, a risetime of $10 \mathrm{~ns}$ or less was preferred, implying a bandwidth an order of magnitude greater than required for the analog signal ( $\simeq 300 \mathrm{MHz}$ versus $\simeq 30 \mathrm{MHz})$. At the time the RCDL system was designed, an analog optical link with this capability was not available. It was determined that by operating the optical link in a saturated mode for the digital signals, a marked increase in risetime would be possible. We discussed these issues with Meret, Inc. Meret believed that it could supply a system to meet all of our requirements, including an analog bandwidth of $1 \mathrm{MHz}$ to $130 \mathrm{MHz}$, an operating frequency range of $20 \mathrm{kHz}$ to $200 \mathrm{MHz}$, and a system dynamic range of at least $40 \mathrm{~dB}$.

\section{Command Trigger Synchronization}

The command trigger console system consists of a fastrise pulse generator, a variable-delay generator with tapped delay 1 ines, two optical uplinks, and support circuitry such as power supplies. The optical trigger signal is converted to an electrical signal on the simulator, and this triggers the AESOP Trigger System (ATRIS). A high-voltage resistive monitor on the output of each trigger generator supplies a signal to the RCDL onboard optical transmitter. This signal is shaped to provide a fast-rise, narrow-width pulse ( $<5 \mathrm{~ns}$ rise, 20 ns width), which drives the transmitter in a saturated mode. The narrow pulse is necessary because the Marx generator waveform follows this pulse by about 50 ns. When the pulse is received by the RCDL console optical receiver, it is fed through a high-pass filter which blocks the Marx erection waveform. The pulse is then applied to a highspeed comparator, which is set to a threshold above the normal wideband noise generated by the optical system. The comparator provides a logic-level output pulse, which is fed to the delay generator to remove any delay that was dialed in initially.

At this point, the two pulses are checked for coincidence through a logic gate. If they are within $\pm 5 \mathrm{~ns}$, an output from the gate activates further logic and pulse stretcher circuitry. This culminates in a side-one/side-two 
timing O.K. light and an interlock signal to the TEMPS command and control, which allow the Marx generator charging sequence to start. An additional feature is a misfire detector activated any time trigger pulses are received without a command trigger initiation. This misfire detector automatically aborts any charging or firing sequences of the AESOP pulser.

\section{Marx Generator Waveform Transmission}

The Marx generator waveforms are detected by E-field plates inside the main vessel. These plates are approximately $1 \mathrm{ft}^{2}$ in area and have an attenuation factor of approximately 20,000:1, yielding about 150 volts peak into 50 ohms at the normal operating level of AESOP. This voltage is attenuated further to about 1.5 volts (100:1) and applied to a power tee into the onboard optical downlink transmitter. Both signals are then transmitted to the RCDL optical receivers. The receiver output directly drives an oscilloscope output, and the oscilloscopes record the waveforms in real time.

As anticipated, several problems were encountered with the data acquisition and recording scheme which were difficult to solve in a high EMP environment. The output level of the Marx generator waveforms is normally about 500 millivolts peak, and any leakage of EMP disturbs these waveforms considerably.

\section{EMP Shielding}

The AESOP simulator successfully generates a very high EMP field environment. Because of this, double shielding of all circuits and optical isolation techniques are used whenever possible. The RCDL console has RFI shielding enclosures for all power supplies, optical receivers and transmitters, and logic circuitry. Optoisolators are used to interface with the AESOP control console, and internal ac power wiring and relay logic is kept to a minimum. Interface with the oscilloscopes is through double-shielded cable to the oscilloscope screenbox. It is this run that appears to pick up most of the noise affecting waveform fidelity. This area can be improved by alternate shielding methods, or relocation of the RCDL into the screenbox. Inside the Faraday cage of the pulser structure, the RCDL onboard electronics are in a much less severe environment. Even so, the same extensive methods of shielding are used.

\section{Operation of the RCDL}

The RCDL system streamlines the tedious task of determining whether the AESOP simulator is operating properly. 
It also makes possible shot-to-shot timing adjustments that keep the simulator at peak efficiency.

A normal discharge cycle begins by arming the AESOP simulator from the control console. Five seconds after turning the arm key, the Fire Ready light on the RCDL console lights up, and the operator presses the Fire button. This directs the ATRIS to check out synchronization. If the coincidence criterion is met, the Sl/S2 Timing O.K. and the Armed indicators on the AESOP console light up. Marx charging can begin shortly after the Charge complete indicator lights, and the RCDL Fire Ready light will come on again. Pressing the Fire button this time will erect the Marx generators and $\overline{l a u n c h}$ the EMP pulse. The Marx generator waveforms are recorded as the simulator fires. The operator can discern from the oscillographs proper synchronization and overall pulser performance.

\section{Future Uses of RCDL Concept}

The most exciting prospect for the use of the RCDL concept is multiple synchronous or ripple EMP wave generation by several simulators controlled by an RCDL system. The diagnostic problems associated with these simulator systems would be greatly simplified. The RCDL can monitor on each shot and eliminate downtime used by traditional diagnostic methods.

The RCDL could also be employed to control an entire pulsed power system through fiberoptics coupled to a microprocessor-based data acquisition and control system. Simulator performance, test subject response, and other parameters could then be integrated into one set of data for study and recording. The RCDL could streamline many facets of pulsed power system testing and operation, while keeping sensitive data acquisition and measurement systems electrically isolated from high EMP or RFI environments.

\section{Acknowledgements}

The author would like to acknowledge the assistance of J. Stewart and W. Petty, EMP Simulation Group, Lab 1000 , Harry Diamond Laboratories, Adelphi, Maryland; J. Keating and $D$. Medved of Meret, Inc., Santa Monica, California; and J. Dempsey of Physics International Company. 
A FIBER OPTIC MONITORING SYSTEM FOR ANTARES PULSE POWER SYSTEM*

\author{
M. E. Thuot and W. R. Scarlett \\ University of California \\ Los Alamos Scientific Laboratory \\ P.0. Box 1663 \\ Los Alamos, NM 87545
}

Antares is a $40 \mathrm{~kJ} \mathrm{CO}_{2}$ laser system being built for inertial confinement fusion studies. The electron beam controlled discharge in the laser power amplifiers is driven by eight $1.2 \mathrm{MV}, 300 \mathrm{~kJ}$, 2 microsecond pulse generators. Each electron gun produces a 5 microsecond pulsed beam of $550 \mathrm{keV}$ electrons with $50 \mathrm{ma} / \mathrm{cm}^{2}$ current density and total beam area of $9.3 \mathrm{~m}^{2}$.

An electro-optic interface to a computer based control and data acquisition system has been developed to provide a capability for the measurement and operational monitoring of pulsed analog waveforms and high voltage system timing information.

Each interface channel consists of a signal powered transmitter, a fiber optic cable, and an optical receiver which interfaces through a CAMAC compatible module to the control system. The data channel is capable of rejecting $600 \mathrm{kV}$ of common mode voltage, and operating in an electromagnetic interference environment of $500 \mathrm{kV} / \mathrm{m}$ and $50 \mathrm{kA} / \mathrm{m}$ at $140 \mathrm{kHz}$.

The analog transmitters send $10 \mathrm{MHz}$ bandwidth amplitude modulated information to transient waveform digitizers in the data system. During operation about 100 data channels monitor voltage and current in each pulser and anode current density in the laser pcwer amplifier. These devices have also been used to measure electron beam current density during electron gun testing.

Timing information of 2 ns resolution originates at each stage of trigger amplification and main pulse output in the ten power amplifier pulsers. The 120 channels of information are processed to provide a complete prefire diagnostic of each pulser.

The design, calibration, and performance of this system and its components will be presented.

\footnotetext{
ॠWork performed under the auspices of the U. S. Department of Energy.
} 


\section{Discussion Session 6}

Bob Hebner (NBS). I would like to ask either or both of the first two speakers about the pulse voltage measurement system that they have described. They showed what they characterized as an electric field pick-up, a relatively sophisticated transmission system to get the waveform back down to ground potential and then reconstruct it. But, what I was curious about was the design accuracy of that system, the achieved accuracy, and the method of verification.

Stan Lyons (Physics International). We felt that the waveform fidelity was much more important than the absolute voltage value. Since this is a real time system it was easy to compare the reconstructed waveform on the ground with the on-board diagnostic pictures we were taking with the screen box. So, we felt that linearity within one or two percent was really not necessary. We were after relative value changes in waveform from shot to shot as long as they reproduce within 5 or 10 percent we found that that was quite acceptable.

Bill Boyer (Sandia). I have a question for Stan also. I was wondering about the quality of the fiber optic data link. Is it a 130 megahertz analog link, how was it to work with, how did it perform?

Lyons. The entire system was a system supplied to us by Meret, Inc., of Santa Monica. We found that it generally met the specifications that we asked for. It seemed to be very insensitive to fiber alignment and things of that nature and we found that it was really a reasonable system to use.

Steve Levy (U. S. Army Electronics \& Technology Laboratory).

My question to the first two authors regards both transmitter and receiver power supply in terms of noise generated in the supplies by the EMP environment. What affect did it have on the response of their optical transmitters and receivers, and on their logic?

Lyons. The power supply ripple and noise was very well filtered before it reached the transmitters or the receivers. In the ground base system we had to take much more care to make it quiet. The transmitters are the main problem in that the diode is biased on all of the time so any change in current or voltage is going to affect the system. I don't believe we saw any effect in regards to EMP or from induced noise.

Levy. I am concerned about the fact that both the stability of the power supply as well as the effect of EMP on the power supply itself. We found that, even though we tried to protect our power supplies in our transient voltage monitors, we had to go to battery operation. That solved our particular problem. Also, TTL logic is very sensitive to noise along the power supply line. Could you analyze the origin of the noise in the receiver end that gave you the problem between the oscilloscope and your optical receiver? 
John Stewart (Harry Diamond Laboratory). Are you speaking of the EMP signal Stan described? We are still working on that problem by going through a process of trying to triple shield and build appropriate shield boxes for the actual component. We are able to live with the EMP induced signal. We are able to identify the operating parameters from the signal that we are getting now.

Mario Di Capua (Physics International). I should have asked this question back at the office, Stan. But what was your bandwidth of your system?

Lyons. We specified initially 200 megahertz bandwidth at the $3 \mathrm{~d} B$ points. It turned out that the best that Meret could give us was 10 kilohertz to 130 megahertz at the $10 \mathrm{~dB}$ point and a usable signal although it was down about $15 \mathrm{~dB}$ at 200 megahertz. The system dynamic range at 130 megahertz ws about $45 \mathrm{~dB}$.

Bob Kolibas (Raytheon Company). A follow up on that same question. What was the distance in the length of the cable and did that affect your bandwidth capability?

Lyons. The length of the cable is 100 meters and the bandwidth capability depends upon whether you use the step index fiber or the graded index fiber. We used the system that Meret suggested which was the step index fiber and it gave us the best overall bandwidth. It turned out that it was flatter over the range than we wanted to use. The graded index had a larger slope on the signal-to-noise than the dynamic range curves.

Richard Malewski (IREQ). You have been measuring voltage and current from the EMP simulator. But you probably also recorded the electromagnetic fields at the ground level nearby the test object with some sort of probe. What is the requirement on the bandwidth of the probes and on your voltage and current monitors? Did you use the same optical fiber system for signal transmission for the probes and for the monitoring devices for voltage and current?

Stewart. We use a differential voltage probe or current probe, depending on the requirement. But we use a different fiber optics data-link system. It's a 150-megahertz bandwidth system with remote control attenuators which was developed by Harry Diamond Laboratory. The fiber optics that are used to generate the signals to the operating system of AESOP are a little different than the data acquisition data-1 ink systems.

Hebner. Before we get off on this, I want to get one further clarification. To my earlier question you answered that you estimated about a 5 percent linearity accuracy. I would like a little further explanation of this. Some of the original voltage waveforms, that I saw very early in the first presentation, had oscillations on them which seemed to come from at least two or three different sources. 
I estimated the magnitude of these oscillations to be between 10 and 20 percent of the peak voltage. Later, I assumed that those resulted from the leakage problems which you talked about but now I realize that those may be real waveforms that result from reflections in your system. Are you measuring those signals to within 5 percent accuracy and are you working at trying to improve the accuracy below 5 percent? If you are, I think that it is remarkable.

Lyons. The high frequency ringing you saw on those waveforms was the EMP Teakage into the system. We ran the system at lower levels so that the EMP generated by the simulator was not as great an amplitude and that is where we compared the down link transmitter waveforms with the on-board diagnostic waveforms. We determined the 5 percent linearity of the waveform by examining the frequency content of the waveform that we were interested in. As we were saying, the noise is still a problem and I don't think that there is anyway that we know of right now to solve that problem completely.

Hebner. By linearity, do you mean the peak voltage as measured? Would you define linearity?

Lyons. OK. I guess maybe I should say waveform fidelity instead. If the pictures were normalized in amplitude and overlayed the perturbations on the waveform were within 5 percent at all points.

Hebner. How did you measure the voltage on the high side to 5 percent? You are comparing it with something else, and these voltage levels of 5 percent measurements are fairly impressive. I was just wondering what device you are comparing your fiber optical system against.

Lyons. The standard was putting the scope in the screen box on board the system and measuring that same waveform without sending it down to the fiber optic system. Again, we were more interested in waveform fidelity than the absolute voltage value.

Hebner. Thank you.

Boyer. Are there any further questions? I have one on the Los Alamos paper. You mentioned early in the paper that you have some sort of monitor to detect the faults in high voltage cables. I was wondering if you would expand on that a little bit.

Bill Scarlett (Los Alamos). What we have is a $\dot{B}$ loop in a high voltage feedthrough insulator. We look for the direction of current flowing in each of six cables feeding each anode. Since the cables are shorted together at the anode, a fault in one of those cables will lead to current flowing in the reverse direction. This leads to an anomaly in the timing of the B signal for the failed cable.

Levy. I have a question on the Antares paper. You mentioned the 
fact that there was a problem of lining up the transmitter fiber optics and in the paper you also mentioned that you had a pigtail lead on the optical transmitter. That means to me then that there was a problem of splicing the two optical fibers, one coming out of the pigtail to the one going into the line. If this is so, could you please explain the problem in a little more detail because this is certainly going to be a fundamental problem that everyone is going to run into if we do go into optical transmission of data. If this is not so, maybe you could explain where the source of error is coming from.

Scarlett. The main source of error is the centering problem in the fiber optic connectors. We measure and transmit a very large number of signals as was indicated on the viewgraph. There are on the order of 100 pulsed quantities and well over 100 timing channels. We use similar types of fibers and terminations in our control channels, so the total number of channels is large. Because of cost considerations, we picked the plastic and connectors, which are one of the cheaper standard connectors available. There are other connectors in which the centering problem has been solved by fairly elaborate means. Unfortunately, these connectors are also fairly expensive. The problem is there, but it can be solved. The main problem occurs with two fibers of similar size. 


\title{
DATA REOORDING TECHNIQUES FOR THE SANDIA PARTICLE BEAM FUSION ACCEUERATOR
}

\author{
William B. Boyer and Eugene L. Neau \\ Sandia National Laboratories \\ Albuquerque, New Mexico 87185
}

\section{ABSTRACT}

A data handling facility has been developed for the Sandia Particle Beam Fusion Accelerator (PBFA-I). The facility records and processes nanosecond diagnostic signals from both machine performance and experiment diagnostic monitors. The system consists of an EMP-shielded cable plant, two minicomputers, coax switches, transient waveform recorders, CAMAC pulse processing modules, and trigger/fiducial timing generators. All components are calibrated under either manual or computer control. Waveforms are recorded and calibrated by the system with an amplitude accuracy of $+1 \%$. Signals from different monitors can be aligned in time to within $\pm .4 \mathrm{~ns}$. The facility is capable of supporting multiple simultaneous users. Applications software has been developed to support calibration, data acquisition, and data reduction. The facility is operational and is supporting two accelerators.

\section{Introduction}

This paper describes an on-line data handling facility for Sandia's Particle Beam Fusion Accelerator, PBEA-I, and the upgrade prototype machine SuperMite. These accelerators are used for research on inertial confinement fusion (ICF) using particle beams. The main main objectives in designing the data acquisition system were:

1. process both experiment and machine performance diagnostic signals,

2. record high signal-to-noise ratio, wideband waveforms in a severe EMP environment,

3. support multiple users recording and analyzing data simultaneously, and

4. provide fast turnaround for experimental results. 
Commercially available equipment is used wherever possible. However, several special purpose devices were developed. This data handling facility is a significant upgrade of an existing system that supports other Sandia particle beam fusion research accelerators.[1]

\section{Accelerators}

PBFA-I has 36 independent beam lines and is capable of delivering 30 terawatts and 1 megajoule in a 2 to 4 megavolt, 35 ns pulse into a target chamber.[2] The accelerator can be configured to generate either electrons or ions. The objective is to deliver sufficient beam energy to demonstrate the scientific feasibility of ICF. The accelerator upgrade, PBFA-II, will provide 3 times as much power in the 1985 time frame. The superMite accelerator is a 4 module prototype used to develop higher power components. Other small accelerarators are also planned for the facility.

\section{Cable System}

Signals to be recorded are fast transient pulses of 10-1000 ns duration and 1-5000 volt peak amplitude. These waveforms nust be recorded in the presence of EMP levels generated by di/dt and $d v / d t$ of $10^{15}$ amps and volts per second. This EMP level requires that signal cables be carefully shielded over the entire run. A picture of the cable plant is shown in Figure 1.

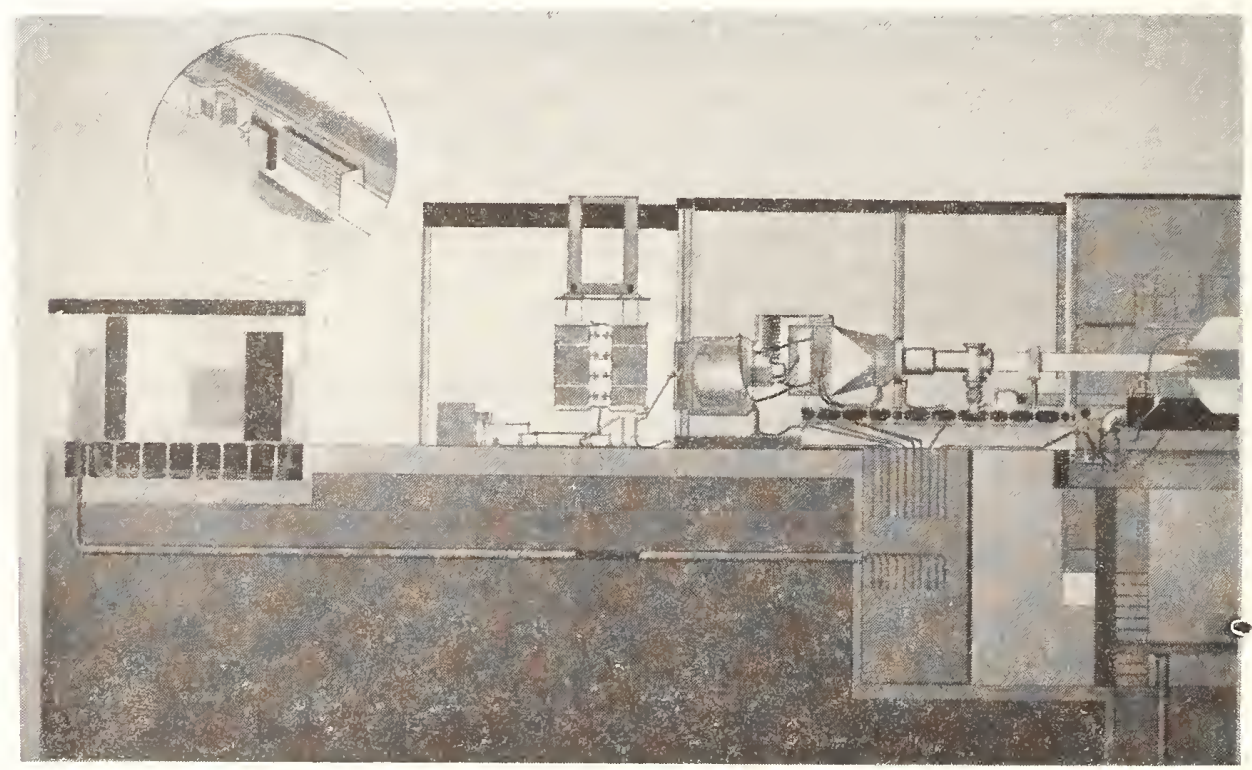

Figure 1. Cutaway view of PBFA-I with fixed cable plant. 
Jumper cables run from diagnostic monitors to feedthru ports near the floor of the tank. These consist of RG-214 cable run inside flexible solid convolute shield tubing. Special adapters were designed to mate the shield conduit to type $\mathrm{N}$ and $\mathrm{HN}$ connectors in an electrically and mechanically sound fashion. Ferrite sleeves are inserted in the adapters to further reduce noise pickup. Figure 2 shows an assembled cable and the convoluted shield. The entire I-beam support structure beneath the tank was made rf-tight. This enclosure contains 800 RG-331 cables which run fran feedthru ports in the bottom of the accelerator to a shielded splice room. All cables serving similar diagnostics were cut to equal lengths to \pm .5 ns. From this point signals can either be recorded and processed Iocally or jumpered to the main screen room for processing. There are 270 RG-331 cables run from the splice roam to the screen room in nine 6-inch diameter conduits. A tenth conduit is available for further expansion. Unshielded RG-214 cables are used as jumpers in both the splice and screen rooms.

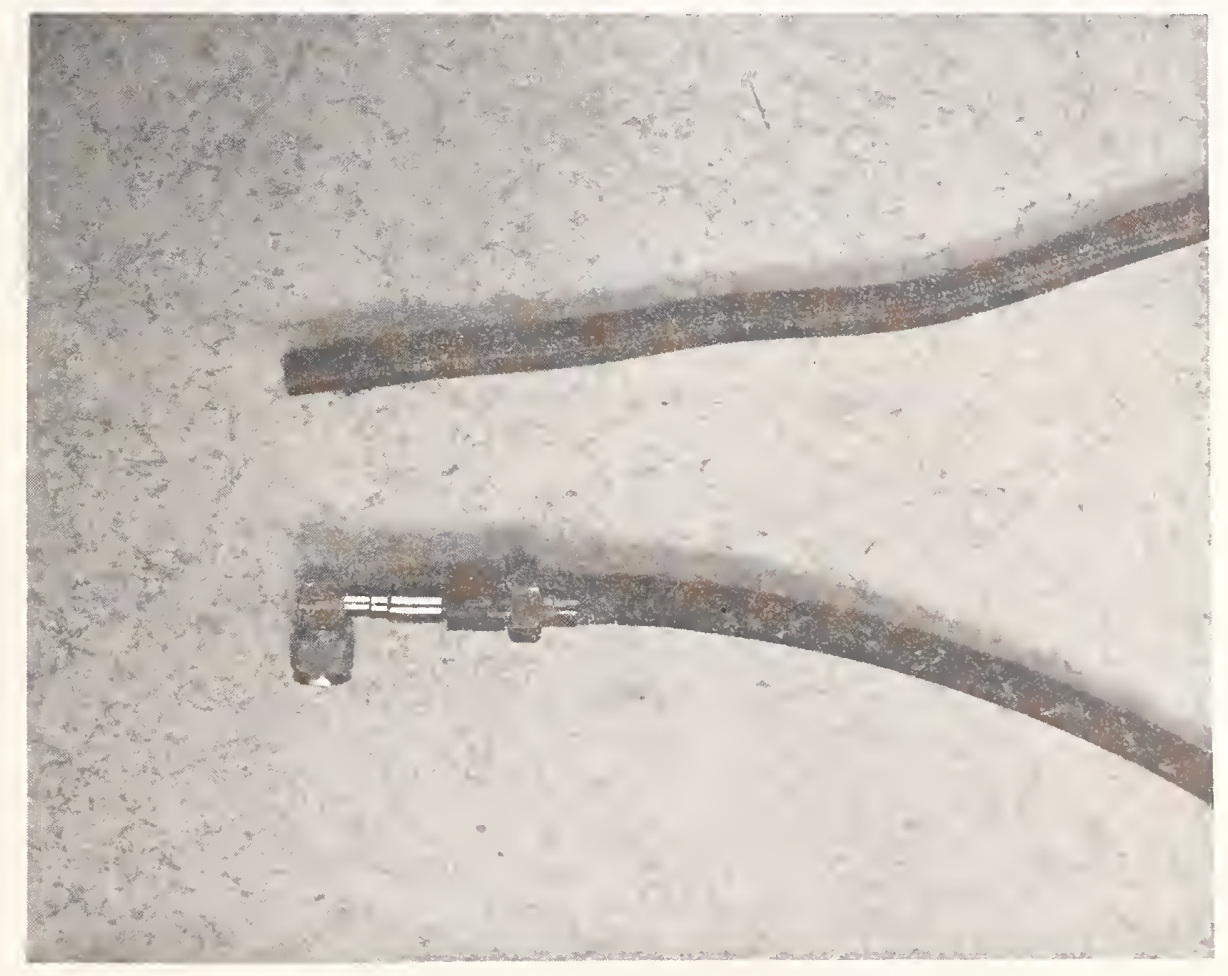

Figure 2. Shielded jumper cable connector assembly and stripped back convoluted shield.

4. Computer Hardware

Must data recording and processing electronics are housed in a high quality double-shielded rf screen room. A photograph is shown in Figure 3. This room is $15^{\prime} \mathrm{W} \times 65^{\prime} \mathrm{L} \times 10^{\prime} \mathrm{H}$ with a raised floor. 


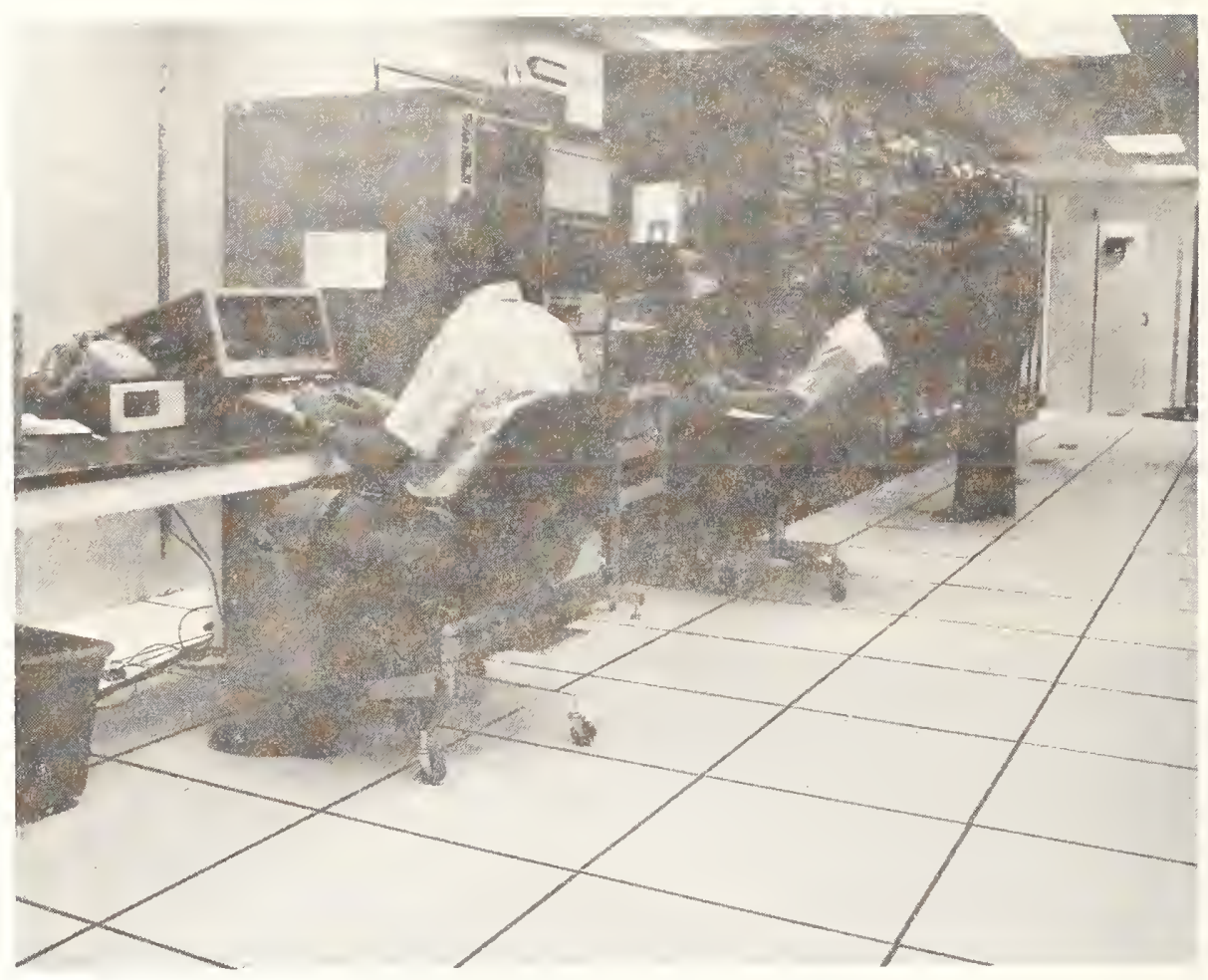

Figure 3. Data aoquisition facility main screen room hardware.

A block diagram of the waveform recording system is shown in Figure 4. The main computer is a Hewlett-Packard HP-1000 system with $256 \mathrm{~Kb}$ of 16 bit memory and a hardware floating point processor. Mass storage consists of a $20 \mathrm{Mb}$ moving head disc drive with a $10 \mathrm{Mb}$ removable cartridge and two $256 \mathrm{~Kb}$ floppy disc drives. A nine track, 45 ips, 800 bpi tape drive is also available. The system console is an $\mathrm{HP}-2648$ graphics terminal with dual cassette tape drives. Two Tektronix 4014 Display Terminals with Enhanced Graphics Modules are available for data acquisition and reduction. Each terminal has a 4631 hard copier with 4-channel input multiplexer. A Versatec 1200A printer/plotter is also available. This device has a Versatec 5-channel input multiplexer to enable the plotter to make high quality hard copies from the Tektronix terminals. The data acquisition HP-1000 is tied via an HP DS-1000 intercamputer link to a similar computer in the PBFA facility control room.

\section{Waveform Digitizing Hardware}

The facility has 44 Tektronix 7912AD Transient Digitizers with $7 \mathrm{Al6P}$ programmable vertical amplifiers and 7B90P programmable time bases. The vertical amplifier has a specified minimum bandwidth of $200 \mathrm{MHz}$. This is adequate for recording virtually all accelerator and experiment diagnostic signals. 


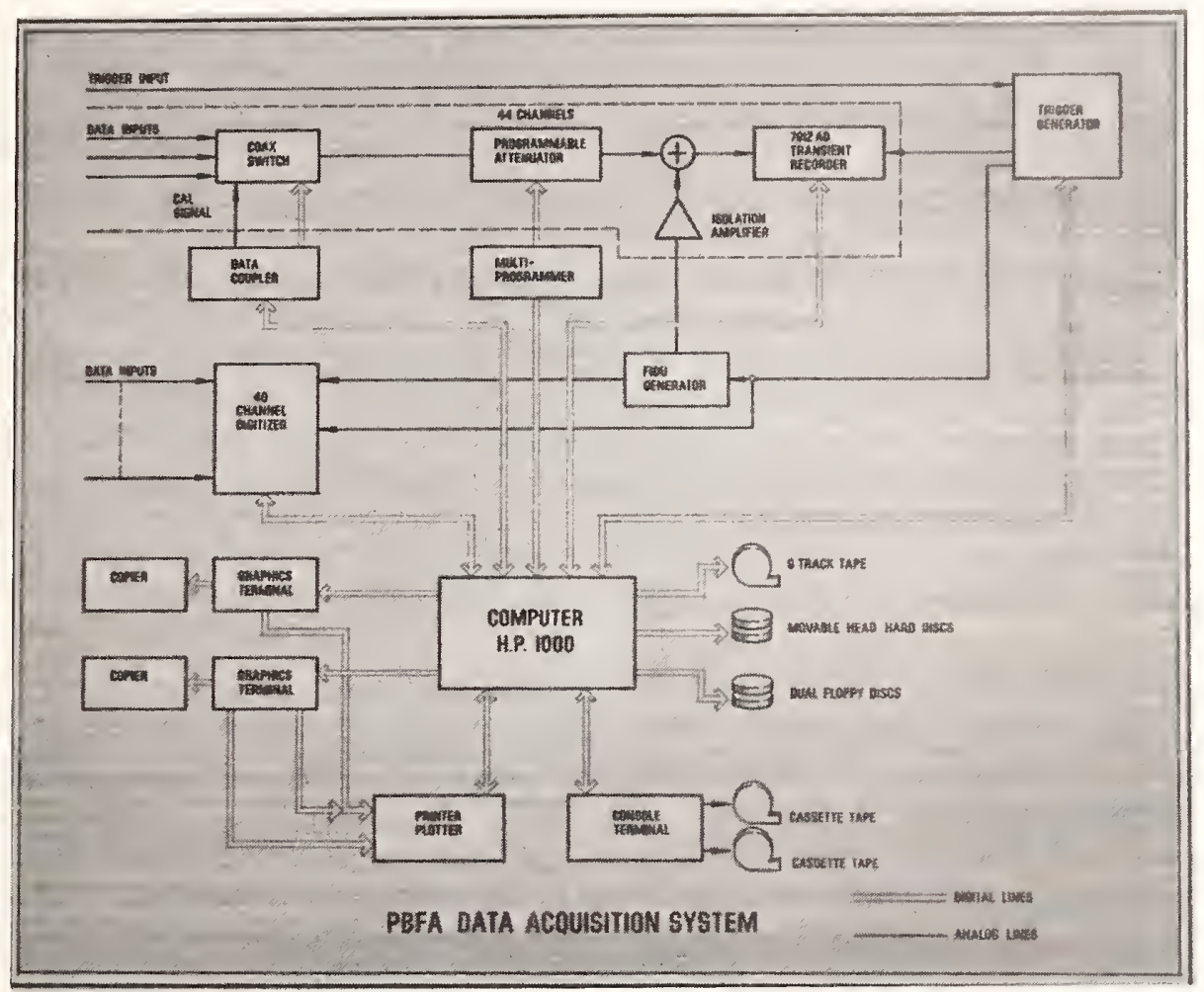

Figure 4. Block diagram of waveform recording system.

The 7912AD's are interfaced to the HP-1000 computer via IEEE 488 General Purpose Instrumentation Buses (GPIB). Each GPIB is specified to handle 15 devices, including the controller. However, the bus would intermittently fail whenever more than eight 7912AD's were powered up on that bus. This problem was solved by installing capacitors on the HP controller cards to slow down the handshaking process. The fastest pulse widths were increased from 300 ns to 600 ns. This enabled bus signal levels to reach a full $2.5 \mathrm{~V}$.

Data signals from up to three different experiments are cabled to the input ports of a computer controlled, four-position coax rotary switch module. Computer generated calibration signals are connected to the fourth port. The coax switches are Tektronix SM-2 matrices with twelve modules per unit. The SM-2's are controlied by 32 bit digital output cards in a Tektronix 1340 Data Coupler. The Data Coupler also houses the vertical and horizontal reference signal generator cards used for autocalibration. The Data Coupler is interfaced to the HP-1000 computer via an RS-232 Iink operating at 1200 baud.

The output signal from the coax switch is fed into a programmable attenuator. A fiducial timing marker pulse can then be added to the 
signal via a resistive power adder. This configuration requires an isolation amplifier in the fiducial signal line to prevent crosstalk among data signals through the fiducial fanout network. In some cases the fiducial signal is routed to the 7912AD vertical amplifier B channel, and timing information is only recorded during the preshot baseline check.

The programmable attenuators are Allen Industries Model 50DA63. Attenuations of 0-63 $\mathrm{dB}$ may be selected in $1 \mathrm{~dB}$ steps by relay closures. They are controlled by standard relay driver cards in Hewlett-Packard Multiprogrammer chassis. The Multiprogrammers are interfaced to the computer over the GPIB. The attenuators can reliably withstand $500 \mathrm{~V}, 100$ ns pulses. Higher energy signals are routed through special fixed attenuators in front of the coax switch. These devices consist of 3 and $8 \mathrm{~W}$ low inductance resistors mounted on $50 \Omega$ stripline boards in a minibox with type $\mathrm{N}$ connectors.

Precision timing is required to record and analyze the diagnostic signals. All cables have been built to \pm .5 ns as measured by a cable length digitizer. Special trigger and fiducial marker pulse generators were developed for this facility.

The trigger generator was built by Science Applications, Inc. (SAI).[3] The unit is fabricated as 6 NIM-type modules. Analog, digital, manual, and internal rep rate inputs are provided. Each analog input has a slope/level discriminator. Minimum delay time is $30 \mathrm{~ns}$ for both analog and digital inputs. Outputs are divided into four groups of 16 each. Each group is separately delayable fram nominal 0-10 $\mu \mathrm{s}$ in 10 ns steps. Asynchronous clocks are used to yield a shot-to-shot jitter of $<1.0 \mathrm{~ns}$. All functions of the trigger generator can be computer controlled via the GPIB.

Programmable functions include: input enable for any or all channels; analog slope and level; digital slope; and output delay.

The fiducial generator was also developed by SAI.[4] This device will trigger on a fixed minimum input pulse level of 2 volts. The unit has ten outputs of 35 volts simultaneous to \pm .1 ns. Output pulse width is variable from 2-20 ns in 2 ns steps. The width may be increased by increasing the length of the internal $10 \Omega$ printed circuit charge line.

The data system has the requirement to record almost 400 diagnostic signals to fully monitor PBFA-I performance. This precludes using $7912 \mathrm{AD}^{\prime} \mathrm{s}$. Sandia is funding development of a multichannel waveform digitizer to record transient signals. The design goals for this device are 40 channels sampled simultaneously at intervals variable from $1-50$ ns and a record length of 240 samples per channel. Full scale amplitude sensitivity is fixed at $2 \mathrm{~V} . \mathrm{A} \pm 1 \mathrm{~V}$ offset is available to allow efficient recording of of unipolar or bipolar signals. 
A block diagram of the multichannel recorder is shown in Figure 5. Input voltage signal channels are converted to light by fast LED's. All light outputs are routed by fiber optics to the photocathode input faceplate of a streak camera tube. The electron beams from the photocathode are accelerated, focused, and swept across the phosphor screen output faceplate. Fiber optic faceplates are used on both input and output to maximize light transfer. The light from the phosphor is carried by a fiber optic reducing bundle to a commercial TV format Charge Coupled Device (CCD) chip. The CCD serves as an analog storage device. Data signals are clocked out from the CCD, digitized, and stored in a digital solid state memory at conventional rates. The multichannel recorder is interfaced to the computer via the GPIB. The initial prototype is scheduled for completion in 1981.

\section{Software}

The HP-1000 computer runs under the RTE-IVA operating system with the Multi-Terminal Monitor. The only modification required on the system software was to enable the GPIB driver to schedule a user program upon receipt of a service request internupt (SRQ). Previously the driver could only be enabled to auto-poll predefined devices. However, the autopolling would not process extended talker and listener devices which require secondary addressing. This feature is used in the 7912AD's to distinguish mainframe, vertical amplifier, and time base commands.

Applications software for the PBFA data handling system is functionally similar to that developed for the previous facility. However, all programs have been rewritten in a structured, tabledriven format to maximize maintainability and expandability. The programs were also designed to be as machine independent as possible. Approximately 22,000 lines of Fortran code have been developed.

Major programs support the following functions:

1. Automatic digitizer set up and data collection.

2. Automatic digitizer calibration.

3. Interactive data reduction.

4. Shot archiving.

Fortran was used because it was the only supported high level language available for the HP-1000 when the project was started. All hardware configuration parameters are placed in tables. Input table data is in ASCII format and may be modified by the source editor. Special programs translate the source tables to mixed-type-variable records and store them in random access files. Special subroutines access these tables for applications programs. Thus hardware capabilities may be modified and expanded without rewriting software. 

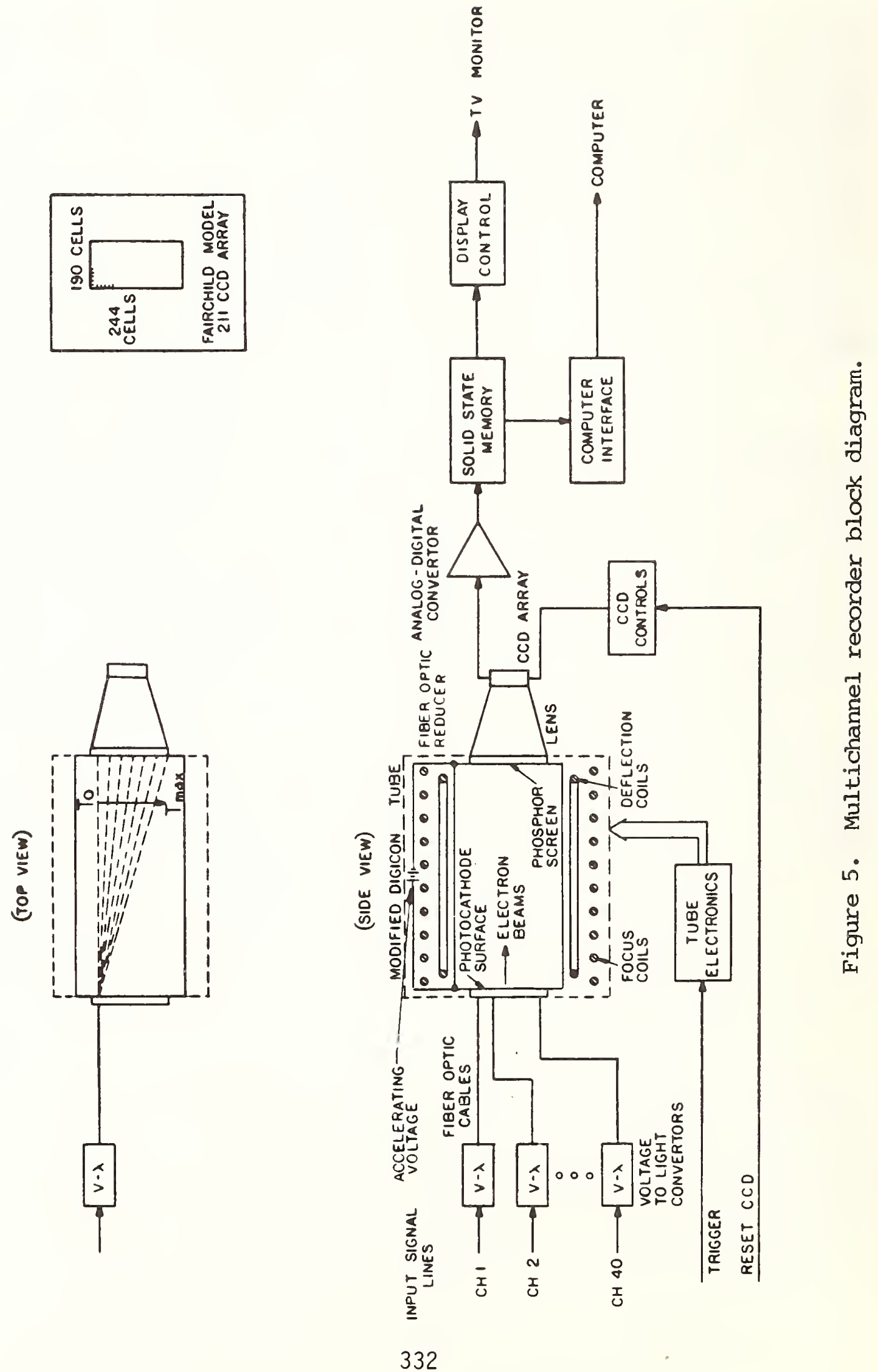
The automatic data acquisition program ( $A Q D$ ) begins by reading and processing a table of signal channel parameters for a given shot. The program uses this and calibration tables to set up all signal recording equipment to be used for the shot. It then digitizes a baseline- and checks each digitizer for proper operation. AQD then arms for the shot. Finally, after the shot, the program reads, calibrates and stores each waveform on the disc. The calibration process consists of:

1. Averaging to center of trace,

2. Multiplying in vertical amplifier gain, attenuations, and gauge scale factors,

3. Correcting for time base sweep nonlinearity,

4. Compensating high frequency cable losses.

After AQD has stored all the waveforms, they are available for user processing. An Interactive Data Reduction (IDR) program is used extensively for manipulating and plotting signals. This program provides the following capabilities:

1. Interactive or procedure file macro-type command execution.

2. Access data from multiple shots on either hard or floppy discs.

3. Array arithmetic operations -- add, multiply, divide, square root, exponentiation, raise-to-power, minimum, maximm, average, integration, differentiation.

4. Fourier transform, convolution, deconvolution, low pass filter.

5. Single, multiple, overlayed, and windowed plots.

6. Cursor control and printout.

7. Control of all programable hardware functions.

Figure 6 shows an example of a waveform processed using IDR commands.

An automatic calibration program ( $A C L$ ) is used to generate and maintain 7912AD and programmable attenuator calibration data. This program generates cal signals and switches them to the appropriate digitizer. It digitizes the resulting waveforms and computes calibration factors. These data are stored on disc for use by programs $A Q D$ and IDR in calibrating signal waveforms.

Accelerator shots are archived on floppy discs. This method is superior to mag tape in that it provides rapid direct access to old shots. The following information is stored for each shot:

Shot Set Up Header

Calibration Data

Baseline Levels

Calibrated and Uncalibrated Cata Records

Cable Campensation Functions 
Figure 6. Data signals processed by program IDR. Vacuum interface $\mathrm{di} / \mathrm{dt}$, integral with respect to time and magnitude of Fourier transform of each in $\mathrm{dB}$.
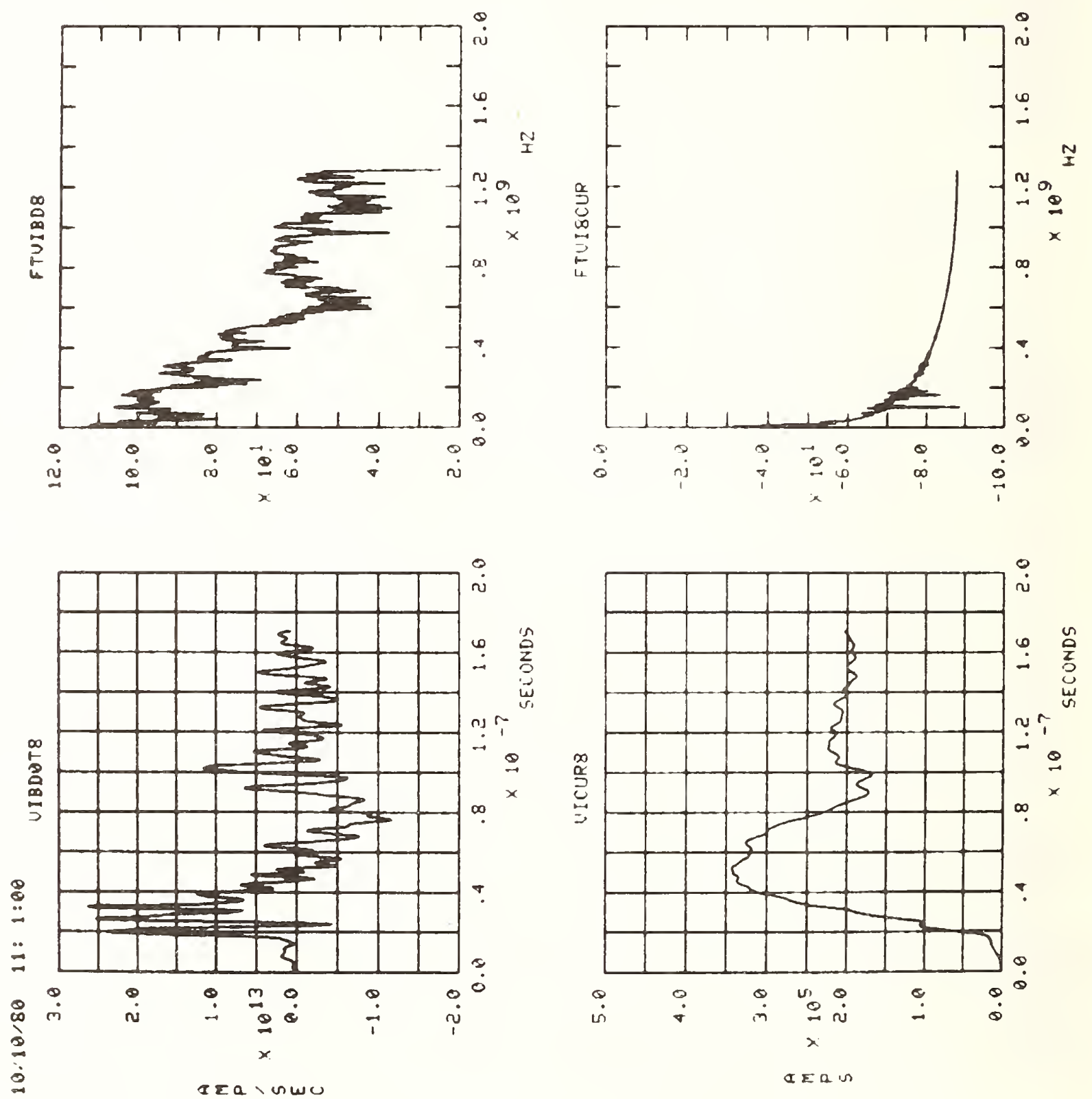

$\stackrel{\infty}{\infty}$

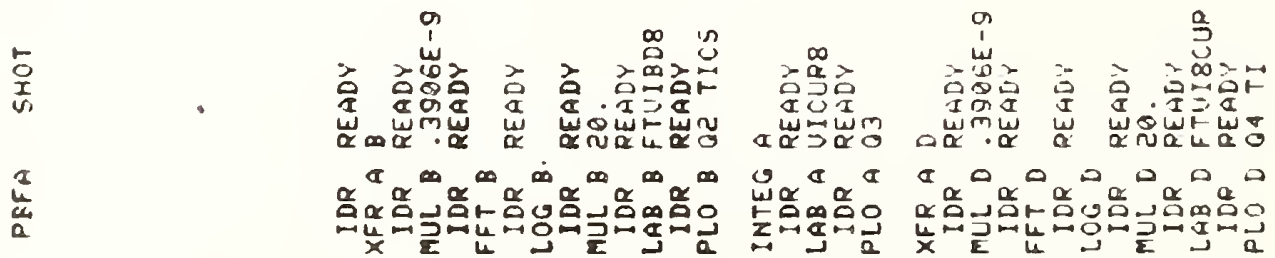




\section{System Performance}

The PBFA-I waveform recording system is a complex combination of hardware and software components. Tests have been performed to determine the overal amplitude and time accuracy of the system. Calibration techniques for the various components that affect the signal amplitude are described below.

The 7Al6P vertical amplifiers are computer calibrated by injecting a single known dc voltage into the input and measuring the deflection. This process is averaged over four tries. Each of the six sections in the programmable attenuators is also computer calibrated by injecting known dc voltages into the unit and measuring output with the associated transient recorder. Fixed attenuators are manually calibrated using dc power supplies and voltmeters. All dc equipment is corrected to $\pm .1 \%$ using a calibrated Fluke $382 \mathrm{~A}$ dc voltage reference source.

Cable runs are calibrated by a hybrid computer/manual technique. A fast rise step pulse is injected directly into a digitizer and recorded. The same pulse is then injected into the cable run to be calibrated and recorded by the transient recorder. A constrained deconvolution process is used to compute a compensating impulse response function for the cable run.

The automatic data acquisition program uses the calibration factors described above to process each recorded waveform. The overall accuracy of this system was checked as follows. A 150V, 50 ns rectangular pulse was recorded by a transient digitizer. This signal was then manually calibrated using deflection factors determined by the $382 \mathrm{~A}$ reference source. The same pulse was injected into the input of a cable run. It was recorded and processed by the data acquisition program: A least squares comparison was made of the two waveforms. The overall scale error was $.4 \%$ and the standard deviation normalized to the peak level was $2.8 \%$. The undegraded signal, recorded degraded signal, and calibrated signal are shown in Figure 7. An overlay plot is shown in the fourth quadrant.

The 7B90p time bases are calibrated by the computer. Periodic waveforms with known frequency are recorded by the 7912AD's. The software locates zero crossings on the waveform and generates a calibration curve of time vs. zero crossing addresses. The data acquisition program uses this curve to process recorded signals. The curve is assumed to be nonlinear. The time at which each sample occurs is computed by a double sliding parabolic interpolation. [5] The signal data points are transformed into an array of data points occurring at a fixed sample rate by linear interpolation.

Accurate alignment in time of signals recorded on different digitizers is critical for analyzing accelerator and experiment performance. Alignment information is recorded by placing a narrow fiducial timing marker on each trace when a baseline is taken. 
Fig. 7. System amplitude and cable compensation test waveforms:

(a) undegraded pulse, (b) recorded pulse, (c) calibrated pulse, and (d) overlay of (a) and (c).
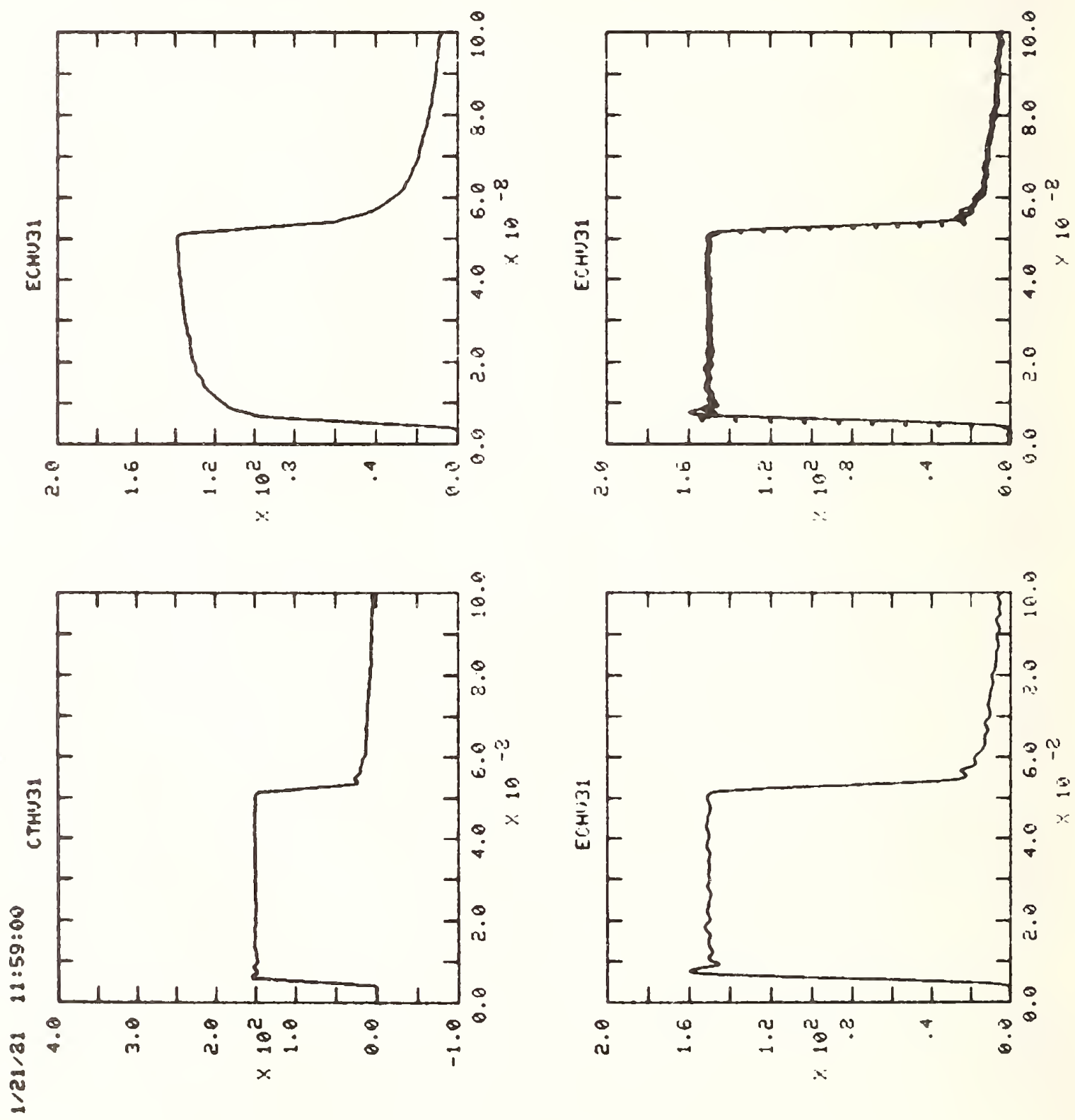

$\omega$

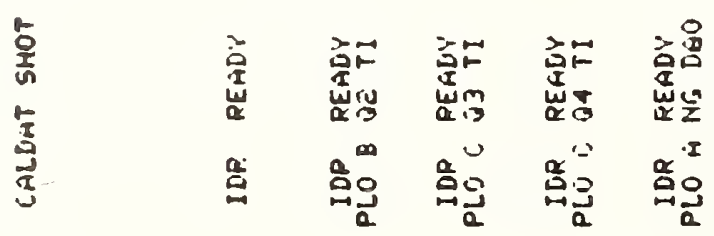


The data acquisition software locates the peak of the fiducial pulse. Ideally the fiducial pulse would be added onto the data signal as it is recorded. However, the lack of a broadband isolating power adding technique precludes this at this time. The only error in taking the fidu location from the baseline trace is caused by jitter in the time base which is specified at .l ns for a .4 ns risetime trigger pulse.

After each signal is time calibrated, the leading edge is truncated at a time equal to the distance to the fidu peak plus one-half of a division. This truncation aligns all signals in time.

Tests have been made to determine accuracy of the data recording system in aligning signals. A pulse generator with ten 20 ns outputs simultaneous to within .1 ns was used. The pulses were injected into the input of the cable nun and recorded by the 7912AD's at $10 \mathrm{~ns} /$ div. The waveforms were processed by the data acquisition software. This process was repeated five times to separate fixed time delays from shot-to-shot jitters. Fixed time delays are caused by unequal cable lengths.

Least squares comparison fits were made of different signals over the same shot. This data was averaged over all shots to get fixed time delays. The fixed time delays were removed and the data was analyzed for different signals over the same shot and for the same signal over different shots. The results are that the expected alignment error for a given shot was .26 ns with a worst case spread of .96 ns. The expected shot-to-shot alignment error was .33 ns with a worst case spread of $1.04 \mathrm{~ns}$.

The most significant source of error is the software process of locating the peak of the fiducial timing marker pulse. Digitization noise results in an expected error of about +1.5 sample. At $10 \mathrm{~ns}$ div (.2ns/sample), this is $\pm .3 \mathrm{~ns}$.

\section{Accelerator Performance Monitors}

Many accelerator performance diagnostic signals may be adequately monitored by a single parameter once the machine operation has been characterized. These parameters include switch closure times, peak amplitudes, and time integrals. A stand-alone accelerator Perfomance Measuring and Evaluation System (PMES) is being developed to record and process single point data. A typical signal channel is shown in Figure 8.

Commercial CAMAC modules are used to record approximately 300 channels of signal parameters. Special NIM-type signal conditioning and trigger modules have been developed to support this system. Inputs to CAMAC time digitizers are buffered by 16-channel avalanche transistor discriminator modules. Inputs to CAMAC gated peak detectors and integrators are processed by modules which can contain 
any combination of attenuators, power splitters, pulse inverters, and integrators. Trigger fanout modules with four TTL input channels and six low level NIM outputs per channel are used to start time digitizers. Gate generator modules with one TTL input and four low level NIM outputs are used to drive gated peak detectors and integrators. Commercial NIM time delay generators are also used in the system.

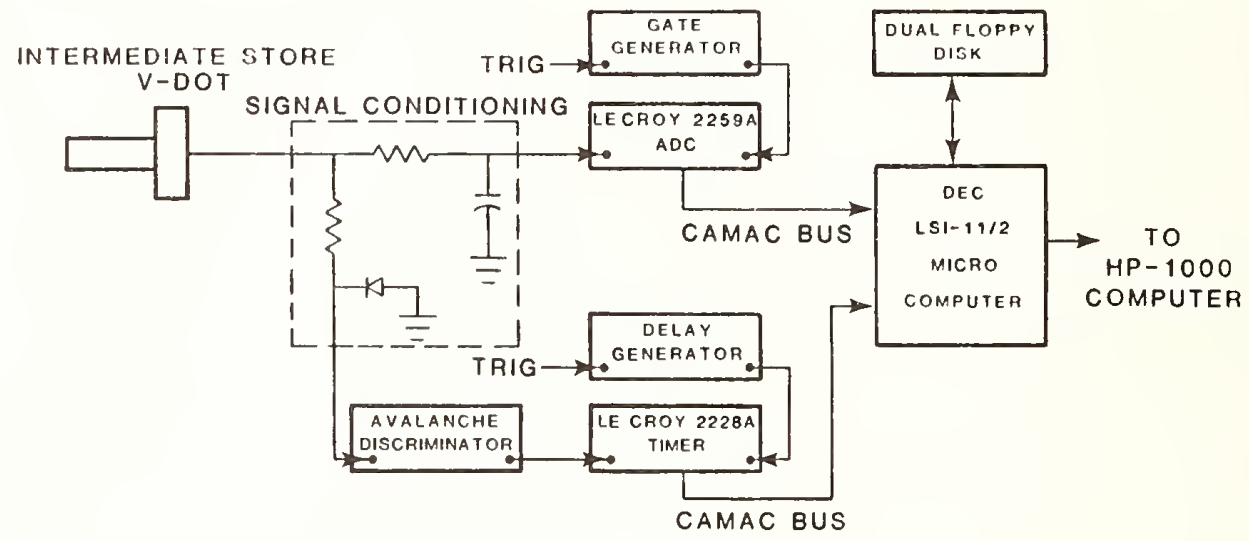

Figure 8. Data channel hardware for recording intemediate store V-dot peak amplitude and gas switch firing time.

The PMES operates in a stand-alone mode with a Standard Engineering MIK-11/2 crate controller with dual double-density floppy discs. The CAMAC system will be located in the cable splice room below the accelerator tank. This location was chosen because of the limited number of cables running to the main screen room. The MIK-11/2 is interfaced to the main facility CPU via an RS-232 fiber optic link.

Software for the PMES system is functionally similar to that for the HP computer. Many routines were directly transportable. Machine dependent subroutines were rewritten. The relatively limited capabilities of the PMES computer forced some simplifications in the software system design. Programs were written to support automatic data acquisition, interactive hardware manipulation, interactive calibration, and transfer of data to and from the HP computer. Programs have been written for the HP computer to enable interactive and automatic data transfers between the two systems.

PMES is currently monitoring the signals shown in Table I. The Marx generator time digitizers are normally triggered by a signal from the Marx pulser unit (MPU) trigger. A second trigger pulse is created by adding signals from all the Marx voltage monitors. This pulse will trigger the time digitizers if one of the Marx generators prefires before the MPU's are triggered. The Marx that prefired can 
be located by noting which of the Marx time digitizers did not receive a stop pulse.

Table I. Accelerator Parameters Measured with PMES

\begin{tabular}{l|l|c}
\hline \multicolumn{1}{c|}{ Monitor Point } & Parameter & Number channels \\
\hline Marx pulser unit & Start time & 8 \\
\hline Marx current & Start time & 36 \\
\hline Marx current & Peak amplitude & 36 \\
\hline Intermediate store $\dot{V}$ & Gas switch trigger time & 36 \\
\hline Intermediate store $\dot{V}$ & Peak amplitude & 36 \\
\hline Line $1 \dot{V}$ & Peak amplitude & 36 \\
\hline
\end{tabular}

9. Conclusion

The data aoquisition and handling system described above is operational and supporting PBFA-I and SuperMite. The 7912AD waveform recording hardware and software have been running since January 1980. The PMES system is currently being brought on line as PBFA-I is characterized. The same system will also support other small accelerators which are planned for the facility.

10. References

[1] W. B. Boyer, "Data Acquisition and Processing on Electron Beam Fusion Accelerators", IEEE Trans. Nucl. Sci., NS-25, No. 1, 501-505 (1978).

[2] G. Yonas, "Pulsed Power Particle Beam Fusion Research", IAEA Technical Committee on Inertial Confinement Fusion, Osaka, Japan, Nov. 1979.

[3] K. R. Sites, A Modular Trigger Generator Unit Operations Manual, Science Applications, Inc., Tech. Man. SAI-81-26-LV, Las Vegas, 1980.

[4] K. R. Sites, SG-111 Fiducial Marker Generator, Science Applications, Inc., Tech. Man. SAI-80-23-LV, Las Vegas, 1979. 
[5] M. E. Bauder, Operations and Maintenance Documentation for Program PLBRD, Sandia TC-TM-70-114, 1970.

12. Sponsor Acknowledgments

This work was supported by the U. S. Department of Energy under contract DE-ACO4-76-DP00789. 
AN EVALUATION OF DIGITAL RECORDING EQUIPMENT AND NUMERICAL CORRECTION TECHNIQUES IN IMPULSE MEASUREMENT

R. Malewski, Hydro Quebec Research Institute, VARENNES, Quebec, Canada, JOL 2P0
T.R. McComb and M.M.C. Collins, National Research Council of Canada, Division of Electrical Engineering, OTTAWA, Ontario, Canada, K1A OR8

The application of analog-to-digital converters to the measurement of short-duration impulses is discussed. The desired accuracy of the measurement is used to derive the performance requirements of the analogto-digital converter. The practical application of this analysis is illustrated by a brief summary of an inter-laboratory comparison of two recorders using both full and chopped lightning impulses with crest values approximately $100 \mathrm{kV}$. One recorder was a micro-processor controlled digital waveform recorder mounted in a special shielding enclosure developed at the Hydro Quebec Research Institute (IREQ); the other was a National Research Council of Canada (NRCC) oscilloscope whose performance had previously been demonstrated in work at NRCC on high voltage impulse measuring standards. The relative accuracy of the two recorders was measured and their ability to resolve glitches on a smooth impulse was investigated. In order to investigate the ability of the IREQ digitizer to correct for errors caused by the response time, comparative measurements were made using two very different impulse voltage dividers connected in parallel. The faster divider was connected to the NRCC oscilloscope and the slower one to the IREQ digitizer. The digital processing capability of the IREQ system was used to calculate an approximation to the original impulse and this reconstructed impulse was compared with the measurement on the faster system. In addition Fourier Analysis was used to reconstruct the impulse and the two methods of reconstruction were compared.

Key Words: impulse measurements; analog-to-digital converters; numerical correction; error caused by response time.

\section{Introduction}

Over the past decade considerable work has been done on the adaptation of digital recorders to high voltage environments. A microprocessor controlled waveform recorder, suitable for use in high voltage laboratories, has been developed at IREQ [1]. While this instru- 
ment has been carefully evaluated there are presently no standards governing the use and performance of digital waveform recorders. As a contribution to the development of such a standard, it was decided to make comparative measurements using the IREQ digital recorder and an impulse oscilloscope developed at NRCC [2]. This oscilloscope's performance had previously been demonstrated in work at NRCC on high voltage measurement standards $[3,4]$. The relative accuracy of the two sets of recording equipment was measured for full and chopped lightning impulses.

Since digital recorders operate by sampling data rather than by continuously recording, doubt has been expressed to to whether fast glitches (such as might be caused by a partial breakdown in a transformer under test) will be recorded. The relative ability of the two sets of recording equipment to resolve glitches on a smooth impulse was investigated using the partial breakdown of an insulator string.

A major advantage of digital recorders is that the record of the impulse is available as a digital file and can be easily processed by computer. It is theoretically possible to reconstruct the input to an impuise measuring system provided that both the output and the step response of the system are known. In practice some information is destroyed and for a sufficiently poor impulse measuring system reconstruction is impossible. However, it is possible that there may be impulse measuring systems which are not sufficiently accurate for a particular type of impulse but whose responses could be corrected by the application of Duhamel's Theorem. Work on evaluating this possibility has been started.

\section{The Application of Digital Recorders to Impulse Measurements}

The use of analog-to-digital converters ( $A D C$ ) in impulse measurements introduces errors because the signal is sampled at discrete points and these errors can be compounded by the limited resolution of the converter. Quantization errors, aperture time and aliasing contribute to the overall error.

Quantization errors of $\pm \frac{1}{2}$ least significant bit are caused by approximating the analog signal by a series of discrete values each of which remains constant for at least one sampling period. This quantization error introduces high frequency noise as the ADC shifts levels abruptly. Care should be taken to ensure that this noise is not accidentally increased during numerical processing of the recorded digital signal.

The time during which the signal is acquired (aperture time) should be short compared with the sampling period since practical ADC cannot sample sufficiently quickly to make use of their full resolution when sampling fast signals.

If the frequency spectrum of the input impulse contains components of significant amplitude at frequencies close to half the sampling rate 
then aliasing may occur. "The frequency spectrum of the input pulse, the sampling rate and the accuracy of the ADC determine whether the error caused by aliasing is significant.

When the rate of change of the input signal is sufficiently low then the signal level can lie half-way between two adjacent digital levels for several or many sample intervals. When this occurs the ADC may change from one level to the next and back again arbitrarily generating an-artificial glitch. An example of this effect is shown in Figure 1. Such glitches can be removed by software filtering but this reduces the response of the system to real glitches [1].

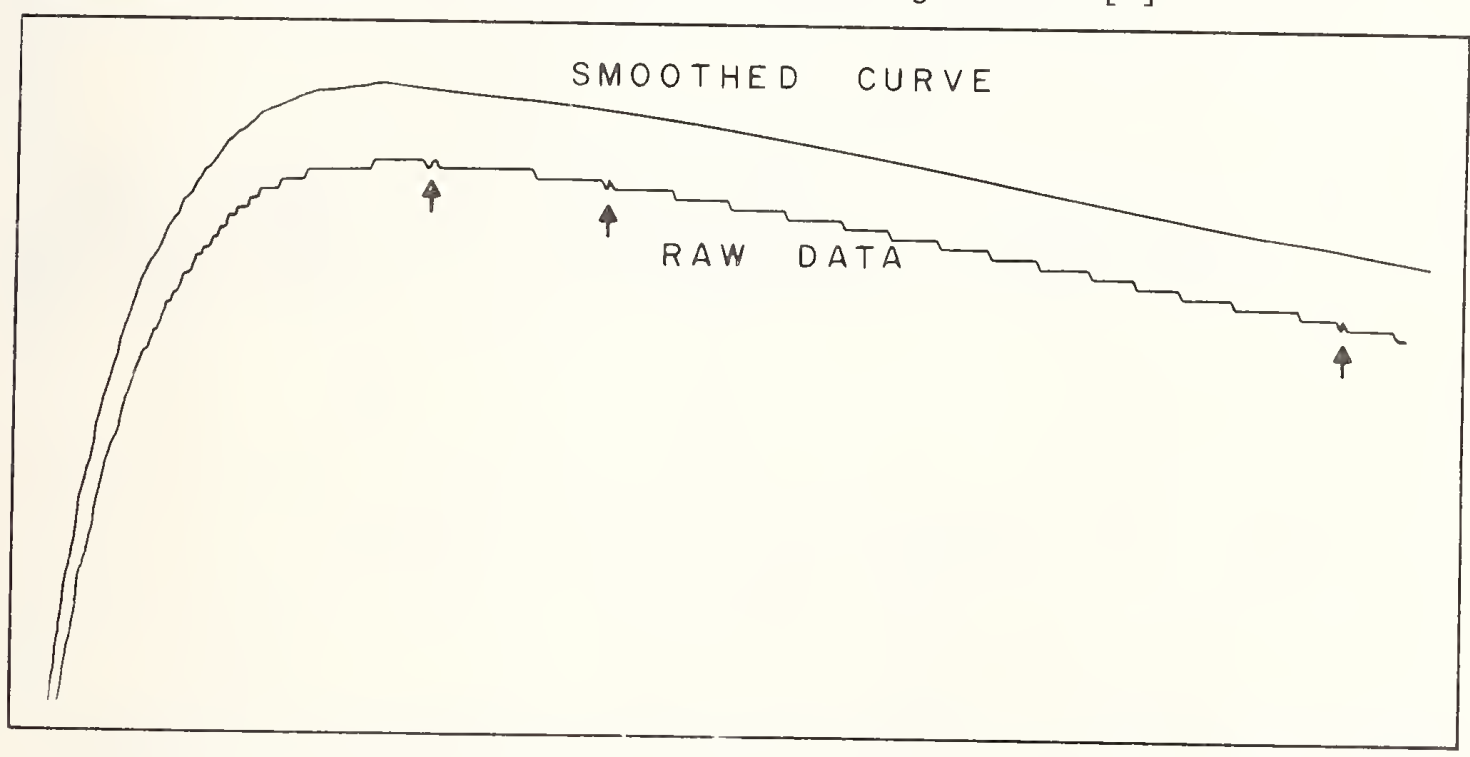

FIGURE 1. FALSE TRANSITION ERRORS ( $\uparrow$ )

When the rate of change of the input signal is sufficiently high most $A D C$ will be subject to skip code errors $[5,6]$ since the least significant bit (LSB) is unable to change quickly enough. For example when an 8 bit $A D C$ is recording a $10 \mathrm{MHz}$ sine wave, whose amplitude is half the full scale deflection, the LSB has to change at approximately $1 \mathrm{GHz}$.

There is presently no generally accepted method of specifying the high frequency performance of $A D C$. One method in use considers the response of the ADC in terms of the sampling error involved in measuring the rate of change of a high frequency sine wave at its zero crossing. This method emphasises the importance of the aperture uncertainty. A more recently developed method is to compare the measured rms error of the digitized high frequency sine wave with the calculated rms noise for an ideal ADC. While these methods are useful for the comparison of various $A D C$ they are not directly applicable to impulse measurements.

Elementary considerations require that if the crest value of an impulse is to be measured to within $x \%$ then at least one sample point must fall within the time interval for which the impulse is expected to 
be within $x \%$ of its crest value. Similarly for a 30 to $90 \%$ risetime (or 10 to $90 \%$ ) to be measured accurately sample points should occur sufficiently close to times of occurrence of these values for interpolation errors to be negligible. The application of these requirements to the measurement of a standard lightning impulse (front time $1.2 \mu \mathrm{s}$, time to half value $50 \mathrm{\mu s}$ ) is illustrated in Table 1 in terms of the sampling rate needed to fully utilize the resolution of the converter for measurement of crest voltages and the rate needed to measure the front time (30-90\% amplitude) within 10\%. When there are oscillations on the impulse a much higher number of samples will be needed to define the oscillations. When the shape of the pulse is known or when numerical correction is used the required number of sample points may be smaller.

\section{TABLE 1 REQUIRED SAMPLING RATES}

$\begin{array}{cc}\text { No. of } & \text { Resolution } \\ \text { Bits } & \text { of Amplitude } \\ & \text { Measurement } \\ & \% \text { Full Scale }\end{array}$

8

10

12
0.4
0.1

0.025
Required Sample Rate For Crest Measurements $(\mathrm{MHz})$

$$
\begin{gathered}
3^{\frac{1}{2}} \\
7^{2}
\end{gathered}
$$

Required Sample Rate For Front Time Measurements $(\mathrm{MHz})$

14
14
14

\section{Inter-Laboratory Comparison of Recorders}

\subsection{Equipment}

The IREQ recorder [1] consisted of two input channels, a fast ADC and memory, and a graphic computer terminal, a 11 housed in an electromagnetic shield and supplied with AC power through RF filters and an isolating transformer. Each input channel consisted of a 1 M $\Omega$ input impedance attenuator followed by a fast buffer circuit to match the attenuator output to the $50 \Omega$ input impedance of the ADC. The ADC had a resolution of 8 bits, a full scale deflection (fsd) of $10 \mathrm{~V}$ on its least sensitive range and a maximum sampling frequency of $10 \mathrm{~ns}$ per sample. Single channel input at the maximum sampling frequency was used throughout this work and each waveform was initially stored in the 2 kilobyte memory. These records were then copied to the intelligent terminal where they were scaled and displayed with appropriate parameters. The amplitude resolution of the instrument was 1 in 256 or approximately $0.5 \%$ fsd which resulted in a maximum possible reading error in amplitude measurements of $0.8 \%$ fsd. The corresponding figures for the time measurements were 1 in 2048 or $0.05 \%$ and $0.1 \%$.

The NRCC oscilloscope was a Southern Instrument transient recorder circa 1960 which had been extensively modified at NRCC to improve its shielding and linearity [7]. The bandwidth was DC to $300 \mathrm{MHz}(-3 \mathrm{~dB})$ and the fsd was $1000 \mathrm{~V}$ for signals input directly to the deflection plates. Each impulse was photographed and the record was read on an 
optical comparator with a resolution of $2.54 \mu \mathrm{m}(0.0001$ inches) corresponding to a resolution of at least $0.2 \%$ for the deflections recorded in this work. The relative accuracy of this type of oscilloscope has been measured by comparing records from two oscilloscopes connected in parallel to one divider: the difference in readings of crest voltage was $0.44 \pm 0.5 \%$ (with a $95 \%$ confidence interva] [8]).

Full and chopped lightning impulses were generated using the circuit shown in Figure 2. The three dividers used in this work were screened resistor dividers which were rated for full lightning impulses with crest values of $900 \mathrm{kV}, 350 \mathrm{kV}$ and $100 \mathrm{kV}$; details of the dividers used are listed in Table 2. Experimental response times of the impulse measuring systems were determined using a low voltage dc power supply and a mercury-wetted relay $[3,4]$; this relay was approximately the same height as the chopping gap and so no additional vertical lead was required. An example of an experimental unit step response for a three component system is shown in Figure 3 . The response times of the three component systems were measured using resistors from $100 \Omega$ to $900 \Omega$ and the response times of two component systems were determined by extrapolating the zero resistance. (Table 3 ).

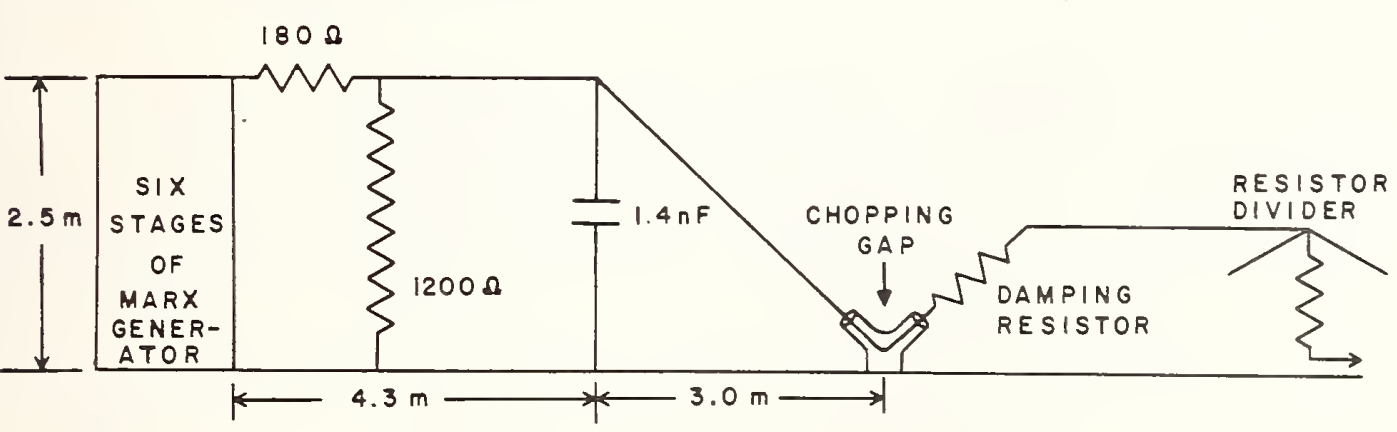

FIG. TWO - CIRCUIT FOR LIGHTNING IMPULSES. DETAILS OF THE IMPULSE MEASURING SYSTEMS AND CHOPPING GAPS ARE GIVEN IN THE TEXT.

TABLE 2 PHYSICAL PROPERTIES OF THE RESISTOR DIVIDERS USED

Rated Withstand

Voltage $(k V)$ *

900

350

100
Insulation

air

air

compressed

nitrogen
Resistance

$(k \Omega)$

30

10

10
Height

(m)

2.7

1.0

0.13

* for full lightning impulses 


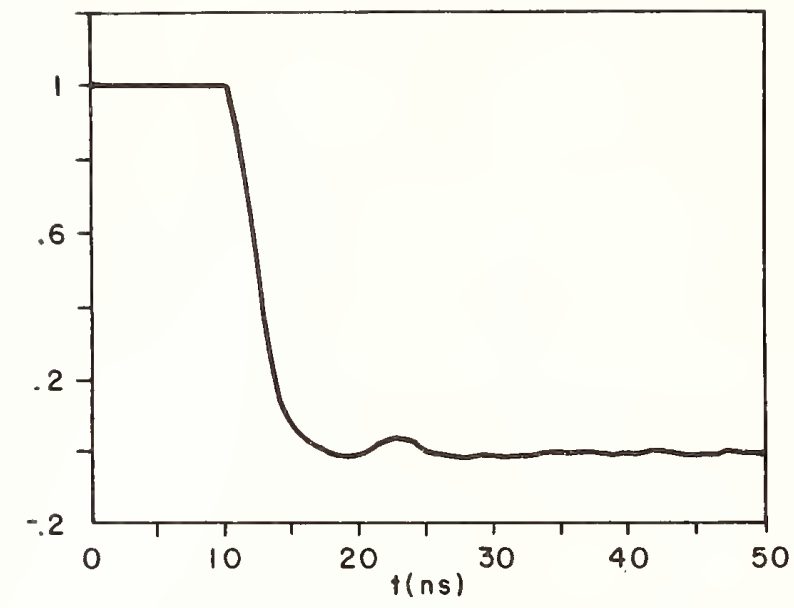

\footnotetext{
FIG. THREE - UNIT STEP RESPONSE OF THE PRESSURIZED DIVIDER IN A THREE COMPONENT SYSTEM; DAMPING RESISTOR

434 OHM,RESPONSE TIME 2.2 NS
}

\section{TABLE 3 SYSTEM RESPONSE TIMES}

Divider

$$
\text { Damping }
$$
Resistor

$$
\text { (ohm) }
$$

$900 \mathrm{kV}$

$900 \mathrm{kV}$

$350 \mathrm{kV}$

$350 \mathrm{kV}$

$100 \mathrm{kV}$

$100 \mathrm{kV}$

$$
877
$$

0

434

0

434

0
Response Time ( $T_{n}$ with 500

MHz Digitizer)

$$
\text { (ns) }
$$

$105^{\star}$
55
$13^{\star}$
2.0
$2.2^{\star}$
-

Calculated Response Times With NRCC Oscilloscope IREQ Digitizer

$$
\text { (ns) }
$$

105

55

13

2.2

2.4

$-4.7 *$ (ns)

112

68

42

40

40

40

* Measured Values

\subsection{Relative Accuracy of Recorders}

The two recorders were connected in parallel so that impulses could be applied to both recorders simultaneously. The NRCC oscilloscope was connected through $30 \mathrm{~m}$ of RGI7 coaxial cable to the base of the high voltage arm of a resistor divider. The low voltage arm was mounted at the NRCC oscilloscope. The IREQ digitizer, whose input impedance was $1 \mathrm{M} \Omega$ in parallel with approximately $50 \mathrm{pF}$, was connected to the input of the NRCC oscilloscope through $2 \mathrm{~m}$ of RG8 cable. Full and chopped lightning impulses were applied to the impulse measuring system.

The results of the comparative measurements are given in Table 4 for full lightning impulses and in Table 5 for chopped lightning impulses. 
TABLE 4 MEASURED VALUES OF THE CREST VOLTAGES OF

FULL LIGHTNING IMPULSES

(Same Input to Both Recorders)

$\begin{array}{cccc}\begin{array}{c}\text { Record } \\ \text { Number }\end{array} & \begin{array}{c}\text { NRCC Value } \\ (\mathrm{kV})\end{array} & \begin{array}{c}\text { IREQ Value } \\ (\mathrm{kV})\end{array} & \begin{array}{c}\Delta^{*} \\ \%\end{array} \\ 5 & 50.6 & 50.9 & +0.6 \\ 5 \mathrm{~A} & 50.8 & 50.9 & +0.2 \\ 8 & 50.8 & 51.5 & +1.4 \\ 9 & 65.4 & 66.0 & +0.9 \\ 10 & 66.6 & 66.0 & -0.9 \\ 11 & 66.7 & 66.6 & -0.2 \\ 403 & 101.9 & 102.7 & +0.2 \\ 557 & 100.4 & 102.1 & +1.7 \\ & & \text { Average } & 0.5 \\ & \text { Standard Deviation } & 1.0 \\ \text { * } & \text { Maximum Possible Reading Error } & 0.6 \\ & & \end{array}$

TABLE 5 MEASURED VALUES OF THE CREST VOLTAGE OF CHOPPED LIGHTNING IMPULSES

(same Input to Both Recorders)

Record

Number

$\begin{array}{rr}22 & 49.4 \\ 23 & 48.7 \\ 24 & 48.3 \\ 25 & 49.2 \\ 64 & 88.0 \\ 400 & 94.0 \\ 402 & 97.5 \\ 501 & 48.6 \\ 503 & 48.9\end{array}$

IREQ Value $(\mathrm{kV})$

$\Delta^{\star}$
$\%$

50.6

49.0

47.1

47.7

86.0

95.8

97.9

50.0

50.0
$+2.4$

$+0.6$

$-2.5$

$-3.0$

$-2.3$

$+1.9$

$+0.4$

$+2.8$

$+2.2$

Average $\triangle$ Standard Deviation

0.3

2.5

Maximum Possible Reading Error 1.6

$\star \Delta=100\left(\frac{\text { IREQ Value }- \text { NRCC Value }}{\text { NRCC Value }}\right)$

Illustrative impulses are shown in Figures 4 and 5 ; on the IREQ records the first $2 \mu \mathrm{s}$ and the first $20 \mu \mathrm{s}$ were plotted. The measurements of crest voltage of full impulses were within $-0.9,+1.7 \%$ and the average difference was $0.5 \pm 0.8 \%$ ( $95 \%$ confidence interval). These results indicate that there was no systematic difference in the results 


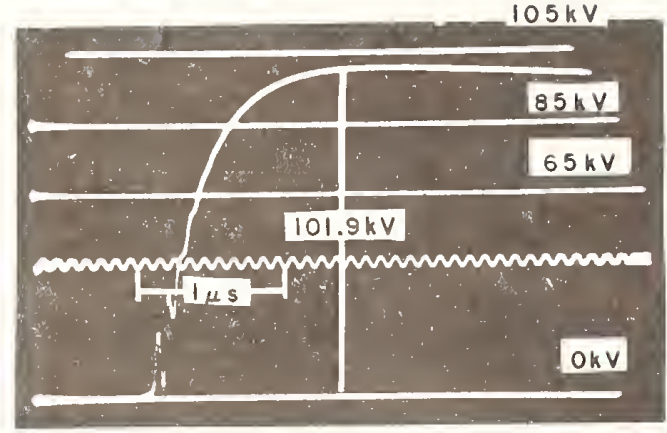

(a) NRC OSCILLOGRAM

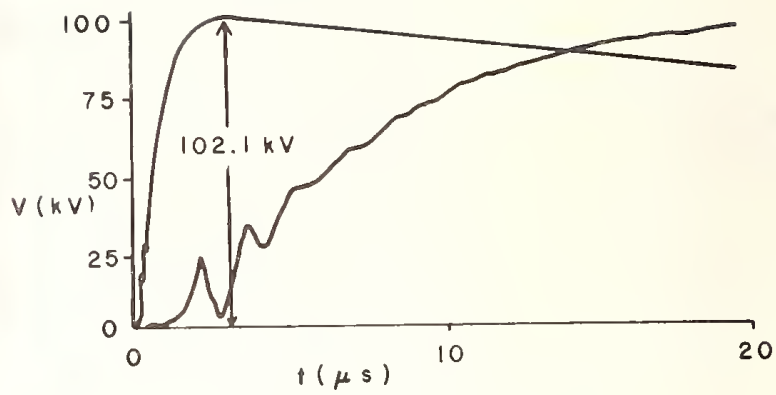

(b) IREQ RECORD

FIG.4-FULL LIGHTNING IMPULSE

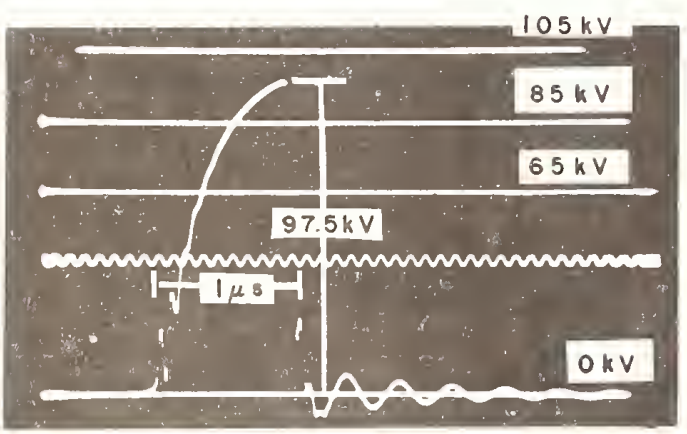

(a) NRC OSCILLOGRAM

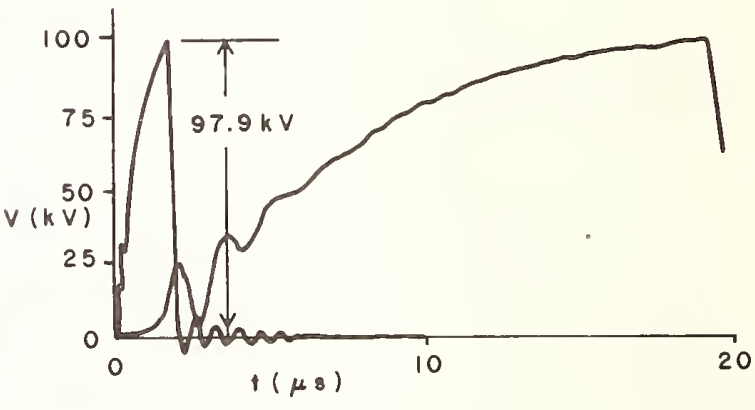

(b) IREQ RECORD

FIG.5-CHOPPED LIGHTNING IMPULSE

from the two recorders, and that the differences in individual readings were within the maximum possible reading error. The measurements of crest voltage of chopped impulses were within $\pm 3 \%$ and the average difference was $0.3 \pm 1.9 \%$ (95\% confidence interval).

\subsection{Glitch Tests}

In order to evaluate the response of the IREQ digital recorder to a fast glitch, the partial breakdown of a transformer under test was simulated by using an insulator string, with one of the insulators paralleled by a smal1 spark gap, as a test object. When a full lightning impulse of suitable amplitude is applied to this test object the spark gap breaks down shorting one insulator and generating a transient. A string of six insulators was used in this test and the spark gap was mounted on the bottom one. The glitches generated in this test were approximately $3 \%$ of the crest voltage. In all cases where a glitch was detected on the oscilloscope a similar glitch was observed on the IREQ recorder. An illustrative response is shown in Figure 6 . 


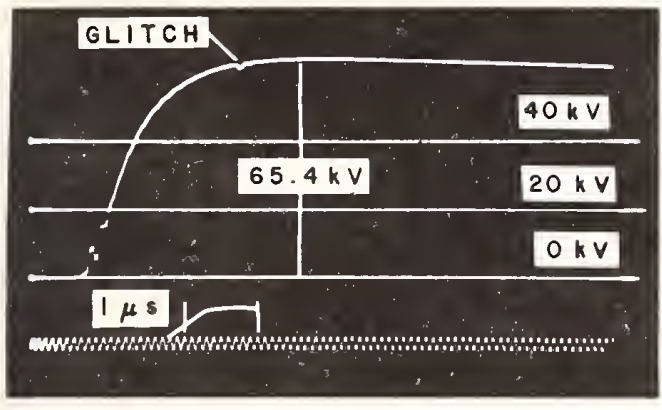

(a) NRC OSCILLOGRAM

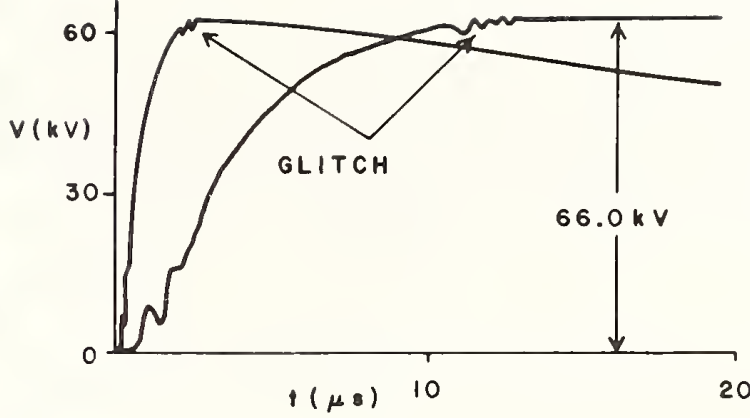

(b) IREQ RECORD

FIG.6 - GLITCH ON FULL LIGHTNING IMPULSE

\section{Numerical Correction Techniques}

\subsection{Reconstruction of Impulses}

Using Duhamel's Theorem (convolution theorem) the response, $r(t)$, of an impulse measuring system to an input, $f(t)$, can be written in terms of the step response of the system, $g(t)$, :

$$
r(t)=\int_{0}^{t} f(t) \frac{d}{d t}\{g(t-\tau)\} d \tau
$$

If $r(t)$ and $g(t)$ were known analytic functions then this integral equation could be solved to find $f(t)$; unfortunately $r(t)$ and $g(t)$ are measured with only limited accuracy ( $\pm 0.5 \%$ of crest value in this work). This inherent inaccuracy in any measuring system leads to a loss of information in the measuring system [9]. Whether this loss is significant depends on the form of $f(t)$ and $g(t)$. One of the aims of high voltage testing standards $[3,4]$ is to set limits on the unit step response of an impulse measuring system so that $r(t)$ will be approximately equal to $f(t)$ (within the accuracy prescribed by the standard); the limits on $g(t)$ depend on the form of $f(t)$ and are prescribed for full and chopped lightning impulses. The purpose of this work was to discover if an impulse measuring system which did not meet the requirements of the standards could be used with numerical correction of the measured output. Two correction techniques were used. One of these was the direct numerical solution of the integral equation given in eq. (1) and the other was the use of the Fast Fourier Transform (FFT) to transform eq. (1) to the frequency domain.

\subsection{Validation of Numerical Correction Techniques}

In order to investigate the accuracy of numerical corrections for errors caused by response time, comparative measurements were made using two very different impulse measuring systems connected in parallel. The faster system (using the $100 \mathrm{kV}$ divider) was connected to the NRCC oscilloscope and the slower system (using the $900 \mathrm{kV}$ divider) was con- 
nected to the IREQ digitizer; the planes of the two impulse measuring systems were at right angles to minimize coupling. Simultaneous measurements were made of impulses chopped on the front and numerical corrections were made to the impulse measured on the slower system. The reconstructed impulse was compared with that measured on the faster system.

\subsection{Numerical Solution of the Integral Equation}

Recently it has been shown that eq. (1) can be solved by expansion into a Taylor series and that $f(t)$ can be expressed as an infinite sum of the derivatives of $r(t)$ [10]. When expressed in terms of finite differences and 1 imited to $2 m+1$ terms this sum can be written:

$$
x_{n}=k_{n-m} y_{n-m}+\ldots k_{n} y_{n}+\ldots k_{n+m} y_{n+m}
$$

In practice it is found that $(2 m+1)$ can be quite smal1 (3 to 7) $[6,11]$. The coefficients, $k_{j}$, are found from the normalised step response and used to calculate $f(t)$ from $r(t)$. It has been found that the convergence of the method can be improved by using a software filter to remove high frequency noise from $r(t)$.

\subsection{Numerical Solution Using the Fast Fourier Transform}

The step response of the slow system ( $900 \mathrm{kV}$ divider) was recorded using a Tektronix WP 2200 system and the impulse response was derived by three point differentiation using Tektronix SPS BASIC Signal Processing Package. The program DCONVL from the Tektronix Software library was used to generate the input waveform by transforming the impulse measured on the slow system and the impulse response into the frequency domain and taking the quotient. This quotient is then transformed back to the time domain to give the reconstructed impulse. The division process wi1] emphasize the effect of high frequency noise and may result in an unrealistic estimate of the input pulse. To reduce the effects of high frequency noise on the reconstruction process a cosine taper is applied to both the measured pulse and the impulse response. This taper can be applied to the edges of the waveforms to smooth 10 to $50 \%$ of each edge. In practice the minimum taper which gives convergence is used.

\subsection{Estimations of Bounds on the Correction Term}

Two simple methods can be used to give approximate correction terms. Since the chopped impulses used had a lower rate of rise immediately before the chop than at the mid-point (i.e. they were concave downwards) aproximating the chopped impulse to a linearly rising pulse with a risetime equal to the virtual risetime [3,4] of the impulse gives an upper bound to the necessary correction. If the convolution of the measured impulse and the impulse response is taken the size of the correction needed for a second pass through the measuring system can be calculated and this operation is easily performed since it reduces the effects of high frequency noise. This correction should always be less than or 
equal to the correction needed for the effects of the first pass through the system.

In practice the uncertainty of the measurement will appear in the calculated corrections. The quoted corrections, which are based on the measured values, are subject to $\pm 2.5 \%$ error from this source in addition to any errors generated in the calculation.

\subsection{Results of Numerical Corrections}

It has been found that both methods of numerical correction overestimate the correction needed. An illustrative result is given below:

Crest voltage measured on fast system

Crest voltage measured on slow system

Standard deviation of crest voltages

Measurement error caused by response time

Correction calculated from integral equation

Correction calculated from FFT

Estimated upper bound

Estimated lower bound

$\begin{array}{cc}156 & \mathrm{kV} \\ 147 & \mathrm{kV} \\ 2.5 & \% \\ 6 & \% \\ 13 & \% \\ 11 & \% \\ 13 & \% \\ 7 & \%\end{array}$

The impulse measured on the fast system is shown in Figure 7.

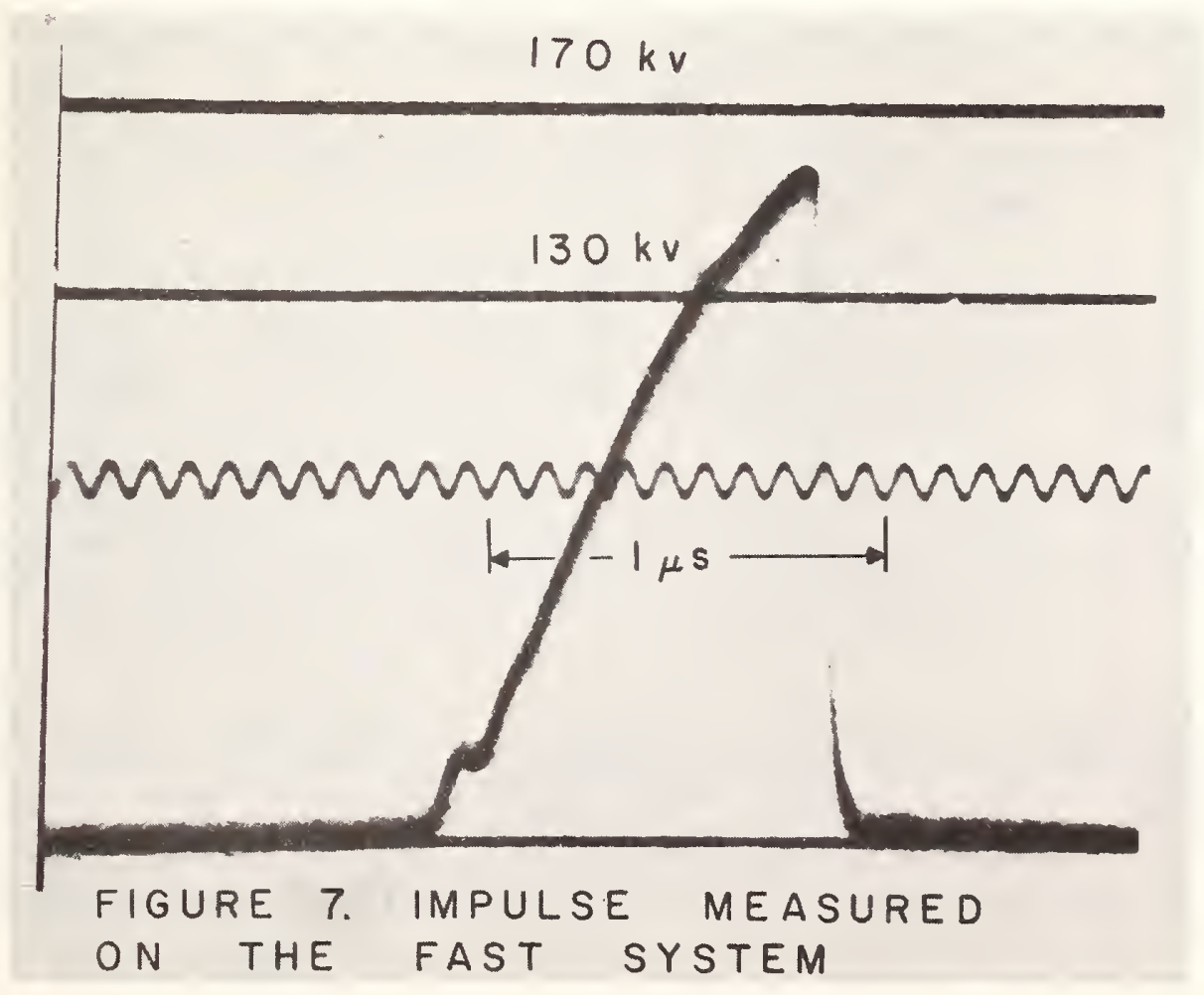


The estimated upper bound for the correction needed to measure this impulse is $0.2 \%$. The measured and reconstructed impulses from the IREQ system are given in Figure 8. In the FFT method, the reconstructed impulse was convolved with the impulse response of the system and the crest value of this calculated output was divided by the crest value of the measured output to give the norilalization factor. The measured output, reconstructed impulse and the calculated output (displaced in time) are shown in Figure 9.

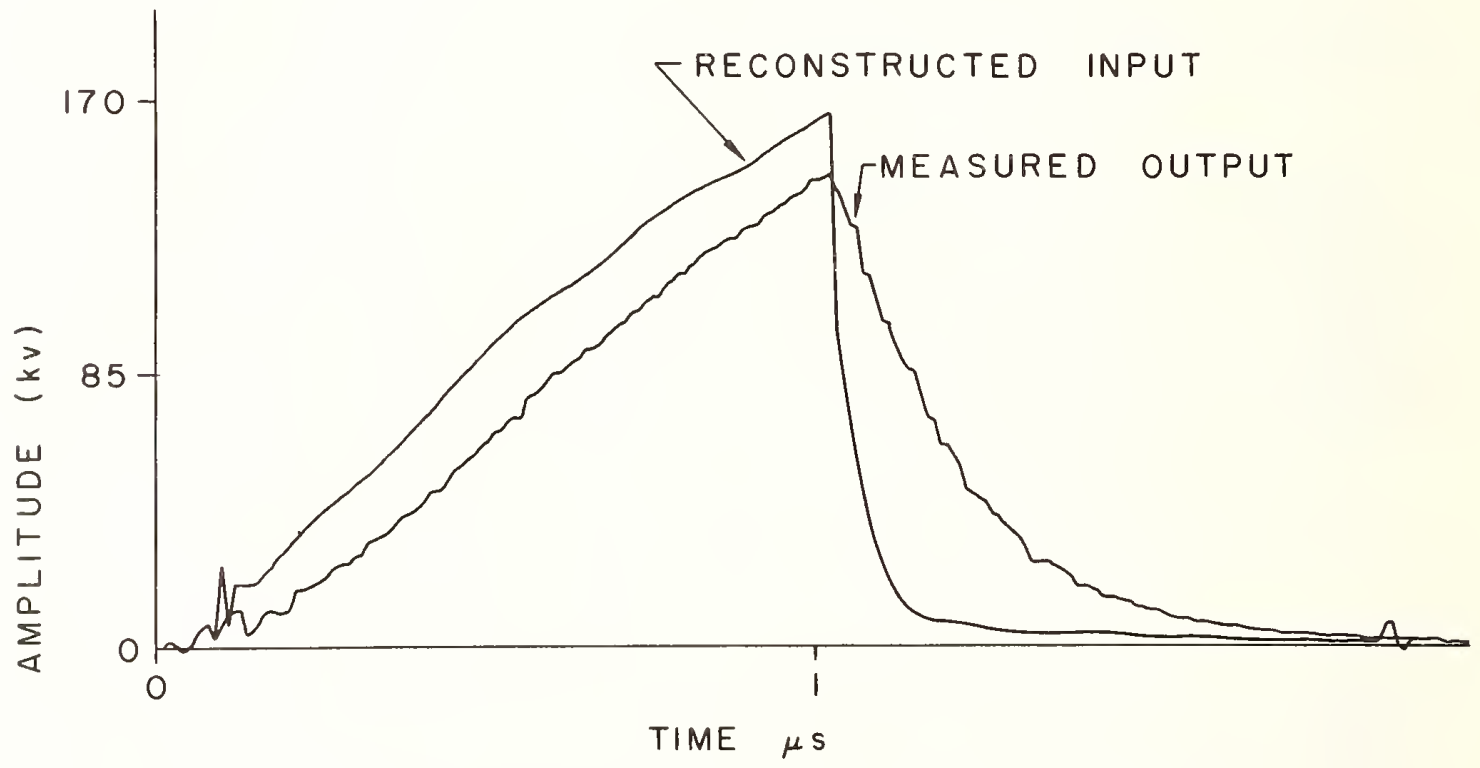

FIGURE 8. RECONSTRUCTED AND MEASURED IMPULSES FROM IREQ SYSTEM

Neither of the calculated input functions faithfully reproduce the measured input. In particular the FFT method shows oscillations not present on the input pulse. While these oscillations can be reduced by increasing the filter weight or the \% of taper applied no a priori criterion has been developed for this. The criterion used in this work was that the calculated output and the measured output pulses should agree.

The measured error caused by response time is equal to the estimated lower bound within the limits of the experimental error ( $\pm 2.5 \%)$. Since both calculated corrections are close to the calculated upper bound it is evident further improvements are needed to the correction techniques. 


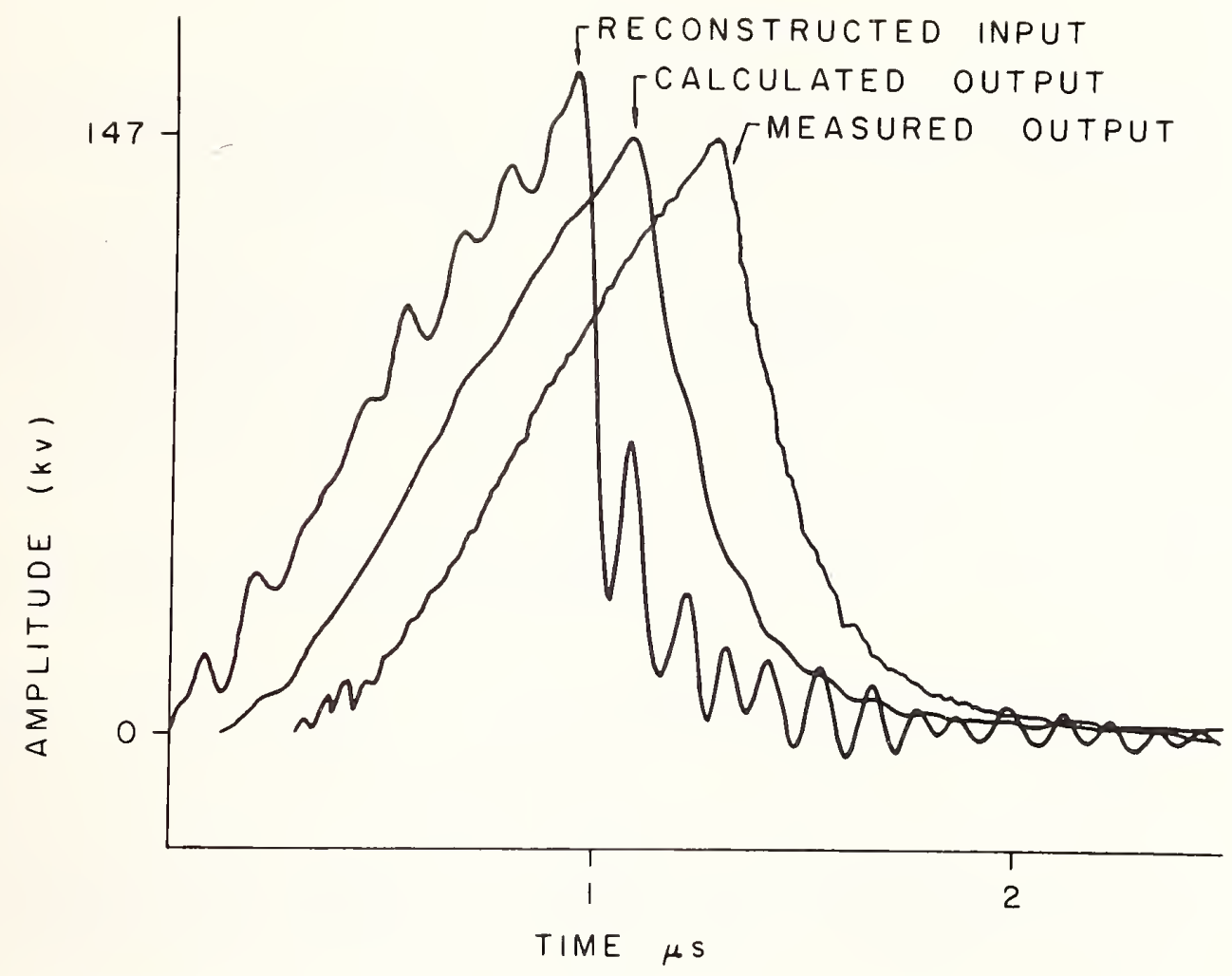

Figure 9. CALCULATED impulses using FFT

5. Conclusions and Future Work

Comparative measurements have shown that the IREQ digitizer was sufficiently accurate to meet the requirements of IEC Standard 60 - High Voltage Test Techniques for the measurement of the crest voltage of full lightning impulses and of the voltage at chopping of chopped lightning impulses.

Measurements of glitches have shown that the IREQ digitizer's sampling resolution was sufficient to resolve glitches caused by the partial breakdown of insulation; an amplitude resolution of more than 8 bits would allow smaller glitches to be measured.

Initial measurements to evaluate techniques of numerical correction of measured impulses have been made. These indicate that the techniques used for correction overestimate the correction needed. It is planned to continue this investigation and to develop both valid numerical correction techniques and criteria to determine when numerical corrections can be applied. 


\section{References}

[1] R. Malewski and A. Dechamplain, 'Digital Impulse Recorder for HighVoltage Laboratories'. IEEE Transactions on Power Apparatus and Systems, Vo1. PAS-99, pp.636-649, March/Apri1 1980.

[2] R. Malewski, T.R. McComb, and M.M.C. Collins, 'An Inter-Laboratory Comparison of Digital and Analog Recording Equipment Used in Impulse Measuring Systems'. Proceedings ofthe Canadian Communications and Power Conference, Montreal, Quebec. October 1980, pp. 60-63.

[3] International Electrotechnical Commission - Standard 60 - HighVoltage Test Techniques.

[4] IEEE Standard 4 - 1978 - IEEE Standard Techniques for High-Voltage Testing.

[5] W.S. Zaeng1, Discussion on Reference 1.

[6] R. Malewski, A. Dechamplain, Closure to Reference 1.

[7] F.C. Creed, 'An Improved Cathode-Ray Oscillograph for Impulse Recording'. Bulletin of the Radio and Electrical Engineering Division, National Research Council of Canada, Vol. 11, pp. 7-11, Oct/Dec. 1961.

[8] International Organization for Standardization 2602-1973 'Statistical Interpretation of Test Results - Estimation of the Mean-Confidence Interval'.

[9] Internationai Research Group Renardieres on Impulse Measuring Systems, 'Facing UHV Measuring Problems'. Electra No. 35, pp. 157-254.

[10] S.E. Kiersztyn, 'Numerical Correction of H.V. Impulse Deformed by the Measuring System'. IEEE Transactions on Power Apparatus and Systems, Vol. PAS-59, No. 5, Sept/0ct. 1980, pp. 1984-1995.

[11] R. Malewski, B. Langlois, Y. Gervais, 'Correction Des Erreurs de Mesure des Chocs Rapides à H.T. par Traitment Numerique'. Proceeding of the Canadian Communications and Power Conference, Montrea1, Quebec, October 1980, pp. 150-153. 


\title{
ANALYSIS OF A POWER SYSTEM TRANSIENT RECORDING LABORATORY
}

\author{
Randall E. Poliner \\ Terry J. Reed \\ Westinghouse Electric Corporation \\ Advanced Systems Technology \\ Pittsburgh, PA 15235
}

Under a project sponsored by the Electric Power Research Institute, two automated Transient Recording Laboratories have been developed to measure and record naturally occurring electrical transient phenomena on transmission lines. The laboratories are now installed and fully operational on the Florida Power and Light transmission network at $500 \mathrm{kV}$ and $138 \mathrm{kV}$ substations. This paper describes noise suppression techniques and methods utilized to insure data integrity in a high voltage substation environment.

\section{Introduction}

The growing concern on the part of equipment manufacturers and utilities as to the nature of transient surges on transmission systems has necessitated the design and development of laboratories capable of recording naturally occurring phenomena under actual operating conditions.[1] To this point, most of the existing knowledge concerning switching surges or fault conditions has been derived from staged tests or analytical studies. To supplement staged test data, the Electric Power Research Institute contracted to produce an instrumentation system to measure and record anomalous power system events with laboratory accuracy and integrity.

\section{Measurement Transducers}

In order to obtain a complete description of each anomalous occurrence relative to the instrumented transmission line, measurements are made of five parameters on each of the three phases (Figure 1)[3]. These include

1) transmission line voltage

2) transmission line voltage as seen by the relay

3) transmission line current

4) transmission line current as seen by the relay

5) substation bus voltage 
The transducers to measure these quantities are up to 300 meters removed from the laboratory itself (Figure 2).

\section{1 Transmission Line and Substation Bus Voltage Transducers}

The line and bus voltage transducers are high accuracy, high bandwidth coupling capacitors. These CCPD's were modified to eliminate the carrier frequency components and transformers, and install a 400 volt tap and capacitive divider network in order to condition the voltage level for input to solid state electronics. The CCPD transducers have been shown in a high voltage laboratory environment to have a bandwidth greater than $1.3 \mathrm{MHz}$ while maintaining an accuracy of better than $7 \%[3]$.

\subsection{Relay Voltage Transducers}

The voltage seen by the relay is monitored in the relay circuit at the control house relay panel. Thus, prior to the laboratory sensor, the signal has passed through the utility relay CCPD and been transmitted to the control house at baseband over nine conductor, Number 10 AWG control cable. At the $138 \mathrm{kV}$ site, the control cable is unshielded, 229 meters in length, and the largest portion of the run is above ground in a cable tray. At the $500 \mathrm{kV}$ site, this control cable is shielded, 305 meters in length, and run in a cable trough under the substation ground grid.

\subsection{Relay Current Transducers}

As with the relay voltage monitoring system, the line current as seen by the relays is monitored in the relay circuit at the control house relay panel. The utility's current transformers are multiratio bushing type with ANSI C-800 ratings. At both sites, the line current is composed of the sum of currents flowing through two circuit breakers, each with an associated current transformer. The current flowing in the transmission line is then the output of the two current transformers on either side of the line termination connected in a differential mode.

The Taboratory transducer located in the control house relay panels monitors the utility current transformer secondary current. The sensor itself is a coaxial current shunt of approximately 0.01 ohms. The shunt has been tested to assure a flat frequency response to $170 \mathrm{kHz}$.

\subsection{Primary Line Current Transducers}

The line current transducer is a free standing device developed specifically for the program. The unique current transformer which serves as the transducer features a special core design, winding technique, and burden selection. The result is a transformer which exhibits a flat frequency response $( \pm 0.3 \mathrm{~dB}$ ) for $1 \mathrm{~Hz}$ to $100 \mathrm{kHz}$. Significant saturation effects for current levels below 169 kA were also 


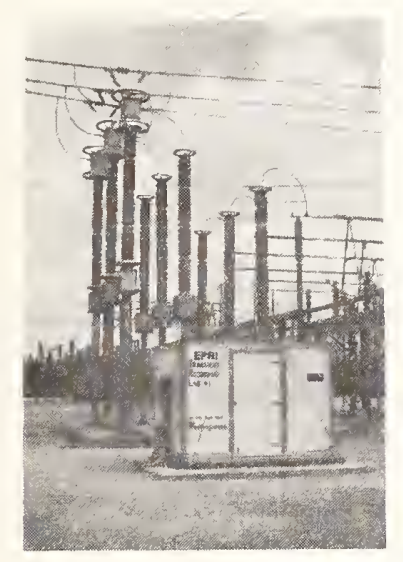

FIGURE 1 - Transient Monitoring Laboratory installed at Florida Power and Light's 138 kV Lauderdale Plant Substation

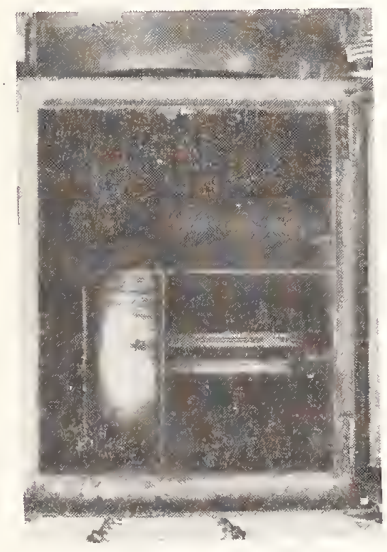

FIGURE 3 - Line Current Transducer housing at line potential containing current transformer, signal conditioning, and signal transmission electronics

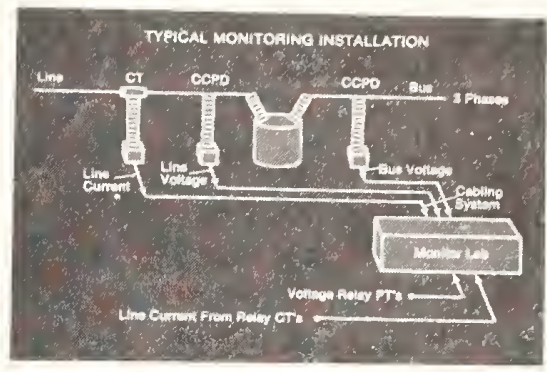

FIGURE 2 - Diagram of Measurement Transducer Locations on each of the Three Phases

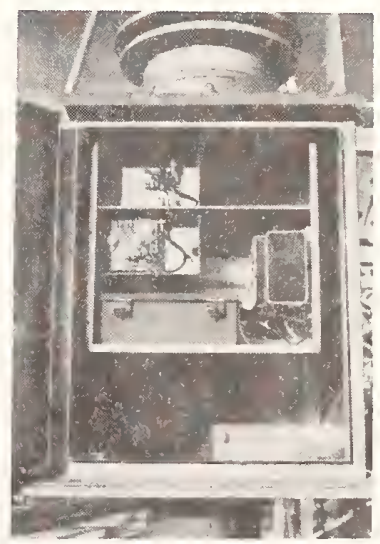

FIGURE 4 - CCPD base housing containing capacitor network, signal conditioning, signal transmission electronics, and power supply 
el iminated except for cases of successive, high magnitude fault currents or transformer inrush currents.

The output signal of the current transformer is cabled to the conditioning and signal transmission electronics, physically adjacent to the transformer. The transformer, signal conditioning, and data transmission electronics all float at line potential within a shielded enclosure (Figure 3). The data transmission electronics are powered by means of ultrasonic rod tapped at each end with piezo-electric transducers and driven by a power supply providing a square wave voltage at approximately $20 \mathrm{kHz}$. In this way, the current monitoring system is not dependent on, or affected by, the measured signal; thus, the system is available to measure energization and de-energization transients. The transformer and associated electronics are supported by a nitrogen filled porcelain column which also contains the ultrasonic rod and a fiber optic cable for data transmission.

\section{Signal Transmission}

The outputs of all primary transducers are conditioned to levels compatible with solid state electronics, frequency modulated, and converted to light signals for transmission over single glass fiber optical cables to the recording laboratories. At the laboratories, the light signals are reconverted to electrical signals, demodulated, and cabled to the digital transient recorder (Figure 4).

\section{1 Modulation and Demodulation}

After conditioning, all signals are frequency modulated prior to conversion to a light signal for transmission. The modulation and demodulation are accomplished via state-of-the-art techniques in order to assure data integrity, low losses, and high inherent signal to noise ratio. Both circuits have been minimized to reduce crosssectional area and minimize lead lengths. Electromagnetic and radio interference are further reduced through appropriate shielding enclosures, conductive gasketing, and uniform copper ground mat.

\subsection{Electrical/Light Conversion}

Conversion of the frequency modulated electrical signal to an infrared light signal is accomplished by a light emitting diode, LED. The LED operates with maximum spectral output at a wavelength of 825 nanometers with full radiated power of nominally 750 microwatts per square centimeter at 150 milliamps. Coupling efficiency into the fiber is approximately $50 \%$ due to the connector, numerical aperature of the fiber, and uniformity of the radiant emittence as the distance from the optical axis increases. Conversion from the light signal back to the frequency modulated electrical signal is accomplished by a silicon PIN photodiode. The photodiode features a typical dark current of 1 nanoamp and rise time of 1 nanosecond. The entire photodiode module is matched to the transmitting LED and features a feedback network to 
compensate for LED power drift, reduce noise susceptability, and enhance data integrity. Both the LED module and PIN photodiode modules have been miniturized into $1^{11} \times 1 / 2^{11} \times 1 / 2^{11}$ hermetically sealed cases. The result is minimized Radio Interference and Electromagnetic Interference. To eliminate capacitive loading and noise pickup, each module is physically enclosed in the same case with the modulation or demodulation circuitry, as appropriate.

\subsection{Optical Fiber}

The optical fiber used for data transmission is used for a variety of purposes. In the case of the primary line current transducer, the non-conducting fiber is used to carry the transmitted data signal from the electronics at line potential to the laboratory at ground potential. The optical fiber also provides for isolation from the various ground potential shifts between transducers and the laboratories that occur in the substation under transient conditions. The optical signals are also immune to the intense electromagnetic noise found within a utility substation on a continuous and transient basis.

Two different types of optical fiber construction were used in installing the laboratory. The first, ten mil fiber with hollow tube construction, was found to be unsuitable for the utility environment where great distances between equipment required enormous tensile strength for pulling purposes. Another fiber of extruded construction was found to have a higher actual tensile strength when terminated. The fiber used is a single fiber, $8 \mathrm{mil}$, step index with attenuation of $5 \mathrm{~dB}$ per kilometer at 900 nanometers. Terminations and splices are accomplished through the use of conical, four rod connectors in order to achieve high coupling ratios and low attenuation at splice points. This splice and termination technique was also found to be most suitable to field installation and repair.

\section{Direct Noise Suppression Techniques}

The utility substation environment is an extremely hostile EMI and RI environment, particularly during transient conditions when ground potential shifts between transducers and recording apparatus may easily reach five kilovolts. [2] Induced lightning and other impulse type surges affect not only the ground plane, but also have a real and measurable affect on the power system. Isolation, particularly of low level electronic signals, can become an extremeiy complex process. Isolation for the Transient Monitoring Laboratories is primarily directed into five areas. These are the power supplies, data transmission channel, grounding techniques, shielding techniques, and laboratory enclosure. The data transmission channel and rationale behind use of the optical fiber was discussed above. 


\subsection{Power Supplies}

A11 power for the laboratory and associated electronics is derived from a battery rack located in the respective site's control house and a $60 \mathrm{~Hz}, 120$ VAC inverter located in the laboratory. A11 power is dispersed from the laboratory to the transducer electronics via shielded cable. Power to each transducer is isolated through instrument quality isolation transformers and dedicated DC power supplies. Power to recording and other laboratory apparatus is also fed through isolation transformers to reduce power supply induced noise from any one component to the other subsystems or from outside the laboratory system. Power to the line current transducer electronics is, in the final analysis, supplied through the use of an ultrasonic rod as discussed in Section 2.4.

\subsection{Grounding Techniques}

It was verified through the course of the project that the inductance of ground leads could lead to high potential shifts during transient conditions which would, in turn, severely affect the noise performance of the system. For this reason, ground leads were kept as short as possible and as continuous as possible. Heavier apparatus, including cabinetry, has been grounded through $20 \mathrm{mil}$ by two inch solid copper ribbon at several points. Circuit card ground planes are accomplished by copper sheets where appropriate. Each enclosure is also grounded with copper ribbon to bleed off extraneous radio frequency signals. The improvement in observable noise due to the strap grounding system was between 6 and $9 \mathrm{~dB}$ depending on the location and frequency of interest. The improvement in signal-to-noise ratio among the data channels was between 2 and $4 \mathrm{~dB}$ of coupled noise.

\subsection{Shielding Techniques}

Basically, each circuit has been shielded from the outside environment as well as each other. Enclosures designed to eliminate radio interference are used to house each critical circuit. All enclosure access covers are sealed, as required, with conductive gaskets. The largest gain observed due to the shielding employed was seen in reduced data channel cross-talk, especially at the demodulation site where several data channels are adjacent to each other.

The laboratory itself is designed as an EMI free enclosure, featuring double walls and copper gasketing around the door. The total shielding system is capable of reducing the noise floor to $-85 \mathrm{~dB}$ so that interference is insignificant compared to the specified $72 \mathrm{~dB}$ signal-to-noise ratio.

\section{Data Transmission Performance}

An exhaustive series of tests were run with known inputs and known power levels of white noise to precisely determine the data integrity 


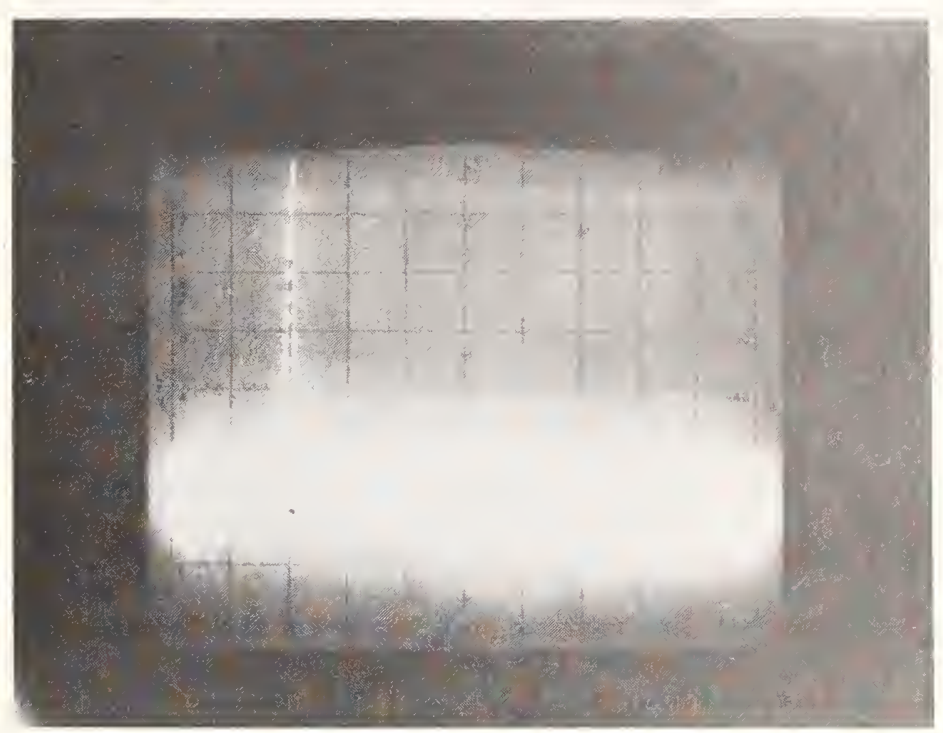

FIGURE 5 - Spectrum Analysis of Transmitted Baseband Signal; Full Scale Input with White Noise at $-50 d B$

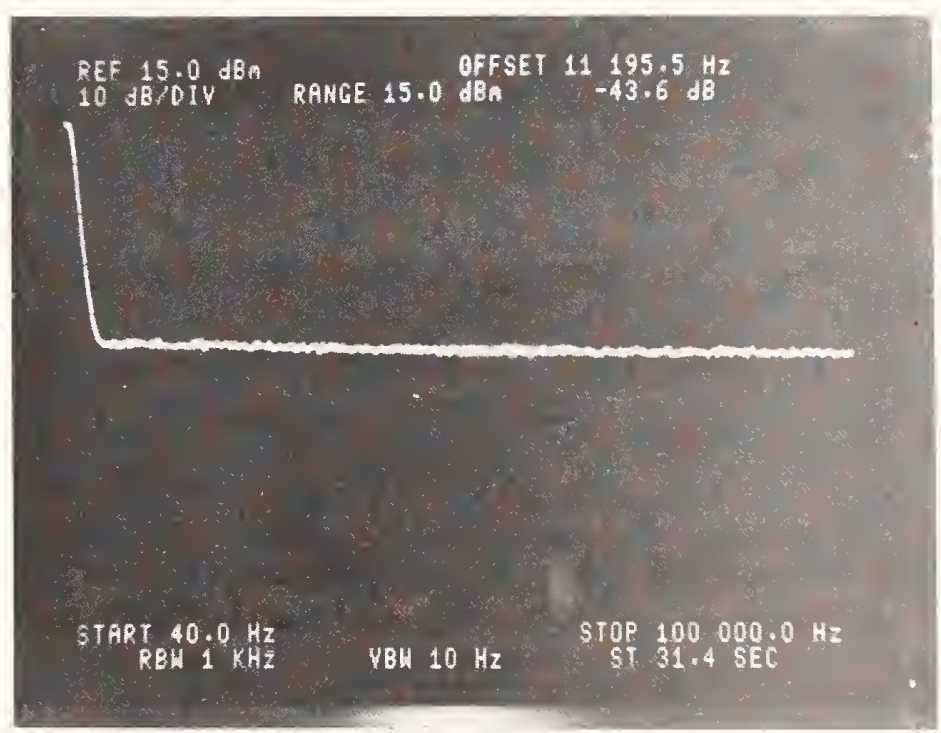

FIGURE 6 - Spectrum Analysis of Received Baseband Signal Using Input of Figure 5 


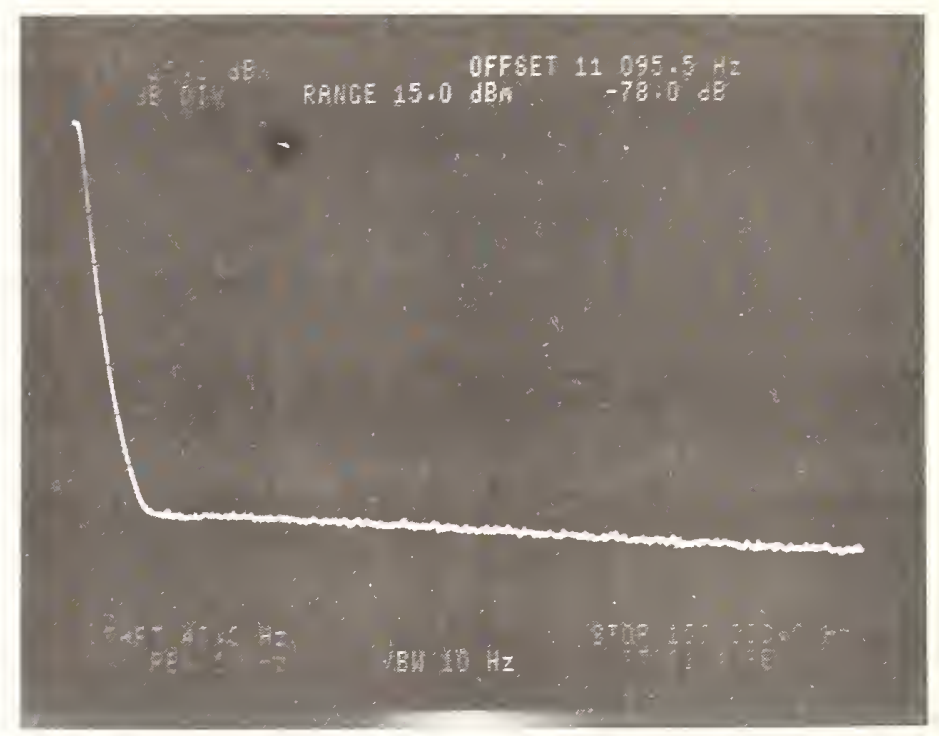

FIGURE 7 - Spectrum Analysis of transmitted baseband signal under operating conditions

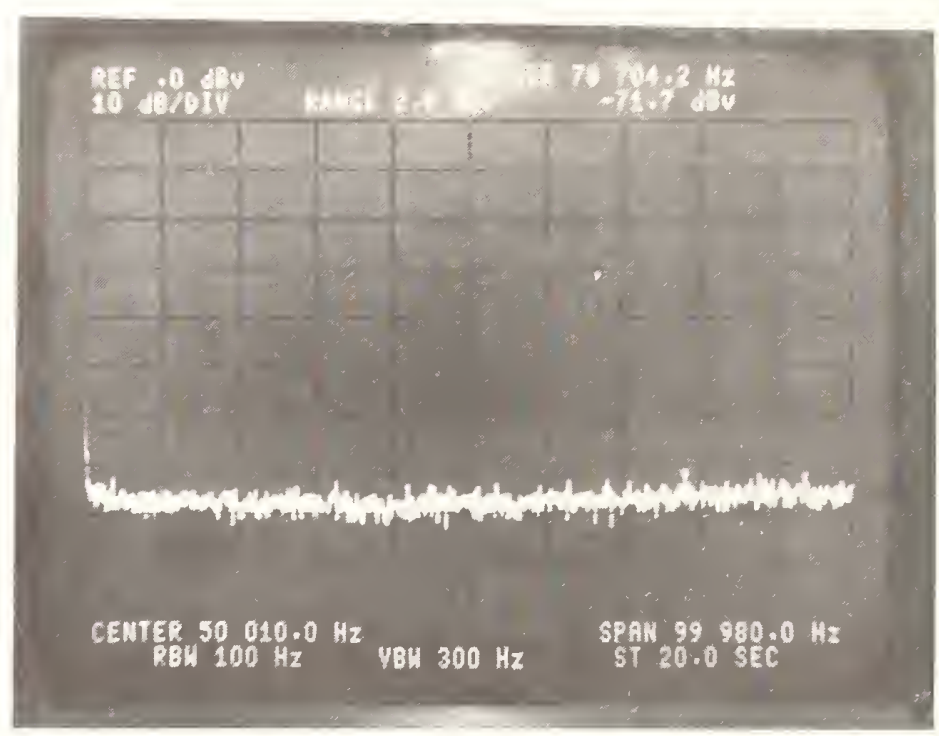

FIGURE 8 - Spectrum Analysis of received baseband signal under operating conditions 
loss in transmission. An example is shown in Figures 5 and 6 for a sample of tests run on a relay voltage channel. The results show approximately a $7 \mathrm{~dB}$ loss in the signal-to-noise ratio due to transmission which is independent of the noise floor in the baseband.

Figures 7 and 8 demonstrate the actual performance of the laboratory communication system under operating conditions. Figure 7 depicts the signal-to-noise ratio and dynamic range as observed at the input to the FM transmitter, just after the transducer. Figure 8 shows the demodulated analog signal in the laboratory. As can be seen, the $7 \mathrm{~dB}$ transmission loss observed above has been verified under operational conditions.

\section{Conclusions}

The resulting field laboratory system is fully capable of monitoring and recording power system transient phenomena with unparalleled data integrity compared to more conventional utility field testing techniques $[1,2]$. The use of fiber optic based data transmission, highly improved primary transducers, and elaborate grounding and shielding techniques has yielded a measurement system with a dynamic range greater than $66 \mathrm{~dB}$ and data of greater than $72 \mathrm{~dB}$ signal-to-noise ratio. The importance of this high bandwidth data to the utility industry becomes readily apparent in developing concepts and designs for:

a) Compact lines, where phase-to-phase switching surges are critical.

b) New, nonlinear arresters, where the frequency and magnitude of overvoltages become important.

c) Ultra-high-speed relays and fault current limiter operating devices, where the signal-to-noise ratio is not well known.

d) Digital or low-energy protective relays, where signal-tonoise ratios are critical; performance evaluation of different digital protective relaying algorithms is also of interest.

e) Current and voltage transformers, which are critical to the pèrformance of high-speed protective relays.

f) Transient analysis programs, where improved fidelity of computational results would be desirable. This could include improved fault models of importance primarily to ultra-high-speed relays and fault current limiter control devices.

The laboratory system is applicable to the direct measurement of extra-high voltage and current phenomena in a variety of areas. While 
the present application will most assuredly advance the understanding of utility power systems under transient conditions, future usage of the laboratories and methodology can be easily transferred to other disciplines where a knowledge of pulse power performance would be of benefit.

\section{Acknowledgements}

The authors wish to express their gratitude for the technical support provided by S. L. Nilsson of EPRI as well as all members of the EPRI Advisory Committee on the Analysis of Transients on Transmission Systems. W. H. Smith of Florida Power \& Light should be recognized for his work in the performance and direction of the design, implementation and installation functions of the project during his association with Westinghouse. The authors also wish to acknowledge the valuable efforts of Messrs. J. S. Barnick, J. E. Lokay, T. E. Mousseau, and $V$. F. Wilreker of the Westinghouse Electric Corporation in analyzing and improving the performance of the laboratories.

\section{References}

[1] IEEE Spectrum, "Technology '80: Power/Energy Developments," January, 1980, page 61.

[2] Shankle, D.F. and Smith, H.M., "Switching Surge Instrumentation and Measurement Techniques for EHV Systems," (AIEE Winter General Meeting, 1963, No. CP 63-75).

[3] Smith, W.H., Reed, T.J., et.al., "New Field Laboratories for the Measurement of Electrical Transients," (CIGRE Session of 1980, No. 34-06). 


\title{
APPLICATION PROBLEMS USING INSTRUMENTATION AMPLIFIERS
} IN THE PULSE PONER ENVIRONMENT

\author{
E. Earl Cunningham \\ Ectron Corporation \\ San Diego, California 92111
}

\section{Abstract}

Difficult and unusual problems arise when low level signals must be accurately measured in the pulse power environment. Usually involved with signals immediately following the pulse, this application demands unique capabilities of the signal processing instrumentation. This paper discusses necessary amplifier and signal conditioner characteristics in addition to shielding and cabling recommendations. Test results involving pulse common mode signals are presented to illustrate the effects of improper shielding and cabling.

\section{The Signal Problem}

Much has been written about the application of instrumentation amplifiers and signal conditioners in conventional data acquisition systems $[1,2,3]$. However, signal conditions present near pulse power systems are very unusual and little material is available to aid the instrumentation eng ineer. [5]

\subsection{Desired Signals}

Generally, when instrumentation amplifiers are used in this application, they are amplifying analog data immediately following the pulse. The desired signals are frequently generated by more or less conventional transducers and, except for the problems associated with the pulse, can be conditioned and amplified using conventional techniques. Therefore, we are dealing with desired signal levels from microvolts up to several hundred millivolts with a frequency range of interest of from $D C$ to $100 \mathrm{kHz}$ or so, and an accuracy of the amplified signal from a few percent down to $0.1 \%$ or somewhat better. 


\subsection{Noise Signals}

Because of unavoidable coupling to the signal leads at the source and into the cabling, plus common mode effects, considerable pulse amplitude is usually present at the input to the amplifier. Depending on a wide variety of factors the amplitude of this pulse can vary from a few hundred up to many thousands of volts with a duration from a few nanoseconds up to several microseconds. At the amplifier this signal can be either common mode (in common with the two signal leads), normal mode (between the two leads) or, more likely, a combination of both.

Other noise signals likely to be present include:

Ring from the main pulse usually highly damped and much higher in frequency than the desired data signal. This signal is usually both CM and NM in nature.

A 1 ine frequency signal sometimes having high frequency components that is predominately CM at the terminals of the amplifier. Its amplitude can be millivolts to volts.

Pulse signals originating with switching devices ranging from SCRs to switching power supplies. These signals, likely to be both CM and NM, are especially troublesome if an A/D converter is used following the amplifier.

Proper care in shielding and cabling such signals between source and amplifier will minimize the effects of these noise signals. However, if signal leads are improperly handled signal and noise can be irretrievably mixed.

\section{Shielding Considerations}

Figure 1 shows an ideal situation where the source, the amplifier and the signal leads are all enclosed within a single continuous shield. This example illustrates a principle of shielding that if all elements of a circuit are totally contained within a single unipotential shield the existence of other electrostatic potentials external to the shieid cannot influence the circuit within.

\subsection{Faraday Cage}

A term used to describe this type of shield is Faraday cage or Faraday shield. In effect, such a shield transposes the circuit from its remote location to the signal source and ground point of the shield. A good shield is one that not only totally encompasses the circuit within but also remains at the same potential. This requirement demands that the primary guard 
shield not be used to bypass drain currents and, of course, requires that this shield be tied to only one ground point. If significant current should flow in the shield it would no longer be at signal potential and rejection of the common mode signal could suffer.

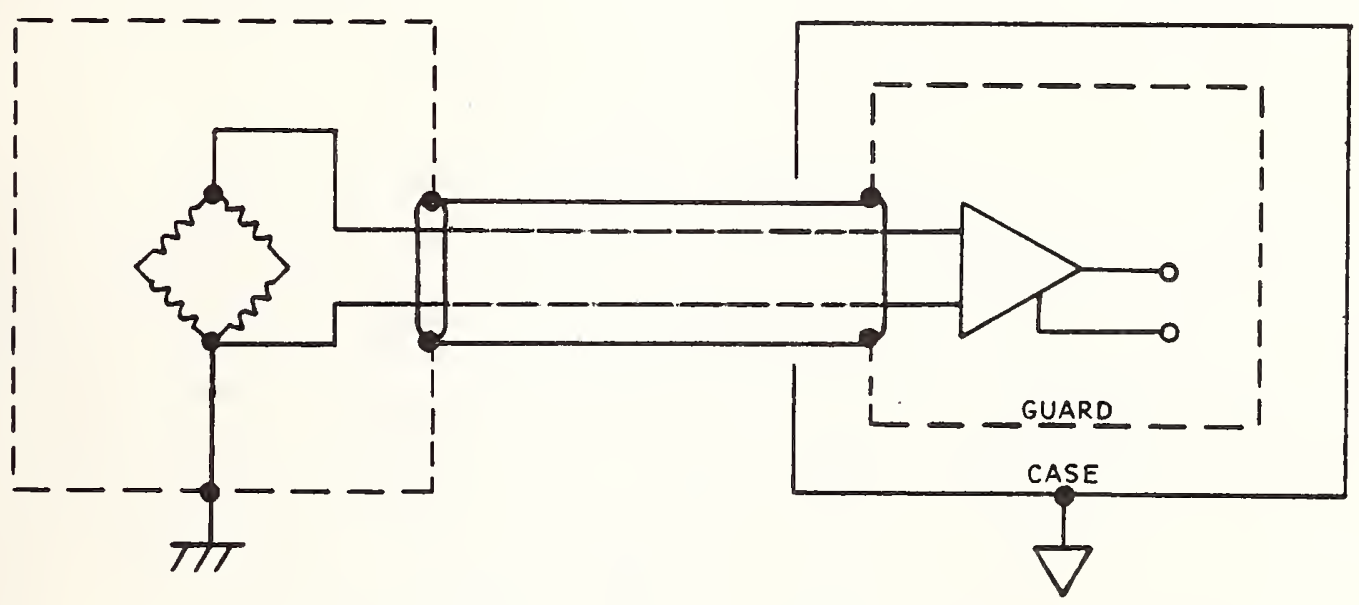

FIGURE 1 IDEAL SHIELD

Note that if the amplifier of figure 1 had any connections external to the shield the integrity of the shield could be impaired. Since an amplifier usually has such connections (to output instruments, to power line and to case) the amplifier itself must maintain appropriate shielding between input and these output connections. The degree to which the input must be isolated is determined by the characteristics of the common mode signal and the level and desired accuracy of the normal mode signal.

\subsection{Voltages}

DC Common mode voltages are usually easily handled in an input signal system. $A C$ CM voltages are more of a problem since shield capacitance to other ground potentials will cause shield currents to flow. However, impulse CM signals are the most difficult since even a small capacitance will cause large shield currrents to flow. Therefore, as will be demonstrated later in this discussion, shield impedance must be kept low both in the cabling and within the conditioner in order to maintain proper Faraday cage conditions. 


\section{Amplifier Characteristics}

Most characteristics of available data amplifiers are acceptable in the pulse power environment. These would include linearity, dc drift, gain accuracy, noise, etc. Specifications of the average amplifier that are not compatible with this application would be common mode rejection at high frequency, overload recovery both NM and CM, and NM and CM breakdown voltage rating.

\subsection{High Frequency CMR}

High frequency common mode rejection is not only required to minimize the effects of the pulse but also to reduce CM noise that follows the pulse. To achieve high CMR at high frequencies requires elaborate guard shielding of all input circuitry within the amplifier. The degree to which all input circuit elements must be guarded can be easily demonstrated by reference to figure 2. C represents the capacitance connecting one input to case or ground at the amplifier. If a CM voltage, Ecm, is present a current will flow through $R_{2}$, the interconnecting cable and $C_{1}$. Because of the voltage dfop across $R_{2}$, the signal from Ecm is no longer equal at the input terminals of the amplifier and, of course, this differential signal is amplified. A simple calculation will show that if Ecm has a frequency of $1 \mathrm{MHz}$ and $R_{2}$ is 100 ohms, $C_{1}$ must be less than 16 femtofarads

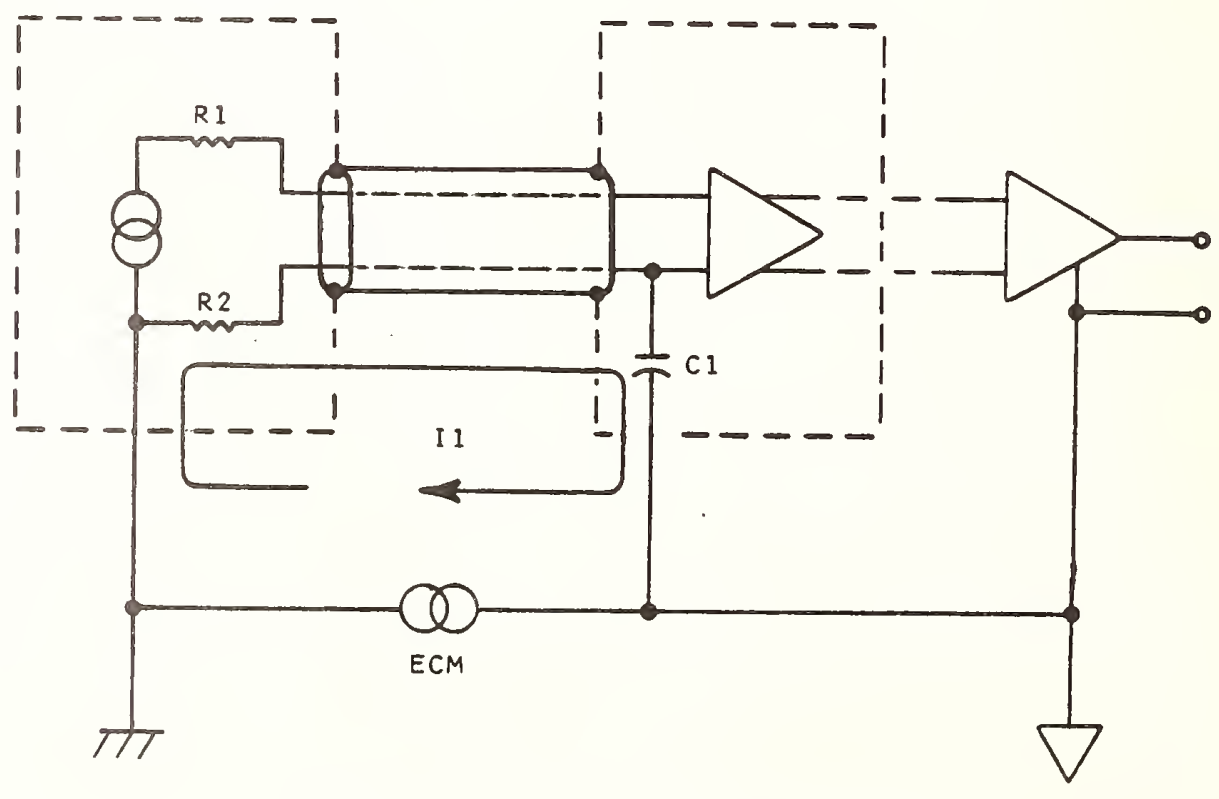

FIGURE 2 LEAKAGE CAPACITANCE 
$\left(10^{-15}\right.$ farads) to provide a CMR of $100 \mathrm{db}$. Obviously, $100 \mathrm{db}$ of rejection is not enough to prevent CM overload during the pulse considering the fact that high frequency components present will far exceed $1 \mathrm{MHz}$ and the amplitude will likely be in the thousands of volts. However, this amount of rejection will minimize the duration of CM overload plus it will provide good rejection of the smaller CM signals that follow the pulse. Although difficult, guard shielding to this degree is possible as illustrated by the Ectron Model 776. This unit's leakage capacity, including its excitation supply, is approximately $10 \mathrm{ff}$ and 1 MHz CMR with 100 ohm unbalance is typically $109 \mathrm{db}$.

\subsection{Maximum CM Voltage}

The maximum common mode voltage rating of an amplifier would normally involve a breakdown specification and, at a lesser voltage, an operating specification. Although it may not be sufficient for many applications in the pulse power field, practical constraints have limited the Ectron unit to \pm 3000 volts breakdown and \pm 1200 volts operating potentials.

\subsection{Maximum NM Voltage}

More difficult to achieve is a comparable normal mode overload characteristic. Since a versatile instrumentation amplifier must perform accurately with gains of 1000 or more an overload specification demands recovery down to the microvolt level in a few microseconds. The 776 specification requires that recovery from an NM overload of 1000 volts will be within $5 \%$ of final value in $10 \mu \mathrm{s}$ and within $0.1 \%$ in $30 \mu \mathrm{s}$. Considerable care must be exercised in selecting components exposed to a wide voltage range not only within the amplifier but also in the input signal system. To quickiy recover from a $1000 \mathrm{~V}$ potential and accurately amplify microvolt level signals, dielectric absorption and thermal emf's must be kept to an absolute minimum. These precautions would apply, for instance, to dividers or filters used ahead of the amplifier.

NM overload is al so a problem in an excitation supply when used in this environment. Protective circuitry must be incorporated in the conditioner's design along with an appropriate recovery capability.

\section{Cabling and Shielding Recommendations}

Because of the breakdown and recovery limitations of the amplifier and signal conditioner, it is essential that pulse and other noise signals be held to an absolute minimum if accurate 
data is to be obtained. Much can be accomplished in reduction of these noise signals by using proper cabling and shielding techniques.

Generally, most of the pulse noise induced into the signal leads will occur at the sensor and on the cabling leading to the sensor since the radiation will be most intense at this point. The sensor itself should have minimum cross sectional area and should be oriented for minimum exposure. Preferably, the sensor should be symmetrical such that the induced pulse will be CM rather than NM.

Similar methods should be used in the selection and routing of the wiring connecting to the sensor. The Los Alamos Scientific Laboratory has done considerable work in developing flat cable for this use, primarily for applications at the Nevada Test Site. When oriented properly, these cables expose a very small cross sectional area to the beam. Again, cable symmetry and routing will reduce NM pickup.

Shielding of the sensor and leads connecting to the sensor is extremely important in order to keep pulse pickup down to tolerable levels. Although frequently limited by constraints of the experiment, liberal use of a shield material effective over the spectrum involved is highly recommended.

Tables 1 and 2 give general cabling and shielding recommendations for signal wiring beyond the sensor area. These suggestions follow the rules of standard practice for instrumentation systems but with modifications to fit the pulse power environment.

Cable selection is of considerable importance in a typical system not only because of the effect on signal quality, but al so because of the significant cost involved when long cable runs and numerous channels are required. Too, this application often demands the highest quality cable.

Note that double shielding of each signal cable is recommended with even triple shields advantageous. Each shield reduces drain problems and offers additional grounding choices. Low shield inductance and resistance is important in being able to drive guard areas in the amplifier/conditioner at pulse frequencies. Foil type shields provide total shield coverage, however, they usually require a separate drain wire because of higher resistance. Use of twisted pair cables having good symmetry is important to minimze the effects of magnetic fields. This characteristic also contributes to making the capacitance of each lead to the shield equal which is necessary to prevent CM to NM conversion within the cable. 


\section{TABLE 1. Cabling Recommendations}

Use twisted pair double shielded cable.

Keep shield inductance and resistance to a minimum.

Capacitance between each conductor and shield should be equal [5].

Do not coil excess cable.

Minimize capacitance between cables and ground surfaces.

Do not use steel core cable.

Voltage rating of the cable must be compatible with the application.

Avoid areas of high magnetic fields when routing cable.

\section{TABLE 2. Shielding Recommendations}

Maintain total shield integrity from source to amplifier. Connect shield to zero signal point having low source impedance. "Ground" shield at only one point. Minimize shield current. Use drain wire or second shield. Each shield should contain only one signal system.

Bundle several cables carrying similar NM and CM signals and cover with overall low inductance sheath.

Use Faraday cage configuration if possible.

\section{CM Pulse Tests}

The following tests, using an Ectron Model $776 \mathrm{~B}$ Conditioner/Amplifier, wiil serve to illustrate the effects of less than ideal shielding and cabling conditions.

Figure 3.1 shows the basic test configuration. A bridge transducer was simulated using four $120 \Omega$ resistors. Two Trompeter Quadrax cables, each 25 meters long, interconnected the excitation supply and amplifier to the bridge. The excitation was set to 10 volts and each of the test configurations was tested at amplifier gains of 20 and 1000 . The pulse generator, simulating a CM signal, produced a 730 ns pulse (FWHM) having an amplitude of 62 volts with rise and fall times of under $1 \mathrm{~ns}$. No attempt was made to terminate the cable.

Figure 3.2 is an oscilloscope trace showing amplifier output with a sensitivity of 1 volt per division, sweep speed of 5 us per division and an amplifier gain of 1000 . These connections, following recommended grounding and shielding procedures, produced an output perturbation of only 0.5 volts settling to $1 \%$ in $4 \mu \mathrm{s}$. (Additional scope pictures were taken at higher sensitivities allowing $1 \%$ measurements to be accurately made.) Resuiting CMR is $102 \mathrm{db}$. Note that increasing the amplitude of the CM pulse to 1000 volts will increase the 
resulting output to approximately 8 volts, still well under overload. Furthermore, if pulse width is decreased from the 730 ns used here, a similar decrease in output amplitude will occur.
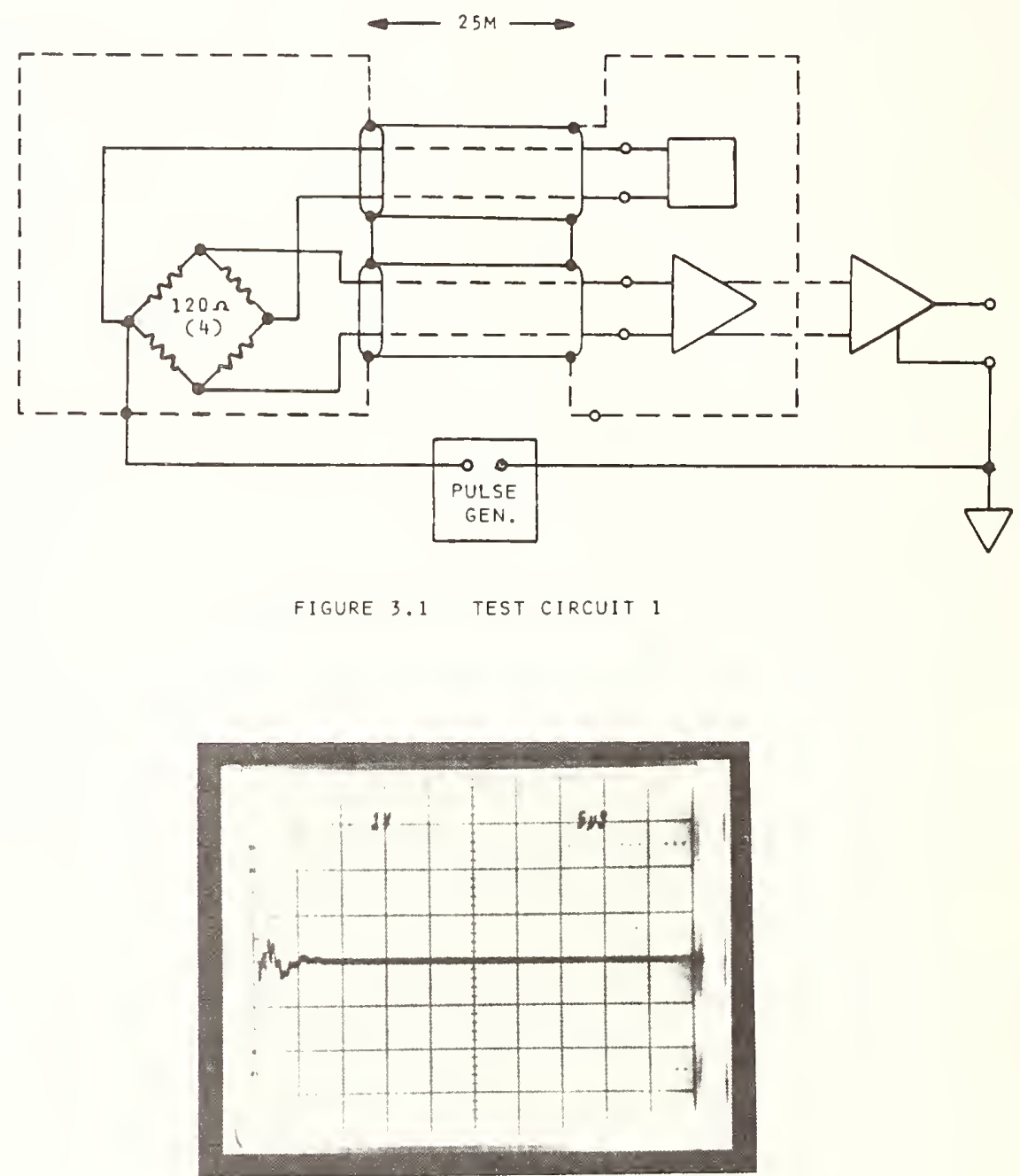

FIGURE 3.2

To illustrate the need to maintain a low impedance connection to the guard of the amplifier, a $10 \Omega$ low inductance resistor was added between cable shields and conditioner guard. This resistor was carefully mounted so that shielding was still complete. Figure 4.1 shows the circuit and figure 4.2 shows the resulting trace. Note that amplitude increased to over 2 volts and recovery to $1 \%$ increased to $8 \mu \mathrm{s}$. These increases are caused by the amplifier's guard shield potential lagging the CM potential of the signal leads causing current to flow in the 
signal leads. This current produces a NM signal which is amplified by the amplifier.
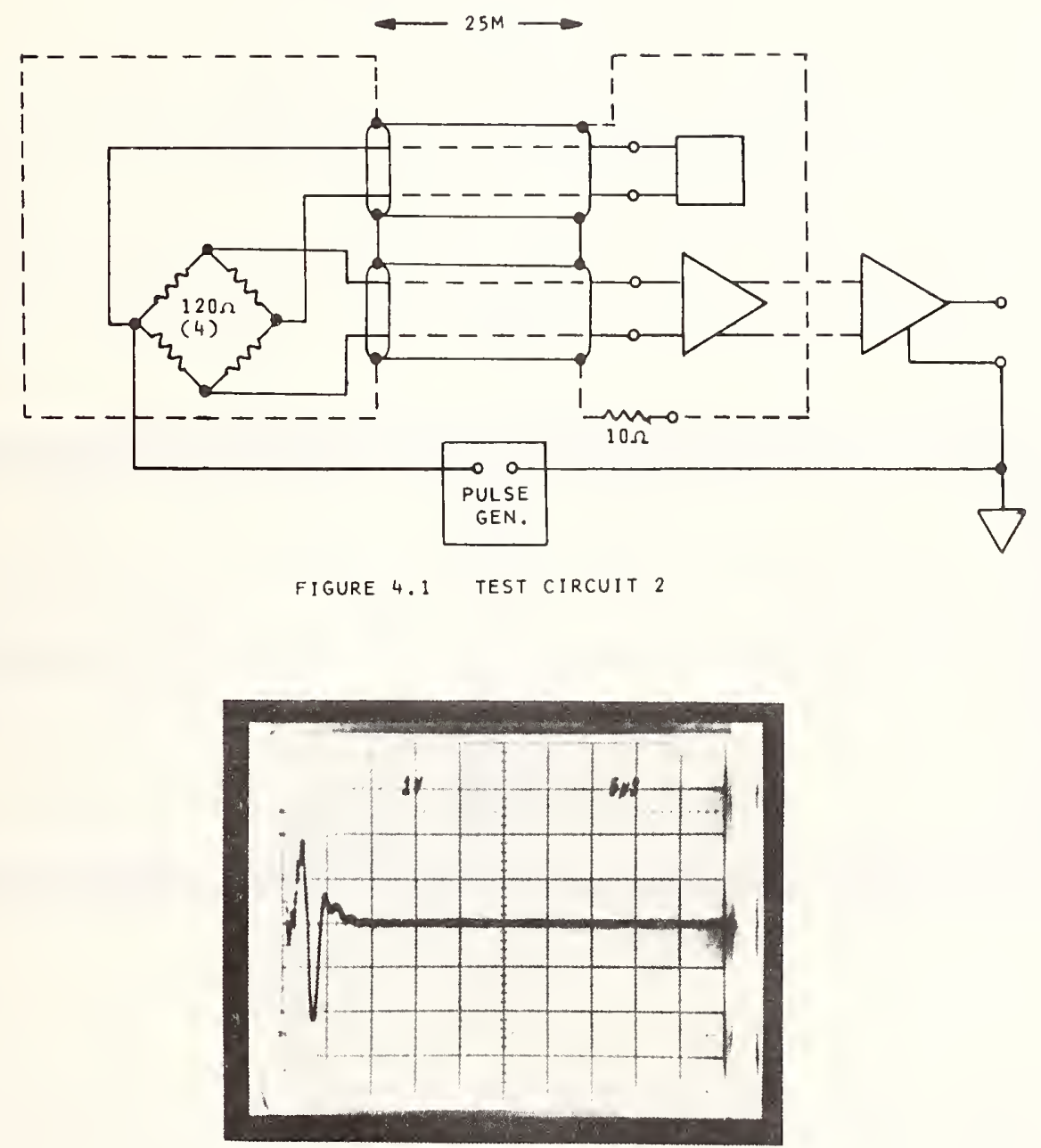

FIGURE 4.2

The need for total guard integrity is illustrated in figures 5.1, 5.2 and 5.3. Here, a 5 picofarad capacitor was connected between one input lead at the amplifier and output ground. This capacitance is similar to running signal leads through a connector whose shell is not connected to guard. The lower sensitivity trace of figure 5.3 shows a maximum amplitude excursion of 5 volts. One percent settling time is $8 \mu \mathrm{s}$. 

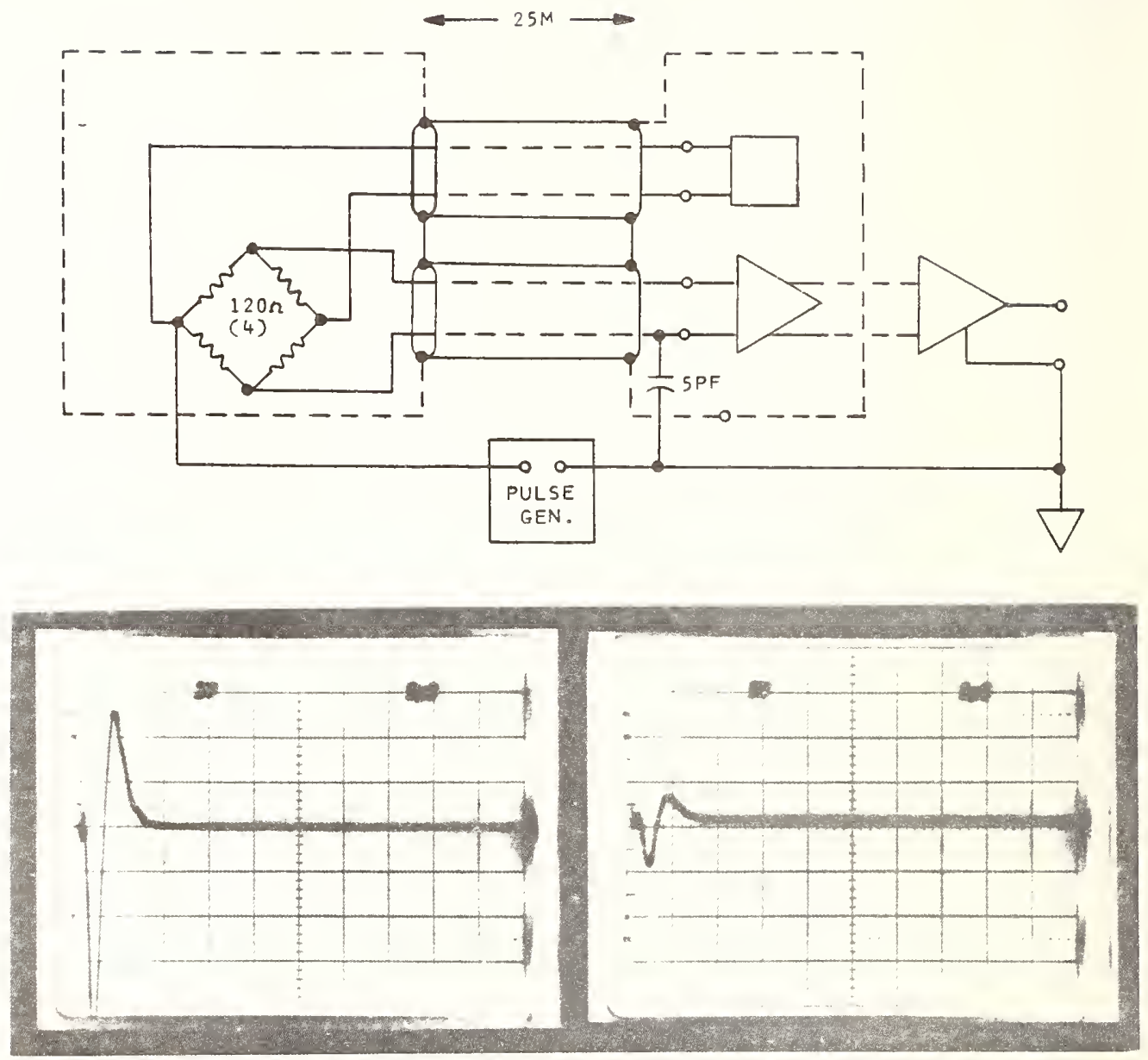

FIGURE 5.2

A fourth test was performed with the guard tied to output ground keeping all other connections as shown in the first test. This arrangment, of course, voids the protective guard shielding within the amplfier, and substantially reduces amplifier CMR. Because of the effects of saturation and considerable ringing, settling time to $1 \%$ increased to $22 \mu \mathrm{s}$. Indicated CMR decreased to $75 \mathrm{db}$ although this figure should be less since saturation $1 \mathrm{imited}$ the true output excursion. When this test was repeated at a gain of 20 , recovery to $1 \%$ improved to $13 \mu \mathrm{s}$ but CMR was only $46 \mathrm{db}$. Again, if pulse amplitude was increased recovery time would proportionally increase and, similarly, if the pulse was narrowed recovery would improve. 
These four tests, summarized in table 3 , illustrate the need to carefully consider the shielding and cabling of signal conditioning equipment when impulsive CM signals are involved. Although each of these tests involved only a single cabling deficiency to best illustrate the results, an actual test configuration can be a combination of these problems with usually an additive effect on recovery and CMR.

\section{TABLE 3. Test Summary}

\begin{tabular}{cccc} 
Test 1 & Test 2 & Test 3 & Test 4 \\
Shield & $10 \Omega$ Shield & & \\
to Guard & to Guard & $5 \mathrm{pf}$ Cap. & Guard/Ground \\
\hline
\end{tabular}

$\begin{array}{lllll}\text { Gain } & \times 1000 & \times 1000 & \times 1000 & \times 1000 \\ \text { Max. Overshoot } & .5 \mathrm{~V} & 2.2 \mathrm{~V} & .5 \mathrm{~V} & 11 \mathrm{~V} \text { (sat) } \\ \text { Time to } 1 \% & 4 \mu \mathrm{s} & 8 \mu \mathrm{s} & 8 \mu \mathrm{s} & 22 \mu \mathrm{s} \\ \text { CMR } & 102 & 89 & 82 & 75\end{array}$

\section{Faraday Cage System}

The Faraday cage concept has been applied to the use of instrumentation amplifiers in the pulse power environment. Although not easy to accomplish, this shielding approach offers significant advantages over more conventional cabling and shielding methods.

Figure 6 shows a typical data channel connected in this manner. The instrumentation is all contained in an isolated enclosure which is cabled to the signal source using proper shielding procedures. Thus, the source, the signal leads and all of the instrumentation are totally encompassed within a single shield system that is strapped together using very low impedance drain connections. As shown, the only connections to ground are the stray capacitances which would normally be located mostly at the source and at the instrument enclosure. Power must be coupled into the enclosure using an isolation transformer and the signal transferred out using suitable isolating techniques such as fiber optics or properly designed signal transformers. The improvement that the Faraday cage offers over a conventional system is in direct relation to the ratio of drain impedance to the impedance of the capacitance of the instrument enclosure. Therefore, it is important to keep the capacitance of this enclosure with respect to ground to an absolute minimum. The use of the Faraday cage system has proven successful in tests both at the Nevada Test Site and at Sandia on the REBA Marx generator. Where the situation permits, this cabling concept is highly recommended. 


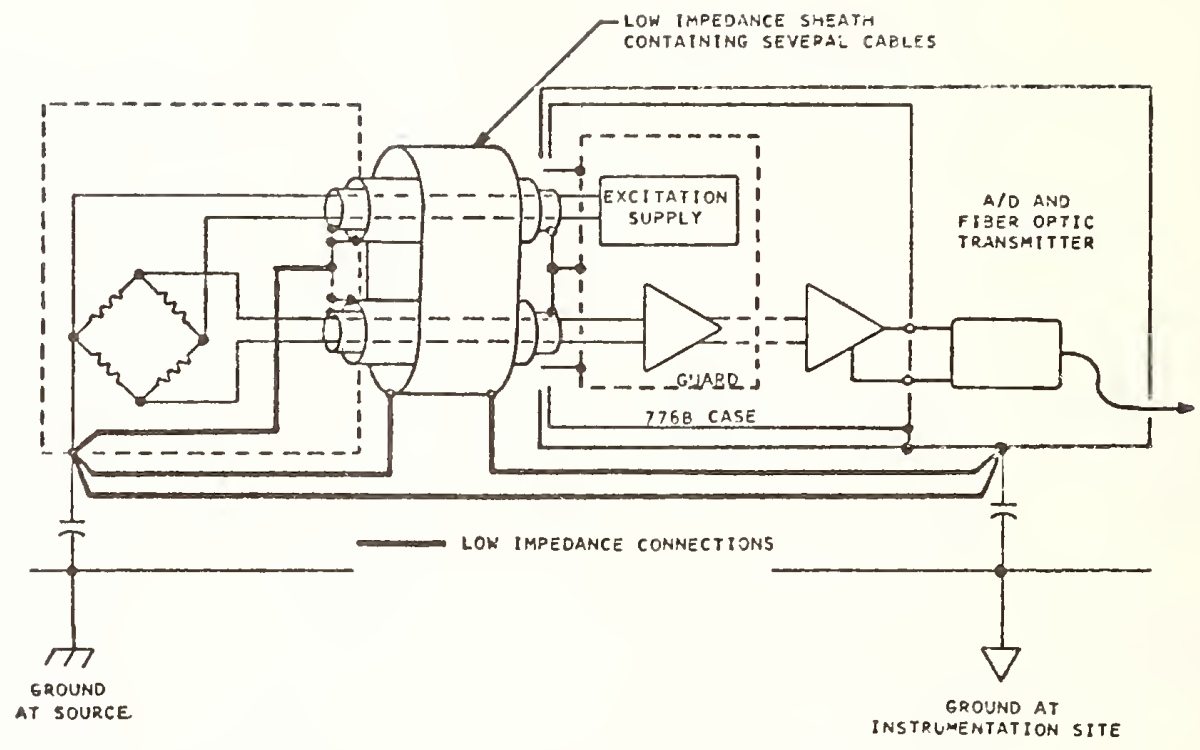

FIGURE 6. FARADAY CAGE SYSTEM

\section{Conclusions}

The amplification of low level signals in the pulse power environment requires special care in cabling and shielding, particularly at the source, to minimize pulse coupling onto the signal leads. The amplifier and signal conditioner used must have unique characteristics to perform well in this environment. Where conditions are particularly poor or where highest performance is required, the use of the Faraday cage is recommended. This approach encloses source, cabling and instrumentation within a single isolated shield system and uses a fiber optic link to couple out the amplified signal. 


\section{Acknowledgements}

R. S. Dingus of Los Alamos Scientific Laboratory contributed information on the use of flat cables and sensor and cable shielding. Raymond M. McNairy of Lawrence Livermore National Laboratory supplied data regarding operation of the Faraday cage system.

\section{References}

[1] Morrison, R., "Grounding and Shielding Techniques in Instrumentation, Wiley and Sons, New York

[2] Harper, C.A., "Handbook of Wiring, Cabling and Interconnecting for Electronics", McGraw-Hill

[3] Engle, C.E., "Techniques to Analyse and Optimize Noise Rejection Ratios of Low Level Differential Data Systems", Dana Laboratories, Inc., Irvine, CA.

[4] Crawford, T., McNairy, R., "High Performance Signal Conditioning Unit", University of California Specification No. J8-76-173, June, 1977

[5] Cunningham, K.E., "Effects of Impulse Common Mode Signals on High Performance Amplifiers", Final Report for LASL Contract No. 4-L60-5011M-1, Ectron Corp., Feb. 1980

[6] Trompeter, E., "Electronic Systems Wiring and Cabling", Pgs 1-7 of Catalog T-13, Trompeter Electronics, Chatsworth,

$\mathrm{Ca}$. 


\section{Discussion Session 7}

Bob Hebner (NBS). Question to Terry McComb. You and I have performed very simitar measurements of waveforms and attempted to reconstruct waveforms. I interpreted what you were saying at the end of your presentation to mean that you felt that the residual disagreement between your calculated and measured outputs was due to a problem in the calculations and that you mentioned that you were going to have to work harder on the calculations. I concluded that our problem was not in the calculations, but in the measurement of the step response. If you give me freedom to choose my step response, I can always make the calculations agree with the measurements. So, my question then is do you indeed feel that your problem is in the calculation rather. than in the measurement?

Terry McComb (NRC, Canada). We11, I think that they are really tied together. One problem was that the wave was relatively long compared to the significant part of the step response. So the step response was changing significantly only for the first part of the sweep. From that point of view then, we are doing the correction in one of the more disadvantageous regions. When we move to faster impulses and we also record the step response on the same scale, then we will have significant information right through the sweep. So our corrections will be bigger, but we would expect them to be more accurate. Another problem was that in order to generate the impulse response, one has to differentiate the step response and this introduces more noise.

Richard Malewski (IREQ). I think that one problem comes from the fact that our A-to-D converter has a finite sampling time. The samples were taken at 10 nanosecond intervals which obviously implies some filtering of the signals. That was one possible source of inaccuracy in reconstruction. We believe that with the new model which now is becoming available with 5 ns sample times, we will be able to produce more exact, more accurate reconstitution of the measured impulses.

Bob Kolibas (Raytheon). Mr. Cunningham, I enjoyed your presentation. On one of your last slides showing the Faraday cage I didn't notice a return to the shield. This implies that it was floating from any return or ground and I am confused. Was it truly grounded some place to drain off the currents?

Earl Cunningham (Ectron Corporation). Maybe I should look carefully at the drawing, but indeed the whole Faraday cage must be isolated from ground at the instrumentation site. The slide in question is figure 6. It is true that the inner shield of the signal cable terminates at the guard of the amplifier and therefore has no return. However, the amplifier can withstand a very high pulse voltage between guard and case or output, plus we have very heavy cabling interconnecting the case that encloses the instrumentation and the low impedance driving 
point at the source. Thus, the common mode signal present on both signal leads and shield at the amplifier will also be applied to the instrumentation case through the heavy cabling and bonding. If this case has low leakage capacitance to ground than the cable driving it will be able to minimize the common mode signal that the amplifier must endure. In other words, if the ratio of capacitive reactance to cable impedance is low then the perturbation between signal input and output common of the amplifier will be low which will minimize the task the amplifier has to perform to recover quickly and to produce accurate data.

Malewski. We once studied this problem. We have two boxes and want to send a signal via coaxial cable from box $A$ to box $B$. We know that there is a common mode interference signal applied to the two boxes that the current circulating in the shield of the cable produces an error. The way of thinking is very much as presented here and is to put in parallel a low impedance conductor which splits this current into two parts. The large current we will call $\mathrm{I}_{\mathrm{g}}$ and small current $I_{C}$. The efficiency $n$ of this system can be expressed with the ratio of $I_{c}$ over $I_{g}$. We looked for the optimal geometry of this ground cable in order to bring this ratio to the low value. The typical configuration was a pipe representing coaxial cable shield and a flat copper shield. We examined two parameters, the distance between the cable and the ground $h$ and the width of the copper strap. As a function of frequency, our results showed that the closer the cable is to the copper strap, the better the system, the lower the $\eta$ parameter. So if we take a different value of $h$ and reduce the width of the copper strap then again the $n$ will increase. The flat bar is more efficient than the round conductor, i.e., for the same amount of copper, we can get much better performance with the flat rather than the round conductor. There is a publication on this subject giving the specific values for the experiment performed. 1

Jack Katzenstein (Maxwell Laboratories). I would like Mr. Poliner to comment on the role of the electro-optic sensors in the utility instrumentation room.

Randy Poliner (Westinghouse). This is a pretty general topic. We see it as the future for the utility environments just because of the isolation and data integrity. We are demanding more and more from our data acquisition systems; particularly advanced relaying schemes have made it almost a necessity to have the isolation gained from electro-optics.

TR. Malewski, G. Nourse, "Transient Measurement Techniques in EHV Systems." IEEE Transactions, Vol. PAS-97, No. 3, May/June 1978, p. 893-902. 
John Anderson (GE). I just had a couple of very short comments. I am wondering whatever happened to all of the ferrite cores that people used to put on cables. It seems to me that this really helps. The second comment is directed to Mr. Poliner. We have used a cascade of ferrite core transformers, to bring power from the ground up to the signal electronics. Working at 30 kilohertz we can get efficiency along the transformer cascade in the order of 80 percent and overall efficiencies of the system of about 40-50 percent from the power line at ground to the electronics at the top. This has been very effective as far as we are concerned. I would like to introduce this as a possible approach for people who want to get power to their electronics in a very very high voltage environment.

Poliner. There are several schemes to get power to floating electronics. The ultrasonic rod we used is, of course, just one of them. People have used wind turbines, solar energy, and some have gone so far as to use broom handles and motor generator sets which work very nicely for some applications. Our particular application required that we be totally isolated from 60 hertz interference so that any pulses that we were trying to measure would not be in turn affecting our power supply. That is one of the main reasons that we did have to go to the ultrasonic rod technique, although admittedly it is a very poor means of transferring power in terms of efficiency. 


\title{
DIGITAL CORRECTION OF CABLE ATTENUATION LOSSES*
}

\author{
C. Trivelpiece, R. Richardson, J. Shannon, J. B. Smith \\ Maxwe11 Laboratories, Inc. \\ 8835 Balboa Avenue \\ San Diego, CA 92123
}

The use of sensitive electronics on large pulsed power generators often necessitates long ( $>50 \mathrm{ft}$ ) signal cables. Many of the signals measured have risetimes less than $10 \mathrm{~ns}$. The long cables cause a significant degradation in the risetime and shape of these signals. Attenuating low frequencies using a passive compensation network is one solution to this cable loss problem. However, when data acquisition is accomplished by computers and high speed transient digitizers, it is possible to accurately correct for cable attenuation without the problems associated with passive compensators. We will discuss a technique for using Fast Fourier Transforms and waveform correction without introducing additional errors.

* Work supported by the Defense Nuclear Agency.

\section{Introduction}

Because of the large amounts of EMI generated by high voltage, high power machines, it is necessary to put the data recording devices such as oscilloscopes and transient digitizers in a screen room. This requires cable runs of 50-100 ft. In addition, there are sometimes short runs of lower quality cable in the screen room to allow for trigger delays, fiducial marks, etc. These cable runs lead to degradation of the high frequencies which lead to risetime and/or peak amplitude degradation.

In the past, pasșive compensation networks were used to correct this problem. At present, the trend for those who can afford it, is to use fast transient recorders and on-line computers to acquire the data. In this situation, many of the frequency compensations, including the cable response, can be handled in the computer software.

This takes less time to achieve the compensation and cable changes can be accomodated by software changes rather than a physical circuit. 
In this paper we describe a method used at Maxwell to correct for cable losses. We will present an analysis justifying the method and present experimental data showing the accuracy of the correction.

\section{Analys is}

In this section the mathematical model used in the analysis will be described. The attenuation in coaxial cables has been treated analyticaliy before [1]. However, it is necessary to develop it here in some detail to illuminate the method used in the correction.

It is well known, that the attenuation (in DB) in coaxial cables is given at sufficiently high frequencies by

$$
D B \sim \sqrt{f},
$$

where $f$ is the frequency. This is a consequence of the copper loss in the cable, since at sufficientiy high frequencies the resistance is proportional to $\sqrt{f}$. In Laplace transform space, the transmission line equations can be written as

$$
\begin{aligned}
& -\frac{d \tilde{V}}{d x}=L^{\prime} \tilde{I}+\alpha \sqrt{s} \tilde{I} \\
& -\frac{d \tilde{I}}{d x}=C^{\prime} s \tilde{V}
\end{aligned}
$$

where $\tilde{V}$ and $\tilde{I}$ are the transformed voltage and current, $L^{\prime}, C^{\prime}$ are the inductance and capacitance per unit length, $\alpha$ is a constant of the cable describing the resistive loss at a "frequency" $s$. The constant $\alpha$ is given by

$$
\alpha=\frac{1}{\ell_{\perp}} \sqrt{\frac{\mu_{0}}{\sigma}}
$$

where $l_{\perp}$ is the total length transverse to the direction of propagation, $\sigma$ is the conductivity of the cable and $\mu_{0}$ is the permeability of free space.

Equation 2 has solutions of the form

$$
\tilde{V}, \tilde{I} \sim e^{ \pm \gamma x}
$$


where $\gamma$ is the propagation constant given by

$$
\gamma=\sqrt{\frac{s^{2}}{u^{2}}+\frac{\alpha s^{3 / 2}}{z_{0} u}}
$$

where $u$ is the speed of propagation in the cable and $z_{0}$ is the impedance of the cable given by

$$
\begin{gathered}
u=\frac{1}{\sqrt{L^{\prime} C^{\prime}}} \\
z_{0}=\sqrt{\frac{L^{\prime}}{C^{\prime}}}
\end{gathered}
$$

For sufficiently high frequencies, $\gamma$ can be expanded to give,

$$
\gamma=\frac{s}{u}+\frac{\alpha s^{1 / 2}}{2 z_{0}}-\frac{1}{8} \frac{\alpha^{2} u}{z_{0}^{2}}+\ldots
$$

For any practical cable and reasonable frequency, all terms except the first two can be neglected.

In this case, a wave propagating down the line has the form

$$
\tilde{V}, \tilde{I} \sim \exp \left[-\frac{s x}{u}-\frac{\alpha s^{1 / 2}}{2 Z_{0}} x\right] .
$$

The first term in the argument is the result of the delay time along the cable and the second term gives rise to the dispersion.

In actual fact, one must be a bit more careful here because of the input and output impedance at the ends of the cable. Because of the frequency dependent series resistance the characteristic impedance defined by the motion of the voltage and current wave is not $z_{0}$ but 


$$
z=\frac{\tilde{V}_{+}}{\tilde{I}_{+}}=z_{0} \sqrt{1+\frac{\alpha U}{z_{0} s^{1 / 2}}}
$$

This means that reflections can occur from a line terminated in $Z_{0}$. In most cases of interest at Maxwe11, the line is fed from sensors that effectively have $Z_{0}$ as an internal impedance and are likewise terminated in $Z_{0}$. For this case it can be shown that the output voltage is related to the sensor voltage by

$$
\tilde{v}_{\text {out }}=\tilde{v}_{g} \frac{4 \text { rus }}{(\gamma u+s)^{2}} e^{-\gamma x}
$$

where $\tilde{V}_{g}$ is the sensor voltage normalized to give $\tilde{V}_{\text {out }}$ on a dispersion-less line. If the same expansion of $\gamma$ is used

$$
\begin{aligned}
\tilde{V}_{\text {out }} & =\tilde{V}_{g} \frac{s^{2}\left(1+\frac{\alpha u}{2 Z_{0} \sqrt{s}}+\ldots\right)}{s^{2}\left(1+\frac{\alpha u}{2 Z_{0} \sqrt{s}}+\ldots\right)} \exp \left[-\frac{s x}{u}-\frac{\alpha x s^{1 / 2}}{2 Z_{0}}\right] \\
& \simeq \tilde{V}_{g} \exp \left[-\frac{s x}{u}-\frac{\alpha x s^{1 / 2}}{2 Z_{0}}\right]
\end{aligned}
$$

Hence the dispersion can be described using only the exponential term.

\section{Waveform Correction}

As was seen above, the voltage at the end of a line of length $x$ is related to the input by

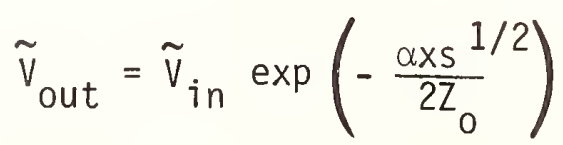


where the delay term $e^{-s x} / u$ has been suppressed. In a straightforward manner we can solve for $V_{\text {in }}$

$$
\tilde{v}_{\text {in }}=\tilde{v}_{\text {out }} \exp \left(\frac{\alpha \times s^{1 / 2}}{2 Z_{0}}\right)
$$

Formally, one would like to transform this to the time domain and convolve $V_{\text {out }}(t)$ with the inverse of the exponential term and get $v_{i n}(t)$. Unfortunately, $\exp \left(\alpha x s^{\frac{1}{2}} / 2 z_{0}\right)$ is not mathematically suitable for transforming to the time domain although its product with $\widetilde{V}_{\text {out }}$ is transformable.

If onty modest corrections to the waveform are required (<20 percent or so) there is an approximate way of finding $V_{i n}(t)$. A small correction in the time domain means that in the frequency domain the argument of the exponential is small. We can then write

$$
\tilde{v}_{\text {in }}=\tilde{v}_{\text {out }}\left(1+\frac{\alpha \times s^{1 / 2}}{2 z_{0}}\right)
$$

This can be written,

$$
\tilde{v}_{\text {in }}=\tilde{v}_{\text {out }}+\left(s \tilde{v}_{\text {out }}\right)\left(\frac{\alpha x}{2 z_{0} s^{1 / 2}}\right)
$$

We notice that multiplying by $s$ is the same as taking the derivative in the time domain and that the inverse Laplace transform gives

$$
\mathscr{L}^{-1}\left[\mathrm{~s}^{-1 / 2}\right]=\frac{1}{\sqrt{\pi t}}
$$

in the time domain. Equation 15 then transforms to: 


$$
v_{\text {in }}(t)=V_{\text {out }}(t)+\int_{0}^{t} k(t-\tau) \frac{d V_{\text {out }}}{d \tau} d \tau
$$

where

$$
\begin{array}{rlrl}
K(t) & =\frac{\alpha x}{2 Z_{0}} \frac{1}{\sqrt{\pi t}} & & t>0 \\
& =0 & t \leq 0 .
\end{array}
$$

Equation 17 is a straight forward operation with the software of the most modest computer. It will be seen below that the approximations made leading to Eq. 17 are reasonable and this correction procedure works remarkably well.

\section{Explanation of Computer Code}

The waveform data from the digitizers is a series of 512 points. A mathematical model is a series of delta functions separated by a constant time interval $\Delta t$. Also, the vertical resolution of the digitizers is at most 512 points but in practice probably around 200-400 points. This tends to cause a staircase representation of the signal. When the derivative is taken a lot of spikes and apparent noise are generated. A three point average is done on the waveform before the derivative is taken to cut down on the noise produced. The three point average was done by averaging each point with the points on either side of it. If the end points of an array are not zero they will produce large spikes when the derivative is taken. This can be eliminated on the derivative waveform by making the last three end points equal to the fourth point in from the end.

The correction table is generated and the convolution integral performed. The correction is then added to the original waveform.

10 REM THIS CODE WILL PERFORM A CABLE CORRECTION

20 WAVEFORM II IS I(511), DI, HI\$,VI\$

30 WAVEFORM VV IS V(511), DV, HVS, VV\$

40 WAVEFORM 00 IS O(511),DO,HO\$,VO\$

50 WAVEFORM GG IS G(1023), DG, HG $\$, V G \$$

60 DIM IP(511)

70 REM II IS THE INPUT WAVEFORM

$80 \quad \mathrm{IP}=\mathrm{I}$

90 REM DO A 3 POINT AVERAGE TO SMOOTH WAVEFORM

$100 \mathrm{I}(1: 510)=(\mathrm{I}(0: 509)+\mathrm{I}(2: 511)+\mathrm{I}(1: 510)) / 3$ 


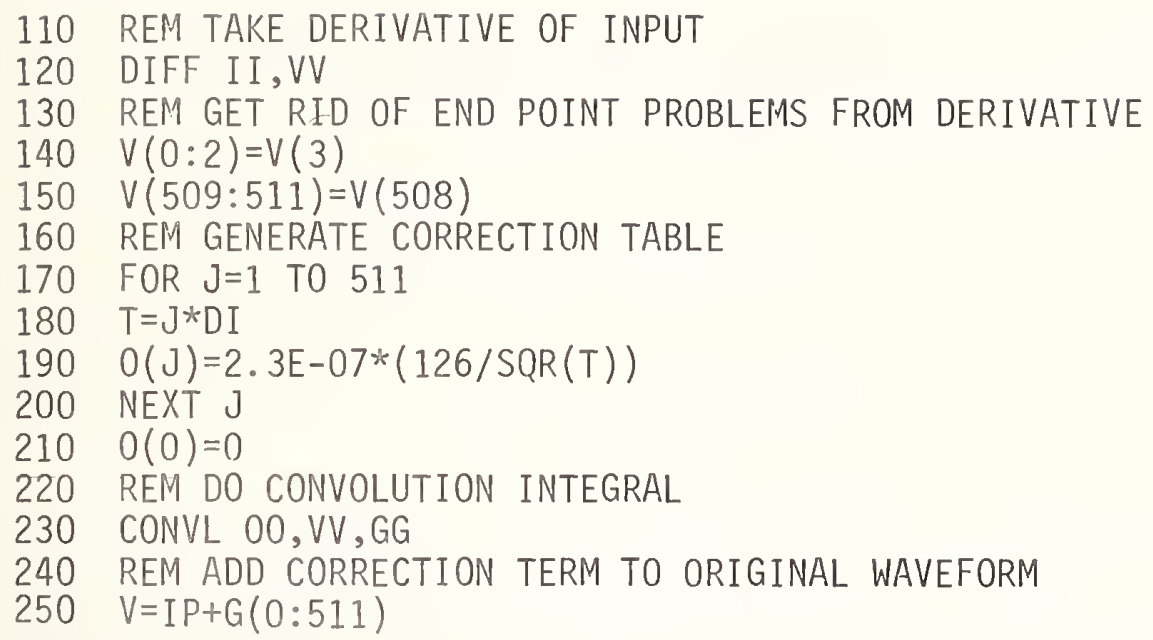

\section{Implementation of Computer Code}

To generate the correction table a few physical factors about the cable must be found. The length can be easily measured and the impedance can be looked up in a book. Now a constant for the attenuation of the cable is needed. Although the correct number for the attenuation must be found experimentaliy, an approximate number can be found using the attenuation versus frequency curves published for cables. This calculated factor agrees closely with the empirically determined one and provides a useful starting point for the experimental determination.

To find the approximate attenuation factor for the corrections the slope in $\ln$ (In/output) versus $\sqrt{\omega}(\omega=2 \pi f)$ must be found from the $\mathrm{db}$ loss curve. Since the slope is a straight line in the frequency range of the tables only two points are necessary. Example: RG-58 has a $15 \mathrm{db} / 100 \mathrm{ft} 10 \mathrm{ss}$ at $540 \mathrm{MHz}$ and a $3 \mathrm{db} / 100 \mathrm{ft}$ loss at $35 \mathrm{MHz}$. Transforming these into the natural $\log$ of the attenuation and $\sqrt{\omega}$ gives

$$
\begin{aligned}
& \frac{15 \mathrm{db}}{100 \mathrm{ft}} \rightarrow \frac{1.72}{100 \mathrm{ft}} \rightarrow \frac{0.0172}{\mathrm{ft}} \text { o } 540 \mathrm{MHz} \rightarrow 5.82 \times 10^{4} \\
& \frac{3 \mathrm{db}}{100 \mathrm{ft}} \rightarrow \frac{0.345}{100 \mathrm{ft}} \rightarrow \frac{0.00345}{\mathrm{ft}} \text { o } 35 \mathrm{MHz} \rightarrow 1.48 \times 10^{4} \\
& \text { s1ope }=\frac{1.72 \times 10^{-2}-3.45 \times 10^{-3}}{5.82 \times 10^{4}-1.48 \times 10^{-4}}=\frac{3.16 \times 10^{-7}}{\mathrm{ft}}
\end{aligned}
$$




$$
\begin{aligned}
& \text { Attenuation } \\
& \text { constant }
\end{aligned}=\sqrt{\frac{2}{\pi}} \times \frac{\text { slope }}{\mathrm{ft}}=\frac{2.52 \times 10^{-7}}{\mathrm{ft}}
$$

the correction table has the form

$$
K(t)=2.52 \times 10^{-7} \times \frac{\text { Length }(\mathrm{ft})}{\sqrt{T}}
$$

The value used in this paper for a length of $126 \mathrm{ft}$ of RG-58 was $2.3 \times 10^{-7}$. A value for RG-213 was computed to be $9.49 \times 10^{-8}$. The factor for the loss in two different types of cables that are hooked together is approximately the sum of their lengths times their respective attenuation constants. The correction table for $126 \mathrm{ft}$. of RG-58 is shown in Figure 1 .

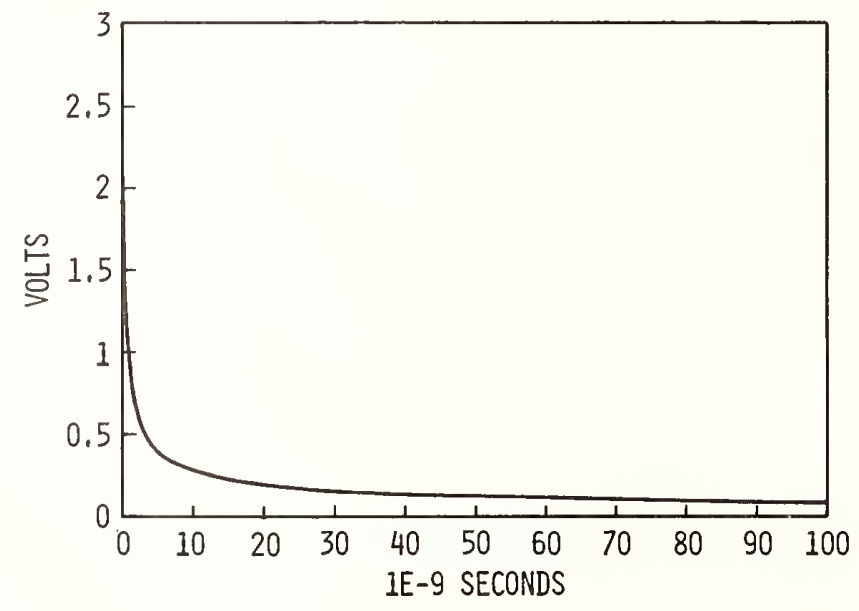

Figure 1. Correction table for 126' of RG-58.

The test setup used is illustrated in Figure 2. 


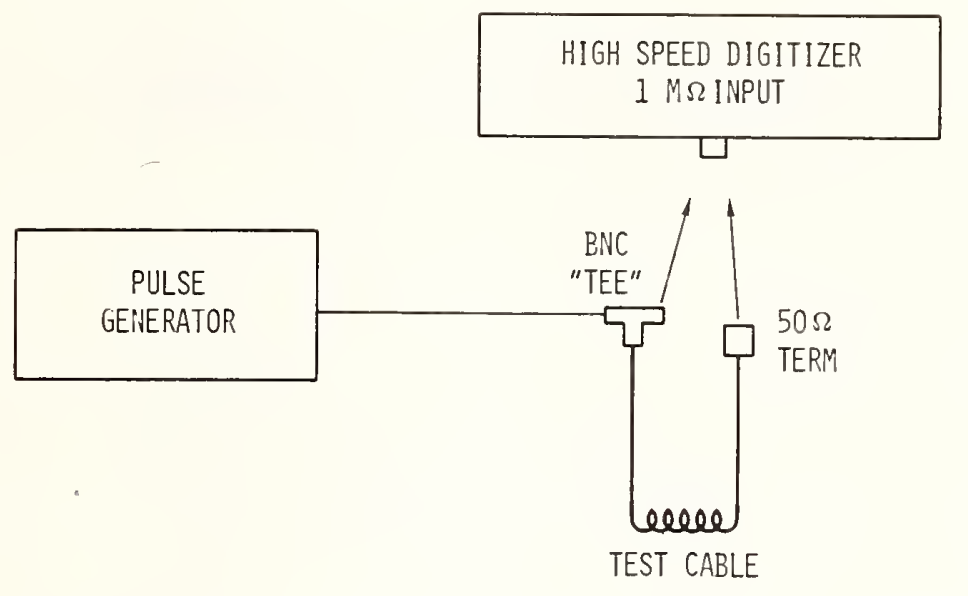

Figure 2. Test set-up for measuring cable response.

The vertical amplifier has a $1 \mathrm{~m} \Omega$ input impedance and the "TeE" is a regular BNC "Tee". This way the input and output of the cable can be measured without affecting the circuit.

A series of pulses are recorded and the attenuation factor is changed until the best fit is obtained. For best results the test should be done at the same sweep speed that will be used to collect data.

\section{Results}

In Figure 3 a fast rise square wave was put into a $126 \mathrm{ft}$ cable of RG-58 and the output was measured (dotted waveform). The correction was applied to the output of the cable and the waveform with squares was produced.

Risetime

Input

Output

Corrected
$1.82 \mathrm{~ns}$

$12.6 \mathrm{~ns}$

$1.89 \mathrm{~ns}$
Peak Value

$100 \%$

$82.5 \%$

$99.3 \%$
Integral

$100 \%$

$82.8 \%$

$98.2 \%$ 


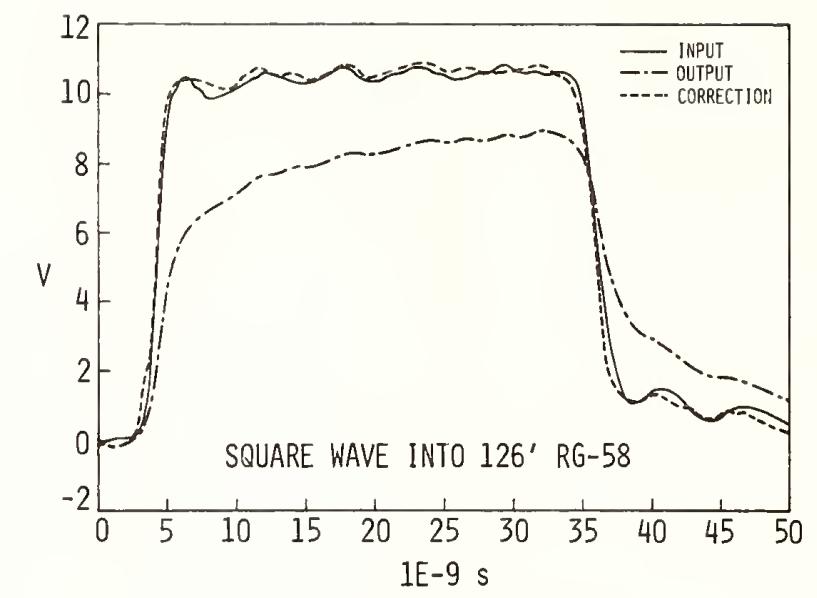

$\begin{array}{lrrr} & \text { RISETUME } & \text { PEAK VALUE } & \text { INTEGRAL } \\ \text { INPUT } & 1.82 \mathrm{NS} & 100.0 \% & 100.0 \% \\ \text { OUTPUT } & 12.60 \mathrm{NS} & 82.5 \% & 82.8 \% \\ \text { CORRECTED } & 1.89 \mathrm{NS} & 99.3 \% & 98.2 \%\end{array}$

Figure 3. Cable correction for fast square wave.

Figure 4 shows the correction that was added to the output of the cable to simulate the input.

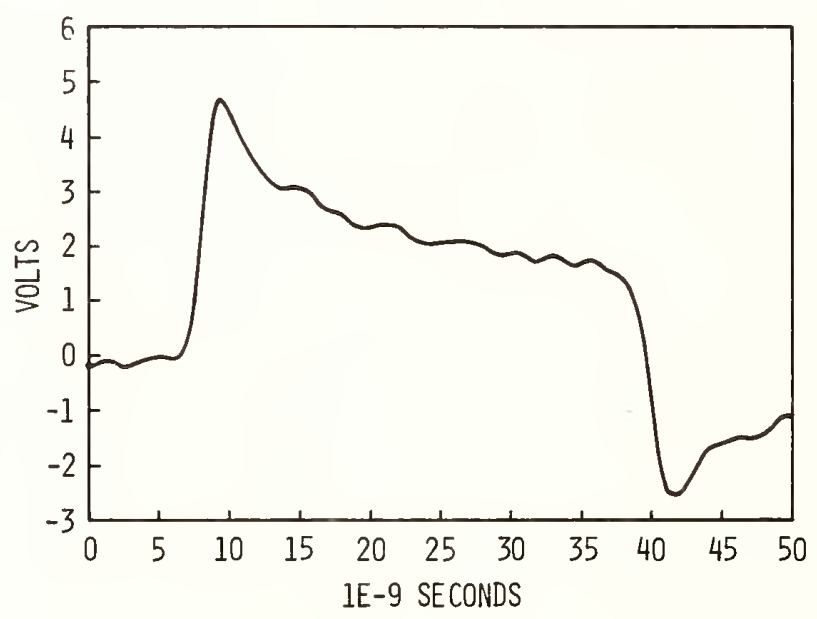

Figure 4. Correction that was added to output in Figure 3. 
Figure 5 shows a more gentle pulse passing through the cable.

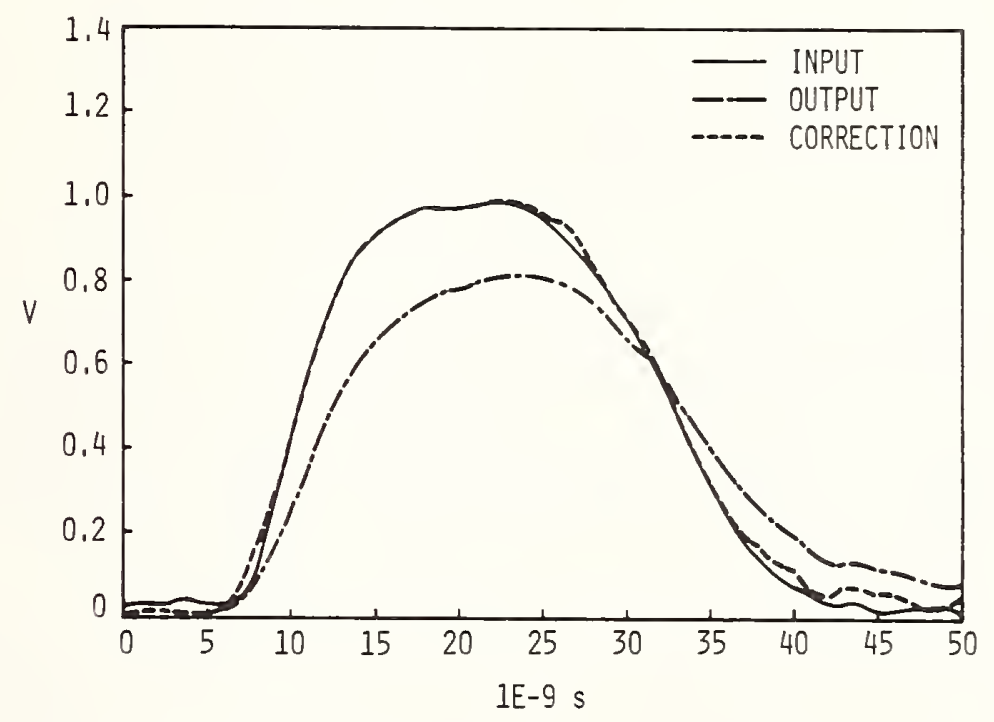

Figure 5. Pulse into $112^{\prime}$ RG-58.

\section{Summary}

For data collection systems that are using high speed digitizers and digital computers, this method provides a convenient and accurate way of compensating for cable losses and distortions. This correction is accurate for a wide range of cable lengths and pulse shapes.

\section{Reference}

[1] R. L. Wilmington and N. S. Nahman, "Transient Analysis of Coaxial Cables Considering Skin Effect", Proc. IRE pp 166-174; February, 1957. 


\title{
PRECISION PICOSECOND-MICROSECOND ELECTROMAGNETIC WAVEFORM MEASUREMENTS AT NBS
}

\author{
R. A. Lawton
}

Electromagnetic Technology Dvision

National Bureau of Standards

Boulder, Colorado 80303

Continuing research in the measurement science of electromagnetic waveforms has resulted in the development of an Automated Pulse Measurement System (APMS). This system consists of a sampling oscilloscope mated with a minicomputer to provide for automatic acquisition and processing of repetitive pulses with durations and transition times in the picosecond to microsecond time frame. In addition, work has been started to add single transient capability to the APMS.

The development of this capability has required the careful development of the electronic circuit interfacing between the sampling oscilloscope and the minicomputer and the development of the appropriate software to allow the scope to be properly controlled by the computer.

Vertical and horizontal calibration techniques have been developed which provide an order of magnitude improvement in accuracy.

Reference waveform generators have been developed and new ones are contemplated to serve as a check on the complete measurement system.

Finally, a measurement system analysis has been performed which includes system modeling, signal parameter characterization and optimal data processing algorithm identification.

With the APMS we are now able to characterize noise free, repetitive time domain waveforms with an uncertainty of $1 \%$ of full scale in amplitude and $0.5 \%$ of full scale on the time axis.

In addition, with our best waveform generators, we can do characterization in the frequency domain over the frequency range $5 \mathrm{MHz}$ to $10 \mathrm{GHz}$. 


\title{
PRECISION PICOSECOND-MICROSECOND ELECTROMAGNETIC WAVEFOPM MEASUREMENTS AT NBS
}

\author{
R. A. Lawton \\ Electromagnetic Technology Dvision \\ National Bureau of Standards \\ Boulder, Colorado 80303
}

Since time resolved waveform measurements are currently assuming increased importance in the electromagnetic community, we at the National Bureau of Standards (NBS) have an on-going measurement program to develop the tools needed to put waveform measurements on a firm foundation.

Much of the development work at NBS has been concentrated on repetitive pulses and low energies [1,2], but the needs for high energy single transient measurements have not been forgotten $[3,4]$.

The development of modern waveform measurement instruments such as sampling oscilloscopes and transient digitizers have greatly aided the waveform development program at NBS, however, the development of modern, dedicated, interactive computers for data acquisition and processing has been of equal importance. These computers have made possible the acquisition of 1024,2048 and 4096 etc. point waveforms with comparative ease and speed - in a fraction of a second. This acquisition time is so quick that the same waveform may be reacquired hundreds of times to allow for signal averaging all in just a few seconds.

I will now describe some of the measurement systems developed at NBS for the precision measurement of time domain waveforms. This will include a description of the waveform acquisition equipment and its modeling, data processing and signal analysis.

The most highly developed waveform measurement system at NBS is the Automatic Pulse Measurement System (APMS) which consists of a sampling oscilloscope controlled by a minicomputer for making measurements on repetitive pulses. The block diagram of this system is shown in figure 1 (PTR denotes paper tape reader and PTP denotes paper tape punch) and a photograph of the system in operation is shown in figure 2 . The A/D converter digitizes the amplitude of each sample which is then stored in memory after which the computer through the D/A commands the oscilloscope to take another sample. This system can accurately record waveforms which have transition times as short as 20 picoseconds. The measurement precision is determined by the amplitude noise and timing jitter of the system. This system has been characterized for these parameters and found to have standard deviations of 2 millivolts of amplitude noise and 6 picoseconds of timing jitter for $10 \mathrm{mV} / \mathrm{cm}$ vertical sensitivity and $200 \mathrm{ps} / \mathrm{cm}$ horizontal sensitivity, using a $200 \mathrm{MHz}$ precision oscillator as a clock. The APMS is capable of measuring pulses with amplitudes in the neighborhood of 1 to $500 \mathrm{mV}$. Higher amplitude signals may be measured at reduced accuracy using calibrated attenuators. 


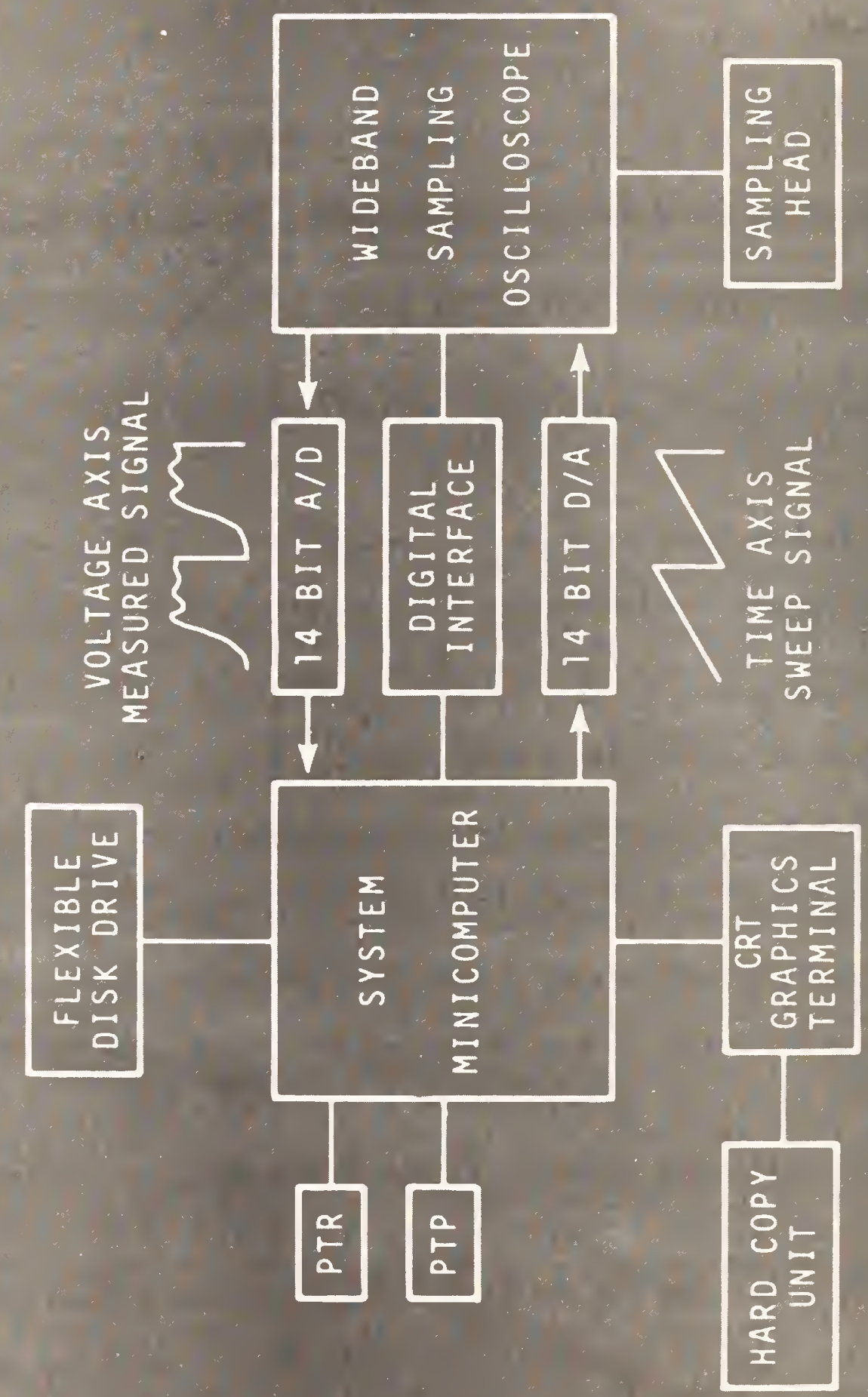

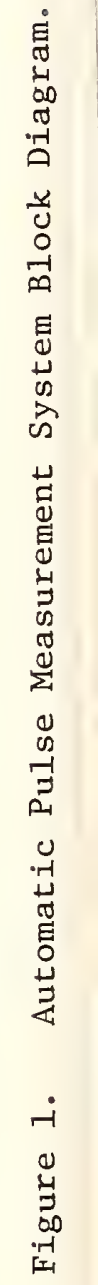




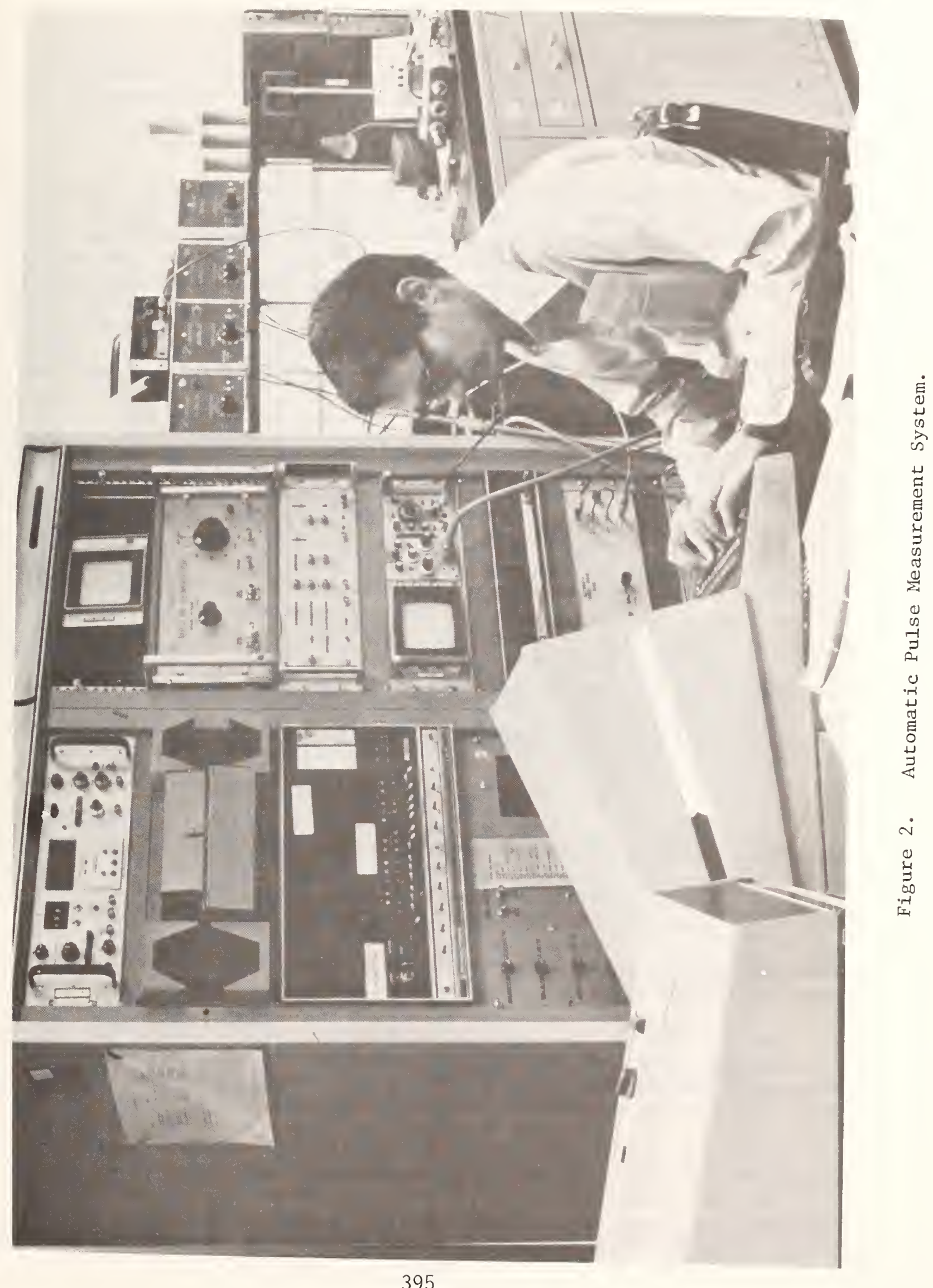


Once the data is acquired and stored, data processing is available in the form of the fast Fourier transform (FFT) to obtain frequency domain information, deconvolution to remove distortion introduced by the measuring instrument, and signal averaging to extract low amplitude signals from noise.

Examples of these types of operations will now be given. Figure 3 illustrates the acquired waveform of an NBS pulse generator and its spectrum obtained by the FFT. Figure 4 shows the impulse and step response of a 65 nanosecond coaxial delay line used with pulse generators where no pretrigger pulse is available. Figure 5 shows the waveform of a pulse fed through this delay line before and after the distortion due to the line has been removed by deconvolution. Finally, figure 6 shows a waveform that has been attenuated below the noise level (upper trace), and the result of signal averaging of many repetitions of the same waveform (lower trace).

Now how does this capability apply to high energy transient measurements with waveforms that are frequently of a non-repeating character? The answer lies first in the ability to accurately determine impulse responses with the APMS as was done for the delay line in the example just given. More appropriate to high energy measurements, the impulse response of attenuators can be obtained and then used to deconvolve the distortion of the attenuator from the transient measurement. Secondly, we have made an initial approach to developing standards for single transient measurements. This has consisted of acquiring a low light level vidicon which among other things can be used to digitize the trace of a fast, real time oscilloscope as shown in figure 7. The acquired waveform can then be stored on a disk and is available for future processing in the same fashion as the repetitively pulsed signals described earlier (except for signal averaging) or for display on a monitor like the one shown in figure 8 .

We have also done some signal processing analysis of a commercial transient digitizer in connection with our development of a laser pulse power meter. This power meter has been described elsewhere [3], but consists of a box housing an optical diffuser type attenuator and PIN photodiode detector shown in figure 9 and a transient digitizer shown in figure 10. This description includes the determination of the system impulse response, attenuation factor and a routine for calibrating the transient digitizer response for both amplitude and time base.

Data processing that has been used at NBS with the transient digitizer includes numerical integration in calculating pulse energy in the attenuator measurement, automatic calibration correction, and computed input waveform in terms of power versus time.

To improve the accuracy of both repetitive and single transient measurements we have developed an automated calibrator for pulse amplitude and time scales [5], standard waveform generators [6] to test pulse measurement systems, system modeling and deconvolution techniques [7], signal parameter characterization techniques, and optimal data processing 

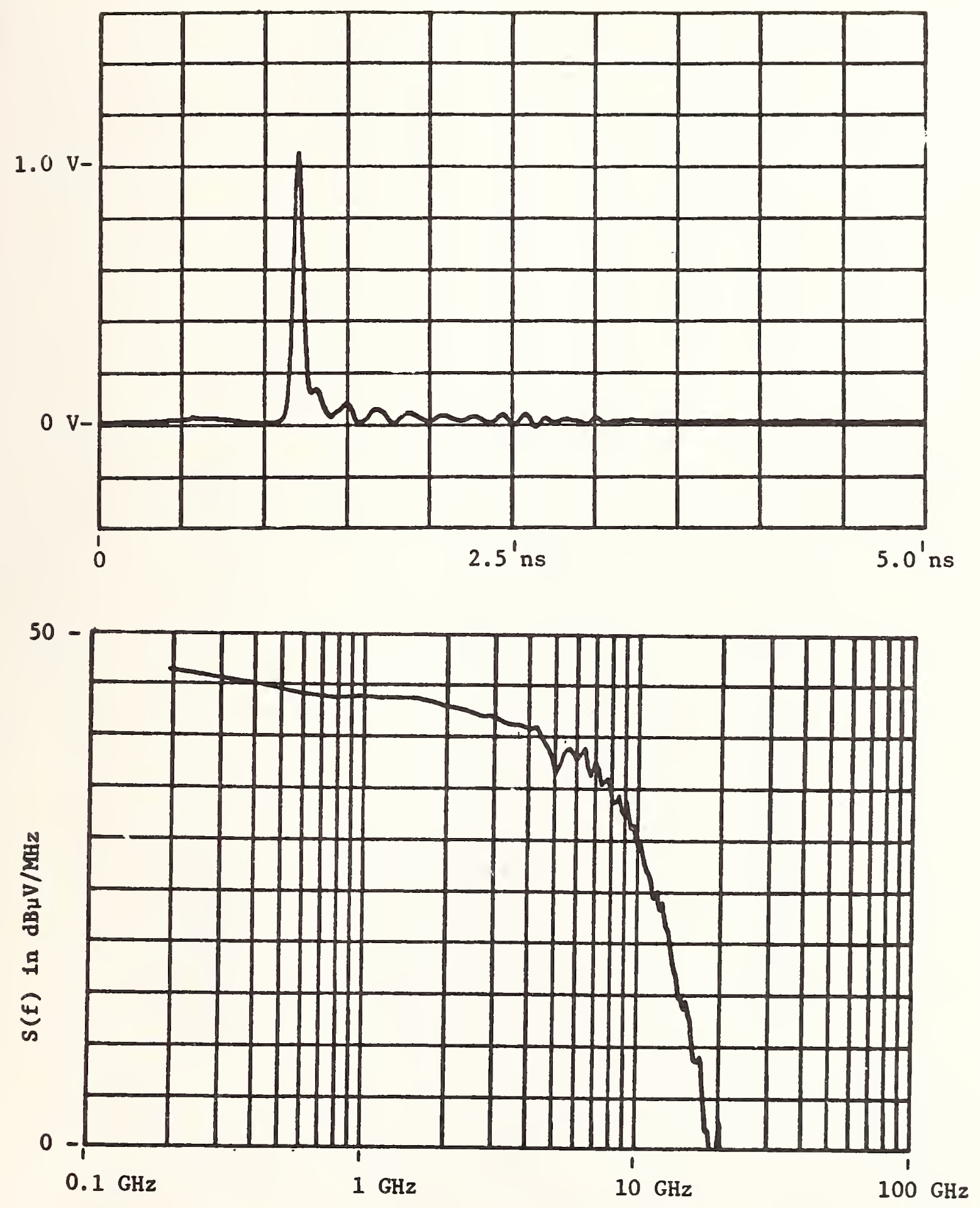

Figure 3. Acquired Waveform (top) and Spectrum (bottom) of Pulse Generator. 

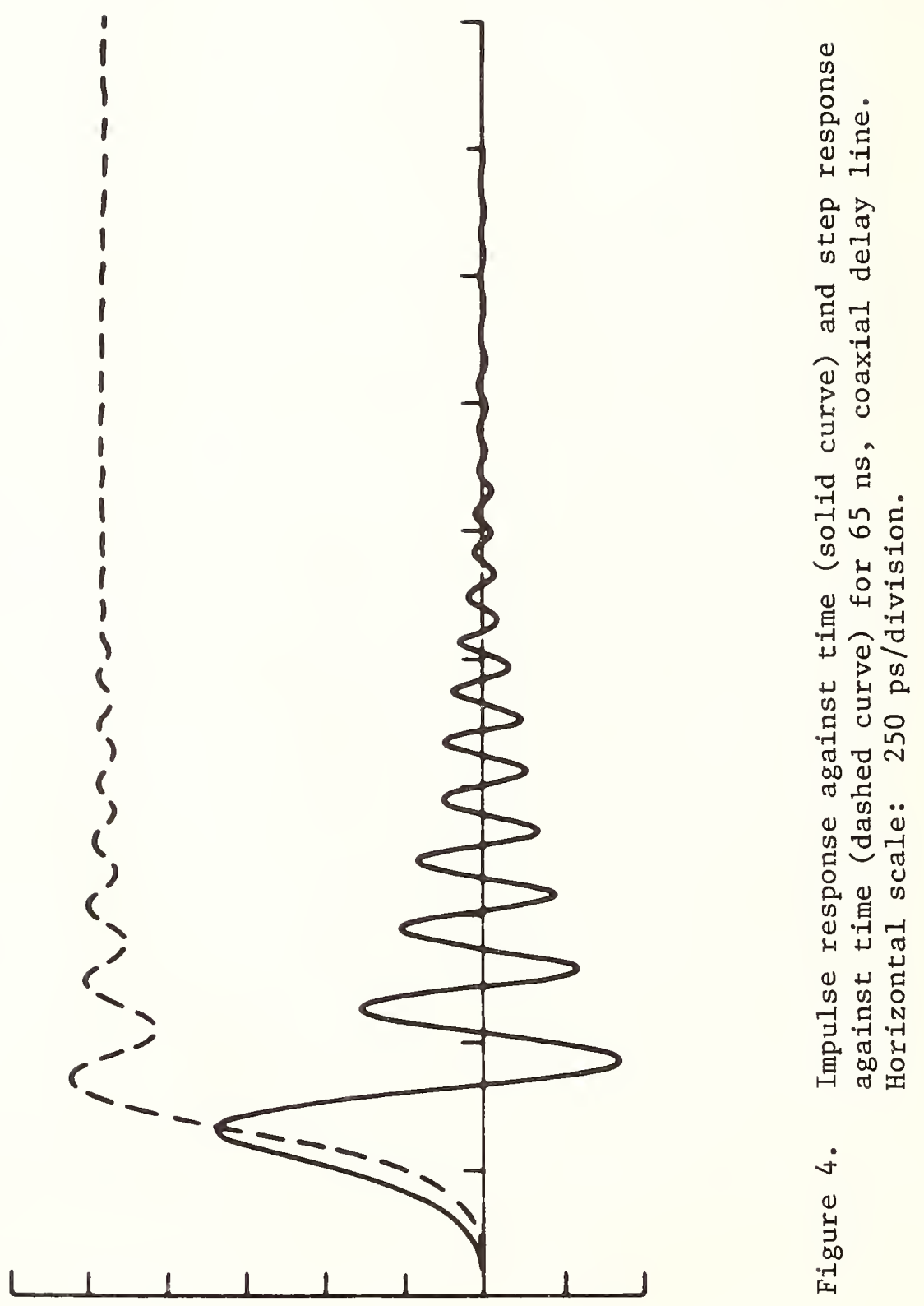


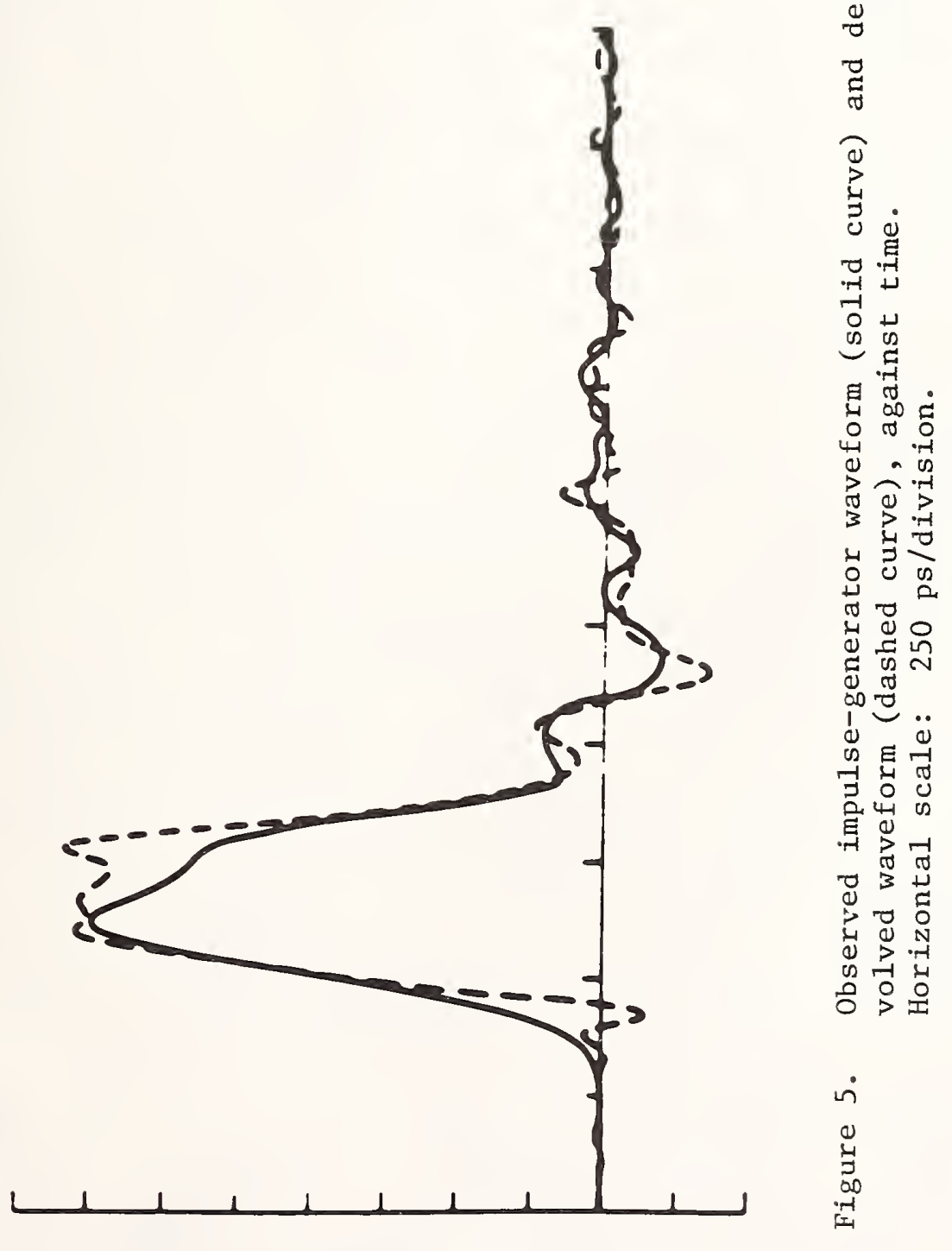



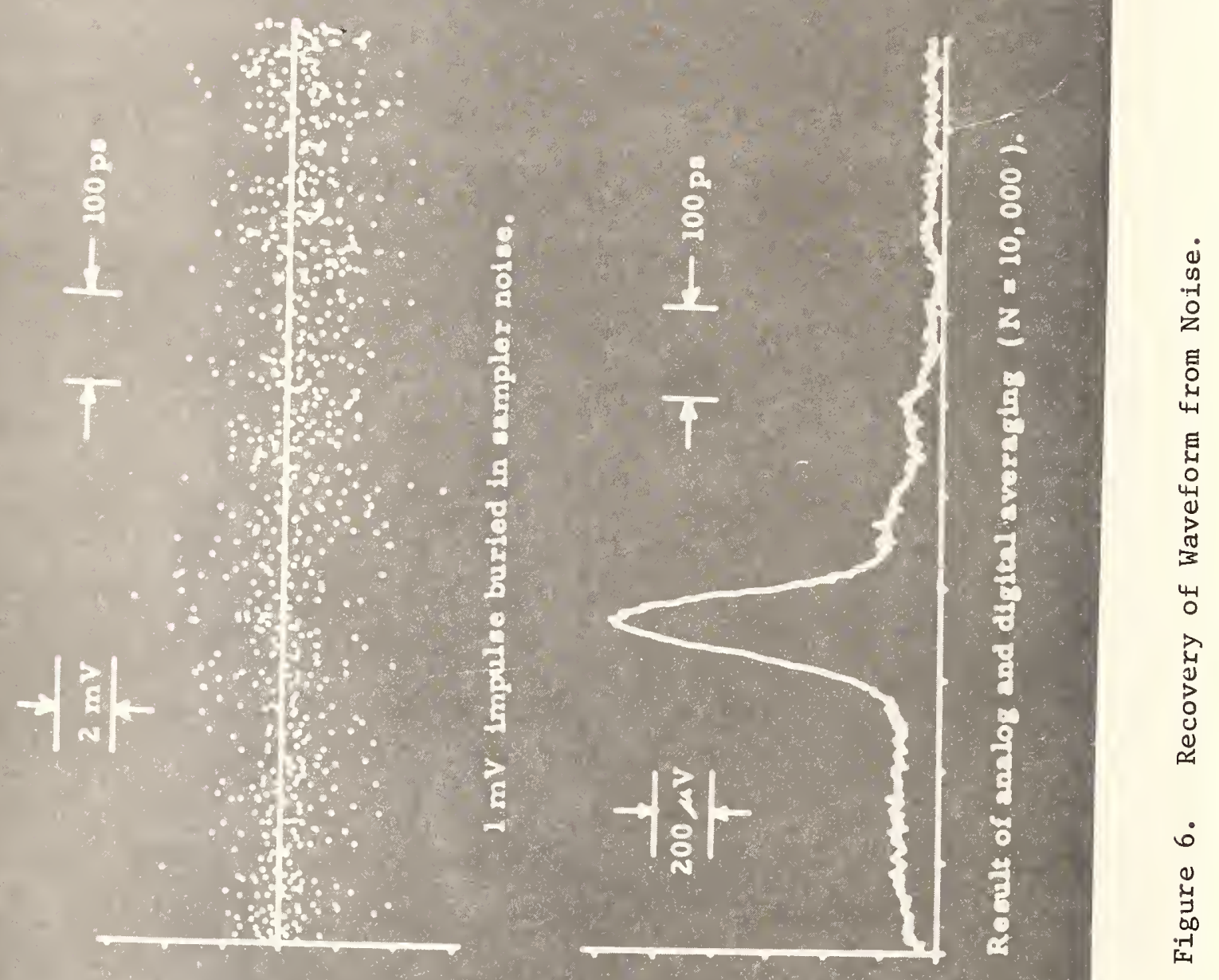


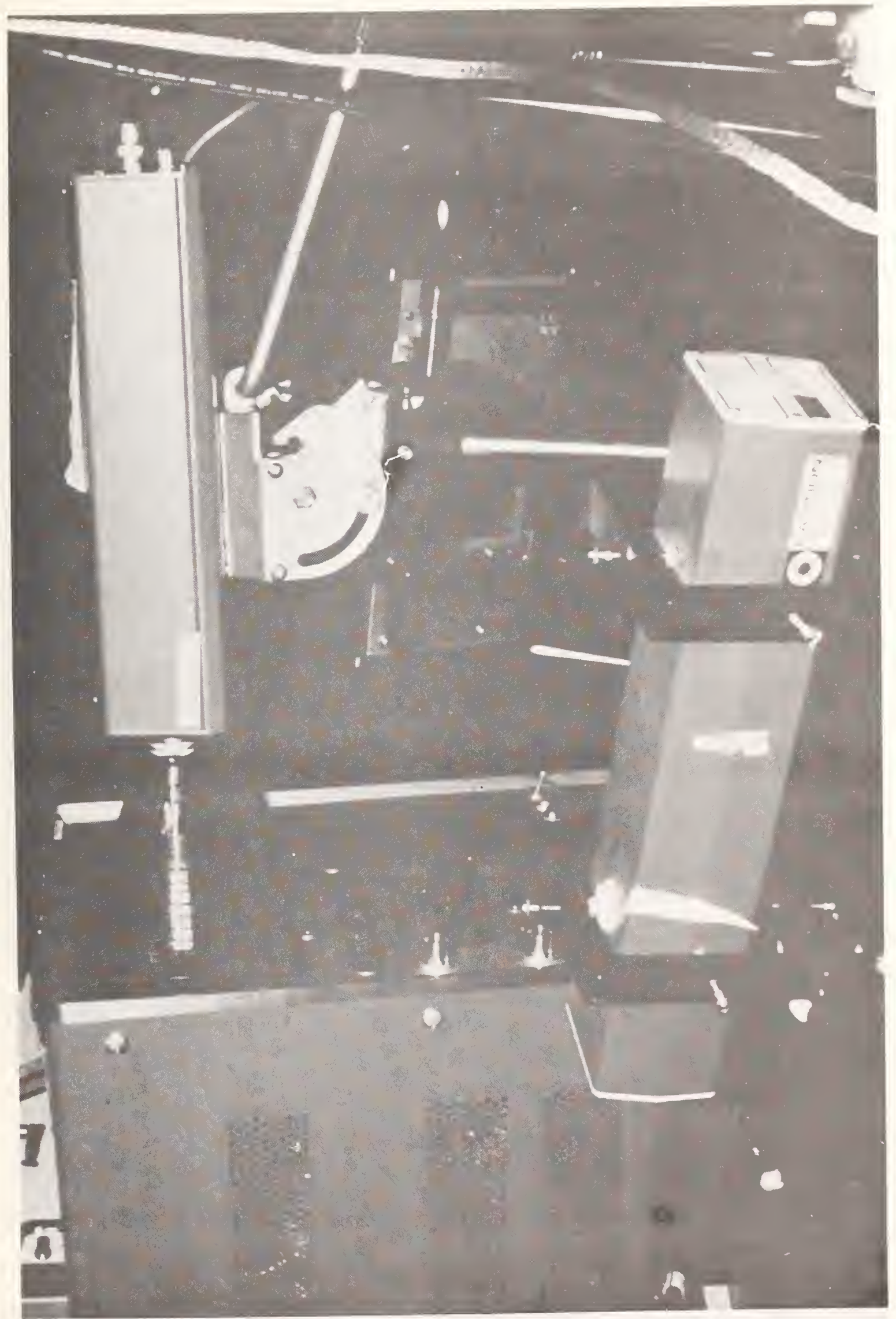

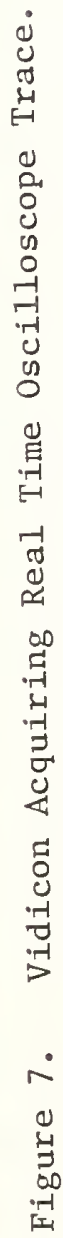




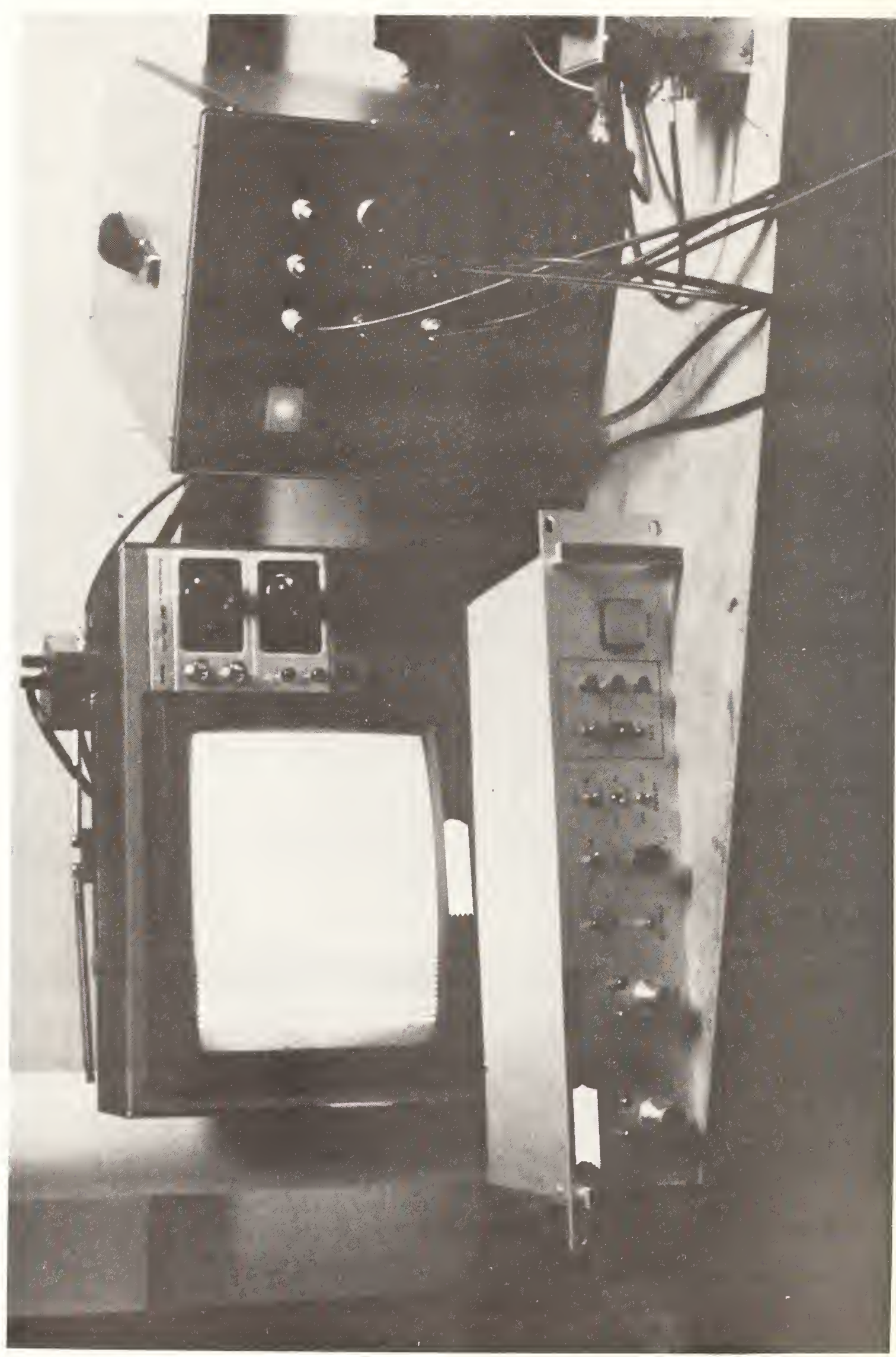

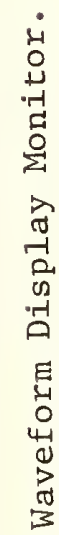

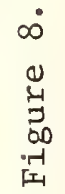




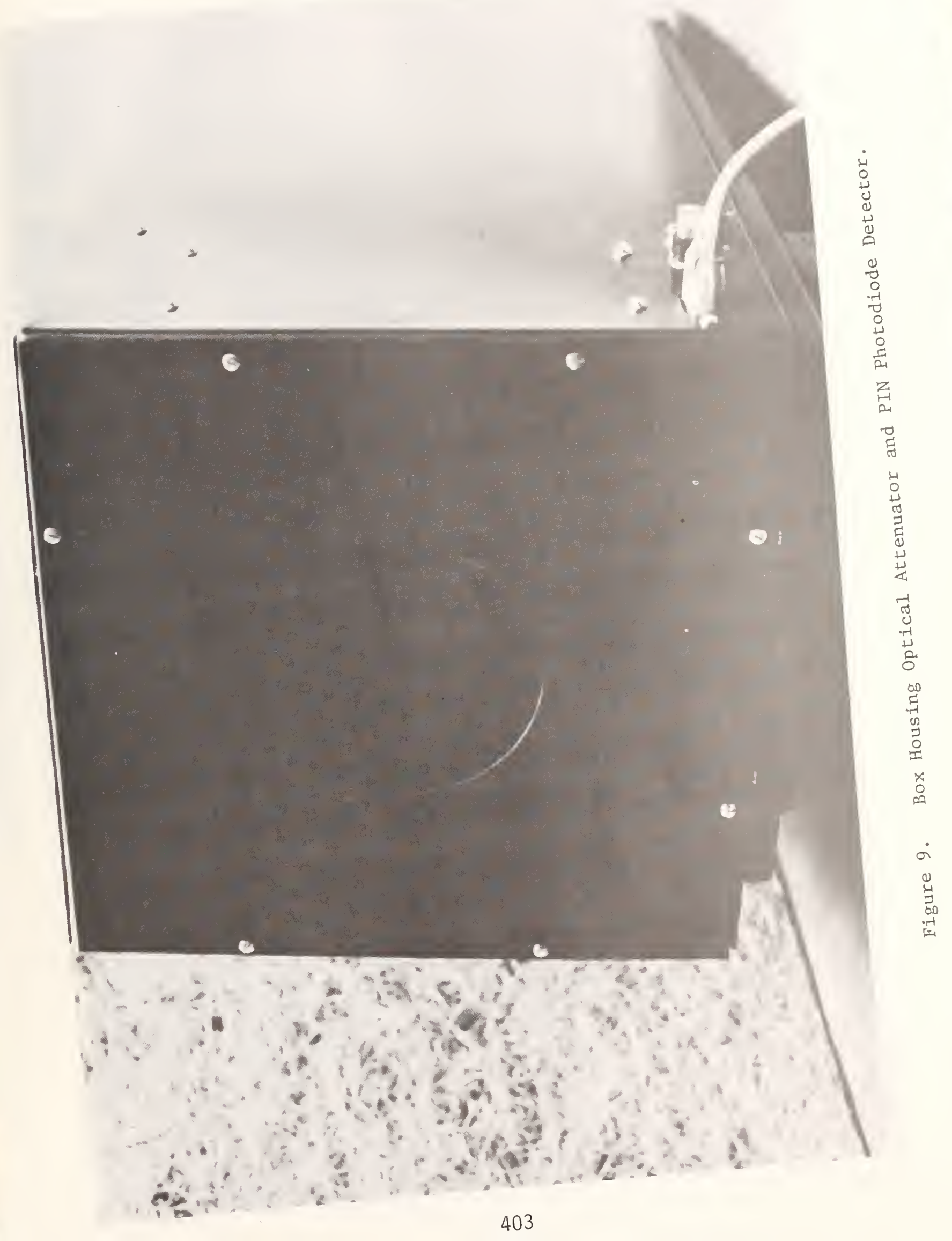



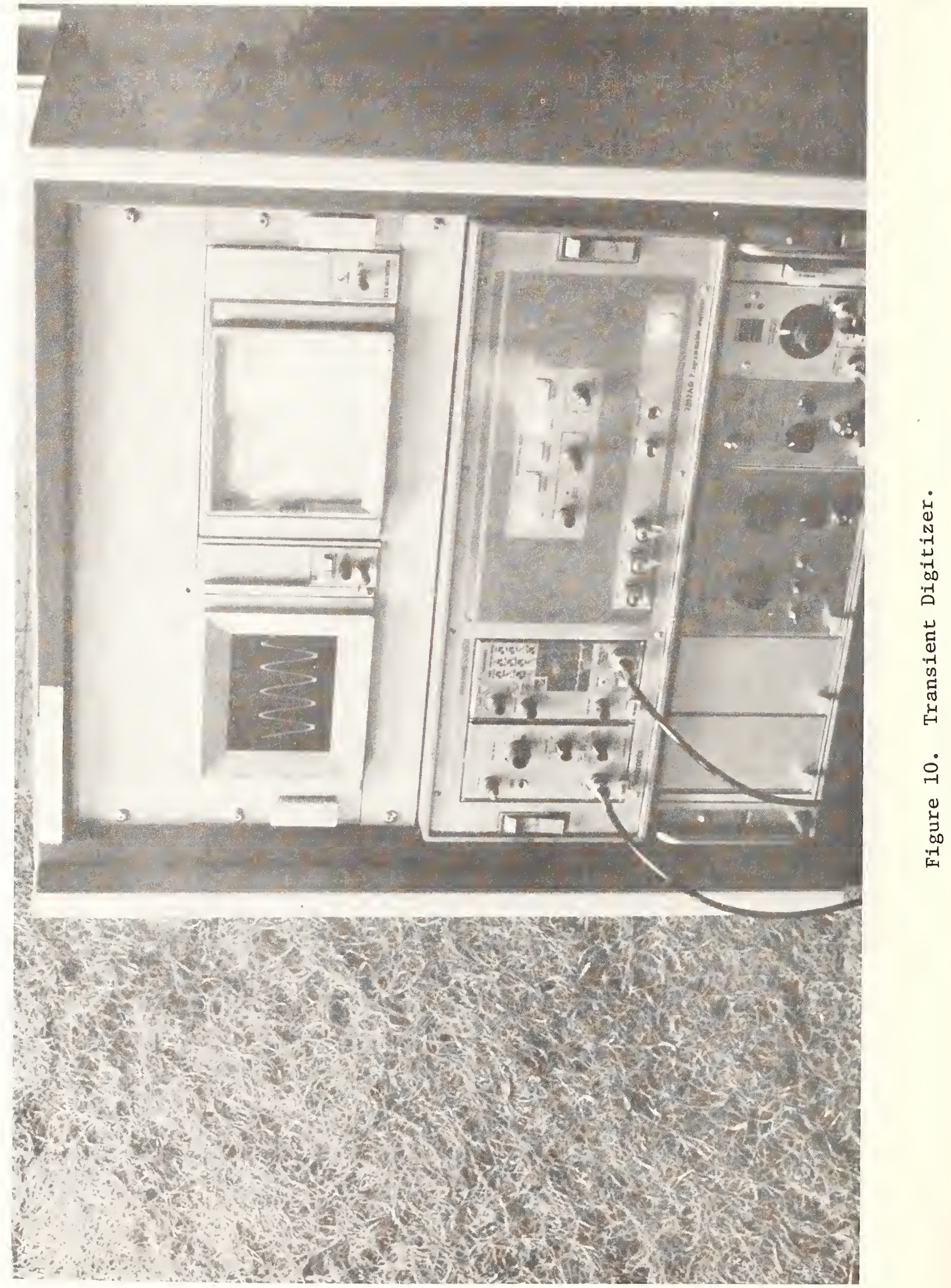
algorithm selection techniques [8].

It is not my purpose here to describe these techniques in detail. I will leave that for those who look up the references. However, I will make a few comments about certain of these techniques.

By using the automatic calibrator the uncertainty in amplitude has been reduced to $<1 \%$ of full scale and the uncertainty in time has been reduced to $<.5 \%$ of full scale. The use of reference waveform generators which have a smooth gaussian transition from the 0 to $100 \%$ level without any overshoot or ringing make it possible now to characterize other waveforms over the entire observation time window instead of only specifying the initial $10 \%$ to $90 \%$ transition time (rise time). In addition the specification of the $10 \%$ and $90 \%$ points is unambiguous for this type of waveform, an example of which is shown in figure 11. By applying system modeling and deconvolution techniques we have been able to extend the range over which we can characterize pulse generators in spectrum amplitude from a previous maximum frequency of $3 \mathrm{GHz}$ to $10 \mathrm{GHz}$ at present.

The goal of all of our measurement activity is to be able to provide for you, the customer, measurement services. These most frequently take the form of calibrations. At present NBS offers calibrations in transition duration (risetime) of both pulse generators and low-pass filters, pulse delay, spectrum amplitude, and wideband attenuation.

Various research efforts are underway to improve our waveform measurement and calibration services. Present research efforts include further refinement of sampler modeling and real time data acquisition research to select optimum filtering algorithms for the deconvolution process, research in jitter characterization and new sampling technique development using Josephson Junction switches and reference waveform development using photoconductive switches and other solid state devices.

Using all of the above developments and resources at the disposal of NBS and that of our colleagues, waveform measurements are beginning to reach the status of a measurement science with a firm foundation. 


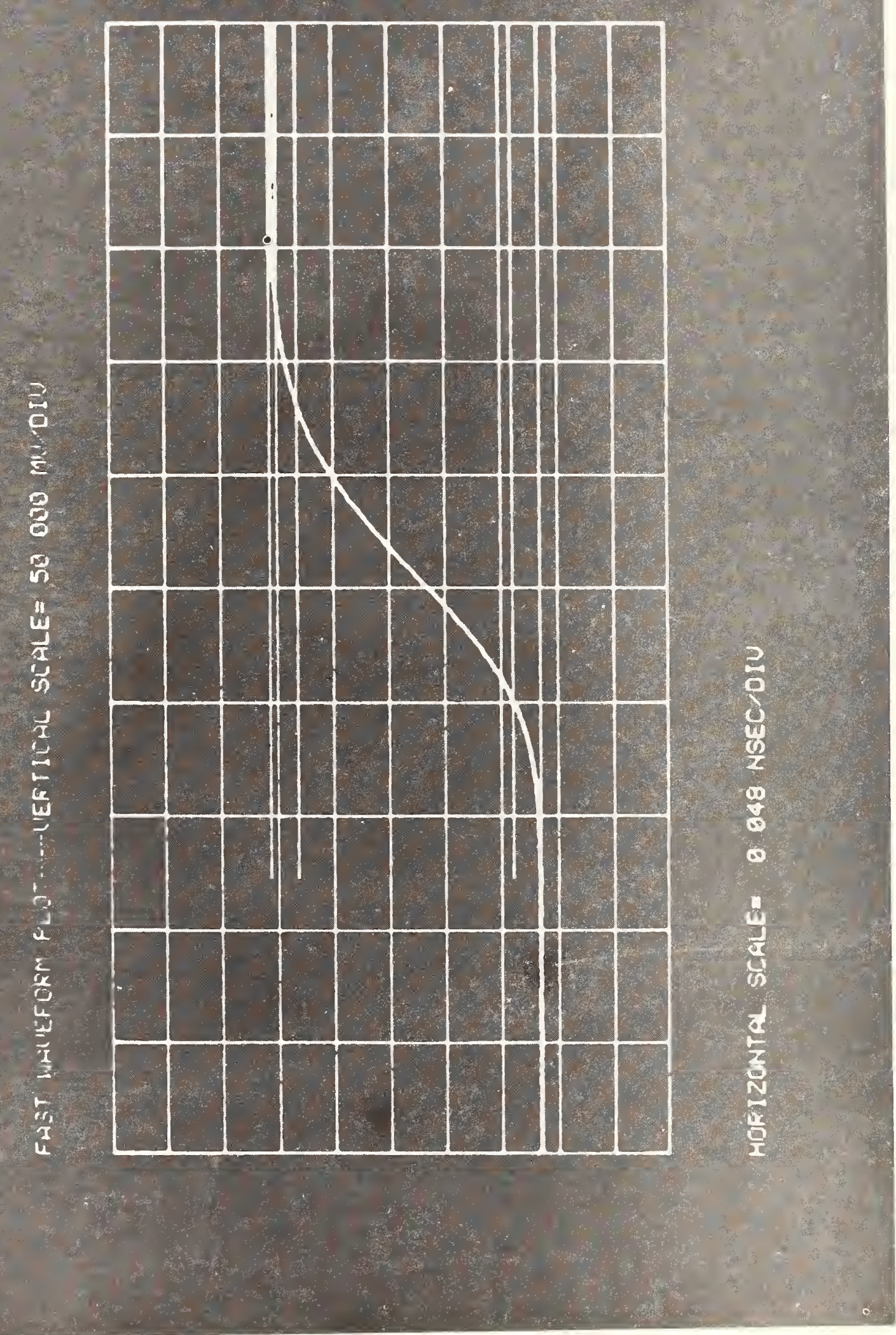

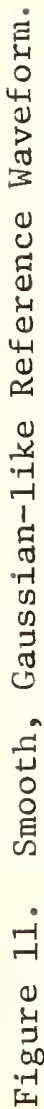




\section{REFERENCES}

[1] Gans, William L., "Present Capabilities of the NBS Automatic Pulse Measurement System," IEEE Trans. Instrum. Meas., IM-25, pp. 384-388, (Dec. 1976).

[2] Andrews, James R., "Automatic Network Measurements in the Time Domain,", Proc. IEEE, Vo1. 66, pp. 414-423, (April 1978).

[3] Young, M., and R. A. Lawton, "Measurement of Pulsed-Laser Power," NBS-TN-1010, (Feb. 1979).

[4] Nahman, Norris S., "Picosecond-Domain Waveform Measurements," Proc. IEEE, Vo1. 66, pp. 441-454, (April 1978).

[5] Andrews, J. R., and E. E. Baldwin, "Amplitude Calibrator for Oscilloscopes," To be published.

[6] Andrews, J. R., "Pulse Reference Waveform Standards Development at NBS," (Invited), Proc. ATE Seminar/Exhibit, Pasadena, CA, pp. IV13-19, (Jan. 1981).

[7] Nahman, N. S., and M. E. Guillaume, "Instrument Modeling and Deconvolution Improve Time Domain Calibrations," NBS Dimensions. . To be published.

[8] Nahman, N. S., and M. E. Guillaume, "Deconvolution of Duration Limited Waveforms," Digest of Papers, URSI/U.S. National Radio Science Meeting, Boulder, Co., (Jan. 1981). 


\section{Discussion Session 8}

Bob Hebner (NBS). I have a left-over comment and question about this morning's paper. The comment that I wish to make first was on Dr. Malewski's review of his paper in which he had studied the magnetic coupling effects over a thin plate as opposed to a wire. He came to the conclusion that a strap was a better way of grounding when you are trying to get good signal characteristics. I think that he owes it to $\mathrm{Mr}$. Poliner to at least acknowledge that that was exactly the point that Mr. Poliner had made earlier in his presentation. Then I wish to turn around and ask a question of Mr. Poliner. He mentioned in his presentation that the limiting accuracy that he had now was in the A-to-D converters. I assume that means the limiting accuracy you have in the low voltage part of measurement system. It seems to me that at least in the voltage channels the accuracy limitations would be imposed by the divider that you are using to get from high voltage to low voltage.

Randy Poliner (Westinghouse). I appreciate your pointing out the fact about copper strapping. I would like to ackowledge Bob Hebner's aid in describing that phenomena as a member of our Electric Power Research Institute (EPRI) review committee. As far as the absolute accuracy of the system, you are correct in assuming that the $A$ to $D$ accuracy mentioned is in the low voltage section. In the case of the voltage transducers, however, the primary transducer and low voltage section accuracies are about equat.

Neville Harris (Ion Physics). Just a comment with regard to the correction of cable losses. My experience with cables has been a bit sadder. I haven't so much worried about attenuation, but what has concerned me are reflections within the cables. Eccentricities are sometimes introduced in the manufacturing process and the impedance variation of cables along their length can be very significant, particularly in the very low gigahertz region. The impedance variation can be either random or periodic in nature and I have had many sad experiences with cables. I was wondering whether you tested each cable separately and didn't just correct the loss but also threw out bad cable?

Craig Trivelpiece (Maxwe11 Laboratories). We11, you know I tested a series of cables. I didn't run across any of those problems. Perhaps we had a slightly lower frequency range. Most of our pulses have risetimes of 10 to 50 nanoseconds so I guess that it just didn't show up.

Bill Boyer (Sandia). We do test all of our cable with a TDR and we don't use the bad ones. We have half-inch foam flex cables going to the screen room. We pulled bundles of thirty cables around four-foot 
radius bends in 6 -inch conduit. That would seem to give a high probability of damage. In all we pulled 270 cables, but only damaged a couple. I have a question for Bob Lawton. You showed a TV camera to monitor an oscilloscope and I was wondering if there is any limitations on writing rate for that system.

Bob Lawton (NBS). You have me at a loss as to what the exact writing rate is. We found one of the prime characteristics was to have it sensitive enough that it could see the writing rate of the oscilloscope and we chose an RCA Starlite TV camera for that application. It was adequate for the real time scopes that we had in our laboratory.

Terry McComb (NRC, Canada). A question for Bob Lawton as regards your system accuracy of 1 percent in amplitude measurements. However, you used a 14 bit converter. That seemed inconsistent to me. I wonder if you could explain why it was a 14 bit.

Lawton. Is the question that the 14 bit is not enough to give one part in one hundred or that it is too much? The uncertainty in the system is not determined by the A-to-D converter. There are many other factors which one must control, not the least of which is the fact that it is OK to make a dc measurement but not clear how one relates that to waveforms in the high frequency range. There is a related development we have made which we call a flat pulse generator to help us make an even more accurate determination of the voltage level of the oscilloscope at the time frames of interest and be able to relate those back to dc levels. So the A to D converter is not the limiting thing there, but we find that compared to the laboratories that have not used this high an accuracy converter, we have better control of noise problems.

Richard Malewski (IREQ). I think that the idea of using flat straps rather than round conductors is a generally known observation among the measuring people. Everybody knows that it is more efficient to use a flat strap rather than a round conductor. For the first time we have been trying to get some quantitative information because there is a real difference between knowing that it is better to use flat straps than round conductors and knowing what is the difference in terms of percentages. And it is a detail, but one of importance. I just wanted to say that we learned about how much better it is.

Hebner. We11, I want to thank you for your attendance, for your interest, and for your comments. I want to thank all of the people who helped make the conference a success. I would like to specifically acknowledge and thank those people who volunteered to be session chairmen and kept the meeting running almost on schedule for three days. I think that we have accomplished something during the course of these three days. I have heard discussions of transformers at breakfast and fiber-optic communication systems at cocktails, and I have heard as much intense measurement discussion in the past two and a half days as I could probably tolerate. I think that to most people 
the conference was a success and the final pay-off will be when we all go back to the institutions which sent us here and perform and insist upon if not better measurements, at least better characterized measurement. Thank you all for coming and I now close the session. 
NBS.114A (REV. 2-8C)

\begin{tabular}{|c|c|r|r}
\hline $\begin{array}{r}\text { U.S. DEPT. OF COMM. } \\
\text { BIBLIOGRAPHIC DATA } \\
\text { SHEET (See instructions) }\end{array}$ & $\begin{array}{c}\text { 1. PUBLICATION OR } \\
\text { REPORT NO. } \\
\text { NBS SP-628 }\end{array}$ & 2. Performing Organ. Report No. 3. Publication Date & June 1982 \\
\hline
\end{tabular}

4. TITLE AND SUBTITLE

Measurement of Electrical Quantities in Pulse Power Systems

Proceedings of the Workshop on Measurement of Electrical Quantities in Pulse Power

Systems held at the Mational Bureau of Standards, Boulder, Colorado, on March 2-4, 1981

5. $\operatorname{AUTHOR}(S)$

R. H: McKnight and R. E. Hebner, Jr., Editors

6. PERFORMING ORGANIZATION (If joint or other than NBS, see instructions)

7. Contract/Grant No.

MATIONAL BUREAU OF STANDARDS

DEPARTMENT OF COMMERCE

WASHINGTON, D.C. 20234

8. Type of Report \& Period Covered

FINAL

9. SPONSORING ORGANIZATION NAME AND COMPLETE ADDRESS (Street, City, stote, The Air Force Office of

Scientific Research; the Defense Advanced Research Project Agency, Directed Energy

Office; the Department of Energy, Office of Inertial Fusion; the Naval Surface Weapons

Center; the U.S. Army, Harry Diamond Laboratories; and the National Bureau of Standards,

Center for Electronics and Electrical Engineering.

10. SUPPLEMENTARY NOTES

\section{Library of Congress Catalog Card Number: 82-600535}

Document describes a computer program; SF-185, FIPS Software Summary, is attached.

11. ABSTRACT (A 200-word or less factual summary of most significant information. If document includes a significant bibliogrophy or literoture survey, mention it here)

The Workshop on Measurement of Electrical Quantities in Pulse Power Systems addressed measurements in pulse systems having the characteristics of pulse duration less than a millisecond, system voltages greater than 10 kilovolts, and system currents greater than 10 kiloamperes. The presented papers were divided into four categories: voltage measurements; current measurements; power and energy measurements, and data acquisition. Included are discussions of applications of conventional measurement techniques and state-of-the-art systems.

12. KEY WORDS (Six to twelve entries; alphabetical order; capitalize only proper names; and seporate key words by semicolons) current measurement; electrical measurements; electromagnetic pulse; fusion; nuclear effects simulation; particle beam technology; pulse power; transients; voltage measuremeits.

13. AVAILABILITY

X Unlimited

For Official Distribution. Do Not Release to NTIS

X] Order From Superintendent of Documents, U.S. Government Printing Office, Washington, D.C. 20402.

Order From National Technical Information Service (NTIS), Springfield, VA. 2216I

14. NO. OF

PRINTED PAGES

$\$ 20$

15. Price

$\$ 9.50$ 



\section{NBS TECHNICAL PUBLICATIONS}

\section{PERIODICALS}

JOURNAL OF RESEARCH-The Journal of Research of the National Bureau of Standards reports NBS research and development in those disciplines of the physical and engineering sciences in which the Bureau is active. These include physics, chemistry, engineering, mathematics, and computer sciences. Papers cover a broad range of subjects, with major emphasis on measurement methodology and the basic technology underlying standardization. Also included from time to time are survey articles on topics closely related to the Bureau's technical and scientific programs. As a special service to subscribers each issue contains complete citations to all recent Bureau publications in both NBS and nonNBS media. Issued six times a year. Annual subscription: domestic $\$ 18$; foreign $\$ 22.50$. Single copy, $\$ 4.25$ domestic; $\$ 5.35$ foreign.

\section{NONPERIODICALS}

Monographs-Major contributions to the technical literature on various subjects related to the Bureau's scientific and technical activities.

Handbooks-Recommended codes of engineering and industrial practice (including safety codes) developed in cooperation with interested industries, professional organizations, and regulatory bodies.

Special Publications-Include proceedings of conferences sponsored by NBS, NBS annual reports, and other special publications appropriate to this grouping such as wall charts, pocket cards, and bibliographies.

Applied Mathematics Series-Mathematical tables, manuals, and studies of special interest to physicists, engineers, chemists, biologists, mathematicians, computer programmers, and others engaged in scientific and technical work

National Standard Reference Data Series-Provides quantitative data on the physical and chemical properties of materials, compiled from the world's literature and critically evaluated. Developed under a worldwide program coordinated by NBS under the authority of the National Standard Data Act (Public Law 90-396).

NOTE: The principal publication outlet for the foregoing data is the Journal of Physical and Chemical Reference Data (JPCRD) published quarterly for NBS by the American Chemical Society (ACS) and the American Institute of Physics (AIP). Subscriptions, reprints, and supplements available from ACS, II55 Sixteenth St., NW, Washington, DC 20056.
Building Science Series-Disseminates technical information developed at the Bureau on building materials, components, systems, and whole structures. The series presents research results, test methods, and performance criteria related to the structural and environmental functions and the durability and safety characteristics of building elements and systems.

Technical Notes-Studies or reports which are complete in themselves but restrictive in their treatment of a subject. Analogous to monographs but not so comprehensive in scope or definitive in treatment of the subject area. Often serve as a vehicle for final reports of work performed at NBS under the sponsorship of other government agencies.

Voluntary Product Standards-Developed under procedures published by the Department of Commerce in Part 10. Title 15, of the Code of Federal Regulations. The standards establish nationally recognized requirements for products, and provide all concerned interests with a basis for common understanding of the characteristics of the products. NBS administers this program as a supplement to the activities of the private sector standardizing organizations.

Consumer Information Series-Practical information, based on NBS research and experience, covering areas of interest to the consumer. Easily understandable language and illustrations provide useful background knowledge for shopping in today's technological marketplace.

Order the above NBS publications from: Superintendent of Documents. Government Printing Office, Washington, DC 20402.

Order the following NBS publications-FIPS and NBSIR's-from the National Technical Information Services, Springfield, VA 22161

Federal Information Processing Standards Publications (FIPS PUB)-Publications in this series collectively constitute the Federal Information Processing Standards Register. The Register serves as the official source of information in the Federal Government regarding standards issued by NBS pursuant to the Federal Property and Administrative Services Act of 1949 as amended, Public Law 89-306 (79 Stat. 1127), and as implemented by Executive Order 11717 (38 FR 12315, dated May 11, 1973) and Part 6 of Title I5 CFR (Code of Federal Regulations).

NBS Interagency Reports (NBSIR) - A special series of interim or final reports on work performed by NBS for outside sponsors (both government and non-government). In general, initial distribution is handled by the sponsor: pubic distribution is by the National Technical Information Services, Springfield, VA 22161, in paper copy or microfiche form 
U.S. DEPARTMENT OF COMMERCE

National Bureau of Standards

Washington, 0.C. 20234

POSTAGE ANO FEES PAIO

OFFICIAL BUSINESS

U.S. OEPARTMENT OF COMMERCE

COM-215

Penalty for Private Use. $\$ \mathbf{\$ 3 0 0}$

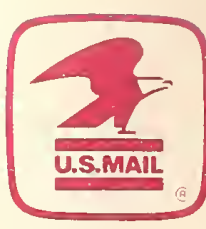

SPECIAL FOURTH-CLASS RATE BOOK 